\title{
BIOLOGY AND MEDICINE DIVISION ANNUAL REPORT 1986
}

LBL --22300

DE87 009280

\section{Lawrence Berkeley Laboratory University of California Berkeley, California 94720}

\author{
DISCLAIMER
}

\begin{abstract}
This report was prepared as an account of work sponsored by an agency of the United States Government. Neither the United States Government nor any agency thereof, nor any of their employees, makes any warranty, express or implied, or assumes any legal liabiltty or responsibility for the accuracy, completeness, or usefulness of any information, apparatus, product, or process disclosed, or represents that its use would not infringe privately owner rights. Reference herein to any specific commercial product, process, or service by trade name, trademark, manufacturer, or otherwise does not necessarily constitute or imply its endorsement, recommendation, or favoring by the United States Government or any agency thereof. The views and opinions of authors expressed herein do not necessarily state or reflect those of the United States Government or any agency thereof.
\end{abstract}

This work was supported by the Office of Health and Environmental Research of the United States Department of Energy under Contract DE-ACO3765F00098. Portions of this work were also supported by the National Institutes of Health, Department of Health and Human Services, the Armed Forces Radiobiology Research Institute, the National Aeronautics and Space Administration, the Nuclear Regulatory Commission, the Office of Naval Research, International Business Machines Corporation (IBM Instruments), the Electric Power Research Institute, Liposome Technologies Inc., and Monsanto. The contracts and grants with the above and subcontracts with the University of California San Francisco, the University of Southern California Medical Center, the University of Colorado, and Stanford University are listed in Appendix A. 


\title{
CONTENTS
}

\section{INTRODUCTION}

Paul $H$. Silverman

\section{SECTION 1. RESEARCH MEDICINE}

RESEARCH MEDICINE PROGRAM OBJECTIVES AND COMPOSITION

T.F. Budinger

\section{Hematology and Atherosclerosis}

\author{
RADIOLABELING OF METALLOPORPHYRINS FOR IMAGING VASCULAR \\ PLAQUES AND LESIONS \\ Yukio Yano, Mohindar Singh, Kathleen M. Brennan, \\ Kanu B. Dalal, Chester A. Mathis, Shirley N. Ebbe, \\ and Thomas F. Budinger
}

MORPHOLOGICAL AND KINETIC ABNORMALITIES OF PLATELETS IN

HYPERCHOLESTEROLEMIC RABBITS

Elisabeth Mazoyer, Shirley Ebbe, Kanu Dalal,

Robert Leven, Bernard Mazoyer, Kathleen Brennan,

Dorothy Carpenter, and Tamlyn Yee

THE EFFECTS OF HYPERCHOLESTEROLEMIA ON RABBIT PLATELETS:

FUNCTIONAL AND BIOCHEMICAL ALTERATIONS

Kanu Dalal, Elisabeth Mazoyer, Shirley Ebbe,

Dorothy Carpenter, and Tamlyn Yee

INCREASE IN MEGAKARYOCYTE SIZE WITHIN PLOIDY GROUPS

AND SHIFT IN PLOIDY IN THROMBOCYTOPENIC MICE

Shirley Ebbe, Dorothy Carpenter, and Tamlyn Yee

MEGAKARYOCYTE FRAGMENTATION AND PLATELET FORMATION

Robert M. Leven

Nuclear Medicine: Blood Flow and Metabolism

${ }^{123}$ |-LABELED RADIOPHARMACEUTICALS FOR IN VIVO

IMAGING OF LOW-DENSITY LIPOPROTEIN METABOLISM

Stephen M. Moerlein, Kanu B. Dalal, Shirley N. Ebbe,

Yukio Yano, Kathleen M. Brennan, Tamlyn Neben,

Julia A. Twitchell, Dorothy A. Carpenter, and Thomas F. Budinger

POSITRON EMISSION TOMOGRAPHY FOR DIAGNOSIS OF

RADIATION NECROSIS AND RECURRENT BRAIN TUMOR

Peter E. Valk and Thomas F. Budinger

BRAIN BLOOD FLOW IN ALZHEIMER'S DISEASE MEASURED

WITH IODINE-122 AND POSITRON EMISSION TOMOGRAPHY

Thornton Sargent III, Chester A. Mathis, William I. Jagust,

Natalia Kusubov, and Thomas F. Budinger 
REGIONAL CEREBRAL BLOOD FLOW AND METABOLISM IN STROKE

William J. Jagust, Chester A. Mathis, Thomas F. Budinger,

Peter E. Valk, and Philip R. Weinstein

SINGLE PHOTON EMISSION COMPUTED TOMOGRAPHY: A PRACTICAL

CLINICAL MODALITY FOR THE LABORATORY DIAGNOSIS OF DEMENTIA William J. lagust, Thomas F. Budinger, Bruce R. Reed,

J. Philip Seab, and Miguel M. Colina

USE OF GENERATOR-PRODUCED ${ }^{122} \mid$ FOR STUDIES OF HEART AND

BRAIN FUNCTION

Chester A. Mathis, Alexander T. Shulgin, Steven M. Moerlein,

Yukio Yano, Kathleen M. Brennan, Thornton Sargent III,

and Thomas F. Budinger

INITIAL RESULTS FROM THE DONNER 600-CRYSTAL

POSITRON TOMOGRAPH

Stephen E. Derenzo, Ronald H. Huesman, John L. Cahoon,

Allen B. Geyer, Donald C. Uber, Tony Vuletich,

and Thomas F. Budinger

Nuclear Magnetic Resonance Studies

FLUORODEOXYGLUCOSE EFFECTS ON GLUCOSE METABOLISM IN

NUCLEAR MAGNETIC RESONANCE (NMR) STUDIES

Kathleen $M$. Brennan, Thomas F. Budinger, Julia A. Twitchell,

and Kay S. Bristol

AN INSTRUMENT CONTROL AND DATA ANALYSIS PROGRAM

FOR IMAGING AND IN VIVO SPECTROSCOPY

Mark S. Roos, Richard A. Mushlin, Eugene Veklerov,

John D. Port, Carol Ladd, and Colin G. Harrison

EFFECTS OF SLICE SELECTION AND DIFFUSION ON $T_{2}$ MEASUREMENT

Sam T. Wong and Mark S. Roos

\section{SECTION 2. PHYSIOLOGY}

INTRODUCTION

HEMATOZOA AND ERYTHROPOIESIS: EFFECTS OF MALARIA INFECTION Paul H. Silverman and Lynn !. Mahlmann

STROMAL CELL CHANGES IN MICE INFECTED WITH MURINE MALARIAS

John C. Schooley, Karen L. Smith, and Birgitta Kullgren

INHIBITION BY INTERLEUKIN-1 OF THE ACTION OF ERYTHROPOIETIN

OF ERYTHROID PRECURSORS AND ITS POSSIBLE ROLE IN

THE PATHOGENESIS OF HYPOPLASTIC ANEMIAS

John C. Schooley, Birgitta Kullgren, and Anthony C. Allison

ONE TYPE OF PLURIPOTENTIAL. HEMOPOIETIC STEM CELLS PROVIDES

PERMANENT, ANOTHER TEMPORARY BONE MARROW REPOPULATION

George Brecher 
IMMUNOREACTIVE ERYTHROPOIETIN CONCENTRATIONS IN

NEONATAL RATS AND THE EFFECTS OF HYPOXIA

Gisela K. Clemons, Sherry L. Fitzsimmons, and Darlene DeManincor

FFFECTS OF OZONE AND HYPOXIA ON THYROID HORMONE RECEPTORS

David E. Erkenbrack and Gisela K. Clemons

BIOLOGICAL EFFECTS OF MAGNETIC FIELDS

Thomas S. Tenforde, Cornelius T. Gaffey, Robert P. Liburdy,

Chu-Chung Lo, Allen B. Geyer, and Lynette Levy

ELECTROMAGNETIC FIELDS AND THE INDUCTION OF PROTEIN SHEDDING

Robert P. Liburdy

INITIAL DISTRIBUTION OF NEPTUNIUM-237 IN A MONKEY

Patricia W. Durbin, Nylan Jeung, and Jerome |. Bucher

NEW SEQUESTERING AGENTS FOR THE ACTINIDES: REMOVAL OF

PLUTONIUM-238 FROM MICE BY ORALLY ADMINISTERED LIGANDS

Patricia W. Durbin, Nylan Jeung, Frederick L. Weitl,

David L. White, Petra Turowski, Kenneth N. Raymond,

and Charles T. Schmidt

\section{SECTION 3. RADIATION BIOPHYSICS}

INTRODUCTION

\section{Radiologicial Physics and Chemistry}

INFLUENCE OF DNA STRUCTURE IN CHEMICAL REACTIONS WITH

HYDROXYL RADICALS

Aloke Chatterjee, Patrice Koehl, John L. Magee,

and William R. Holley

LET DEPENDENCE OF CROSS SECTIONS FOR DNA STRAND

BREAKS BY DIRECT EFFECTS OF CHARGED PARTICLES

William Holley, Aloke Chatterjee, and John L. Magee

AN EXPERIMENT TO MEASURE THE MULTIPLE COULOMB SCATTERING

OF HEAVY CHARGED PARTICLES

Mervyn Wong, Walter Schimmerling, Bernhard A. Ludewigt,

Mark H. Phillips, Rochelle Frank, Stanley B. Curtis,

Cornelius A. Tobias, John T. Walton, Heinrich A. Sommer,

Donald A. Landis, Norman W. Madden, and Fred S. Goulding

COMPARISON OF DOSE LOCALIZATION PROPERTIES OF CARBON

AND PROTON BEAMS

Eleanor A. Blakely, William T. Chu, Bernhard A. Ludewigt,

Mark H. Phillips, and Cornelius A. Tobias 
Molecular and Cellular Radiobiology and Mutagenesis

CELL CYCLE DEPENDENT X RAY OER: ROLE OF ENDOGENOUS GLUTATHIONE

Eleanor A. Blakely, Ruth J. Roots, Polly $Y$. Chang,

Leora Lommel, Laurie M. Craise, Edwin H. Goodwin,

and Elaine Yee

LESIONS IN CHO CELL CHROMATIN PRODUCED BY VERY

HIGH LET HEAVY IONS

Edwin H. Goodwin, Eleanor A. Blakely, and Cornelius A. Tobias

DNA LESIONS IN CELL NUCLEI FOLLOW NEYMAN A DISTRIBUTION

Cornelius A. Tobias, Edwin H. Goodwin, and Eleanor A. Blakely

RADIOSENSITIZATION PRODUCED BY IODODEOXYURIDINE IN HEAVY ION BEAMS David E. Linstadt, Eleanor A. Blakely, Theodore L. Phillips, Joseph R. Castro, Polly Y. Chang, and Leora Lommel

THE IMPORTANCE OF TRIGGERING DOSE AND CONDITIONS OF SPLIT

DOSE INCUBATION TO THE DEVELOPMENT OF THERMOTOLERANCE

Polly Y. Chang and Eleanor A. Blakely

DOSE PROTRACTION STUDIES WITH LOW AND HIGH LET

RADIATIONS ON NEOPLASTIC CELL TRANSFORMATION IN VITRO

Tracy Chui-hsu Yang, Laurie M. Craise, Man-tong Mei,

and Cornelius $\mathrm{A}$. Tobias

INDUCTION OF THYMIDINE-KINASE GENE MUTATION IN MAMMALIAN

CELLS BY LOW- AND HIGH-LET RADIATION.

Tracy Chui-hsu Yang, Carl F. Perez, Man-tong Mei,

Laurie M. Craise, and Cornelius A. Tobias

EFFECTS OF HIGH ENERGY URANIUM PARTICLES ON SOMATIC

CELL MUTATION AND DNA TRANSFECTION

Tracy C. Yang, Lausie M. Craise, Gerhard Kraft,

and Cornelius $\mathrm{A}$. Tobias

\section{Tumor and Tissue Effects of Heavy Particles}

THE SANDWICH SYSTEM AS A TUMOR ANALOG

Lynn Hlatky and Edward L. Alpen

TUMOR RADIOBIOLOGY STUDIES WITH HEAVY CHARGED PARTICLE BEAMS S.M. Javed Afzal, Thomas S. Tenforde, Kris Kavanau, and Stanley B. Curtis

COMPARISON OF DAMAGE AND RECOVERY IN QUIESCENT RAT LENS CELLS

FOLLOWING IRRADIATION WITH HIZE PARTICLES AND X RAYS

Edgar F. Riley, Alice L. Lindgren, and E. John Ainsworth

RESPONSE OF MOUSE MARROW STEM CELLS (CFU-S) TO HEAVY

CHARGED PARTICLES

E. John Ainsworth, John C. Prioleau, and Lynn J. Mahlmann 
THE RELATIVE BIOLOGICAL EFFECTIVENESS OF $670 \mathrm{MeV} / \mathrm{A}$ NEON AS A

FUNCTION OF DEPTH IN WATER FOR A TISSUE MODEL

Walter Schimmerling, Edward L. Alpen, Patricia Powers-Risius,

Meryyn Wong, and Marwin Rapkin

NEON IRRADIATION OF THE RAT SPINAL CORD

Adrian Rodriguez, Edward L. Alpen, and Randy J. DeGuzman

INDUCTION OF HARDERIAN GLAND TUMORS IN MICE BY

HEAVY ION IRRADIATION

Edward L. Alpen, Patti Powers-Risius, R.J. Michael Fry,

John Ainsworth, Randy J. De Guzman, Virginia Havens,

and Kris Kavanau

LIFE SHORTENING EFFECTS OF HEAVY CHARGED PARTICLES AND PHOTONS

E. John Ainsworth, John C. Prioleau, and Lynn J. Mahlmann

\section{Heavy Ion Therapy Radiosurgery and Therapy Physics}

\section{BEAM DELIVERY SYSTEMS FOR HEAVY CHARGED PARTICLE THERAPY}

AT THE BEVALAC

Edward L. Alpen, William T. Chu, Bernhard A. Ludewigt,

Maurice B. McEvoy, Mark A. Nyman, Timothy Renner,

and Rajinder $P$. Singh

RADIATION THERAPY TOLERANCE DOSES FOR TREATMENT PLANNING

John T. Lyman

\section{RADIOTHERAPY PHYSICS}

J. Michael Collier, Sheri D. Hender son, Samuel Pitluck, Paula Petti, Marc Kessler, and Kari Baken

\section{HEAVY CHARGED PARTICLE RADIOTHERAPY}

Joseph R. Castro, Theodore L. Phillips, David Linstadt,

Anthony Berson, Kay H. Woodruff, Grant E. Gauger,

Philip H. Gutin, J. Michael Collier,Sheri D. Henderson,

Paula L. Petti, Timothy R. Renner, Kari Baken,

Robert E. Walton, Niedra Dodson, Charles R. Pascale,

Elizabeth C. Williamson, Marc Kessler, Monica Reimers,

lames Judnick, and lacquelyn I. Iler

\section{STEREOTACTIC HEAVY CHARGED PARTICLE BRAGG PEAK RADIOSURGERY}

Jacob I. Fabrikant, Kenneth A. Frankel, Mark H. Phillips,

John T. Lyman, Edward L. Alpen, Neela B. Manley,

Richard P. Levy, Eng Lo, Myrtle L. Foster,

George T. Hampton, Frederick W. Yeater, Leal Kantstein,

Dresden Van Deusen, Barbara Modlinski (in collaboration with

Gerald D. Silverberg, John W. Hanbery, Charles B. Wilson,

Yoshio Hosobuchi, William H. Marshall, Dieter Enzmann,

Thomas H. Newton, and David Norman

THE FUTURE FOR RESEARCH WITH ACCELERATED NUCLEI: BUG AND LIBRA

The LIBRA Study Group: Cornelius A. Tobias, Richard A. Gough,

David A. Shirley, $\dagger$ Gerd M. Rosenblatt, $\uparrow$ Paul H. Silverman,

Klaus H. Berkner, Thomas F. Budinger, Joseph R. Castro,

and Jacob I. Fabrikant 


\section{Lipoproteins}

ANALYTIC ULTRACENTRIFUGATION OF LIPOPROTEINS:

SOME CURRENT COLLABORATIONS

Frank T. Lindgren

LARGE HIGH DENSITY LIPOPROTEINS: THEIR APOLIPOPROTEIN

SPECIFICITY AND ORIGINS

Elaine L. Gong, Alex V. Nichols, Trudy M. Forte,

and Patricia J. Blanche

LIPOPROTEIN COMPLEX FORMATION

Thomas A. Musliner, and Ronaid $M$. Krauss

LOW DENSITY LIPOPROTEIN SUBCLASSES AND RISK OF

MYOCARDIAL INFARCTION

Melissa A. Austin and Ronald M. Krauss

A NEW ANIMAL MODEL FOR GENETIC HYPERCHOLESTEROLEMIA

Wendy L. Fitch, Ronald M. Krauss, William J. Checovich,

and Alan D. Attie

SYNTHESIS OF LECITHIN: CHOLESTEROL ACYLTRANSFERASE BY

THE HUMAN LIVER CELL LINE

Trudy M. Forte, Richard T. Thrift, Barbara E. Cahoon,

Ching-Hong Chen, and John I. Albers

CHARACTERIZATION OF HIGH DENSITY LIPOPROTEIN SUBCLASSES

ISOLATED FROM THE HUMAN HEPATOMA DERIVED CELL LINE, HEP G2

Mark R. MCCall, Trudy M. Forte, and Virgie G. Shore

CACO-2 CELLS: A HUMAN INTESTINAL CELL LINE WITH

THE CAPACITY FOR SECRETING LIPOPROTEINS

Trudy M. Forte, Thomas E. Hughes, W. Vodek Sasok,

Jose M. Ordovas, Stefania Lamon-Fava and Ernst J. Schaefer

\section{Structural Biology}

IMPROVEMENT IN HIGH RESOLUTION IMAGE QUALITY OF RADIATION

SENSITIVE SPECIMENS ACHIEVED WITH REDUCED SPOT SIZE OF

THE ELECTRON BEAM

Kenneth $H$. Downing and Robert $M$. Glaeser

IMPROVED SUPPORT FILMS FOR ELECTRON MICROSCOPY OF BEAM

SENSITIVE SPECIMENS

Joseph R. Taylor and Robert M. Glaeser

THE MOLECULAR DESIGN OF MEMBRANE CHANNELS

Bing K. Jap 
LOW TEMPERATURE SCANNING ELECTRON MICROSCOPY OF

FROZEN HYDRATED LUNG

lacob Bastacky, Gregory R. Hook, Gregory L. Finch,

Jon Goerke, John Clements, John Gilbert,

Rob Conhaim, Charles Lee, Boris Rubinsky,

Josephine Barr, and Thomas L. Hayes

HIGH VOLTAGE ELECTRON MICROSCOPY OF UNSECTIONED HUMAN LUNG

ALVEOLAR WALLS

Jacob Bastacky, Thomas L. Hayes, David W. Ackland,

Saundra Parra, and Timothy Takaro

CIRCULAR DIFFERENTIAL SCATTERING AND CIRCULAR DIFFERENTIAL

ABSORPTION OF DNA PROTEIN CONDENSATES AND OF DYES

BOUND TO DNA PROTEIN CONDENSATES

Cynthia L. Phillips, William Mickols, Marcos F. Maestre,

and Ignacio Tinoco, Jr.

\section{SECTION 5. CELLULAR AND MOLECULAR BIOLOGY}

INTRODUCTION

\section{DNA Replication and Recombination}

THE CROI GENE OF SACCHAROMYCES CEREVISIAE CONTROLS MITOTIC

CROSSING OVER, CHROMOSOMAL STABILITY AND SPORULATION

Michael S. Esposito, Dimitrios T. Maleas, Kathleen A. Bjornstad,

and Libby L. Holbrook

PARTIAL PURIFICATION OF HOLLIDAY IUNCTION RESOLVASE ACTIVITY

FROM SACCHAROMYCES CEREVISIAE

Libby Litzenberger Holbrook, Kathleen A. Bjornstad, Dimitrios T. Maleas,

and Michael S. Esposito

SINGLE STRAND SPECIFIC DNA BINDING PROTEINS OF

SACCHAROMYCES CEREVISIAE

Junko Hosoda, Maren Bell, Kathleen A. Bjornstad,

Midori M. Hosobuchi, Herbert W. Moise, and Michael S. Esposito

\section{DNA Repair}

GENETIC AND PHYSICAL STUDIES OF REPAIR AND

RECOMBINATION IN YEAST

John C. Game, Robert K. Mortimer, David Schild,

Vincent Cook, and Shirley Sutton

MOLECULAR ANALYSES OF YEAST RECOMBINATION-REPAIR GENES

Robert K. Mortimer, David Schild, Mari Aker,

Gary Cole, John Game, Susan Lovett,

and Karen Sitney

INDUCED EXCISION REPAIR IS REQUIRED FOR REPAIR OF

LESIONS IN THE VICINITY OF DNA REPLICATION FORKS

Priscilla K. Cooper, Sherry Gee, and Vincent Ling 
REPAIR OF DNA DAMAGE IN THE HUMAN METALLOTHIONEIN

GENE FAMILY

Steven $A$. Leadon and Margaret $M$. Snowden

234

\section{Carcinogenesis}

ALKYLATION OF POLYNUCLEOTIDES IN VITRO AND IN VIVO

Bea A. Singer, Sylvia J. Spengler, Frank Chavez,

Heinz Fraenkel-Conrat, and Jarek T. Kusmierek

EFFECTS OF ALKYLATING CARCINOGENS ON HUMAN TUMOR CELLS

IN CULTURE

Regine Goth-Goldstein and Mildred Hughes

\section{CARCINOGENIC POTENCY}

Lois Swirsky Gold, Bruce N. Ames, Renae I. Magaw,

Georganne M. Backman, Thomas H. Slone, Joan Schwalbe,

Maria Da Costa, Susan Eisenberg, Paul Chous,

and Veronica Cabras

TISSUE TROPISM AND TEMPORAL EXPRESSION OF ROUS SARCOMA

VIRUS IN EFMBRYONIC AVIAN LIMB IN VIVO AND AFTER

TRANSFER TO CELL CULTURE

Anthony R. Howlett, Betsey L. Cullen, Mark D. Hertle,

and Mina J. Bissell

245

QUANTITAT: IMMUNOCYTOCHEMICAL ASSAY FOR AVIAN RETROVIRUSES

Andrew W. Stoker and Mina J. Bissell

DEVELOPMENTAL REGULATION OF TYROSINE PHOSPHORYLATION

SUBSTRATES CALPACTIN 1 AND VINCULIN IN EMBEYYONIC

AVIAN LIMB AND EXPRESSION IN CULTURED LIMB CELLS

V. Celeste Carter, Anthony R. Howlett, G. Steven Martin,

and Mina J. Bissell

HUMAN MAMMARY EPITHELIAL CELLI. CULTURE

Martha Stampfer and lack Bartley

EXPRESSION OF KNOWN ONCOGENES IN HIJMAN MAMMARY EPITHELIAL CELLS Martha Stampfer, Ming Li, Kari Koszdin,

and Mina Bissell

PRODUCTION OF OXIDATIVE BASE DAMAGE BY BENZO(a)PYRENE METABOLISM AND IONIZING RADIATION IN HUMAN MAMMARY EPITHELIAL CELLS

Steven A. Leadon, Martha Stampfer, Tracy Yang,

and lack Bartley

\section{Differentiation}

EXPRESSION OF DIFFERENTIATED FUNCTION IN HUMAN MAMMARY EPITHELIAL

CELLS IN CULTURE Jack Bartley, Gordon Parry, Gerri Levine, and Martha Stampfer 
SPECIALIZED MEMBRANE BIOGENESIS IN MAMMIARY EPITHELIAL CELLS

Gordon Parry, Betsey L. Cullen, and Lenny Moss

INFLUENCE OF EXTRACELLULAR MATRIX AND ITS COMPONENTS ON

CASEIN GENE EXPRESSION IN MOUSE MAMMARY EPITHELIAL CELLS

Ming Liang Li, Judith Aggeler, Deborah A. Farson,

Carroll Hatier, and Mina J. Bissell

HIGH SERUM LEVELS INTERFERE WITH THE NORMAL DIFFERENTIATED

STATE OF AVIAN TENDON CELLS BY ALTERING TRANSLATIONAL

REGULATION

Janice Valmassoi and Richard 1. Schwarz

\section{APPENDICES}

Appendix A. List of Contacts and Grants Supporting

Portions of Work Presented in This Annual Report ............................. 267

Appendix B. 1986 Publications

Appendix C. Biology and Medicine Division Staff 


\section{INTRODUCTION}

During 1986 a number of eyents occurred that are destined to have a profound influence on the future or the Biology and Medicine Division. Director David Shirley appointed a Panel to Explore New Directions (PEND) for the entire Lawrence Berkeley Laboratory. A subcommittee of PEND chaired by Martha Stampfer has proposed important new initiatives for the life sciences. During 1987 the feasibility of and means for implementing these new directions will be determined.

in early November an intensive review of the Division's programs, organization, and management was carried out by an external committee of distinguished scientists invited for this purpose by Director Shirley. The results of that review are expected to be available in early 1987 and will contribute to future changes.

Other important events included a series of workshops concerned with genetic engineering. cellular and molecular biology, and biotechnology. The workshops, which included LBL life scientists from several divisions, industry, and UC campuses, are described by Jack Bartley in his introduction to the Cellular and Molecular Biology Group report.

During early December an international meeting on biomedical accelerators, jointly sponsored with the Accelerator and Fusion Research Division, brought together colleagues from the U.S.A., Japan, England, France, Germany, and Canada to share experiences and plans for future new machines. LBL participants described a proposal by a potential consortium that includes UCSF and Merritt Peralta Medical Center for a Light Ion Biomedical Research Accelerator (LIBRA), which is described by Cornelius Tobias in this annual report.

In Algust, a change in leadership of the Division took place with the appointment of Paul Silverman as Acting Associate Director of LBL, Acting Head of the Biology and Medicine Division, and Acting Director of the Donner Laboratory. In Eddition to the appointment of Paul Silverman, Igor Blake was appointed Division Administrator, replacing Baird Whaley, who retired August 31, 1986.

The Biology and Medicine Division continues to make important contributions in scientific areas in which it has a long-established leadership role. For 50 years the Division has pioneered in the application of radioisotopes and charged particles to biology and medicine. The quality of its science is evidenced by the outstanding support provided by the National Institutes of Health award of grants through the intensively competitive peer review process. Of the approximately $\$ 20$ million budget in FY-1986, 65\% was derived from NIH and $31 \%$ from DOE.

As described in this annual report, there is a growing emphasis on cellular and molecular applications in the work of all the Division's research groups. The powerful tools of genetic engineering, the use of recombinant products, the analytical application of DNA probes, and the use of restriction fragment length polymorphic DNA are described and proposed for increasing use in the future. It is clear that with these new tools, the life sciences are on the verge of a revolution that may provide both an understanding of essential biological processes and the means for controlling them. The prospects for understanding development and senescence, disease and aging, the effects of environment and pollution, and how we can prevent and reverse adverse processes are on the horizon. Unraveling the factors that control the expression of genes and finding how specific products are produced offer the hope of generating important new therapeutic agents and nutrients through biotechnology.

The role that the Biology and Medicine Division will play in the realization of these exciting prospects depends on how well we marshall our resources and focus our future efforts. During 1987 we will continue the activities begun in 1986, which are aimed at enhancing our effectiveness through increased programmatic cooperation and collaboration. To resolve the scientific problems that we are confronting requires a multi-disciplinary and multi-investigator approach. Just as the science of physics, originally based on the work of individual investigators, now requires teams working cooperatively to achieve results that would have been impossible by members working independently, so too, the important advances in life sciences are increasingly the result of team efforts. With the current strengths of the Division and the exciting new directions being identified, we look forward to a promising future. 


\section{SECTION 1. RESEARCH MEDICINE}

\section{RESEARCH MEDICINE PROGRAM OBJECTIVES AND COMPOSITION}

\section{T.F. Budinger}

The major emphasis of the Research Medicine Group is the application of new instruments and procedures for the evaluation of the physiological state of normal and pathological processes, with particular emphasis on atherosclerosis and mental disorders. The Research Medicine Group of the Donner Laboratory evolved from the early work by Dr. Lawrence on the use of phosphorous-32 for the treatment of polycythemia vera, the development of the gamma camera and other nuclear detection instruments, and the use of proton and helium-ion beams for the treatment of pituitary diseases. The instrumentation for nonirvasive studies includes high resolution positron einission tomography, practical single-photon-emission tomography, and very high field NMR in vivo spectroscopy systems. The group consists of approximately 40 scientists, technicians, and students. A natural division of effort is shown below (Fig. 1). The four categories have approximately equal emphasis and priority.

\section{RECENT ACCOMPLISHMENTS}

1. Establishment of a new method for brain blood-flow measurements in Alzheimer's disease and stroke.

2. Development of a method for labeling human blood platelets so that early signs of stroke can be detected.

3. Development of a method of evaluation of coronary artery disease using positron tomography and a rubidium-82 generator device developed at $\mathrm{LBL}$.

4. Development of an instrument control and data analysis program for NMR imaging and spectroscopy, in collaboration with IBM and the Bitter National Magnet Laboratory at MIT.

5. Discovery of a remarkably abnormal behavior of platelets in hypercholesterolemic rabbits.

b. Discovery of a method for distinguishing radiation for necrosis from tumor recurrence or distinguishing Pick's disease from Alzheimer's disease using positron tomography with ${ }^{8 !} \mathrm{Rb}$ and ${ }^{18} \mathrm{FDG}$.

\begin{tabular}{|c|c|c|c|}
\hline $\begin{array}{c}\text { PHYSICS } \\
\text { Detector } \\
\text { Instrumentation }\end{array}$ & $\begin{array}{c}\text { CHEMISTRY } \\
\text { Radiopharmaceutical } \\
\text { Tracers }\end{array}$ & $\begin{array}{l}\text { MATHEMATICS } \\
\text { Computer Based } \\
\text { Algorithms }\end{array}$ & $\begin{array}{l}\text { BHOLOGY/MEDICINE } \\
\text { Basic Clinical } \\
\text { Science }\end{array}$ \\
\hline $\begin{array}{l}\text { Positron Emission } \\
\text { Tomograph }\end{array}$ & $\begin{array}{l}\text { - Brain \& Heart } \\
\text { Flow \& Metabolism }\end{array}$ & $\begin{array}{l}\text { Reconstruction } \\
\text { Methods }\end{array}$ & - Blood Clotting \\
\hline $\begin{array}{l}\text { Nuclear Magnetic } \\
\text { Resonance }\end{array}$ & - Neuroreceptors & $\begin{array}{l}\text { Statistical } \\
\text { Analysis }\end{array}$ & \begin{tabular}{|l} 
Atherosclerosis \\
- Coronary \\
Artery \\
Disease \\
- Platelets
\end{tabular} \\
\hline $\begin{array}{l}\text { - Single Photon } \\
\text { Tomograph }\end{array}$ & - New Generators & - Kineli us Modeling & $\begin{array}{l}\text { Alzheimer's } \\
\text { - Diagnosis } \\
\text { - Cause(s) }\end{array}$ \\
\hline Squid Detector & L NMR Probes & $\begin{array}{l}\text { NMR Imaging } \\
\text { Systems }\end{array}$ & $\begin{array}{l}\text { - Schizophrenia } \\
\text { - Metabolism } \\
\text { Brain Tumor }\end{array}$ \\
\hline
\end{tabular}

Fig. 1. 
7. Development of a new mathematical method for assessing all the optimum protocols for data collection in noninvasive tracer studies.

8. Discovery of a difference in kinetics of low density lipoprotein turnover in the liver and blood pool in rabbits on a hypercholesterol vs. normal diet.

9. Development of the ${ }^{122} \mid$ positron emitter (3.6-min half-life) generator and associated radionuclide-labeled blood pool '(albumin), heart blood flow, and brain perfusion (HIPDM) agents for cyclotron-free use of positron tomography.
10. Verification of the abnormal pattern of methionine metabolism in schizophrenia.

11. Development of a method for analysis of the in vivo metabclism of radiolabeled ligands using a manifold of HPLC methods with an integrated spectrometer for data analysis.

12. Completion of the highest resolution PET instrument ( $2.3 \mathrm{~mm}$ center), with subsecond complete data acquisition times.

13. Completion of the first epidemiological survey of long term magnet-field effects, which showed no health effects in workers exposed to magnetic fields for years.

\title{
Hematology and Atherosclerosis
}

\section{RADIOLABELING OF METALLOPORPHYRINS FOR IMAGING VASCULAR PLAQUES AND LESIONS}

\author{
Yukio Yano, Mohindar Singh, Kathleen M. Brennan, Kanu B. Dalal, \\ Chester A. Mathis, Shirley N. Ebbe, and Thomas F. Budinger
}

Porphyrins and metalloporphyrins have been shown to accumulate in atheromatous plaques and lesions. The accumulation of hematoporphyrin derivative (HPD) in atheromatous plaques in the aorta of hyperlipidemic rabbits and monkeys was detected by fluorescence, while no fluorescence was seen in aortic segments free of plaque.' Similar results were obtained when sections of human aorta containing atheromatous plaques were incubated with HPD. ${ }^{2}$

Paramagnetic manganese as the tetrakis(psulfophenyl) porphyrin (MnTPPS ${ }_{4}$ ) was used to enhance proton relaxation in nuclear magnetic resonance (NMR) studies that showed selective uptake of $\mathrm{MnTPPS}_{4}$ in atheromatous plaques. ${ }^{3}$

The sensitivity of radiolabeled porphyrin and positron emission tomograpy (PET) would be much greater for localizing vascular lesions than NMR studies. The development of a high resolution PET system with a resolution of about $2 \mathrm{~mm}$ has greatly increased the potential for detecting atheromata or vascular lesion. The purpose of the present study was to !abel various porphyrin compounds with radionuclides and to determine their uptake in nor$\mathrm{mal}$ and balloon-catheter de-endothelialized rabbit aortas or in hyperlipidemic-rabbit aortas. Another means of imaging vascular lesions is by single photon emission computed tomography (SPECT) for application to more widely uselul nuclear medicine studies.

The choice of the most useful radionuclide for these studies is dependent upon availability, decay mode, useful photon energy, and half-life of the radionuclide as related to the biological clearance and uptake of the radioporphyrin and the resultant radiation dose to the patient. Some potentially useful radionuclides and their physical decay properties are shown in Table 1 . In this study we used ${ }^{68} \mathrm{Ga}$, ${ }^{67} \mathrm{Ga}$, and ${ }^{57} \mathrm{Co}$. These radionuclides were labeled to the porphyrin compounds shown in Fig. 1.

In general the method of radiolabeling porphyrins was as follows: The $\mathrm{Ga}$ radionuclides were purified by ether extraction from $6 \mathrm{~N} \mathrm{HCl}$ and reconstituted in acetic acid that was added to 1 $\mathrm{mg} / \mathrm{ml}$ of the porphyrin reagent in various solvent such as water, acetic acid, or acetate buffer. The $\mathrm{pH}$ was adjusted to 2.5-7.0 and the reaction mixture was heated by refluxing the solvent at elevated temperature or by autoclaving the sealed reaction vial. Analysis of the radioporphyrin and the radiolabeling yield were determined by HPLC and ITLC methods. The results are summarized in Table 2, 
Table 1. Radionuclides for labeling porphyrins.

\begin{tabular}{ccccc}
\hline Nuclide & Half life & Decay mode \% & Gamma (MeV) & Production \\
\hline${ }^{45} \mathrm{Ti}$ & $3.08 \mathrm{~h}$ & $\beta^{+}(85), \mathrm{EC}(15)$ & $1.2-1.7$ & ${ }^{45} \mathrm{Sc}(\mathrm{p}, \mathrm{n})$ \\
${ }^{55} \mathrm{Co}$ & $17.5 \mathrm{~h}$ & $\beta^{+}(81), \mathrm{EC}(19)$ & 0.932 & ${ }^{56} \mathrm{Fe}(\mathrm{p}, 2 \mathrm{n})$ \\
${ }^{57} \mathrm{Co}$ & $270 \mathrm{~d}$ & $\mathrm{EC}(100)$ & $0.122,0.136$ & Commercial \\
${ }^{66} \mathrm{Ga}$ & $9.4 \mathrm{~h}$ & $\beta^{*}(55), \mathrm{EC}(45)$ & 1.04 & ${ }^{65} \mathrm{Cu}(\alpha, 3 \mathrm{n})$ \\
${ }^{67} \mathrm{Ga}$ & $78.3 \mathrm{~h}$ & $\mathrm{EC}(100)$ & $0.093,0.184,0.396$ & Commercial \\
${ }^{68} \mathrm{Ga}$ & $68.2 \mathrm{~m}$ & $\beta^{+}(90), \mathrm{EC}(10)$ & 1.07 & ${ }^{60} \mathrm{Ge}$ generator \\
${ }^{99 m} \mathrm{Tc}$ & $6.0 \mathrm{~h}$ & $\mathrm{IT}(100)$ & 0.140 & ${ }^{99}$ Mo generator \\
${ }^{111} \mathrm{In}$ & $2.83 \mathrm{~d}$ & $\mathrm{EC}(100)$ & $0.171,0.245$ & Commercial \\
\hline \hline
\end{tabular}

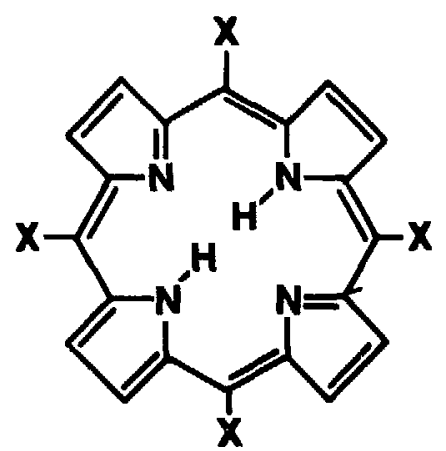

(1) $\mathrm{X}=\mathrm{O}-\mathrm{OCH}_{3}$ (TKMPP)

(2) $x=0,(T P P)$

(3) $x=$ EN, (TP,P)

(4) $X=\mathrm{GN}_{\mathrm{N}}-\mathrm{CH}_{3},\left(\mathrm{TMP}_{y} \mathrm{P}\right)$

(5) $x=(0)-\mathrm{SO}_{3}{ }^{-}$, (TPPS $\left._{4}\right)$

(6) $\mathrm{X}=\left(\mathrm{O}-\mathrm{N}-\left(\mathrm{CH}_{3}\right)_{3}(\right.$ TTAP)

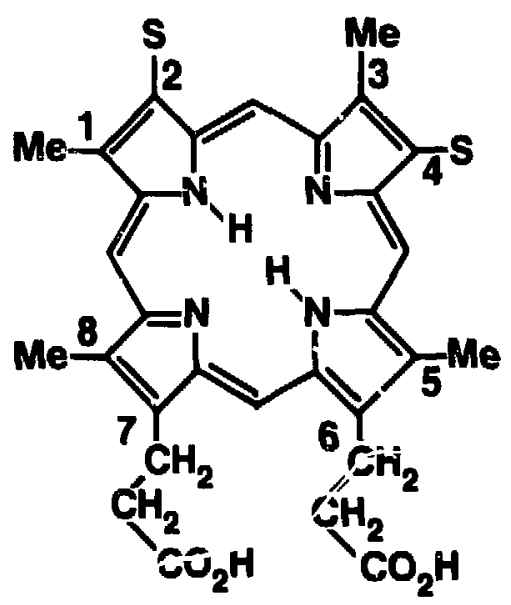

(7) $S=-H$, (Deut $-I X)$

(8) $\mathrm{S}=-\mathrm{CH}=\mathrm{CH}_{2}$, (Proto-IX)

(9) $\mathrm{S}=-\mathrm{SO}_{3}-,\left(\mathrm{DPS}_{2}\right)$

(10) $\mathrm{S}=-\mathrm{CH}(\mathrm{OH}) \mathrm{CH}_{3}$ (Hemato-IX)

Fis. 1. Porphyrin compounds used in these studies are shown with their chemical structure. The 10 porphyrin compounds studied were:
1. tetrakis-(p-methoxyphenl)porphyrin (TKMPP)
2. tetraphenylporphyrin(TPP)
6. tetra(N,N,N-trimethylanilinium)porphyrin (TTAP)
3. tetra(4-pyridyl)porphyrin (TPyP)
7. deuteroporphyrin-IX (Deut-IX);
8. protoporphyrin-IX di-Na (Proto- $(X)$
4. tetra(N-methylpyridyl)porphyrin (TMPYP)
5. tetra(4-sulfonatophenyl)porphyrin (TPPS,
9. deuteroporphyrin disulfonate $\left(D P S_{2}\right)$
10. hematoporphyrin-IX (Hemato-IX).

Porphyrins 4, 5, 6, and 9 were purchased from Midcentury Chemical Co. The other porphyrin compounds were purchased from Aldrich Chemical $\mathrm{Co}$. 
Table 2. Radiolabeling porphyrins.

\begin{tabular}{|c|c|c|c|c|c|c|}
\hline Porphyrin (mg) & Solvent & $\mathrm{pH}$ & isotope & Conditions & Analysis & Purity (\%) \\
\hline TKMPP $(0.25)$ & HAC & 5.0 & ${ }^{68} \mathrm{Ga} /{ }^{57} \mathrm{Co}$ & $121^{\circ} / 20^{\prime}$ & ITLC & $98 / 96$ \\
\hline $\operatorname{TPP}(3.0)$ & HAC & 2.5 & ${ }^{60} \mathrm{Ca}$ & $121^{\circ} / 60^{\prime}$ & ITLC & 95 \\
\hline TPyP (3.0) & HAC & 2.5 & ${ }^{60} \mathrm{Ga}$ & Reflux $60^{\prime}$ & ITLC & 80 \\
\hline TMPyP (1.0) & $\mathrm{HAC} / \mathrm{AC}$ & 4.5 & ${ }^{67} \mathrm{Ga}$ & $121^{\circ} / 20^{\prime}$ & HPLC/Ext & 99 \\
\hline TPPS $_{4}(1.0)$ & $\mathrm{H}_{2} \mathrm{O}$ & $3.5-7.0$ & ${ }^{67} \mathrm{Ga} /{ }^{68} \mathrm{Ga}$ & $121^{\circ} / 30^{\prime}$ & Exi & 97 \\
\hline TTAP (2.0) & AC & 4.5 & ${ }^{67} \mathrm{Ga} /{ }^{68} \mathrm{Ga}$ & $121^{\circ} / 30^{\prime}$ & Ext & 99 \\
\hline Deut-IX $(0.75)$ & HAC & 2.5 & ${ }^{67} \mathrm{Ga}$ & $121^{\circ} / 30^{\prime}$ & ITLC & 99 \\
\hline Proto-IX (3.0) & $\mathrm{HAC} / \mathrm{H}_{2} \mathrm{O}$ & 5.0 & ${ }^{68} \mathrm{Ga}$ & $121^{\circ} / 30^{\prime}$ & ITLC & 80 \\
\hline $\mathrm{DPS}_{2}(1.0)$ & $\mathrm{H}_{2} \mathrm{O}$ & 5.0 & ${ }^{67} \mathrm{Ga} /{ }^{68} \mathrm{Ga}$ & $121^{\circ} / 30^{\prime}$ & Ext & 99 \\
\hline Hemato-IX (0.5) & $A C$ & 5.0 & ${ }^{67} \mathrm{Ga} /{ }^{68} \mathrm{Ga}$ & $121^{\circ} / 30^{\prime}$ & Ext & 99 \\
\hline
\end{tabular}

$\mathrm{HAC}=$ acetic acid

$A C=$ acetate buffer

Ext $=$ solvent extraction

and show the autoclaving method for heating and acetic acid as the solvent of choice to produce the best labeling yields.

Various porphyrins labeled with ${ }^{67} \mathrm{Ga}$ and ${ }^{68} \mathrm{Ga}$ were administered to rabbits after balloon-catheter de-erdothelialization and to hyperlipidemic rabbits, and the uptake in various organs and in normal or damaged aorta are shown in Tables 3 and 4 respectively. These results show that in the ballooncatheter-scraped aorta there is a 2- to 3-fold greater uptake of ${ }^{67} \mathrm{Ga}$-tetrakis(p-sulfonylphenyl)porphyrin than in the normal aorta. However, in the hyperli- pidemic rabbits there was no significant increase in the uptake of ${ }^{67} \mathrm{Ga}-\mathrm{TPPS}_{4}$ in the aorta as compared to the normal aorta.

These results indicate that radioporphyrins will be useful for visualizing vascular lesions with highresolution PET studies. Further evaluation of the most suitable porphyrin compound and radionuclide for PET imaging studies remains to be determined in animal model studies of vascular lesions or atheromata. The optimum time for accumulation and imaging after administration of the radioporphyrin remains to be determined.

Table 3. Distribution of ${ }^{57 / 60}$ Ga-porphyrins in rabbit scraped aorta."

\begin{tabular}{|c|c|c|c|c|c|c|c|c|c|}
\hline Porphyrin & Blood & Heart & Lungs & Liver & Kidney & Spleen & n-Aorta & s-Aorta & Ratiob \\
\hline${ }^{\circ} \mathrm{Ga}-\mathrm{TKMPP}$ & 0.12 & 0.02 & 1.52 & 0.23 & 0.08 & 0.09 & 0.004 & 0.008 & 2.0 \\
\hline${ }^{67} \mathrm{Ga}-\mathrm{TMP} y \mathrm{P}^{\mathrm{d}}$ & 0.07 & 0.03 & 0.06 & 0.08 & 0.43 & 0.09 & 0.07 & 0.11 & 1.6 \\
\hline${ }^{67} \mathrm{Ga}-\mathrm{DPS}{ }_{2}^{\mathrm{e}}$ & 0.07 & 0.03 & 0.04 & 0.09 & 0.06 & 0.12 & 0.02 & 0.04 & 2.0 \\
\hline "Ga-TTAP' & 0.02 & 0.02 & 0.04 & 0.11 & 0.20 & 0.09 & 0.04 & 0.08 & 2.0 \\
\hline \multirow[t]{2}{*}{${ }^{67} \mathrm{Ga}-\mathrm{TPPS}_{4}{ }^{8}$} & 0,17 & 0.12 & 0.15 & 0.08 & 0.13 & 0.13 & 0.08 & 0.19 & 2.4 \\
\hline & 0.34 & 0.12 & 0.25 & 0.18 & 0.16 & 0.19 & 0.08 & 0.24 & 3.0 \\
\hline${ }^{67} \mathrm{Ga}-\mathrm{Clt}$ rate & 0.18 & 0.04 & 0.07 & 0.06 & 0.24 & 0.08 & 0.08 & 0.08 & 1.0 \\
\hline
\end{tabular}

- Percent I.D.

- Ratio = scraped aorta/normal aorta

c Tetrakis methoxy phenyporphyrin at $30 \mathrm{~min}$

detra( $\mathrm{N}$-methyl-4-pyridyl)porphyrin at $4 \mathrm{~h}$

- deuteroporphyrin-disulfonate at $5 \mathrm{~h}$

- tetra(N,N,N-trimethylanilinium) porphyrin at $4 \mathrm{~h}$

- tetra(4-5ulfonatophenyl)porphyrin at $5 \mathrm{~h}$ or (tetrakis(p-sulfonylphenyl)porphyrin) 
Table 4. Uptake of ${ }^{67} \mathrm{Ga}-\mathrm{TPPS}$, in hyperlipidemic rabbits.

\begin{tabular}{rccccccc}
\hline Rabbit & Blood & Heart & Lungs & Liver & Kidney & Spleen & Aorta \\
\hline $5 \mathrm{~h}^{\mathrm{a}}$ & 0.23 & 0.09 & 0.10 & 0.12 & 0.10 & 0.10 & 0.09 \\
$5 \mathrm{~h}^{\mathrm{b}}$ & & 0.75 & 2.34 & 21.1 & 2.08 & & \\
$24 \mathrm{~h}^{\mathrm{a}}$ & 0.13 & 0.06 & 0.09 & 0.09 & 0.12 & 0.07 & 0.11 \\
$24 \mathrm{~h}^{\mathrm{b}}$ & & 0.36 & 2.55 & 12.8 & 2.46 & & \\
\hline
\end{tabular}

\% I.D. $/ \mathrm{g}$

b \% I.D./organ

\section{REFERENCES}

1. Spears, J.R., Serur, J., Shropshire, D., and Paulin, S. Fluorescence of experimental atheromatous plaques with hematoporphyrin derivative. I. Clin. Invest. 71, (395-399) 1983.

2. Kessel, D., and Sykes, E. Porphyrin accumulation by atheromatous plaques of the aorta.
Photochem. Photobiol. 40, (59-61) 1984.

3. Sohn, M., Roberts, M.F., Spokojny, A.M., Sinclair, I.N., Mulcahy, J., Serur, J.R., Davis, M., Adains, D.F., and Spears, J.R. Metalloporphyrins: MRI contrast agents for atherosclerotic vascular disease. Magnetic Resonance Imaging Meeting, August 19-23, 1985, Abstract P902.

\title{
MORPHOLOGICAL AND KINETIC ABNORMALITIES OF PLATELETS IN HYPERCHOLESTEROLEMIC RABBITS
}

\author{
Elisabeth Mazoyer, Shirley Ebbe, Kanu Dalal, Robert Leven, Bernard Mazoyer, \\ Kathleen Brennan, Dorothy Carpenter, and Tamlyn Yee
}

Hypercholestrolemia was produced by feeding rabbits diets supplemented with cholestrol and peanut oil. Arterial blood was collected and analyzed at 1-2 week intervals for 8-12 weeks from rabbits on the special diets and from control rabbits that were fed a normal diet for laboratory rabbits. Serum cholestrol concentrations increased rapidly in rabbits fed a diet with $1 \%$ cholesterol and $4 \%$ peanut oil and reached about $1800 \mathrm{mg} / \mathrm{dl}$ at 8 weeks while they remained at the normal level $(<100 \mathrm{mg} / \mathrm{dl})$ in the controls (Fig. 1). The hypercholesterolemic rabbits developed aortic atherosclerosis and fatty infiltrates of tissues.

Platelet volumes were measured electronically in samples of whole blood and in platelet-rich plasma (PRP) prepared from the same samples. Measurements in PRP were corrected for possible interference from lipid droplets in the lipemic plasma, but, in fact, platelet-poor plasma (PPP) from lipemic rabbits did not give different results than PPP from the controls. By both techniques, plate- lets from hypercholesterolemic rabbits became smaller than those from controls and smaller than they had been in the same rabbits before the diet was started (Fig. 2). This change occurred at the end of the second week of the experiments when serum cholesterol levels were about $700 \mathrm{mg} / \mathrm{dl}$. When the diet was modified to include only $0.5 \%$ cholesterol and $2 \%$ peanut oil, serum cholesterol levels increased more slowly (Fig. 1). Platelet microcytosis occurred later, becoming significant after 8 weeks of the diet (Fig. 3), at a time when serum cholesterol values were about $900 \mathrm{mg} / \mathrm{dl}$. These findings indicate that platelet microcytosis was a consistent accompaniment to distary hypercholesterolemia in rabbits and suggest that the hypercholesterolemia must be severe $(>600$ $\mathrm{mg} / \mathrm{dl}$ ) before it develops. Platelet counts showed no consistent relationship to platelet volumes, so microcytosis could not be perceived as a homeostatic adjustment intended to maintain platelet mass. Since platelet counts did not differ from 


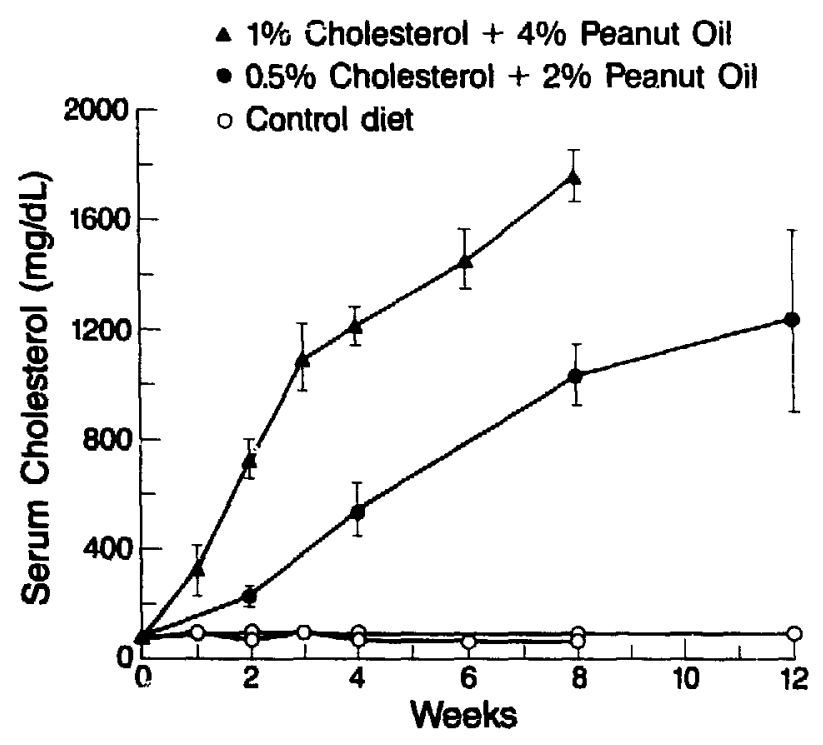

Fi. 1. Serum cholesterol concentrations in rabbits fed diets supplemented with cholesterol and peanut oil or a normal laboratory diet. Each point is the mean \pm SEM for 8 or 24 rabbits on the $1 \%$ cholesterol diet, 4 rabbits on the $0.5 \%$ cholesterol diet, or 4-14 controls.

$(X B L-8611-6501)$ control values at most time points, it could be concluded that, in general, platelet mass (platelet count $X$ platelet volume) was less in hypercholesterolemic rabbits than in controls.

Platelets from hypercholesterolemic and control rabbits were indistinguishable by transmission electron microscopy, indicating that production of subcellular organeiles was normal in spite of membrane biochemical abnormalities that may have accounted for the small size (see accompanying report by Dalal et al.).

Platelet survival studies were done to determine if evidence for intrinsic platelet abnormalities or platelet interaction with atherosclerotic vessels would be uncovered. Platelets from three types of donors were labeled with ${ }^{51} \mathrm{Cr}$ and transfused into completely normal recipients. Donors were either hypercholesierolemic as a result of having been fed the higher cholesterol diet, normocholesterolemic controls that had been bled on the same schedule as the hypercholesterolemic rabbits, or completely normal (new rabbits that had not been previously studied and were about 8 weeks younger than the experimental animals). It was found that platelets from hypercholesterolemic donors were destroyed

\section{Whole Blood}

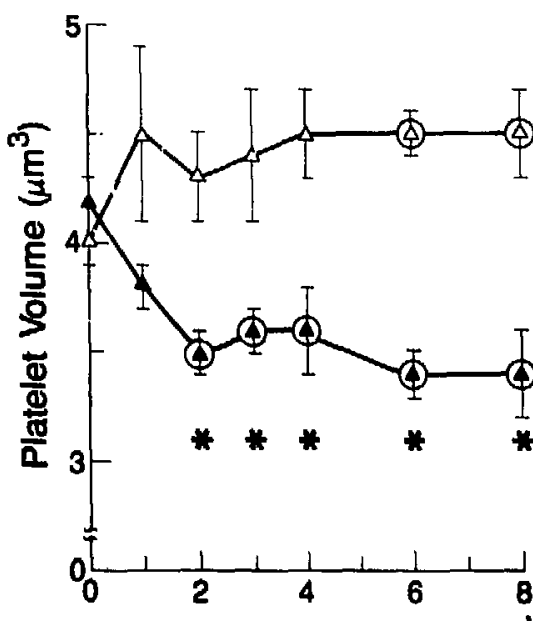

\section{PRP}

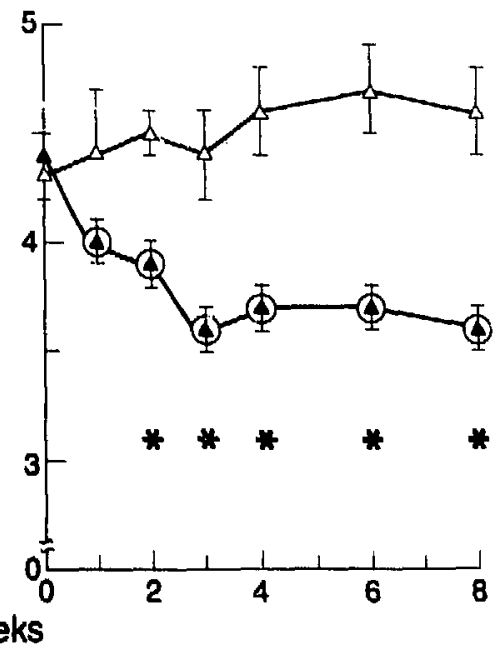

Fig. 2. Platelet volumes (mean \pm SEM) as measured in samples of whole blood (left panel) or platelet-rich plasma (right panel). Solid symbols represent rabbits on a diet supplemented with $1 \%$ cholesterol and $4 \%$ peanut oil; open symbols represent controls. Circled values are significantly different from values at time 0 in the same rabbits. Asterisks indicate significant differences between hypercholesterolemic and control rabbits.

(XBL 8611-6505) 


\section{Whole Blood}

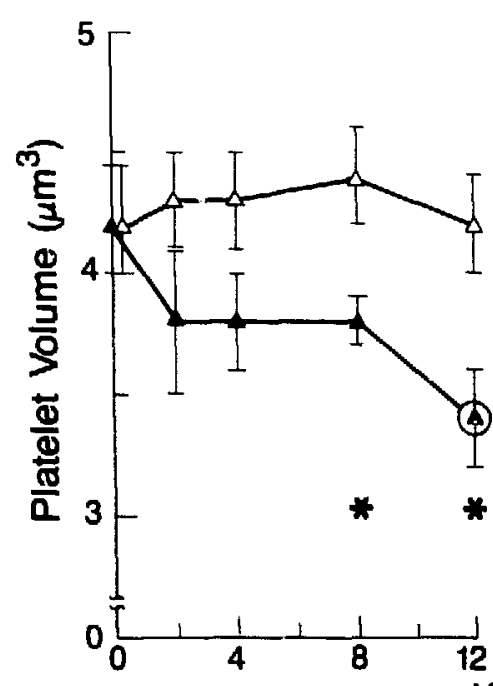

PRP

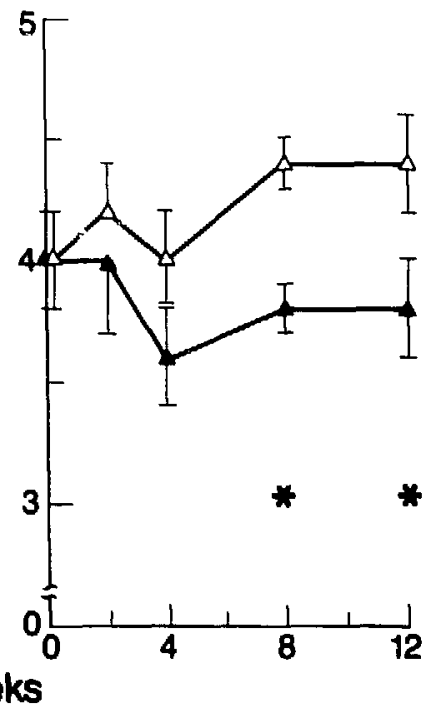

Fig. 3. Platelet volumes (mean \pm SEM) as measured in samples of whole blood (left panel) or platelet-rich plasma (right panel). Solid symbols represent rabbits on a diet supplemented with $0.5 \%$ cholesterol and $2 \%$ peanut oil; open symbols represent controls. The circled value is significantly different from the value at time 0 in the same rabbits. Asterisks indicate significant differences between hypercholesterolemic and control rabbits.

(XBL 8611-6498)

more rapidly than those from controls or from normal donurs. This finding suggested that the microcytic platelets may have had structural abnormalities that limited their ability to survive in the circulation. In converse experiments, ${ }^{51} \mathrm{Cr}$-labeled no:mal platelets were transfused intr; recipients of each of the three types; their survival was less in both hypercholesterolemic recipients and their controls than in normal donurs. The mechanism responsible for the rapid destruction of normal platelets was not clear. And since there was no difference between hypercholesterolemic recipients and their controls in this respect, a specific effect of the atherosclerosis (which was present ouly in hypercholesterolemic rabbits) on platelet survival was not apparent.

\section{THE EFFECTS OF HYPERCHOLESTEROLEMIA ON RABBIT PLATELETS: FUNCTIONAL AND BIOCHEMICAL ALTERATIONS}

\section{Kanu Dalal, Elisabeth Mazoyer, Shirley Ebbe, Dorothy Carpenter, and Tamlyn Yee}

Experimental evidence points to hypercholesterolemia as an important risk factor for atherosclerosis and related occlusive vascular disorders. In spite of considerable support implicating cholesterol, the mechanism of its effect on this group of disorders is not entirely clear.

Accumulation of fat and cholesterol in the platelets could influence the development of the initial fatty atheromatous lesions in human beings and animals. Since platelets may have a critical role in atherogenesis, we have examined the mechanisms underlying the relationship among platelets, cholesterol (C), phospholipid (PL) metabolism, and platelet function in rabbits fed diets supplemented with cholesterol ( $1 \%$ and $0.5 \%)$ and peanut oil ( $4 \%$ and $2 \%$ ).

At 2-week intervals, complete blood counts, serum cholesterol, platelet volume and platelet 
lipids and protein were determined. The rabbits fed a high (1\%) cholesterol diet became hypercholesterolemic (HC), with serum cholesterol increasing to $1800 \mathrm{mg} \%$ after 8 weeks (normal $<100 \mathrm{mg} \%$ ) while those fed a low $(0.5 \%)$ cholesterol diet showed a serum cholesterol level of $1030 \mathrm{mg} \%$ (normal $=91 \mathrm{mg} \%$ ). Lipids and fatty-acids of washed platelets were separated by one-dimensional thin-layer chromatography. HC platelets contained $25-30 \%$ more lipid than their normal counterparts due to an increase in free and esterified cholesterol. This was associated with a progressive reduction in total phospholipids. Analyses of individual phospholipids in $H C$ platelets showed sequential increases in the level of lysolecithin (LL) and phosphatidylinositol (PI), and a progressive decrease in phosphatidylcholine (PC). The cholesterol/phospholipid molar ratio of $\mathrm{HC}$ platelets increased from $0.49+0.06$ to $0.9 \pm 0.07$ $(P<0.05)$ in 8 weeks. HC plateiets (six determinations, each containing pooled aliquots of platelets) had $99 \%$ more arachidonic acid (AA) in the PI than normal platelets (Table 1).
Platelet function was monitored by the uptake and release of ${ }^{14} \mathrm{C}$-5-hydroxytryptamine $\left({ }^{14} \mathrm{C}-5 \mathrm{HT}\right)$ in platelet-rich plasma (PRP), using varying concentrations of collagen as an aggregating agent. The average uptake of ${ }^{14} \mathrm{C}-5 \mathrm{HT}$ in $\mathrm{HC}$ and normal platelets ranged from 80 to $92 \%$. The percent of ${ }^{14} \mathrm{C}$ $5 \mathrm{HT}$ released from normal and $\mathrm{HC}$ platelets was proportional to the concentration of collagen. However, HC platelets were hyperreactive to low concentrations of collagen. Incorporation of $50-\mu \mathrm{M}$ acetylsalicylic acid into the aggregating medium suppressed the release of ${ }^{14} \mathrm{C}-5 \mathrm{HT}$ in normal PRP by more than $90 \%$ but had only a partial effect on HC PRP. These results show that changes in platelets may be involved in the structure and/or function of the sites that modulate response to aggregating agents. Furthermore, a greater content of $\mathrm{LL}$, $\mathrm{Pl}$, and $\mathrm{AA}$, along with the lower levels of PC, could be related to associated abnormalities of platelet size and survival, and to the in vivo behavior of platelets under certain abnormal circumstances such as stroke and deep-vein thrombosis.

Table 1. Biochemical alterations in hypercholesterolemic rabbit platelets.

\begin{tabular}{|c|c|c|c|c|c|c|}
\hline \multirow[b]{2}{*}{ Days on diet } & \multicolumn{2}{|c|}{$C / P L$ (molar ratio) } & \multicolumn{2}{|c|}{$P L\left(\mu g / 10^{\circ}\right.$ platelets) } & \multicolumn{2}{|c|}{ AA (weight $\%$ of TFFA) } \\
\hline & Control & $\operatorname{Diet}^{b}$ & Control & Diet & Control & Diet \\
\hline 0 & $\begin{array}{l}0.49 \pm \\
0.06\end{array}$ & $\cdots$ & $\begin{array}{c}38.0 \pm \\
3.0\end{array}$ & $\cdots$ & $\begin{array}{l}22.0 \pm \\
2.0\end{array}$ & -- \\
\hline 57 & $\begin{array}{l}0.43 \pm \\
0.05\end{array}$ & $\begin{array}{l}0.90 \pm \\
0.07\end{array}$ & $\begin{array}{c}40.0 \pm \\
5.0\end{array}$ & $\begin{array}{c}80.0 \pm \\
7.0\end{array}$ & $\begin{array}{c}20.5 \pm \\
4.0\end{array}$ & $\begin{array}{l}39.0 \pm \\
6.0\end{array}$ \\
\hline $\begin{array}{l}\text { Number of } \\
\text { - Number of }\end{array}$ & $\begin{array}{l}\text { animals }=1 \\
\text { animals }=2\end{array}$ & & & & & \\
\hline
\end{tabular}




\section{INCREASE IN MEGAKARYOCYTE SIZE WITHIN PLOIDY GROUPS AND SHIFT IN PLOIDY IN THROMBOCYTOPENIC MICE}

\section{Shirley Ebbe, Dorothy Carpenter, and Tamlyn Yee}

Mechanisms by which megakaryocytes become macrocytic in response to immunothrombocytopenia were evaluated in mice. Acute or chronic immunothrombocytopenia was induced by a single injection or daily injections of guinea pig antimouse platelet serum. Sequential measurements of megakaryocyte size and ploidy were made in cohorts of mice. Areas of mature megakaryocytes were measured microscopically in smears of bone marrow stained with polychromatic stains. Ploidies of the same cells were then measured by twowavelength microspectrophotometry after destaining and restaining by the Feulgen technique. The amount of chromophore in smail segmented nuclei in each smear served as the $2 \mathrm{~N}$ reference.

Relative amounts of chromophore in normal megakaryocytes were distributed about three major peaks corresponding to $8 \mathrm{~N}, 16 \mathrm{~N}$, and $32 \mathrm{~N}$, with $65-80 \%$ being $16 \mathrm{~N} ; 64 \mathrm{~N}$ and $4 \mathrm{~N}$ megakaryocytes were infrequent. Mean sizes of megakaryocytes increased with increasing ploidy, but sizes of megakaryocytes of different ploidies overlapped.

With acute immunothrombocytopenia, platelet counts of $1-4 \%$ of normal were followed by gradual recovery and rebound thrombocytosis. Megakaryocyte mean size increased abruptly on day 2 of recovery from acute immunothrombocyto- penia due to 1) a shift in ploidy with a decrease in the proportion of $16 \mathrm{~N}$ and an increase in $32 \mathrm{~N}$ and 2) an inconstant increase in size of megakalyocytes within the $32 \mathrm{~N}$ group.

With chronic immunothrombocytopenia, platelet counts of $1-9 \%$ of normal were maintained. Megakaryocyte mean size was increased on days 3-6 of chronic immunothrombncytopenia due to 1) a decreased proportion of $8 \mathrm{~N}$ and $16 \mathrm{~N}$ cells, increased $32 \mathrm{~N}$ and $64 \mathrm{~N}$, and appearance of $128 \mathrm{~N}$ cells and 2) a consistent increase in size within the $16 \mathrm{~N}$ group and inconstant size increases within $8 \mathrm{~N}$ and $32 \mathrm{~N}$ groups. Signs of hemorrhage and anemia developed in some mice with chronic immunothrombocytopenia, but the findings did not differ in mice that did not become anemic.

The fact that megakaryocyte ploidy increases in response to thrombocytopenia has been know/n from the work of others. The present results confirm the conclusion that the shift in ploidy distribution is a major mechanisrn responsible for macromegakaryocytosis in both acute and chronic immunothrombocytopenia. In addition, they also describe a second, previously unrecognized mechanism that regularly contributes to macrocytosis of megakaryocytes in prolonged immunothrombocytopenia, namely an increase of cell size out of proportion to ploidy.

\section{MEGAKARYOCYTE FRAGMENTATION AND PLATELET FORMATION}

\section{Robert M. Leven}

The mechanism by which megakaryocytes fragment into blood platelets is poorly understood. It is believed that these cells form long cytoplasmic processes that break into small fragments, the circulating blood platelets. Since discovering that fractionated plasma from thrembocytopenic rabbits stimulates megakaryocyte fragmentation in vitro, ${ }^{1}$ investigation of the underlying cellular mechanisms involved in this process has been continued.

We have measured the number of cells that are stimulated to fragment by thrombocytopenic rabbit plasma (TRP) or the $60-80 \%$ ammonium sulfate fraction (TRP $60-80$ ) of this plasma. In addition, the inhibition by colchicine and vincristine has been quantitated (Table 1). By two independent techniques, trypan blue exclusion and ${ }^{51} \mathrm{Cr}$ release, we have demonstrated that this plasma fraction has no obvious effect on cell viability (Table 2 ).

Using immunofluorescence microscopy for the visualization of microtubules we have found that the cytoplasmic processes formed during fragmentation contain many microtubules. Cytoplasmic fragments released from megakaryocytes may contain microtubule rings, which are structural components characteristic of normal circulating platelets. 
Table 1. Effect of TRP and TRP 60-80 on megakaryucyte fragmentation."

\begin{tabular}{lc}
\hline Culture condition & $\begin{array}{c}\text { Percent of megakaryocyte } \\
\text { forming processes }\end{array}$ \\
\hline Control (No additions) & 0 \\
NRP (10\%) & $0.1 \pm 0.14^{\mathrm{b}}$ \\
TRP $(10 \%)$ & $5.4 \pm 0.58$ \\
NRP 60-80 $(1.0 \mathrm{mg} / \mathrm{ml})$ & $1.0 \pm 0.82$ \\
TRP $60-80(1.0 \mathrm{mg} / \mathrm{ml})$ & $18.4 \pm 1.58$ \\
TRP $60-80+1 \mathrm{mg} / \mathrm{ml}$ colchicine & $5.0 \pm 0.69$ \\
TRP $60-80+10 \mu \mathrm{g} / \mathrm{ml}$ vincristine & $3.7 \pm 0.80$ \\
\hline \hline
\end{tabular}

- Data are the averages from three experiments in which a minimum of 500 cells were counted for each condition. Each experiment was from a different megakaryocyte preparation. Colchicine and vincristine were added $1 \mathrm{hr}$ prior to addition of TRP $60-80$. Counts were made $3 \mathrm{hr}$ after NRP, TRP or TRP 60-80 addition. TRP 60-80 was significantly greater than NRP $(P<0.001)$. TRP $60-80$ was signficantly greater than NRP 60-80 ( $P<0.005$ ). Inhibition was significant by both colchicine $(P<0.01)$ and vincristine $(P<0.005)$ as compared to TRP 50-80 alone.

- Standard error of the mean.

Ultrastructural examination of the cytoplasmic processes has shown that the cytoplasm appears similar to that of normal platelets. A very dense cytoplasm with typical cytoplasmic granules is apparent (Fig. 1). Although the original observations of this phenomenon were made with guinea pig megakaryocytes, we have now found that rat megakaryocytes also fragment in vitro when incubated with TRP 60-80 (Fig. 2). In addition we

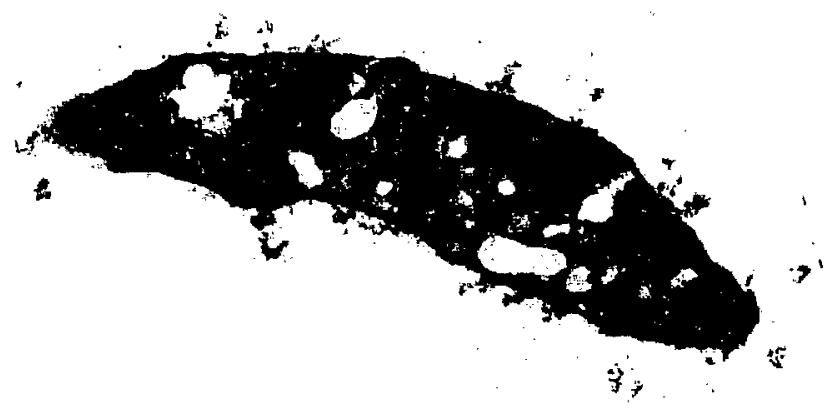

Fig. 1. Transmission electron micrograph of part of a megakaryocyte cytoplasmic process. Alpha granules can be seen as well as open space of what may be the developing platelet surface connected canalicular system.

(XBB 860-9673)

Table 2. Effect of NRP 60-80, TRP 60-80 colchicine and vincristine on megakaryocyte viability.

\begin{tabular}{|c|c|c|c|}
\hline Trypan blue exclusion & & Percent viable & \\
\hline & $\begin{array}{l}\text { Control (No addition) } \\
\text { NRP } 60-80(1.0 \mathrm{mg} / \mathrm{ml}) \\
\text { TRP } 60-80(1.0 \mathrm{mg} / \mathrm{ml}) \\
\text { Colchicine }(1.0 \mathrm{mg} / \mathrm{ml}) \\
\text { Vincristine }(10 \mu \mathrm{g} / \mathrm{ml})\end{array}$ & $\begin{aligned} 86.5 & \pm 1.36^{b} \\
83 & \pm 0.94 \\
85 & \pm 0.95 \\
80 & \pm 0.77 \\
86 & \pm 1.63\end{aligned}$ & \\
\hline \multirow[t]{2}{*}{${ }^{51}$ Chrominum release } & & Specific Toxicity & \\
\hline & $\begin{array}{l}\text { NRP } 60-80(1.0 \mathrm{mg} / \mathrm{ml}) \\
\operatorname{TRP} 60-80(1.0 \mathrm{mg} / \mathrm{ml})\end{array}$ & $\begin{array}{l}\text { Expt } 1 \\
0 \\
0\end{array}$ & $\begin{array}{l}\text { Expt } 2 \\
0 \\
0.4\end{array}$ \\
\hline
\end{tabular}

- Data from the trypan blue exclusion experiments are average results of three experiments. Measurements are made in iriplicate for each condition in each experiment. Viability in NRP 60-80 and TRP 60-80 with trypan blue was determined after $3 \mathrm{hi}$ incubation. Viability in colchicine and vincristine was determined $3 \mathrm{hr}$ after drug addition. None of the groups show any significant difference from the control $(P>0.05)$. The data show the results of two experiments in which measurements for each condition were made in triplicate. Cr release was measured after $3 \mathrm{hr}$ incubation in TRP 60-80 or NRP 60-80.

b Standard error of the mean. 
have found that the fragmenting cytoplasm of the rat megakaryocytes spreads onto the culture substratum when exposed to adenosine diphosphate or when placed on a collagen surface, indicating that the surface of the cytoplasmic processes and fragmenting cytoplasm is reactive to extracellular stimuli as are normal megakaryocytes and platelets.

Our understanding of the mechanism of platelet formation is still limited, but our recent experiments are beginning to shed light on the subcellular events that occur during platelet formation in vitro. The data we now have indicate that the cytoplasm that we observed fragmenting shares ultrastructural

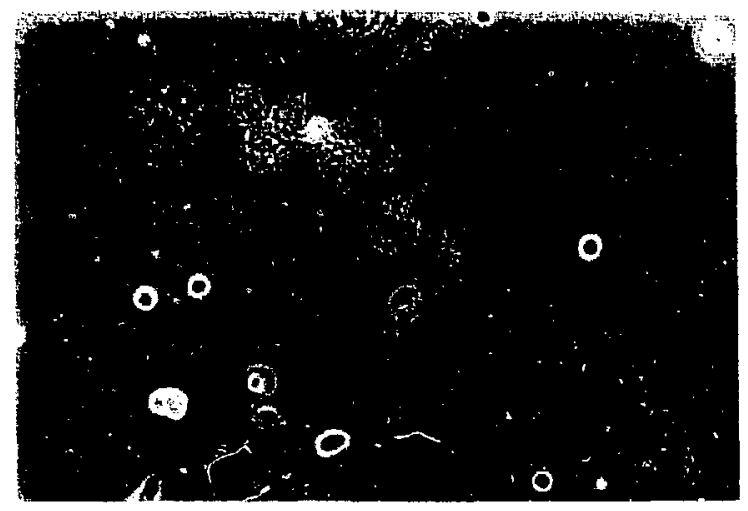

Fig. 2(a). The long intrizate processes of a fragmenting rat megakaryocyte are seen as well as two spread cells and several unreactive cells which remain spherical. and physiological properties with normal platelets; consequently we believe that stimulated megakaryocyte fragmentation may accurately represent the normal platelet formation mechanism. At present a working model has been formulated in which it is postulated that a breakdown of microfilamentous structures leads to a weakening of the cortical region of the megakaryocytes. The internal cytoplasm, divided by the demarcation membrane system, then separates into long microtubule based fragments, or proplatelets. The proplatelets finally break, by an as yet undescribed mechanism, into individual platelets.

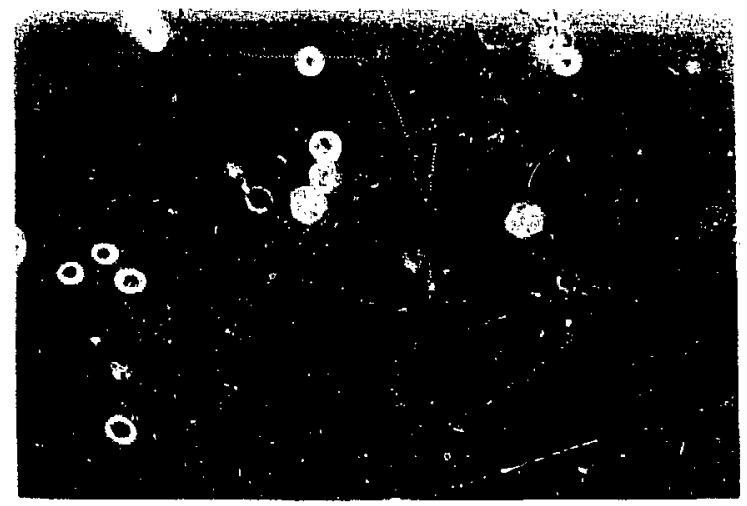

Fig. 2(b). Several small cyloplasmic fragments that have troken off of the long proplatelet processes of a fragmenting megakaryocyte.

(XBB 860-10031)

and partially fractionated thrombocytopenic plasma: A model system for the study of platelet formation. Blood, in press. 


\title{
Nuclear Medicine: Blood Flow and Metabolism
}

\author{
${ }^{123}$ I-LABELED RADIOPHARMACEUTICALS FOR IN VIVO IMAGING OF LOW-DENSITY \\ LIPOPROTEIN METABOLISM
}

\author{
Stephen M. Moeriein, Kanu B. Dalal, Shirley N. Ebbe, Yukio Yano, \\ Kathleen M. Brennan, Tainlyn Neben, Julia A. Twitchell, Dorothy A. Carpenter, \\ and Thomas F. Budinger
}

Low density lipoprotein (LDL) is the maior transport protein for cholesterol and is an important determinant of human health and disease. Elevated serum levels of LDL have been implicated as a major predisposing factor in the genesis of atherosclerotic lesions in animals and man. Deviant blood concentrations of this protein can result from primary (genetic) defects, such as familial hypercholesterolemia, or be acquired secondary to such altered metabolic states as cirrhosis, nephrotic syndrome, diabetic acidosis, and thyroid disease. Because of the involvement of LDL in these disease states, we have commenced a study of the behavior of radiolabeled LDL analogues using nuclear imaging methods. lodine- 123 was chosen as a label for LDL due to its useful decay characteristics for this purpose $\left(159-\mathrm{keV} \gamma, \mathrm{t}_{1 / 2}=13.2 \mathrm{hr}\right)$.

The objective of this investigation was to find a tracer of LDL that will remain in tissue cells as a function of metabolic activity. The use of LDL labeled in the conventional manner by direct radioiodination may be misleading in this application because the radiolabel can be metabolized off the plasma protein and thereafter have a biodistribution that is particular to radioiodide and not LDL. A radioiodinated tyramine-cellobiose adduct of $L D L$ (TC-LDL) has advantages as a radiopharmaceutical tracer of LDL metabolism because the radioiodine label is intracellularly trapped following degradation of the protein.' We have compared the in vivo characteristics of 123 /-labeled TC-LDL and LDL to assess the utility of these compounds as radiopharmaceuticals for the noninvasive study of this plasma protein. Due to the exquisite sensitivity of the rabbit to changes in blood cholesterol concentrations, this animal was employed as a preclinical model of human disease.

The radiosynthesis of $\left[{ }^{123} \mid\right) L D L$ and $\left.\left|{ }^{123}\right|\right]$ TC-LDL was optimized for radiopharmaceutical production. ${ }^{2}$ As shown in Scheme 1, $\left.\right|^{123}|| L D L$ was prepared in $40 \%$ radiochemical yield within 30 minutes by direct iodination of $\mathrm{LDL}$ with exchange-labeled

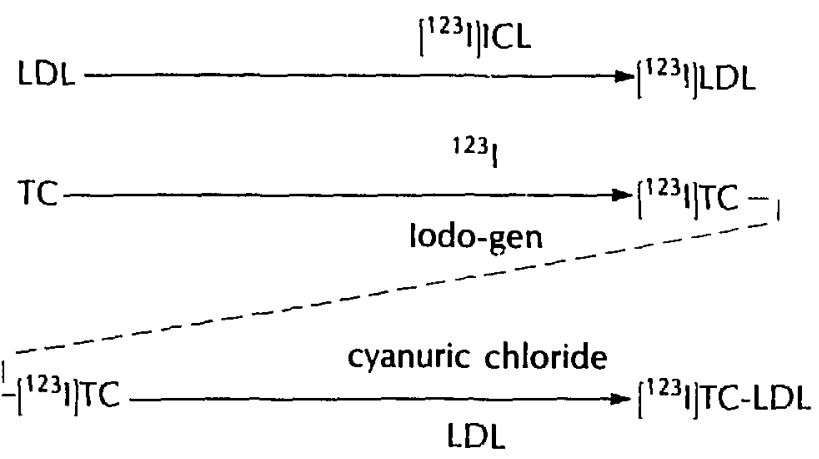

SCHEME 1

[123||IC!. Tyramine-cellohiose (TC) was radioiodinated with no-carrier-added ${ }^{123}$ I using Idogen as an in situ oxidant, and subsequently coupled to LDL using cyanuric chloride as a cross-linking agent to produce $\left[{ }^{123} \mid\right.$ TC-LDL in $30 \%$ radiochemical yield and an overall preparation time of 75 minutes. Both [ ${ }^{123}$ ]]LDL and [ ${ }^{123}$ ] TC-LDL were purified by gel permeation chromatography prior to in vivo application, and examination of the labeled derivatives by gradient gel electrophoresis indicated that both compounds were very similar in character to native LDL. ${ }^{2}$

The tissue distribution of the radioiodinated LDL derivatives was determined in control and hypercholesterolemic rabbits via surgical dissection. The hypercholesterolemic rabbits were fed special diets that contained additional cholesterol and peanut oil. ${ }^{3}$ These diets were found to induce metabolic changes in the rabbits that were similar to hypercholesterolemia in humans. The animals were under the professional care of a licensed veterinarian at all times.

Table 1 shows the tissue concentrations of radioiodinated $\mathrm{LDL}$ and $\mathrm{TC}-\mathrm{LDL}$ in control rabbits 24 hours postinjection. The tissue distribution of radioiodinated TC was also determined to see whether it differed from that of radioiodinated TC- 
Table 1. Tissue concentrations in normal rabbits. ${ }^{3}$

\begin{tabular}{|c|c|c|c|}
\hline Organ & $\left.\right|^{131} ! \mid L D L$ & (131//TC-LDL & {$[31]$ / } \\
\hline Liver & 0.10 & 1.94 & 0.14 \\
\hline Spleen & 0.23 & 4.80 & 0.01 \\
\hline Lungs & 0.25 & 0.09 & 0.01 \\
\hline Kidneys & 0.04 & 0.04 & 0.19 \\
\hline Heart & 0.02 & 0.04 & 0.00 \\
\hline Thyroid & $>12.92$ & 0.52 & 2.77 \\
\hline
\end{tabular}

a Data reported as \% injected dose-kg/g at 24 hr postinjection.

LDL. In these studies, the longer-lived ${ }^{131}$ I isotope was used for experimental convenience. As can be seen, the tissue distribution of $\left[{ }^{131}\right.$ I]TC-LDL was distinct from that of $\left[{ }^{131}|| T C\right.$, so the large accumulation of radioactivity in the liver following administration of this $\mathrm{LDL}$ analogue can be attributed to the predominance $^{4}$ of this organ in the catabolism of LDL and not merely due to the tissue distribution of the radioiodinated meiabolic end-product. In contrast to $\left[{ }^{131} \mid\right] T C-L D L,\left[{ }^{131} \mid\right] L D L$ showed relatively little localization in the liver, and very nigh accumulation in the thyroid. The latter tissue has a high affinity for sequestration of iodide, and the large localization of radioactivity in this organ rather than the liver indicates that loss of the radioiodine label from $\left[{ }^{131}\right.$ I]LDL occurs independently of LDL metabolism.

This conclusion is further supported by the plasma clearance of these radioiodinated compounds (Table 2). Although the elimination halflives of $\left.\left[{ }^{131}\right]\right] L D L$ and $\left.\left[{ }^{131}\right]\right] T C-L D L$ from the plasma

Table 2. Plasma clearance in normal and hypercholesterolemic rabbits.

\begin{tabular}{|c|c|c|}
\hline \multirow{2}{*}{$\begin{array}{c}\text { Radiolabeled } \\
\text { compound }\end{array}$} & \multicolumn{2}{|c|}{ Plasma half-life (hr) } \\
\hline & Control & Hypercholesterolemic \\
\hline${ }^{131}|| L D L$ & 19.5 & 9.4 \\
\hline${ }^{131} \mid \mathrm{TTC}-\mathrm{LDL}$ & 23.7 & 36.2 \\
\hline$\left[{ }^{13 t}\right.$ I]TC & 5.0 & 4.5 \\
\hline
\end{tabular}

- Determined by regression analysis of data from plasma samples taken 2-24 hr postinjection. are very simi'ar for normal rabbits, the clearance rate of the latter compound is decreased in hypercholesterolemic animals whereas the former derivative has enhanced plasma clearance. The in vivo behavior of ${ }^{131}$ ||TC-LDL may be explained by a decreased fractional metabolic rate of the lipoprotein, which results from the saturation of LDL receptors in the liver by elevated plasma levels of LDL. On the other hand, the faster elimination rate of $\left.{ }^{131} /\right\} L D L$ in the hypercholesterolemic rabbit is not representative of the biochemistry of $L D L$, but rather is the result of unknown enzymatic deiodination processes that are brought about by the altered metabolic state of the animals. For the case of $\left[{ }^{131}\right.$ i]TC, the clearance half-lives were similar in both control and diet rabbits, and significantly shorter than those of $\left[{ }^{131} I\right] T C-L D L$. These results show that the tissue distribution of radioiodinated TC-LDL is indicative of the sites of LDL metabolism rather than the relative organ affinity of a metabolic endproduct.

Dynamic imaging of $\left[{ }^{123} \mid\right] \mathrm{LDL}$ and [1231]TC-LDL in control and hypercholesterolemic rabbits generated data that were in good agreement with the preliminary surgical experiments. Over $35 \%$ of the injected dose of $\left[{ }^{123}\right] \mid T C-L D L$ accumulated in the liver of a control rabbit, a value that decreased to about $20 \%$ for a hypercholesterolemic rabbit. By contrast, less than $20 \%$ of the injected dose of $\left[{ }^{123} I\right] L D L$ was accumulated by the liver of a normal rabbit, while the liver of a diet animal sequestered only about $10 \%$ of the injected dose. These data indicate that superior counting statistics are achieved with ${ }^{123}$ |TC-LDL as a radiopr rmaceutical as compared to [ $\left.{ }^{123}\right]$ ] LDL. The blood clearance of the two radioiodinated lipoprotein derivatives as determined noninvasively with a gamma camera was similar to the trends tabulated in Table 1 . As anticipated, a high degree of deiodination of [123] LDL took place during these imaging experiments, as the scintigrams in Fig. 1 vividly illustrate.

In summary, we have shown using a rabbit model of hypercholesterolemia, that $\left.\right|^{123} \mid$ TC-LDL is superior to [1231]LDL as a radiopharmaceutical for noninvasively studying the metabolic fate of this plasma protein. Future applications of this agent include the imaging of various patient populations to better understand the physiological mechanism of LDL catabolism in human health and disease, 
SCINTIGRAMS OF HYPERCHOLESTEROLEMIC RABBITS

$[123]$ LOL
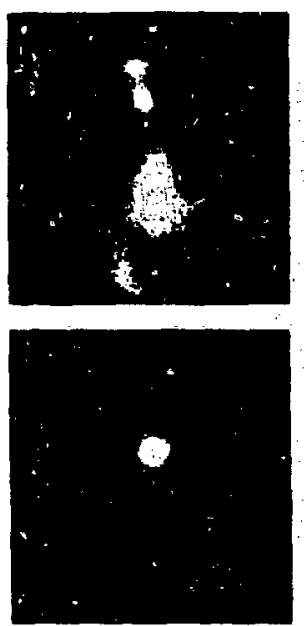

$\left[{ }^{123}\right]$ TC-LDL
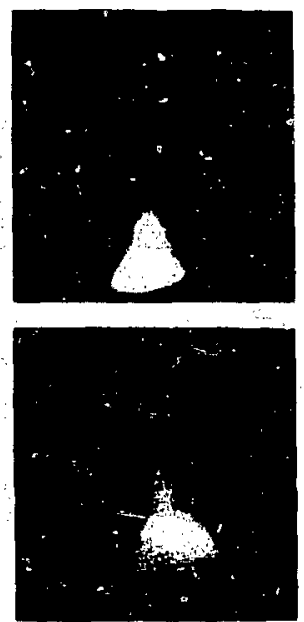

Fig. 1. Scintigrams of hypercholesterolemic rabbits at 2 and 24 $h r$ postinjection. Note the preferential accumulation and retention of radioactivity in the liver of the scintiglam for $\left[{ }^{123} / T C\right.$. $L D L$, and the relatively minor liver uptake and overwheiming localization of activity in the thyroio of the scintigram for [123|lL!

(XBB 860-8880)

and use of the rabbit model to evaluate the effect of pharmacological intervention on the metabolic rate.

\section{REFERENCES}

1. Carew, T.E., Pittman, R.C., Marachand, E.R., and Steinberg, D. Measurement in vivo of irreversible degradation of low density lipoprotein in the rabbit aorta. Arteriosclerosis 4, 214-224 (1984).

2. Moerlein, S.M., Dalal, K.B., and Yano, Y. Radiosynthesis of ${ }^{123}$ - and ${ }^{131}$ I-labeled derivatives of Jow density lipoprotein for radiopharmaceutical use. J. Lab. Comp. Radiopharm. (submitted 1986).

3. Mazoyer, E., Ebbe, S., Dalal K., et al. Morphological and kinetic abnormalities of platelets in hypercholesterolemic rabbits. Blood (abstract, in press).

4. Margolis, S. and Capuzzi, D. Serumlipropotein synthesis and metabolism. In Blood Lipids and Lipoproteins: Quantitation, Composition, and Metabolisni, Nelson, G.J. (Ed.). Wiley-Interscience, New York, pp. 825-880 (1972).

\section{POSITRON EMISSION TOMOGRAPHY FOR DIAGNOSIS OF RADIATION NECROSIS AND RECURRENT BRAIN TUMOR}

\section{Peter E. Valk and Thomas F. Budinger}

High-dose radiation therapy is the most effective treatment for high-grade primary brain tumors, but is associated with a risk of radiation necrosis, which can itself be fatal. ${ }^{1}$ The clinical features of radiation necrosis are very similar to those of tumor recurrence and differentiation of the two is frequently difficult. Appearances of the two conditions on $x$-ray $C T$, magnetic resonance imaging, and angiography can be identical, and differential diagnosis frequently requires surgical biopsy. Functional imaging by positron emission tomography (PET) provides a new approach to this diagnostic problem and we have used PET with rubidium-82 $\left({ }^{82} \mathrm{Rb}\right)^{2}$ and ${ }^{18} \mathrm{~F}$-2-fluoro-2-deoxyglucose $\left({ }^{18} \mathrm{~F} \text {-FDG }\right)^{3}$ to distinguish recurrent tumor from necrosis in this patient group.

All patients studied have had high-grade gliomas, which had bzen treated with external irradia- tion and interstitial implantation of ${ }^{125}$ seeds. Following an initial response to treatment, the patients had presented again with clinical or radiologic deteriuration, resulting from radiation necrosis or tumor recurrence. Imaging was performed with the Donner 280 -Crystal Tomograph. ${ }^{42} \mathrm{Rb}$ is a bloodbrain barrier tracer and cerebral uptake is seen only when this is disrupted by a pathologic process, such as tumor or necrosis. ${ }^{82} \mathrm{Rb}$ uptake was used to demonstrate the precise site of the abnormality and ${ }^{18} \mathrm{~F}$-FDG uptake was used to determine the level of glucose metabolism at this site. In most cases of tumor, lesion uptake of ${ }^{18} \mathrm{~F}$-FDG was found to be equal to or greater than uptake in the immediately adjacent tissue, while in necrosis it was less (Fig. 1). The PET diagnosis was made by visual assessment of this lesion to background ratio. In the first 9 patients, uptake within and around the 
(a)
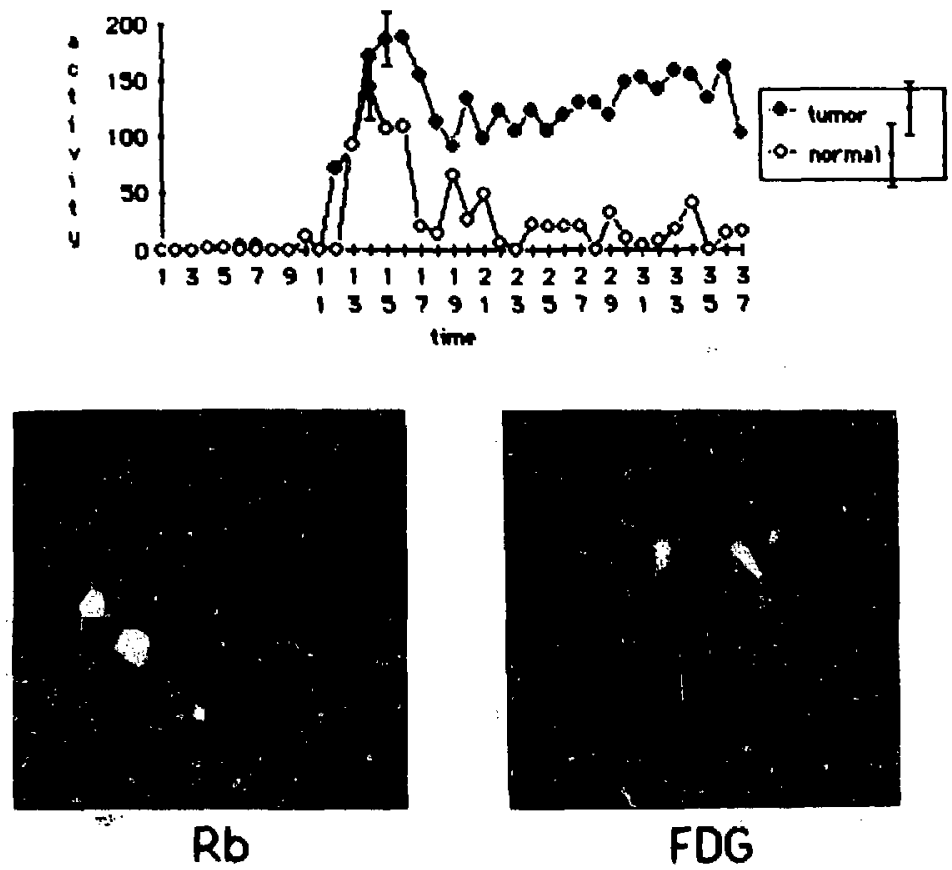

FDG

(b)

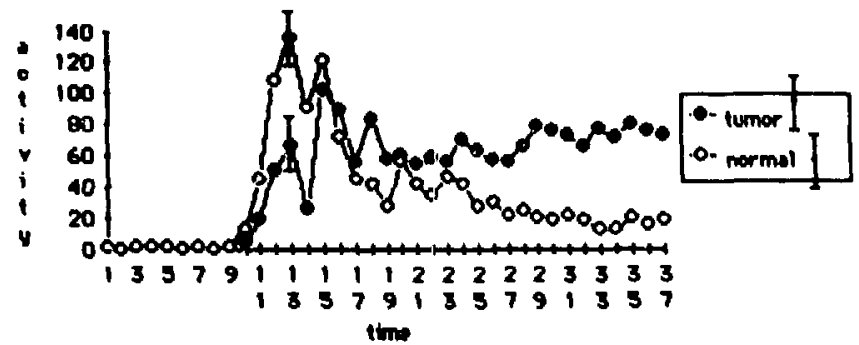

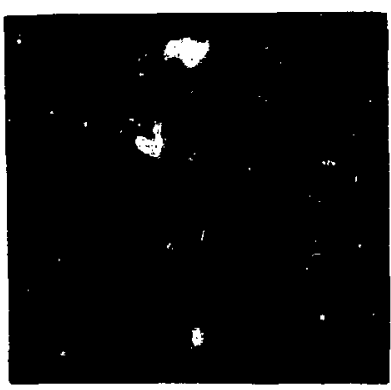

$\mathrm{Rb}$

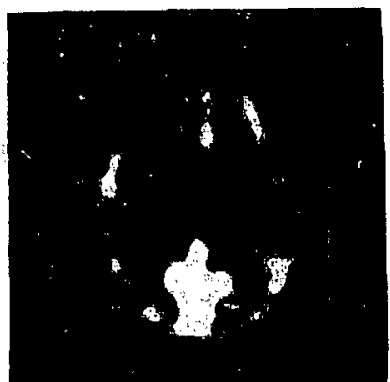

FDG

Fig. 1. (a) Tumor recurrence in a patient with glioblastoma multiform. The patient had been treated with external and interstitial radiation and 6 months later presented will recurrence of symploms and an enlarging mass on CT examination. The ${ }^{14}$ F.FDG image showed more uptake of tracer in the region of EBB abnormality than in the immediately adjacent tissue, indicating actively metabolizing lumor. Confirmed histologically. (b) Radiation necrosis in a patient with anaplastic astrocytoma. The patient had an enlarging mass on CT examimation 8 months after interstitial irradiation. No ${ }^{18} F$.FDG was seen in the area of abnormal BBB, indicating absence of metabolism. Necrosis confirmed histologically. (a and b) the ${ }^{n 2} R b$ time-activity curves compare activily in the lesions with activity in homologous contralateral regions, during the first 3 min after injection. 
lesion was quantitated and a calculated lesion to background ratio was also obtained. In these patients, ${ }^{82} \mathrm{Rb}$ time-activity curves for the lesion and for a contralateral homologous region were also plotted.

Thirty seven studies were performed in thirtytwo patients. A subsequent histologic diagnosis was obtained in 18 ( 2 were reported as tumor only, 12 as tumor plus necrosis, and 3 as necrosis only). In one case, hematoma was the unexpected finding. Using the above criteria, the 5 cases of tumor or necrosis only were correctly identified by PET, and tumor was correctly identified in 9 of the 12 cases of mixed pathology. In 3 cases of coexistent tumor and necrosis, the PET study failed to detect the presence of tumor. The hematoma was diagnosed as "necrosis," as would be expected. Where quantitation of uptake in and around the lesion was done, the diagnosis based on the calculated ratio agreed with the visual findings in all cases. ${ }^{82} \mathrm{Rb}$ dynamic studies in 9 patients showed that tumor first-pass peak activity exceeded normal in all cases. However, this finding was not specific, as this pattern was also seen in one case of necrosis.

These studies have established PET as a reliable means of differentiating recurrent brain tumor from radiation necrosis, following high-dose radiation therapy of high-grade gliomas. The criteria described here for radiation injury of the brain are being evaluated for post-radiation follow-up of other patient groups.

\section{REFERENCE}

1. Sheline, G.E., Wara, W.M., and Smith, V. Therapeutic irradiation and brain injury. Int. I. Radiat. Oncol. Biol. Phys. 6, 1215-1228 (1980).

2 Yen, C.K., and Budinger, T.F. Evaluation of blood-brain barrier permeability changes in rhesus monkeys and man using rubidium-82 and positron emission tomography. 1. Comput. Assist. Tomogr. 5, 792-799 (1981).

3. Reivich, M., Kuhl, D., Wolf, A., et al. $|F-18|$ Fluorodeoxyglucose method for measurement of local cerebral glucose utilization in man. Circ. Res. 44, 127-137 (1979).

4. Derenzo, S.E., Budinger, T.F., Huesman, R.H. et al. Imaging properties of a positron tomograph with 280 BGO crystals. IEEE Trans. Nucl. Sci. NS. 28, 81-89 (1981).

\section{BRAIN BLOOD FLOW IN ALZHEIMER'S DISEASE MEASURED WITH IODINE-122 AND POSITRON EMISSION TOMOGRAPHY}

\section{Thornton Sargent III, Chester A. Mathis, William J. Jagust, Natalia Kusubov, and Thomas F. Budinger}

In recent reports we have described the development of a ${ }^{122} \times \mathrm{Xe}^{122}$ I generator for the production of ${ }^{122}$ I for use in positron emission tomography (PET). This isotope has the advantage of being a positron emitter with a short half-life (3.6 $\mathrm{min}$ ), and is derived from a generator system containing the longer-lived parent, ${ }^{122} \times \mathrm{Xe}$ (half-life 20 $\mathrm{hr}$ ). The parent ${ }^{122} \mathrm{Xe}$ can be produced at a distant cyclotron and shipped to PET centers, where the ${ }^{122}$ I can be drawn off every 20 minutes for repeated use. We have reported a number of isomers of our original iodinated dimethoxylated amphetamine, each of which we have succeeded in labeling with ${ }^{122}$ I in less than one half-life. When imaged with PET after injection into a dog, these radiopharmaceuticals are extracted on first pass through the brain, and thus behave as extracted flow tracers. These compounds are presently under detailed study to verify their accuracy and validity in flow measurements compared to microspheres, to be foliowed by pharmacotoxicity studies, prior to application in human studies. Meanwhile, we have utilized another amphetamine-type flow agent, HIPDM, which was developed for use with ${ }^{123}$ in single-photon tomographic studies by Dr. Hank F. Kung of the Veteran's Administration Medical Center, Buffalo, N.Y.

In last year's report, we described the labeling of HIPDM with ${ }^{122}$ and its use in PET imaging of a patient with stroke. This patient was injected first with ${ }^{122}$ I-HIPDM, and 20 minutes later when imaging was complete and the ${ }^{122}$ I had decayed, with the patient in exactly the same position in the PET camera, ${ }^{18} \mathrm{FDG}$ was injected. A more detailed analysis of the data from this patient was per- 
FDG with ROI's

\section{Patient \#1}
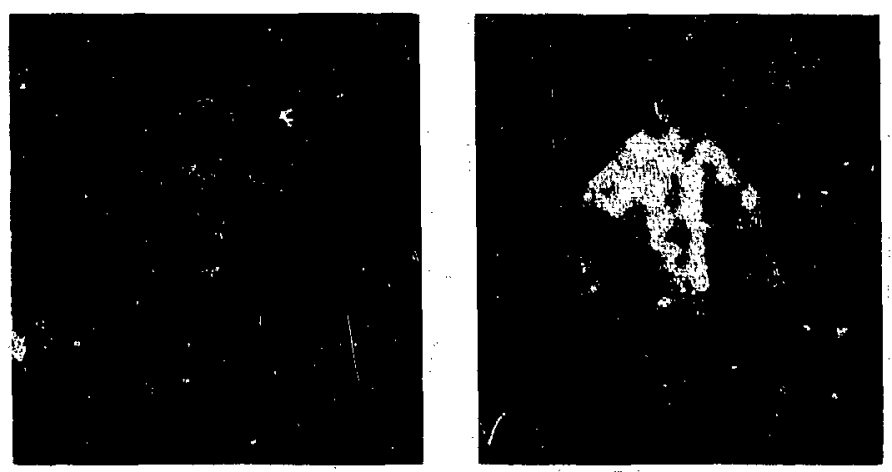

Patient \#2
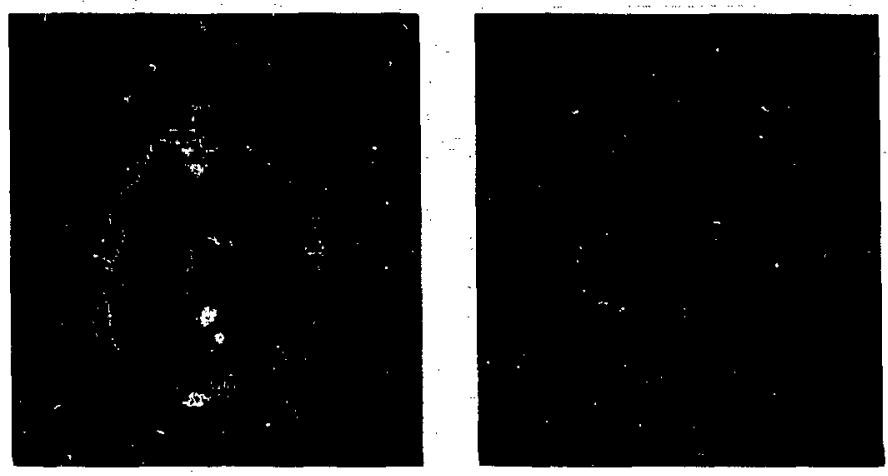

\section{Patient \#3}
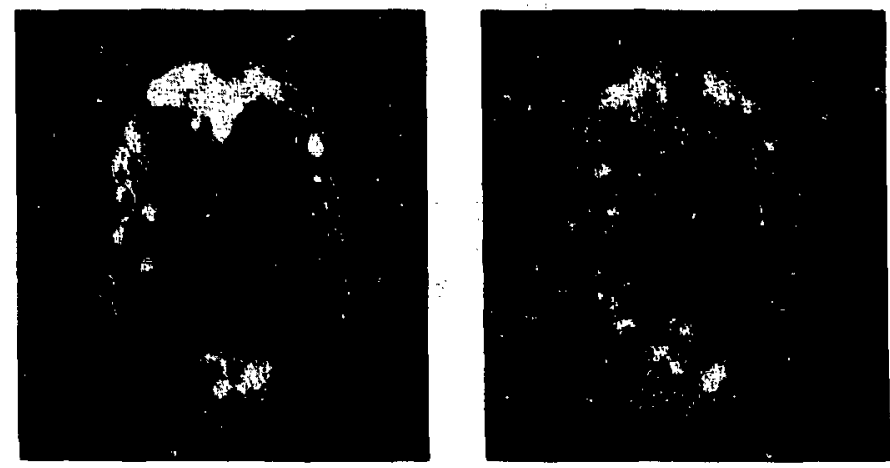

Fig. 1. Regional blood flow and metabolism in patients with Alzheimer's disease.
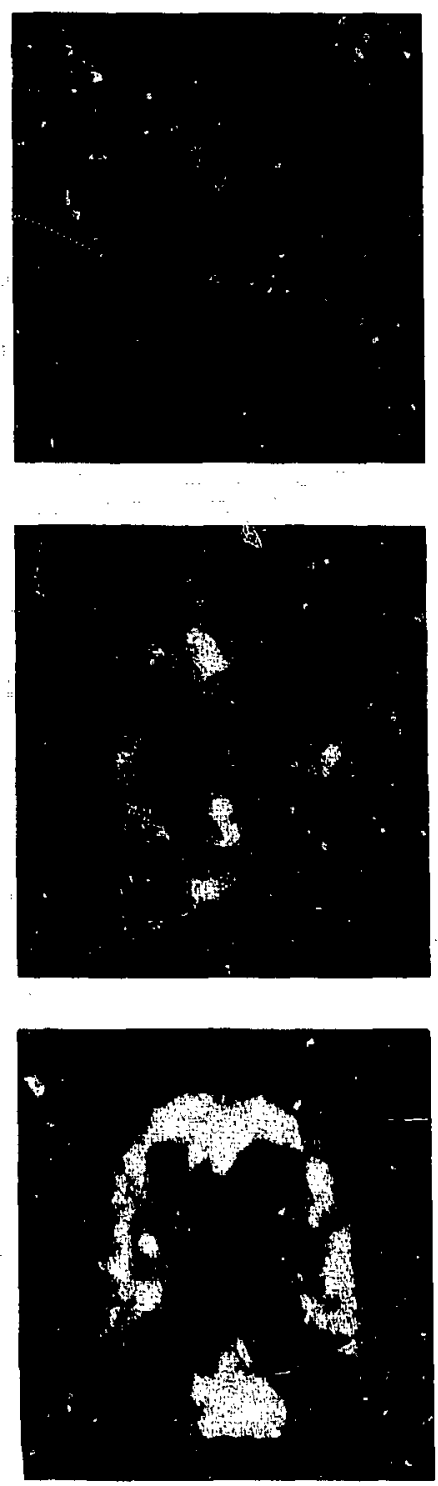

122-I-HIPDM

18-FDG

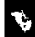

(XBB 850-10251) 
formed, and the results are described in the follo:ving report.

In previous reports other workers in our group have described the redured metabolic rate observed in the parietotemporal regions of patients suffering from Alzheimer's disease. We have studied three such patients with the flow-metabolism technique described above using ${ }^{122}$ !-HIPDM and ${ }^{18} \mathrm{FDG}$, and obtained the images shown in Fig. 1. The middle column of images shows the metabolism as measured by ${ }^{18} \mathrm{FDC}$, and the first column shows the regions of interest (ROIs) drawn over the ${ }^{18} \mathrm{FDG}$ image from which the numerical data were taken. The third column shows the 1221-HIPDM images; the same ROls were superimposed over these images because they preceded the ${ }^{18} \mathrm{FDC}$ with the patient in the same position.

In each region, from the PET data and from the measured plasma input function of each isotope, we calculated the regional cerebral metabolic rate (rCMR) from the ${ }^{18} \mathrm{FDG}$ data, and the regional cerebral blood flow (rCBF) from the 122/-HIPDM data. Because an extracted tracer acts as a molecular microsphere, the rCBF is calculated using the conservation of mass relationship; the rCMR was calculated from the steady state equation for glucose metabolic rate. The rCBF and rCMR were calculated as described above, using mean values of $k_{1}$, $k_{2}$, and $k_{3}$ from Alzheimer's patients obtained as described in last year's report, for the rCMRs. Again as in previous reports, the parietotemporal regions were compared to the frontal regions on each side by the following expression, in which the percent difference between the two regions, $\% F>T P$, is calculated by:

$\% \mathrm{~F}>\mathrm{TP}=\frac{\text { frontal }- \text { temporoparietal }}{1 / 2 \text { (frontal }+ \text { temporoparietal })} \times 100$

These patients, especially \#2 and \#3, were only mildly affected by the disease, but differences are seen when the rCBF and rCMR are calculated, as shown in Table 1 . In patient \#1 there are large differences in both $\mathrm{rCBF}$ and $\mathrm{rCMR}$, amounting to about $50 \%$ on both sides. Patient $\# 2$ has a small difference on the left side, but an insignificant difference on the right. Patient \#3 has differences on both sides of 14.1 to $30.6 \%$. It can be seen that the differences in rCBF are rather closely followed by the $r C M R$, and this is shown by the ratios $\mathrm{rCBF} / \mathrm{rCMR}$, which remain constant in all regions. This coupling ratio has the units of $\mathrm{ml}$ blood/mg glucose, and represents the $\mathrm{ml}$ of blood required to transport one $\mathrm{mg}$ of glucose to the brain in a region.

It appears from this preliminary group of three patients that regional cerebral brain blood flow is closely coupled to regional metabolic rate in Alzheimer's disease. If this proves to be the case with a larger series of patients, the rCBF measurement could be used to supplant the more expensive and time consuming ${ }^{18}$ FDG studies. The method should also have utility in measurement of flow-metabolism coupling in a great variety of other brain disorders, or of flow alone, the latter especially in studies on normal subjects in which brain function is manipulated experimentally to measure flow changes during normal brain function.

Table 1. Values for $\mathrm{CCBF}$ and $\mathrm{CCMR}$ obtained in three patients with Alzheimer's disease, and percent differences between frontal and parietofemporal regions.

\begin{tabular}{|c|c|c|c|c|c|c|}
\hline & \multicolumn{3}{|c|}{ Left } & \multicolumn{3}{|c|}{ Right } \\
\hline & Frontal & $\begin{array}{c}\text { Temporo- } \\
\text { parietal }\end{array}$ & $\% F>T P$ & Frontal & $\begin{array}{c}\text { Temporo- } \\
\text { parietal }\end{array}$ & $\% F>T P$ \\
\hline \multicolumn{7}{|l|}{ Patient \#1 } \\
\hline $\begin{array}{l}\text { rCBF } \\
\text { rCMR } \\
r C B F / r C M R\end{array}$ & $\begin{array}{l}78.1 \\
8.00 \\
9.8\end{array}$ & $\begin{array}{c}46.7 \\
4.57 \\
10.2\end{array}$ & $\begin{array}{l}50.3 \\
54.6\end{array}$ & $\begin{array}{c}88.4 \\
8.99 \\
9.8\end{array}$ & $\begin{array}{c}53.4 \\
5.90 \\
9.1\end{array}$ & $\begin{array}{l}49.4 \\
41.3\end{array}$ \\
\hline \multicolumn{7}{|l|}{ Patlent "12 } \\
\hline $\begin{array}{l}\text { rCBF } \\
\text { rCMR } \\
\text { rCBF/rCMR }\end{array}$ & $\begin{array}{l}60.5 \\
6.62 \\
9.1\end{array}$ & $\begin{array}{c}55.0 \\
5.85 \\
9.4\end{array}$ & $\begin{array}{r}9.5 \\
12.2\end{array}$ & $\begin{array}{c}76.4 \\
7.76 \\
9.8\end{array}$ & $\begin{array}{l}72.7 \\
7.87 \\
9.2\end{array}$ & $\begin{array}{r}5.0 \\
-1.4\end{array}$ \\
\hline \multicolumn{7}{|l|}{ Patient $\# 3$} \\
\hline $\begin{array}{l}\text { rCBF } \\
\text { rCMR } \\
\text { rCBF/rCMR }\end{array}$ & $\begin{array}{c}82.6 \\
9.18 \\
9.0\end{array}$ & $\begin{array}{c}71.7 \\
7.33 \\
9.8\end{array}$ & $\begin{array}{l}14.1 \\
22.4\end{array}$ & $\begin{array}{r}88.8 \\
9.9 \\
8.9\end{array}$ & $\begin{array}{c}65.2 \\
7.95 \\
8.2\end{array}$ & $\begin{array}{l}30.6 \\
22.2\end{array}$ \\
\hline
\end{tabular}




\title{
REGIONAL CEREBRAL BLOOD FLOW AND METABOLISM IN STROKE
}

\author{
William J. Jagust, Chester A. Mathis, Thomas F. Budinger, Peter E. Valk, and \\ Philip R. Weinstein*
}

Positron emission tomography (PET) is capable of making quantitative measurements of processes including cerebral blood flow and metabolism that have important implications for the study of stroke. The most common form of stroke is cerebral infarction, which is caused by occlusion of cerebral blood vessels. This occlusion results in diminished regional blood flow, which makes it difficult for brain cells to extract enough glucose and oxygen (the brain's source of energy) to meet their metabolic needs. The exact relationship between blood flow and metabolism is of crucial importance in brain tissue, since at some low flow values tissue may still be able to meet its metabolic needs by extracting more glucose from the blood. This introduces the concept of the "ischemic penumbra," which defines the relationship between flow and metabolism in the area around an infarct. The center of the infarct represents a region with so little blood flow that the tissue dies because it is unable to extract enough glucose and oxygen. Surrounding this area is a region of brain tissue which has diminished blood flow and is at risk of becoming infarcted, but is able to meet its metabolic needs by extracting more glucose and oxygen per flow volume. The fate of this brain region may be of critical importance in determining the ultimate outcome: if flow diminishes slightly, the tissue may become irreversibly infarcted, while if flow stabilizes or is increased, the tissue may be saved. The identification of this ischemic penumbra is thus of great importance, because therapeutic measures that may increase blood flow to this region can potentially diminish the size of the final infarct, resulting in a better clinical outcome for the patient. In addition, our ability to map the area of the ischemic penumbra is important in predicting the outcome of stroke and in better understanding the abnormal physiological processes that contribute to the ultimate clinical outcome.

At LBL our analysis of this problem has made use of two PET tracers, one for measuring blood flow and one for measuring metabolism. The flow tracer is the radiopharmaceutical HIPDM labeled

"Department of Neurosurgery, University of California San Francisco. with ${ }^{122} \mathrm{I}$. This -tracer has the advantage that the radionuclide, ${ }^{122} \mathrm{l}$, can be produced from a generator containing the parent radionuclide ${ }^{122} \mathrm{Xe}$, obviating the need for an on-site cyclotron. HIPDM is a "molecular microsphere" that crosses the intact blood brain barrier and is distributed in the brain in proportion to blood flow. By using PET to map this radioactivity distribution, and in conjunction with blood sampling and an appropriate physiological model, we can calculate regional rates of cerebral blood blow. Because of the short half-life of ${ }^{122}$, repeated scans can be performed at one sitting, oi more importantly, a metabolic study may be performed immediately following a flow study. The metabolic tracer that we have utilized is $\left({ }^{18} \mathrm{~F}\right.$ ffluorodeoxyglucose (FDG), which allows us to measure regional rates of glucose metabolism.

In order to evaluate the utility of this technique, we performed a flow and metabolism study in a 60-year-old male who had recently suffered a left-hemisphere cerebral infarct. The study was performed on the Donner 280-Crystal Tomograph (resolution $8 \mathrm{~mm}$ FWHM). After taking transmission measurements to correct for photon attenuation (a technique that improves quantitative accuracy), the HIPDM study was performed. Ten $\mathrm{mCl}$ of ${ }^{122}$ HIPDM were injected, and subsequent serial scans were taken at one tomographic level in the brain. At the same time, blood samples were withdrawn in order to obtain a time course of the plasma activity. The entire scan took 20 minutes. Following this, FDG was injected, and serial brain scans were taken in conjunction with sampling of blood to obtain the time course of blood FDG activity. We subsequently calculated regional rates of blood flow and glucose metabolism for multiple brain regions.

Figure 1 is a schematic diagram of the results of this experiment. In the unaffected right hemisphere, regioral cerebral blood flow (rCBF) ranges from $48 \mathrm{ml} / 100 \mathrm{~g} / \mathrm{min}$ in the posterior portion of the brain, to $57 \mathrm{ml} / 100 \mathrm{~g} / \mathrm{min}$ anteriorly. Regional cerebral glucose metabolism (rCMR) is 8.2-8.3 $\mathrm{mg} / 100 \mathrm{~g} / \mathrm{min}$ in all regions, and the ratio of flow to metabolism ( $\mathrm{rCBF} / \mathrm{rCMR}$ ) is $5.8-6.9 \mathrm{ml} / \mathrm{mg}$. In the infarcted hemisphere, both flow and metabolism are at their minimum in the center of the infarct and gradually increase with increasing dis- 


\section{Regional Blood Flow and Metabolism in Stroke}

\begin{tabular}{|c|c|c|c|c|c|}
\hline $\begin{array}{l}\frac{\mathrm{rCBF}}{\mathrm{rCMR}} \\
(\mathrm{ml} / \mathrm{mg})\end{array}$ & $\begin{array}{c}\text { rCMR } \\
(\mathrm{mg} / 100 \mathrm{~g} / \mathrm{m})\end{array}$ & $\begin{array}{c}\mathrm{rCBF} \\
(\mathrm{ml} / 100 \mathrm{~g} / \mathrm{m})\end{array}$ & $\begin{array}{c}\mathrm{rCBF} \\
(\mathrm{ml} / 100 \mathrm{~g} / \mathrm{m})\end{array}$ & $\begin{array}{c}\text { rCMR } \\
(\mathrm{mg} / 100 \mathrm{~g} / \mathrm{m})\end{array}$ & 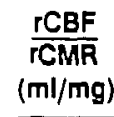 \\
\hline & & & & 6.7 & 6.7 \\
\hline \multirow[t]{2}{*}{6.9} & 8.3 & & -26 & 4.8 & 5.4 \\
\hline & & & & 3.5 & 5.7 \\
\hline \multirow[t]{2}{*}{6.6} & 8.2 & & & 2.7 & 5.6 \\
\hline & & & & 4.9 & 5.1 \\
\hline \multirow[t]{3}{*}{5.8} & 8.3 & & & 6.1 & 6.2 \\
\hline & & & & 7.3 & 6.3 \\
\hline & & & & 7.6 & 6.4 \\
\hline
\end{tabular}

Fig. 1. A schematic diagram of regional cerebral blood flow ( $\mathrm{CCBF}$ ) and metabolism (rCMR) in multiple brain regions in a patient with a left-hemisphere infarct (right and left are reversed, such that the left hemisphere is to the viewer's rightl. The infarcted area is shown in black, and progressively lighter shaded areas represent tissue progressively more distant from the left hemisphere infarct. The right side of the brain is divided into three brain regions, one of these is placed symmetrically to the infarct.

(XBL B62-8646B)

tance from the center. Most importantly, the flow to metabolism ratio is distinctly diminished in a brain region next to the infarcted area, while most other brain regions show a value that is similar to the normal ratio seen on the other side. This reduced ratio (5.1), suggests that this brain region is extracting more glucose per $\mathrm{ml}$ of blood flow, and thus represents the ischemic penumbra, which is at risk for further infarction, or which may potentially be salvaged.

The potential utility of this type of study is important for several reasons. The ability of the clinician to know whether a patient has a large potentially viable region of brain can clearly guide therapy, whether medical, as in prescribing anticoagulants or platelet antagonists, or surgical, such as carotid artery surgery. In addition, by studying many patients with stroke we may be capable of ascertaining the relationship of the size of the ischemic penumbra to ultimate clinical outcome, thus improving our ability to prognosticate. Finally, by accurately quantitating cerebral blood flow and metabolism in multiple brain regions during stroke, our basic understanding of the abnormal physiological processes occurring during stroke will be improved. 


\title{
SINGLE PHOTON EMISSION COMPUTED TOMOGRAPHY: A PRACTICAL CLINICAL MODALITY FOR THE LABORATORY DIAGNOSIS OF DEMENTIA
}

\author{
William J. Jagust, Thomas F. Budinger, Bruce R. Reed," J. Philip Seab, and \\ Miguel M. Colina
}

The diagnosis of dementia, a common condition of older age that results in the progressive loss of intellectual function, often presents the clinician with significant difficulty. The most common cause of dementia, Alzheimer's disease (AD), cannot be positively diagnosed in the laboratory except by brain biopsy, and it may be difficult to distinguish from other causes of dementia as well as from normal aging. At LBL we have discovered a specific pattern of brain function that differentiates $A D$ patients from healthy control subjects. This function, brain glucose metabolism, was quantitated by using positron emission tomography (PET) with the tracer ${ }^{18} \mathrm{~F}$-fluorodeoxyglucose (FDG).

PET studies using FDG in $A D$ patients have shown deficits in brain glucose metabolism that are most severe in temporoparietal cortex. it has been suggested that this finding would have potential diagnostic utility. However, although PET provides a method for accurate quantitation of tracer uptake, its clinical utility is limited because the technique requires the availability of a regional or local cyclotron or positron emitter generator system to provide the needed radionuclides. Single photon emission computed tomography (SPECT), however, utilizes radionuclides of the sort employed in current routine clinical nuclear medicine studies and therefore offers a technique that is potentially more widely available.

This study was designed to investigate whether SPECT measurements of regional cerebral blood flow (rCBF) are capable of demonstrating the same temporoparietal deficits that have been seen in PET studies of glucose metabolism, and to evaluate the utility of SPECT in differentiating patients with varying clinical severities of $A D$ from healthy control subjects and patients with the second most common cause of dementia, multiple stroke or multiinfarct dementia (MID). Single photon-emitting radiopharmaceuticals that are capable of measuring cerebral function have recently been developed and are commercially available. One such tracer, $\mathrm{N}$-isopropyl-p-iodoamphetamine (IMP), labeled with

\footnotetext{
-Department of Neurology, Martinez V.A. Medical Center and
} University of California, Davis
${ }^{123} 1$ is distributed in brain in proportion to blood flow and when scanned tomographically, can provide three-dimensional information about $\mathrm{rCBF}$.

We studied nine patients with $A D$, all of whom met current research criteria for $A D$. All subjects were free of significant medical illnesses, including hypertension, were taking no medications, and had experienced a progressive loss of cognitive functioning for at least one year, with memory loss as the most significant symptom. Neuropsychological testing was performed on all subjects and the Mini Mental Status Questionnaire (MMSQ) scores were used as a measure of disease severity.

Five control subjects were free of all significant medical, neurological, and psychiatric illnesses. All were functioning at normal intellectual levels, and were taking no medications. These subjects were spouses of the demented patients.

Two patients with MID were studied. These patients had histories of multiple strokes, focal neurological signs, and showed $x$-ray computed tomography $(C T)$ evidence of cerebral infarction. In addition, CT in both patients demonstrated marked periventricular lucencies. One patient had a nuclear magnetic resonance (NMR) imaging study that demonstrated markedly increased signal in the periventricular regions on a $\mathrm{T} 2$ weighted image.

SPECT imaging was performed on the Donner Laboratory's modification of the Cleon multidetector focused collimator system, which has a resolution of $14 \mathrm{~mm}$ full-width at half-maximum (FWHM) in the plane of the section, and a slice thickness of $13 \mathrm{~mm}$. The instrument collects data for one brain level at a time, using $12 \mathrm{Nal}$ crystals that tangentially scan the head.

Patients were positioned in the scanner with eyes and ears unoccluded, in a quiet room, with the plane of section parallel to the canthomeatal line. The blood flow tracer, $N$-isopropyl-piodoamphetamine was injected as a $5-\mathrm{mCi}$ i.v. bolus. Scanning began 10 minutes following injection and continued for 60 minutes. Each brain level was scanned for 20 minutes, and generally three levels were scanned for each patient. We quantitatively analyzed data obtained only at the midventricular level for this study. Regions of 
interest were drawn with reference to a standard CT brain anatomic atlas in right and left frontal (F) and temporoparietal (TP) cortex as well as around the whole tomographic slice (WS). The system software then calculated the regional radioactivity count density in the form of counts $/ \mathrm{cm}^{3}$ for the tissue volume chosen. These activity densities were then used to construct a ratio of activity of each brain region to that of the activity in the entire tomographic slice. We calculated the ratio TP/WS by taking the mean activity density of left and right TP cortex for the numerator, and derived a similar ratio for $F / W S$. Thus, quantitative analysis was based on evaluation of amounts of activity in one brain region relative to activity in the entire slice.
Figure 1 shows SPECT-IMP images for an AD patient and a control subject at the mid-ventricular and basal ganglia levels. The AD patient demonstrates diminished radionuclide uptake in temporoparietal cortex bilaterally. This pattern was visually apparent in all of our patients with $A D$. All control subjects had a homogeneous cortical pattern of tracer distribution. Patients with MID demonstrated diminished tracer uptake in both frontal lobes.

The TP/WS ratio differentiated all $A D$ patients from controls and MID, without any overlap. The difference between $A D$ and controls was significant $(p<0.0005)$, as was the difference between $A D$ and MID patients $(p<0.005)$. The clinical evaluation of the disease severity, as measured by the
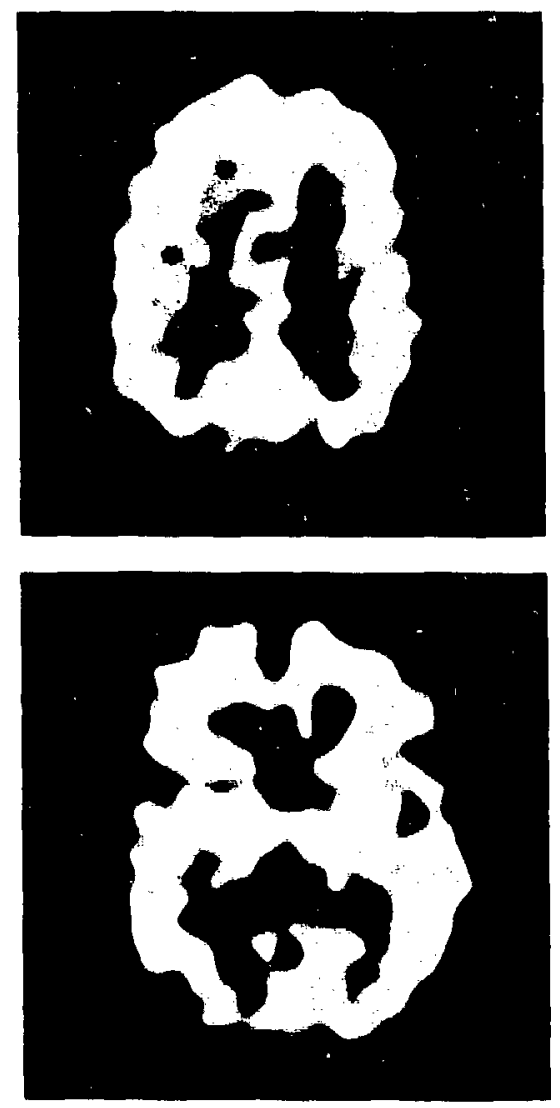

Control
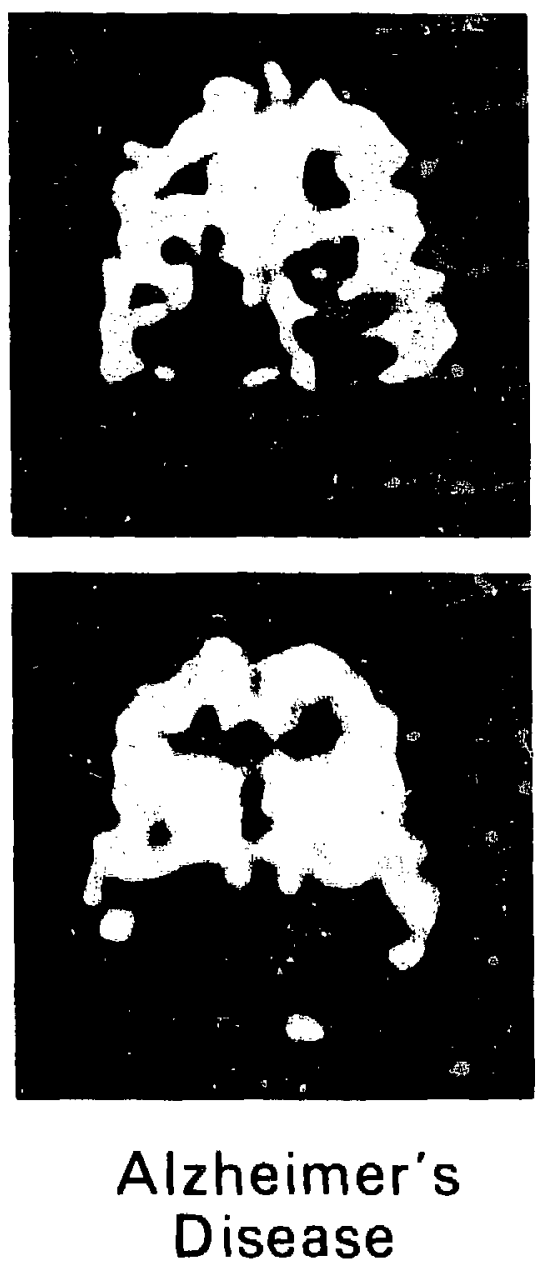

Fig. 1. Representative SPECT-IMP images from a patient with Alzheimer's disease and a control subject. Each image represents 20 minules of data collection. The AD patient shows diminished uplake in temporal and parietal cortical brain regions, while the control subject shows and even cortical distribution of tracer.

(XBB 869-7044) 
MMSQ, correlated highly with this ratio in the $A D$ group (Pearson $r=0.82, p<0.01$ ). Controls and MID patients did not differ on this measure. Evaluation of the F/WS ratio showed that MID patients had lower values for this ratio than both controls and $A D$ patients, reflecting the diminished blood flow seen visually in frontal cortical regions. The MID group is significantly lower than the $A D$ group for this ratio ( $p<0.05$ ), while other comparisons are not statistically significant.

The primary finding to emerge from this study is that the rCBF pattern of temporoparietally diminished blood flow measured using SPECT and IMP is similar to the pattern seen using PET with a variety of metabolic and flow tracers. Furthermore, this pattern is sufficiently distinctive to allow differentiation of $\mathrm{AD}$ patients both from healthy controls and from patients with MID without any overlap between the $A D$ patients and either of the other groups. The lack of overlap between the AD and control groups is important because three of the $A D$ patients with MMSQ scores of 26,27 , and 28 were very mildly affected. In each case either a family member or a family physician had recently thought the patient normal. The ratio of TP to whole slice activity also correlated strongly with disease severity measured neuropsychologically. The ability of SPECT to discriminate these patients from normal aged individuals suggests that this imaging modality is sensitive in early stages of the disease. These findings taken together suggest that SPECT will be of utility in diagnosing dementia, and will also provide a method for estimating the severity of the disease.

\title{
USE OF GENERATOR-PRODUCED ${ }^{122}$ I FOR STUDIES OF HEART AND BRAIN FUNCTION
}

\author{
Chester A. Mathis, Alexander T. Shulgin, Steven M. Moerlein, Yukio Yano, \\ Kathleen M. Brennan, Thornton Sargent III, and Thomas F. Budinger
}

The ability to quantitate radiotracers in vivo following injection of labeled compounds is a unique feature of positron-emission tomography (PET). The nonavailability of useful positron emitting radionuclides for PET studies has contributed to limiting the application of this technique to a relatively few PET centers. Due to their short half-life, most positron emitting radionuclides must either be produced on-site by a cyclotron or harvested from long-lived parent radionuclides in a generator system. There are a number of positron generator sys. tems that contain parent radionuclides whose halflives are long enough to permit shipment from the site of production to a distant PET facility. Most of these generator systems produce metal ions such as ${ }^{68} \mathrm{Ga}^{+3},{ }^{82} \mathrm{Rb}^{+},{ }^{52 m} \mathrm{Mn}^{+2},{ }^{62} \mathrm{Cu}^{+2}$, and ${ }^{128} \mathrm{Cs}^{+}$, which are not ideal for incorporation into radiopharmaceuticals used to evaluate brain and heart function. We have explored the potential usefulness of the ${ }^{122} \mathrm{Xe} /{ }^{122}$ generator system because the half-life of the parent ${ }^{122} \mathrm{Xe}(20 \mathrm{hr})$ is long enough to permit shipment, and the daughter's radionuclidic chemical properties allow rapid incorporation into a variety of organic radiopharmaceuticals. In addition, the short half-life of ${ }^{122}$ ( $\left.3.6 \mathrm{~min}\right)$ makes possible repeated injections in the same subject due to its rapid decay and relatively low radiation dose per injection.

\section{DEVELOPMENT OF THE ${ }^{122} \times E /{ }^{122} \mid$ GENERATOR}

A ${ }^{122} \mathrm{Xe} /{ }^{122} 1$ generator system (Fig. 1) has been developed to rapidly separate the ${ }^{122}$ I positron emitter from the parent ${ }^{122} \mathrm{Xe}$ gas and allow the rapid incorporation of the ${ }^{122} \mid$ daughter inio several useful radiopharmaceuticals. ${ }^{1}$ The ${ }^{122}$ Xe gas is produced at Crocker Nuclear Laboratory, UC Davis in collaboration with Dr. M. Lagunas-Solar, via the $(p, 6 n)$ reaction of $68-\mathrm{MeV}$ protons on a molten sodium iodide target. The ${ }^{122} \mathrm{Xe}$ is shipped to LBL in a lead-shielded drum and transferred into a heavily shielded glove box by cryogenic pumping at liquid nitrogen temperatures. Once contained insicle the generator, the ${ }^{122}$ / can be separated from the ${ }^{122} \mathrm{Xe}$ by cryogenic transfer of the ${ }^{122} \mathrm{Xe}$ to a storage vessel leaving behind the ${ }^{122}$-iodide on the the walls of the reaction vessel. The ${ }^{122}$-iodide can then be incorporated into radiopharmaceuticals by several different reaction techniques.

Typical 6-hour cyclotron bombardments at Crocker Nuclear Laboratory produce approximately $400 \mathrm{mCi}$ of ${ }^{122} \mathrm{Xe}$, which is utilized for PET studies by the Research Medicine Group on the following day. The time required to harvest the ${ }^{122}$ |-iodide from the ${ }^{122} \mathrm{Xe}$ is approximately 90 seconds, and the efficiency of the harvest procedure is approximately $60 \%$. In practical terms, the generator sys- 


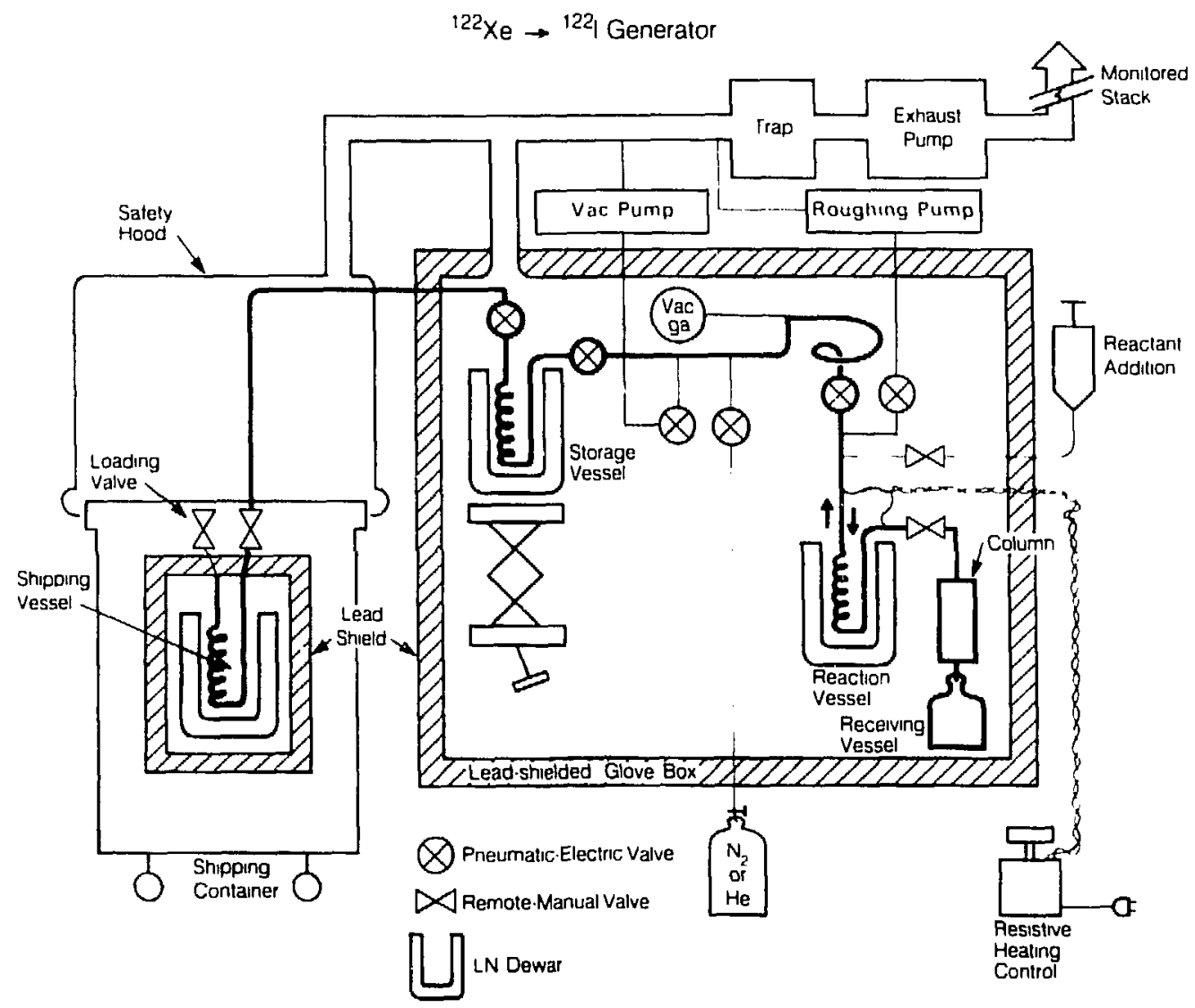

Fig. 1. Schematic drawing of the ${ }^{122} X \mathrm{f}^{122} \mathrm{I}$ generator system. The ${ }^{122} \mathrm{Xe}$ is transferred from the shipping drum to a storage vessel located inside a lead-shielded glove box. Once loaded into the generator system, the ${ }^{122} X e$ can be cryogenically transferred to the ingrowih vessel and back to the storage vessel every 30 minutes 10 provide ${ }^{122}$ l for radiochemical incorporalion into compounds for use with PET studies.

(XBL 840.7160)

tem is capable of producing $40 \mathrm{mCi}$ of ${ }^{122}$-iodide from $100 \mathrm{mCi}$ of ${ }^{122} \mathrm{Xe}$ every 30 minutes for the entire day. Due to the short half-life of 122 this permits repeat 122 studies in the same subject every 30 minutes.

\section{I RADIOPHARMACEUTICAL DEVELOPMENT}

Successful application of the ${ }^{122} \mathrm{Xe} /{ }^{122}$ generator requires labeling techniques that give high radiochemical yields within the short half-life of ${ }^{122}$ I. To produce useful quantities of radiopharmaceuticals for PET studies, we have found that the effective limitations imposed on the labeling efficiency and speed are a minimum of $50 \%$ radiochemical yields within 120 seconds. There are several rapid labeling techniques that are available to the radiochemist, and we have critically evaluated them in labeling a variety of radiopharmaceuticals. These labeling lechniques include direct labeling of highly chemically activated precursors, rapid exchange labeling of ${ }^{122}$-iodide for cold iodide $\left({ }^{127} 1\right)$ or bromine, and displacement of metallic groups from precursor molecules to produce the radiopharmaceutical. We have successfully labeled several compounds that are inherently chemically activated; these include dimethoxyamphetamine derivatives for brain ${ }^{2,3}$ and heart blood flow studies and phenylpiperazinium compounds for heart blood flow studies. Rapid exchange of ${ }^{122}$ | for ${ }^{127}$ i has been utilized to label ${ }^{122}$ |-HIPDM (see articles in this and the previous (1985) Annual Reports) for cerebral blood flow sludies. However, the reliance upon the use of highly activated molecules for direct and exchange labeling with ${ }^{122}$, severely limits the types of radiopharmaceuticals 
that one can employ for PET studies. Thus, we were prompted to examine the use of metallated precursors to determine their suitability for use with ${ }^{122}$ I, with the desire to extend the use of this radionuclide to many nonactivated compounds.

Useful radioiodination yields with ${ }^{12 \mathrm{~J}} \mathrm{l},{ }^{125} \mathrm{I}$ and ${ }^{131}$ I were reported by other investigators using reactions that involve displacement of aromatic tin, silicon, boron, germanium, and mecury groups. The reaction times reported in these studies exceed the practical limits for labeling with ${ }^{122} \mathrm{I}$, prompting us to examine the use of these precursors at short reaction times to examine their suitability for use with ${ }^{122}$ I. As shown in Table 1, aromatic iododestannylation and iododemercuration resulted in the greatest labeling yields. Radioiodination yields via destannylation and demercuration reactions were also impervious to the effects of ring activation (Fig. 2), with high yields achieved from substrates activated $\left(\mathrm{OCH}_{3}\right)$, nonactivated $(\mathrm{F})$ and deactivated $\left(\mathrm{CF}_{3}\right)$ towards iodination. We believe that the regiospecificity (i.e., putting ${ }^{122}$ I where one wants it to go on the molecule), high radiochemical yields, and lack of solvent or substituent effects make iododestannylation the method of choice for labeling aromatic sites with ${ }^{122}$. The use of mercurated or germylated precursors may also be useful for the production of ${ }^{122}$ I-labeled radiopharmaceuticals under some conditions such as in acidic reaction media or when utilizing activated compounds that could lose the metallated grcup to electrophiles other than ${ }^{122}$ I. We plan to utilize various stannylated precursors to produce new useful ${ }^{122}$ labeled compounds for PET applications.

Table 1. Radiochemical yields for the ${ }^{122}$ I-iododemetallation reactions.

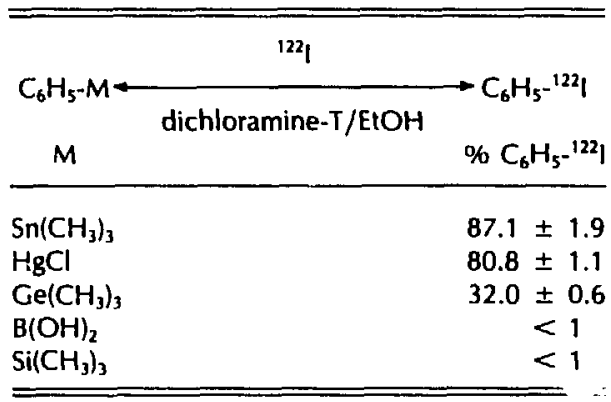

\section{No-Carrier-Added Aromatic lododemetallation} of Stannylated and Mercurated Arenes

\section{lododestannylation:}

Relative

Isomeric Yield<smiles>COc1ccccc1OS(=O)(=O)c1ccccc1</smiles>

Total Aromatic Substitution Yield ( $=60$ s, DCT/EtOH)

$\begin{array}{ll}131 \mathrm{l} & 93.8 \pm 2.1 \\ 122 \mathrm{l} & 94.2 \pm 0.7 \\ \text { Melative } & \end{array}$

Total Aromatic Substitution Yield ( $t=60 \mathrm{~s}, \mathrm{DCT} / \mathrm{EtOH})$

$$
\text { 131 I: }
$$

$67.4 \pm 1.1$

$93.7 \pm 1.2$

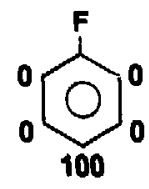<smiles>O=C1C=CC(C(F)(F)F)C(O)C1</smiles>

$74.9 \pm 2.0$

$65.6 \pm 2.0$

$92.6 \pm 1.4$

Fig. 2. Relative and absolute isomerir yields of various $1 \%$ and ${ }^{122} /$-labeled compounds following 30-second reaction times at room temperature in ethanol solvent using dichloramine-T (DCT) oxidant. The tin $\left(-\mathrm{Sn}\left(\mathrm{CH}_{3}\right)_{3}\right)$ derivatives resulted in high, regiospecific yields in all cases. 


\section{USE OF ${ }^{122}$-LABELED RADIOPHARMACEUTICALS FOR BRAIN AND HEART FUNCTION STUDIES}

Studies to evaluate several ${ }^{122}$ l-labeled dimethoxyamphetamine compounds ${ }^{2,3}$ as cerebral blood flow agents continue. The criteria that must be satisfied include low lung uptake of the compound, high first-pass cerebral extraction (>90\%) of the radiotracer from the brain blood supply, a low metabolic degradation rate of the compound, and retention of the compound by the brain (low egress rate). We have synthesized and evaluated a number of compounds that satisfy these requirements to varying degrees in animals. Until the time that an "ideal" cerebral blood flow agent is synthesized for use with ${ }^{122} \mathrm{l}$, we will continue to employ ${ }^{122}$ I-HIPDM for cerebral blood flow studies in human applications that include stroke patients [see this and previous (1985) Annual Reports], Alzheimer's disease (see this Annual Report), and tumor patients. While ${ }^{122}$-HIPDM does not completely satisfy all the criteria of an ideal cerebral blood flow agent, it does successfully meet most requirements and can be utilized for quantitative studies with PET if adjustments to the blood flow equation are made for its nonideal behavior.

Recently we have found that ${ }^{122}$-labeled quaternary nitrogen compounds such as 2,4dimethoxy-N,N-dimethyl-N-ethyl-5-iodoamphetaminium (Figs. 3 and 4) may be useful as myocardial blood flow (MBF) agent. In collaboration with Dr. R. Hanson of the Northeastern University Pharmacology Department, we have also investigated the potential of iodophenylpiperazinium compounds as MBF agents (Fig. 4). Preliminary indications in rats and dogs are that these classes of compounds are rapidly taken up by the myocardium, clear the blood pool, and remain in the heart for several hours after intravenous injection. We are continuing to evaluate these agents for use as quantitative MBF agents for future PET applications in patients with myocardial infarcts. Another potential MBF agent available from a generator system $\left({ }^{82} \mathrm{Rb}^{+}\right)$has been shown to exhibit a decreased extraction efficiency at elevated flow rates relative to low flow values. This variation in extraction efficiency makes quantitative MBF determinations complicated. We are seeking a MBF agent whose extraction efficiency does not vary with flow and exhibits the other requisite properties of an ideal MBF agent.

To date, we have utilized ${ }^{122}$ /-labeled compounds for cerebral blood flow studies in animals and human subjects and myocardial blood flow studies in animals. Future applications of ${ }^{122}$ I include labeling compounds for cerebral and myocardial blood volume studies (such as albumin) and use of iodophenyl fatty acids for myocardial metabolism assessment.
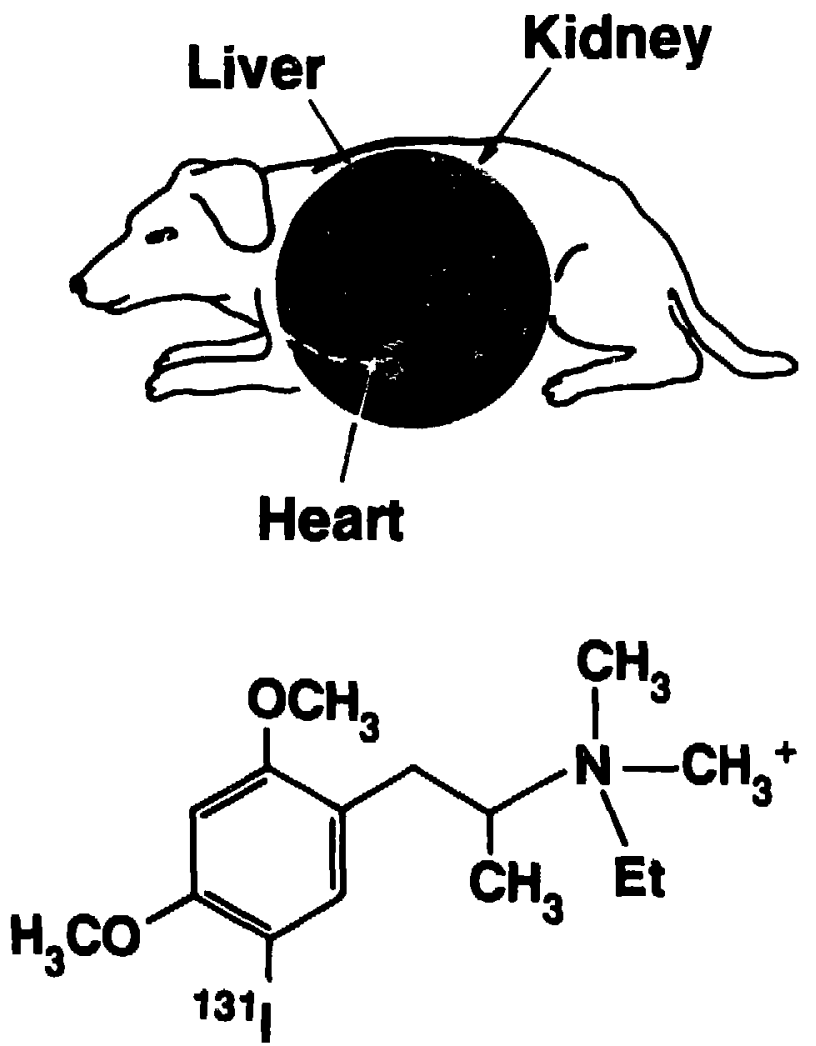

2,4-Amphetaminium

Fig. 3. Anger camera image of a dog heart about $t$ hr after the injection of the $13 /$ labeled compound whose structure is indicated. The image shows that a large amount of the injected activity remains in the normal heart at times as long as $1 \mathrm{hr}$ postinjection.

(XBB 860-8900) 
${ }^{122}$ I-Phenylpiperazinium

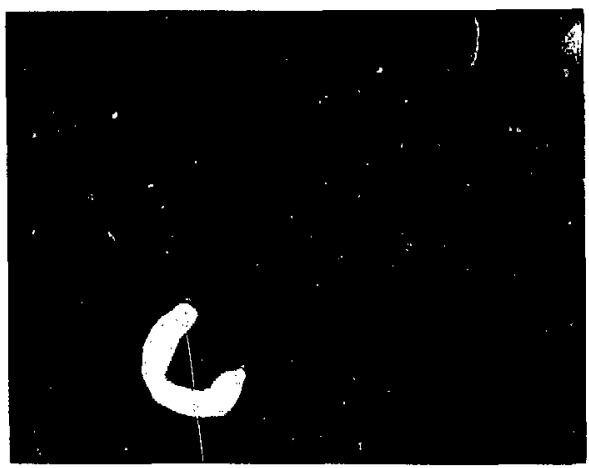

${ }^{122}$ I-Amphetaminium

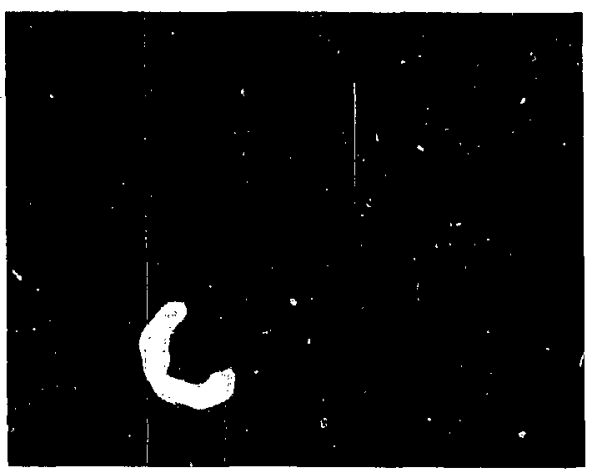

$$
\nabla=\text { myocardium } \bullet=\text { blood pool }
$$

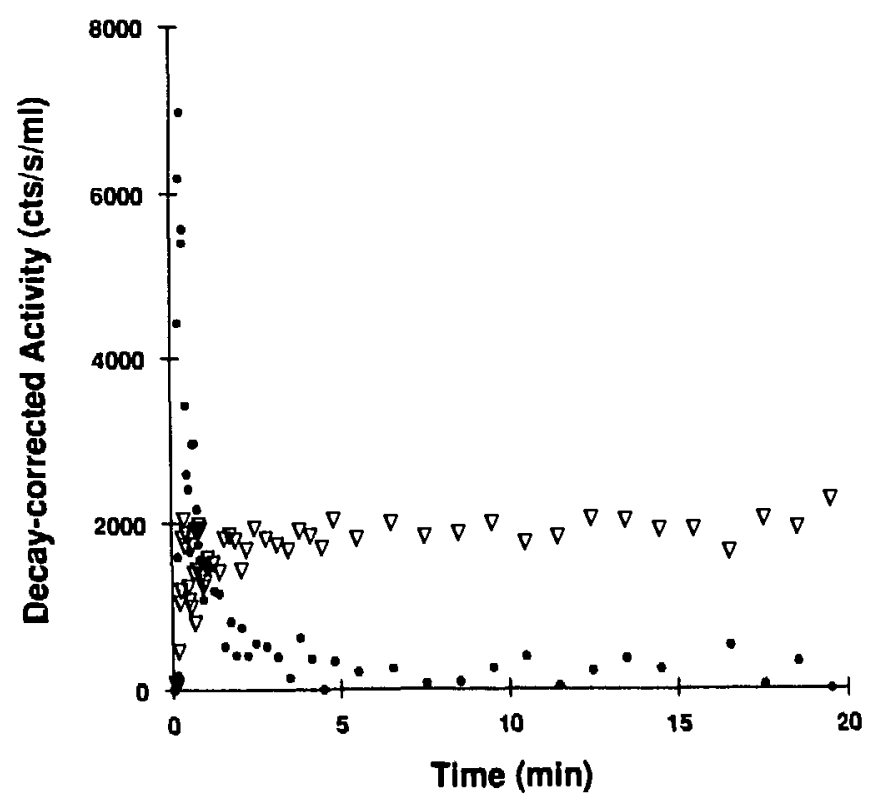

Fig. 4. Cross-sectional PET images of the normal dog mjocardium after injection of ${ }^{122}$ /-labeled 2,4-dimethoxy-5iodoamphetaminium (struclure shown in Fig. 3) and a ${ }^{122}$-labeled phenylpiperazinium derivative. Dynamic decaycorrected PET data of the myocardial uptake and retention and left ventricular blood pool ciearance of the ${ }^{122}$ amphetaminium are shown in the bottom panel. This compound has good uptake and retention in the myocardium and rapid clearance from the blood indicating its potential as an MBF agent.

(XBB 868-6901A) 


\section{REFERENCES}

1. Mathis, C.A., Lagunas-Solar, M.C., Sargent III, T., Yano, Y., Vuletch, A., and Harris, L.). A ${ }^{122} \mathrm{Xe}^{-}{ }^{122} \mathrm{I}$ generator for remote radioiodinations. Appl. Radiat. Isot., Int. J. Radiat. Appl. Instrum., Part A 37, 258-260 (1986).

2. Mathis, C.A., Shulgin, A.T., and Sargent III, T. Synthesis of ${ }^{122}$ - and ${ }^{125}$ /-labelled metadimethoxy-N,N-dimethyliodophenylisopropylamines. J. Label Cmpd. Radiopharm. 23,
115-125 (1986).

3. Mathis, C.A., Sargent III, T., and Shulgin, A.T. lodine-122-labeled amphetamine derivative with potential for PET brain blood-flow studies. J. Nucl. Med. 26, 1295-1301 1985.

4. Moerlein, S.M., Mathis, C.A., and Yano, Y. Comparative evaluation of electrophilic aromatic iododemetallation techniques for labeling radiopharmaceuticals with iodine-122. Appl. Radiat, Isot., Int. J. Radiat. Appl. Instrum., Part $A$ (in press).

\title{
INITIAL RESULTS FROM THE DONNER 600-CRYSTAL POSITRON TOMOGRAPH
}

\author{
Stephen E. Derenzo, Ronald H. Huesman, John L. Cahoon, Allen B.Geyer," \\ Donald C. Uber," Tony Vuletich," and Thomas F. Budinger
}

\section{INTRODUCTION}

There is considerable interest in improving the spatial resolution in positron emission tomography for the brain and neck, because many important functional regions here are too small to be investigated quantitatively with $10-$ to $20-\mathrm{mm}$ resolution. For example, a spatial resolution of 2 to $3 \mathrm{~mm}$ FWHM will permit measurement of the dynamics of blood flow in the cerebral arteries and the uptake and disappearance of labeled tracers in small functionally distinct brain nuclei.

This work shows that 3-mm-wide BGO crystals can provide excellent resolution with good efficiency, in spite of factors such as the range of positrons in tissue, deviations from $180^{\circ}$ emission, offaxis penetration through the crystals, and Compton scattering resulting in multiple crystal interactions. This tomograph is designed for quantitation of tracer activity in 5-mm regions, which requires a spatial resolution of 2-3 $\mathrm{mm}$, this in turn requiring sampling the projections with a frequency of one per $\mathrm{mm}$ or finer.

\section{DETECTORS AND GANTRY}

Each BGO crystal is individually coupled to a 14-mm-diameter phototube (Fig. 1, Table 1). The BGO crystals have three different shapes, depending on the orientation of the associated phototube. These shapes were chosen experimentally to max-

*Engineering Division, LBL. imize the light output and pulse height resolution. Of the 600 crystals, 240 are $10 \times 3 \times 30 \mathrm{~mm}$ deep with a $45^{\circ}$ cut (crystals $A$ and $E$ in Fig. 1), 240 are $10 \times 3 \times 23 \mathrm{~mm}$ deep (crystals $B$ and $D$ in

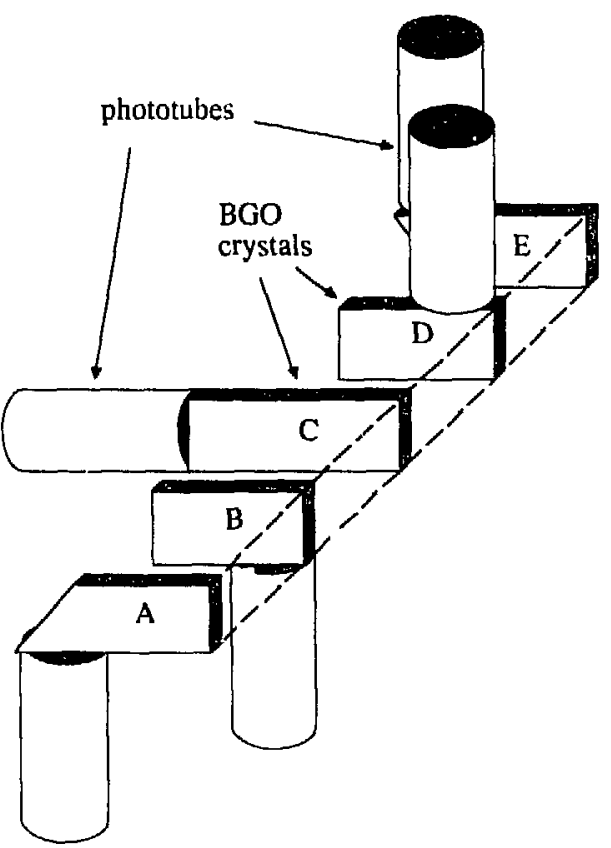

Fig. 1. Sketch of defector configuration. Each BGO crystal is coupled individually to a 14 -mm-diameter phototube. Crystals $A$ and $E$ are $30 \mathrm{~mm}$ long, $10 \mathrm{~mm}$ high, and $3 \mathrm{~mm}$ thick, cut at a $45^{\circ}$ angle to improve light collection efficiency. Crystals $B$ and $D$ are $23 \mathrm{~mm}$ long, $10 \mathrm{~mm}$ high, and $3 \mathrm{~mm}$ th ick. Crystal $C$ is $30 \mathrm{~mm}$ long, $10 \mathrm{~mm}$ high, and $3 \mathrm{~mm}$ thick.
(XBL 8610-3942) 
Table I. Tomograph data.

\begin{tabular}{lc}
\hline Number of BGO crystals & 600 \\
Detector ring diameter & $60 \mathrm{~cm}$ \\
Patient port diameter & $30 \mathrm{~cm}$ \\
BGO crystal size & $3 \mathrm{~mm} \times 10 \mathrm{~mm}$ \\
& $\times 23$ to $30 \mathrm{~mm}$ deep \\
BGO crystal & $3.14 \mathrm{~mm}$ \\
Center to center spacing & 2 \\
Number of sampling positions & 120,000 \\
\hline
\end{tabular}

Fig. 1), and 120 are $10 \times 3 \times 30 \mathrm{~mm}$ deep (crystal $C$ in Fig. 1). Only certain regions of the crystals are coupled to the phototubes and the other crystal surfaces are polished and coated with dry $\mathrm{MgO}$ reflector. The reflector coating between the crystals is thin, and the center-to-center crystal spacing is $3.14 \mathrm{~mm}$. Since the phototubes are coupled to three sides of the crystal array, this approach is limited to a single ring of crystals. The detectors have a pulse height resolution of about $25 \%$ FWHM for $511-\mathrm{keV}$ annihilation photons.

The lead shielding has a gap that is adjustable from 1 to $10 \mathrm{~mm}$, an inner diameter of $30 \mathrm{~cm}$, and an outer diameter of $60 \mathrm{~cm}$. The section thickness is $5 \mathrm{~mm}$, corresponding to the geometric axial response.

The detectors are mounted on two C-shaped arms that are hinged together at one point on the detector ring. A cam mechanism, opposite the hinge, can open the arms $3.14 \mathrm{~mm}$, which is the detector center-to-center spacing. When the arms are shut, the detector ring forms a circle of 600 closely packed detectors. When the arms are opened, the detector ring approximates a 601 crystal ring with one detector missing. The combined data set provides 120,000 projection measurements with 100 rays at each of 1200 angles. Lateral spacing is staggered in angle, resulting in a linear sampling of $0.79 \mathrm{~mm}$, which is one-fourth of the center-to-center crystal spacing.'

Reconstructions were performed using the convolution method on $1.79-\mathrm{mm}$ projection bins. The reconstructed images of the $0.35-\mathrm{mm}$-diameter ${ }^{22} \mathrm{Na}$ wire solurce at the center of the tomograph have a PSF that is circular with a FWHM of $2.9 \mathrm{~mm}$ (Fig. 2). At a distance of $8 \mathrm{~cm}$ from the center of the gantry, the PSF is elliptical with a radial FWHM of $4.0 \mathrm{~mm}$ and a tangential FWHM of $3.0 \mathrm{~mm}$. The ${ }^{22} \mathrm{Na}$ source used here (and the ${ }^{18} \mathrm{~F}$ source used in brain studies) has positron energies below $600 \mathrm{keV}$, and the blurring caused by the positron range is less than $1 \mathrm{~mm}$.,3 The radial elongation visible in Fig. 2 for sources far from the gantry center is due to crystal penetration.
Table 2. Results.

\begin{tabular}{ll}
\hline Timing resolution (first photoelectron) & $5 \mathrm{nsec}$ FWHM \\
Coincidence window (90\% acceptance) & $9 \mathrm{nsec}$ \\
Photopeak resolution & $20-30 \%$ FWHM \\
Detector pair resolution & $2.4 \mathrm{~mm}$ FWHM \\
Reconstructed image resolution: & \\
$\quad$ at $0 \mathrm{~cm}$ & $2.9 \mathrm{~nm} \mathrm{FWHM}$ \\
at $8 \mathrm{~cm}$ & $3.0 \times 4.0 \mathrm{~mm} \mathrm{FWHM}$ \\
\hline
\end{tabular}

These results can be understood in terms of three factors (Table 3): 1) the detector size, which contributes a triangular PSF with $1.5 \mathrm{~mm}$ FWHM at the center of the gantry, 2) the deviations from $180^{\circ}$ emission, which contribute a nearly Gaussian PSF with $1.3 \mathrm{~mm}$ FWHM, 3) the range of the positrons in tissue, which we approximate here $\left({ }^{18} \mathrm{~F}\right.$, ${ }^{22} \mathrm{Na}$ ) to be $0.5 \mathrm{~mm}$. The resultirig detector-pair resolution is $2.0 \mathrm{~mm}$ FWHM, which is degraded by about $45 \%$ in the reconstructed image to $2.9 \mathrm{~mm}$ FWHM. We expect an improvement in the reconstructed image resolution when convolutions are performed on $0.79 \mathrm{~mm}$.

Figure $3(a)$ is a sketch of the pie-shaped hotspot phantom previously reported. ${ }^{4}$ Figure 3(b) shows an image of 200 million ${ }^{18} \mathrm{~F}$ events taken with the Donner 280-Crystal Positron Tomograph using clam sampling and reconstrucled with $2.5-\mathrm{mm}$ projection bins. Figure 3(c) shows an image of 35 million ${ }^{68} \mathrm{Ga}$ events taken with the Donner 600 Crystal Positron Tomograph in stationary mode and reconstructed with $1.57-\mathrm{mm}$ projection bins. The

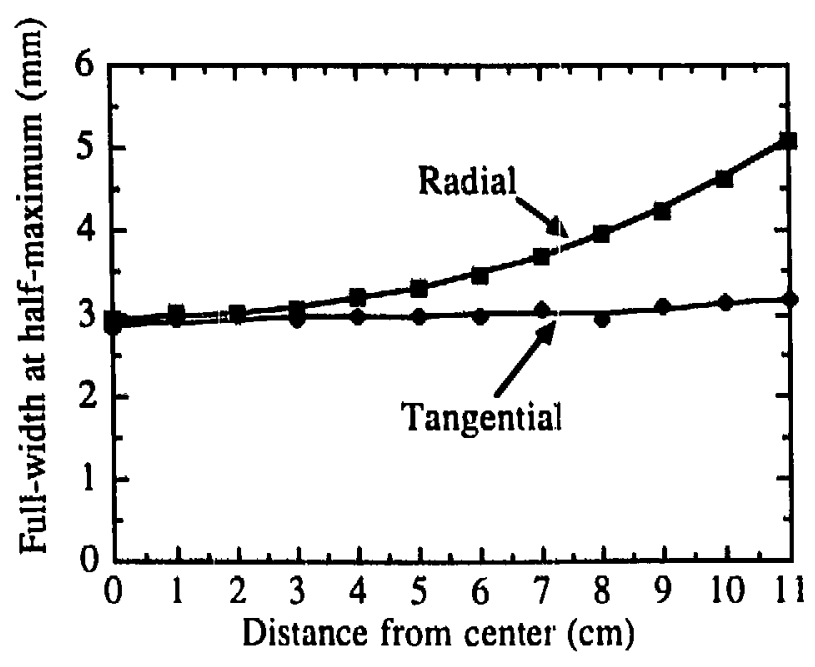

Fig. 2. Resolution in the reconstructed image of a $0.35-\mathrm{mm}$. diameter ${ }^{22} \mathrm{Na}$ wire as a function of the distance to the center of the tomograph. Clam motion was used with $1.57 \mathrm{~mm}$ projection bins, and the image was a superposition of the clam open and clam shut backprojections

(XBL $8610-3948$ ) 

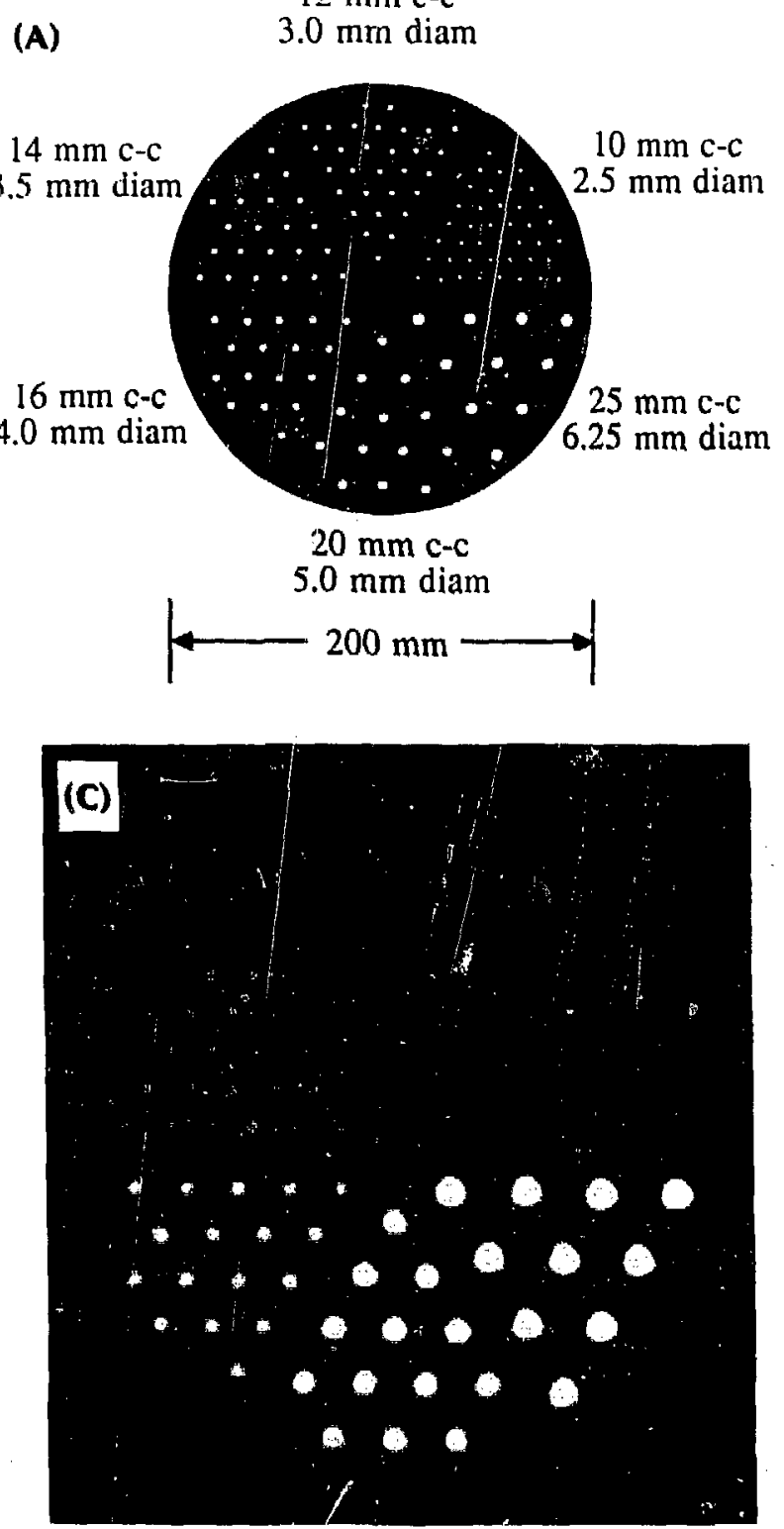

improvement resulting from reducing the crystal size from $9.5 \mathrm{~mm}$ to $3 \mathrm{~mm}$ is quite evident.

A new phantom was designed [Fig. 4(a)| to challenge the resolution of the new tomograph containing six sectors with circular channels whose centerto-center spacing is $12,10,8,6,5$, and $4 \mathrm{~mm}$. In each case the diameter of the channel is one-fourth the center-to-center spacing. Figure $4(b)$ shows an image of 120 million ${ }^{18} \mathrm{~F}$ events taken with the Donner 600-Crystal Positron Tomograph using clam sampling and reconstructed with $1.57-\mathrm{mm}$ projection bins.

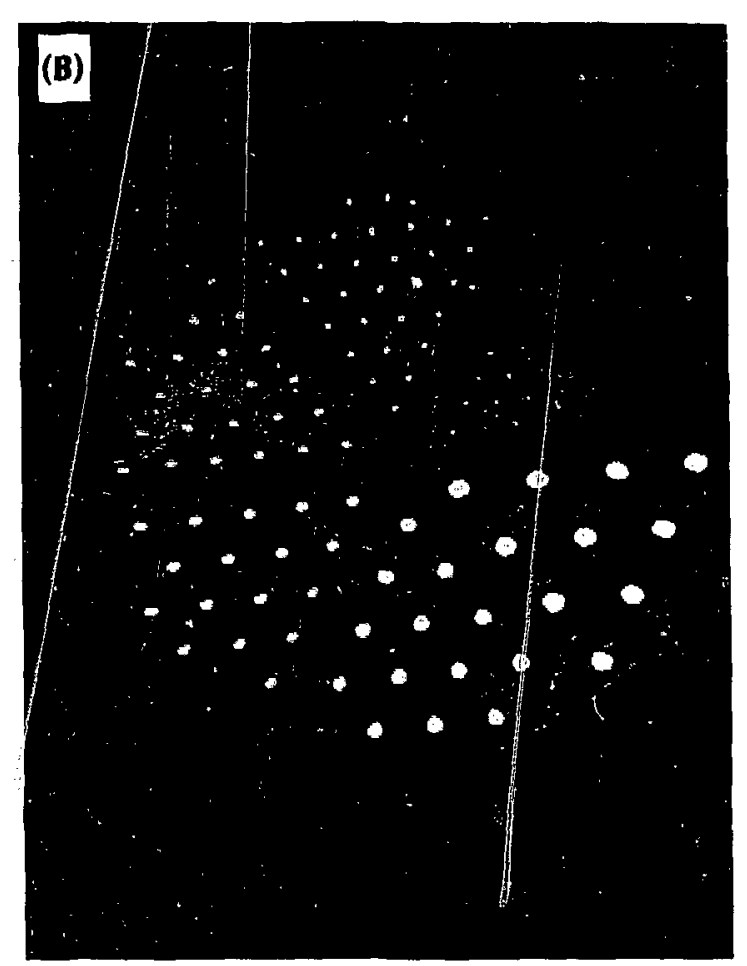

Fig. 3. (A) Sketch of hot-spot phantom fabricated by drilling channels in a solid cylinder of plexiglass. (B) Image of $200 \mathrm{mil}$ lion 'F events taken with the Donner 280-Crystal Positron Tomograph using clam sampling and reconstructed with $2.5-\mathrm{mm}$ projection bins. (C) Image of 35 million ${ }^{38} \mathrm{Ga}$ events taken with the Donner 600-Crystal Positron Tomograph in stationary mode and reconstructed with $1.57-\mathrm{mm}$ projection bins.

(A: XBL 8610-4031; B: XBB 826-5465A; C: XBB 8610-8420)

Table 3. Anticipated resolution (FWHM).

\begin{tabular}{ll}
\hline Factor & \multicolumn{1}{c}{ Contribution } \\
\hline Detector size & $1.5 \mathrm{~mm}$ (triangular) \\
Deviations from $180^{\circ}$ & $1.3 \mathrm{~mm}$ (Gaussian) \\
$\begin{array}{l}\text { Positron Range }\left(^{18} \mathrm{~F}\right) \\
\text { Detector pair resolution }\end{array}$ & $0.5 \mathrm{~mm}$ (sharply peaked) \\
(added in quadrature) & $2.0 \mathrm{~mm}$ \\
Reconstructed Image Resolution & $2.6 \mathrm{~mm}$ \\
(30\% increase due to filter) & \\
\hline
\end{tabular}

- Calculated as 2.35 times the measured rms deviation from Ref. 2 


\title{
Nuclear Magnetic Resonance Studies
}

\section{FLUORODEOXYGLUCOSE EFFECTS ON GLUCOSE METABOLISM IN NUCLEAR MAGNETIC RESONANCE (NMR) STUDIES}

\author{
Kachleen M. Brennan, Thomas F. Budinger, Julia A. Twitchell, and Kay S. Bristol
}

\section{INTRODUCTION}

We embarked on studies of dose vs effect of deoxyglucose on brain metabolism because NMR of ${ }^{19} \mathrm{~F}$-deoxyglucose ( $\left.{ }^{19} \mathrm{FDG}\right)$ showed results different from positron emission tomography (PET) studies that used the radioactive ${ }^{18} \mathrm{~F}$-deoxyglucose ( $\left.{ }^{18} \mathrm{FDG}\right)$. ${ }^{18} \mathrm{FDG}$ has been used as a metabolic tracer for monitoring the local cerebral metabolic rate of glucose utilization measured by PET for over 7 years in over 1,000 human and animal studies. The validity of these studies depends on the assumption that the tracer is trapped in the FDG-6-phosphate form and that FDG or FDG-6-P are not substantially metabolized along the other known metabolic pathways for glucose.

However, NMR ${ }^{19} \mathrm{~F}$ spectroscopic studies showed metabolic products beyond ${ }^{19} \mathrm{~F}$ deoxyglucose phosphorylation. Doses used in the NMR experiments are usually at least 100 times greater than the FDG doses given during a PET tracer study. The major goal of the PET study utilizing both radioactive ${ }^{18} \mathrm{FDC}$ and cold ${ }^{19} \mathrm{FDG}$ was to determine if the doses used in NMR studies have a toxic effect on brain metabolism.

\section{MATERIALS AND METHODS}

In male New Zealand white rabbits under anesthesia using ${ }^{18} \mathrm{FDG}$ as a very-low dose-tracer with PET and the nonradioactive ${ }^{19} \mathrm{FDC}$ in doses below or near those of the NMR investigators, we were able to study the effect of tissue dose on brain deoxyglucose kinetics. Two separate studies were done, each involving a ${ }^{19} \mathrm{FDG}$-loaded rabbit and a control rabbit. Two rabbits were given respectively $70 \mathrm{mg} / \mathrm{kg}$ and $210 \mathrm{mg} / \mathrm{kg}$ loading doses of ${ }^{19} \mathrm{FDG} 20$ minutes prior to ${ }^{18} \mathrm{FDG}$ injections; the two control rabbits received no ${ }^{19} \mathrm{FDG}$ prior to ${ }^{18} \mathrm{FDG}$ injections. Radioactive ${ }^{18} \mathrm{FDG}$ is produced at the 76-inch Crocker cyclotron at the University of California at Davis using the LBL targets and procedures.
For each study a ${ }^{19}$ FDG-loaded rabbit and a control rabbit were positioned on their sternums side by side on the 280-crystal positron emission tomograph bed (built at LBL) in order that both brains could be imaged simultaneously in corona! cross-section (Fig. 1). Identical doses ( $8.5 \mathrm{mCi}$ ) of ${ }^{18} \mathrm{FDG}$ were given simultaneously to both rabbits in i.v. boluses. Dynamic brain images were obtained continuously at 2.5-30 second intervals for the first 4 minutes, then at 60-second intervals until 10 minutes postinjection, followed by 5 -minute intervals until 40 minutes postinjection. Data were also taken at the liver 40 -minutes postinjection.

Immediately after ${ }^{18}$ FDG injection, arterial inpui function samples were obtained as rapidly as possible for the first 2 minutes then at progressively longer intervals to 40 minutes after ${ }^{18} \mathrm{FDG}$ injection. These blood samples were then counted in a TM Tracor gamma-well counter and the results were decay corrected back to the time of injection. The PET dynamic data were used along with arterial blood concentration data to derive the kinetic constants for the model in Fig. $2{ }^{B}$

\section{RESULTS (EFFECTS OF ${ }^{19} \mathrm{FDG}$ ON ${ }^{18} \mathrm{FDG}$ KINETICS)}

In both studies, regions of interest were drawn over each rabbit's brain and the time-activity curves compared for the ${ }^{19} \mathrm{FDG}$-loaded rabbit and the respective control rabbit. Results are shown in Fig. 3 and Tables 1 and 2 .

In the study where a loading dose of $70 \mathrm{mg} / \mathrm{kg}$ ${ }^{19}$ FDG was used, both rabbit brains showed an identical ${ }^{18}$ FDG uptake out to 15 minutes followed by a progressive decrease in activity in the ${ }^{19} \mathrm{FDC}$ rabbit brain out to 40 minutes. At 40 minutes the ${ }^{19} \mathrm{FDG}$ brain had a decrease of $22 \%$ in the ${ }^{18} \mathrm{FDG}$ activity compared to its respective control rabbit.

For the study where $210-\mathrm{mg} / \mathrm{kg}{ }^{19} \mathrm{FDG}$ was used, uptakes of ${ }^{18}$ FDG were only similar for the 


\section{${ }^{19}$ FDG AFFECTS ON ${ }^{18}$ FDG KINETICS}

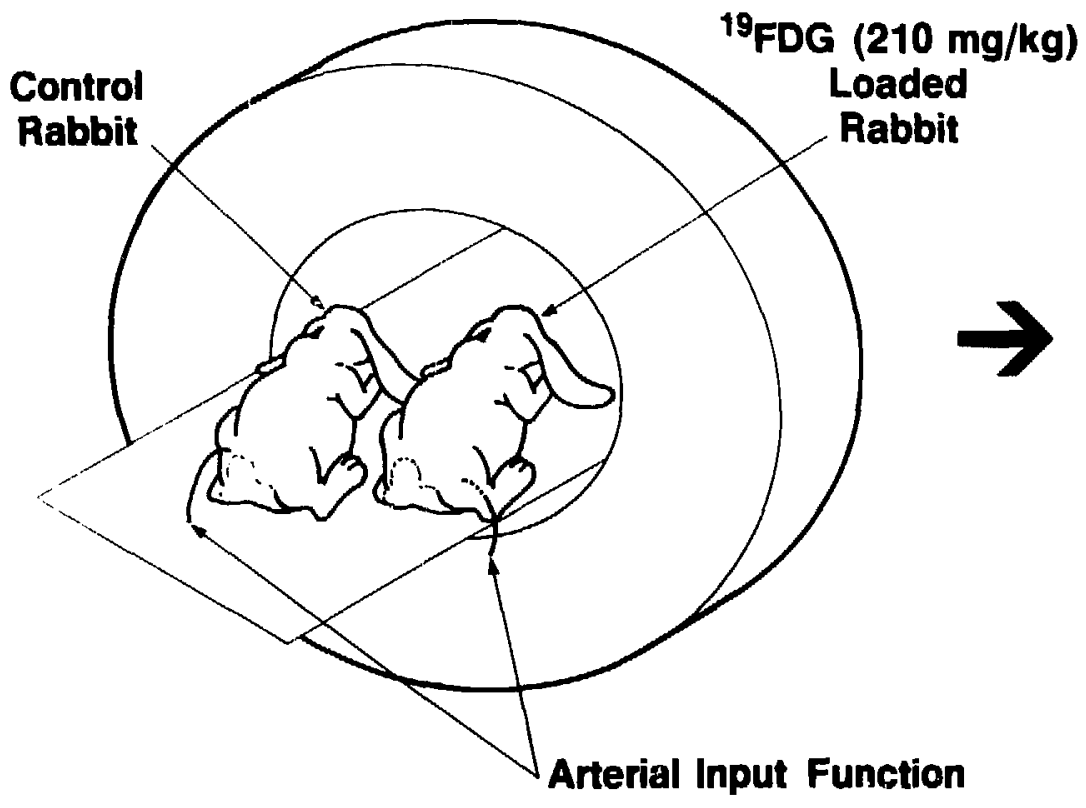

PET ${ }^{18}$ FDG
Emission Data

Control 19FDG Loaded

Rabbit

Rabbit

Fig. 1. Experimental setup for the positron emission tomography study. Control and ${ }^{19} F D G-l o a d e d$ rabbits are imaged simultaneously in the PET ring.

(XBB 868-6466)

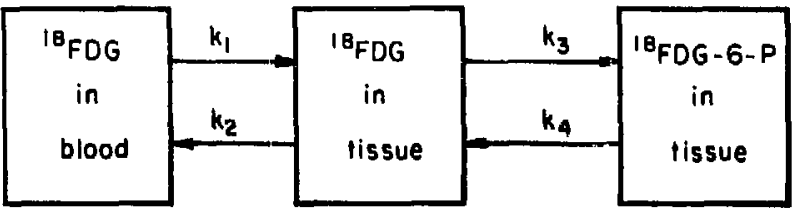

Fig. 2. Three compartment kinetic model used for halysis of positron emission tomography data. Values of the kisetic constants $k_{1.4}$ are found in Table 2. The vascular fraction (percent of activity in a given organ due to blood) is represented by $f_{v}$

(XBL B24-3766)

first 2 minutes followed by an even more dramatic decrease in the overall activity in the ${ }^{19} \mathrm{FDC}$ rabbit brain with the final activity being $43 \%$ less than the respective control brain.

In both studies, liver activity was found to be $20 \%$ greater in the ${ }^{19}$ FDG-loaded rabbits compared to their respective controls. Arterial input functions measuring ${ }^{18}$ FDG were identical for both rabbits in each study.
Table 1. Comparison of relative organ uptakes of ${ }^{18} F D C$ in control and ${ }^{19} F D G$ loaded rabbits.

\begin{tabular}{|c|c|c|c|}
\hline Organ & Control & ${ }^{19} \mathrm{FDC} 70 \mathrm{mg} / \mathrm{kg}$ & ${ }^{19} \mathrm{FDG} 210 \mathrm{mg} / \mathrm{kg}$ \\
\hline Brain & $100 \%$ & $78 \%$ & $57 \%$ \\
\hline Liver & $100 \%$ & $1.20 \%$ & $120 \%$ \\
\hline
\end{tabular}

Table 2. Comparison of kinetic rate constants (see model in Fig. 2) in conirol and ${ }^{19}$ FDG loaded rabbits

\begin{tabular}{cccc}
\hline \hline Parameter & $\begin{array}{c}{ }^{19} \mathrm{FDG} 210 \mathrm{mg} / \mathrm{kg} \\
\text { rabbit }\end{array}$ & $\begin{array}{c}\text { Control } \\
\text { rabbit }\end{array}$ & $\begin{array}{c}\text { \% difference } \\
{ }^{19} \mathrm{FDC} / \text { control }\end{array}$ \\
\hline$k_{1}$ & 0.092 & 0.089 & $+3 \%$ \\
$k_{2}$ & 0.239 & 0.154 & $+55 \%$ \\
$k_{3}$ & 0.027 & 0.042 & $-36 \%$ \\
$k_{4}$ & 0.00015 & 0.0043 & $-97 \%$ \\
$f_{4}$ & 0.183 & 0.146 & $+25 \%$ \\
\hline
\end{tabular}




\section{Comparison of 18-FDG Uptake \\ in \\ 19-FDG Loaded Rabbits}

- $0 \mathrm{mg} / \mathrm{kg} 19-\mathrm{FDG}$

$\begin{array}{rll}\Delta-70 & \text { " } \\ 0-210 & \text { " }\end{array}$

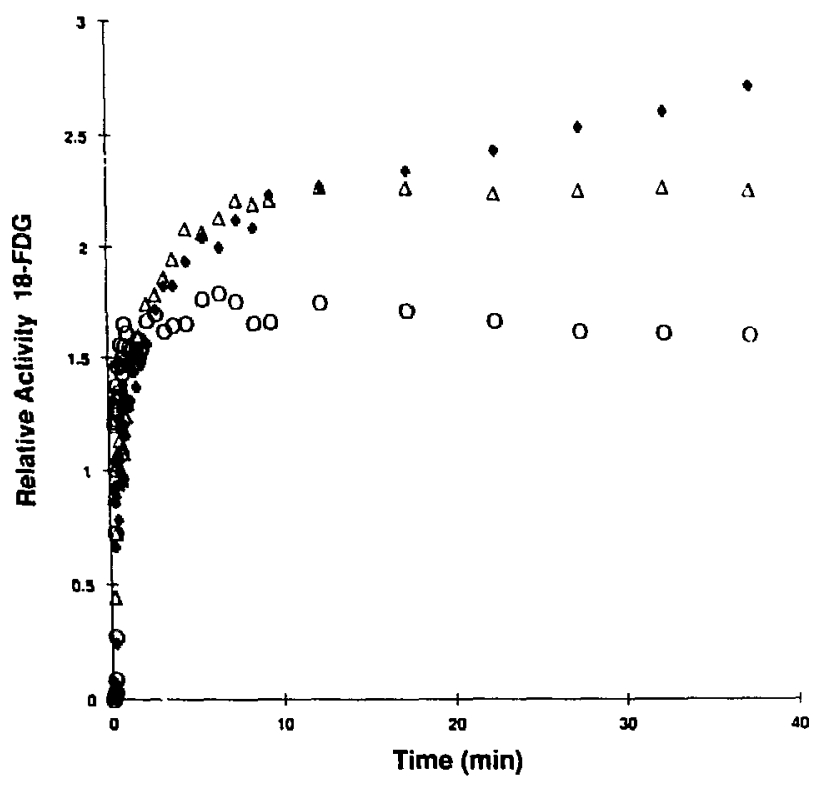

Fig. 3. ${ }^{18} F D G$ uptake curves comparing the relative activity time course for control and ${ }^{19}$ FDG-loaded rabbils. (XBL 8610-9008)

\section{DISCUSSION}

The PET studies clearly show an effect of ${ }^{19} \mathrm{FDG}$ loading on ${ }^{18} \mathrm{FDG}$ kinetics. There was a decrease in brain accumulation of ${ }^{18}$ FDC in animals who received a loading dose of ${ }^{19} \mathrm{FDG}$ and the rate of washout for ${ }^{18} \mathrm{FDG}$ is greater in the loaded than in the control animals. These animals were under anesthesia, thus the results may not exactly reflect metabolic changes in the unanesthetized animal. However, the amount of ${ }^{19} \mathrm{FDG}$ given in previous NMR studies is 2 to 3 times higher than the doses used in these studies $(132 \mathrm{mg} / \mathrm{kg}$ in Ref. 2, $250 \mathrm{mg} / \mathrm{kg}$ in Refs. 3 and $4,400 \mathrm{mg} / \mathrm{kg}$ in Ref. 5). The fact that even at these lower doses we find an effect on the kinetics of FDG, which is counter to our experience in human, dog, cat and rabbit studies as well as biochemical studies by others, $(6,7)$ argues for caution in conclusions regarding glucose metabolism and biochemistry in tissues exposed to the stated doses of fluorodeoxyglucose. This work is the basis for a study of fluorodeoxyglucose metabolism as a function of fluorodeoxyglucose tissue concentration. ${ }^{19} \mathrm{~F},{ }^{31} \mathrm{P}$ and proton (1H) NMR spectroscopy of the brain extracts are currently in progress to determine the metabolic fate of FDC when given at these elevated doses for NMR study. ${ }^{B}$

\section{REFERENCES}

1. Sokoloff, L., Reivich, M., Kennedy, C., Des Rosiers, M.H., Patlak, C.S., Peltigrew, K.D., Sakurada, O., and Shinohara, $M$. I. Neurochem. 28, 897-916 (1977).

2. Babcock, E.E., and Nunnally, R.L. Fourth Annual Meeting, Society of Magnetic Resonance in Medicine Vol 2, 751 (1985).

3. Berkowitz, B.A., and Ackerman, J.J.H. Fourth Annual Meeting, Society of Magnetic Resonance in Medicine Vol 2, 759 (1985).

4. Wyrwicz A.M., Murphy, R., Prakash, l., Moriarty, R.M., and Dougherty, T. Fourth Annual Meeting, Society of Magnetic Resonance in Medicine Vol 2, 827 (1985).

5. Nakada, T., Kwee, I., and Conboy, C.B. I. Neurochem. 46, 198-201 (1986).

6. Nelson, T., Lucignani, G., Atlas, S., Crane, A.M., Dienel, G.A., and Sokoloff, L. Science 229, 60-62 (1985).

7. Nelson T., Lucignani, G., Goochee, J., Crane, A.M., and Sokoloff, L. J. Neurochem. 46, 905-919 (1986).

B. Bolo, N.R., Brennan, K.M., Jones, R.M., and Budinger, T.F. Ann. NY Acad. Sci. (submitted) (1986). 


\title{
AN INSTRUMENT CONTROL AND DATA ANALYSIS PROGRAM FOR IMAGING AND IN VIVO SPECTROSCOPY
}

\author{
Mark S. Roos, Richard A. Mushlin, Eugene Veklerov, ${ }^{\star}$ John D. Port, ${ }^{\dagger}$ Carol Ladd, $^{\dagger}$ \\ and Colin G. Harrison ${ }^{\dagger}$
}

\section{MOTIVATION}

An instrument control and data analysis program has been implemented for use in research laboratories where development of new techniques for NMR imaging and in vivo spectroscopy are the primary activities. Traditional control software for such instruments suffers from three major limitations: 1) Decisions about the timing and sequence of real-time operations such as data transfer are not made by the user but are built into system primitives and interrupt service routines; 2) Automation sequences in the command language are basic batch processors with restricted command sets and limited inputs and outputs; 3) Data structures have little dynamic character. In this environment, the real-time operations are programmed in a high-level language. The timing and mix of data acquisition, processing, storage and display functions are selected by the user to customize data flow for such diverse applications as spectroscopy and fast imaging. The primary programming structure (macro) of this high-level language provides passby-reference parameters, user-defined variables of local or global scope, and unlimited nesting. Multidimensional array structures are dynamically allocated and removed at will, and may be passed to macros or stored on disk.

Users spend most of their time on the system creating or running macros. The primitive command set is designed primarily for writing macros rather than for direct execution from the console (although this is supported). The commands are somewhat lower level than in most spectrometer control software. The system derives its personality more from the macros available to the user than from the primitive command set. Thus, by choosing to load different sets of macros, the system can be customized for different applications. This process is automated so that the user may select a system customized for spectroscopy, imaging, etc., at run time. Indeed, each user can have a customized environment.

"Engineering Division, (Real Time Systems Group) LBL.

IIBM Instruments, Inc.
PROGRAM ARCHITECTURE AND IMPLEMENTATION

Execution of a command or macro is controlled by an executive that is also responsible for management of user-defined variables. To respond quickly enough to control data acquisition from a high-level user-defined macro, command primitives must be resident in memory when invoked. Primitives are Pascal subroutines compiled and linked into the program, with their start addresses stored in a command table. Parameters are not passed to the commands as Pascal arguments, but rather through a set of global argument pointer variables. When a command is invoked from the keyboard, it is located in the command table, its arguments are parsed by the executive, resolved, and the argument pointers are set. Execution then jumps to the start address of the command. Error detection is incorporated in each command subroutine, which may also write responses on the screen and prompt for missing arguments.

Macros reside on disk as text files and may be edited from within the program. Our experience indicated that a simple interpreted batch command processor would not be fast enough for real-time control. At the time a macro is loaded into memory, all symbolic references to primitive commands, other macros, parameters, variables of local or global scope, and constants are resolved and stored in compact form. The macro storage data structure is a linked list or records, one record per instruction. When the user invokes the macro, the executive simply loads the argument pointers for the first command, jumps to the start address, and repeats the process on return until the macro is completed. This approach yields a command execution overhead (in a macro) of about $150 \mu \mathrm{sec}$. There is no limitation on macro nesting, but recursive calls are prohibited.

The program is currently implemented with three tasks: the main lask, the acquisition task, and a simple task supporting interaction with the image display through a digitizing tablet. Figure 1 shows the relationship of the three tasks. The main and acquisition tasks are controlled by identical execu- 


\section{Program Task Structure}

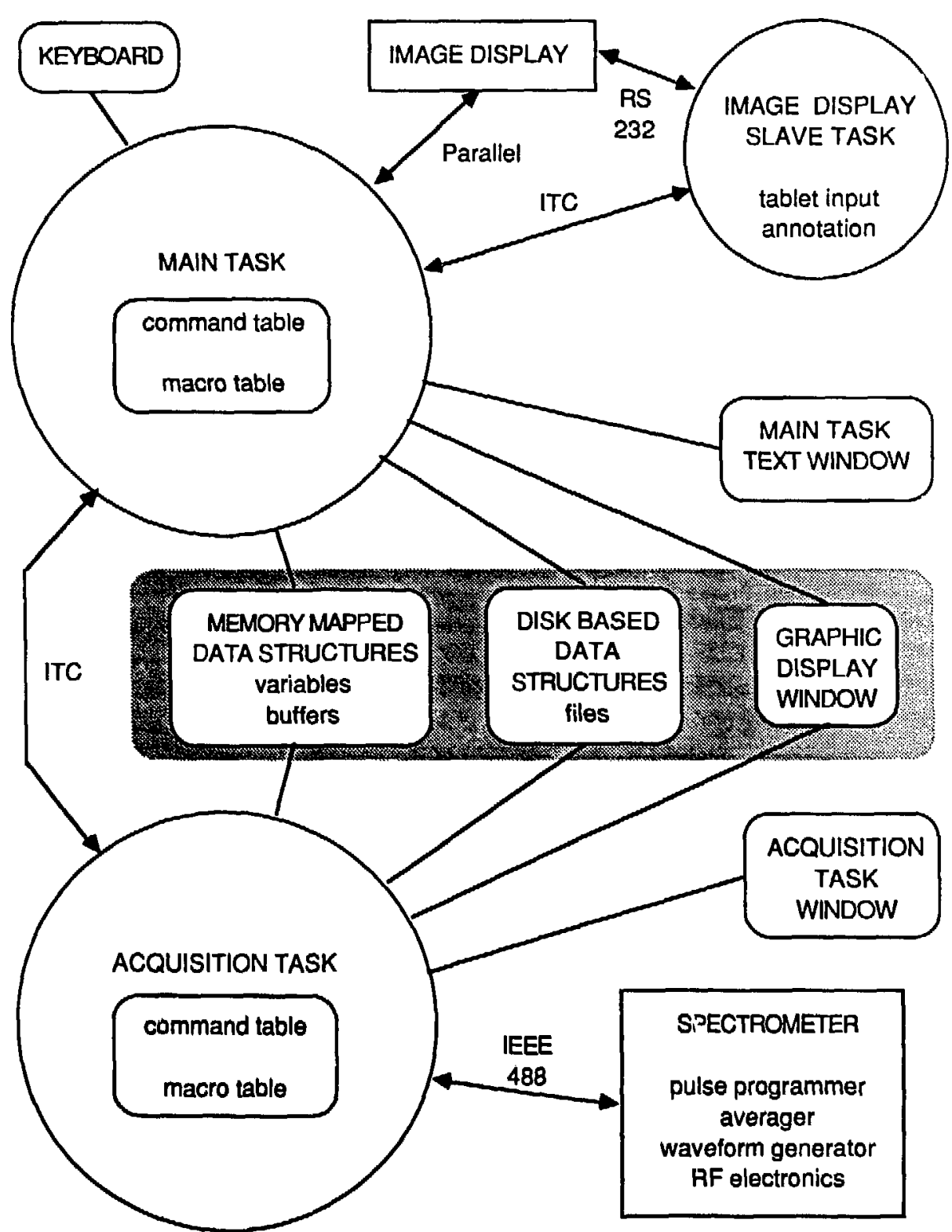

Fig. 1. System resources are allocated to three tasks according to task function: the main task handles data processing the keyboard and system utilities; the acquisition task controls the spectrometer; and the image display is manipulated using a slave task. The shaded area shows resources shared between the main and acquisition tasks. Commands are transmitted among the lasks over intertask communication channels, while data is passed through shared memory.

(XBL 8610-9009)

tives of the type described above. The main task receives its input from the keyboard, while the acquisition task receives input from an intertask communication channel connecting it with the main task.

\section{THE COMMAND SET}

The command set contains over 200 primitives. Some primitives exist in only the main or acquisition task, while some are duplicated, as shown 
diagramatically in Fig. 2. Scalar arithmetic, branching, operations involving creation of variables, buffers, files, and graphics display operations are all performed by the host and are available in either task. Commands involving buffer mathematics are implemented using the array processor and are contained exclusively in the main task. All commands requiring direct communication with the spectrometer instruments are installed in the acquisition task.

An extensive set of commands for mathematical manipulation of buffers is implemented in the main task using the SKY array processor, supplemented with some assembly code for byte operations. Special functions for NMR and almost all the routines in the SKY Pascal library are installed as command primitives. A syntax was developed that uses wildcard characters to support vector operations with implied loops over multiple array dimensions. This syntax frees the user from repeatedly typing range specifications, and facilitates writing general purpose macros that operate on buffers of arbitrary size.

The primitives controlling data acquisition are divisible into two categories: those that communicate directly with the instrument control Z-B0 microprocessors and those that do not. All com- mands of the first type exist exclusively in the acquisition task and perform such functions as moving data from the averager, loading waveforms, synthesizer tables, and pulse programs, and controlling the timing of experiments. These commands directly initiate communications over the instrument control bus that need to be carefully synchronized to ensure correct experimental results and good data throughput. Since the commands are in the acquisition task only, they are inaccessible from the keyboard or main task macros while a detached acquisition task macro is running. Thus unintentional interference with a running experiment is prevented. Commands of the second type are used to set instrument parameters from both the main and acquisition tasks. These parameters, such as pulse lengths and phases, need to be adjusted during experiments while detached acquisition macros are running. When commands of this type are executed, packets of update information are assembled and queued for transmission to the various instruments. A primitive of the first type (acquisition task only) causes these queues to be flushed. The timing of all instrument control bus transactions is determined by the sequence of commands in a (detachable) acquisition task macro written by the user.

editor

operating system utilities (COPY, DIR,FREE)

MAIN TASK buffer operations using the array processsor

parameter adjustment during experiments

image display

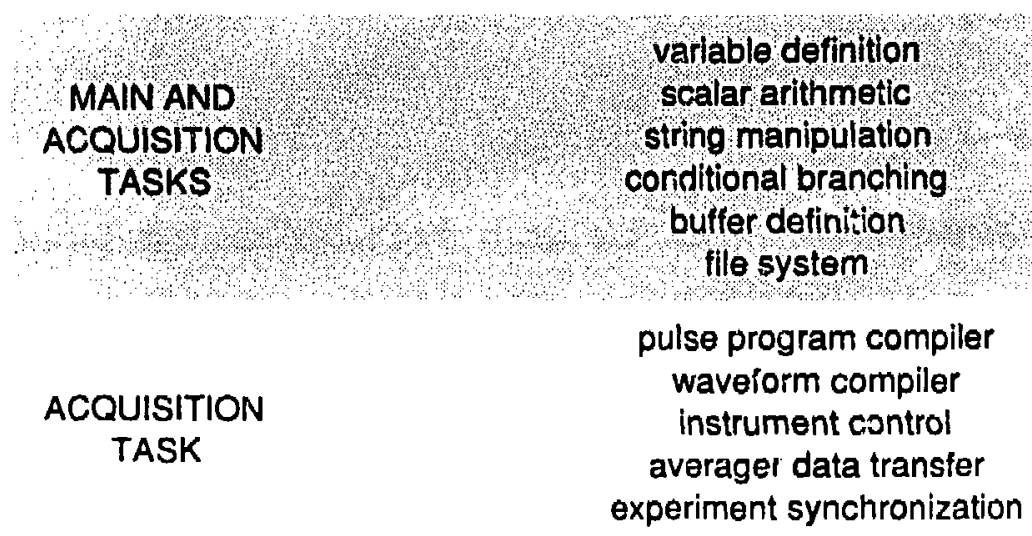

Fig. 2. The command set is divided among the main and acquisition tasks as shown, with commands duplicated in both tasks listed in the darker region.

iXBL 8610-9010) 


\section{CONCLUSION}

The spectrometer control and data processing environment described above and the libraries of macros designed to support imaging and in vivo spectroscopy are currently in use at the Lawrence Berkeley Laboratory, the Massachusetts Institute of Technology, and the Brigham and Women's Hospital in systems ranging from a 2- $T$ animal spectrometer to a 0.5-T whole body imager. Experiments that have been successfully implemented include multislice, multi-echo imaging, fast steady state free precession imaging, and ${ }^{31} \mathrm{P}$ spectroscopy. The flexibility of the macro programming structure allowed very rapid development of these macro libraries.
We have demonstrated that instrumentation developed around standard hosts, buses, and operating systems can yield research tools with performance comparable to highly specialized systems. The combination of low macro instruction overhead, provision for user access to system internals, and a rich command set controlling basic acquisition and processing functions provides a foundation on which libraries of macros may be built to serve a broad range of users, perhaps more easily than a system with larger sets of less primitive commands and a more limited batch processor. Well defined program interfaces for macros and for installing commands, as well as the ability to modify instrument control code significantly broaden the range of experiments accessible to the researcher.

\section{EFFECTS OF SLICE SELECTION AND DIFFUSION ON $T_{2}$ MEASUREMENT}

\section{Sam T. Wong and Mark S. Roos}

Proton NMR imaging is a sensitive indicator of brain pathology that is being used at LBL to study radiation effects and aging in human subjects. In the nuclear magnetic resonance (NMR) studies we examine the distributions of spin-spin relaxation $\left(T_{2}\right)$ and spin-lattice $\left(T_{1}\right)$ relaxation time constants in different regions of the brain by a multi-echo spinwarp imaging sequence using selective excitation and selective refocusing if pulses. Unfortunately, estimates of these parameters from images are subject to systematic errors resulting from the imaging technique. In addition it is importa is distinguish among the various physical processes: "tat contribute to the observed $T_{2}$. We condicis an experimental study that confirms theoretical results' suggesting that the use of selective refccusing pulses causes bias in such estimates of $T_{2}$. This apparent change in $T_{2}$ has been previously attributed to diffusion ${ }^{2}$. With normal imaging gradient strength and duration, classical NMR diffusion theory suggests that suppression of signal due to diffusion during slice selection is insignificant. We verified that diffusion effects are small and that the apparent change in $T_{2}$ is mainly due to resonance offset effects. In addition, we have demonstrated a method to correct for the $T_{2}$ bias.

We assume a mono-exponential relaxation model (one relaxation rate per pixel), thin slices with insignificant partial volume effect, and that all gradients have durations and strengths found in normal spin-warp imaging experiments. The $T_{2}$ image, $T_{2}^{\prime}(x, y)$, calculated from the ratio of two magnitude images reconstrucled from consecutive echoes is related to the true image by

$$
\frac{1}{T_{2}^{\prime}(x, y)}=\frac{1}{T_{2}(x, y)}-\frac{1}{2 \tau} \log _{e} R_{t} \text {. }
$$

where $2 T$ is the echo-to-echo time and $R_{l}$ is a constant depending on the imaging parameters and the slice profiles excited by the of envelope. Equation (1) shows that $T_{2}^{\prime}(x, y)$ is an unbiased estimate of $T_{2}(x, y)$ only when the echo-to-echo time approaches infinity or $R_{1}=1$. Also, when $T_{2}$ is small the $\frac{1}{T_{2}}$ term may dominate and $T_{2}^{\prime} \approx T_{2}$. On the other hand, when $T_{2}$ is large, the second term dominates and $\mathrm{T}_{2}{ }_{2}$ may be drastically different from $T_{2}$. In most circumstances the slice profiles are independent of $T_{2}$, so $R_{1}$ is just a characteristic of the sequence that can be found experimentally. Equation (1) then provides a simple way to correct $\mathrm{T}_{2}^{\prime}$.

There are two ways to make $R_{\mid}$approach unity and so reduce the bias in $T^{\prime}{ }_{2}$. One way is to use an if modulation function that gives a more rectangular distribution of flip angles. Experimental results show that the slice profiles become nar- 
rower for later echoes and the difference between two echo profiles is mainly in the transition regions of distribution of flip angles. The value of $R_{i}$ is directly related to this difference. Therefore, it is better to use a sinc if envelope than a Gaussian if envelope as the sinc produces narrower transition regions. The bias in $\mathrm{T}^{\prime}{ }_{2}$ is illustrated for two iypes of excitation pulses in Fig. 1. For large $T_{2}$ the bias in $\mathrm{T}_{2}$ may be as large as $75 \%$ for the Gaussian envelope. The other way to increase $R_{l}$ is to increase the bandwidth (in resonance offset) of the $180^{\circ}$ pulses relative to the $90^{\circ}$ pulse. However, the ratio of refocusing and excitation pulse bandwidths is limited to the range of 1 to 2 in multi-slice imaging to avoid slice interference. This results in $T_{2}$ bias as high as $40 \%$ even with a sinc if envelope. Therefore, $T_{2}$ correction using Eq. (1) is usually necessary for measurements with selective if pulses.

In a previous investigation, ${ }^{2}$ slice selection gradients were observed to cause reductions in $T_{2}$ that were attributed to diffusion. This effect was not observed for readout and phase encoding gradients. This finding was puzzling because the slice selection gradient usually has strength comparable to the readout and phase encoding gradients, and its duration is usually much shorter than the readout gradient. According to classical NMR diffusion theory, the slice selection gradient should have even smaller diffusion effects unless there are unexpected interactions between the of pulse and the diffusing spins. Our experiments show that diffusion effects are indeed insignificant under normal imaging conditions and that the previously reported effect was probably due to the thin phantom employed.

The materials chosen for the experimental study were doped water with $T_{2}=135$ milliseconds, glycerol with $T_{2}=16.5$ milliseconds and water heavily doped to give a $T_{2}$ of 14.9 milliseconds, comparable to that of glycerol. Because the diffusion coefficient of glycerol is two orders of magnitude smaller than that of water, the role of diffusion can be determined by comparing data for glycerol and the heavily doped water sample. Table 1 shows the ratio $R_{1}$ for the three samples at three different slice selection gradient strengths. The difference in $R_{1}$ between the three samples is insignificant at any gradient strength. The apparent change in $R_{1}$ as the gradient is increased is due to eddy rurrent effects. This was confirmed experimentally. By increasing the timing between the if pulses and the readout gradient we were able to suppress the change in $R_{l}$ for the water sample with the higher $T_{2}$. This was not done for the other samples due to poor signal to noise ratios. (a)

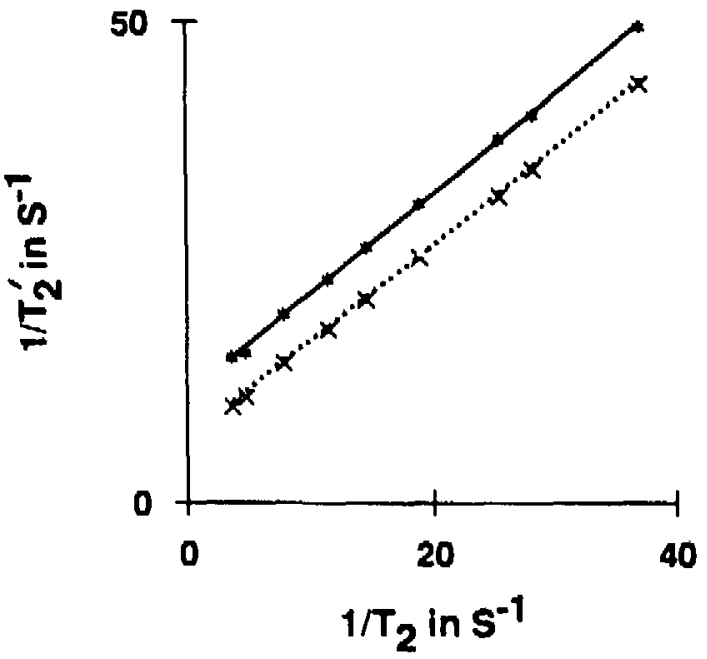

(b)

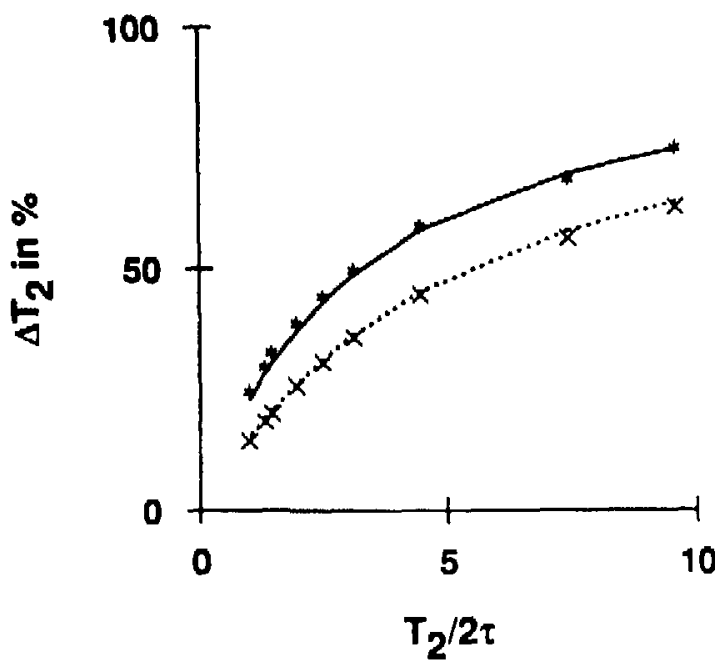

Fis. 1. (a) Plot of data for a phantom consisting of vials containing $\mathrm{CuSO}_{4}$ doped water. $\mathrm{T}_{2}$ is estimaled from image dala, while $T_{2}$ is obtained using a CPMC sequence with hard if pulses. (b) The fractional bias, $\Delta T=\left(T_{2}-T_{2}^{\prime}\right) / T_{2}$, is plofled versus $T_{2} / 2+$ for the same set of data $\left.2 \tau=27.5 \mu \mathrm{sec}\right)$. The solid and dotted lines are theoretical curves with $R_{1}=0.74$ and 0.83 respectively. The symbol (") indicates data points obtained with Gaussian of envelopes, while $(x)$ indicates data points obtained with sinc if envelopes.

(XBL-8610-9011) 
Table 1. $R_{1}$ of three different phantoms at different slice selection gradient strengths.

\begin{tabular}{lccc}
\hline $\mathrm{G}_{2}(\mathrm{G} / \mathrm{cm})$ & \multicolumn{1}{c}{$\mathrm{R}_{1}$} \\
\cline { 2 - 4 } & $\mathrm{H}_{2} \mathrm{O}\left(\mathrm{T}_{2}=135 \mathrm{msec}\right)$ & glycerol $\left(\mathrm{T}_{2}=16.5 \mathrm{msec}\right)$ & $\mathrm{H}_{2} \mathrm{O}\left(\mathrm{T}_{2}=14.9 \mathrm{msec}\right)$ \\
\hline 0.2 & $0.84 \pm 0.02$ & $0.82 \pm 0.02$ & $0.82 \pm 0.02$ \\
0.4 & $0.81 \pm 0.03$ & $0.82 \pm 0.02$ & $0.84 \pm 0.04$ \\
0.6 & $0.75 \pm 0.04$ & $0.78 \pm 0.03$ & $0.78 \pm 0.05$ \\
0.8 & $0.66 \pm 0.04$ & $0.69 \pm 0.03$ & $0.65 \pm 0.05$ \\
\hline
\end{tabular}

\section{REFERENCES}

1. Majumdar, S., and Gore, J.C. Effects of selec-

2. Wesbey, G.E., Moseley, M.E., and Ehman, R.L. tive pulses on the measurement of $T_{2}$ and apparent diffusion in multiecho MRI. Submitted to Magnetic Resonance in Medicine (1986). Translational molecular self-diffusion in magnetic resonance imaging, I. Effects on observed spin-spin relaxation. Invest. Rad. 19, 484-490 (1984). 


\section{SECTION 2. PHYSIOLOGY}

\section{INTRODUCTION}

The primary research objective of the Physiology Group is the elucidation of molecular, cellular, and tissue regulatory mechanisms underlying physiological functions in normal and perturbed states. This program includes the analysis of changes that occur in physiological control mechanisms in response to nonionizing and ionizing radiation, and various classes of infectious agents and chemicals. This year's annual report for the Physiology Group describes progress in research on hematozoan infections, hematopoiesis, the endocrine system, nonionizing radiation bioeffects and dosimetry, and transuranium element biokinetics and clearance techniques.

Previous studies on the pathogenesis of the anemia that accompanies malarial infection have shown that stem cell precursors (CFU-S) in the bone marrow are reduced significantly during advanced stages of parasitemia in mice. This reduction in CFU-S occurs at the same time as the levels of biologically active erythropoietin (Ep) and colony-stimulating factors (CSF) increase in the plasma of infected murine hosts. These observations suggest that the anemia that accompanies advanced stages of malarial infection may result from several factors, including the lack of erythroid progenitor cell production, the inhibition of progenitor cell differentiation, and alterations in the marrow stromal cell content that is essential for the development of blood cells. Research by Silverman. Schooley, and their associates described in this year's annual report indicate that all of these factors may play a role in the dyserythropoiesis that accompanies malarial infection. The interesting new observation was made by Silverman and Mahlmann that the infusion of mice with tumor necrosis factor (TNF), which is produced by macrophages and/or monocytes during the processing of malarial antigen, leads to a reduction of CFU-S and the migration of hematopoietic stem cells from the bone marrow into the spleen and circulation. These effects of TNF correspond to the stem cell alterations that have been observed in mice infected with three strains of murine malaria. The results of Schooley and his associates also indicate that the glycoprotein interleukin-1 (IL-1), which is produced by macrophages and monocytes in response to foreign antigens, inhibits the production of Ep-responsive erythroid progenitor cells. In studies on mice with a phenylhydrazine-induced anemic state, Schooley, Kullgren, and Allison demonstrated that IL-1 blocked the effects of Ep in stimulating DNA synthesis by erythroid precursor cells. They have concluded that IL-1 may play an important role in the pathogenesis of chronic anemic states such as those induced by malarial infection, lupus erythematosus, and graft-versushost disease.

Using two sublines of CBA mice whose cells can be distinguished by the presence of electrophoretically distinct alloenzymes of phosphoglycerate kinase (PGK-A and PGK-B), Brecher has been studying the identity of transplanted bonemarrow stem cells that repopulate the marrow of lethally irradiated mice. After an injection of 100,000 cells he observed $100 \%$ survival of the recipient mice. Forty percent of the recipients contained blood cells with the PGK-A marker, and $60 \%$ contained either $\mathrm{B}$ or $\mathrm{AB}$ markers. When the transplanted inoculum of marrow cells was reduced to 50,000 cells, $40 \%$ of the lethally irradiated recipient mice survived and all contained only the donor PGK-A marker in their blood cells. Because the PGK-A/PGK-B ratio in the marrow donors was $7: 1$, the observed mixture of $A$ and $B$ cells following these two transplanted inocula conformed to theoretical predictions. Brecher also found that after a uniformly lethal radiation dose, the transplantation of 20,000 marrow cells with PGK-A marker led to a $20 \%$ survival of the recipient mice. Initially the repopulating blood cells in the survivors contained the donor PGK-A marker, but later acquired the identity of the original host cells. This finding indicates that transplanted marrow cells in irradiated recipients can provide temporary maintenance of hematopoiesis, which is later reestablished by cells of host origin.

In other hematology research, Clemons and her associates have used a sensitive radioimmunoassay to study renal and hepatic production of $\mathrm{Ep}$ in rats during the first six weeks of neonatal life. In rormal rats the liver has been found to be the major source of Ep until the tenth day of neonatal life, at which time the kidney becomes the predominant 
source of this hormone. In rats subjected to severe hypoxia the switchover from hepatic to renal sources occurs on the second day after birth. Studies with nephrectomized rats have demonstrated that the liver synthesis of Ep can compensate for the loss of renal sources during the first three weeks of neonatal life. No differences in male and female production of Ep were noted until day 42, at which point the level of this hormone in males was found to exceed that in females.

Research by Clemons and her associates during the past several years has demonstrated that the exposure of rats to $1 \mathrm{ppm}$ of ozone leads to a significant decrease in the levels of circulating thyroid hormones. It has also been demonstrated that the binding affinity of serum proteins for thyroid hormones increases as a result of ozone exposura, and this effect leads to an accelerated clearance of the hormones from the circulation. In this year's annual report Erkenbrack and Clemons describe a new set of experirnents that indicate that the exposure of rats to $1 \mathrm{ppm}$ of ozone for one day produces a substantial increase in the level of triiodothyronine (T3) nuclear receptors in lung cells. After one week of exposure the lung T3 receptor level is elevated more than tenfold relative to the level in control animals. The mechanism underlying this effect of ozone is not known, but may relate to oxidative effects of ozone on the intermediates that regulate the turnover of $\mathrm{T} 3$ receptors in the lung.

Studies on the biological interactions and dosimetry of static and $60-\mathrm{Hz}$ magnetic fields are described in the chapter by Tenforde and his associates. Recent experiments with a 9-tesla (90,000 gauss) superconducting magnet have demonstrated that significant alterations occur in the permeability properties of lipid bilayer membranes in response to static magnetic field exposure. It was observed that the release of a chemotherapeutic drug (ARA-C) encapsulated in unilamellar liposomes is facilitated by magnetic field deformational forces when the exposure occurs at temperatures immediately below the thermal phase transition point. The apparent link between magnetic field sensitivity and phospholipid phase transitions in bilayer membranes has been explained on the basis of the structural instabilities that occur in lipid bilayers at temperatures in the prephase transition range. In other magnetic field research, a compact microprocessor-controlled dosimeter has been developed for monitoring human exposure to $60-\mathrm{Hz}$ fields in residential and occupational environments. The initial prototype dosimeter has been completed, and the results of field tests are presented in this year's report.

Research on the interaction of $2450-\mathrm{MHz}$ microwaves with the membranes of rabbit and human erythrocytes is described in the chapter by Liburdy. He has observed that exposure of erythrocyte suspensions to nonthermal levels of microwave radiation leads to the release of several species of membrane proteins. As in the static magnetic field interaction with bilayer membranes described above, the alteration of membrane structure induced by microwaves is most pronounced at temperatures approaching the thermal phase transition region. Using polyacrylamide gel electrophoresis to characterize the proteins shed from erythrocyte membranes during microwave exposure, Liburdy has characterized 11 species of released proteins that range in molecular weight from 8 to 31 kilodaltons. The larger protein species that are released depend on $\mathrm{Ca}^{2+}$ bridges for stable attachment to the membrane. Studies using high performance liquid chromatography have demonstrated that microwaves cause the shedding of relatively cationic and nonpolar protein species from erythrocyte membranes.

Two chapters by Durbin and her associates in this year's annual report describe new studies on actinide element biokinetics and clearance. The first of these papers reports the results of initial studies on the clearance kinetics and tissue distribution of ${ }^{237} \mathrm{~Np}$ in a monkey. These studies are of importance because ${ }^{237} \mathrm{~Np}$ contributes significantly to the radiation hazard of old nuclear fission waste materials. It was found that the tissue absorption of ${ }^{237} \mathrm{~Np}$ was complete in 4 days after intramuscular injection, and that approximately $40 \%$ of the initial material was excreied via the renal pathway. Approximateiy one-half of the absorbed ${ }^{237} \mathrm{~Np}$ was deposited in bone, and the liver uptake of this element was very low. These findings with a monkey are similar to earlier observations made on rats injected with ${ }^{237} \mathrm{~Np}$. The results of this preliminary work indicate that the ICRP general actinide metabolic model, developed primarily from biological data on ${ }^{238} \mathrm{Pu}$, may not provide a good prediction of the tissue distribution and dose resulting from the uptake of ${ }^{237} \mathrm{~Np}$.

For the last several years Durbin has been working in collaboration with scientists from the LBL Materials and Molecular Science Division and the UC Berkeley Department of Chemistry to develop new classes of chelating agents that have appropriate molecular dimensiors for the efficient binding of ${ }^{238} \mathrm{Pu}(\mathrm{IV})$. A long-range gcal of this pro- 
gram is to develop chelating agents with a low biological toxicity that can be administered orally to eliminate actinides and chemically similar metals from the body. In this year's annual report Durbin and her associates describe the results of tests on the clearance of ${ }^{238} \mathrm{Pu}(\mathrm{IV})$ from mice that were orally administered six different chelating agents. The injected ligands were from three classes of chemical compounds: 1) polyhydroxamic acid derivatives, 2) polycarboxylic acid derivatives, and 3) tetracatechoylamides with solubilizing carboxylic and sulfonic acid constituents on the catechol ring. It was found that one member of the third class of compounds, a sulfonated derivative denoted as 3,4,3-LICAM(S), was especially efficient in facilitating the clearance of ${ }^{238} \mathrm{Pu}(\mathrm{IV})$ when administered orally. This catechoylamide derivative promoted the clearance of $59 \%$ of the injected dose of ${ }^{238} \mathrm{Pu}(\mathrm{IV})$, and was also the most effeclive ligand for reducing the body uptake of this element. A major effect of the orally administered 3,4,3-LICAM(S) was a significant reduction in the liver uptake of ${ }^{238} \mathrm{Pu}(\mathrm{IV})$. This effect of the ligand was attributed to its strong binding affinity for ${ }^{238} \mathrm{Pu}(\mathrm{IV})$ and its rapid absorption into the gastrointestinal tract.

\section{HEMATOZOA AND ERYTHROPOIESIS: EFFECTS OF MALARIA INFECTION}

\section{Paul H. Silverman and Lynn I. Mahlmann}

Anemia and immunosuppression are complications associated with malaria infection. The biological mechanisms that lead to these disease characteristics are only partly understood. Numerous studies have demonstrated that red cell destruction by the plasmodial parasite is inadequate to account for the severity and extent of anemia caused by malaria. It has been suggested that anemia and immunosuppression might be due to the impairment of progenitor hemopoietic cells or an imbalance in the competition between erythropoietic and immune cell lineages that are derived from a common pluripotent stem cell (CFU-s).

During the first year of our work we demonstrated that infection with three species of murine malaria resulted in substantial quantities of erythropoietin (Ep) being produced and available in the circulating plasma. Ep is the hormone responsible for regulating red-cell production by committed erythron progenitors. It has also been found that colony-stimulating factors (CSF) that regulate the proliferation and differentiation of granulocytic blood cells is stimulated in increased quantities during infection with malaria. These findings suggest that the dyserythropoiesis observed in malariainfected hosts might be caused by events inhibiting progentior differentiation and amplification or the lack of progenitor-cell production by stem cells. Our results, reported here and by Dr. I.C. Schooley indicate that both situations occur during malaria infection.
We have reported, previously, that infection with two lethal strains of murine malaria, Plamodium berghei and $P$. vinckei result in significant depletion of pluripotent stern cells in the bone marrow. 1,2 We have now demonstrated that similar depletion occurs with a nonlethal strain of malaria, $P$. chabaudi adami. Maximum depletion of bone marrow stem cells coincides with the maximum parasitemia. As the self-limiting infection with $P$. chabaudi adami runs its course, stem cell levels and the total cellularity of the bone marrow return to normal (Table 1). ${ }^{3}$

Although stem cells and total cellularity have returned to normal or above normal levels when red-cell parasitemia becomes negative, evidence remains that a substantial migration of stem cells has occurred (Table 2). The number of stem cells observed in the spleen and circulating in the blood may account for the bone marrow depletion. However, whether splenic and circulating stem cells are functionally equivalent to bone marrow stem cells remains to be determined. Similar observations were made in mice infected with lethal infections of $P$. berghei and $P$. vinckei prior to death or the host. These observations of $P$. chabaudi adami enabled more extensive evaluations to be made of stem cell kinetics. Determination of the rate of stem cell proliferation by assessing the killing effects of hydroxyurea indicated that DNA synthesis in stem cells in bone marrow or spleen were comparable to that which occurs in normal mice 
Table 1. Changes in female balb/c mice after inoculation with $1 \times 10^{5}$ red blood cells infected with murine malaria."

\begin{tabular}{|c|c|c|c|c|c|c|c|c|}
\hline $\begin{array}{l}\text { Donor } \\
\qquad N\end{array}$ & $\begin{array}{l}\text { Post- } \\
\text { inoculation } \\
\text { (day) }\end{array}$ & $\begin{array}{l}\text { Parasitemia } \\
\qquad(1 / 1)\end{array}$ & $\begin{array}{l}\text { Cells/ } \\
\text { femur } \\
\left(\begin{array}{ll}\times & \left.10^{6}\right)\end{array}\right.\end{array}$ & $\begin{array}{l}\text { CFU-s/ } \\
10^{6} \mathrm{BMC}^{\mathrm{b}}\end{array}$ & $\begin{array}{l}\text { CFU-s/ } \\
\text { femur }\end{array}$ & $\begin{array}{l}\text { Hematocrit } \\
(\%)\end{array}$ & $\begin{array}{l}\text { Body } \\
\text { weight } \\
\text { (g) }\end{array}$ & $\begin{array}{l}\text { Spleen } \\
\text { weight } \\
\text { (g) }\end{array}$ \\
\hline \multicolumn{9}{|c|}{ Uninfected controls: } \\
\hline 16 & - & - & $13.7 \pm 0.50$ & $285 \pm 7.0$ & $3900 \pm 86$ & $47 \pm 0.04$ & $20.7 \pm 0.19$ & $0.10 \pm .001$ \\
\hline \multicolumn{9}{|c|}{ Plasmodium chabaudi adami: } \\
\hline 4 & 6 & $5.5 \pm 0.98$ & $9.4 \pm 0.26$ & $420 \pm 21$ & $4000 \pm 194$ & $40 \pm 1.4$ & $20.0 \pm 0.48$ & $0.41 \pm 0.01$ \\
\hline 4 & 7 & $15.3 \pm 1.1$ & $8.2 \pm 0.75$ & $340 \pm 13$ & $2800 \pm 93$ & $36 \pm 2.5$ & $21.5 \pm 0.29$ & $0.68 \pm 0.12$ \\
\hline 4 & 14 & $<1 \%$ & $11.1 \pm 0.93$ & $220 \pm 11$ & $2400 \pm 155$ & $39 \pm 1.9$ & $22.0 \pm 0.91$ & $1.10 \pm 0.03$ \\
\hline 4 & 23 & zero & $13.8 \pm 0.70$ & $315 \pm 21$ & $4400 \pm 270$ & $48 \pm 0.48$ & $22.5 \pm 0.50$ & $0.34 \pm 0.01$ \\
\hline
\end{tabular}

* All values are expressed as the mean $上$ S.E.M.

b $B M C=$ bone marrow cells

(Table 2, column 4). Similar observations will need to be undertaken with stem cells recovered during active infections before the impact on UNA synthesis can be fully evaluated.

In our previous report, we proposed that stem cell depletion in bone marrow is induced by a diffusable substance. Several candidate substances have been identified, including tumor necrosis factor (TNF). This TNF (or cachectin) is produced by macrophages and/or monocytes during the processing of malaria antigen. It has been reported that TNF inhibits adipocyte gene expression. ${ }^{4.5}$ Adipo- cytes are a major cellular component of the adherent stromal bone marrow and provide an essential environment for renewal and differentiation of the nonadherent hemopoietic stem cells. The inhibitory action of malaria-induced TNF might account for both the drop in total cellularity and the depletion of marrow hemopoietic stem cells. We report here, for the first time, the effects of recombinant human TNF on stem cells in a murine model. ${ }^{6}$

Because of the short half life in vivo of TNF, mini-osmolic pumps containing rHuTNF in RPMI

Table 2. Bone marrow stem cell (CFU-S) content in temale balb/c mice infecled with Plasmodium chabaudi adami."

\begin{tabular}{|c|c|c|c|c|c|c|}
\hline & $N$ & $\begin{array}{c}\text { cells } \times 10^{-6} / \\
\text { organ }\end{array}$ & CFU-S $/ 10^{6} \mathrm{NC}^{6}$ & CFU-S/organ & $\% \mathrm{pRBC}^{\mathrm{C}}$ & $\begin{array}{l}\text { Surviving } \\
\text { fractiond }^{d}\end{array}$ \\
\hline \multicolumn{7}{|l|}{ Marrow: } \\
\hline Normal & 9 & $14 \pm 01.1$ & $260 \pm 23$ & $3600 \pm 120$ & 0 & $0.74=0.034$ \\
\hline Day 17-18 & 4 & $20 \pm 0.92$ & $170 \pm 12$ & $3550 \pm 180$ & 0 & $0.69 \pm 0.057$ \\
\hline Day 24 & 7 & $15 \pm 0.78$ & $290 \pm 20$ & $4350 \pm 190$ & 0 & $0.87 \pm 0.069$ \\
\hline \multicolumn{7}{|l|}{ Spleen: } \\
\hline Normal & 5 & $130 \pm 3.4$ & $15 \pm 0.79$ & $2000 \pm 93$ & 0 & $0.77 \pm 0.059$ \\
\hline Day $17-18$ & 4 & $900 \pm 61$ & $45 \pm 4.8$ & $40200 \pm 2300$ & 0 & $0.79 \pm 0.065$ \\
\hline \multirow[t]{2}{*}{ Day 24} & 5 & $400 \pm 17$ & $29 \pm 2.0$ & $11800 \pm 670$ & 0 & $0.68 \pm 0.068$ \\
\hline & $N$ & $\mathrm{NC} \times 10^{-\mathrm{b}} / \mathrm{ml}$ & CFU $-5 / 10^{7} N C$ & $\mathrm{CFU} \cdot \mathrm{S} / \mathrm{ml}$ & $\%$ pRBC & \\
\hline \multicolumn{7}{|l|}{ Whole Blood: } \\
\hline Normal & 10 & $8.5 \pm 0.71$ & $4.2 \pm 1.5$ & $3.4 \pm 1.3$ & 0 & \\
\hline Days $17-18$ & 4 & $11.0 \pm 0.75$ & $280 \pm 30$ & $300 \pm 24$ & 0 & \\
\hline Day 24 & 4 & $4.9 \pm 0.26$ & $43 \pm 5.0$ & $21 \pm 2.4$ & 0) & \\
\hline
\end{tabular}

- All values are expresserl as the mean \pm 5.L.M.

- $\mathrm{NC}=$ nucleated cells

- $P R B C=$ parasitized red blood cells

- Fraction surviving after injection with hydroxyurea 
1640 tissue culture medium were implanted subcutaneously into female Balb/c mice. At an infusion rate of $1 \mu \mathrm{l} / \mathrm{hr}$ the estimated plasma concentration of TNF was 13,200 units. Control mice were infused with RPMI 1640. After six days of infusion the total CFU-s content of the blood, bone marrow and spleen was determined using the spleen colony assay.

In many respects, after six days of infusion, the TNF-induced cellular changes in mice are very similar to those observed in malaria-infected animals. The total number of bone marrow nucleated cells is decreased, a significant decrease in the number of bone marrow stem cells occurs, stem cell levels in the spleen are greatly increased, and evidence of stem cell migration is apparent by their presence in large numbers in the circulating blood (Table 3 ). Much more needs to be done to determine the specific cellular elements affected by rHuTNF in the murine model and also how this compares with murine TNF.

The relevance of these observations to the production of TNF during malaria infection was tested by injecting monoclonal antibody prepared against rHuTNF into mice prior to infecting with $P$. vinckei and $P$. berghei. Booster doses of $10 \mu \mathrm{g}$ of the monoclonal antibody (anti-TNF) were given every
72 hours until the mice were tested for CFU-S content. It was anticipated that the antibody might have a sparing, effect on the bone marrow stem cell population, and such results were seen in mice infected with $P$, vinckei. Although early observations indicated more dramatic protection effects, replicate experiments resulted in the data summarized in Table 4. It is highly probable that antigenic differences occur between human and murine TNF which might account for the lack of activity with the monoclonal antibody.

In addition to the potential role of TNF/cachetin in the pathogenicity of malaria, other work (reported by J.C. Schooley) has identified interleukins as contributors to the regulation of hemopoietic activity. The insights gained by analysis of this model system will have application to a basic understanding of hemopoiesis and might lead to manipulation of differentiation and proliferation of various cell lineages.

\section{REFERENCES}

1. Silverman, P.H. Schooley, J.C., and Mahlmann, L.J. Erythropoietin and bone marrow stem cell responses to murine malaria. 60th Annual Meeting American Society of

Table 3. Effect of iniused rHuTNF on CFU-5 content in marrow, spleen, and blood in balb/c mice. ${ }^{b}$

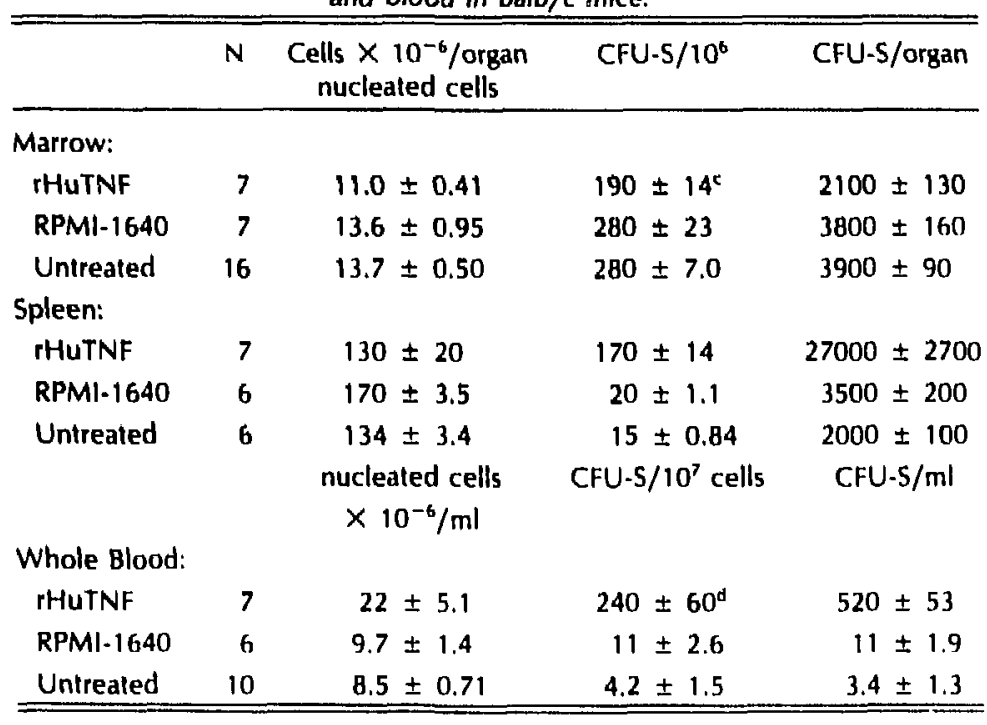

" Supplied by Genentech

b All values are expressed as the mean \pm S.E.M. Untreated mice did not have subcutaneous implants of mini-osmotic pumps.

- $P<0.01$

d $P<0.001$ 
Table 4. Effect of anti-TNF monoclonal antibody on bone marrow CFU-S in balb/C mice infected with murine malaria."

\begin{tabular}{lccccc}
\hline & $\%$ pRBC & CFU-S/10 BMC & CFU-S/femur & cells $\times 10^{-6} /$ femur & $N$ \\
\hline Control & 0 & $290 \pm 7.0$ & $3900 \pm 86$ & $14 \pm 0.50$ & 16 \\
Anti-TNF & 0 & $290 \pm 16$ & $3500 \pm 270$ & $13 \pm 0.39$ & 4 \\
$\begin{array}{l}\text { Plasmodium vinckei vinckei (Day } 8 \text { ): } \\
\text { Infected }\end{array} 74 \pm 3.9$ & $280 \pm 10.0$ & $2400 \pm 100$ & $8.2 \pm 0.49$ & 8 \\
+ Normal sera & $76 \pm 15$ & $300 \pm 18$ & $2500 \pm 140$ & $8.4 \pm 0.36$ & 4 \\
+ Anti-TNF & $70 \pm 7.8$ & $360 \pm 12$ & $3100 \pm 130$ & $8.9 \pm 0.79$ & 7 \\
$\begin{array}{l}\text { Plasmodium berghei (Day 10): } \\
\text { Infected }\end{array} 50 \pm 6.8$ & $190 \pm 12$ & $1300 \pm 90$ & $6.4 \pm 0.35$ & 8 \\
+ + Normal sera & $56 \pm 11$ & $280 \pm 25$ & $1760 \pm 160$ & $6.3 \pm 0.19$ & 3 \\
+ Anti-TNF & $61 \pm 2.3$ & $150 \pm 15$ & $1300 \pm 150$ & $7.4 \pm 0.60$ & 8 \\
\hline
\end{tabular}

2 Supplied by Chiron

b All values are expressed as the mean \pm S.E.M.

c $\mathrm{pRBC}=$ parasitized red blood cells

Parasitologists, Abstract No. 150, August 4-8, 1985, Athens, GA.

2. Maggio-Price, L., Brookhoff, D., and Weiss, L. Changes in hematopoietic stem cells in bone marrow of mice with Plamodium berghei malaria. Blood 66, 1080 (1985).

3. Silverman, P.H., Schooley, J.C., and Mahlmann, L.J. Murine malaria decreases hemopoietic stem cells. Blood, in press.

4. Torti, F.M., Dieckmann, B., Beutler, B., Cerami, A., and Ringold, G.M. A macrophage factor inhibits adipocyte gene expression: an in vitro model of cachexia. Science 229, 867 (1985).

5. Seutler, B., Milsark, I.W., and Cerami, A.C. Passive immunization against cachectin/tumor necrosis factor protects mice from lethal effect. of endotoxin. Science 229, 869 (1985).

6. Silverman, P.H., Schooley, J.C., Mahlmann, L.J., and Smith, K.L. Mechanisms of hemopoietic suppression by murine malaria. 35th Annual Meetirig of the American Society of Tropical Medicine and Hygiene, Abstract No. 121, December 7-11, 1986, Denver, CO.

\section{STROMAL CELL CHANGES IN MICE INFECTED WITH MURINE MALARIAS}

\section{John C. Schooley, Karen L. Smith, and Birgitta Kullgren}

The supportive cells of tissues (the stromal cells) not only provide a structural matrix, but also modulate by their synthetic activity the proliferation, differentiation, and gene expression of the nonstromal cells in many tissues. For example, the stromal cells of the hematopoietic tissues-the fibroblasts or fibroblast-like cells, endothelial cells and adipocytes-secrete various structural proteins such as collagen, proteoglycans, fibronectin, and also synthesize various cytokines that modulate the activities of the precursors of myeloid and lymphocytic cells and their progeny. Conversely, the hematopoietic cells secrete cytokines that modulate the activities of the stromal cells. Some of these complex interrelationships between the various cells of the hematopoietic system and the various cytokines that they produce are shown in Fig. 1. Megakaryocytes produce platelets, and the platelets release platelet-derived growth factor (PDGF) which stimulates fibroblast proliferation. Macrophages and lymphocytes produce fibroblast-activating factors as well as TNF $\alpha$ and TNF $\beta$ which play an important role in regulating the conversion of preadipocytes (a fibroblast subpopulation?) into adipocytes in some tissues, although the details of this process in hematopoietic tissue have not yet been resolved. 


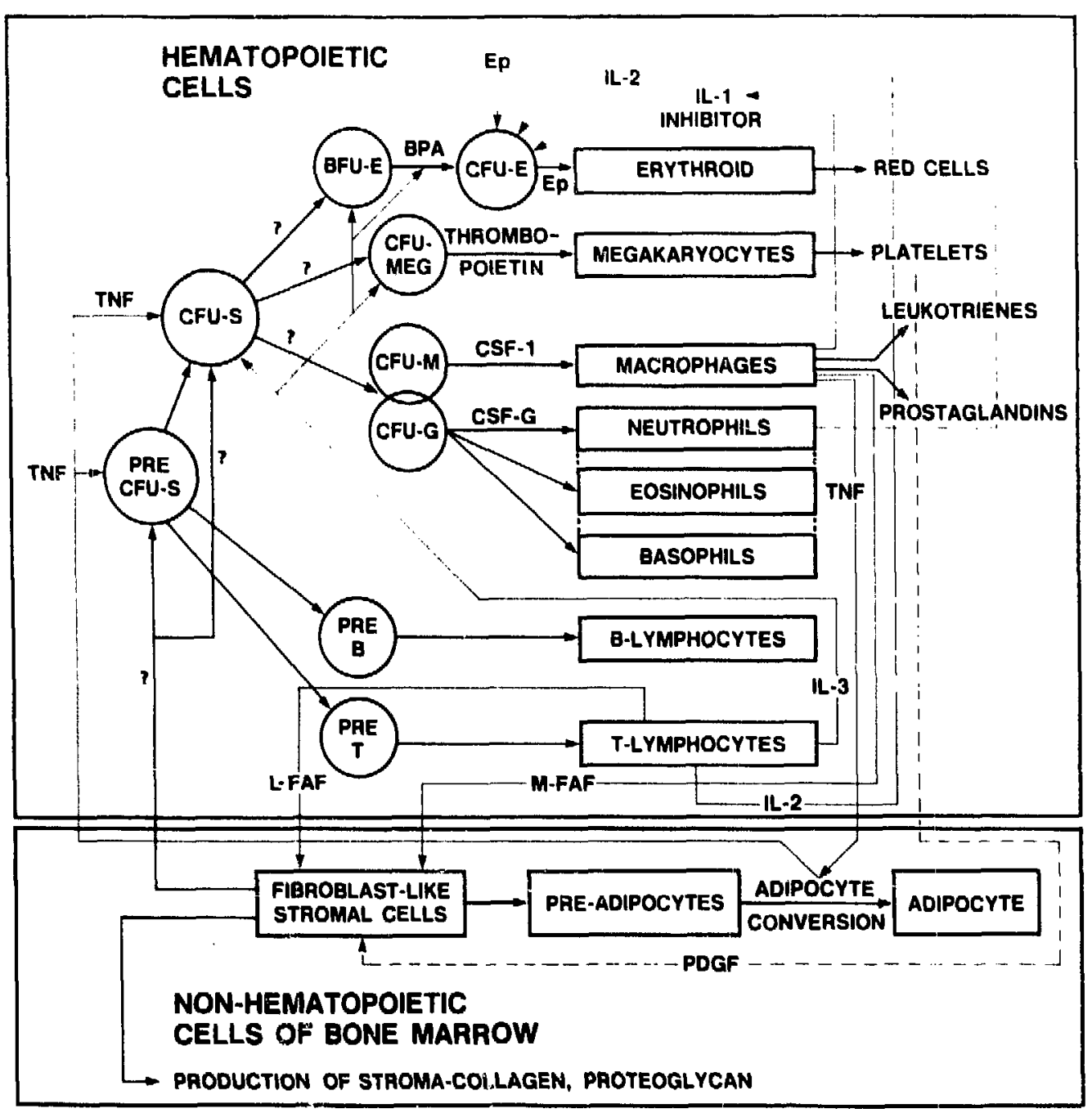

Fig. 1. Complex interrelationships between various cells of the hematopietic system and the various cytokines produced, TNF = tumor necrosis factor; FAF = fibroblast activating factor; BPA = burst promoting activity; CSF = colony stimulating factor; PDGF = platelet derived growth factor.

(XBL 8610-3867A)

There is evidence that implicates adipocytes in hematopoiesis, especially in erythropoiesis, and we have considered that alterations in the conversion of fibroblast precursors to adipocytes as well as the production of fibroblasts themselves may play an important role in the dyserythropoiesis of chronic disease.

We have used mice infected with murine malaria as a model of chronic disease and have determined the numbers of fibroblast-like progenitor cells (CFU-F) in the hematopoietic tissues during the early stages of infection using an in vitro cloning procedure to enumerate the cells. We find that the CFU-F in the bone marrow of the tibia of mice are significantly reduced $(\mathrm{P}<0.001)$ by the sixth day after infection with murine malaria. The results of one representative experiment are shown in Table 1. As can be seen from this table, the CFU-F of $P$. vinckei-infected animals, the more virulent of the two malarial species tested, was significantly reduced by the third day of infection: $214 \pm 17$ CFU-F/tibia as opposed to $364 \pm 15 \mathrm{CFU}-\mathrm{F} / \mathrm{tibia}$ in the control animals. By day 6 of infection, animals infected with either $P$. vinckei or $P$. berghei were both significantly depressed from normal levels. By day 10, $P$. berghei infected animals consistently showed CFU-F values far exceeding those of normal animals. In Table 1 they had reached $230 \% \pm 13 \%$ of normal values. The significance of this abortive attempt at reestablishment of 
Table 1

\begin{tabular}{|c|c|c|c|c|c|}
\hline Organism & $\begin{array}{l}\text { Day of } \\
\text { infection }\end{array}$ & $\begin{array}{l}\text { Nucleated cells } \\
\left(\times 10^{6}\right) / \text { TIBIA }\end{array}$ & $\begin{array}{l}\text { CFU-F/ } \\
\text { TIBIA }\end{array}$ & $\begin{array}{l}\text { Percent of } \\
\text { Normal CFU-F }\end{array}$ & $\begin{array}{c}\mathrm{P} \\
\text { value }\end{array}$ \\
\hline Normal & & $16.2 \pm 0^{a *}$ & $364 \pm 15$ & & \\
\hline P. berghei & 3 & $15.1 \pm 0.7$ & $378 \pm 20$ & $104 \pm 7$ & $N S^{b}$ \\
\hline P. vinckei & 3 & $12.6 \pm 1.2$ & $214 \pm 17$ & $59 \pm 5$ & $P<0.001$ \\
\hline Normal & & $15.3 \pm 0.8$ & $237 \pm 18$ & & \\
\hline P. berghei & 6 & $10.7 \pm 0.7$ & $64 \pm 6$ & $27 \pm 3$ & $P<0.001$ \\
\hline$P$. vinckei & 6 & $13.3 \pm 0.6$ & $126 \pm 10$ & $53 \pm 6$ & $P<0.001$ \\
\hline Normal & & $15.5 \pm 0.8$ & $395 \pm 17$ & & \\
\hline P. berghei & 10 & $15.7 \pm 0.7$ & $910 \pm 31$ & $230 \pm 13$ & $P<0.001$ \\
\hline
\end{tabular}

- Mean \pm standard error of the mean.

- Not significant.

stromal cells is only conjecture at this time, but may reflect the fact that this is the period during infection during which erythropoiesis is maximally stimulated. These animals generally die at 14-18 days postinfection while $P$. vinckei-infected animals seldom live longer than 8 or 9 days. The results of three experiments are consistent with those of the experiment depicted in Table 1 and show that the CFU-F/tibia of mice infected with $P$. berghei range from $16 \%$ to $24 \%$ of normal at day 6 of the infection while those from animals infected with $P$. vinckei were $30 \%$ to $53 \%$ of normal at the same point in the infection. We have found in other studies that the total numbers of pluripotential hematopoietic stem cells (C.FU-S) are also reduced at this time. In contrast, the total numbers of CFU-GM, early progenitors of granulocytic and macrophage cells, are significantly increased at this time. An increase in CFU-GM would be expected since we find that the concentration of the glycoprotein colony stimulating factor (CSF) is elevated in the serum on the sixth day postinfection. The concentration of erythropoiatin (Ep), the hormone that regulates red blood cell formation, is also significantly elevated in the serum, but the mice are severely anemic, and the numbers of recognizable erythroid cells are reduced in the bone marrow as are the total numbers of erythropoietin-responsive cells.

We conclude that mice with malaria have an elevation in the serum level of CSF and Ep, the factors that regulate the differentiation of two hematopoietic cell lines, the granulocytic and the erythroid, and the granulocytic system is stimulated while the erythroid is inhibited. The concept that the dyserythropoiesis of malaria directly results from a reduction in CFU-S appears untenable, since ultimately both granulocytes and erythroid cells are derived from the multipotential progenitor. We have suggested that the dyserythropoiesis may result because the production of erythroid cells from early erythroid progenitors is blocked by some soluble agent generated by mononuclear cells (probably interleukin 1). The data presented here suggest that an additional disturbance in erythropoiesis may result from the reduction of CFU-F and, ultimately, adipocytes in the bone marrow animals infected with either $P$. vinckei or $P$. berghei were both significantly depressed from normal levels. By day $10, P$. berghei infected animals consistently showed CFU.F values far exceeding those of normal animals. In Table 1 they had reached $230 \% \pm 13 \%$ of normal values. The significance of this abortive attempt at reestablishment of stromal cells is only conjecture at this time, but may reflect the fact that this is the period during infection during which erythropoiesis is maximally stimulated. These animals generally die at 14-18 days postinfection while $P$. vinckei-infected animals seldom live longer than 8 or 9 days. The results of three experiments are consistent with those of the experiment depicted in Table 1 and show that the CFU-F/tibia of mice infected with $P$. berghei range from $16 \%$ to $24 \%$ of normal at day 6 of the infection while those from animals infected with $P$. vinckei were $30 \%$ to $53 \%$ of normal at the same point in the infection. We have found in other studies that the total numbers of pluripotential hema- 
topoietic stem cells (CFU-S) are also reduced at this time. In contrast, the total numbers of CFU-GM, early progenitors of granulocytic and macrophage cells, are significantly increased at this time. An increase in CFU-GM would be expected since we find that the concentration of ihe glycoprotein colony stimulating factor (CSF) is elevated in the serum on the sixth day postinfection. The concentration of erythropoietin (Ep), the hormone that regulates red blood cell formation, is also significantly elevated in the serum, but the mice are severely anemic, and the numbers of recognizable erythroid cells are reduced in the bone marrow as are the total numbers of erythropoietin-responsive cells.

We conclude that mice with malaria have an elevation in the serum level of CSF and Ep, the factors that regulate the differentiation of two hematopoietic cell lines, the granulocytic and the erythroid, and the granulocytic system is stimulated while the erythroid is inhibited. The concept that the dyserythropoiesis of malaria directly results from a reduction in CFU-S appears untenable, since ultimately both granulocytes and erythroid cells are derived from the multipotential progenitor. We have suggested that the dyserythropoiesis may result because the production of erythroid cells from early erythroid progenitors is blocked by some soluble agent generated by mononuclear cells (probably interleukin 1). The data presented here sugge it that an additional disturbance in erythropoiesi, may result from the reduction of CFU-F and, ultimately, adipocytes in the bone marrow which may limit the energy source for erythropoiesis.

Our understanding of the events that lead to these complex alterations in early hematopoietic progenitors, their progeny, the stromal populations and the hormones and cytokines that regulate their proliferation, differentiation, and biochemical activity is slowly advancing. It appears clear that the dyserythropoiesis of pathophysiological conditions such as malaria is multifactorial, resulting from alterations in stromal cells and stem cells, and the production of erythropoietin-responsive cells, and rational therapeutic approaches to managing this aspect of the disease must consider this complexity.

\title{
INHIBITION BY INTERLEUKIN-1 OF THE ACTION OF ERYTHROPOIETIN OF ERYTHROID PRECURSORS AND ITS POSSIBLE ROLE IN THE PATHOGENESIS OF HYPOPLASTIC ANEMIAS
}

\author{
John C. Schooley, Birgitta Kullgren, and Anthony C. Allison*
}

\section{SUMMARY}

Highly purified and cloned preparations of interleukin-1 (IL-1) were found to antagonize the capacity of erythropoietin (Ep) to stimulate the proliferation of mouse spleen and bone marrow erythroid precursor cells (EPC) in culture. Cloned murine $\mathrm{IL}-1$ and purified and cloned human $\mathrm{L}-1 \alpha$ and $\mathrm{IL}-1 \beta$ were approximately equipotent in this assay. IL-1 inhibited the proliferation response of EPC even when added as long as 17 hours after Ep, suggesting that $\mathrm{LL}-1$ does not affect binding of Ep to receptors or biochemical events following shortly thereafter. Indomethacin did not influence the inhibitory effect of IL-1 on Ep-induced proliferation, and $\mathrm{PGE}_{2}$ had no demonstrable effect on the pro-

Institute of Biological Sciences, Syntex Research, Palo Alto, CA, U.S.A. cess. Tumor necrosis factor $\alpha$ and interferons did not affect Ep-induced proliferation.

It is suggested that IL-1-mediated antagonism of the effects of Ep on erythroid precursors is a factor in the pathogenesis of many types of hypoplastic anemia, including those associated with infections, rheumatoid arthritis and systemic lupus erythematosus, giant-cell arteritis, graft-versus-host disease, and disorders associated with lymphocyte-mediated suppression of erythropoiesis.

Pure red-cell aplasia is a syndrome characterized by normochromic normocytic anemia, reticulocytopenia, and deficiency of erythroblasts in otherwise normal bone marrow. It may be congenital (Diamond-Blackfan syndrome) ${ }^{\prime}$ or associated with chronic infections, ${ }^{2}$ rheumatoid arthritis, ${ }^{3}$ systemic lupus erythematosus ${ }^{4}$ or giant cell arteritis. ${ }^{5}$ Anemia 
in the presence of other abnormalities is associated with graft-versus-host reactions ${ }^{6}$ and ill-defined disorders responding to therapy with immunoglobulin against thymocytes. ${ }^{7}$ Previous attempts to find a common factor underlying the pathogenesis of these hypoplastic anemias have failed. In this paper we present evidence that two varieties of interleukin-1 (IL-1 $\alpha$ and IL-1 $\beta$ ) inhibit the action of erythropoietin (Ep) on Ep-responsive cells (ERC) recovered from mouse spleens or bone marrows, and we discuss the possible relevance of this finding to the pathogenesis of hypoplastic anemias.

IL-1 formation by monocyles, macrophages and perhaps other cell types can be induced by microbial products such as lipopolysaccharide endotoxins $^{8}$ or the muramyl dipeptide components of bacterial cell walls. ${ }^{9} \|-1$ formation can also be stimulated by products of activated T- or B-lymphocytes. Macrophages can be induced to produce a thymocyte-mitogenic factor, now termed IL-1, by $T$-lymphocytes in a cell-contact-dependent interaction requiring la-identity ${ }^{10}$ or through the release by T-lymphocytes of mediators such as GM-colonystimulating factor that can act on macrophages without direct contact in a genetically unrestricted way. ${ }^{11}$ Immune complexes and self-associating immunoglobulins such as $\lg G$-anti-lgC, which are found in patients with rheumatoid arthritis, can induce the production of $\mathrm{IL}-1$ by human monocytes. $^{12}$ The action of interferon- $\gamma$ (IFN- $\gamma$ ) and lipopolysaccharide endotoxin (LPS) on human monocytes provides an example of a synergistic action of a product of activated T-lymphocytes and a microbial product in the induction of IL- 1 synthesis and release. IFN- $\gamma$ does not by itself induce $I L-1$ formation, but it sensitizes the monocytes so that they produce much more IL-1 when exposed to LPS. ${ }^{13}$ We suggest that production of IL-1 by microbial products, or by an ongoing T- or B-lymphocytemediated immune response, or by synergistic effects of these two mechanisms, may be a common factor underlying the pathogenesis of many types of hypoplastic anemia.

Chronic infections such as tuberculosis, brucellosis, osteomyelitis, and systemic fungal diseases are often associated with a normochromic normocytic anemia resistant to iron therapy. The pathogenesis of anemia associated with infections is not well understood. Decreased erythropoiesis is the main cause of the anemia, and this is found despite high serum Ep levels in many patients ${ }^{2}$. Serum iron concentrations are often low, apparently because of iron accumulation in phagocytic cells. Adherent cells from the bone marrow of patients with the anemia of chronic disease have been reported to produce a factor or factors suppressing erythropoiesis in vitro. ${ }^{14,15}$

Analysis of anemia associated with infection is facilitated by the use of fully characterized microbial products and mediators. LPS markedly inhibits erythropoiesis. Following LPS administration to mice there is reduction in the number of erythroid burst-forming units (BFU-E) $)^{16}$ and colony-forming units (CFU-E) and recognizable nucleated erythroid cells in hematopoietic tissues. ${ }^{17.18}$ Although deficiency of circulating Ep may contribute to this effect, ${ }^{17}$ there is also evidence for active suppression of erythropoiesis. Mouse peritoneal macrophages cultured in the presence of LPS release a factor that inhibits the growth and differentiation of erythroid precursors in vitro. ${ }^{19}$

The objective of our research has been to define monocyte or macrophage-derived mediators that can suppress erythropoiesis and could therefore contribute to anemia associated with infection. Four glycoprotein products of activated human monocyles or macrophages have been sequenced and cloned: interferon- $\alpha(\mathrm{IFN}-\alpha){ }^{20}$ two varieties of IL-1 (IL- $1 \alpha$ and IL-1 $\beta)^{21-24}$ and tumor necrosis factor $\alpha($ TNF $-\alpha){ }^{25}$ We have found that both IL-1 $\alpha$ and IL-1 $\beta$ inhibit the action of Ep on ERC in culture, whereas TNF- $\alpha$ and interferons have no demonstrable effect. Prostaglandins do not measurably contribute to the suppressive effects of IL-1.

We therefore suggest that IL-1 may be a major mediator of the inhibition of erythropoiesis that follows LPS administration, and in the discussion we summarize evidence supporting the view that IL-1 may be a common mediator of many types of hypoplastic anemia. Inhibition of IL-1 production may explain the beneficial affects of glucocorticoid therapy in the Diamond-Blackfan syndrome ${ }^{\prime}$ and anemia associated with giant-cell arteritis, ${ }^{5}$ and may be one factor in the favorable response to immunosuppressive therapy that can occur in hypoplastic anemias associated with T-lymphocyte activation. ${ }^{7}$

\section{MATERIALS AND METHODS}

Mice. Two- to three-month old female BALB/C BK mice, obtained from Bantin and Kingman, Fiemont, CA, weighing $22-25 \mathrm{~g}$, were made anemic by two consecutive daily intraperitoneal injections of a neutralized solution of phenylhydrazine hydrochloride in er-minimal essential medium ( $\alpha-M E M)$ $(0.3 \mathrm{ml}$ of a $6 \mathrm{mg} / \mathrm{ml}$ solution). 
Cell Cultures. Hypertrophied spleens and femurs enriched in ERC were removed 3 days after the second phenylhydrazine injection, and single-cell suspensions were prepared in $\alpha$-MEM and diluted to a cell concentration of about $6 \times 10^{6}$ cells $/ \mathrm{ml}$. Similar single-cell suspensions were made of the spleen and femoral contents of normal mice. Ten $\mathrm{ml}$ of these cell suspensions contained $2.5 \mathrm{ml}$ of fetal bovine serum (FBS) (Flow Laboratories, Inglewood, $C A$; lot \#29101676), $0.1 \mathrm{ml}$ of penicillinstreptomycin solution (Gibco, cat. \#600-5245), and $6 \mu \mathrm{l}$ of a 7 -mercaptoethanol (ME) solution $(14 \mu \mathrm{l}$ ME added to $1 \mathrm{ml} \alpha-M E M)$.

Chemicals and Biological Reagents. Phenylhydrazine and ME were obtained from Sigma Cheinicals (St. Louis, MO). Human urinary Ep (specific activity $>500$ IU/mg protein) was obtained from Alpha Therapeutics (Los Angeles, CA) and recombinant Ep was obtained from AmGen Biologicals (Thousand Oaks, CA) (specific activity $>70,000 \mathrm{IU} / \mathrm{ml}$ protein). These commercial Ep preparations were calibrated against the International Reference Preparation of human urinary Ep using the previously described post-carbon-monoxide plethoric mouse assay. ${ }^{26}$ and no difference was observed between the behavior of the two Ep preparations in the in vitro assay system. Human $\mathrm{IL}-1$ purified from activated human peripheral blood monocytes, ${ }^{27}$ consisting almost entirely of the glycosivated $\mathrm{IL}-1 \beta$ form, and human recombinant $\mathrm{LL}-1 \alpha$ isid $\mathrm{IL}-1 \beta^{23}$ were from Immunex Corporation, Seattle, WA. One unit of IL-1 activity (stimulation of IL-2 production by the murine T-cell line LBRM-33-1A5) corresponds to $0.1 \mathrm{pg}$ protein. Recombinant murine IL-1 was provided by Dr. P. Lomedico, Hoffman La Roche, Nutley, N). Recomb: nant TNF$\alpha$ and murine IFN- $\gamma$ were provided by conentech, Inc., South San Francisco, CA. All preparations were obtained under conditions designed to exclude LPS, and did not have LPS detectable by the limulus lysate assay or immunoassay.

Assays for Response to Ep. The in vitro assay using spleen or bone marrow suspensions was essentially that described by Krystal ${ }^{28}$ and is illustrated diagrammatically in Fig. 1. Briefly, $20 \mu \mathrm{l}$ of each dilution of Ep with or without IL-1 or TNF- $\alpha$ was added to $80 \mu \mathrm{l}$ of the single cell suspensions in a 96-well culture tray (Coslar, Cambridge, MA) (approximately $5 \times 10^{5}$ cells/well). The cells were incubated for $22 \mathrm{hr}$ at $37^{\circ} \mathrm{C}$ in a humidified atmosphere of $5 \% \mathrm{CO}_{2}$ and $95 \%$ air. Then $20 \mu \mathrm{l} \alpha$ -

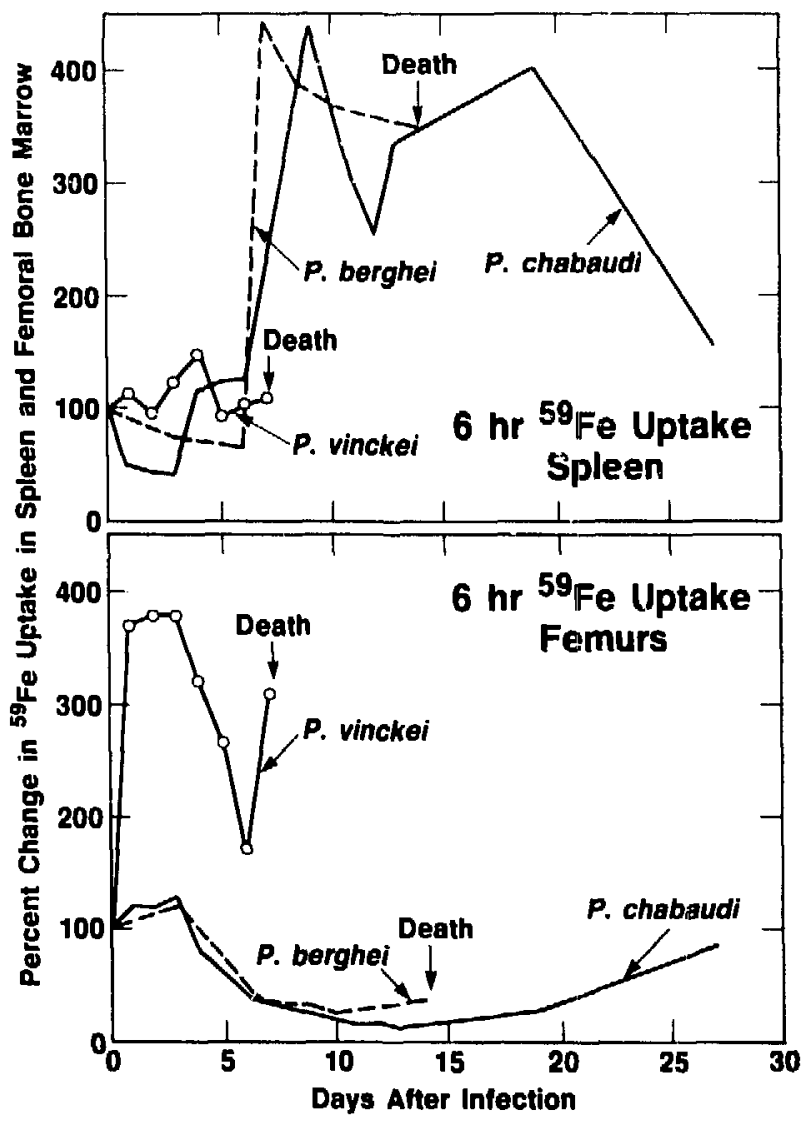

Fig. 1. Diagram of the procedure used to measure the in vitro uplake of ${ }^{3} \mathrm{H}$-h hymidine induced by erythropoietin acting on suspensions of bone marrow or spleen cells.

(XBL 8512-8619)

MEM containing $1.0 \mu \mathrm{Ci}$ of methyl- ${ }^{\mathrm{H}} \mathrm{H}$-thymidine (1 $\mathrm{mCi} / \mathrm{ml}, 10 \mathrm{Ci} / \mathrm{mmole}$, New England Nuclear) was added to each well, and after a further incubation for $2 \mathrm{hr}$ the cells were harvested onto glass-fiber filters using a Skatron Microharvester (Sterling, VA). The glass-fiber discs were punched out and counted using Econofluor scintillation fluid (New England Nuclear) in a Beckman LS9000 counter. Each determination has been expressed as the mean \pm standard deviation (SD) of the mean of the ${ }^{3} \mathrm{H}$-thymidine uptake by the cells in $6-8$ individual wells. When appropriate, as indicated in the text, the uptake of thymidine by cells without the addition of Ep has been subtracted from the total uptake to show the net uptake induced by the presence of added Ep. Examination of autoradiographs of cytospin preparations of the Ep-stimulated spleen suspensions indicated that the label was primarily in early proerythroblast cells. 


\section{RESULTS}

In Table 1 the responses of normal and anemic spleen cells to partially purified Ep $(125 \mathrm{mU} \mathrm{Ep} / \mathrm{ml})$ in the presence of various doses of IL-1 purified from activated human peripheral blood cells is given. Normal spleen cells show little uptake of ${ }^{3} \mathrm{H}$-thymidine (Row A), and uptake is slightly increased by the presence of $20 \mathrm{pg} / \mathrm{ml}$ of $\mathrm{IL-1}$; this effect is presumably due to stimulation of nonerythroid cells. Addition of Ep more than doubles the uptake of ${ }^{3} \mathrm{H}$-thymidine (Row B compared to Row A), and IL-1 has no significant effect on this response, perhaps because inhibition of erythroid cells is balanced by stimulation of nonerythroid cells. Suspensions of anemic spleen cells show an uptake of ${ }^{3} \mathrm{H}$-thymidine more than twice that observed with an equal number of normal spleen cells (Row C compared to Row A). As expected, the addition of Ep markedly increases DNA synthesis by spleen cells recovered from anemic mice (Row D, from 5.59 to $70.9 \times 10^{3} \mathrm{cpm}$ ). This increase, triggered by the presence of Ep, was reduced from 70.9 to $24 \times 10^{3} \mathrm{cpm}$ by the addition of $20 \mathrm{pg} / \mathrm{ml}$ of IL-1.

The effect of Ep and IL-1 on DNA synthesis by suspensions of normal femoral bone marrow cells and bone marrow cells obtained from mice mad: anemic by phenylhydrazine injections (anemic bone marrow) is shown in Table 2. Normal bone marrow cells exhibit a rather high uptake of ${ }^{3} \mathrm{H}$-thymidine in the absence of Ep (Row A). The addition of Ep to these cells (Row B) further increases DNA synthesis approximately 2.5-rold above the control levels. The addition of IL- 1 , especially in higher doses, leads to a significant decrease in Ep-induced DNA synthesis of normal bone marrow cells. Anemic bone marrow cells behave similarly, responding to Ep with an increased ${ }^{3} \mathrm{H}$-thymidine cellular incorporation (Row D compared to Row C). This DNA synthesis is significantly reduced at IL-1 concentrations of $20 \mathrm{pg} / \mathrm{ml}$ and, in many cases, even at the lower concentrations of 10 and 5 $\mathrm{pg} / \mathrm{ml}$. In our experience, although normal spleen and bone marrow and anemic bone marrow do respond to Ep, only anemic spleen cells consistentiy respond to the hormone in a dose-dependent manner. We have, therefore, emphasized the data accumulated with anemic spleen cells in this paper.

The effect of recombinant human IL- $1 \alpha$ and IL$1 \beta$ on DNA synthesis by anemic spleen cells in response to doses of Ep varying from 31 to 500 $\mathrm{mU} / \mathrm{ml}$ is shown in Fig. 2 . It is evident that either form of IL-1 significantly ( $p<0.001$ ) inhibits the Ep-generated response except at the highest (500 $\mathrm{mU} / \mathrm{ml}$ ) Ep dose. With low Ep doses the inhibition is potent. The effect of various concentrations of recombinant $\|-1 \alpha$ and IL-1 $\beta$ on reducing the uptake of ${ }^{3} \mathrm{H}$-thymidine in suspensions of anemic spleen cells induced by $125 \mathrm{mU} / \mathrm{ml}$ of recombinant produced Ep is shown in Table 3. Two separate experiments on the effect of IL-1 $\beta$ are indicated. It is also clear from the data in Table 3 that TNF- $\alpha$ over a 100-fold range in concentration has no demonstrable effect on Ep-induced DNA synthesis by suspensions of spleen cells obtained from mice made anemic by phenylhydrazine injections.

The histograms in Fig. 3 demonstrate that IL-1 $\alpha$ (upper histograms) and IL-1 $\beta$ (lower histograms) sig-

Table 1. Effect of $1 \mathrm{~L}-1$ on the uptake of ${ }^{3} \mathrm{H}$-thymidine

by spleen cells obtained from normal and anemic mice cultured with Eip.

\begin{tabular}{|c|c|c|c|c|c|}
\hline \multirow{2}{*}{\multicolumn{2}{|c|}{ Spleen }} & \multicolumn{4}{|c|}{ 2-hr ${ }^{3} \mathrm{H}$-thymidine incorporation $\left(\times 10^{-3} \mathrm{cpm}\right)$} \\
\hline & & Without IL-1 & $\begin{array}{c}\mid \mathrm{LL}-1 \\
20 \mathrm{pg} / \mathrm{ml}\end{array}$ & $\begin{array}{c}\mid \mathrm{L}-1 \\
10 \mathrm{pB} / \mathrm{ml}\end{array}$ & $\begin{array}{c}\mid \mathrm{L} \cdot 1 \\
5 \mathrm{pg} / \mathrm{ml}\end{array}$ \\
\hline A. & Normal spleen & $1.74 \pm 0.597^{4}$ & $5.01 \pm 0.81$ & $3.18 \pm 0.49$ & $2.93 \pm 0.96$ \\
\hline B. & $\begin{array}{l}\text { Normal spleen } \\
\text { with Ep }\end{array}$ & $8.97 \pm 1.2$ & $10.4+1.9$ & $8.75 \pm 0.81$ & $8.34 \pm 0.53$ \\
\hline C. & Anemic spleen & $5.59 \pm 1.5$ & $4.7 i \pm 1.5$ & $3.98 \pm 1.1$ & $3.68 \pm 0.68$ \\
\hline & $\begin{array}{l}\text { Anemic spleen } \\
\text { with Ep }\end{array}$ & $70.9 \pm 12$ & $24.0 \pm 2.4$ & $21.3 \pm 1.8$ & $23.2 \pm 3.0$ \\
\hline
\end{tabular}

- Mean \pm standard deviation of the mean of 6-8 determinations. $125 \mathrm{mU} / \mathrm{ml}$ of Ep. 
Table 2. Effect of $\mathrm{L}-1$ on the uptake of ${ }^{3} \mathrm{H}$-thymidine by bone marrow cells obtained from normal and anemic mice cultured with Ep.

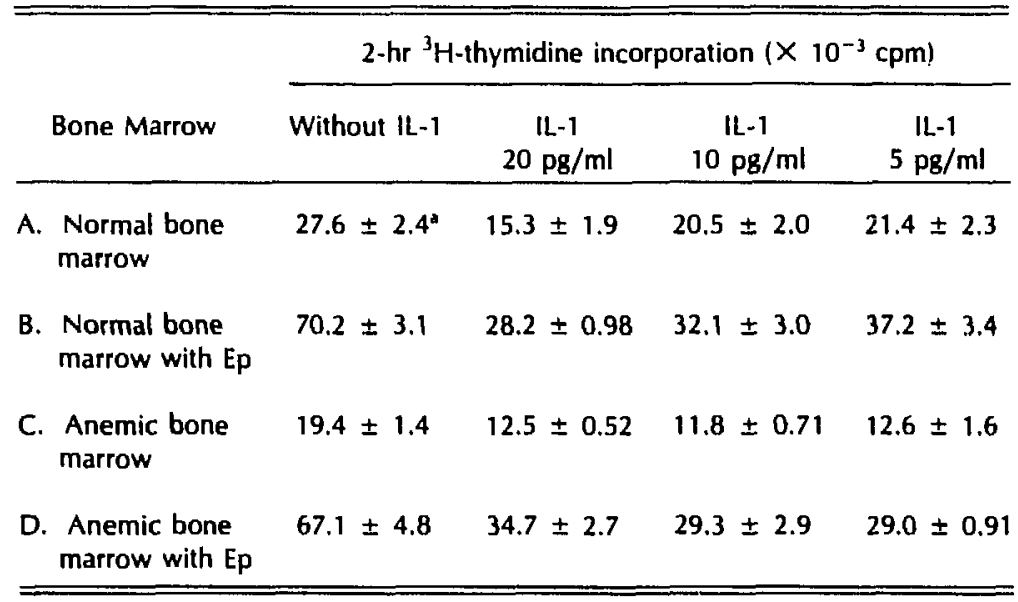

- Mean \pm standard deviation of the mean of $6-8$ determinations. $125 \mathrm{mU} / \mathrm{ml}$ of Ep.

nificantly reduce ( $p<0.001$ ) the magnitude of the DNA synthesis induced by various concentrations of Ep when added to cultures at the same time as the hormone and 7 or $17 \mathrm{hr}$ later. In contrast, the

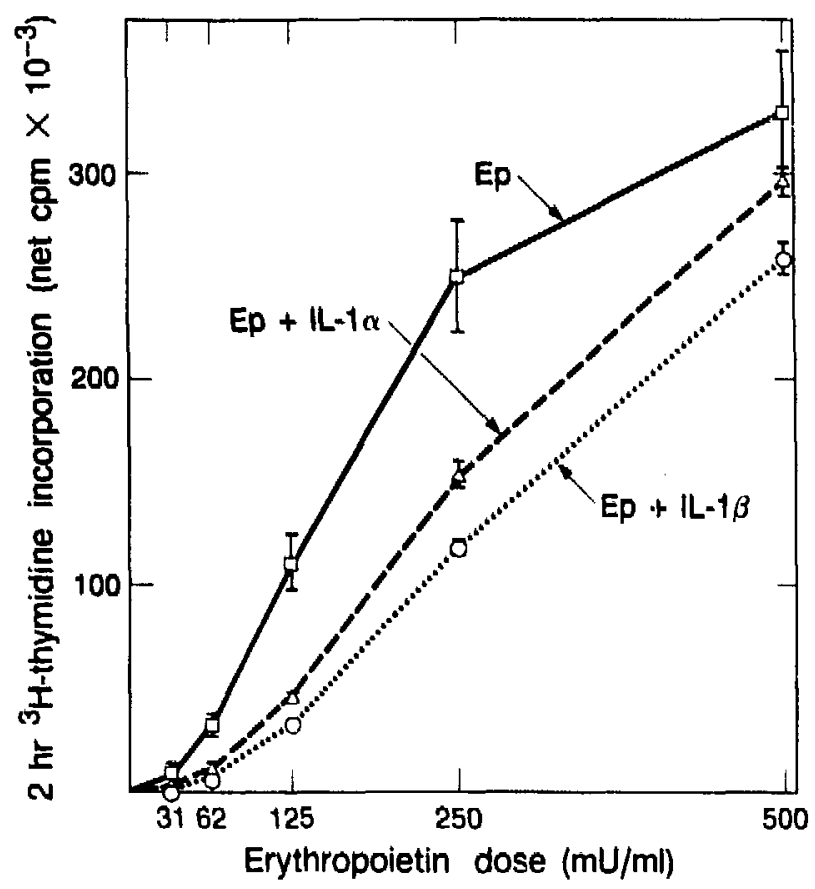

Fig. 2. Graph of the 2-hr ${ }^{3} \mathrm{H} \cdot$-thymidine incorporation induced by the action of various concentrations of erythropoietin ana ils inhibilion by $1 \mathrm{~L}-1 \mathrm{r}$ and $/ \mathrm{L}-1 \mathrm{~B}(40 \mathrm{pg} / \mathrm{m} /)$. addition of either form of IL-1 $22 \mathrm{hr}$ after the addition of Ep, at the same time as the ${ }^{3} \mathrm{H}$-thymidine, has no influence on the Ep-induced incorporation of the DNA precursor. Except for the highest dose of Ep used $(500 \mathrm{mU} / \mathrm{ml}$ which, as shown previously, is on the plateau of the response in this system) there is a tendency at each dose of Ep for less of a reduction in the DNA synthesis the longer the interval between the additions of Ep and IL-1 to the culture.

In additional experiments (Table 4), the basal and Ep-induced ${ }^{3} \mathrm{H}$-thymidine incorporation by spleen cells from anemic mice was found not to be reduced by $\mathrm{PGE}_{2}$ in the concentration produced by the spleen cells $\left(\sim 10^{-5} \mathrm{M}\right)$ or by a concentration of indomethacin sufficient to inhibit by more than 90\% PGE 2 production under comparable culture conditions $\left(\sim 10^{-5} \mathrm{M}\right)$. Furthermore, indomethacin had no demonstrable effect on IL- $1 \beta$-induced inhibition of the response to Ep. It therefore seems unlikely that the inhibitory effects of IL-1 varieties on responses to Ep are mediated by prostaglandin production.

In view of reports that IFN- $\gamma$ inhibits erythropoiesis, ${ }^{14,29}$ we tested the effect of recombinant murine IFN- $\gamma$ on the response of spleen cells from anemic mice to Ep. As shown in Table 4, no significant inhibition was observed. In other comparable experiments, murine IFN-b had no demonstrable effect on the response of ERC-enriched spleen cells to Ep (data not shown). 
Table 3. The inhibition of the Ep-induced incorporation of ${ }^{3} \mathrm{H}$-thymidine in spleen cells obtained from anemic mice by various concentrations of $I L-1 \alpha$ and $I L-1 \beta$ compared to the effect of TNF- $\alpha$.

\begin{tabular}{|c|c|c|c|c|}
\hline \multirow[b]{2}{*}{$\begin{array}{c}\mathrm{IL}-1 \text { concentration } \\
(\mathrm{pg} / \mathrm{ml})\end{array}$} & \multicolumn{4}{|c|}{ 2-hr ${ }^{3} \mathrm{H}$-thymidine incorporation (net $\mathrm{cpm} \times 10^{-3}$ ) } \\
\hline & None & 20 & 40 & 60 \\
\hline A. Ep with $\operatorname{LL}-1 \alpha$ & $8.3 \pm 7.0^{2}$ & $18.4 \pm 2.5$ & $32.7 \pm 3.8$ & $24.0 \pm 3.6$ \\
\hline $\begin{array}{l}\text { B. Decrease in Ep } \\
\text { response (\%) }\end{array}$ & & $78 \pm 3 \%$ & $61 \pm 6 \%$ & $71 \pm 5 \%$ \\
\hline C. Ep with IL-1 $\beta$ (a) & $76.6 \pm 6.2$ & $28.4 \pm 2.8$ & $20.4 \pm 2.1$ & $13.2 \pm 1.9$ \\
\hline (b) & $84.4 \pm 5.0$ & $44.7 \pm 2.7$ & $37.3 \pm 2.6$ & $26.9 \pm 2.6$ \\
\hline $\begin{array}{l}\text { D. Decrease in Ep (a) } \\
\text { response }(\%)\end{array}$ & & $63 \pm 5 \%$ & $73 \pm 3 \%$ & $83 \pm 3 \%$ \\
\hline (b) & & $47 \pm 4 \%$ & $56 \pm 4 \%$ & $68 \pm 4 \%$ \\
\hline
\end{tabular}

\begin{tabular}{lcccc} 
& \multicolumn{4}{c}{ 2-hr ${ }^{3} \mathrm{H}$-thymidine incorporation (net cpm $\times 10^{-3}$ ) } \\
\cline { 2 - 5 } $\begin{array}{c}\text { TNF- } \alpha \text { concentration } \\
\text { (units/ml) }\end{array}$ & None & 50 & 1250 & 5000 \\
\hline E. Ep with TNF- $\alpha$ & $83.0 \pm 5.5$ & $84.5 \pm 6.4$ & $80.7 \pm 6.4$ & $84.6 \pm 6.1$ \\
\hline \hline
\end{tabular}

- Mean \pm standard deviation of the mean of 8 determinations. Final concentration of Ep $125 \mathrm{mU} / \mathrm{ml}$.

\section{DISCUSSION}

A gene for murine IL-1 and two genes for human IL-1 have been cloned and expressed in $E$. coli. $^{21-24}$ In all cases the primary gene products are proteins with relative molecular masses $\left(M_{p}\right)$ in the range 30,000 to 31,000 , which are cleaved to form, from the carboxyl terminal region of the precursor, biologically active proteins of $M_{r}$ about 17,500 . The two human products are termed IL-1 $\alpha$ and IL-

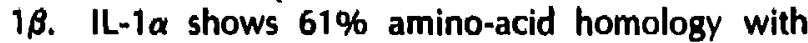
murine IL- 1 whereas IL- $1 \beta$ shows only $26 \%$ homology with IL-1 $\alpha$ and $27 \%$ homology with the murine molecule. Nevertheless, IL-1 $\alpha$ and $\mathrm{IL}-1 \beta$ bind to the same receptors on several cell types ${ }^{30}$ and share many biological activities. These include pyogenic effects and stimulation of the formation of acute-phase proteins by hepatocytes. ${ }^{8}$ The selectivity of this effect is illustrated by the observations that IL-1 increases the synthesis of mRNA of some acute-phase proteins while inhibiting the production of specific albumin mRNA by the same cell $5^{31}$ IL-1 is synthesized by many cell types activated by diverse stimuli. Among the bacterial products inducing IL-1 formation by monocytes or macrophages are $\mathrm{LPS}^{\mathrm{B}}$ and the muramyl peptide components of bacterial cell walls. ${ }^{9}$ The only small molecules known to inhibit IL-1 formation by such cells are glucocorticoids. ${ }^{32}$ Dexamethasone is reported to augment the number of human BFU-E and the colony size. ${ }^{33}$

The experiments described in this report show that the ability of spleen cells enriched in ERC to respond to highly purified recombinant Ep by DNA synthesis is significantly depressed in the presence of low concentrations of IL-1. The antagonism of Ep is comparable with cloned murine IL-1 or human IL-1 $\alpha$ or IL-1 $\beta$ (nonglycosylated) or IL-1 purified from activated human peripheral blood mononuclear cells (glycosylated). Hence the inhibitory effect of IL-1 on erythropoiesis is neither species-specific nor dependent on glycosylation.

The depression of Ep-induced DNA synthesis by ERC cells in culture occurs even when addition of $\mathrm{IL}-1$ is delayed as long as 17 hours after the addition of Ep. However, addition of IL-1 22 hours after Ep has little inhibitory effect. Presumably the ERC in culture include both BFU-E and CFU-E, but 


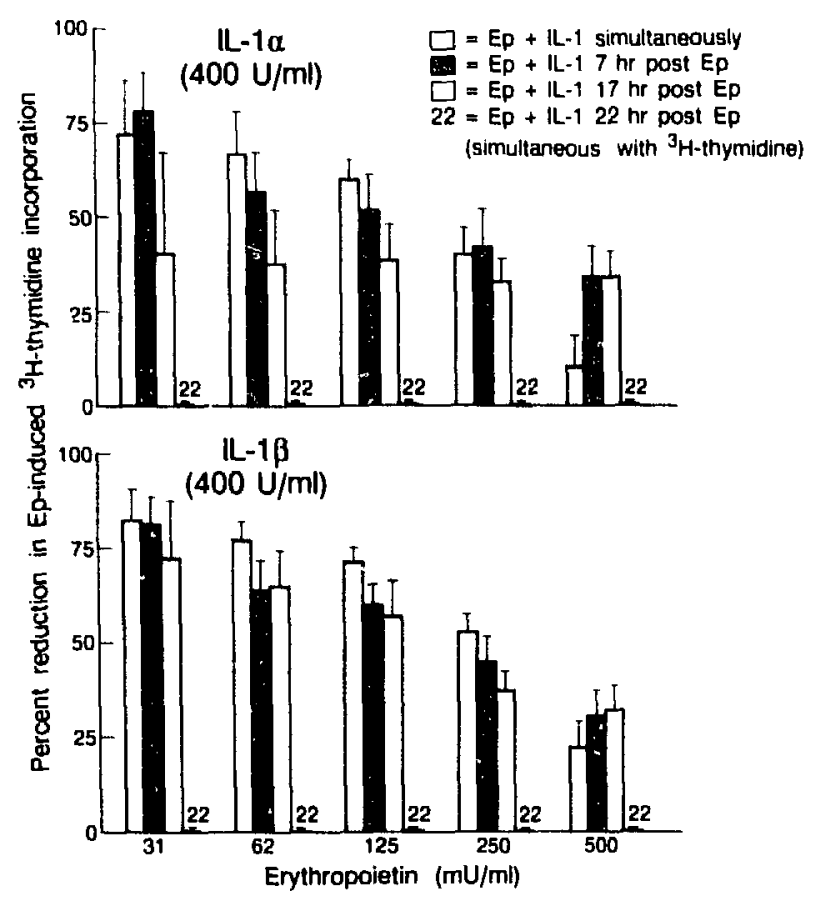

Fig. 3. Histograms of the inhibitory activity of $1 \mathrm{~L}-1 \mathrm{\alpha} \quad(400$ $U / \mathrm{ml}=40 \mathrm{pg} / \mathrm{ml}$, upper $)$ and $l L-1 \beta(400 \mathrm{U} / \mathrm{ml}=40 \mathrm{pg} / \mathrm{ml}$. lower) on the DNA synthesis induced by various concentrations of erythropoietin. The results are expressed as the percent reduction of the response induced by a dose of erythropoietin when IL-I is added to the cultures simultaneously with erythropoietin and at various later times.

(XBL 862-8654)

the kinetics of development of these erythroid protors in suspension cultures are unknown. Because IL-1 is active long after addition of $E p$, it is unlikely that IL-1 interferes with binding of Ep to specific receptors or events immediately following such binding. IL-1 appears to inhibit a late stage in the sequence of events leading to DNA synthesis in erythroid precursors. The inhibitory effect of IL-1 on erythropoiesis is not blocked by indomethacin, so it is unlikely to be mediated by prostaglandins.

Sassa et al. ${ }^{19}$ reported that medium from cultures of endotoxin-stimulated mouse peritoneal macrophages inhibits the growth and differentiation of Friend virus-transformed erythroleukemic cells. Partially purified preparations of this factor were found to lower lipoprotein lipase activity of 3T3-11 cells, an activity associated with TNF- $\alpha,{ }^{34}$ Responses of Friend virus to Ep are variable, depending on the strain of virus used. ${ }^{35}$ TNF- $\alpha$ inhibits the growth of several virus-transformed and other tumor cells, ${ }^{36}$ and effects on Friend cells may have a similar basis. Our observations show no effect of TNF- $\alpha$ on the response to Ep of ERC from spleens of anemic mice.
Table 4. Lack of effect of IFN- $\gamma$. PGE 1 and indomethacin on the response of spleen cells of anemic mice to Ep.

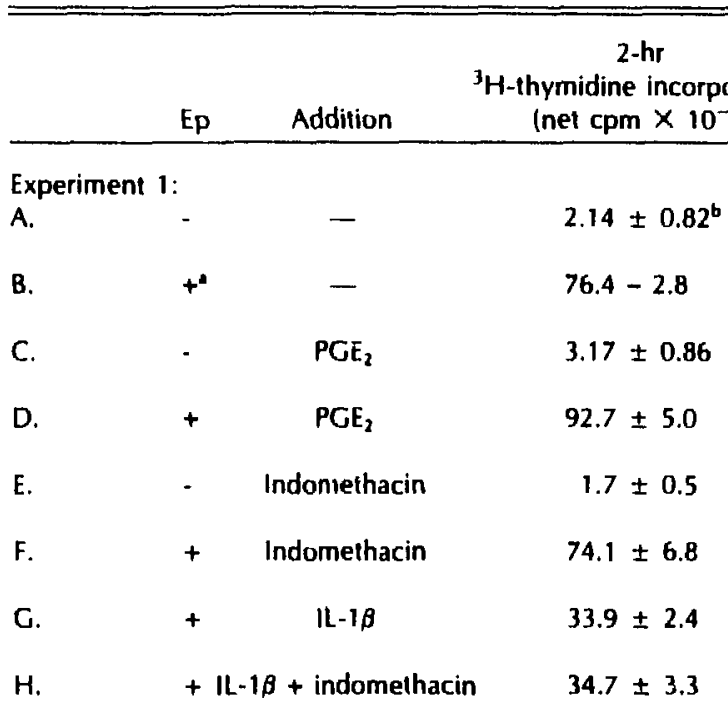

Experiment 2:

$\begin{array}{llll}\text { A. } & + & - & 84.0 \pm 4.0 \\ \text { B. } & + & \text { IFN- } \gamma & 82.0 \pm 4.3\end{array}$

- The cultures marked + were exposed to a final concentration of $125 \mathrm{mU} \mathrm{Ep} / \mathrm{ml}$.

- Mean \pm standard deviation of the mean of 8 determinations. IC to $\mathrm{H}, \mathrm{PGE}_{2}$ and indomethacin final concentrations $10^{-5} \mathrm{M}$. $1 \mathrm{GH}$, IL-1 $\beta$ firal concentration $60 \mathrm{pg} / \mathrm{ml}$. 2B, IFN- $\gamma$ final concentration 100 units/ml.

Another product of activated monocytes or macrophages is IFN- $\alpha$. Indeed, IFN- $\alpha$ is often found in bone marrow sera of normal individuals, and has little, if any, effect on human erythropoiesis. ${ }^{29}$ In our hands IFN- $\gamma$ and IFN-b likewise had little or no depressive effect on erythropoiesis.

The observations with LPS reviewed above show effects of a microbial product in inducing IL-1 formation $^{8}$ and inhibition of erythropoiesis. ${ }^{18,19}$ The hypoplastic anemia following intravenous injection of Mycobacterium bovis BCG into mice ${ }^{37}$ is thymus dependent and presumably requires the production by activated T-lymphocytes of mediator or mediators that inhibit the effects of the raised Ep levels seen in these animals. The human counterpart is pure red cell aplasia associated with disseminated BCG infection. ${ }^{38}$ The muramyl dipeptide component of mycobacterial cell walls itself induces IL1 formation, ${ }^{9}$ which might be augmented by IFN- $\gamma$, GM-CSF or other lymphokines. 
As a model for the anemia associated with chronic infection, rats or dogs injected with Freund's complete adjuvant have been used. ${ }^{39}$ These animals show reduced serum iron and increased serum copper, changes similar to those observed in animals inoculated with leukocyte endogenous mediator, which is now thought to be the same as $\mathrm{IL}-1 .{ }^{40}$

Another model for hypoplastic anemia associated with T-lymphocyte activation is that occurring in mice following transplantation of bone marrow mismatched at the M1s locus but compatible at major histocompatibility loci. ${ }^{41}$ These are the conditions required for optimum interaction of activated donor lymphocytes and host macrophages to produce IL-1. ${ }^{10} \mathrm{It}$ is also noteworthy that $\mathrm{C} 3 \mathrm{H} / \mathrm{He}$ donor cells were found to induce anemia whereas $\mathrm{C} 3 \mathrm{H} / \mathrm{Hel}$ donor cells, which are unresponsive to endotoxin, did not. ${ }^{41}$ This observation is consistent with IFN- $\gamma$-primed, endotoxin-stimulated IL-1 production as a mediator of the hypoplastic anemia. Graft-versus-host reaction in children following intrauterine and exchange transfusions that include leukocytes can also produce hypoplastic anemia. ${ }^{6}$ The influence of the microbial flora on the incidence and severity of graft-versus-host disease in human allogeneic bone marrow transplantation is well documented. ${ }^{42}$

This leads into the complex problem of the role of activated T-lymphocytes in congenital and acquired red cell aplasias and effects of immunosuppressive regimes and bone marrow transplantation. It is well established that some patients benefit from treatment with anti-thymocyte globulin. ${ }^{7}$ Co-culture experiments have sometimes provided clear evidence of T-lymphocyte-mediated suppression of erythropoiesis. ${ }^{43}$ However, this is not found consistently, perhaps because T-lymphocytes can have complex indirect effects on erythropoiesis. Anti-thymocyte globulin may not only have immunosuppressive effects: activation of human peripheral blood lymphocytes from hypoplastic anemia patients by anti-thymocyte globulin leads to the production of IL-2 and a factor or factors stimulating erythropoiesis. ${ }^{44}$

The inhibitory factor that has altracted most attention is IFN- $\gamma$. Culture supernatants of $\mathrm{T}$. lymphocytes with receptors for FclgG have long been reported to inhibit erythropoiesis. ${ }^{45}$ A Leu $2^{+}$ subset of T-lymphocytes bearing activation markers (HLA-DR and Tac) from some hypoplastic anemia patients has been found spontaneously to produce IFN- $\gamma$ and to inhibit hemopoiesis. ${ }^{29}$ Recombinant human IFN- $\gamma$ was reported to suppress BFU-E and CFU-E in human bone marrow cultures. ${ }^{14}$ However, the inhibitory effects of IFN- $\gamma$ were mediated through accessory cells, including monocytes. Aithough neither IFN- $\alpha$ nor IFN- $\gamma$ directly induces the production of $\mathrm{IL}-1$ by human monocytes, they markedly augment $\mathrm{IL}-1$ production by small doses of endotoxin, ${ }^{13}$ and doubtless other microbial products that are frequent contaminants of culture media and elicit IL- $I$ formation. ${ }^{46}$ These observations arrd our own negative data with IFN- $\gamma$ reported in this paper suggest that the effect of IFN- $\gamma$ on erythropoiesis may be indirect and produced by another mediator such as IL-1.

Rheumatoio arthritis is not infrequently associated with pure red-cell aplasia. ${ }^{3}$ Rheumatoid synovial tissues produce large amounts of IL-1, which is believed to play an important role in the pathogenesis of the disease. ${ }^{47}$ The high levels of acutephase reactants in rheumatoid arthritis, and hypoplastic anemia associated with the disease, may be due to systemic effects of IL-1.

A cell line with promonocytic characteristics from a child with congenital pure red-cell aplasia (Diamond-Blackfan syndrome) has been found to produce IL-1 constitutively. ${ }^{48}$ It is tempting to speculate that in this syndrome there is defective control of $11-1$ production in the bone marrow, leading to suppression of erythropoiesis. Production of IL-1 is inhibited by flucocorticoids, ${ }^{32}$ which often relieve the anemia in children with this syndrome. ${ }^{1}$

Another hypoplastic anemia that is improved by glucocorticoid therapy occurs in elderly humans in association with iever, increased plasma viscosity, and giant cell arteritis. ${ }^{5}$ In this condition cells of the monocyte-macrophage lineage invade arterial walls, and they may produce $\mathrm{L}-\mathrm{T}$, which has pyrogenic effects and stimulates acute-phase protein synthesis. Following glucocorticoid therapy the fever disappears, plasma viscosity falls and the anemia improves.

Now that sensitive immunoassays and bioassays for the two varieties of human $\mathrm{L}-1$ are becoming available, it should be possible to detect them in peripheral and bone-marrow plasma in different diseases. Furthermore, we have produced monoclonal antibodies inhibiting the effects of $\mathrm{IL}-1 \beta$. It will therefore be possible to lest directly the hypothesis advanced in this paper, that IL-1 is a major factor in the pathogenesis of hypoplastic anemias.

The findings now reported may help to explain another remarkable property of IL-1, its capacity when administered before potentially lethai doses of whole-body radiation to protect mice. ${ }^{49}$ This is due to preservation of hemopoietic progenitor cells, 
including erythroid precursors, in the bone marrow. The fact that IL-1 antagonizes the effect of Ep on erythroid precursors implies that cycling of these cells is inhibited, which may be related to their increased resistance to radiation. The possible use of $\mathrm{IL}-1$ to decrease myelotoxicity in cancer therapy is under investigation.

\section{REFERENCES}

1. Diamond, L.K., Wang, W.C., and Alter, B.P. Advances in Pediatrics 22, 349-378 (1976).

2. Douglas, S.W., and Adamson, J.W. Blood 45, 55-65 (1975).

3. Desspyris, E.N., Baer, M.R., Sergent, J.S., and Krantz, S.B. Annals of Internal Medicine 100, 202-206 (1984).

4 Stricker, R.B., and Shuman, M.A. American Journal of Hematology 17, 193-201 (1984).

5. Allison, M.C., and Gough, K.R. Postgraduate Medical Journal 61, 501-503 (1985).

6. Parkman, R., Mosier, D., Umansky, I., Cockran, W., Carpenter, C.B., and Rosen, F.S. New England Journal of Medicine 290, 359-363 (1974).

7. Doney, K., Martin, P., Storb, R., Whitehead, J., Smith, A., Hansen, J.A., Appelbaum, F., Buckner, C.D., and Thomas, E.D. Experimental Hematology 13, 520-524 (1985).

8. Gery, I., and Lepe-Zuniga, J.L. In Lymphokines (Ed. E. Pick) Academic Press, New York 9, 109-143 (1984).

9. Oppenheim, 1.J., Togawa, A., Chedid, L., and Naizel, S. Cellular Immunology 50, 71-81 (1980).

10. Farr, A.G., Kiely, 1.-M., and Unanue, E.A. Journal of Immunology 122, 2395-2404 (1979).

11. Moore, R.N., Oppenheim, J.J., Farrar, J.J., Carter, C.S. Ir., Waheed, A., and Shadduck, R.K. Journal of Immunology 125, 1302-1305 (1980).

12. Nardella, F.A., Dayer, J.-M., Roelke, M., Krane, S.M., and Mannik, M. Rheumatology International 3, 183-186 (1983).

13. Arenzana-Seisdedos, F., Virelizier, I.L., and Fiers, W. Journal of Immunology 134, 24442448 (1985).

14. Zanjani, E.D., McGlave, P.B., Davies, S.F., Banisadre, M., Kaplan, M.E., and Sarosi, G.A. Hematology 50, 479-490 (1982).
15. Roodman, G.D., Horadam, V.W., and Wright, T.L. Blood 62, 406-412 (1983).

16. Pololi-Anagnoustou, L., Lad, T., Schade, S., and Anagnoustou, A. Clinical Research 28, 730A (1980).

17. Schade, S.G., and Fried, W. American Journal of Physiology 231, 73-76 (1976).

18. Udupa, K.B., and Lipschitz, D.A. Blood 59, 1267-1271 (1982).

19. Sassa, S., Kawakami, M., and Cerami, A. Proceedings of the National Academy of Sciences USA 80, 1717-1720 (1983).

20. Pestka, S. Archives of Biochemistry and Biophysics 221, 1-37 (1983).

21. Lomedico, P.T., Gubler, V., Hellman, C.P., Dukovitch, M., Giri, J.G., Pan, Y.-C.E., Collier, K., Semionow, R., Chua, A.O., and Mizel, S.B. Nature 312, 458-462 (1984).

22. Auron, P.E., Webb, A.C., Rosenwasser, L.J., Mucci, S.F., Rich, A., Wolff, S.A., and Dinarello, C.A. Proceedings of the National Academy of Sciences USA 81, 7907-7911 (1984).

23. March, C.j., Mosley, B., Larsen, A., Cerretti, D.P., Braedt, G., Price, V., Gillis, S., Henney, C.S., Kronheim, S.R., Grabstein, K., Conlan, P.J., Hopp, T.D., and Cosman, D. Nature 315, 641-647 (1985).

24. Gubler, V., Chua, A.O., Stern, A.S., Hellmann, C.P., Vitek, M.P., Dechiara, T.M., Benjamin, W.R., Collier, K.J., Dukovitch, M., Familletti, P.C., Fielder-Nagy, C., Jensen, J., Kaffka, K., Kibian, P.L., Stremlo, D., Wittreich, B.H., Woehle, D., Mizel, S.B., and Lomedico, P.T. Journal of Immunology 136, 2492-2497 (1986).

25. Pennica, D., Nedwin, G.D., Hayflick, J.S., Seeburg, P.H., Derynck, R., Palladino, M.A., Kobr, W.J., Aggarwal, B.B., and Goeddel, D.V. Nature 312, 724-729 (1984).

26. Schooley, I.C., and Mahlmann, L.J. Clinical Medicine 78, 765-770 (1971).

27. Kronheim, S.P., March, C.J., Erb, S.K., Conlan, P.J., Mochizuki, D.Y., and Hopp, T.P. Experimental Medicine 161, 490-502 (1985).

28. Krystal, G. Experimental Hematology 11, 649-660 (1983).

29. Zoumbos, N., Raefsky, E., and Young, N. Progress in Hematology 14, 201-234 (1986).

30. Dower, S.K., Kronheim, S.R., March, C.J., Conlan, P.J., Hopp, T.P., Gillis, S., and Urdal, D.L. Journal of Experimental Medicine 162, 501-515 (1985). 
31. Ramadori, G., Sipe, I.D., Dinarello, C.A., Mizel, S.B., and Colten, H.R. Journal of Experimental Medicine 162, 930-942 (1985).

32. Snyder, D.S., ard Unanue, E.R. Journal of Immunology 129, 1803-1805 (1982).

33. Steinberg, M.H., Coleman, M.B., Graver, F.A., Grenett, H.E., Pressley, A., and Adams, I.G. American Journal of Hematology 10, 37-45 (1981).

34. Beutler, B., Greenwald, D., Hulmes, I.D., Chang, M., Pan, Y.C.E., Mathison, J., Ulevitch, R., and Cerami, A. Nature 316, 552-554 (1985).

35. Koury, M.J., Sawyer, S.T., and Bondurant, M.C. Journal of Cellular Physiology 121, 526-532 (1984).

36. Shalaby, M.R., Pennica, D., and Palladino, M.A., Ir. Springer Seminars in Immunopharmacology: New Perspectives in Immunotherapy 1986.

37. Marchal, G., and Milon, G. British Journal of Hematology 48, 551-560 (1981).

38. Long, H.J. Military Medicine 147, 1067-1070 (1982).

39. Feldman, B.F., Kaneko, I.J., and Farver, T.B. American Journal of Veterinary Research 42, 1109-1113 (1981).

40. Kampschmidt, R.F. Journal of Leukocyle Biol- ogy 36, 341-355 (1984).

41. Knospe, W.H., Steinberg, D., and Speck, B. Experimental Hematology 11, 542-552 (1983).

42. Schmeiser, T., Kurrle, E., Arnold, R., Heit, W., Krieser, D., Kubanek, B., and Heimpel, $H$. Progress in Clinical and Biological Research 181, 433-438 (1985).

43. Hanada, T., Aoki, Y., Ninomiya, H., and Abe, T. British Journal of Hematology 63, 69-74 (1584).

44. Gascon, P., Zoumbos, N.C., Scala, G., Djeu, I.Y., Moore, J.G., and Young, N.S. Blood 65, 407-413 (1985).

45. Bacigalupo, A., Pedesta, M., Frassoni, F., Piaggio, G., Van Lint, M.F., Repetoo, M., and Marmont, A.M. British Journal of Hematology 59, 611-616 (1985).

46. Usinger, W.R., Clark, G.C., Gottschalk, E., Holt, S., and Mishell, R.I. Current Microbiology 12, 203 (1985).

47. Wood, D.D., Ihrie, E.J., and Hamerman, D. Arthritis and Rheumatism 28, 853 (1985).

48. Butler, R.H., Revoltella, R.P., Musiani, P., and Piantelli, M. Cellular Immunology 78, 368-374 (1983).

49. Neta, R., Douches, S., and Oppenheim, J.J. Journal of Immunology 136, 2483-2485 (1986).

\section{ONE TYPE OF PLURIPOTENTIAL HEMOPOIETIC STEM CELLS PROVIDES PERMANENT, ANOTHER TEMPORARY BONE MARROW REPOPULATION}

\section{George Brecher}

The use of isogeneic bone marrow transfusions in the study of stem cell kinetics has been greatly aided during the past five years by the introduction of a new strain of mice with two variants of the enzyme phosphoglycerate kinase, referred to as PGK-A and PGK-B. The enzyme is present in every cell in the body. Hence the progeny of A cells transfused into a $B$ host can be traced in the peripheral blood of individual animals over prolonged periods, the ratio of donor to host enzyme being determined electrophoretically. Since PGK is an $X$-linked enzyme, males with a single $X$ chromosome can only iriherit either the $A$ or $B$ enzyme. Females, with two $X$ chromosomes, can be $A A, B B$, or $A B$. In an $A B$ female, however, individual cells must be either $A$ or $B$, because in each cell one of the Iwo $X$ chromosomes is inactivated randomly early in development. When $A B$ marrow is transfused after destroying the host's own stem cells by irradiation, the host marrow will become $A B$, because multiple cells are usually transfused including both $A$ and $B$ stem cells. However, if hosts should be repopulated by a single stem cell, the marrow will be either $A$ or $B$.

To test whether single stem cells can indeed repopulate the entire marrow of a mouse, lethally irradiated animals were given 500,000 cells, 100,000 cells, or 50,000 cells from marrows of $A B$ females. Survival was $100 \%$ after transfusion of 500,000 cells and all animals had both $A$ and $B$ enzymes. After 100,000 cell transfusions survival was still $100 \%$. However, $40 \%$ of the hosts had 
been repopulated by $A$ cells only, $60 \%$ by $A$ and $B$ cells. When the number of marrow cells transfused was reduced to 50,000 , only $40 \%$ of animals survived and the survivors had $A$ cells only, indicating that single stem cells were responsible for the repopulation of the entire animal. The repopulation from a single cell was permanent. One year later, the four survivors of 10 lethally irradiated animals transfused with 50,000 AB cells were still alive and entirely $A$. The predominance of $A$ cells when single cells repopulated the entire marrow is based on the genetically fixad $70: 30$ ratio of $A: B$ enzymes in our strain. The probabilities of the entire marrow being $A, B$, or $A B$ are indicated in the nomogram of Fig. 1. It is based on the Poisson distribution of cells actually transplanted when the average number of stem cells in a given volume of marrow is 0.1 to 13. The relative probabilities of $A, B$, or $A B$ cells among the stem cells are computed from the binomial distribution and the incidence of $A: B$ cells of 70:30 in the donor marrow. For an average of one stem cell transplanted with 50,000 cells, the probability of no cells being transplanted is $40 \%$, predicting a mortality of that order. The probability of the entire marrow being $A$ is also $40 \%$, with the probability of the entire marrow being $B$ or $A B$ being $10 \%$ each. With only four survivors in our experiment, both the mortality of the group and the predominance of $A$ cells only are thus within the statistical predictions. For 100,000 cells and an assumed average number of two stem cells transplanted, the expected mortality is $10 \%$, with $41 \%$

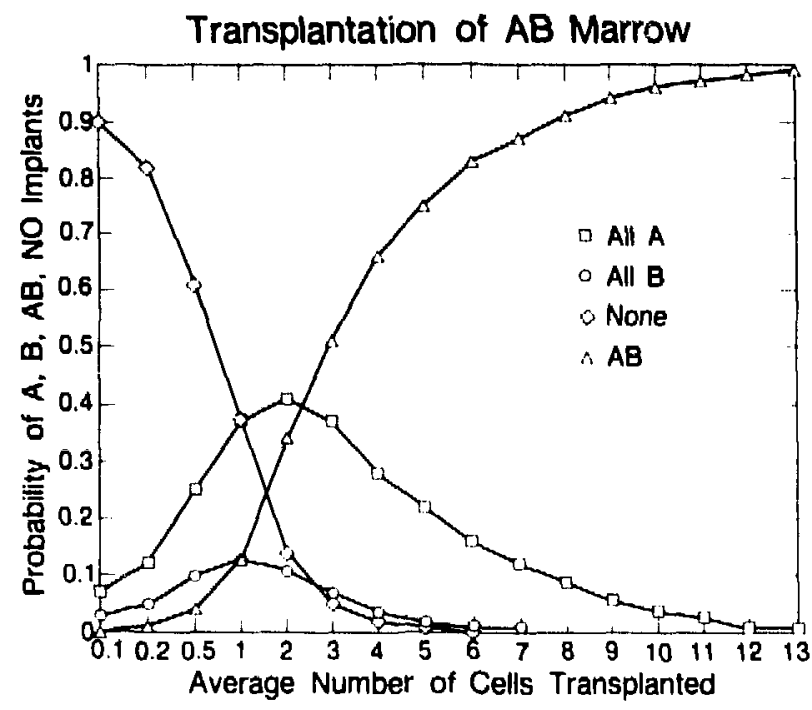

Fig. 1. Probabilities of repropulation with all $A$, all $B$, or $A B$ cells when an average of 0.1 10 $13.3 \mathrm{AB}$ cells are transplanted. of animals being all $A, 10 \%$ all $B$, and $37 \% A B$. The observations were in agreement with the predicted distributions.

The results also agree with those of H.S. Micklem who used a different approach to determine the number of cells that repopulate a lethally irradiated animal. The number of clones or "founder cells" responsible for repopulation after transfusion of 100,000 cells into lethally irradiated hosts was computed by Micklem as 2.5 , very close to the 2 cells seeding after transfusion of 100,000 cells derived from our observations. One might deduce from our results that 50,000 marrow cells is the absolute minimum of donor cells allowing some survival in the lethally irradiated hosts. However, Cronkite gave transfusions of 10,000 and 20,000 cells and obtained survival of $20 \%$ of mice given a uniformly lethal dose. When the experiment was repeated in the $P G K / A, B$ mice in which the contribution of host and donor cells to repopulation can be determined, cells of donor origin were noted to repopulate the marrow partially beginning immediately after irradiation. They are gradually replaced by host cells. At 12 to 27 weeks host cells constituted the entire marrow and remained the sole marrow population indefinitely (Fig. 2). Apparently the donor cells maintain the animal long enough so that the host cells can recover sufficiently from the radiation injury to take over.

The data indicate that the donor stem cells present in 20,000-cell inocula have a limited selfrenewal, can repopulate host marrow only tem-

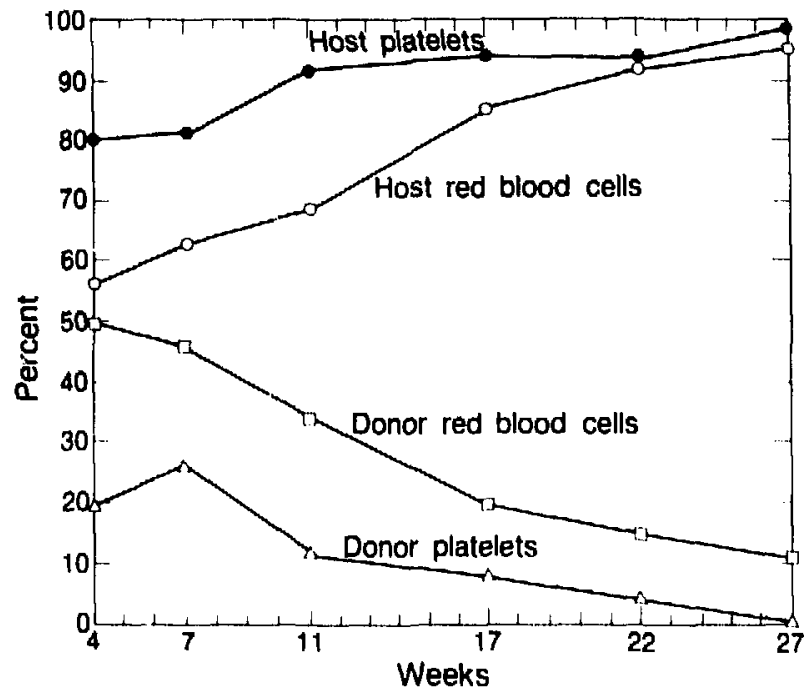

Fig. 2. Recovery of platelets and red b/ood cefls in host mice given 20,000 marrow cells atter lethal irradiation $(10.5 \mathrm{G} \%)$ Cells of donor and host origin were defermined independently. (XBL 8611-9061) 
porarily, and thus differ from the stem cells present in 50,000-marrow-cell and larger inocula that can repopulate irradiated marrow permanently. Following Micklem, who predicted the existence of at least two types of pluripotential stem cells, the two types described here may be called $A$ and $B$. Micklem has already shown that stem cells with "greater" self-renewal replace stem cells with "lesser" self-renewal. Hence the fact that B type stem cells with limited self-renewal have not been observed before is presumably due to the large inocula, usually in excess of 500,000, used customarily to restore lethally irradiated animals. With those large inocula, type A cells are always transfused and replace any type $B$ cells present.
In summary, we have demonstrated the permanent repopulation of the marrow of lethally irradiated mice by single donor stem cells. The incidence of these cells in donor marrow is of the order of 1 in 50,000 . Transfusions of 10 and 20,000 marrow cells are also capable of ensuring the recovery of about $20 \%$ of lethally irradialed mice. Donor cells temporarily repopulate the host marrow and enable the irradiated host cells to maintain the animal indefinitely. The transfused donor stem cells are not capable of permanent repopulation of the host because their self-renewal is limited. The two types of pluripotential stem cells are referred to as type $A$ and $B$, following Micklem who predicted their existence.

\section{IMMUNOREACTIVE ERYTHROPOIETIN CONCENTRATIONS IN NEONATAL RATS AND THE EFFECTS OF HYPOXIA}

\section{Gisela K. Clemons, Sherry L. Fitzsimmons, and Darlene DeManincor}

Immunoreactive erythropoietin (Ep) was measured daily in intact neonatal normoxic and hypoxic rats during the first three weeks of life and was compared to adult levels. Plasma and tissue Ep levels were determined by radioimmunoassay, a method that provides the high sensitivity necessary to determine normal and below normal values in unmanipulated small animals. In addition, the determinations require a small sample size and are not as costly as the in vivo bioassay.

Several studies have shown that the neonatal rat is able to increase circulating $\mathrm{Ep}$ levels in response to hypobaric hypoxia. On the other hand, erythropoiesis cannot be further stimulated in the newborn rat by exposure to hypoxia or exogenous Ep administration, nor can erythropoiesis be suppressed in these young animals by starvation, bilateral nephrectomy, or hypertransfusion. Since the kidney is considered to be the primary organ responsible for Ep production in adult mammals, several authors concluded that erythropoiesis in the neonatal rat may be independent of renal Ep during the first three weeks of life. These studies were carried out in several different laboratories, using animals at different ages and atmospheric pressures for varying lengths of time, and the samples were analyzed primarily in the in vivo polycythemic mouse assay.
We attempted to find answers to the following questions: What are the circulating, hepatic and renal Ep levels in normal neonates measured daily through the first three weeks of life and when they attain sexual maturity (day 42)? How are these Ep levels altered by exposure to hypoxia? Can immunoreactive Ep be measured in milk obtained from hypoxic lactating rats, and can it be transferred to the nursing neonatal animals? Since we established earlier that in the fetal rat the liver is the main Ep producing tissue, we attempted to determine the age at which the switch from liver to kidney occurred in both the normal and the hypoxic neonatal rat.

Mothers and their pups were exposed to a reduced atmospheric pressure $(0.5 \mathrm{~atm})$ for 18 hours. ( 3 p.m.to 9 a.m.) while for each day an aged matched group served as a control. From day 1 to day 21 six pools were generated daily for the control group and six for the hypoxic group; in the latter group six pools were obtained for males and females separately from day 10 to 21 .

Plasma Ep levels in normoxic neonatal rats vary profoundly during the first three weeks of life (Fig. 1). With the exception of days 1 and 9, the circulating levels were significantly higher than those measured in 42-day-old and adult rats. The initial rise was attributable to hepatic Ep synthesis. 

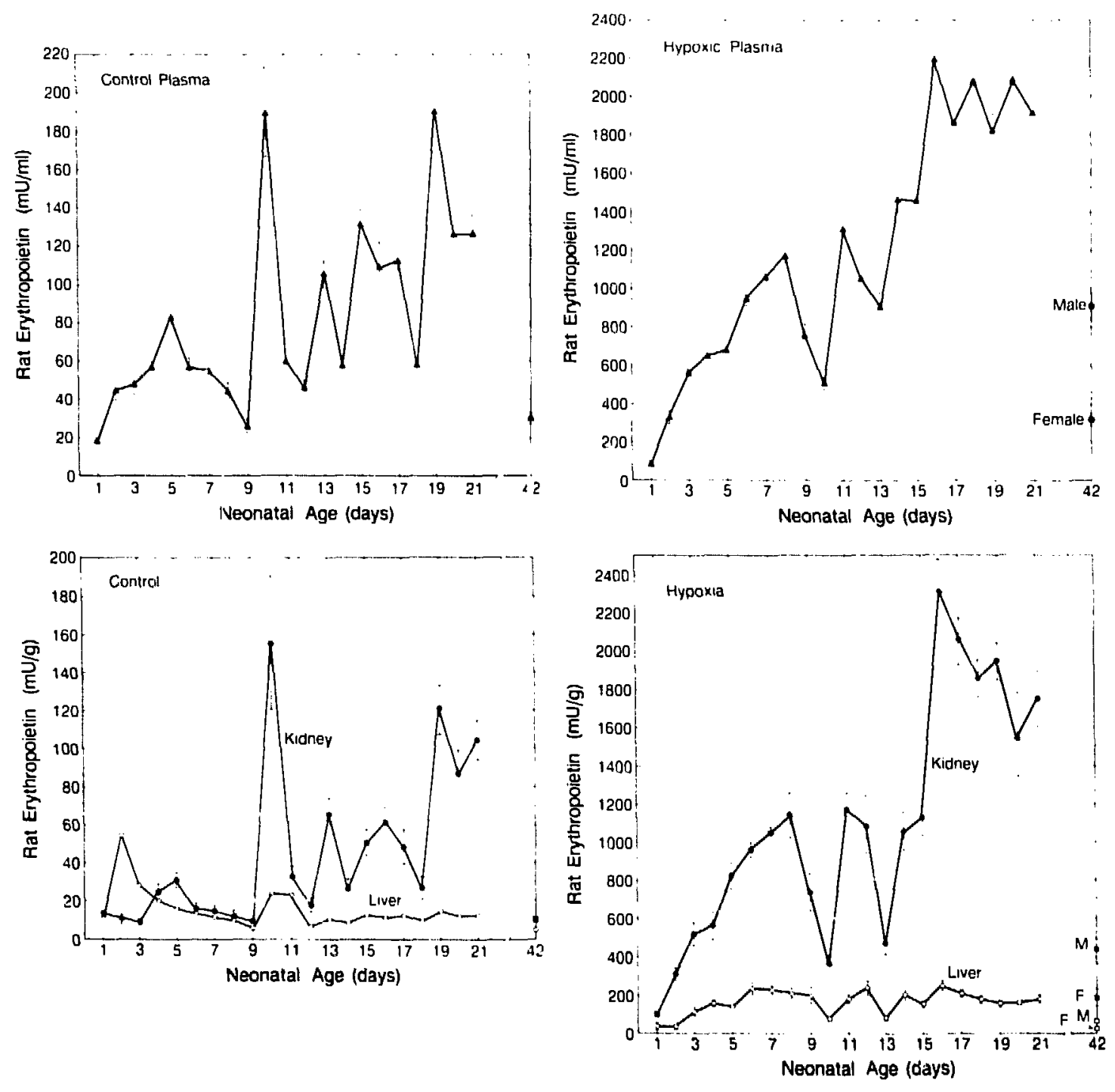

Fig. 1. Immunoreaclive erythropoietin levels in plasma (upper panels) and tissues (lower panels) of normoxic and hypoxic neonatal rats.

(XBL 8512-8607B)

Renal Ep content was reflected in the plasma levels from 10 days of age. The liver continued to be a contributor until days 10 and 11 .

Hypoxic exposure showed that neonatal rats increase Ep production as early as the first day of life, and the increase in circulating Ep levels closely resembled the increase in renal Ep concentrations. While on the whole Ep production increased with age, it is of interest that on days 10 and 13 we observed sharp declines in renal and plasma Ep at a time when unstimulated control animals showed unexpected peaks. Nevertheless, the Ep levels in the hypoxic groups were still significantly higher than those measured in the controls. Liver Ep con- centrations fluctuated relatively little. No difference in the response of males and females could be documented from day 10 through weaning, but at day 42 , the levels in male rats were significantly higher than in female rats and were similar to those measured in adult rats of both sexes.

We have shown earlier that the fetal rat kidney is able to synthesize $\mathrm{Ep}$ and respond to maternal hypoxia two days before birth, but that during the last week of fetal life the liver is the main Ep producing tissue in the rat fetus. Taking into account tissue weights and their Ep concentrations, it is possible to calculate how much of the hormone is available from renal or hepatic sources. Figure 2 


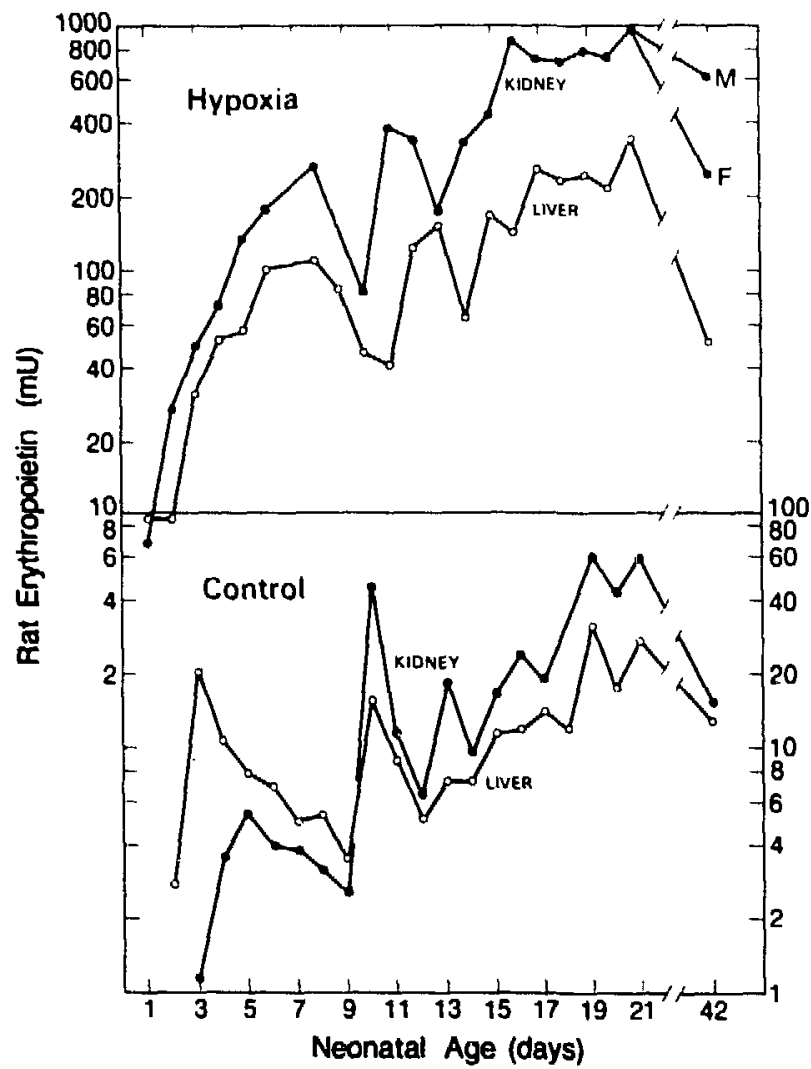

Fij. 2. Total liver and kidney tissue erythropoietin content ( $m U \times$ tissue weight) in the neonatal rat as a function of age under hypoxic (upper panel) and normoxic (lower panel) conditions. ( $M=$ Male; $F=$ Female)

(XBL 8512-8611)

shows that in the normoxic neonatal animal more Ep originates from the liver than the kidneys until day 10 . The presence of the profound increase in day 10 seems to be connected with the liver to kidney switch of Ep production in the normal rat, because thereafter the availability of Ep from the kidney is always higher than that from the liver. During hypoxic conditions, however, the switch occurred as early as 2 days after birth, and in spite of increased liver weight from day 2 on, the kidney provided more Ep.

The earlier reported conclusions that Ep production in significant measure is independent of renally produced Ep during the neonatal period in the rat appear not to be correct when applied to the intact rat, and only true in the nephrectomized neonatal rat. The effects observed with nephrectomized rats were rather due to the fact that during the first three weeks of life the liver retains the ability to synthesize Ep, and it fully compensates in the absence of the kidneys. This ability is mostly lost in the adult, although the liver has been found to be the principal site of extrarenal Ep production, and it is able to maintain baseline quantities of Ep in the absence of the kidneys.

This present study could neither confirm the presence of Ep in milk obtained from normoxic and hypoxic mothers nor establish if the Ep levels were elevated in the newborns nursed by hypoxic mothers. Lactating rats exposed to the same hypoxia usually have plasma levels of approximately $300-400 \mathrm{mU} / \mathrm{ml}$, and we were not able to show any transfer from the mother to the young.

The age of 7 days was chosen for this particular experiment because at this time endogenous renal and hepatic Ep levels are low and the plasma levels are declining and still separated from the 10-day peak by 3 days (Fig. 1). It also appears unlikely that Ep was inactivated by acid hydrolysis in the stomach because the $\mathrm{pH}$ in the neonatal rat stomach is reported not to fall below 6 before day 10, and we have found that Ep becomes immunologically unreactive below $\mathrm{pH} 4.5$.

While it is important to be able to measure circulating plasma and tissue concentrations and to determine the time of liver to kidney switch in the developing rat, no explanation can be offered at the present time to the following question: Why would an animal whose erythropoiesis cannot be stimulated further by exogenous Ep administration or hypoxia maintain higher than adult levels during the first three weeks of life, and during hypoxic conditions elaborate even more than is necessary for the adult to reach erythropoietic homeostasis? This is especially puzzling because it has been shown that very small increases in the levels of Ep (approximately $5 \mathrm{mU} / \mathrm{ml}$ ) are capable of significant increases in red blood cell production in the adult rat. The possibinty has to be considered that the function(s) of Ep in neonatal rats may be multifold and not just limited to those necessary for ery:t poiesis. Rather, Ep may work in conjunction vis:other growth factors until the adult homeostasis is achieved, a fact that would also support the earlier suggestions that erythropoiesis in the neonatal rat is governed differently than from that in the adult. 


\section{EFFECTS OF OZONE AND HYPOXIA ON THYROID HORMONE RECEPTORS}

\section{David E. Erkenbrack and Gisela K. Clemons}

For almost a decade this laboratory has been examining the acute effects of ozone on the economy of several endocrine systems. Ozone is one of the most significant of air pollutants because of its toxicity and ubiquity: concentrations in urban areas frequently reach levels that have been shown to have pathological effects on lung tissue, while substrates of the photochemical reactions resulting in its formation are present in the smog of virtually every urban area on the globe. Of the many injurious effects that have been reported in regard to this remarkably toxic gas, few are as striking as its perturbation of the thyroid hormone economy. Ozone exposures at levels that approach those found during severe smog inversions (1 ppm) resulted in rapid $(24 \mathrm{hr})$ and highly significant decreases in serum levels of thyroxine (T4), triiodothyronine (T3), and thyroid stimulating hormone as well as effects on the serum binding proteins for T4 indicative of an increased clearance rate for this hormone. These effects have been interpreted to be a protective adaptation to ozone exposure since the toxicity of such exposure has been conclusively shown to be potentiated by concomitant administration of thyroid hormone; conversely, hypothyroidism induced by hypophysectomy, thyroidectomy, or goitrogens results in a marked tolerance of otherwise lethal ozone exposure.

This potentiation of ozone toxicity by thyroid hormones is consistent with the general catabolic effects of overconveyance of these hormones seen most strikingly in thyrotoxicosis. Although the synergism of ozone and these hormones has been known for decades, further understanding of the phenomenon became possible only after the elucidation of the intracellular mechanism of action of the thyroid hormones in recent years. All effects of these hormones thus far examined have been found to be mediated by receptors, primarily those in the nucleus. Utilizing a sensitive assay for characterizing these receptors,' we decided to explore this intriguing interaction between the thyroid hormones and ozone loxicity in lung tissue. The results of this work thus far appear to partially resolve the mechanism of this interaction. Ozone exposure causes prompt and sustained increases in lung nuclear T3 receptor levels (Fig. 1). Scatchard analysis of the data reveals no significant change in receptor affinity, indicating that ozone does not affect the basic structure or conformation of the receptor molecule. In contrast to the ozone effect on serum proteins mentioned above, the increase in capacity is not construed to be an effect secondary to hormone clearance since the experimental procedure ensures that no endogenous hormone is carried into the assay. ${ }^{\prime}$ An increase in receptor levels was not seen in parallel studies done on liver receptors in which both control and ozone exposed groups had normal affinities and capacities. In view of the catabolic effects of saturating a tissue's thyroid hormone receptors (as in thyrotoxicosis), it seems likely that some of the catabolic effects of ozone exposure are mediated through an increase in receptor levels. Such a mechanism would explain the above mentioned protective adaptation since both the potentiation of ozone damage due to hormone administration (seen as an overreactior. to the hormone caused by hypersensitivity of th:tissue) and the protective effect caused by hypothyroidism (seen as a withholding of the catabolic

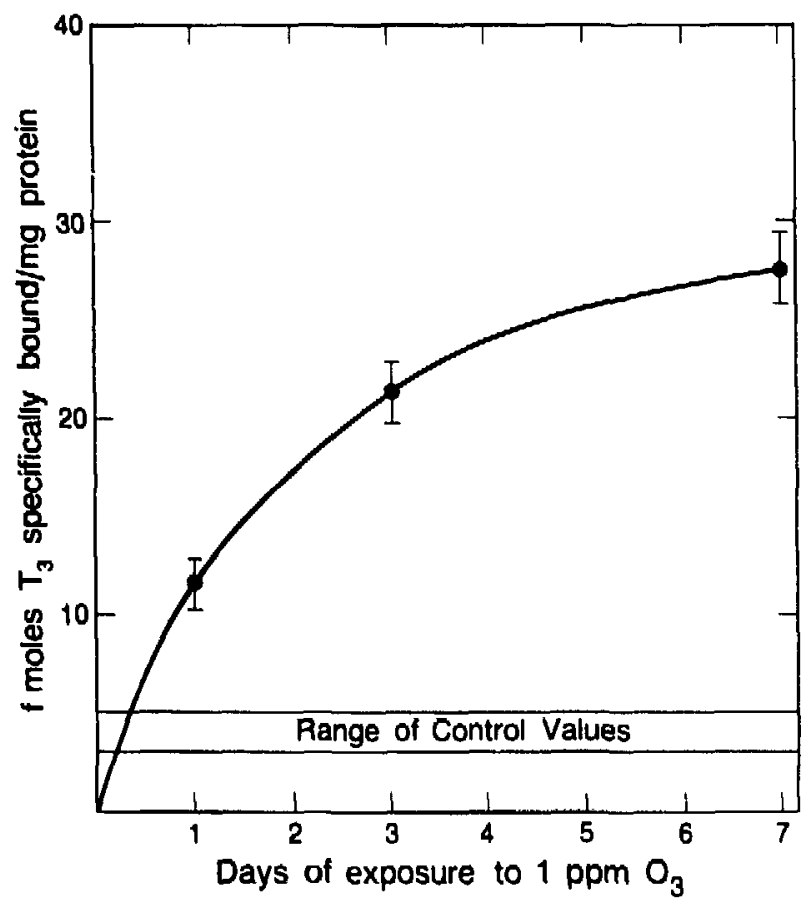

Fig. 1. Rat lung $T 3$ receptor maximal binding capacity (MBC) after exposure to 1 ppm ozone for periods indicated. Points are mean values, error bars $\pm 5 E M$. MBC was derived from Scaichard analysis and is equivalent to the number of receptors present per unit protein.
(XBL 869-8975) 
agent that actually causes the damage when present at otherwise normal levels, since the tissue has become hypersensitive) can be viewed as events secondary to an increase in receptor levels. The reason that ozone causes this increase is problematic. A possible explanation may be that one or more of the intermediates regulating the production (or clearance) of the receptor is easily oxidized to a nonfunctional state, or otherwise susceptible to the perturbation of the cellular environment which must follow the introduction of significant quantities of such a strong oxidant. This is consistent with reports of the protective effects of reducing agents such as vitamin $E$ or glutathione during ozone exposure.

If thyroid hormone receptors are truly involved in the mediation of ozone injury, blocking the receptor should obviate some or all of the damage. For this reason we have begun experiments utilizing the dextrorotatory stereoisomer of $T 3$, which has an affinity for the receptor similar to that of the native L-form with only a fraction of its bioactivity. Similar studies are planned using methylene-bridged $\mathrm{T} 3$ (i.e., a form of $\mathrm{T} 3$ in which a methylene linkage is substituted for the ether linkage between the phenolic and nonphenolic rings), which is a compound possessing a much more favorable disparity between affinity and bioactivity. It is also possible that this research could contribute to the ongoing search for an effective antagonist for thyroid hor- mones, a therapeutic agent that would be a important clinical tool for treating hyperthyroid states. The thyroid hormone nuclear receptor assay is also being used to continue this laboratory's longstanding investigation of the mechanism of action of erythropoiesis. Direct thyroid hormone effects on bone marrow erythroid cell differentiation have been reported by several investigators, so that it seems likely that the influence of erythropoietin on these blood forming elements could be mediated at least in part by an effect of erythropoietin on the receptors for thyroid hormone. Preliminary results in which a significant increase in binding capacity caused by a 4-day exposure to hypoxia (1840 \pm $30 \mathrm{fmole} / \mu \mathrm{g}$ protein as opposed to $690 \pm$ $70 \mathrm{fmole} / \mu \mathrm{g}$ in control animals) are consistent with the interpretation that an increase in circulating levels of erythropoietin (caused by hypoxia) acted to increase thyroid hormone receptor levels. Further exploration of this area is planned, including an investigation of the ontogeny of these receptors in the neonate.

\section{REFERENCE}

1. Erkenbrack, D.E., and Rosenberg, L.L. Binding of thyroid hormones to nuclear extracts of thyroid cells, Endocrinology 119(1), 311-317 (1986).

\section{BIOLOGICAL EFFECTS OF MAGNETIC FIELDS}

\section{Thomas S. Tenforde, Cornelius T. Gaffey, Robert P. Liburdy, Chu-Chung Lo," Allen B. Geyer," and Lynette Levy}

The primary objectives of the magnetic field research program are 1) the development of a detailed theoretical and experimental understanding of the mechanisms through which these fields interact with biological systems, 2) the acquisition of laboratory data on the response of living systems to magnetic fields at the cellular, tissue, and animal levels, 3) the development of advanced microprocessor-controlled dosimeters for characterizing human exposure to static and time-varying magnetic fields, and 4) the assessment of possible health risks to humans exposed to high-intensity

-Engineering Division, LBL magnetic fields in various industrial, research, and medical technologies. Progress in three selected areas of the magnetic field research program is described in this year's annual report.

\section{ELECTROPHYSIOLOGICAL RESEARCH}

Sensitive electrical recording techniques are being used to delect functional alterations in the cardiovascular, neural and visual systems of laboratory animals exposed to high-intensity magnetic fields. Research efforts have been focused on these specific systems because they involve ionic conduction processes that undergo electrodynamic interac- 
tions with an applied magnetic field. A major finding in this research area has been the observation of large magnetically induced potentials associated with pulsatile blood flow in the presence of a magnetic field.' ${ }^{1}$ These electrical potentials can be detected in the externally recorded electrocardiogram (ECG), as shown in Fig. 1 for a Macaca monkey exposed to a 1.5 -tesla field ( 1 tesla $=10^{4}$ gauss). The largest magnetically induced potential is associated with pulsatile blood flow into the aortic vessel, and is detectable as a superimposed electrical signal at the locus of the T-wave in the ECG. This induced potential has been shown to be a linear function of the magnetic flux density, and its appearance within the ECG is completely reversible upon termination of the magnetic field exposure.

Using a combination of phonocardiography and echocardiography to study the temporal sequence of cardiac valve displacements in relation to the timing of signals recorded in the ECG (see Fig. 1), it has been possible to identify magnetically induced potentials that are associated with pulsatile blood flows through the mitral, tricuspid, aortic, and pulmonary valves. The correlation between magnetically induced poientials detectable in the ECC and specific pulsatile blood flows within the heart and the major vesseis of the central circulatory system provides a useful noninvasive method for the study of cardiovascular dynamics.

Magnetohydrodynamic effects on the rate of arterial blood flow and intra-arterial blood pressure have also been studied in laboratory animals exposed to high-intensity magnetic fields. The electrodynamic interaction between an applied magnetic field and a flowing electrolyte solution such as blood creates a net volume force within the fluid.

\section{Macaca Monkey Cardiovascular Parameters}
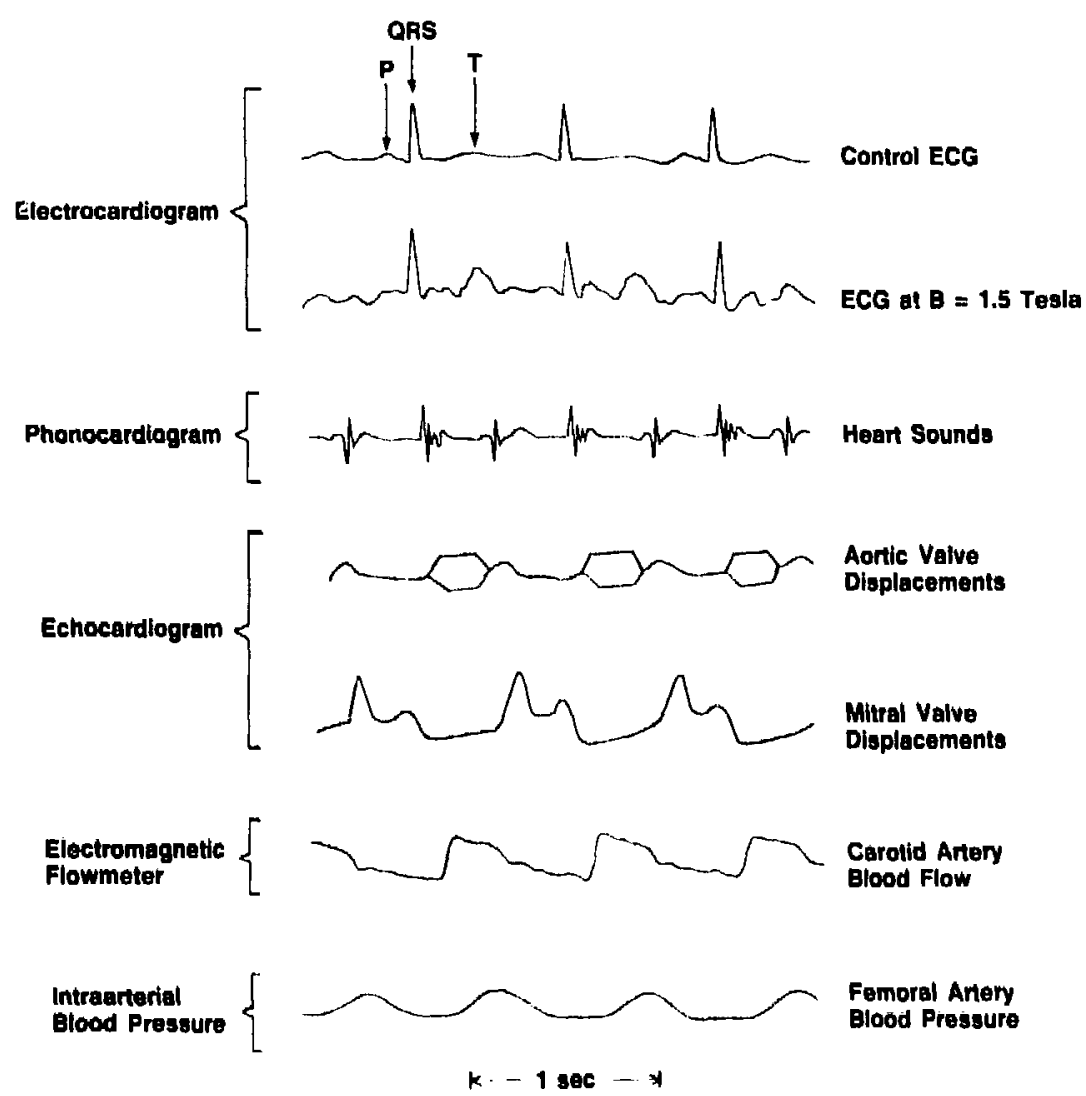

Fig. 1. Illustration of the cardiovascular paramelers measured in Macaca monkeys and beagle dogs exposed lo static magnetic fields with flux densilies up to 1.5 tes/a. The second trace from the top demonstrates the superimposed blood flow potentials that are recorded in the ECG during exposure of animals to high-intensity magnetic fields. The most prominent magnetically induced potential appears at the locus of the 7 -wave in the ECG, and is associaled with pulsatile dortic blood flow in the presence of a magnetic field. 
The magnetohydrodynamic consequence of this electrical force is a reduction in the axial flow velocity of the fluid. A combination of arterial blood flow velocity measurements and intra-arterial blood pressure measurements have been carried out in beagle dogs and Macaca monkeys exposed to static magnetic fields with flux densities up to 1.5 tesla (Fig. 1). In accord with theoretical predictions, ${ }^{2}$ these experimental results have demonstrated that magnetohydrodynamic interactions in a 1.5-tesla field do not produce a measurable alteration in blood flow dynamics.

\section{MEMBRANE INTERACTIONS WITH MAGNETIC FIELDS}

Diamagnetically anisotropic assemblies of molecules such as membrane phospholipids experience an orientational torque in strong magnetic fields and adopt an equilibrium configuration that minimizes their energy within the field. For thin nematic liquid crystals, a 0.3-tesla field applied parallel to the crystal plane was shown to significantly alter the rate of diffusion of tritium gas across the film as a consequence of magneto-orientation of the lipoidal constituents. ${ }^{3}$ Recent light scattering and magnetic birefringence studies have demonstrated that the orientational effects of strong magnetic fields on phospholipid bilayers are temperature dependent, with the most pronounced effects occurring in the pretransition and the main structural transition regions. 4.5

Experiments completed in our laboratory during the past year have demonstrated that the permeability properties of phospholipid bilayer membranes are also significantly influenced by exposure to static magnetic fields at temperatures in the structural phase transition region. ${ }^{6}$ Homogeneous magnetic fields with flux densities up to 7.55 tesla were found to increase the rate of transport of a chemotherapeutic drug, tritiated cytosine arabinofuranoside ( ${ }^{3} \mathrm{H}-\mathrm{ARA}-\mathrm{C} ; \mathrm{MW}=243$ ), through the phospholipid bilayer membranes of unilamellar liposomes when the exposure occurred at temperatures approaching the phase transition region (Fig. 2). The accelerated release of solute in these experiments was not associated with disruption of the liposome membranes, as indicated by the fact that no appreciable loss of ${ }^{14} \mathrm{C}$-labeled membrane phospholipids occurred from the exposed liposomes. The observed increase in ${ }^{3} \mathrm{H}-\mathrm{ARA}-\mathrm{C}$ permeability was found to have a sigmoidal dependence on magnetic flux density, with the $50 \%$ effect level occurring at 0.015 tesla. In the prephase transition temperature range from 40.0 to $40.5^{\circ} \mathrm{C}$, the pres-

\section{Effect of 7.5 Tesla DC Magnetic Field} on Liposome Permeability: Temperature Dependence

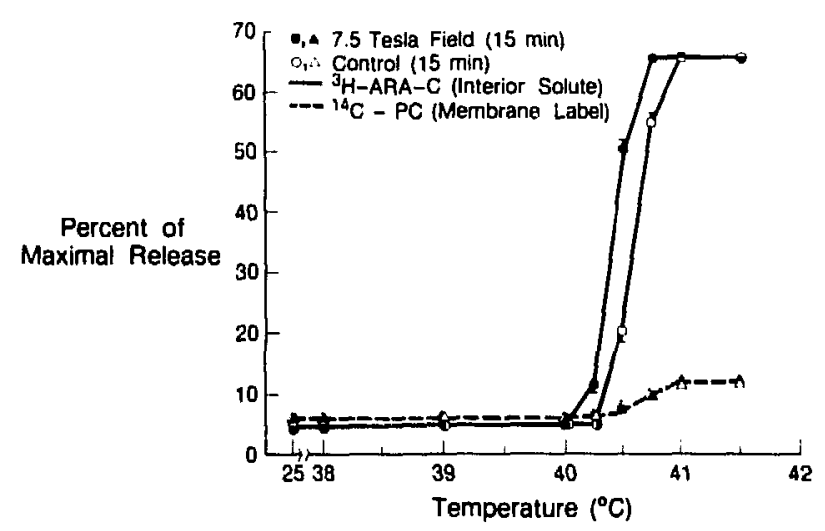

Fig. 2. Effect of a 7.55.tesla static magnetic field on liposome permeability to ${ }^{3} \mathrm{H}-A R A-C$ is plotted as a function of the sample temperature. Also shown is the release of ${ }^{14} \mathrm{C}$-labeled dipalmity!phosp tatidylcholine from liposome membranes as a function of tempersture. Error bars represent 1 S.E.M. [XBL 859-8498]

ence of magnetic fields greater than 0.01 tesla led to a significant enhancement of ${ }^{3} \mathrm{H}$-ARA-C release from liposomes within one minute (Fig. 3).

The results of these studies thus indicate that static magnetic fields of moderate intensity exert a rapid effect on the permeability of liposome bilayer membranes to a low-molecular-weight solute, and

Effects of 7.5 Tesla DC Magnetic Field on Liposome Permeability: Kinetics of Drug Release at $40.5^{\circ} \mathrm{C}$

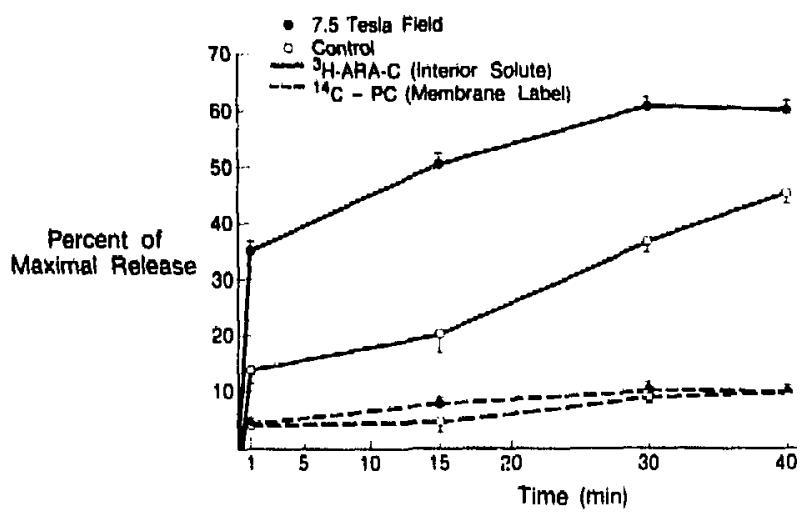

Fig. 3. The release of ${ }^{3}$ H-ARA-C from liposome vesicles is plotted as a function of time for control samples and for samples exposed to a 7.55-tesla static magnetic field at $40.5^{\circ} \mathrm{C}$. Also shown is the release of ${ }^{14} \mathrm{C}$-labeled dipalmitylphosphatidylcholine from liposome membranes as a function of time during exposure at $40.5^{\circ} \mathrm{C}$. Error bars represent 1 S.E.M.

$|X B L \quad 859-8499 A|$ 
that this effect is linked to changes in the molecular organization of membrane phospholipids that occur at temperatures in the prephase transition range. In developing a theoretical model of this magnetic field interaction, we have incu porated the fact that domains (clusters) of phospholipids form within a bilayer membrane in the prephase transition region? (Fig. 4). The physical model that has been developed is based on an earlier theoretical description of the elastic properties of spherical lipid bilayer vesicles, in which the local radius of curvature of patches of membrane phospholipids was assumed to differ from that of the liposome vesicle. ${ }^{8}$ In our model these patches of phospholipids correspond to domain structures that form in the prephase transition temperature range. By using the calculus of variations to derive a criterion for minimizing the total elastic and magnetic energy within a bilayer membrane, the following equation was derived for the deformation of a spherical liposome in the presence of a magnetic field:

$$
\frac{\Delta R}{R}=\frac{-\left(\chi_{n}-\chi_{1}\right) R^{2} B^{2}}{3 K[6-(R / \rho)] \mu_{0}}
$$

Where $\Delta R$ is the change in radius of a spherical liposome with an undeformed radius $R,\left(\chi_{n}-\chi_{t}\right)$ is the difference in magnetic susceptibility normal and tangential to the plane of the membrane bilayer ($1.1 \times 10^{-6}$ M.K.S, units), $B$ is the magnetic flux density, $K$ is the bilayer elastic modulus $\left(10^{-11} \mathrm{~N}\right), p$ is the local radius of curvature of phospholipid domain structures within the bilayer membrane, and $\mu_{0}$ is the magnetic permeability of free space

\section{Phosphollpid Bilayer Phase States}
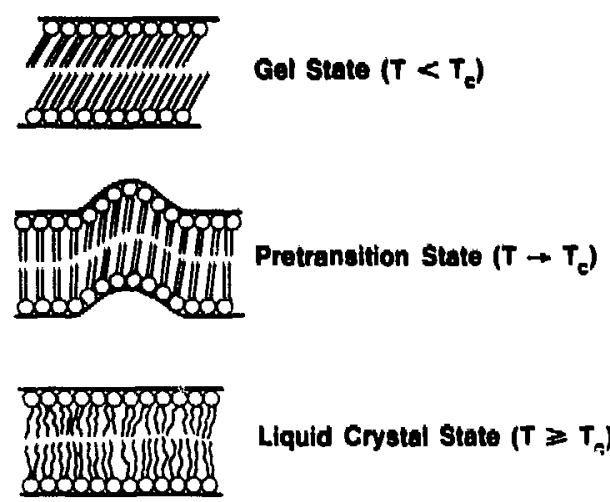

Liquid Crystal state $\left(T \geq T_{f}\right.$ )

Fig. 4. Schematic diagram of the clustering at prephase transition temperatures of "tilted" and "nontilted" phospholipid domains in a bilayer membrane. $T_{t}$ represents the midpoint temperattere of the main phase transition region. [XBL B63-8681] $\left(4 \pi \times 10^{-7} \mathrm{~N} / \mathrm{A}^{2}\right)$. The interesting feature of this result is the prediction that the magnetic deformational stress exerted on a bilayer membrane increases rapidly when the local radius of curvature of the phospholipid domains approaches the critical value R/6. From Eq. (1) it is predicted that the magnetic deformation will elongate a liposome vesicle into a prolate spheroidal form and subsequently compress it into an oblate spheroid as the radius of curvature of the phospholipid domains passes through the singular point at $\rho=R / 6$. This predicted deformational behavior of liposome membranes exposed to static magnetic fields is supported by recent studies on the magnetic birefringence of aqueous suspensions of liposome vesicles. ${ }^{5}$ The magnetic birefringence was observed to exhibit a rapid increase, followed by a rapid decrease, as the temperature of the solution was increased through the prephase transition region. This change in birefringence is consistent with the deformations that are predicted from Eq. (1) to occur in the presence of a magnetic field. Another consequence of the magnetic deformational stresses exerted on liposome bilayers as $\rho$ approaches the value $R / 6$ is that a rapid change in pressure occurs between the internal and external aqueous compartments. This pressure change is predicted to cause a release of encapsulated solutes, as observed in our experiments on the loss of ${ }^{3} \mathrm{H}$ ARA-C from liposomes during magnetic field exposure in the prephase transition temperature range.

\section{DOSIMETER DEVELOPMENT}

An important aspect of the magnetic field program since 1980 has been the development of compact, microprocessor-controlled dosimeters for monitoring human exposure to magnetic fields. A miniature 230-g dosimeter for recording static magnetic fields was completed in 1983, and subsequently used to characterize the exposure of workers to high-intensity magnetic fields in numerous industrial and laboratory facilities throughout the United States. During the past year a portable dosimeter was developed for monitoring human exposure to power-frequency magnetic fields in residential and occupational environmentc, ${ }^{9}$ This compact dosimeter has been designed to measure $60-\mathrm{Hz}$ fields with flux densities ranging from $20 \mu \mathrm{G}$ to $600 \mathrm{mG}$, thereby covering the broad range of field levels generally encountered in households and occupational settings. Schematic diagrams of the design and operational features of the dosimeter are presented in Figs. 5 and 6, and pholographs of its components are shown in Fig. 7. 


\section{0-Hz MAGNETIC FIELD DOSIMETER OPERATION}
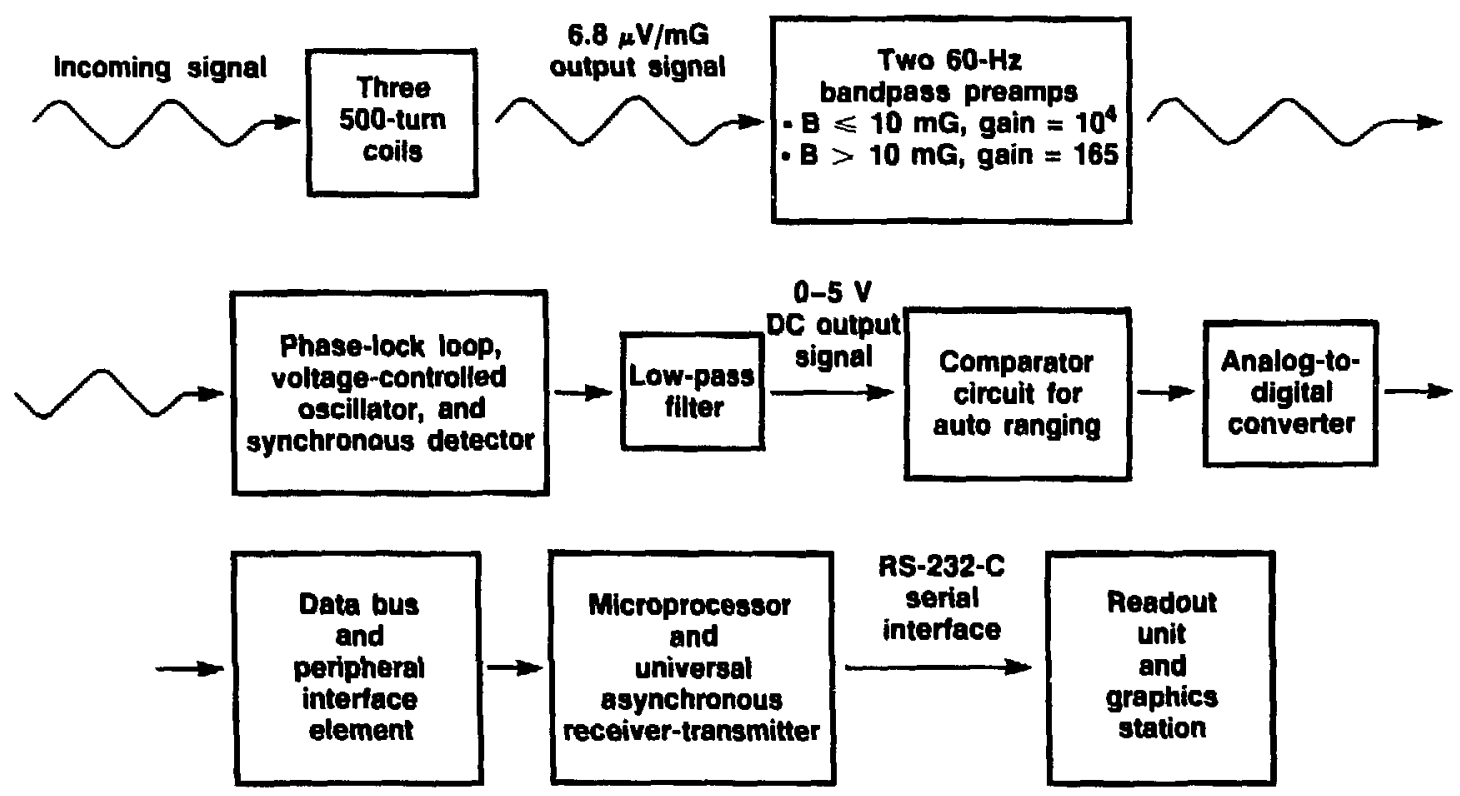

Fig. 5. Schematic diagram of the operational features of the 60-Hz magnetic field dosimeter. [XBL 8510-8523]

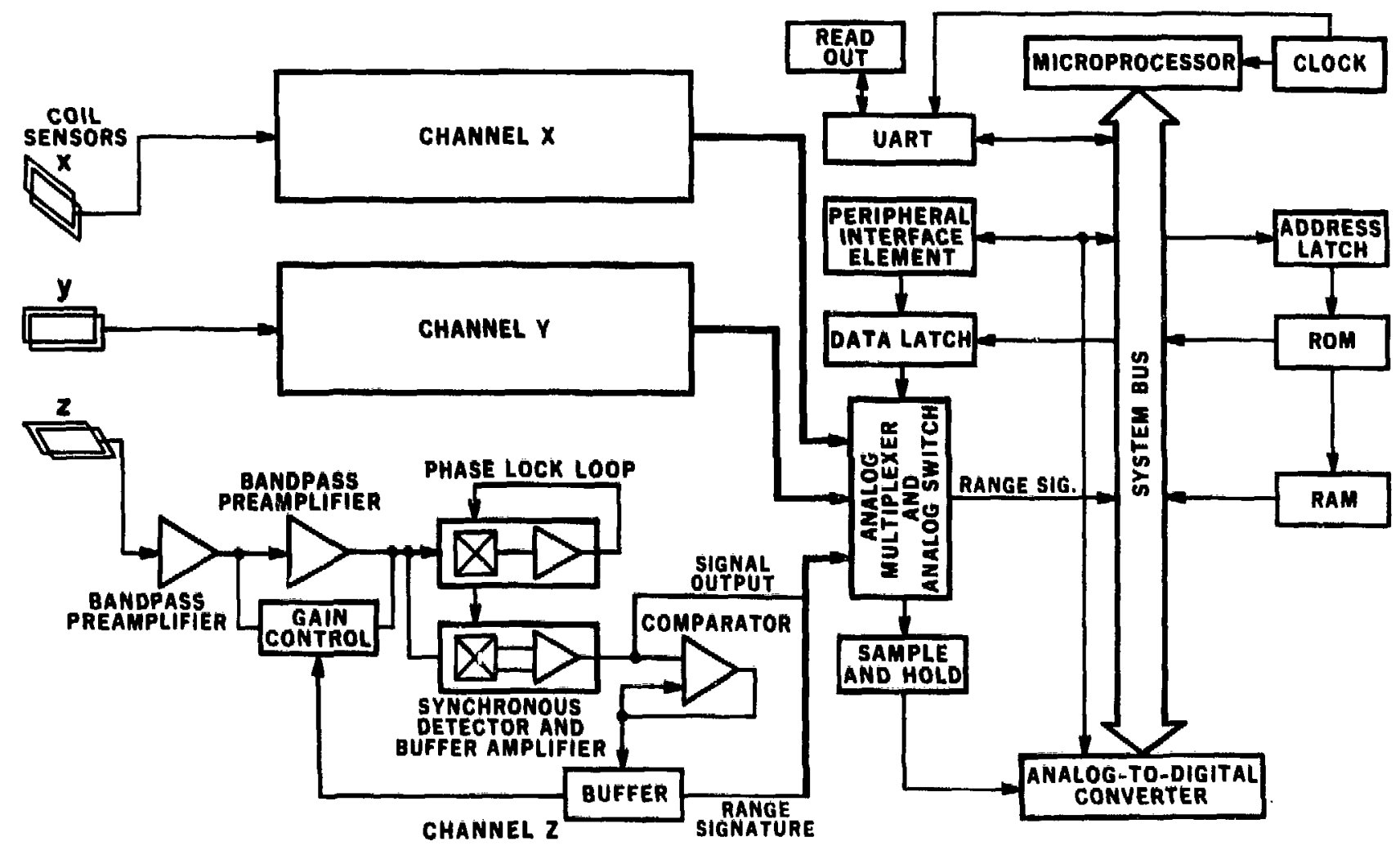

Fig. 6. Block diagram of the portable $60 \cdot \mathrm{Hz}$ magnelir field dosimeter. 


\section{0- $\mathrm{Hz}$ Magnetic Field Dosimeter}

A

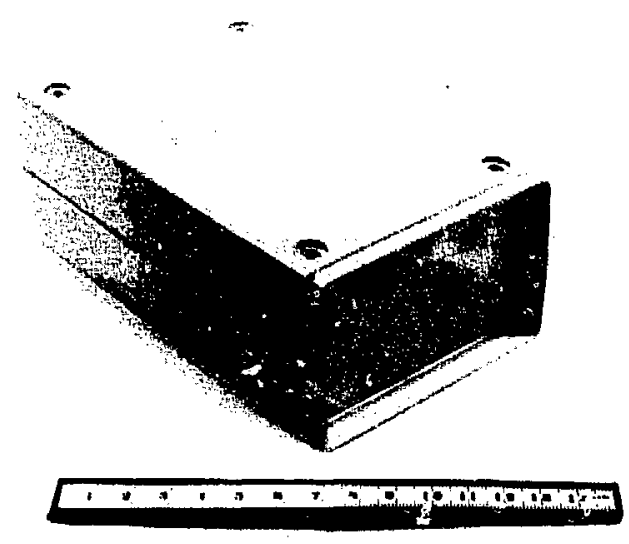

C

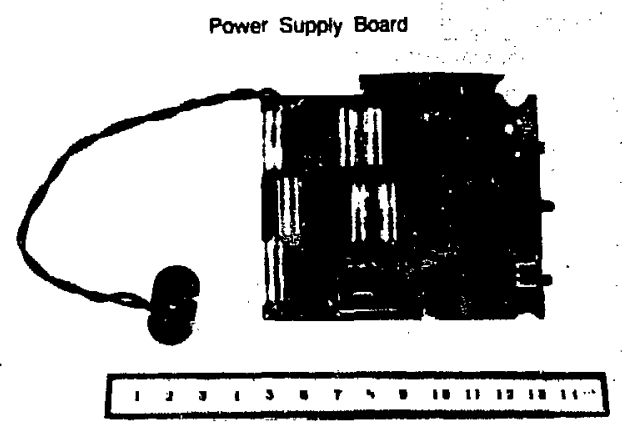

$\mathbf{E}$

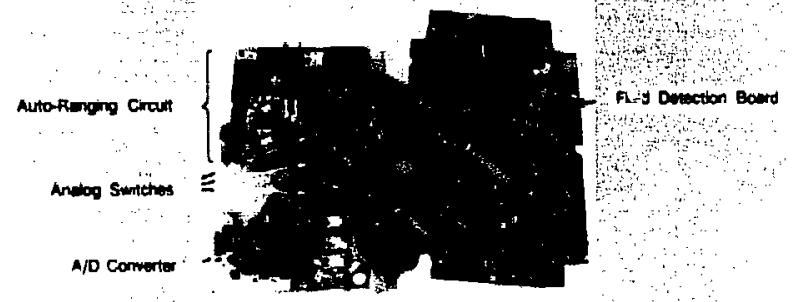

B

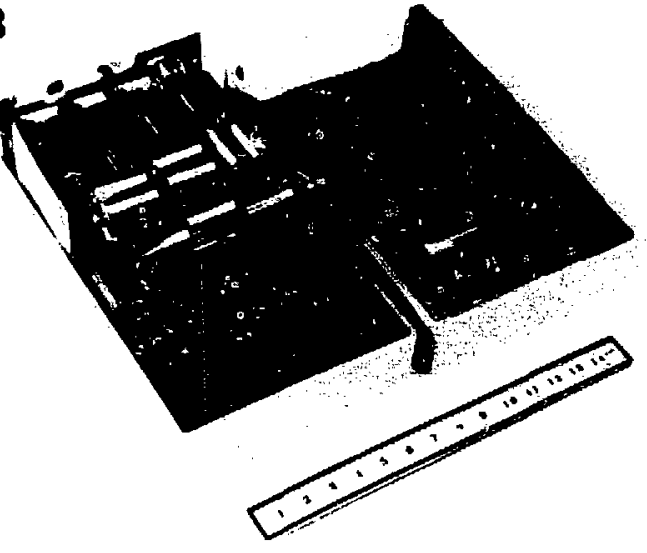

D

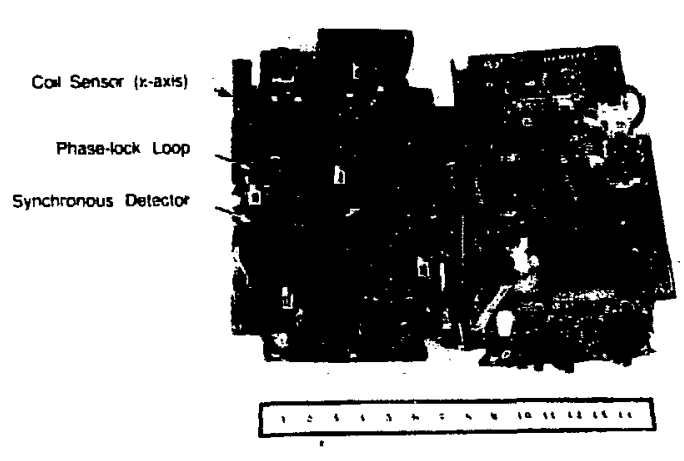

$\mathbf{F}$

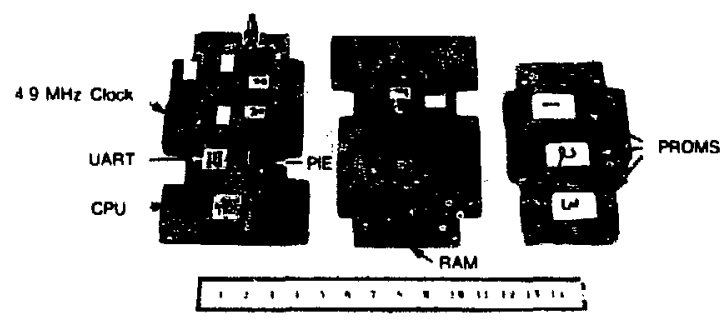

Fig. 7. Photographs of the porable 60.Hz magnetic field dosimeter and its six circuit boards. 
Field detection by the dosimeter is accomplished by three 500-turn coils, which are arranged along orthogonal axes to monitor the $x, y$ and $z$ components of $60-\mathrm{Hz}$ ambient magnetic fields. Since the coils are also sensitive to electric fields, they are shielded within individual copper chambers. The signal output from each coil is amplified by two $60-\mathrm{Hz}$ bandpass preamplifiers in cascade. At field levels of $10 \mathrm{mG}$ or lower, the total maximum gain of the two active filters is set at 10,000 so that a $50-\mu \mathrm{G}$ field will yield a $3.4-\mathrm{mV}$ signal and a $10-\mathrm{mG}$ field will yield a $680-\mathrm{mV}$ signal a! the output of the second active filter. When the field being monitored exceeds $10 \mathrm{mG}$, the total gain of the two active filters is automatically decreased to 165 . A 20-mG field will thus yield a 22.4-mV signal and a 500-mG field will yield a $560-\mathrm{mV}$ signal at the output of the second active filter.

The second active filter supplies amplified signals to a phase-locked loop (PLL) and synchronous detector. The PLL phase locks with the incoming signal and produces a quadrature voltage-controlled oscillator (VCO) output that is in phase with the incoming signal. Th. quadrature VCO output is then used as the demodulating signal for the synchronous detector. The demodulated output is passed through a low-pass filter suppressing all harmonics except the dc component, which is determined only by the amplitude of the incoming 60$\mathrm{Hz}$ magnetic field. The dc output of the synhcronous detector is amplified to be compatible with the 0 to 5 volt range of the arialog to digital converter $(A D C)$ in the data acquisition section of the dosimeter. The amplified detector output and a comparator circuit are used for automatic ranging between the high-gain $(10,000)$ and low-gain (165) signal processing modes, which are used in the 20 $\mu \mathrm{G}$ to $10-\mathrm{mG}$ and $10 \cdot \mathrm{mG}$ to $600-\mathrm{mG}$ field ranges respectively.

Three analog switches are used to transfer data to the ADC. Digitization of the three orthogonal axes is done sequentially. A 12-bit successive approximation $A D C$ is used in the uniroslar mode providing $150 \mu \mathrm{G} /$ bit and $2.5 \mu \mathrm{G} /$ bit in the lowgain and high-gain modes respectively. A programmable interface element interfaces the ADC and the universal asynchronous receiver-transmitter (UART) used for serial data transmission to the microprocessor. The UART interfaces the microprocessor with an HP-85 compute: 1 at is used for initialization and data readout.

Calibration tesis for the dosimeter's deteclion circuitry were carried out with a standard coil that provided a known magnetic flux density as a function of the applied current. The coil was placed inside a Mumetal shield to reduce the background $60-\mathrm{Hz}$ magnetic field to approximately $20 \mu \mathrm{G}$. Linearity of the detection system was observed to be within $2 \%$ over the range of field intensities from $50 \mu \mathrm{G}$ to $600 \mathrm{mG}$, as shown in Fig. 8. Contamination of the main $60-\mathrm{Hz}$ signal by harmonics was found to be less than $1 \%$.

The integrated data acquisition and recording systems of the prototype dosimeter have also been subjected to calibration tests and to field tests in both laboratory and residential environments. The results of two 4-hour field tests of the dosimeter's performance while being worn on the belt of an engineer working in a laboratory at LBL Building 29 and in a private residence are shown in Figs. 9 and 10. These $60-\mathrm{Hz}$ magnetic field exposure profiles clearly indicate that the peak transient exposure resulting from the switching on of equipment can significantly exceed the average exposure level recorded over a 5-minute time interval.

The development of this type of miniature personal dosimeter has become of increasing importance because of several recent epidemiological surveys that have found that a relationship may exist between cancer risk and residential or occupational exposures to power-frequency magnetic fields. ${ }^{10} \mathrm{~A}$ major deficiency in these epidemiological studies was the failure to use quantitative dosimetry procedures for characterizing magnetic field exposure levels. Our future plans in magnetic field dosimetry involve the development $\mathrm{c} f$ an advanced prototype dosimeter that can be commercially produced in large numbers for use in epidemiological

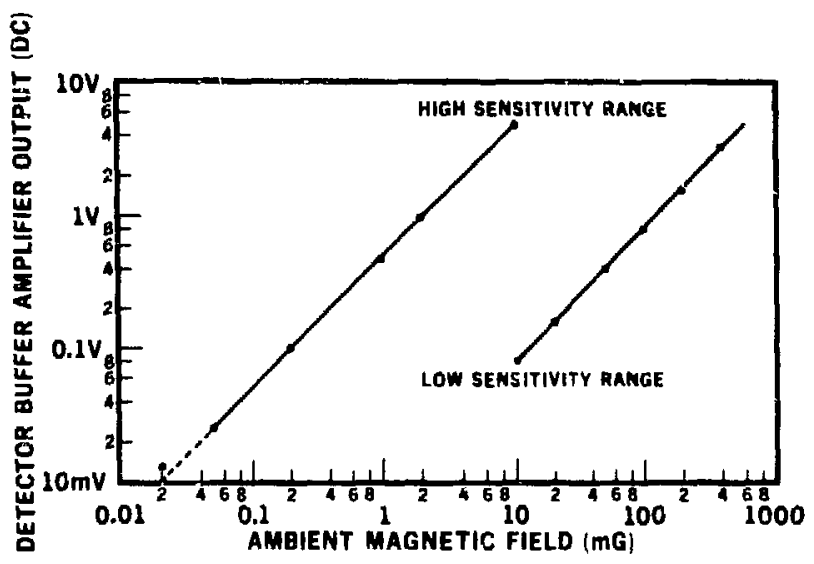

Fig. 8. Plots of the amplified detector output in the $60-\mathrm{Hz}$ dosimeter as a function of the magnetic flux density.

$|X B L \quad 8510-4193|$ 


\section{Field Test of 60-Hz Dosimeter in a Laboratory}
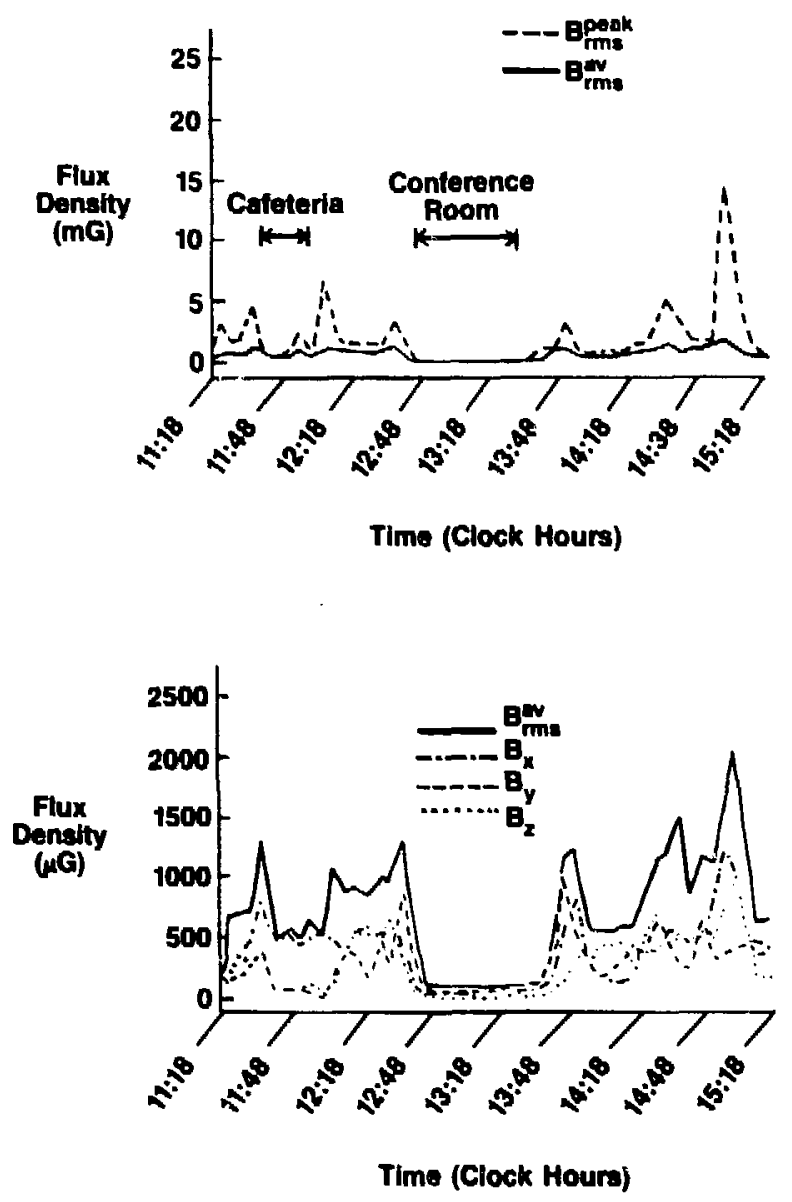

Fig. 9. Results of a 4-hour test of the 60.-Hz magnetic field dosimeter's performance while worn on the belt. Except for a 20-minute period spent in the LBL cafeferia, the remainder of the field lest was performed in Building 29. [XBL 861i-6491]

studies of workers in electrical occupations. This advanced dosimeter will incorporate many of the design features of the prototype model described here, but will be further miniaturized and will contain an extended memory capability to permit continuous field monitoring over periods of several days duration.

\section{REI : :CES}

1. Tenforde, T.S., Gaffey, C.T., and Raybourn, M.S. Influence of stationary magnetic fields on ionic conduction processes in biological systems. In Proceedings of the Sixth Syrnposium and Technical Exhibition on Electromagnetic

\section{Field Test of $60-\mathrm{Hz}$ Dosimeter in a Residence}

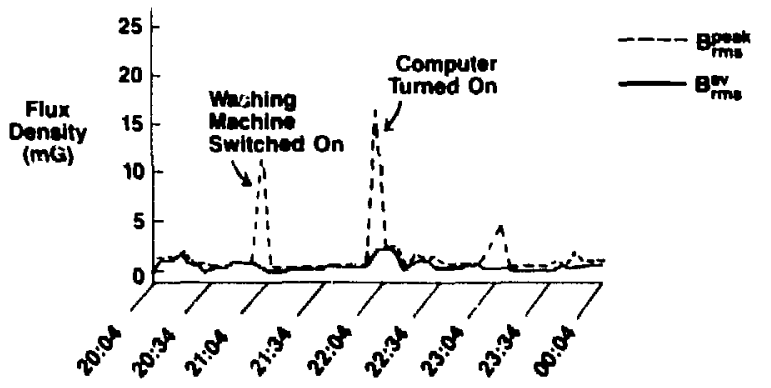

Time (Clock Houre)

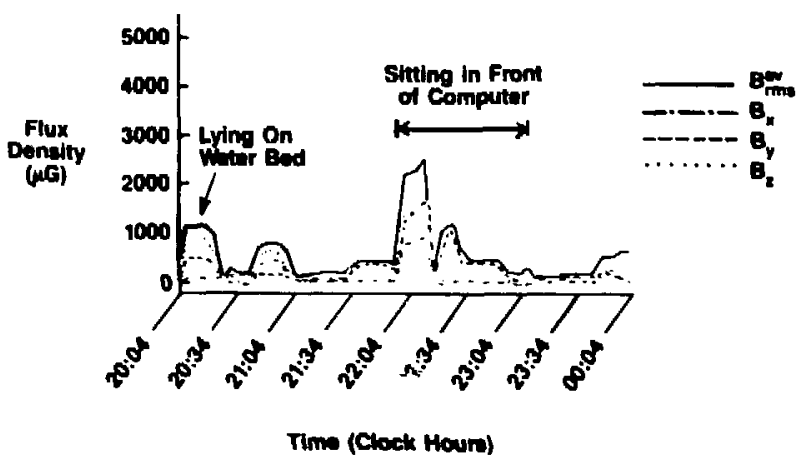

Fig. 10. Results of a 4-hour test of the $60-\mathrm{Hz}$ magnetic field dosimeter's performance while worn as a belt-mounted unit in a privale residence. Large Iransient values of the magnetic $h u x$ density were associated with the switching on of a washing machine and the disk drive of an IBM personal computer.

$\mid X B L$ 8611-6490|

Compatibility, March 5-7, 1985, pp. 205-210 (T. Dvorak, Ed.) Zurich, Switzerland.

2. Tenforde, T.S. Mechanisms for biological effects of magnetic fields. In Biological Effects and Dosimetry of Non-lonizing Radiation: Static and ELF Electromagnetic Fields, pp. 71-92. (M. Grandolfo, S.M. Michaelson, and A. Rindi, Eds.) Plenum Press, New York (1985).

3. Teucher, I., Baessler, H., and Labes, M.M. Diffusion through nematic liquid crystals. Nature Phys. Sci. 229, 25-26 (1971).

4. Braganza, L.F., Blott, B.H., Coe, T.J., and Melville, $D$. The superdiamagnetic effect of magnetic fields on one and two compone it multilamellar systems. Biochim. Biophys. Acta 801, 66-75 (1984). 
5. Maret, G., and Dransfeld, K. Biomolecules and polymers in high magnetic fields. Topics Appl. Phys. 57, 143-204 (1985).

6. Liburdy, R.P., and Teniorde, T.S. Magnetic field-induced drug permeability in liposome vesicles. Radiat. Res. 108, 102-111 (1986).

7. Nagle, J.F. Theory of the main lipid phase transition. Ann. Rev. Phys. Chem. 31, 157-195 (1980).

8. Helfrich, W. Elastic properties of lipid bilayers: Theory and possible experiments. Z. Natur- forsch. 28C, 693-702 (1973).

9. Lo, C.C., Fujita, T.Y., Geyer, A.B., and Tenforde, T.S. A wide dynamic range portable 60-Hz magnetic dosimeter is 'h data acquisition capabilities. IEEE Trans. Nucl. Sci. 33. 643-646 (1986).

10. Tenforde, T.S. Interaction of ELF magnetic fields with living matter. In CRC Handbook of Biological Effects of Electromagnetic Fields, pp. 197-225. (C. Polk and E. Postow, Eds.) CRC Press, Inc., Boca Raton, Florida (1986).

\section{ELECTROMAGNETIC FIELDS AND THE INDUCTION OF PROTEIN SHEDDING}

\section{Robert P. Liburdy}

The cell membrane is considered to be the biological structure most likely to be responsive to electromagnetic fields, and recently we have demonstrated that both natural as well as synthetic membranes maintained in the presence of an electromagnetic field experience increases in bilayer permeability. ${ }^{1,2}$ Soúlium cation permeability increases are seen in erythrocytes and lymphocytes and a permeability increase is also observed when drug-loaded liposomes are field-exposed. One striking feature of these membrane responses is that the permeability increases are temperature dependent. the electromagnetic field elicits the effect at temperatures associated with a phase transition in the respective membrane. This suggests that physical structure of the membrane is important for sensitivity to the electromagnetic field.

Recently we initiated a series of studies to test whether physical changes to the cell membrane occur in the presence of electromagnetic fields. ${ }^{3}$ Our approach to this problem has been to employ two techniques, polyacrylamide gel electrophoresis (PAGE) using sensitive silver staining, and high pressure liquid chromatography (HPLC), to detect the presence of proteins shed or released into the cellfree supernatant following exposure to electromagnetic fields. We have observed that short-term exposures result in protein shedding of at least 11 species of $\leqslant 31 \mathrm{kDa}$. This effect is oxygen and temperature dependent, involves unique protein species $\leqslant 14 \mathrm{kDa}$ associated with a pl of 6.3-7.0, and is linked to the disruption of calcium bridges that act to stabilize membrane-associated proteins. HPLC studies further indicate that electromagnelic field-treatment is uniquely associated with the shedding of a class of relative cationic and nonpolar protein species.

In these experiments erythrocytes from adult male donors or from adult male New Zealand rabbits were employed, and pure erythrocyte suspensions were treated with $2450-\mathrm{MHz}$ electromagnetic fields for 30 minutes in a temperature- $\left( \pm 0.05^{\circ} \mathrm{C}\right)$ and $\mathrm{pO}_{2}$-controlled waveguide device. ${ }^{1.2}$ Onedimensional PAGE was performed in $12.5 \%$ polyacrylamide slab gels with $\mathrm{NaDodSO}_{4}$ present. Two dimensional PAGE involved isoelectric focusing in the first dimension followed by $\mathrm{NaDodSO}_{4}-$ PAGE. HPLC analyses were conducted using Synchrom/Synchropak anion exchange and reverse-phase columns. ${ }^{4}$

Detection of at least 11 protein species of molecular weight $\leqslant 35 \mathrm{kDa}$ following electromagnetic field treatment $(M)$ at $17^{\circ} \mathrm{C}$ is depicted in Fig. 1. Exposures were conducted at $17^{\circ} \mathrm{C}$ since increases in $\mathrm{Na}^{+}$permeability were reported at this temperature, which corresponds to a membrane phase transition.' Sham-exposures (5) were conducted at identical temperatures and were observed to lead to a distinctly different pattern of protein release. For example, field treatment results in protein shedding with pronounced staining of species of 28-, 15-, 14-, 13-, 11-10- and 8-kDa molecular weight, whereas sham-treated cells exhibit pronounced staining of 20 - and $18-\mathrm{kDa}$ species. The band observed at $68 \mathrm{kDa}$ is a marker protein, bovine serum albumin (BSA), present in the Ringer's buffer used. A temperature study has demonstrated that such protein shedding is res- 


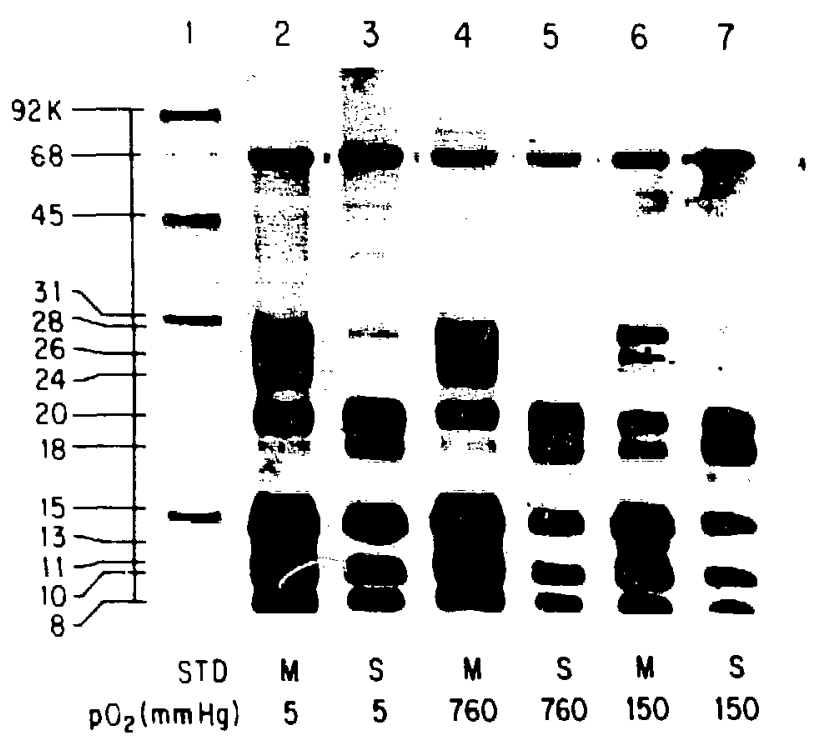

Fig. 1. Electromagnetic field-induced protein shedding in the erythrocyte: Oxygen dependence at $T_{c}=17^{\circ} \mathrm{C}$. Electrophoretograms of cell-free supernatants following microwave $(M)$ or sham (S) exposure for 30 minutes at hypoxic $(5 \mathrm{~mm} \mathrm{Hg})$. hyperoxic $(760 \mathrm{~mm} \mathrm{Hg})$, or almospheric $(150 \mathrm{~mm} \mathrm{Hg})$ oxygen rension. Rabbit erythrocytes exposed to $2450-\mathrm{MHz}$ microwaves at $100 \mathrm{~mW} / \mathrm{g}$ for 30 minutes. Sham exposures at identical temperatures maintained by conventional heating. Erythrocytes at $10^{\prime a}$ cells $/ \mathrm{ml}$ in Ringer's buffer, $0.1 \%$ BSA, $\mathrm{pH}=7.4$. Mean \pm S.D. $n=6$.

(XBB 860-8228)

tricted to the special temperature range of $17-21^{\circ} \mathrm{C}$ where a membrane phase transition is found. ${ }^{3}$ Figure 1 also illustrates that when oxygen tension is varied a marked alteration in protein release occurs. In particular, relative-hypoxia-enhanced protein shedding suggesting that oxygen, and perhaps membrane-associated hemoglobin, play a significant role in mediating this effect.

Following electromagnetic-field or sham treatments erythrocytes appear as normal morphological discocytes, and this suggests that protein shedding may involve the release of proteins that are only loosely bound to the cell surface. To test this hypothesis. cells were treated at $18^{\circ} \mathrm{C}$ with EDTA to chelate calcium and the cell-free supernatant was analyzed. EDTA is a mild chaotropic agent that acts to release proteins from the red cell membrane that are highly water soluble, do not span the membrane, and, importantly, are electrostatically bound via divalent calcium bridges. ${ }^{3}$ Figure 2 indicates that a 30 minute treatment in the presence of EDTA and Ringer's buffer without calcium (E), in contrast to Ringer's buffer with calcium present (R), results in at least five prominent bands at 25 $31 \mathrm{kDa}$. The band observed at $14 \mathrm{kDa}$ is the dissociated tetramer of hemoglobin. A comparison of

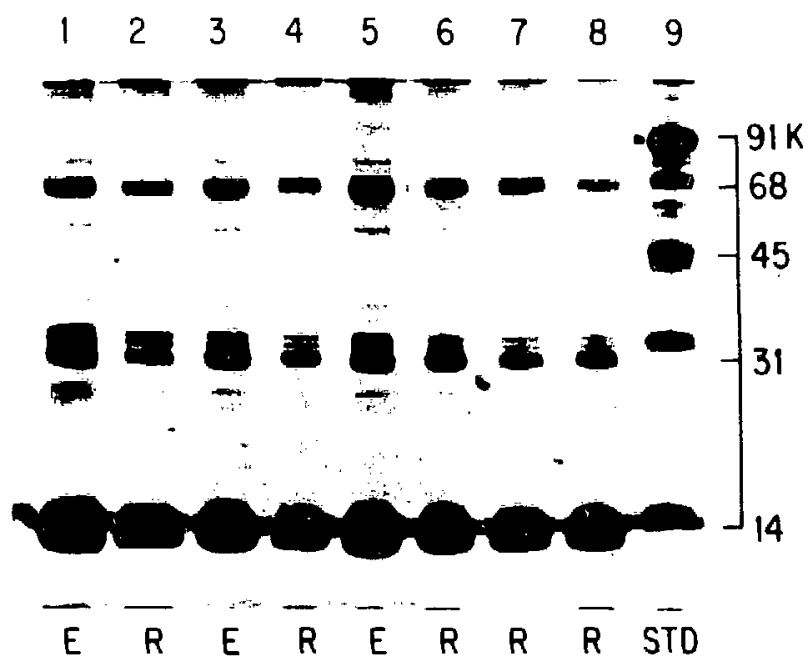

Fig. 2. Protein shedding induced by divalent cation chelation. Cell-free supernatants analyzed after erythrocytes were convenlionally heated at $T_{c}=17^{\circ} \mathrm{C}$ for 30 minutes in Ringer's buffer $(R)$, or in Ringer's buffer prepared without calcium and magnesium but with 25-mM EDTA present (E). Enythrocytes used at $10^{10}$ cells $/ \mathrm{m} /$ with $0.01 \%$ BSA, $\mathrm{pH}=7.4$ and atmospheric $\mathrm{pO}_{2}$.

(XBB 86I-48)

lanes in Figs. 1 and 2 associated with field $(M)$ and EDTA (E) treatments, respectively, reveals that both result in the release of protein species in the $25-31 \mathrm{kDa}$ range. These findings suggest that the electromagnetic field acts to destabilize calcium divalent bridges associated with anchoring these proteins to the cell membrane.

A two-dimensional PAGE characterization involving isoelectric point focusing was performed to identify differences in net protein charge. Figure 3(A) depicts the two-dimensional separation of proteins released during sham treatments at $18^{\circ} \mathrm{C}$. The majority of proteins, including the marker protein BSA(pl 5.1, $68 \mathrm{kDa})$ focused at $\sim \mathrm{pH}$ 5.1. When a similar analysis was performed on field-treated samples, protein species of $25 \mathrm{kDa}$ and $\leqslant 14 \mathrm{kDa}$ were observed to focus at $\mathrm{pH}$ 6.8-7.3. These species are unique to the field-treated samples and this indicates that relatively neutral charged species are released at phase-transition temperatures in response to 2450 $\mathrm{MHz}$.

Characterization by anion exchange and reverse-phase HPLC revealed additional differences in the nature of the proteins released during field treatments. Anion exchange separations were carried out using a 300-A spherical silica derivatized with a bonded polymeric layer of amine, and a mobile phase of $50 \mathrm{mM}$ Tris, $\mathrm{pH} 8.0$ in combination with a linear gradient of increasing $\mathrm{NaCl}$ 

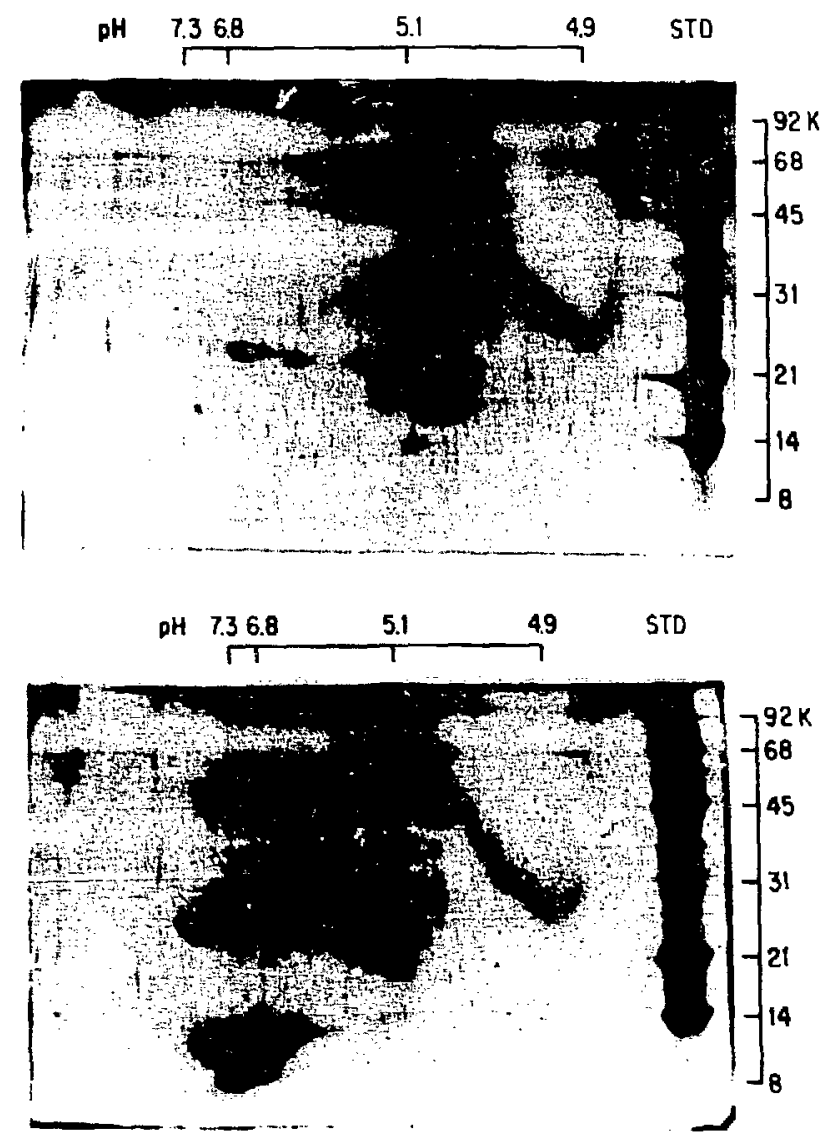

Fig. 3. Isoelectric point $(\mathrm{pl})$ characterization of electromagnetic field-induced protein shedding at $T_{\mathrm{c}}=17^{\circ} \mathrm{C}$. Erythrocytes were sham (3A) or field-exposed (3B) according to conditions in Fig. 1 at atmospheric $\mathrm{pO}_{2}$. Proteins released into the cell-free supernatants were separated by isoelectric focusing in the $x$ direction (pl) and by standard PAGE in the y-direction (molecular (veight).

(XBB 861-49) (XBB 860-8229)

$(0.0-0.05 \mathrm{M})$ over 20 minutes. Figure 4 illustrates chromatographs for sham and field-treated (MW) samples with retention times shown in minutes for each peak; peak areas were integrated to quantitate relative protein mass. Major differences in protein elution occurred during the first 6 minutes of separation during which relatively anionic species are retained. Six peaks appear in field-ireated samples that correspond to $-87 \%$ or the total protein mass. In contrast, for sham samples only three/four peaks are resolved during this time period and they are associated with $-68 \%$ of the total protein mass. In particular, a major peak at 3.78 minutes $(\sim 47 \%$ protein mass) appears in the field-treated sample that is absent in the sham samples.

HPLC analyses were also performed using C-8 reverse phase columns which separate proteins on the basis of hydrophobic/nonpolar interactions. A

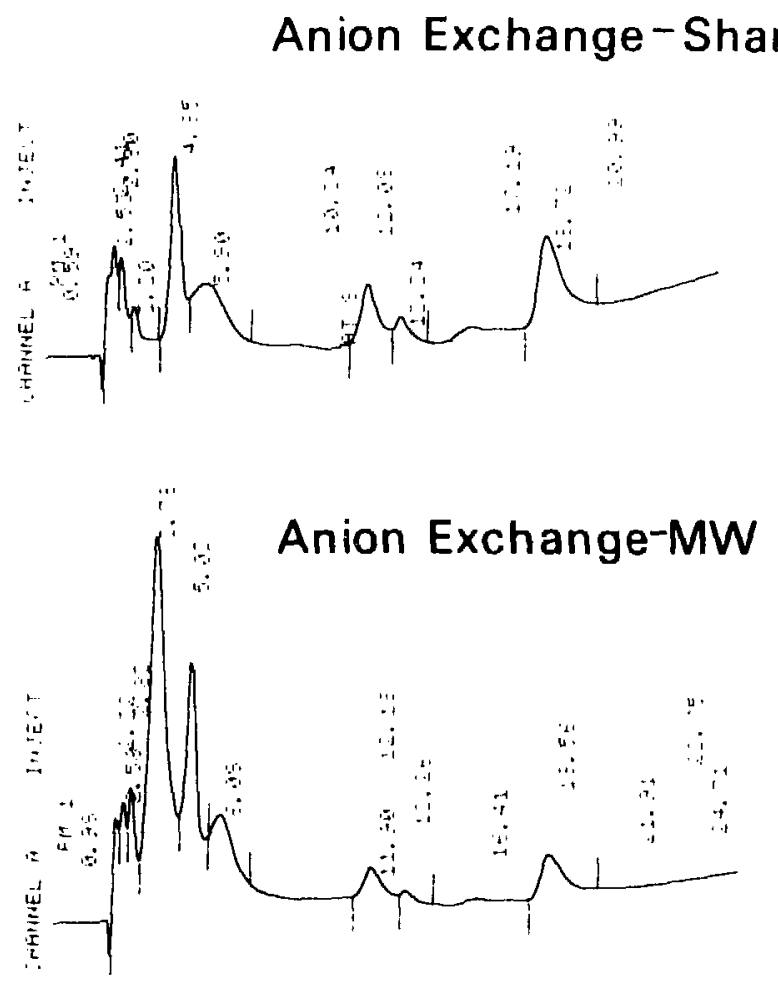

Fig. 4. Anion exchange HPLC characterization of electromagnetic field-induced protein shedding at $T_{c}=17^{\circ} \mathrm{C}$. Erthrocytes were sham or field-exposed as in Fig. 1 at atmospheric $\mathrm{pO}_{2}$. Proteins released into the cell-free supernatants were loaded onto Synchropak AX300 $(250 \times 4.1 \mathrm{~mm})$ columns at $20 \mu \mathrm{g}$ in $50 \mu \mathrm{l}$ in $50-\mathrm{mM}$ Tris $\mathrm{pH} 8.0$, at $0.5 \mathrm{ml} / \mathrm{min}$. A linear gradient from $0.100 \%$ \% $0.5 \mathrm{~N} \mathrm{NaCl}$ was run from 6 to 26 minutes. Absorbance was at $280 \mathrm{~nm}, 0.05$ units full scale. Retention time in minutes.

(XBL 865-1897)

mobile phase of $95 \% \mathrm{H}_{2} \mathrm{O}$ and $5 \%$ acetonitrile with $0.1 \%$ trifluoroacetic acid was used with a 20 -minute linear gradient that achieved $95 \%$ acetonitrile and $5 \% \mathrm{H}_{2} \mathrm{O}$. Figures $5(A)$ and $5(B)$ depict separations for sham and field-treatment, respectively. Significant differences are observed in major protein peaks appearing at 20-22 minutes that are relatively nonpolar in nature since they elute well into the acetonitrile gradient. For the field-treated samples these proteins represent $46 \%$ of the total protein mass, whereas proteins with identical elution times in the sham sample correspond to $4.3 \%$ of the protein mass. Differences were also observed in relatively polar proteins that elute early in the separation at $\sim 5.6$ and $\sim 6.5$ minutes. Fieldtreated samples display protein peaks at these times that correspond to $9.8 \%$ and $4.7 \%$ of the total protein mass, respectively. In contrast, sham-treated samples exhibit co-eluting peaks that are associated 

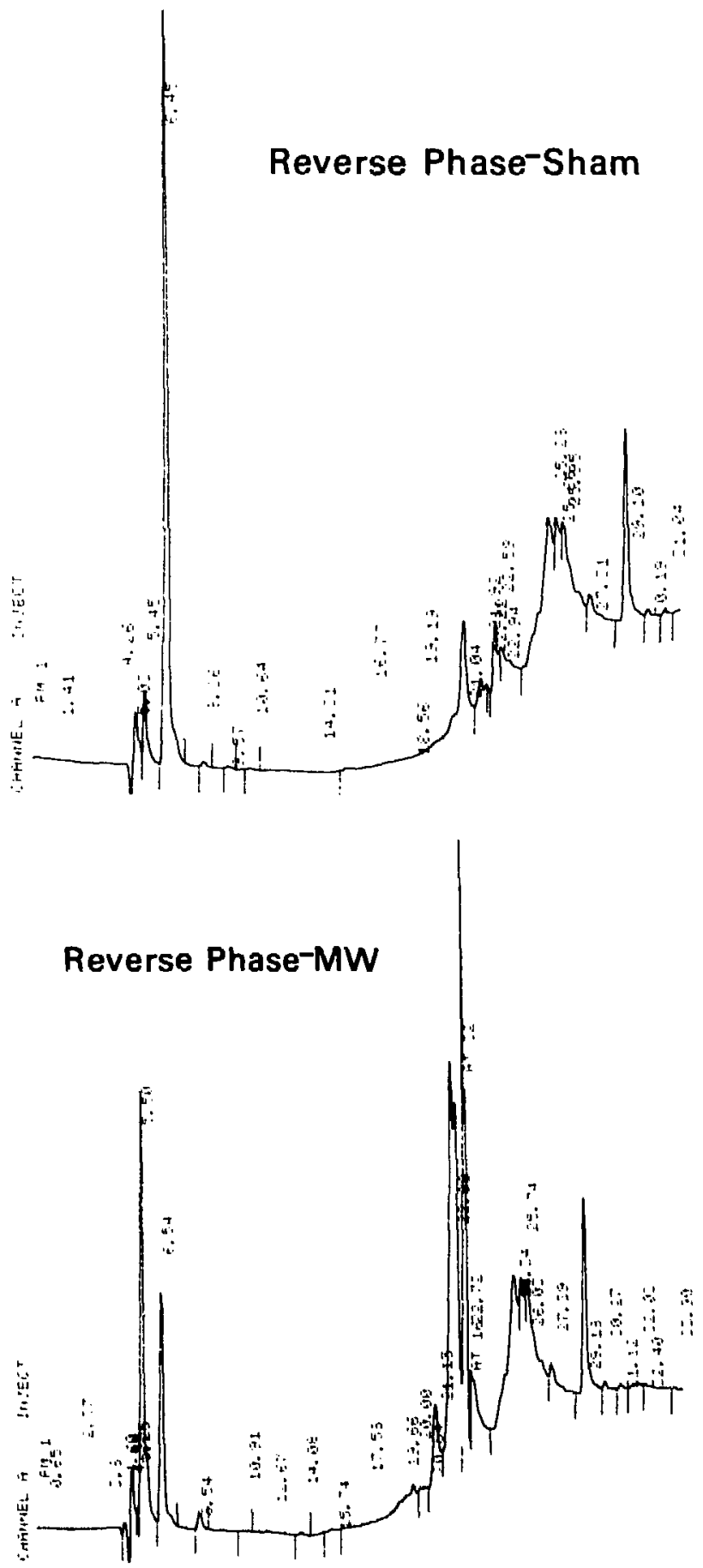

Fig. 5. Reverse phase HPLC characterization of electromagnetic field-induced protein shedding at $T_{\mathrm{c}}=17^{\circ} \mathrm{C}$. Erythrocytes were sham (5A) or field-exposed (5B) as in Fig. 1 at atmospheric $p \mathrm{O}_{2}$. Proteins released into the cell-free supernatants were loaded onto Synchropak RP-8 $(250 \times 4.1 \mathrm{~mm})$ columns at $20 \mathrm{\mu g}$ in $50 \mu \mathrm{l}$ in $\mathrm{H}_{2} \mathrm{O}$ plus $0.1 \%$ TFA, at $0.5 \mathrm{ml} / \mathrm{min}$. A linear gradient from $95 \% \mathrm{H}_{2} \mathrm{O} / 5 \%$ acefonitrile $105 \% \mathrm{H}_{2} \mathrm{O} / 95 \%$ acetonitrile was run from 0.2 to 20.2 minutes. Absorbence was at $280 \mathrm{~nm}$, 0.05 units full scale. Retention time in minutes.

(XBL 865-1896) (XBL 865-1899) with $5 \%$ and $21 \%$ of the total protein mass, respectively.

The results presented above indicate that electromagnetic fields operate to influence the protein composition of erythrocyte. This phenomenon of protein shedding is observed to occur over a restricted temperature range of $17-21^{\circ} \mathrm{C}$ which corresponds with the presence of a membrane phase transition. It is likely that conformational changes associated with the phase transition, e.g., membrane phospholipid chain and head-group reorientation and protein vertical extrusion away from the plane of the cell riembrane, may significantly increase the absorption of electromagnetic energy in the erythrocyte membrane and thereby facilitate the release of protein. ${ }^{1,2,3}$ This is supported by recent measurements of energy absorption in unilamellar liposome vesicles that demonstrate that electromagnetic energy absorption is markedly increased at the membrane phase transition. ${ }^{5,6}$ The characterization of physical changes that we observe during field treatment involve the shedding of at least 11 protein species, which takes place in a temperature- and oxygen-dependent manner, and the release of 28-31 kDa species that are stabilized by divalent calcium bridges. Field treatment is also associated with the shedding of major protein components that possess relatively cationic and/or nonpolar domains.

\section{REFERENCES}

1. Liburdy, R.P., and Vanek, P.F., Jr. Microwaves and the cell membrane. II. Temperature, plasma, and oxygen mediate microwaveinduced membrane permeability in the erythrocyte. Radiation Research 102, 190-205 (1985).

2. Liburdy, R.P., and Magin, R.L. Microwavestimulated drug release from liposomes. Radiation Research 103, 266-275 (1985).

3. Liburdy, R.P., and Vanek, P.F., Ir. Microwaves and the cell rnembrane. III. Protein shedding is oxygen dependent and temperature dependent. Evidence for cation bridge involvement. Radiation Research, in press.

4. Liburdy, R.P., Burgess, N.L., and Mindich, B.P. Quantitative HPLC analyses of human plaque proteins expressed in coronary and thoracic aorta arteries(ABS). Tenth International Symposium on Column Liquid Chromatography, San Francisco, CA, May 1986.

5. Liburdy, R.P., Magin, R.L., Niesman, A., Surowiec, and Stuckly, S. Measurements of 
the dielectric properties of liposome vesicles at the membrane phase transition (ABS). Eighth Annual Meeting of the Bioelectromagnetics Society, Madison, Wl, June 1986.
6. Liburdy, R.P., and Tenforde, T.S. Membrane responses to magnetic and electromagnetic fields. In Biophysical Effects of Static Magnetic Fields, 44-53, Springer-Verlag, NY, 1986.

\title{
INITIAL DISTRIBUTION OF NEPTUNIUM-237 IN A MONKEY
}

\author{
Patricia W. Durbin, Nylan leung, and lerome I. Bucher*
}

Long-lived ${ }^{237} \mathrm{~Np}\left(\mathrm{~T}_{1 / 2}=2.14 \times 10^{6}\right.$ years) may be a significant contributor to the biological hazard of very old fission wastes. 1.2 Thermodynamically, the stable state of $\mathrm{Np}$ under the mild conditions of $\mathrm{pH}$ (3.5 to 8.5 ) and $\mathrm{eH}(0.5$ to $-0.5 \mathrm{~V}$ ) in ground and surface waters ${ }^{3}$ is expected to be the dioxo cation, $\mathrm{NpO}_{2}^{+}$. Because $\mathrm{NpO}_{2}^{+}$forms soluble compounds and weak complexes with the abundant anions in soils and natural waters, ${ }^{4}$ and does not sorb strongly to mineral or sediment surfaces ${ }^{5}$ except those with high carbonate content, ${ }^{6}$ it has been assumed that $\mathrm{NpO}_{2}^{+}$will eventually migrate from high level waste disposal sites ${ }^{2,5}$ and enter human food chains. ${ }^{7}$

Neptunium was the first transuranic element to be investigated in an animal; the initial studies of ${ }^{239} \mathrm{~Np}$ distribution in rats were completed in $1943 .^{8}$ In the intervening years no kinetic studies of $\mathrm{Np}$ have been performed in animals larger than rats, except to measure gastrointestiral absorption. ${ }^{10.11}$ Technical difficulties in detection of the sparse alpha emissions of low-specific activity ${ }^{237} \mathrm{~Np}$ and the short half-lives or poor availability of other $\mathrm{Np}$ isotopes led in many instances to administration of 0.5 to $15 \mathrm{mg} / \mathrm{kg}$ of ${ }^{237} \mathrm{~Np},{ }^{9,12}$ amounts far greater than would ever be taken into the body in contaminated food or water. Furthermore, most of those experiments are confounded by slow absorption from injection sites, in vivo colloid formation, or chemical toxicity.

In the earlier studies, the oxidation state of $\mathrm{Np}$ in the administered solutions was uncertain. When citrate buffers were present, it is likely that instead of $\mathrm{NpO}_{2}^{+}$, the $\mathrm{Np}$ was partly or wholly $\mathrm{Np}$ (IV), which would be expected to behave like Pu(IV). Strong oxidants like $\mathrm{Cl}_{2}$ oxidize $\mathrm{NpO}_{2}^{+}$to $\mathrm{NpO}_{2}^{+2}, 10$ the closest analogue of uranyl ion. In the presence of reductants like $\mathrm{Fe}^{+2} 11$ and strongly binding chelating agents, $\mathrm{NpO}_{2}^{+}$can be reduced and stabil-

\footnotetext{
- Materials and Molectilar Research Division, LBL.
}

ized as $\mathrm{Np}(\mathrm{IV}),{ }^{11}$ the closest analogue of $\mathrm{Pu}(\mathrm{IV})$. Citrate ion, an abundant ligand in mammalian body fluids, is a special case, because not only does its great affinity for actinides in the IV state facilitate reduction of $\mathrm{NpO}_{2}^{+}$, it also acts as a reducing agent, and by itself can both promote reduction of $\mathrm{NpO}_{2}^{+}$ to $\mathrm{Np}$ (IV) and stabilize it. ${ }^{13}$ At $\mathrm{pH} 7.4$ and the mild redox conditions of mammalian body fluids, ${ }^{14}$ administered $\mathrm{NpO}_{2}^{+2}$ is likely to be rapidly reduced to $\mathrm{NpO}_{2}^{+}$, and the important strongly binding biological ligands, like citrate ion, transferrin, and possibly phosphates, may facilitate at least partial reduction of $\mathrm{NpO}_{2}^{+}$and stabilize it in some tissues as $\mathrm{Np}$ (IV). In the absence of adequate data for $\mathrm{Np}$, the International Commission on Radiological Protection (ICRP) recommends that the general actinide metabolic model, developed largely from biological data for Pu(IV), be applied to Np regardless of its stable state in the body. ${ }^{15}$

This pilot study of the initial distribution of ${ }^{237} \mathrm{~Np}(\mathrm{~V}, \mathrm{IV})$ in an adult Macaque monkey was undertaken 1) to begin upgrading the biological data for Np using an animal phylogenetically close to man, 2) to demonstrate that it was possible to measure small amounts of ${ }^{237} \mathrm{~Np}$ in biological samples accurately by means of its photon emissions, 3) to provide detailed initial distribution data in soft tissues and within the skeleton for an actinide element with a stable (V) state, for comparison with similar data we have obtained for ${ }^{90} \mathrm{Sr}(I I),{ }^{241} \mathrm{Am}$ (III), ${ }^{238} \mathrm{Pu}(\mathrm{IV})$, and ${ }^{233} \mathrm{U}(\mathrm{IV})$ in adult Macaques.

\section{METHODS}

An 11-year-old male cynomolgus monkey (Macaca fasicularis) weighing $4.25 \mathrm{~kg}$ was given ${ }^{237} \mathrm{~Np} \mid 43 \mu \mathrm{g} / \mathrm{kg}, 0.031 \mu \mathrm{Ci}(1122 \mathrm{~Bq})$ per $\mathrm{kg} \mid$ by intramuscular injection in the right thigh. The ${ }^{237} \mathrm{~Np}$ (in equilibrium with its ${ }^{233} \mathrm{~Pa}$ daughter) was received as $\mathrm{NpO}_{2} \mathrm{Cl}$ in $0.01 \mathrm{M} \mathrm{HCl}$, but just before the injection, it was diluted with $0.08 \mathrm{M}$ citrate 
buffer $(\mathrm{pH} 3.5)$ and forced through a $0.22-\mu \mathrm{m}$ Millipore filter. Thus, some of the Np may have been reduced to $N p(I V),{ }^{13}$ and the possible presence of a mixture of oxidation states is acknowledged by the designation $V_{p}(V, I V)$. Separated excreta were collected daily, and the monkey was killed by exsanguination at 4 days. All soft tissues, the skin, muscle and bones of the injected and uninjected legs (which were managed as separate samples) and the cleanly defleshed skeletal parts (subdivided as described earlier) ${ }^{16}$ were weighed, dried, and dryashed at $600^{\circ} \mathrm{C}$. Excreta and skeletal parts were reweighed after ashing. The ash of small soft tissues was dissolved in acid and evaporated on tared glass planchets for alpha measurement. Large soft tissues, excreta, and bone samples were dissolved in minimal amounts of dilute acid and transferred to 60-ml screw-cap plastic bottles for detection of photons. Photons were detected in the 16-, 30-, and $86-\mathrm{keV}$ peaks with a pair of large diameter, thin Nal crystals mounted coaxially in a lead box. Appropriate self-absorption corrections were applied to measurements of the alpha particles and the two lower energy photons. Those procedures have been described in detail. ${ }^{16}$ Data from the three photon measurements agreed closely with one another when reduced to percent injected activity per sample, and the results are reported as means of the three measurements. The results shown in the tables are expressed as percent of injected dosage (\% ID) normalized to $100 \%$ absorption and $100 \%$ material recovery.

\section{RESULTS}

During the interval in which the samples were being counted, 21 to 40 days after the injection, the net count rate in the higher energy region $(60$ to $100 \mathrm{keV}$, dominated by the emissions of ${ }^{233} \mathrm{~Pa}$ ) was increasing by about $4 \%$ per day, and at 8 months after the injection the net count rate in that region was 2.7 times what it had been on the day of injection. It appeats that, fortuitously, increasing the $\mathrm{pH}$ by dilution in the citrate buffer caused most of the ${ }^{233} \mathrm{~Pa}$ in the stock ${ }^{237} \mathrm{~Np}$ solution to be held back on the surfaces of the injection-solution bottle, the Millipore filter, and the syringes and needles used for filtering and injecting. Thus, the animal probably received little if any ${ }^{233} \mathrm{~Pa}$, and the short 4-day interval between injection and sac:ifice would have allowed little in vivo ingrowth of ${ }^{133} \mathrm{~Pa}$ that would have been metabolized independently. There are three pieces of evidence suggesting that the soluble ${ }^{237} \mathrm{~Np}$ had been effectively separated from the readily hydrolyzable ${ }^{233} \mathrm{~Pa}$. If a significant amount of ${ }^{233} \mathrm{~Pa}$ had been injected 1) there would have been a measureable excess of counts, especially in the $86-\mathrm{keV}$ region, in the samples from the injected leg, because intramuscularly injected $\mathrm{Pa}$ is poorly absorbed ${ }^{8}$; 2) the counts in the three photon peaks would not have yielded the same results (\% ID/sample); 3) there would have been less ingrowth of photon activity in the higher energy region, if a significant amount of ${ }^{233} \mathrm{~Pa}$ had been present in the solution injected into the animal and used to prepare the counting standards. We are reasonably confident that these results represent the behavior of ${ }^{237} \mathrm{~Np}$ and not a mixture of $\mathrm{Np}$ and $\mathrm{Pa}$.

Detailed results are shown in Fig. 1 (excreta), Table 1 (soft tissues) and Table 2 (bones). Material recovery was $96.2 \%$ ID. Absorption was nearly complete in 4 days; only $0.72 \%$ ID remained at the injection site.

Blood was sampled only at autopsy. At that time, there was about $0.8 \%$ ID in plasma and $0.25 \%$ associated with red cells. The Np content of monkey plasma at 4 days was about one-half that obtained for Pu $\{1.6 \%$ ID (Ref. 16) $\}$ and nine

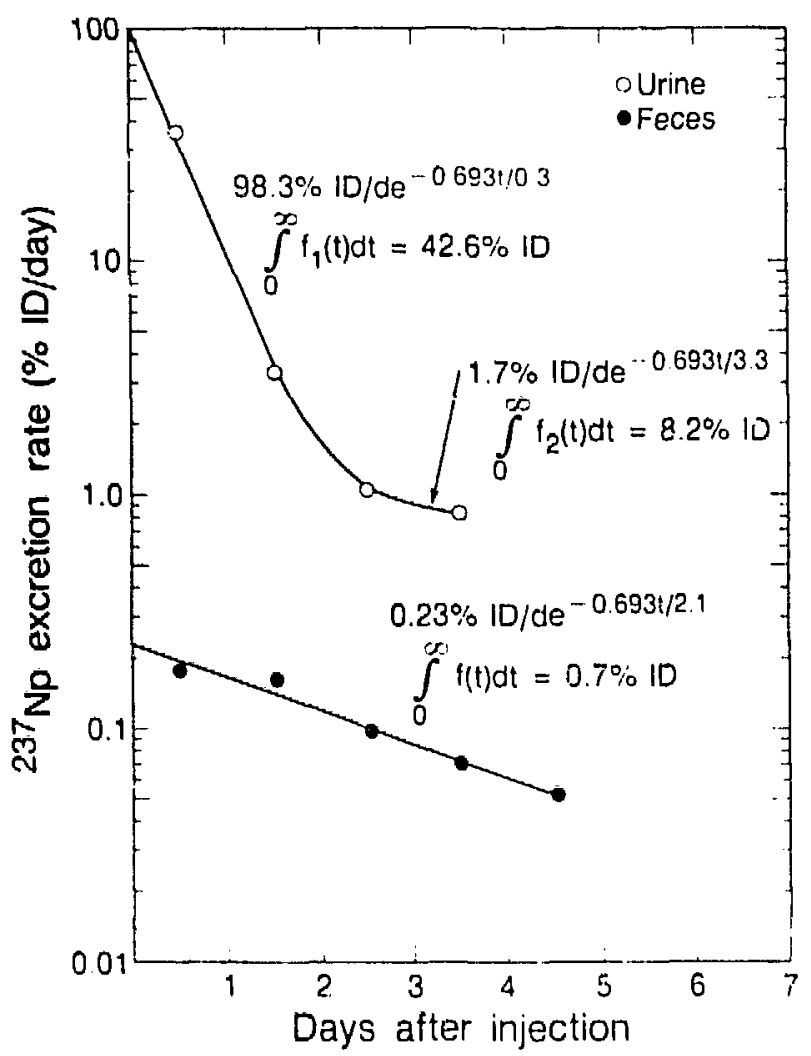

Fig. 1. Excretion of intramuscularly injected "Np(V,IV) by an adult male cynomolgus monkey. 
Table 1. Distribution and concentration of ${ }^{237} \mathrm{~Np}(\mathrm{~V}, \mathrm{IV})$ in soft tissues

of an adult male cynomolgus monkey at 4 days after intramuscular injection.

\begin{tabular}{|c|c|c|c|c|c|}
\hline Tissue & $\begin{array}{l}\text { Content } \\
\text { (\% ID) }\end{array}$ & $\begin{array}{l}\text { Concentration } \\
(\% 10 / 8 \text { wet) }\end{array}$ & Tissue & $\begin{array}{l}\text { Content } \\
\text { (\% ID) }\end{array}$ & $\begin{array}{l}\text { Concentration } \\
\text { (\% ID/g wet) }\end{array}$ \\
\hline Liver & 8.94 & 0.124 & Salivary gland & 0.025 & 0.0028 \\
\hline Kidney & 1.20 & 0.065 & Adrenal & 0.0018 & 0.0027 \\
\hline Trachea & 0.088 & 0.046 & Lymph node & -- & 0.0027 \\
\hline Aorta & 0.034 & 0.020 & Seminal vesicle & 0.014 & 0.0026 \\
\hline Pituitary & 0.0007 & 0.012 & Urinary bladder & 0.016 & 0.0025 \\
\hline Larynx & 0.033 & 0.011 & Mesentery ${ }^{b}$ & 0.10 & 0.0021 \\
\hline Gall bladder & 0.0053 & 0.0078 & Gastrointestinal $\operatorname{tract}^{\mathrm{c}}$ & 0.19 & 0.0020 \\
\hline Spleen & 0.075 & 0.0067 & Sweat gland & 0.0084 & 0.0016 \\
\hline Bile & $\cdots$ & 0.0065 & Thyroid & 0.001 & 0.0015 \\
\hline Lung & 0.11 & 0.0060 & Epididymis & 0.0066 & 0.0014 \\
\hline Plasma & 0.81 & 0.0051 & Pelt & 1.17 & 0.0013 \\
\hline Vas deferens & 0.0024 & 0.0047 & Eyes & 0.0072 & 0.0012 \\
\hline Pancreas & 0.013 & 0.0033 & Striated muscle ${ }^{e}$ & 2.03 & 0.0012 \\
\hline Prostale & 0.012 & 0.0031 & Testis & 0.013 & 0.00050 \\
\hline Red cells & 0.25 & 0.0031 & Brain & 0.013 & 0.00024 \\
\hline Heart & 0.047 & 0.0030 & & & \\
\hline
\end{tabular}

- For Macaca, plasma mass $(\mathrm{g})=37.4 \mathrm{~g} / \mathrm{kg}$ body weight; red cells mass $(\mathrm{g})=19.4 \mathrm{~g} / \mathrm{kg}$ body weight. Body weight $=4.25 \mathrm{~kg}$.

b Weight of mesentery $=$ fresh weight minus weight of fat decanted after drying at $100^{\circ} \mathrm{C}$.

- Contents removed from Gl tract.

Includes ears and ischial callosities.

- All soft tissue removed during dissection of skeleton plus tongue and diaphragm.

times that for Am $\mid 0.09 \%$ ID (Ref. 17)| The Np content of red cells was about $40 \%$ of the value expected, if the Np concentration in red-cell water were in equilibrium with plasma, and the red cell membrane were freely permeable to the circulating Np complexes.

In 4 days, 39.6\% of the injected $\mathrm{Np}$ was excreted. Of that, 98.6\% was renal excretion, and $86.4 \%$ of the renal excretion of $\mathrm{Np}$ occurred in the first 24 hours. The first phase $\left(T_{1 / 2}=0.3\right.$ days), which probably represents filtration of a soluble $\mathrm{Np}$ complex by analogy to $\mathrm{UO}_{2}{ }^{2+}$, most likely $\mathrm{NpO}_{2} \mathrm{CO}_{3}^{-}$(Ref, 18)] from plasma, is in keeping with the rapid absorption from the injection site. The second phase ( $T_{y_{2}}=3.3$ days, defined by only two data points), presumably represents feedback from extracellular fluid (ECF). Prompt fecal excretion of $\mathrm{Np}$ was negligible, suggesting impeded passive transfer from plasma into digestive tract secretions. The Np concentration in bile at 4 days $(0.0065 \%$ ID/g) suggests that some biliary excretion of $\mathrm{Np}$ can be expected.

The data for $N p$ in soft tissues and internal fluids are arranged in Table 1 in descending order of $\mathrm{Np}$ concentration ( $\%$ ID/g wet weight). Tissues with $\mathrm{Np}$ concentrations greater than plasma were excretory organs (liver, gall bladder, kidneys), organs with large complements of reticuloendothelial (RE) cells (spleen, pituitary), tissues that are to some degree mineralized (trachea, larynx, lung), and specialized blood vessels (aorta). In the bulk of soft tissue, the $\mathrm{Np}$ concentration was less than that of plasma. The $\mathrm{Np}$ concentration in testis, only $10 \%$ of that in plasma, was among the lowest-only brain was lower. The $\mathrm{Np}$ content of monkey testis $(0.013 \%$ ID) is about one-fourth of the initial $\mathrm{Pu}$ or $\mathrm{Am}$ concentration in monkey testis |0.06\% ID (Ref. 16)|.

The distribution and concentration of ${ }^{237} \mathrm{~Np}(\mathrm{~V}, \mathrm{IV})$ in structurally distinctive monkey bone parts are shown in Table 2. Similarly to all boneseeking cations, the greatest initial $\mathrm{Np}$ concentrations were in specimens comprised mainly of fine cancellous bone and red marrow (large s/v ratio, small ash fraction), and the lowest were in specimens comprised mainly of compact bone (small $5 / \mathrm{v}$ ratio, large ash fraction), reflecting the fact that the first point of contact with bone is the mineral 
Table 2. Distribution and concentration of ${ }^{237} \mathrm{~Np}(\mathrm{~V}, \mathrm{IV})$ in mineralized tissues of an adult male Cynomolgus monkey at 4 days and after intramuscular injection.

\begin{tabular}{|c|c|c|c|c|}
\hline & $\begin{array}{l}\text { Content } \\
(\% \text { ID) }\end{array}$ & $\begin{array}{l}\text { Concentration } \\
\text { (\% ID/g ash) }\end{array}$ & $\begin{array}{c}\text { Relative } \\
\text { concentration }\end{array}$ & $\begin{array}{c}\text { Ash Fraciion } \\
(\%)^{b}\end{array}$ \\
\hline Temporal bones & 1.81 & 0.21 & 0.82 & 33.5 \\
\hline Zygomatic arches & 0.40 & 0.30 & 1.15 & 35.9 \\
\hline Mandible & 1.96 & 0.18 & 0.69 & 44.4 \\
\hline Scapulae-head & 0.74 & 0.34 & 1.30 & 37.1 \\
\hline superior border & 0.45 & 0.31 & 1.20 & 37.2 \\
\hline spine & 0.46 & 0.20 & 0.77 & 46.7 \\
\hline Clavicles-shaft & 0.092 & 0.12 & 0.45 & 47.0 \\
\hline sternal end & 0.090 & 0.75 & 2.90 & 15.4 \\
\hline acromial end & 0.060 & 0.60 & 2.32 & 19.2 \\
\hline Sternum & 0.67 & 1.06 & 4.09 & 14.5 \\
\hline Ribs - sternal third & 0.76 & 0.46 & 1.79 & 29.3 \\
\hline - central third & 0.67 & 0.28 & 1.10 & 37.8 \\
\hline - spinal third & 1.31 & 0.44 & 1.68 & 29.4 \\
\hline - LR-5 (whole) & 0.27 & 0.36 & 1.30 & 32.3 \\
\hline Cervical vert. - arches & 0.49 & 0.28 & 1.08 & 29.8 \\
\hline - bodies & 0.43 & 0.57 & 2.20 & 31.8 \\
\hline Thoracic vert. - arches & 1.18 & 0.32 & 1.23 & 31.0 \\
\hline - bodies & 3.04 & 1.06 & 4.08 & 22.4 \\
\hline Lumbar vert. - arches & 1.19 & 0.19 & 0.71 & 33.8 \\
\hline - bodies & 5.76 & 0.88 & 3.40 & 22,0 \\
\hline Sacrum & 1.71 & 0.56 & 2.16 & 22.5 \\
\hline Pelvis-iliac crest & 0.34 & 0.46 & 1.76 & 22.3 \\
\hline sacro-ilium & 0.93 & 0.29 & 1.10 & 40.3 \\
\hline ilium remainder & 0.23 & 0.15 & 0.59 & 49.0 \\
\hline acetabulum & 1.29 & 0.29 & 1.13 & 37.3 \\
\hline ishium & 0.68 & 0.27 & 1.04 & 27.3 \\
\hline pubis & 0.30 & 0.35 & 1.36 & 28.6 \\
\hline Humeri-shaft & 0.66 & 0.075 & 0.29 & 50.7 \\
\hline proximal end & 1.34 & 0.66 & 2.54 & 28.8 \\
\hline distal end & 0.45 & 0.24 & 0.92 & 378 \\
\hline Radii-shafit & 0.23 & 0.051 & 0.20 & 49.2 \\
\hline proximal end & 0.13 & 0.37 & 1.44 & 30.4 \\
\hline distal end & 0.14 & 0.21 & 0.82 & 26.7 \\
\hline Ulnae-shaft & 0.38 & 0.080 & 0.31 & 50.0 \\
\hline proximal end & 0.38 & 0.24 & 0.92 & 36.5 \\
\hline distal end & 0.047 & 0.21 & 0.82 & 27.1 \\
\hline Carpals & 0.21 & 0.18 & 0.68 & 24.4 \\
\hline Melacarpals & 0.24 & 0.12 & 0.48 & 29.0 \\
\hline Hand phalanges & 0.23 & 0.12 & 0.48 & 27.1 \\
\hline
\end{tabular}


Table 2. (continued)

\begin{tabular}{lllll}
\hline \hline & $\begin{array}{c}\text { Content } \\
(\% \text { ID })\end{array}$ & $\begin{array}{c}\text { Contentration } \\
\text { (\% ID/g ash) }\end{array}$ & $\begin{array}{c}\text { Relative } \\
\text { concentration }\end{array}$ & $\begin{array}{c}\text { Ash Fraction } \\
(\%)^{\mathrm{b}}\end{array}$ \\
\hline Femora-shaft & 0.42 & 0.044 & 0.17 & 50.4 \\
proximal end & 0.99 & 0.26 & 1.02 & 34.8 \\
distal end & 0.71 & 0.32 & 1.24 & 28.6 \\
Tibiae-shaft & 0.35 & 0.050 & 0.20 & 46.4 \\
proximal end & 0.40 & 0.23 & 0.65 & 27.0 \\
distal end & 0.15 & 0.15 & 0.57 & 31.7 \\
Fibulae-shaft & 0.086 & 0.045 & 0.17 & 52.4 \\
ends & 0.12 & 0.25 & 0.95 & 25.3 \\
Patellae \& fabellae & 0.10 & 0.20 & 0.77 & 28.0 \\
Tarsals & 0.53 & 0.15 & 0.58 & 30.5 \\
Metatarsals & 0.48 & 0.15 & 0.60 & 32.2 \\
Foot phalanges & 0.28 & 0.16 & 0.63 & 25.6 \\
Hyoid bone & 0.053 & 0.59 & 2.25 & 8.6 \\
Costal cartilages & 0.10 & 0.08 & 0.31 & 17.8 \\
Teeth & 0.33 & 0.048 & 0.18 & 65.0 \\
Calvaria & 0.99 & 0.16 & 0.62 & 53.9 \\
Facial bones & 4.39 & 0.32 & 1.21 & 31.5 \\
\hline \hline
\end{tabular}

- Relative concentration $=(\% \mathrm{ID} / \mathrm{B}$ ash, sample $) /(\% 10 / 8$ ash, skeleton).

Ash fraction $(\%)=\mid$ ash weight $(g) \times 100) /$ fresh weight $(g) \mid$.

surface-fluid interface. For adult Macaques, specific surfaces of compact and fine cancellous bone are 26 and $194 \mathrm{~cm}^{2} / \mathrm{g}$ ash, respectively ${ }^{19}$ and a compact/cancellous concentration ratio of $C R=26 / 194=0.13$ implies a uniform distribution on all anatomical bone surfaces.

\section{COMPARISON WITH OTHER BONE SFEKERS}

Data sets for the same 53 monkey bone parts were available for ${ }^{238} \mathrm{Pu}(\mathrm{IV}),{ }^{241} \mathrm{Am}$ (III) and ${ }^{90} \mathrm{Sr}(\mathrm{II})$. Normalized nuclide concentrations (footnote $e$, Table 3 ) and relative concentrations (footnote a, Table 2) in those bone parts were calculated. Table 3 contains the mean $\pm S . D$. for 1) each entire set of normalized nuclide concentrations (not mass weighted), 2) the relative concentrations (mass weighted) in typical compact bone (long bone shafts) and typical fine cancellous bone in red marrow (sterrum, thoracic and lumbar vertebral bodies), and 3 ) the relative concentrations in the major segments of the skeleton.
The degree of irregularity of nuclide distribution on bone surfaces can be estimated from the sets of normalized and relative nuclide concentrations. The normalized nuclide concentrations in fine cancellous bone in red marrow were in the proportion, Pu:Np:Am::1.0:0.47:0.59, indicating the tendency of $\mathrm{Np}$ to deposit on those surfaces at about the same intensity as Am, but less than that of $\mathrm{Pu}$. In compact bone, the normalized concentrations were in the proportion, Pu:Np:Am::1.0:1.25:2.3, indicating that $\mathrm{Np}$ deposition on those surfaces is more restricted than that of $\mathrm{Am}$, but less so than $\mathrm{Pu}$. The mean normalized $\mathrm{Np}$ concentration (not mass weighted) for 53 skeletal parts was $0.14 \pm 0.10 \% \mathrm{ID} / \mathrm{g}$ ash. The dispersion of the $\mathrm{Np}$ concentrations about the mean was S.D. $=76 \%$, which was close to that for Am (78\%), somewhat greater than for $\mathrm{Sr}(60 \%)$, and much less than for $\mathrm{Pu}(133 \%)$. The compact/cancellous concentration ratios (long bone shafts)/(sternum and vertebral bodies)| for $\mathrm{Pu}, \mathrm{Np}$ and $\mathrm{Am}$ were $0.026,0.06$ and 
Table 3. Gross initial intraskeletal distribution parameters of parenterally injected " $\left.N P(V, I V),{ }^{28} \mathrm{Pu}(I V),{ }^{241} \mathrm{Am}, i l l\right)$ and ${ }^{11} \mathrm{Sr}(I I)$ in adult monkeys.

\begin{tabular}{|c|c|c|c|c|}
\hline Nuclide & ${ }^{218} \mathrm{Pu}(\mathrm{IV})$ & ${ }^{237} N p(V, I V)^{2}$ & ${ }^{241} \mathrm{Am}(I 11)$ & ${ }^{\text {T) }} \mathrm{Sr}(111)$ \\
\hline Monkey no. & C145M & C143M & $\mathrm{C} 155 \mathrm{~F}$ & R161F \\
\hline Days after injection & 7 & 4 & 8 & 8 \\
\hline Skeleton', wet (g) & 664 & 462 & 435 & 670 \\
\hline $\operatorname{ash}(\mathrm{g})$ & 236 & 161 & 161 & 265 \\
\hline mean conc. (1//1 $10 / 8$ ash $)$ & 0.075 & 0.26 & 0.13 & 0.053 \\
\hline \multicolumn{5}{|l|}{ Relative concentrations } \\
\hline$\Sigma$ long bone shafts ${ }^{r}$ & 0.17 & 0.22 & 0.40 & 0.52 \\
\hline I sternum, TV \& LV bodies & 6.52 & 3.64 & 3.84 & 2.23 \\
\hline \multicolumn{5}{|l|}{ Ratio of relative concentrations ${ }^{c}$} \\
\hline ( $\Sigma$ LBS/ $\Sigma$ si, TVB, LBV) & 0.026 & 0.060 & 0.104 & 0.23 \\
\hline $\begin{array}{l}\text { Concentrations in skeletal parts }{ }^{\text {d.e }} \\
\qquad(\% 10 / 8 \text { ash), mean } \pm \text { S.D. }\end{array}$ & $0.15 \pm 0.20$ & $0.14 \pm 1.10$ & $0.13 \pm 0.10$ & $0.15 \pm 0.09$ \\
\hline \multicolumn{5}{|c|}{ Comparison with ${ }^{237} \mathrm{NP}(\mathrm{V}, \mathrm{IV})^{\mathrm{d}}$} \\
\hline $\begin{array}{l}\text { Ratios of relative concentrations } \\
\qquad(\mathrm{Np} / \text { nuclide), inean } \pm \mathrm{S} . \mathrm{D}\end{array}$ & $1.31 \pm 0.48$ & $\cdots$ & $1.06 \pm 0.40$ & $0.92 \pm 0.39$ \\
\hline $\begin{array}{l}\text { Relative concentration }{ }^{c} \text { differences } \\
\qquad \text { (Np - nuclide), mean } \pm S . D \text {. }\end{array}$ & $0.51 \pm 0.82$ & $\cdots$ & $0.31 \pm 0.29$ & $0.48 \pm 0.51$ \\
\hline \multicolumn{5}{|c|}{ Relative nuclide concentrations in major skeletal segments ${ }^{c}$} \\
\hline Head & 0.57 & 0.90 & 0.91 & 0.81 \\
\hline Thorax & 1.59 & 1.40 & 1.56 & 1.23 \\
\hline Spine & 2.91 & 2.12 & 1.91 & 1.46 \\
\hline Pelvis & 0.96 & 1.09 & 1.06 & 1.47 \\
\hline Upper limbs & 0.48 & 0.57 & 0.61 & 0.76 \\
\hline Lower limbs & 0.32 & 0.49 & 0.50 & 0.81 \\
\hline
\end{tabular}

- Nuclides were given intramuscularly in $0.08 \mathrm{M}$ Na citrate buffer at $\mathrm{pH} 3.5$. Because $K_{1}$ for $\mathrm{Np}(\mathrm{IV})-\mathrm{cit}>\mathrm{K}_{f}$ for $\mathrm{Np}(\mathrm{V})-\mathrm{cit}$ and the mole ratio was large, (citrate)/(Np) $-4.5 \times 10^{5}$. dilution of $\mathrm{Np}(\mathrm{V})$ in the citrate buffer may have caused partial reduction to $\mathrm{Np}(\mathrm{IV}){ }^{13}$

b Skeleton does not include teeth, tail, hyoid bone or costal cartilages.

- See footnote a Table 2.

d Comparisons available for 53 skeletal parts.

- Concentrations in bone ash are normalized to the averages for four nuclides - $23.6 \%$ ID in skeleton and total skeletal ash $=206 \mathrm{~g}$.

0.104, respectively. If all of those nuclides are initially restricted to bone surfaces, then one infers that Am labels bone surfaces almost uniformly (the Am deposit on fine cancellous surfaces is somewhat more intense than on compact bone surfaces), while $N p$ deposition on those cancellous surfaces is about twice as intense and that for $\mathrm{Pu}$ is about 4.5 times as intense as on compact surfaces.

Sample-by-sample comparisons of the ratios and absolute differences in the relative concentrations for the 53 bone parts between $\mathrm{Np}$ and the other three nuclides (Table 3) indicated a better match of the $\mathrm{Np}$ bone data to $\mathrm{Am}$ and $\mathrm{Sr}$ than to Pu. 
The gross initial distribution and excretion in adult monkeys of ${ }^{237} \mathrm{~Np}(\mathrm{~V}, \mathrm{IV})$ is compared in Table 4 to four other bone-seeking radioelements with different principal oxidation states. Urinary excretion of these elements with increased binding to serum pioteins decreased from $\mathrm{UO}_{2}{ }^{2+}(86.6 \%$ ID excreted), which forms a filterable biscarbonate complex in plasma, ${ }^{18}$ to $\mathrm{Pu}$ (IV) $(2.1 \%$ ID excreted), which circulates almost entirely as a nonfilterable transferrin complex. ${ }^{16}$ The rate at which these elements were absorbed from an i.m. injection site decreased with increasing tendency to hydrolyze from $\mathrm{Sr}$ (II) (completely absorbed in 8 days) to $\mathrm{Pu}$ (IV) (5\% ID unabsorbed in 8 days). Deposition in liver, ranging from $0.64 \%$ ID for $U(I V)$ to $62.2 \%$ ID for $\mathrm{Pu}(\mathrm{IV})$, is in roughly the same order as the stability of the complexes of these metals with organic ligands. Bone deposition, which is the net result of competition with liver deposition and excretion, cannot yet be identified with one chemical property. The chemical reactions of $\operatorname{Sr}(\mathrm{II})$ and $\mathrm{UO}_{2}{ }^{+2}$ with bone crystals are well established, ${ }^{20}$ but in spite of considerable work and even more speculation, the fundamental reactions of Am(IiI) and $\mathrm{Pu}(\mathrm{IV})$ with the mineral and/or organic constituents of bone remain unsettled. While the specific chemical reactions of $\mathrm{Np}$ as $\mathrm{NpO}_{2}^{+}$or $\mathrm{Np}$ (IV) with the bone constituents are not known, we believe that the available data support the following working hypothesis: Actinides in high valence states have a great affinity for coordination to oxoanions, e.g., hydroxide, carbonate, phosphate and organic acids, and hydroxy acids. The effective charge of $\mathrm{NpO}_{2}^{+}$is about 1.5 , and its linear structure, $0-\mathrm{M}-0$, is the same as $\mathrm{UO}_{2}{ }^{+2}{ }^{4}$ Therefore, we expect that the initial reaction of $\mathrm{NpO}_{2}^{+}$in bone will resemble $\mathrm{UO}_{2}^{+2}$-ion exchange at crystal surfaces with displacement of $\mathrm{Ca}^{+2}$ and immobilization of $\mathrm{PO}_{4}{ }^{-3} \cdot{ }^{20}$ However, the large fractional deposit of $\mathrm{NpO}_{2}^{+}$in the skeleton [compared to $\mathrm{UO}_{2}^{+2}$ and $\mathrm{Sr}(\mathrm{II})$ and its failure to be released promptly la large fraction of the initial bone deposit of $\mathrm{UO}_{2}^{+2}$ or $\mathrm{Sr}$ (II) if promptly released by reverse exchange| suggest that in the presence of a local excess of organic acids (citrate, lactate) ${ }^{20}$ and a strongly binding anion (phosphate), the $\mathrm{NpO}_{2}^{+}$that is deposited on bone surfaces is rapidly reduced in situ and immobilized. In that case, Np, like Pu(IV), would only be released by structural remodeling, but the possibility remains that a fraction of $N p$ (IV) released by remodeling and returned to the well-oxygenated central circulation may be reoxidized to $\mathrm{NpO}_{2}^{+}$and excreted before being redeposited in bone.

Original data from two early studies of Np distribution in rats were obtained from the Crocker Laboratory archives., ${ }^{8,21}$ They were recalculated, and appropriate corrections were applied for absorption from the i.m. injection site and for selfabsorption of the alpha particles of ${ }^{237} \mathrm{~Np}$. The gross distributions of absorbed $N p$ in rats and the monkey are compared in Table 5. Entire skeletons were measured, and material recoveries approached

Table 4. Inirial partitioning of some bone-seeking radionuclides in tissues and excreta of adult Macaques.

\begin{tabular}{|c|c|c|c|c|c|}
\hline \multicolumn{6}{|c|}{ Nuclide distribution ( $\%$ absorbed dosage) } \\
\hline Nuclide & ${ }^{23)} \mathrm{U}(\mathrm{VI})$ & ${ }^{90} \mathrm{Sr}(\mathrm{II})^{4}$ & ${ }^{237} \mathrm{~Np}(\mathrm{~V}, \mathrm{YV})$ & ${ }^{241} \mathrm{Am}(I I I)^{19}$ & ${ }^{230} \mathrm{Pu}(\mathrm{IV})^{1 \mathrm{~h}}$ \\
\hline Dosage $(\mu \mathrm{g} / \mathrm{kg})$ & 37 & Tracer & 42 & 0.087 & 0.017 \\
\hline Days after injection & 3 & 8 & 4 & 8 & 7.5 \\
\hline No. of animals & 1 & 1 & 1 & 3 & 7 \\
\hline Liver & 0.64 & & 8.9 & 53.6 & 62.6 \\
\hline Kidneys & 8.4 & 0.72 & 1.2 & 0.55 & 0.62 \\
\hline Other soft tissues & $\therefore$ & & 4.3 & 5.3 & 5.0 \\
\hline Bones and teeth & $<3.5^{\mathrm{b}}$ & 14.5 & 42.0 & 278 & 27.4 \\
\hline Urine & 86.6 & 72.3 & 40.7 & 9.6 & 2.1 \\
\hline Feces & 1.0 & 10.8 & 0.56 & 1.8 & 1.9 \\
\hline Injection site $(m / 4)|D|$ & $\therefore$ & -0 & 0.72 & 1.0 & 5.0 \\
\hline
\end{tabular}

- Unanalyzed carcass contains less than $3.5 \%$ ID.

Unnublished data 
Table 5. Initial distribution of $N p(V, I V)$ in al: adult male cynomolgus monkey compared to measurements in rats and the general actinide distribution model recommended by ICRP for Reference man. Values are expressed as percent of absorbed dosage normalized to $100 \%$ material recovery.

\begin{tabular}{lcccc}
\hline & \multicolumn{4}{c}{ \% Absorbed dosage } \\
\cline { 2 - 5 } & Monkey & & Rat & Reference Mand $^{\text {d }}$ \\
\hline Principal valence state & (V) & (IV) & (V) & $\ldots$ \\
Days after injection & $4^{\mathrm{a}}$ & $4^{\mathrm{b}}$ & $1^{\mathrm{c}}$ & $\mathrm{T}(0)$ \\
Bones and teeth & 43.7 & 52.6 & 51.9 & 45 \\
Liver & 9.3 & 4.1 & 6.8 & 45 \\
Non-liver tissues & 5.7 & 2.4 & 8.8 & 5 \\
Testes & 0.013 & 0.035 & --- & 0.05 \\
Excretion & 41.2 & 40.9 & 32.6 & 5 \\
\hline \hline
\end{tabular}

a ${ }^{237} \mathrm{~Np}(\mathrm{~V}, \mathrm{IV}), 43 \mu \mathrm{g} / \mathrm{kg}$; i.m. in $0.08 \mathrm{M} \mathrm{Na}$ citrate, $\mathrm{pH} 3.5$.

b ${ }^{239} \mathrm{~Np}$ (IV), tracer; i.m. in $0.08 \mathrm{M} \mathrm{NaCl}$ and $2 \times 10^{-4} \mathrm{M} \mathrm{Fe}(\mathrm{II})$, $\mathrm{pH} 2.6^{\mathrm{B}}$

c ${ }^{237} \mathrm{~Np}(\mathrm{~V}, \mathrm{IV}) 1 \mathrm{mg} / \mathrm{kg}$ i i.m. in $0.1 \mathrm{M} \mathrm{Na}$ citrate; females. ${ }^{21}$ :

d ICRP Pub. 30, part 2 (1980). ${ }^{15}$

$100 \%$ in all three studies. The two rat studies agree with each other, with the data for the monkey, and with the combined results of other comparable studies of $\mathrm{Np}$ in rats, ${ }^{9}$ regardless of the mass or the most likely oxidation state of the administered $\mathrm{Np}$. In each case 30 to $40 \%$ of the absorbed $\mathrm{Np}$ was promptly excreted in urine, about one-half of the absorbed $\mathrm{Np}$ was deposited in bone, and the liver $\mathrm{Np}$ content was less than $10 \%$. Combined, the three data sets suggest a preferred oxidation state (or mixture of oxidation states) for $\mathrm{Np}$ at the $\mathrm{pH}$ and $\mathrm{eH}$ of mammalian body fluids. This description of the initial distribution of $\mathrm{Np}$ differs substantially from the ICRP's geneial actinide model, in that the initial liver deposit of $\mathrm{Np}$ is about one-tenth and the early urinary excretion is about ten times the respective value recommended by ICRP. New human data support the description of $\mathrm{Np}$ distribution obtained from the animal studies. $^{22}$ At the low concentrations of $\mathrm{Np}$ and $\mathrm{Pu}$ in atmospheric fallout, $\mathrm{Np}$ was transported out of the lung much more rapidly than $\mathrm{Pu}$, and the $\mathrm{Np}$ content of liver was only $4 \%$ of the value predicted by $\mathrm{Pu}$ data. The low Np content of human liver suggests that the amount initially accumulated was small (compared to $\mathrm{Pu}$ ) and/or $\mathrm{Np}$ in human liver has a much shorter half-time than the 40 years incorporated in the ICRP general actinide model.
Two important pieces of information are needed to complete a realistic biokinetic model for $\mathrm{Np:}$ 1) there is no description of the timedependent changes in the microscopic distribution of the $\mathrm{Np}$, which was initially deposited nearly uniformly on bone surfaces, and 2) there is not even an estimate of the net half-time of the Np deposited in the skeleton. Clearly, unless all of the $\mathrm{Np}$ in the body at times longer than a few days after intake has been permanently reduced to $\mathrm{Np}(\mathrm{IV})$, the biological half-time of $\mathrm{Np}$ in bone will be considerably shorter than the 100 years recommended by ICRP.

\section{REFERENCES}

1. Pigford, T.H., and Ang, K.P. The plutonium fuel cycles. Health Phys. 29, 451-468 (1975).

2. Cohen, B.L. Effects of ICRP Publication 30 and the 1980 BEIR Report on hazard assessments of high level waste. Health Phys. 42, 133-143 (1982).

3. Garrels, R.M., and Christ, C.L. Solutions, Minerals and Equilibria, pp. 122-143. Harper and Row, New York (1965).

4. Cotton, F.A., and Wilkinson, G. Aquivanced Inorganic Chemistry 4th Ed. pp. 1005-1046. John Wiley and Sons, New York (1980). 
5. Burkholder, H.C., Cloninger, H.O., Baker, .A., and Jansen, $G$. Incentives for Partitioning High-Level Waste, Battelle Pacific Northwest Laboratory report BNWL-1927 (1975).

6. Fowler, S.W., and Aston, S.R. Application of ${ }^{235} \mathrm{~Np}$ in experimental aquatic radioecology: Preliminary ouservations on neptunium behavior in sea water, sediments and zoo plankton. Health Phys. 42, 515-520 (1982).

7. Schreckhise, R.G., and Cline, J.F. Comparative uptake and distribution of plutonium, americium, curium and neptunium in four plant species. In: Transuranic Elements in the Environment (W.C. Hanson, editor) pp. 361-370. U.S. Dept. of Energy (1980).

8. Lanz, H., Scott, K.G., Crowley, !., and Hamilton, J.E. The Metabolism of Thorium, Protoactinium and Neptunium in the Rat. Metallurgical Laboratory report $\mathrm{CH}-3606$ (1946).

9. Thompson, R.C. Neptunium-The neglected actinide: $A$ review of the biological and environmental literature. Radiat. Res. 90, 1-32 (1982).

10. Ralston, L.G., Cohen, N., Bhattacharyya, M.H., Larsen, R.P., Ayres, L., Oldham, R.D., and Moretti, E.S. The metabolism and gastrointestinal absorption of neptunium and photoartinium in adult baboon. In Speciation-85 Seminar: Speciation of Fission and Activation Products in the Environment, Oxford, April 16-19, 1985.

Metivier, H., Bourges, J., Fritsch, P., Nolibe, D., and Masse, R. Jastrointestinal absorption of neptunium in primates: Effect of ingested mass, diet and fasting. Radiat. Res. 106, 190-200 (1986).

12. Moskalev, Yu.l. (․․). Tuxicology and Radiobiology of ituptunium-237. Atomizdat Publishers, Moss ow (1979). English translation, ORNL-tr-4is 15.

13. Sevost'yanova, E.i. Stability of $N p(I V), N p(V)$, and $N p(V I)$ in citric acid solutions.
Radiokhimiya 25, 340-345 (1983). English transiation, Plenum Publishing Corp. (1984).

14. Raymorid, K.N., and Carrano, C.J. Coordination chemistry and microbial iron transport. Acc. Chem. Res. 12, 183-190 (1979).

15. International Commission on Radiological Protection, Limits for Intakes of Radionuclides by Workers, ICRP Pub. 30, part 2. Ann. ICRP 4, $3 / 4$ (1980).

16. Durbin, P.W., Jeung, N., and Schmidt, C.T. ${ }^{238} \mathrm{Pu}(\mathrm{IV})$ in Monkeys: Overview of Metabolism. U.S. Nuclear Regulatory Commission report NUREG/CR-4355, Vol. 1 (1985).

17. Durbin, P.W. Metabolism and biological effects of the transplutonium elements. In Uranium, Plutonium, Transplutonic Elements (H.C. Hodge, J.N. Stannard, and J.B. Hursh, Eds.), Handbook of Experimental Pharmacol:ogy, vol. 36, pp. 739-928. Springer-Verlag, Berlin (1973).

18. Durbin, P.W. Metabolic models for uranium. In Biokinetics and Analysis of Uranium in Man (R.H. Moore, editor), pp. F-1 to F-65. Hanford Environmental Health Foundation repont USUR-05 HEHF-47 (1984).

19. Durbin, P.W., and Schmidt, C.T. The U.S.Transuranium Registry report on the ${ }^{241} \mathrm{Am}$ content of a whole body. Part V. Implications for metabolic modeling. Health Phys. 49, 623-661 (1985).

20. Neuman, W.F., and Neuman, M.W. The Chemical Dynamics of Bone Mineral. Univ. hicago Press, Chicago (1958).

21 Hamilton, 1.G. The metabolic properties of plutonium and allied materiais. In: University of California Radiation Laboratory Medical and Health Physics Quarterly Report UCRL41.4, pp. 4-24 (1949).

22. Efurd, D.W., Perrin, R.E., and Mclnroy, J.F. Neptunium-237 in human tissue samples. Health Phys. 51, 665-666 (1986). 


\title{
NEW SEQUESTERING AGENTS FOR THE ACTINIDES: REMOVAL OF PLUTONIUM-238 FROM MICE GY ORALLY ADMINISTERED LIGANDS
}

\author{
Patricia W. Durbin, Nylan Jeung, Frederick L. Weitl," David L. White," \\ Petra Turowski," Kenneth N. Raymond," and Charles T. Schmidt ${ }^{\dagger}$
}

The actinides are radioactive and carcinogenic, and an excess of chemically similar Fe(III) is toxic. The only known way to reduce the risks to health when these metals are deposited in the body is to enhance their excretion with chelating agents.

The purpose of this program is to provide biological data needed to guide the design of safe and effective chelating agents for actinides and chemically similar elements. Macromolecules have been synthesized containing multiple catechoylamide (CAM), hydroxamate (X), hydroxypyridinone (HOPO) and carboxyl (C) functional groups, either all with the same functional groups |e.g., the tetrameric carboxycatechoylamide, 3,4,3-LICAM(C) or with different functional groups (e.g., DFOHOPO, which contains three hydroxamate and one hydroxypyridinone groups). All of those functional groups, in a variety of molecular arrangements, form remarkably stable complexes with Pu(IV) and $\mathrm{Fe}(\mathrm{III})$ at $\mathrm{pH}$ 7.4, and when administered by parenteral injection, promote significant excretion of circulating $P u(I V)$ in mice ${ }^{1-4}$ and dogs. ${ }^{5}$ Oral administration is the ideal mode of ligand intake for convenience, patient comfort, and maintenance of an effective ligand concentration in body fluids, but neither of the ligands that are clinically accepted for removal of iron (desferrioxamine, DFOM) or for removal of actinides (diethylenetriaminepentaacetic acid, $\mathrm{CaNa}_{3}-\mathrm{DTPA}$ ) are very effective when given by mouth. One of the goals of this program is the development of potent actinide and ironsequestering agents that are effective at moderate dosages when given orally. This report presents the results of a preliminary test of the oral effectiveness of several representative new ligands for promoting excretion of circulating Pu(IV) from mice.

A significant fraction of intravenously injected Pu(IV) is cleared fairly rapidly from the circulation of the mouse and is deposited in liver and skeleton. $^{6}$ The procedure used here is an attempt to maximize the effect of the ligand by placing it in the animal's stomach at a time when a significant

"Materials and Molecular Research Division, LBL, and Dept. of Chemistry, University of California, Berkeley.

'Medical Services, LBL. amount of $\mathrm{Pu}$ is still circulating. Mice were fasted 17 to 18 hours. Under ether anesthesia groups of five mice received an intravenous injection of ${ }^{238} \mathrm{Pu}(\mathrm{IV})$ in citrate buffer, and immediately afterwards $30 \mu$ mole $/ \mathrm{kg}$ of a ligand in a volume of 0.5 $\mathrm{ml}$ was given by stomach tube. A small amount of food was provided from 4 hours after the administration of $\mathrm{Pu}$ and ligand. The mice were killed 24 hours after the $\mathrm{Pu}$ injection, frozen and dissected after partial thawing. Separated excreta were collected at 4 and 24 hours. The ${ }^{238} \mathrm{Pu}$ in skeleton, tissues, and excreta was determined by counting the ${ }^{234} \mathrm{U} \mathrm{L} \times$ rays. Details of the procedures have been published. ${ }^{1,2}$ The solubility of the six orally tested ligands was determined in $0.13 \mathrm{M} \mathrm{HCl}(\mathrm{pH}$ 0.9 ), which is at the upper end of the range of acidity of fasting human gastric juice $[\mathrm{pH} 0.9$ to 1.2.]. One-half $\mathrm{ml}$ of each of the ligand solutions administered orally to mice $(\mathrm{pH} 7.1)$ and $1 \mathrm{ml}$ of $0.2 \mathrm{M}$ $\mathrm{HCl}$ were mixed with stirring in clear glass tubes. After standing for $\mathbf{3 0}$ minutes at room temperature they were examined for turbidity. All except 3,4,3-LICAM $(\mathrm{C})$ were soluble at $\mathrm{pH} 0.9$, and titration with dilute base to $\mathrm{pH} 3$ promptly redissolved 3,4,3-LICAM(C).

The effectiveness of six orally administered ligands $(30 \mu \mathrm{mole} / \mathrm{kg}$ immediately after injection of $\mathrm{Pu}$, designated hereinafter as oral administration) for promoting $\mathrm{Pu}$ excretion from mice is shown in Table 1 (Pu excretion) and Table 2 (Pu distribution in the body at 24 hours). For comparison, the tables include data for Pu distribution and excretion in mice given the same ligands by intraperitoneal (i.p.) injection 1 hour after the $\mathrm{Pu}$ at the standard dosage, (30 $\mu \mathrm{mole} / \mathrm{kg}$, designated hereinafter as standard i.p. administration) and the lower dosage that yielded the same reduction in body Pu content as the oral administration (designated hereinafter as the oral equivalent i.p. dosage), and Pu-injected control mice killed at 1 and 24 hours. Previously published results are noted in the tables. Data for low dosages of i.p. injected ligands were available from dosage-effectiveness (D-E) studies. In two cases, DFOM and DFO-HOPO, the oral equivalent i.p. dosage was a match by one D-E data point. The oral equivalent i.p. dosage for the other four 
Table 1. Promotion of ${ }^{23.9} \mathrm{Pu}$ excretion in mice by orally or intraperitoneally administered ligands."

\begin{tabular}{|c|c|c|c|c|c|c|c|c|}
\hline \multirow[b]{3}{*}{ Ligand } & \multicolumn{7}{|c|}{ Pu excretion ( $\%$ injected dosage $)^{b}$} & \multirow{3}{*}{$\begin{array}{l}\text { Effective nel urinary } \\
\text { excretion half-time } \\
\text { (Tu eff }), h r^{\circ} \\
0-4 \mathrm{hr}\end{array}$} \\
\hline & \multicolumn{2}{|c|}{ Urine } & \multicolumn{2}{|c|}{ Gl tract $(0-24 \mathrm{hr})$} & \multicolumn{3}{|c|}{ Net excretion } & \\
\hline & $0-4 \mathrm{hr}$ & $4-24 \mathrm{hr}$ & Feces & Contents & Urine & $\begin{array}{c}\mathrm{Gl} \\
\text { tract }\end{array}$ & $\begin{array}{l}\text { Urine } \\
\text { fraction }^{d}\end{array}$ & \\
\hline \multicolumn{9}{|c|}{ Oral (30 $\mu \mathrm{mole} / \mathrm{kg}$ ) } \\
\hline $\begin{array}{l}\text { DFOM } \\
\text { DFO-HOPO } \\
\text { CaNa,-DTPA } \\
\text { ZnNa,-DTPA-DX } \\
\text { 3,4,3-LICAM(C) } \\
\text { 3,4,3-LICAM(S) }\end{array}$ & $\begin{array}{c}4.5 \\
21 \\
4.2 \\
4.9 \\
30 \\
53\end{array}$ & $\begin{array}{c}2.0 \\
8.0 \\
5.3 \\
5.7 \\
10 \\
11\end{array}$ & $\begin{array}{c}1.9 \\
10 \\
1.9 \\
9.0 \\
3.3 \\
1.9\end{array}$ & $\begin{array}{l}3.0 \\
1.2 \\
3.1 \\
1.9 \\
1.0 \\
1.1\end{array}$ & $\begin{array}{l}3.1 \\
26 \\
6.2 \\
7.3 \\
36 \\
59\end{array}$ & $\begin{array}{l}0 \\
6.2 \\
0 \\
5.9 \\
0 \\
0\end{array}$ & $\begin{array}{l}1.00 \\
0.81 \\
1.00 \\
0.55 \\
1.00 \\
1.00\end{array}$ & $\begin{array}{l}2.0 \\
2.1 \\
6.7 \\
5.7 \\
2.0 \\
1.5\end{array}$ \\
\hline \multicolumn{9}{|l|}{$\begin{array}{l}\text { Intraperitoneal } \\
\qquad(30 \mu \mathrm{mole} / \mathrm{kg})\end{array}$} \\
\hline $\begin{array}{l}\text { DFOM } \\
\text { DFO-HOPO } \\
\text { CaNa,-DTPA } \\
\text { ZnNa-DTPA-DX } \\
\text { 3,4,3-LICAM(C) } \\
\text { 3,4,3-LICAM(S) }\end{array}$ & $\begin{array}{l}32< \\
63 \\
13 \\
39 \\
52\end{array}$ & $\begin{array}{l}0> \\
7.8 \\
2.6 \\
8 \\
11 \\
10\end{array}$ & $\begin{array}{l}9.7 \\
37 \\
- \\
34 \\
14 \\
0.4\end{array}$ & $\begin{array}{c}2.9 \\
9.7 \\
4.0 \\
10 \\
7.3 \\
3.0\end{array}$ & $\begin{array}{l}37 \\
36 \\
61 \\
18 \\
47 \\
57\end{array}$ & $\begin{array}{c}7.6 \\
42 \\
0 \\
39 \\
16 \\
0\end{array}$ & $\begin{array}{l}0.83 \\
0.46 \\
1.00 \\
0.32 \\
0.75 \\
1.00\end{array}$ & $\begin{array}{l}1.8 \\
1.2 \\
0.54 \\
2.8 \\
1.3 \\
1.1\end{array}$ \\
\hline \multicolumn{9}{|c|}{$\begin{array}{l}\text { Intraperitoneal } \\
\text { (dosage equivalent to } \\
30 \mu \text { mole/kg oral) }\end{array}$} \\
\hline $\begin{array}{l}\text { DFOM } \\
\text { DFO-HOPO } \\
\text { CaNa }{ }_{3} \text {-DTPA } \\
\mathrm{ZnNa}^{3} \text {-DTPA-DX } \\
\text { 3,4,3-LICAM(C) } \\
\text { 3,4,3-LICAM(S)' }\end{array}$ & $\begin{array}{r}< \\
6.7 \\
52<\end{array}$ & $\begin{array}{l}.4> \\
3> \\
3> \\
10\end{array}$ & $\begin{array}{c}1.1 \\
14.9 \\
1.2 \\
11 \\
2.4 \\
0.4\end{array}$ & $\begin{array}{l}1.2 \\
2.9 \\
4.6 \\
6.8 \\
2.0 \\
3.0\end{array}$ & $\begin{array}{l}4.4 \\
25 \\
10 \\
5.7 \\
19 \\
57\end{array}$ & $\begin{array}{c}0 \\
13 \\
0.8 \\
13 \\
0 \\
0\end{array}$ & $\begin{array}{l}1.00 \\
0.66 \\
0.92 \\
0.30 \\
1.00 \\
1.00\end{array}$ & $\begin{array}{l}- \\
- \\
- \\
-\end{array}$ \\
\hline \multicolumn{9}{|c|}{${ }^{210}$ Pu-injected controls } \\
\hline $\begin{array}{l}\text { Kill } 1 \mathrm{hr} \\
\text { Kill } 24 \mathrm{hr}\end{array}$ & $\begin{array}{l}1.1 \\
2.1\end{array}$ & $\overline{1.2}$ & $\overline{3.7}$ & $\begin{array}{l}5.1 \\
1.3\end{array}$ & - & - & - & - \\
\hline
\end{tabular}

- 230Pu(IV) citrate injected i.v.; ligands administered orally immediately thereafter, or intraperitoneally at $1 \mathrm{hr}$; mice killed at $24 \mathrm{hr}$.

b Urine and feces were collected from each group of five mice caged together; the GI Iract(full) samples from those five mice were also pooled for $\mathrm{Pu}$ analysis, and the $\mathrm{Pu}$ attributable to tissues and $\mathrm{Gl}$ contents was cal. culated. ${ }^{2}$ Results for each experimental group were normalized to $100 \%$ material recovery; discrepancies are due to rounding.

- Net excretion - (treated - 24-hr control).

d Net urine fraction = net urine/[net urine + net(feces + Gl contents)]. Where total Gl tract excretion is less than $5 \%$ ID, net is shown as zero, and material balance is preserved by subtracting the difference from total urine.

- Effective excretion half-time $=0.693 / \lambda_{w}$ where net cum ur fr $=1.0\left(1-e^{-\lambda} u^{t}\right), 1-$ net cum ur fr $=e^{-\lambda} u^{t}$ and $\ln (1$ - net cum ur fr $) / 1=-\lambda_{u}$. For example, for $30 \mu \mathrm{mole} / \mathrm{kg}$ of $\mathrm{ZnNa-DTPA}$.DX i.p. at $1 \mathrm{hr}$, net cum ur fr $=$ $10.9 /(10.9+9.8)=0.526,-\lambda_{u}=-0.746 / 3=0.249 \mathrm{hr}^{-1}, \mathrm{Tu}_{\mathrm{eff}}=0.693 / 0.249=2.8 \mathrm{hr}$.

1 Fraction of total urinary excretion in 0.4 and $4-24 \mathrm{hr}$ after i.p. injection of $30 \mu \mathrm{mole} / \mathrm{kg}$ of CaNa - DTPA estimated from Ref. (B) and of 3,4,3-LICAM(5), from our unpublished data for excretion of Pu-3,4,3-LICAM(S).

- Dash indicates no data available.

" Oral equivalent i.p. dosages ( $\mu$ mole $/ \mathrm{kg}$ ) were as follows: DFOM, 3.0; DFO-HOPO, 0.9; CaNa, DTPA, 1.2 to 3.5; ZnNa-DTPA-DX, 0.3 to 3.0; 3,4,3-LICAM(C), 0.2 to $0.8 ; 3,4,3-\operatorname{LICAM}(\mathrm{S}), 20$ to 30. 
Table 2. ${ }^{230} \mathrm{Pu}$ in tissues of mice given ligands by stomach tube or intraperitoneal injection."

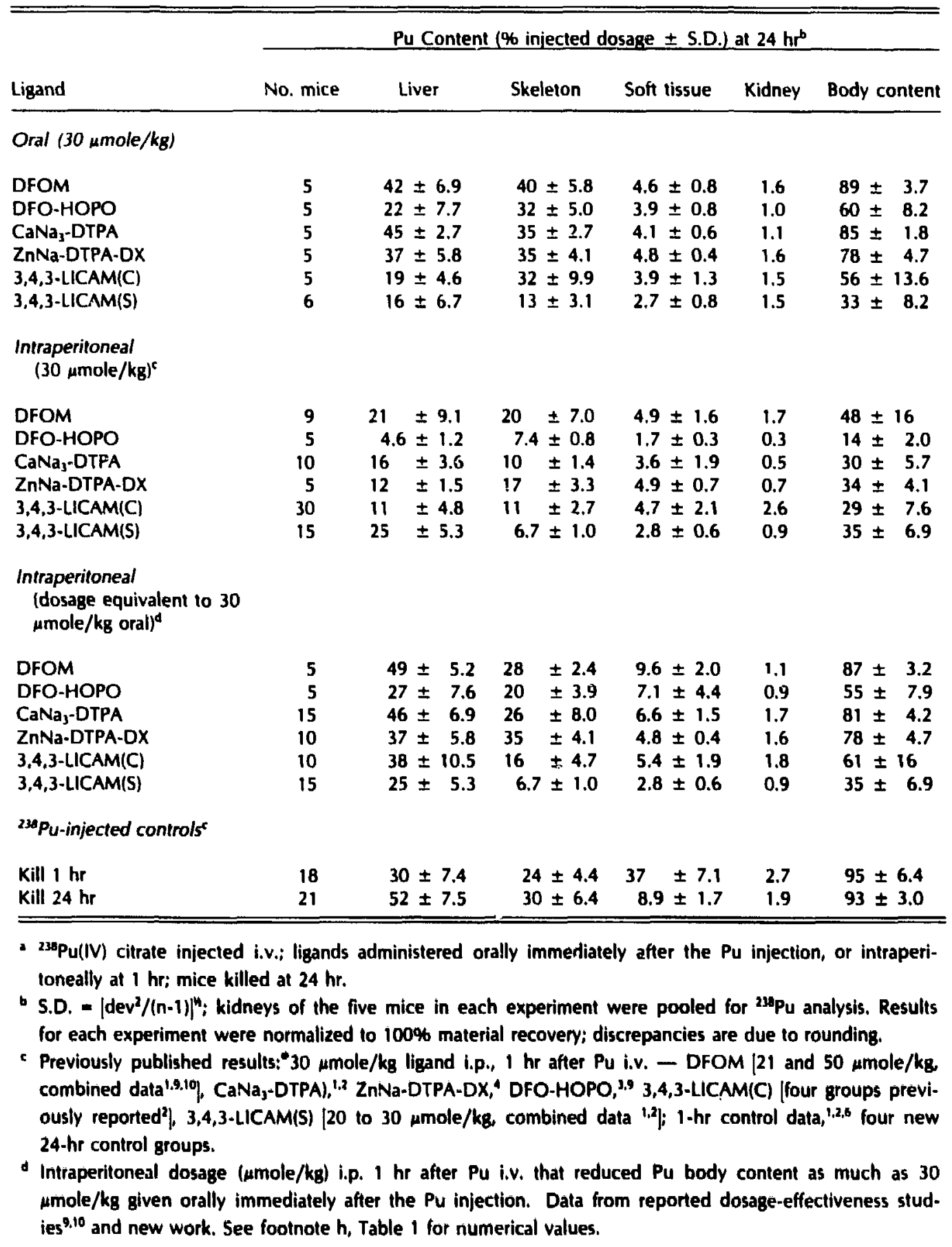

ligands lay between two D-E data points, and the values shown in Table 2 are means for the two groups.

Three classes of ligands were tested: polyhydroxamic acids, DFOM, and its hydroxypyridinone derivative, DFO-HOPO; polycarboxylic acids,
$\mathrm{CaNa}_{3}$-DTPA, and its dihydroxamato derivative, ZnNa-DTPA-DX; tetracatechoylamides with solubilizing constituents on the catechol ring-carboxyl, 3,4,3-LICAM(C), and sulfonate, 3,4,3-LICAM(S). Orally administered DFOM promoted no Pu excretion in excess of controls. The addition of the 
HOPO group, which is deprotonated at $\mathrm{pH} 5.8$, produced a molecule that could be absorbed, and a net $\mathrm{Pu}$ excretion of $36 \%$ ID was observed. Net $\mathrm{Pu}$ excretion promoted by oral $\mathrm{CaNa}_{3}$-DTPA $(6.2 \%$ ID) was significantly different from the 24-hour controls |t-test, $p<0.01 .^{\prime \prime}$ The small amount of $\mathrm{Pu}$ excretion elicited by $\mathrm{CaNa}_{3}$-DTPA was expected, because its absorption from the Gl tract is slow. ${ }^{8}$ Absorption of ZnNa-DTPA-DX was more efficient than the parent compound, because net Pu excretion was doubled $(13.2 \% \quad$ ID). The poly(catechoylamides) were the most effective oral ligands tested; 3,4,3-LICAM(C) promoted net $\mathrm{Pu}$ excretion of $36 \% \mathrm{ID}$, in agreement with other work $^{12}$, and $30 \mu$ mole $/ \mathrm{kg}$ of 3,4,3-LICAM(S) given orally was as effective for $\mathrm{Pu}$ removal (net $\mathrm{Pu}$ excretion $59 \%$ (D) as the standard i.p. dosage. If the oral equivalent i.p. dosage of a ligand is taken as a crude measure of its $\mathrm{Gl}$ absorbed fraction, $\mathrm{Gl}$ absorption of the ligands may be estimated as follows: DFOM, CaNa ${ }_{3}$-DTPA, and ZnNa-DTPA,DX, 5 to $10 \%$ ID data for $\mathrm{CaNa}_{3}$-DTPA are in agreement with direct measurements ${ }^{7}$; 3,4,3-LICAM(C) and DFO-HOPO, about $3 \%$ ID $\mid 3 \%$ absorption of 3,4,3-LICAM(C) has been reported ${ }^{12} \mid$; and 3,4,3LICAM(S), more than 67\% ID. Additional evidence from excretion patterns and distribution of retained Pu (discussed below) support these estimates.

The two most acidic ligands, $\mathrm{CaNa}_{3}$-DTPA and 3,4,3-LICAM(S), promote only urinary Pu excretion (net urine fraction was about 1.0 for each) regardless of dosage or mode of administration. Significant net Pu excretion through the $\mathrm{Gl}$ tract was promoted by the standard i.p. dosage of the less acidic ligands, DFOM, DFO-HOPO, ZnNa-DTPA-DX and 3,4,3-LICAM(C). However, fecal excretion of $\mathrm{Pu}$ promoted by those ligands was reduced (urine fraction increased) when the i.p. dosage was reduced. Promotion of $\mathrm{Pu}$ excretion via the Gl tract appears to depend on both the chemistry of the ligand and its concentration in body fluids. The fecal fraction of net $\mathrm{Pu}$ excretion promoted by those ligands was also reduced when they were administered orally (compared to results for the standard i.p. administration), from which we conclude that their concentrations in the body are low after oral administration, and their $\mathrm{Gl}$ absorbed fractions are small.

The urine data provide preliminary information about the rates of renal excretion of the Pu-ligand chelates. Effective half-times for net renal Pu excretion (UT $T_{\text {eff }}$ ) in the interval 1 to 4 hours after i.p. ligand injection or 0 to 4 hours after oral administration can be estimated from the fraction of net urinary $\mathrm{Pu}$ excreted in the interval (see Table 1). After the standard i.p. injection of the ligands,
UT $_{\text {ett }}$ for the $\mathrm{Pu}$ chelates ranged from most rapid excretion, Pu-DTPA $U T_{\text {eff }}=0.54 \mathrm{hr}(32 \mathrm{~min})$, in agreement with direct measurements of $\mathrm{CaNa}_{3}$ DTPA excretion ${ }^{7}$, to the slowly eliminated PuDTPA-DX chelate $\left(U T_{\text {eff }}=2.8 \mathrm{hr}\right.$ ). In all cases $U T_{\text {eff }}$ was longer when the ligand was administered orally than after i.p. injection, reflecting the delay incurred in passage across the GI tract epithelium.

As noted earlier, within a few hours after i.v. injection. Pu leaves the circulation of the mouse and is deposited, mainly in the skeleton and liver $\mid 36$ and $55 \%$ ID at 1 and 4 hours, respectively|, ${ }^{6}$ so any reduction in body $\mathrm{Pu}$ caused by an orally administered ligand will depend on its rate of $\mathrm{Gl}$ absorption as well as $\mathrm{Gl}$ absorbed fraction. Reduction in body $\mathrm{Pu}$ following oral administration was in the decreasing order: 3,4,3-LICAM(S) > 3,4,3LICAM(C), DFO-HOPO > ZnNa-DTPA-DX > $\mathrm{CaNa}_{3}$-DTPA. The difference in urinary excretion half-times $U T_{\text {eff }}$ (oral) - UT eff(i.p.) was in the exact reverse order, which suggests that that difference is a crude measure of $\mathrm{Gl}$ absorption rate.

The distribution of the $\mathrm{Pu}$ retained in mice 24 hours after receiving an oral ligand both resembled and differed from the $\mathrm{Pu}$ distribution observed in mice given the same ligands i.p., either at the standard or oral equivalent dosages (see Table 2). The $\mathrm{Pu}$ content of soft tissues (other than liver and kidney) was $\leqslant 50 \%$ of 24 -hours $\mathrm{Pu}$-injected controls; and the differences were statistically significant. Kidney $\mathrm{Pu}$ was also reduced. The major effect of the orally administered ligands was reduction of liver $\mathrm{Pu}$ to $\leqslant 24$-hours controls; the degree of reduction was significantly different, except for DFOM and CaNa -DTPA. Only 3,4,3-LICAM(S) reduced bone $\mathrm{Pu}$. In fact lexcept for 3,4,3LICAM(S)|, the bone Pu of mice given ligands orally was greater than that of the 24-hour controls, and for the DFOM-treated mice the apparent increase was significant. "The liver/skeleton Pu ratios are: for mice given $30 \mu$ mole/ $\mathrm{kg}$ of DFOM either orally or by i.p. injection, 1.05; for mice given the lower oral equivalent i.p. dosage and the 24-hour controls, 1.73. The standard i.p. dosage of DFOM significantly reduced the liver/skeleton ratio while promoting excretion of $44 \%$ of the injected $\mathrm{Pu}^{\prime}$ There is no evidence that enough orally administered DFOM was absorbed by the GI tract to change the control partitioning of $\mathrm{Pu}$ between bone and liver. It is more likely that the liver/skeleton $\mathrm{Pu}$ ratio of all the mice that received oral ligands was shifted to greater $\mathrm{Pu}$ deposition in bone and less in liver than occurs in normally fed mice by the 17- to 18-hour fast that preceded the Pu injection. An experiment is scheduled to measure $\mathrm{Pu}$ 
distribution in control mice on the fasting-feeding schedule of the oral administration studies. For the sake of the present argument, if the Pu distribution in the mice given DFOM orally is close to that of fasted 24-hour controls, it appears that the ligands other than 3,4,3-LICAM(S) were absorbed too slowly to establish plasma concentrations great enough to remove any, even loosely bound, $\mathrm{Pu}$ from the skeleton. However, some circulating $\mathrm{Pu}$ was chelated and excreted and some additional late $\mathrm{Pu}$ deposition in liver, and possibly bone, was prevented. In the case of 3,4,3-LICAM(S), and to a lesser degree 3,4,3-LICAM(C) and DFO-HOPO, the ligand concentration in blood was apparently sufficient at early times to remove some loosely bound $\mathrm{Pu}$ from the liver.

Removal of newly injected Pu by orally administered ligands depends on the stability of the Puligand complex as well as the rate of ligand absorption from the $\mathrm{Gl}$ tract. Thus, reasonably good $\mathrm{Pu}$ removal was obtained with the strongly binding ligands, 3,4,3-LICAM(C) and DFO-HOPO, even though available evidence suggests little prompt absorption (about 3\% ID), while Pu removal was poor for the less strongly binding ligands, DFOM, $\mathrm{CaNa}_{3}-\mathrm{DTPA}$ and $\mathrm{ZnNa-DTPA}-\mathrm{DX}$, even though total absorption may be as much as $5 \%$ to $10 \% \mathrm{ID}$. The excellent $\mathrm{Pu}$ removal obtained with 3,4,3LICAM(S) $\mathrm{C}^{-1}$ be attributed to both strong binding and rapid $\mathrm{Gl}$ absorption. The important questions are 1) What chemical properties underlie the differences in $\mathrm{Gl}$ absorption rates among the three strongly binding ligands, and 2) What are the chemical properties of $\mathrm{CaNa}_{3}$-DTPA and DFOM that impede their $\mathrm{Gl}$ absorption. Some time ago it was suggested that when $\mathrm{CaNa}_{2}$-EDTA was mixed with the acidic stomach contents the insoluble free acid, $\mathrm{H}_{4}$-EDTA (and by analogy $\mathrm{H}_{5}$-DTPA), would form and slow absorption would follow its subsequent dissolution at the higher $\mathrm{pH}$ of the intestines. ${ }^{13}$ Precipitation of free acid in the stomach cannot account in part for the slow $G$ l absorption of $\mathrm{CaNa}_{3}$-DTPA and its derivative, ZaNa-DTPA-DX, because at the concentrations of those ligands in the mouse stomach, both are soluble at $\mathrm{pH} 0.9$. Precipitation and dissolution, which delay absorption, may, however, account in part for the poorer Pu removal obtained with oral 3,4.3-LICAM(C) than with its sulfonated analogue.

The apparent singular influence of the sulfonate group on $\mathrm{Gl}$ absorption will be investigated by testing the oral activity (for $\mathrm{Pu}$ removal) of some other poly(catechoylamide) ligands that are available both nonfunctionalized and sulfonated. The role of gastric acidity on absorption of metal-binding agents will be investigated by orally administering DFOHOPO and 3,4,3-LICAMiC) and the baseline ligands, DFOM and $\mathrm{CaNa}_{3}$-DTPA, in solutions sufficiently alkaline to reduce the acidity of the stomach contents to $\mathrm{pH} \mathrm{3}$; and by testing the oral activity of metal romplexes of the more potent ligands, which are expected to dissociate at the $\mathrm{pH}$ of the stomach.

\section{REFERENCES}

1. Durbin, F.W., Jones, E.S., Raymond, K.N., and Weitl, F.L. Specific sequestering agents for the actinides: 4. Removal of ${ }^{238} \mathrm{Pu}$ (IV) from mice by sulfonated tetrameric catechoylamides. Radiat. Res. 81, 170-187 (1980).

2. Durbin, P.W., Jeung, N., Jones, E.S., Weitl, F.L., and Raymond, K.N. Specific sequestering agents for the actinides: 10. Enhancement of ${ }^{238} \mathrm{Pu}$ elimination from mice by poly(catechoylamide) ligands. Radiat. Res. 99, 85-105 (1984).

3. White, D.L., Durbin, P.W., Jeung, N., and Raymond, K.N. Specific sequestering agents for the actinides: 13. Synthesis and initial biological testing of polydentate hydroxypyridinonate ligands. Lawrence Berkeley Laboratory report LBL-21714 (1986). Submitted to /. Med. Chem.

4. Durbin, P.W., Jeung, N., Raymond, K.N., White, D.L., Rodgers, S.J., and Turowski, P. New sequestering agents for the actinides: Effectiveness for removal of Pu from mice of hydroxamic acid derivatives of DTPA, EDTA and desferrioxamine and an $\mathrm{N}$-centered tris(catechoylamide) ligand; graded dosages of desferrioxamine pyridinone oxide (desferriHOPOCAM); protracted administration of 3,4,3LICAM(C), Biology and Medicine Division Annual Report 1985, Lawrence Berkeley Laboratory report LBL-20345, pp. 70-78 (1986).

5. Lloyd, R.D., Bruenger, F.W., Mays, C.W., Atherton, D.R., Jones, C.W., Taylor, G.N., Stevens, W., Durbin, P.W., Jeung, N., Jones, E.S., Kappel, M.J., Raymond, K.N., and Weitl, F.L. Removal of Pu and Am from beagles and mice by 3,4,3-LICAM(C) or 3,4,3-LICAM(S). Radiat. Res. 99, 106-128 (1984).

6. Durbin, P.W., and Jeung, N. Kinetics of plutonium deposition in the mouse. Division of Biology and Medicine Annual Report 1982-1983, Lawrence Berkeley Laboratory report LBL-16840, pp. 56-59 (1984). 
7. Hightower, N.C., Ir., and lanowitz, H.D. Digestion. Best and Taylor's Physiological Basis of Medical Practice, 9th Ed. J.R. Brobeck, Ed., pp. 2-1 to 2-128. The Williams and Wilkins Co., Baltimore (1973).

8. Foreman, $H$. The pharmacology of some useful chelating agents. In Metal Binding in Medicine, M.I. Seven and L.A. Johnson, Eds., pp. 82-94, Lippincott, Philadelphia (1959).

9. Durbin, P.W., Jeung, N., Rodgers, S.J., White, D.L., and Raymond, K.N. New sequestering agents for the actinides: Acute toxicity and effectiveness for removal of $\mathrm{Pu}$ from mice of derivatives of desferrioxamine and of poly(hydroxypyridinone) ligands and their ferric and zinc complexes. Biology and Medicine Division Annual Report 1983-1984, Lawrence Berkeley Laboratory report LBL18393, pp. 59-64 (1985).
10. Durbin, P.W., Jeung, N., Jones, E.S., Raymond, K.N., and Weitl, F.L. Plutonium removal from mice by poly(catechoylamide) ligands, $\mathrm{Ca}$ DTPA, desferrioxamine B, and Tiron: Effect of ligand dosage. Biology and Medicine Division Annual Report. Lawrence Berkeley Laboratory report LBL-16840, pp. 43-48 (1984).

11. Fisher, R.A. Statistical Methods for Research Workers, 12th Ed., Rev., Hafner Publishing Co., New York (1954).

12. Volf, V. Chelation therapy of incorporated plutonium-238 and americium-241: comparison of LICAM $(C)$, DTPA and DFOA in rats, hamsters and mice. Int. J. Radiat. Biol. 49, 449-462 (1986).

13. Foreman, $H_{\text {., Vier, }}$., and Magee, $M$. The metabolism of $\mathrm{C}^{14}$-labelled ethylenediametitraacetic acid in the rat. J. Biol. Chem. 203, 1045-1053 (1953). 


\section{SECTION 3. RADIATION BIOPHYSICS}

\section{INTRODUCTION}

During the last several years heavy accelerated particles have proven to be useful tools for a better understanding of fundaniental biology. The practical results obtained with brain radio-surgery and with heavy-ion therapy for cancer make it urgent that plans be made for increased availability of heavy-ion accelerator facilities in the future, a need that is being recognized by physicians and scientists in a number of countries. We have somewhat arbitrarily divided the field into "light ions" (from proton to neon) and "heavy ions" (from neon to uranium). There are plans to upgrade the Bevalac for greater heavy-ion intensities and versatility (described in the report of the Light Ion Biomedical Research Accelerator (LIBRA) study group). We feel that the time has arrived to transfer light-ion accelerator technology to the private sector. By a coordinated effort between the staff of the MerrittPeraita hospitals in Oakland, the UC Medical School in San Francisco, and LBL, we are planning the LIBRA facility. If we can proceed according to plan, then LIBRA and a hospital laboratory complex may be completed and operational sometime in 1990 , in time to transfer much of the research to this facility while the Bevalac is shut down for the upgrading process. At LIBRA, the full potential of light ions fo: therapy can be fulfilled, while this machine will also continue to be a very useful research tool. In the meanwhile, research is progressing in four different areas as summarized below.

\section{RADIATION PHYSICS AND BIOCHEMISTRY}

With the leadership of Aloke Chatterjee and John Magee, our group is engaged in a combined experimental and theoretical effort on radiation damage to DNA. The circular DNA molecule of the simian virus SV4O is the object of many of these investigations. At the theoretical level the group has been able to model diffusion-controlled free-radical interactions of DNA in aqueous milieu step by step in Monte Carlo processes. Aloke Chatterjee et al. have been able to quantitate the role of radiation-induced $\mathrm{OH}$ radicals in producing DNA damage by an indirect process. William Holley et al. have shown that the cores of the ionizing particle tracks are of the greatest importance when strand breaks are produced by heavy ions. This work has allowed an extension of detailed molecular analysis of radiation and particle effects on DNA-protein complexes.

As light and heavy ions pass through matter, the path of each particle deviates from a mathematically straight line because of multiple scattering on the nuclei of absorbing matter. Although this process is well known for electrons, for heavy nuclei there are some differences because the moving heavy particles often collide with resting nuclei that are lighter. For this reason Mervyn Wong et al. are conducting a detailed experimental and theoretical study of the process. The practical importance of scattering to dose localization in the Bragg peak of particles is illustrated by the studies of Eleanor Blakely et al. They have experimentally verified the notion that carbon and oxygen particles can make sharper and smaller lesions than protons. These results will be of importance for the production of small lesions in human brain and spinal cord.

\section{MOLECULAR AND CELLULAR RADIOBIOLOGY AND MUTAGENESIS}

Dissolved oxygen makes cells and tissues more sensitive to the effects of ionizing radiations. It has now been demonstrated that the magnitude of the oxygen effect changes as human cells progress through the cell division cycle. Eleanor Blakely et al. have related the magnitude of the oxygen effecl to the quantity of intracellular nonprotein sulfhydryls; there is much interest in these findings because they may explain existing variations in the radiosensitivity of tumor cells of various origin. If we could reliably identify which tumor cells are resistant to low-LET radiation, these would then become targets for heavy-ion radiotherapy.

Edwin Goodwin et al. are using an intriguing technique to quantitate the number of chromatin breaks in mammalian cells; the technique is premature chromosome condensation (PCC). The simple result is that individual heavy particles can produce multiple chromatin breaks in single cells, whereas $x$ rays can almost never accomplish this. Cornelius Tobias et al. have theoretically elaborated on this finding by claiming that the statistical distributions 
of lesions exposed to heavy ions must be described by a method originally developed by the late Professor Jerzy Neyman for the spread of contagious processes. The presence of multiple lesions in heavy-ion-irradiated cells explains the high lethal and mutagenic efficiency of these particles. We have previously postulated in the RMR model that it is the misrepair or misrejoining of such lesions that is responsible for some of the effects. The PCC technique has now supplied us with a tool for quantitative study of misrepair. In other related work David Linstadt et al. sensitized human cells with iododeoxyuridine, and Polly Chang and Eleanor Blakely studied the development of thermotolerance that is dependent on protein synthesis.

Tracy Yang and associates present three papers all relating to mutagenesis and cell transformation following heavy-ion irradiation. There is an important difference in the actions of low- and high-LET radiation; if protracted or split doses are administered, then some of the lesions repair in the period between successive doses, whereas splitdose administration of heavy ions with high LET results in enhancement of the cell iransforming effect. Further evidence has been gathered to show that heavy ions are very efficient in producing aberrations and mutations that are usually associated with carcinogenesis. It is important to understand the full explanation for such effects so that we can evaluate the importance of split-dose or low-dose-rate delivery of neutrons near nuclear reactors and of heavy ions in space flight.

\section{TUMOR AND TISSUE EFFECTS OF HEAVY PARTICLES}

The responses of tissues are different from those of isolated cells, and it is important to find techniques that are suitable for quantitative studies where cell populations are involved. Lynn Hlatky et al. have developed a very interesting system consisting of a "tissue culture sandwich," where the cell-to-cell relationships and the diffusion of nutrients can be studied in a thin uniform layer. Javed Afzal and associates compare the consequences of irradiations when lumor cells are exposed in vivo or in vitro. In their most recent studies they are able to quarititate some aspects of the host immune response to irradiated tumors. The role of cellular damage in organized tissue systems is being studied by Edgar Riley et al. in rat lens epithelium and in mouse bone marrow stem cells by John Ainsworth et al.

Walter Schimmerling et al, have analyzed the depth effectiveness of a $670-\mathrm{MeV} / \mathrm{u}$ neon beam and compared their calculations to various available data. Rodriguez et al. are carrying out studies on the effects of neon beams on rat spinal cord. Split doses of neon particles in the Bragg-peak region are much more effective than in the plateau region of the beam. These results are of importance in relation to particle therapy.

Edward Alpen et al. have obtained an impressive array of data on the carcinogenic effects of various heavy particles. The data are also compared to neutron carcinogenesis. The Harderian glands of mice, stimulated by transplanted pituitaries, are the objects of these studies. John Ainsworth et al. are carrying out life span studies on populations of mice exposed to a variety of heavy accelerated ions.

\section{HEAVY ION THERAPY, RADIOSURGERY, AND THERAPY PHYSICS}

In addition to our goal of producing information of fundamental importance, several of the projects in the Radiation Biophysics group have produced methodology and data that are essential for undertaking programs relating to humans. The first human heavy-ion irradiations have been performed by members of our group and we are acknowledged as world leaders in this field.

Our laboratory has developed a variety of physical methods for beam delivery, including wobbling and beam scanning, as described by Edward Alpen et al. Since our laboratory is the only one with experience in this field, the results will be useful wherever that particle accelerators will be used in human therapy. John Lyman has prepared information on the tolerance of various tissues and Michael Collier et al. describe current progress in therapy physics and computerized therapy planning.

The National Cancer Institute has been supporting statistically controlled therapy trials in which two universities and many northern California hospitals are participating. These studies are headed by Joseph Castro et al. While significant results have already been achieved with uveal melanoma and juxtaspinal tumors, this multi-year effort now extends also to certain types of lung and prostate cancer, bone and soft-tissue sarcomas, and also tumors at a number of additional sites.

Another approach to the control of human disease is stereotactic radiosurgery with helium ions, as carried out by Jacob Fabrikant et al. with the collaboration of members of the departments of neurosurgery at UCSF and at Stanford University. About 250 surgically inaccessible cases of intracranial arteriovenous malformations have been treated so far with encouraging clinical follow-ups. 


\title{
Radiological Physics and Chemistry
}

\section{INFLUENCE OF DNA STRUCTURE IN CHEMICAL REACTIONS WITH HYDROXYL RADICALS}

\author{
Aloke Chatterjee, Patrice Koehl, John L. Magee, and William R. Holley
}

It is well known that hydroxyl radicals $(\cdot \mathrm{OH})$ can damage a DNA molecule either by reacting with the bases or with the sugar moiety, and a comprehensive account of these phenomena has appeared in a recently published review article. ${ }^{1}$ In order to understand the biochemical mechanism associated with the reactivity of these radicals, several experimental studies have been made to determine the rate constants of $\mathrm{OH}$ with the various sites in a DNA molecule. For a systematic approach to the solution of this problem, some investigators have used various monomeric components of nucleic acid and polynucleotides of different sizes and structures involving single- and double-stranded molecules and compared their results of the rate constants for the same sites when present in a DNA molecule. ${ }^{2-4}$ For example, Michaels and Hunt observed that at neutral $\mathrm{pH}$, the rate constants for the attack of $\mathrm{OH}$ on monomers were of the order of $5 \times 10^{9} \mathrm{~mole}^{-1} \mathrm{sec}^{-1}$. In contrast, they found that the rate constants, per base, for . $\mathrm{OH}$ attack on single-stranded polynucleotides were approximately a factor of 5 lower. For doublestranded polynucleotides and DNA, these values were even lower than with the monomers by a factor of about 10. Thus it appears that when a given site (sugar moiety or base) is present in an aqueous solution as an isolated molecule, its reactivity is higher than when the same site is present in a polynucleotide or in a DNA polymer. Based on a simple calculation, Michaels and Hunt had made a hypothesis that these differences in rate constant values cannot be attributed to the secondary structure (random coil or base stacking) and tertiary structure (double-stranded helix with hydrogen bonding) of polynucleotides in a significant manner. In order to test this hypothesis we have made theoretical calculations by considering explicitly the secondary and tertiary structures of polynucleotides and their effects on the ability of $\mathrm{OH}$ to react with various sites. Since the chemical reactions are diffusion controlled, we have tried to simulate this process based on a Monte Carlo approach. ${ }^{5}$ This paper presents the calculation procedures and the results obtained.

Without the loss of generality, we have chosen a dilute aqueous solution of SV40 DNA molecules
$(10 \mu \mathrm{g} / \mathrm{ml})$ in Tris buffer $(10 \mathrm{mM})$ irradiated with 14-MeV electrons. The Tris molecules are known to be a very good scavenger of $\cdot \mathrm{OH}$ and for the concentration used, the diffusion-migration distance of a hydroxyl radical to a DNA molecule is only $150 \mathrm{~A}$. One reason for choosing SV40 DNA as our test molecule is because its genome is well characterized, ${ }^{6}$ and in order to incorporate the structure in our calculations we needed this information.

The calculation begins with the consideration of an isolated base in aqueous solution. In order to test the accuracy of the Monte Carlo approach for diffusion calculations, we have compared the rate constant values with an analytical approach. This comparison was possible only in the case of an isolated base. The base was then incorporated in double-stranded polynucleotides of various sizes, whose stereochemical parameters were used to generate a three-dimensional representation of the sensitive sites (bases and sugars) in space. Each hydroxyl radical was then followed along its randomized diffusive path until it reacted, either with a sibling radical, with Tris, or with a reactive site on the polynucleotide. The final calculation was then made by extending the polynucleotide to represent a SV40 DNA molecule in the $B$ form. The rate constant values were then computed for the $\mathrm{OH}$ reactivity in each case beginning with the consideration of an isolated base.

\section{METHOD OF CALCULATION}

There are two main features of our overall procedure, as follows: 1) a Monte Carlo simulation of the diffusive motion of the $\cdot \mathrm{OH}$ radicals, and 2) a geometrical model for chemical reactions with different monomers in a polynucleotide or in a structured DNA molecule. A brief description of these two feature is presented here:

\section{Monte Carlo Simulation}

Hydroxyl radicals are created along with other products such as $\cdot \mathrm{H}, \mathrm{e}_{\mathrm{aq}}^{-}$and $\mathrm{H}_{3} \mathrm{O}^{+}$. Following thermalization these species are positioned in three-dimensional space in a random fashion. However, they have been assumed to follow a Gaussian pattern with a characteristic standard deviation $(\sigma)$ 
value for each of the species. For example, $\sigma \mathrm{H}=$ $26 \mathrm{~A}$ and $\sigma \mathrm{OH}=13 \mathrm{~A}$. The simulation process of actual diffusive motion by a finite number of processes is carried on with each reactant "jumping" with a frequency, $v$, over a distance, I. The frequency and distance are interrelated by the diffusion constant, $D$, such that

$$
D=\left.v\right|^{2} / 6
$$

In our calculations, the jump distance, I, has been taken less than 1 A to ensure that the reactants cannot move far enough to "jump through" each other without having a chance to react. The four species considered react with each other. For example, an $\mathrm{OH}$ radical reacts with an $\mathrm{H}$ radical if they happen to be within $2.83 \AA$. Similarly, ereacts with $H$ if they are within $2.56 \AA$. These reaction radii as well as radii for reactions between other species have been determined from experimentally known rate constants and the model proposed by Smoluchowski. According to the model, two reactants, $A$ and $B$, diffusing in solution with respective diffusion constants, $D A$ and $D B$, react with each other with a unit probability if they are within the reaction radius, $R_{A B}$. The value of $R_{A B}$ is related to the diffusion constants and the rate constant $k_{A B}$ for reaction between $A$ and $B$ as follows:

$$
k_{A B}=4 \pi\left(D_{A}+D_{B}\right) R_{A B}
$$

As will be seen below, the same concept has also been extended to consider reactions between $\mathrm{OH}$ radicals and the various sites on a polynucleotide or on a DNA molecule.

\section{Geometrical Model for Chemical Reactions with Monomers}

Before considering the situation where an $\mathrm{OH}$ radical reacts with the various sites in a DNA molecule, one needs to find out the survival probability of this radical due to the scavenging effects of the Tris buffer molecules. Even if a given $\mathrm{OH}$ radical escapes a reaction with another $\mathrm{OH}$, or a $\mathrm{e}_{\mathrm{aq}}^{-}$, or a $H$ radical, it may very well react with a Tris molecule and hence be lost from the system for having any chance to react with a polynucisotide or a DNA molecule. The rate constant for this reaction is relatively unknown; however, we have estimated it to be $3 \times 10^{9} \mathrm{I} / \mathrm{mole}$ sec. For a solution containing $10 \mathrm{mM}$ Tris, the aver age life time of an $\mathrm{OH}$ radical is $3 \times 10^{-8} \mathrm{sec}$. In order to take into accouilt the effect of the buffer reaction, we have reduced the survival probability of an $\mathrm{OH}$ radical in a "jump" time $\Delta t$, corresponding to the jump distance, I, by a factor given by $\exp (-\Delta t / 3$ $\left.\times 10^{-8}\right)$. Based on this probability, a random number determines whether an $\mathrm{OH}$ radical is not scavenged by a Tris molecule.

In the geometrical modei for reactions with monomers present in a polynucleotide or in a DNA molecule, we have assumed that the Smoluchowski theory holds. This is based on our experience in determining the extent of strand breaks ${ }^{7}$ for a given dose. We therefore atiribute to each sensitive site (sugar or base) in the polynucleotides a reaction radius calculated from Eq. (1) using the rate constant of reactions summarized in Table 1 (also see Fig. 1). The diffusion constant for $\mathrm{OH}$ has been taken to be $2 \times 10^{-5} \mathrm{~cm}^{2} \mathrm{sec}^{-1}$, and we consider no motion of the DNA molecule or the polynucleotide, since any one of these is i much larger molecule than $\mathrm{OH}$. The values for the rate constant shown in Table 1 have been obtained from experimental results for reactions between $\mathrm{OH}$ radicals and isolated sugar and base molecules.

In ordar to position the courdinates of the various sugar molecules and base molecules, we consider the DNA in the B form. The parameters used to simulate this structure are obtained from the crystallographic data from Arnott and Hukins. Molecules of poly dA.dT and poly dG.dC were built, with size varying from 1 base pair up to 13 base-pairs. The SV40 DNA molecule has 5226 base-pairs. It has $60 \%$ A-T and $40 \%$ G-C pairs. Also, for each pair of nucleotides, the position of the purines and pyrimidines on one strand or the other was randomized with equal probability for each strand. About 500,000 trials were made to determine how many times an $\mathrm{OH}$ radical react with a particular site. By knowing this and also from a knowledge of the number of times a particular type of site appears in a large molecule, the rate constant of $\mathrm{OH}$ reaction with either a base or a sugar has been determined.

\section{RESULTS}

Calculations for a simple nucleotide followed by double-stranded polynucleotides (poly dA.dT)

Table 1. Rate constants and reaction radii for DNA-OH reactions.

\begin{tabular}{lcc}
$\begin{array}{l}\text { Reaclion } \\
\text { sites }\end{array}$ & $\begin{array}{c}\text { Rate constant } \\
\left(1 \text { mole }^{-1} \mathrm{sec}^{-1}\right)\end{array}$ & $\begin{array}{c}\text { Reaclion } \\
\text { radius (A) }\end{array}$ \\
\hline Deoxyribose & $1.5 \times 10^{9}$ & 1.0 \\
Adenine & $5.8 \times 10^{9}$ & 3.8 \\
Thymine & $5.8 \times 10^{9}$ & 3.8 \\
Guanine & $8.2 \times 10^{9}$ & 5.4 \\
Cytosine & $5.8 \times 10^{9}$ & 3.8 \\
\hline
\end{tabular}




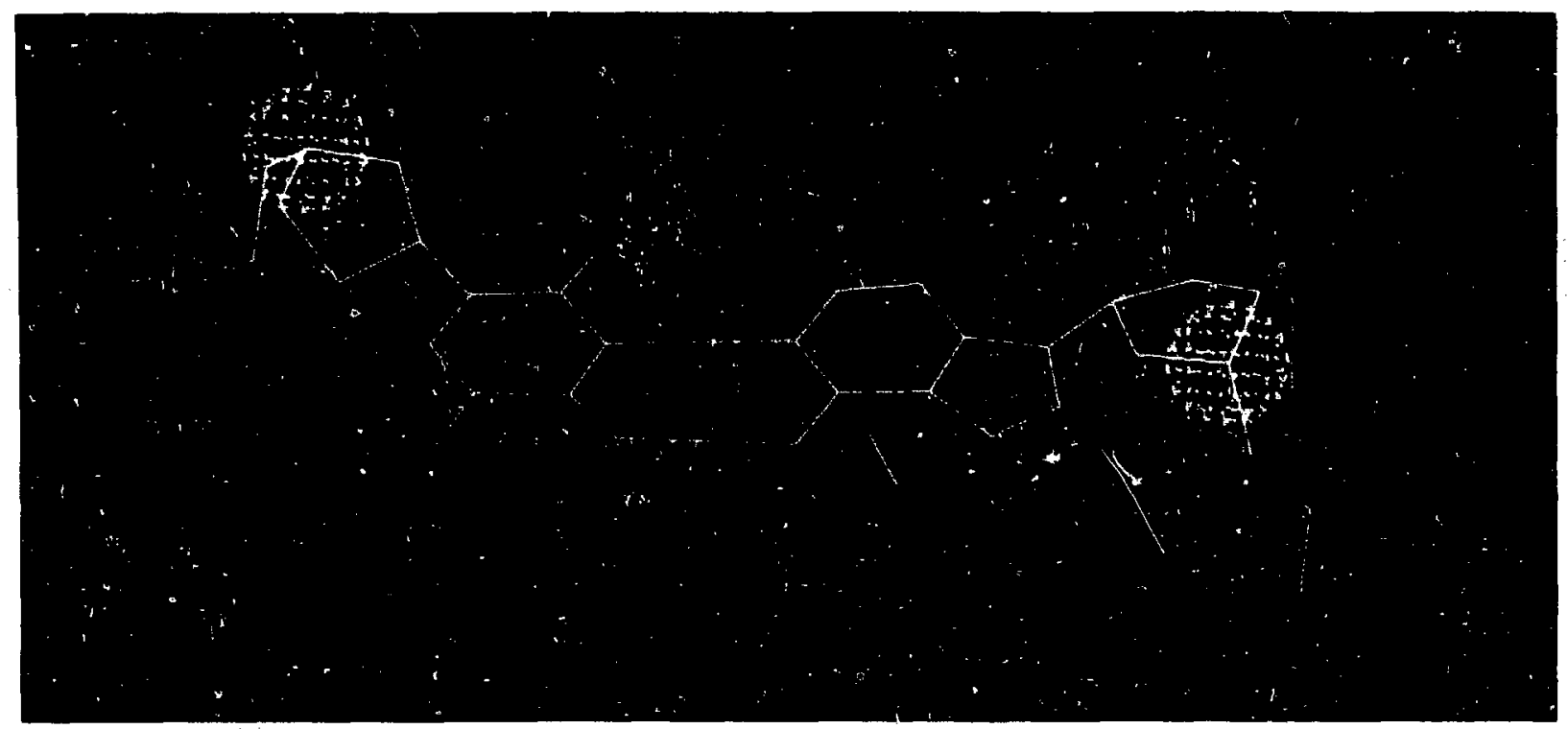

Fig. 1. A three-dimensional configuration of an Adenine-Thymine base pair has been projected on a plane. The spheres of influences (for $H$ reaction) around the reaction sites are indicated by dots over their respective surfaces. In our model, there are two reaction sites on each nucleotide, one on the sugar and the other one on the base.

(CBB 857-5206)

from 1 to 13 base-pairs show a progressive change of the effective rate constant values between $\mathrm{OH}$ and a sugar molecule and $\mathrm{OH}$ and a base molecule. In Fig. 2, the results are summarized and show that the rate of $\mathrm{OH}$-sugar and $\mathrm{OH}$-base (normalized by the isolated molecule rate constant for $\mathrm{OH}$-sugar reaction) against the number of base pairs. On the same figure, the ratio of base to sugar reaction is indicated. The initial increase of this ratio from isolated molecules to one nucleotide can

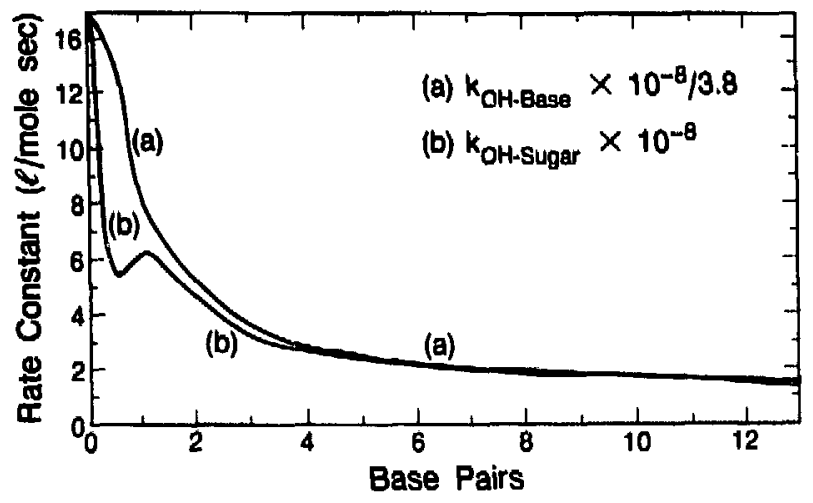

Fig. 2. The variation in rate constants for $\cdot H$-base (a) and $\cdot H$ sugar (b) reactions have been plotted against the number of base-pairs. Since the bases have larger spheres of influence, they control the overall reaclivity.

(XBL 8611-9057) be explained as follows: the bases being more reactive with $\mathrm{OH}$, have a larger reaction-radius and shield the sugar from $\mathrm{OH}$ interaction. When a second nucleotide is added, the bases start shielding each other and thereby reduce their influence in protecting sugar from reaction with $\mathrm{OH}$.

From our calculations we have also found that at $10-\mathrm{mM}$ Tris buffer concentration the rate constant of reaction, $\mathrm{K}_{\mathrm{OH}}$ base is $6.5 \times 10^{8} \mathrm{l} /$ mole $\mathrm{sec}$ in a DNA molecule. This should be compared with the isolated case of a base, which is $6.0 \times 10^{9}$. Hence the DNA structure plays a key role in reducing the rate constant by almost a factor of 10 , as was observed by Michaels and Hunt in their experimental measurements.

\section{CONCLUSION}

From our calculation it is clear that the secondary and tertiary structures of a polymer play a significant role in influencing the rate constant of reactions. For reaction of $\mathrm{OH}$ with a monomer unit in a po!ymer is smaller than that of $\mathrm{OH}$ with the same unit when in isolation. The reason for such a reduction is that the monomer unit in the polymer is shielded by other units in the polymer and hence there is an overall reduction in the reaction possibilities. 


\section{REFERENCES}

1. Huichinson, F. Chemical changes induced in DNA by ionizing radiation, Progress in Nucleic Acid Research and Molecular Biol. 32, 115 (1985).

2. Schoies, G., and Wilson, R.L. Pulse radiolysis of aqueous solutions of deoxyribonucleotides and of DNA: reaction with hydroxy-radicals. Chem. Commun., p. 17 (1969).

3. Michaels, H.B., and Hunt, J.W. Reactions of the hydroxyl radical with polynucleotides, Rad. Res. 56, 57 (1973).

4. Myers, L.S., Holis, M.L., and Theard, L.M. Pulse radiolysis of DNA and related pyrimidine compounds: reactions of the $\mathrm{OH}$. free radical.
In Radiation Chemistry (Advances in Chemistry Series 81) (Ed. R.F. Gould) Vol. 1, pp. 345-367, American Chemical Society, Washington, D.C. (1968).

5. Turner, J.E., Magee, J.L., Wright, H.A., Chatterjee, A., Hamm, R.N., and Ritchie, R.H. Physical and chemical development of electron tracks in liquid water, Rad. Res. 96, 437-449 (1983).

6. Arnott, S., and Hukins, D.W.L. Optimised Parameters for A-DNA and B-DNA. Biochem. Biophys. Res. Comm. 47, 1504 (1972).

7. Chatterjee, A., and Magee, J.L. Theoretical investigation of the production of strand breaks in DNA by water radicals, Radiation Protection Dosimetry, 13 (1-4), 137 (1985).

\section{LET DEPENDENCE OF CROSS SECTIONS FOR DNA STRAND BREAKS BY DIRECT EFFECTS OF CHARGED PARTICLES}

\section{William Holley, Aloke Chatterjee, and John L. Magee}

In an attempt to develop an understanding of the mechanisms of radiation damage to biological material, we start with the physical phenomena of energy deposition by ionizing radiation. In a living cell radiation damage is generally thought to be brought about by a combination of indirect and direct effects. Indirect effects refer to interactions of radiation with the surrounding media (usually water), which create reactive molecules (the most important of which seems to be the hydroxyl radical) that eventually cause the cellular damage. Direct effects, on the cther hand, refer to damage caused by deposition of energy by fast charged particles dirertly onto the biological molecule.

In particular, in the theoretical studies described here, we consider the effects of the direct deposition of energy from the core or penumbra of a fast charged particle onto a DNA molecule in dilute aqueous solution, as indicated schematically in Fig. 1. The actual physical energy deposition processes are statistical or stochastic in natuie. We use stopping power formulas to calculate the average energy absorption of DNA, and Poisson statistics to obtain a detailed description of the resulting states of excitation of the molecule. The average energy of an excitation (ionization) is riur known for molecules like nucleic acids or DNA, but we feel that $30 \mathrm{eV}$ is a reasonable figure (it is obtained from arguments about the absorption spectrum of water). Excitation of the DNA molecule is most likely followed by one of two different types of dissociation, deprotonation or direct dissociation, both of which mav lead to DNA strand breaks.

To make the problem more definite, let us consider a model system consisting of a beam of parallel incident particles impinging on a randomly oriented lincar SV40 DNA molecule. A given track can be characterized by its impact parameter or distance from the DNA central axis, the angle between track and DNA axis, and the track core radius Rc. First consider only the glancing collisions (which take place inside the track core). We use the detailed geometrical coordinates of the DNA to determine which sugar-phosphate molecular groups lie within the core of a track. We use stopping power theory and the Bragg rule to calculate the average glancing energy, $E_{g y}$ deposited in each of these molecular groups. In our present calculation we assume that energy directly deposited on the bases will not lead to strand breaks.

The average number of ionizing reactions at a site is given by $E_{g} / E_{x}$ where $E_{x}$ is the average energy per ionizing reaction $(30 \mathrm{eV})$. Because the energy loss process is stochastic in nature the number of ionizing reactions follows a Poisson distribution, and the probability of one or more reac- 

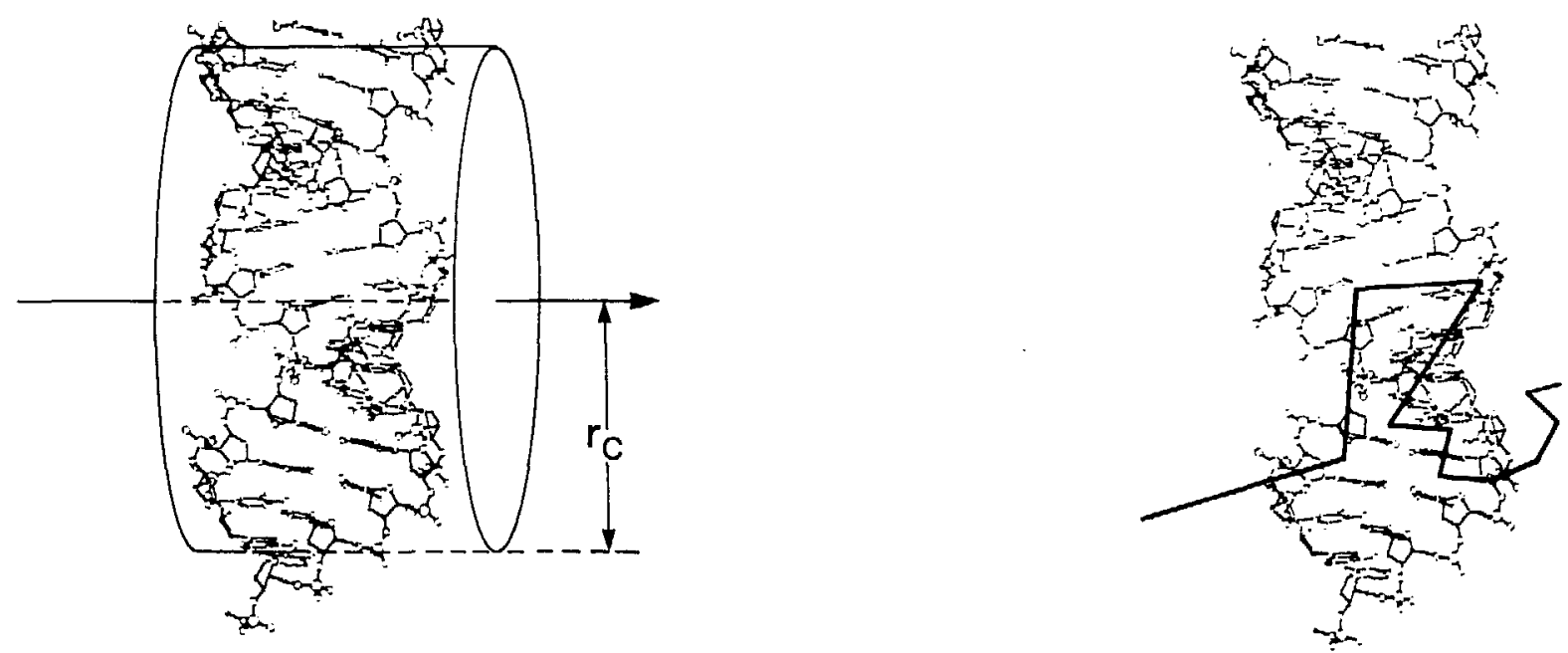

Fig. 1. Type of direct effect on DNA. Left: Excitation of DNA by core of heavy particle track or fast knock-on electron; Right: Excitation of DNA by slow electron in penumbra.

(XBL 864-1358)

tions, $P$, at a particular site, is given by

$$
P=1-\mathbf{e}^{-E_{t} / E_{2}} \text {. }
$$

In our calculations, in which we use Monte Carlo or random-walk techniques, we generate an "event" by choosing a random number, $R$, for each reaction site inside the track core. If $R>P$ we consider an ionizing reaction to have occurred, and we assume that a DNA strand break will occur at that site, either by direct dissociation or deprotonation. Breaks occurring close enough to each other on opposite strands (e.g., within 12 base pairs) are assumed to lead to double strand breaks. Events are then classified into the following catagories: 1) no breaks (NB), 2) one or more single strand breaks (SSB), and 3) one or more double strand breaks (DSB).

If we consider $N$ incident particle tracks uniformly distributed over an area A yielding \#SSB single strand breaks and \#DSB double strand breaks, the corresponding cross sections are given by

$$
\sigma_{\mathrm{SSB}}=\frac{\# \mathrm{SSB}}{\mathrm{N} / \mathrm{A}},
$$

$$
\sigma_{\mathrm{DSB}}=\frac{\# \mathrm{DSB}}{\mathrm{N} / \mathrm{A}}
$$

Up to this point we have just considered the glancing collisions, which only account for half of the direct energy deposition, and have neglected the contributions from the knock-on electrons. As a first approximation, we have included the penumbra effects by using a simple radial energy density distribution for the penumbra electrons to modify $E_{g}$, the average energy deposited at a DNA site.

Preliminary results of our calculated strandbreak cross sections for representative incident particles are plotted versus track LET in Fig 2 . The general behavior can be understood in the following way. First consider single strand breaks. At low LET the total energy deposited in a DNA molecule is small, even for a track at zero impact parameter, and therefore the probability of an ionization reaction and subsequent strand break is small. In this situation it is easily seen from Eq. 1 that the probability of a strand break is proportional to the energy deposited and therefore is proportional to LET as shown by the $45^{\circ}$ slope of the single-strand-break cross sections at low LET. At high LET, at least for 


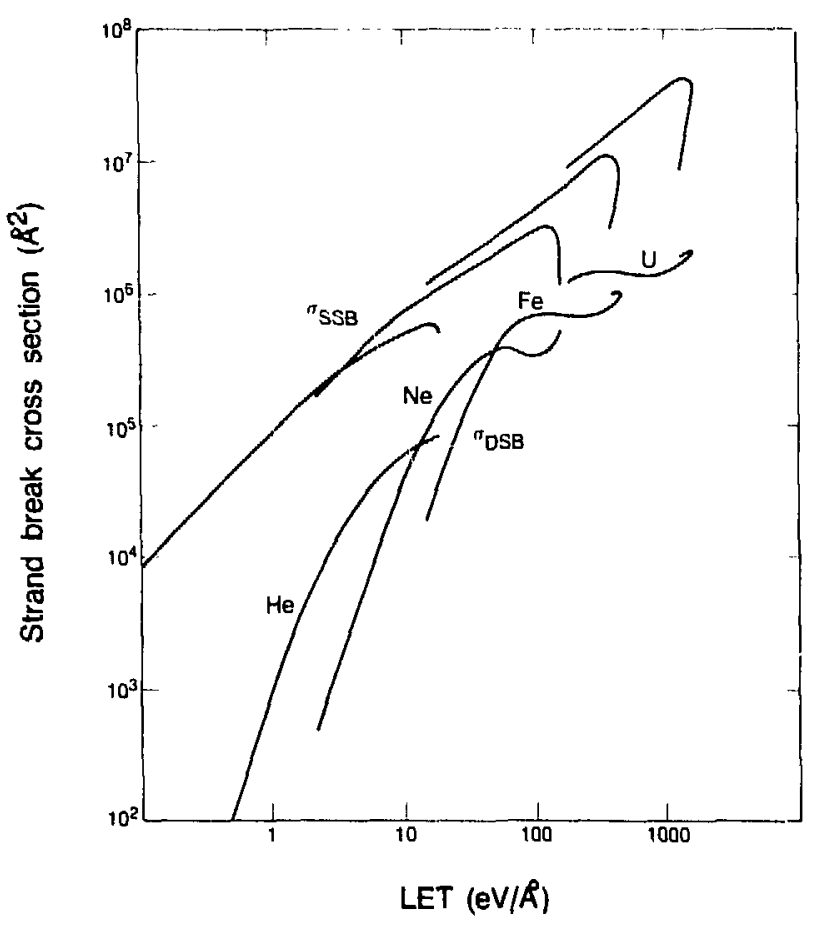

Fig. 2. Calculated single and double strand break cross sections due to direct effects as function of LET. $\quad(\lambda B L$ 854-9171) neon and heavier particles, any time the particle core passes through the DNA more than enough energy is deposited to produce a strand break, and the cross section is limited by the track core size. At this point, since higher LETs for a given particle correspond to lower velocities, the cross section decreases as the core radius shrinks.

On the other hand, double strand breaks produced by the mechanism considered here require two independent single strand breaks. At low LET the probability of two breaks is the product of probabilities of single breaks, and therefore the cross section behaves roughly as (LET) ${ }^{2}$. At high LET the cross sections again saturate due to the shrinking core radius. Near the maximum LET for a given heavy particle the energy density in the penumbra eventually becomes large enough to be effective in producing breaks, causing a moderate increase in the DSB cross section.

Our current effort is directed toward understanding the direct effects of electron tracks at high and low energies, Detailed knowledge of electron effects is needed to study radiation damage such as strand breaking caused by photon and electron beams. We are also using detailed electron-track calculations to treat more realistically the effects of the penumbra electrons from heavy-particle tracks.

\title{
AN EXPERIMENT TO MEASURE THE MULTIPLE COULOMB SCATTERING OF HEAVY CHARGED PARTICLES
}

\author{
Mervyn Wong, Walter Schimmerling, Bernhard A. Ludewigt, Mark H. Phillips, \\ Rochelle Frank, Stantey B. Curtis, Cornelius A. Tobias, John T. Walton," \\ Heinrich A. Sommer," Donald A. Landis," Norman W. Madden," and \\ Fred S. Goulding*
}

We report on an experiment clesigned to meassure the multiple Coulomb scattering of heavy charged particles at high energy. A particle travelsing a target undergoes multiple collisions, which result in deflection of the path of the particle from its original course. This multiple scattering leads to the spreading of a beam of particles as it traverses material, and increases the beam divergence. A consequence of such scatterings is that for any single particle incident on a target, it will emerge from the target along a trajectory somewhat deflected

\footnotetext{
"Office of Electronics Engineering, LBL
}

from its original one. The aim of this experiment is to measure this deflection, as shown in Fig. 1. Also measured is the lateral displacement of the particle before and after scattering. Thus far experiments have been performed for beams of neon, iron, and uranium ions scattered in targets of aluminum, copper, lead, and water.

Four sets of semiconductor position-sensitive detectors are used to determine the position of the particle before and after scattering. The first detector of each set is used to measure the horizontal $(x)$ position and the second detector is used to measure the vertical $(y)$ position. The incident-particle trajectory and the deflected-particle trajectory are 

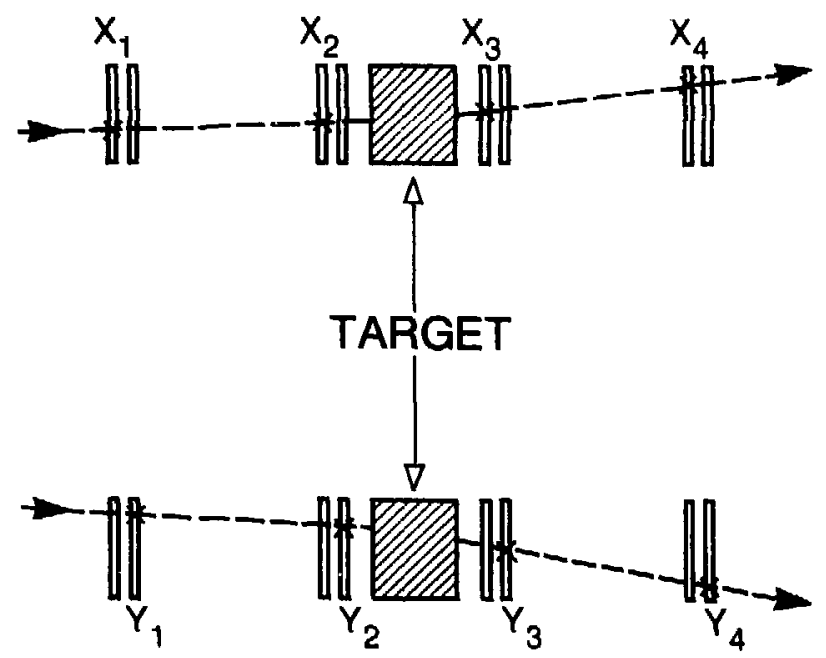

Fig. 1. Principle of the experiment. In the horizontal plane, the lateral position coordinates $x_{1}$ and $x_{2}$ of each particle prior to scattering and the lateral position coordinates $x_{3}$ and $x_{4}$ after scattering are recorded in position-sensitive detectors. In the same way, the lateral position coordinates $y_{1}, y_{2}, y_{3}$ and $y_{4}$ are measured in the vertical plane. For each event, the incidentparticle trajectory and the scattered particle trajectory are reconstructed in three dimensions from the measured particle coordinates. In addition to particle position, the energy deposited in each of the eight detectors by the traversing particle is recorded. In this way, an eightfold fast electronic coincidence requirement can be used to provide an event selection comprising elastically scattered beam particles. The angle through which the incident particle has scattered (the multiple scattering angle) for a particular event is calculated from the reconstructed incident and outgoing particle trajectories for that event.

(XBL 8611-9038)

both reconstructed in three dimensions from the measured particle coordinates.

In addition to the particle position, the energy deposited in each detector by the traversing particle is recorded. In this way an eight-fold fast electronic coincidence requirement can be used to constrain the accepted energy deposition pattern to elastically scattered beam particles.

Figure 2 is a photograph of the four detector sets placed on the optical bench used for the experiment. Figure 3 shows a close-up view of the four detector sets, Each detector set consists of two semiconductor detectors. The front detector measures horizontal position, the rear detector vertical position. The close-up shows in detail how each detector is mounted concentrically. Each detector has its own charge-sensitive preamplifier mounted at the base of the detector housing, as shown. The entire detector housing can be translated vertically and horizontally to position the detector centers on the beam axis.
Each detector is made from $\sim 800-\mu$ m thick silicon wafer, and has a diameter of $\sim 44 \mathrm{~mm}$. The silicon wafers were lithium-drifted to compensate for impurities. A 20- $\mu \mathrm{m}$ lithium layer, which acts as a resistive sheet, is diffused into the front face of each detector. Two aluminum contact electrodes are located at the ends of a diameter on this resistive sheet that serve to collect the charge flowing to them following passage of a charged particle through the detector. The asymmetry in the charge collected by these two electrodes is a measure of the position of traversal of the particle. $A$ detector with its contact electrodes located along a horizontal diameter measures horizontal position; one with electrodes along a vertical diameter measures vertical position.

An approximately 500-angstrom layer of gold was evaporated onto the rear face of each detector. The charge collected on this conducting surface is a measure of the total energy deposited in the detector by the traversing charged particle. A single ring contact electrode on the rear face suffices to collect this charge for preamplification.

All of the lithium-drifted position-sensitive silicon detectors used in this experiment were fabricated by the Instrument Science and Engineering Department at Lawrence Berkeley Laboratory.

Assuming a constant resistivity $\rho_{\mathrm{s}}$ (ohms) across the surface of the detector, the resistance of an area bounded by the circumference and a chord distant $x$ from the center is:

$$
R(x)=\frac{\rho_{s}}{2}\left[\frac{\pi}{2}+\sin ^{-1}\left(\frac{x}{r}\right)\right]
$$

where $r$ is the detector radius. If $q_{E}$ is the charge collected on the east electrode, $q_{w}$ the charge collected on the west electrode, and $Q$ the charge collected on the energy side of the detector, then by considering the charge flow to the position electrodes resulting from traversal of a particle at a horizontal displacement $u$ from the detector center, it can be shown that

$$
\frac{q_{E}-q_{W}}{Q}=\frac{2}{\pi} \sin ^{-1}\left(\frac{u}{r}\right)
$$

from which

$$
u=r \sin \left(\frac{\pi}{2} \frac{q_{E}-q_{W}}{Q}\right) .
$$

Similarly, for the vertical direction, 


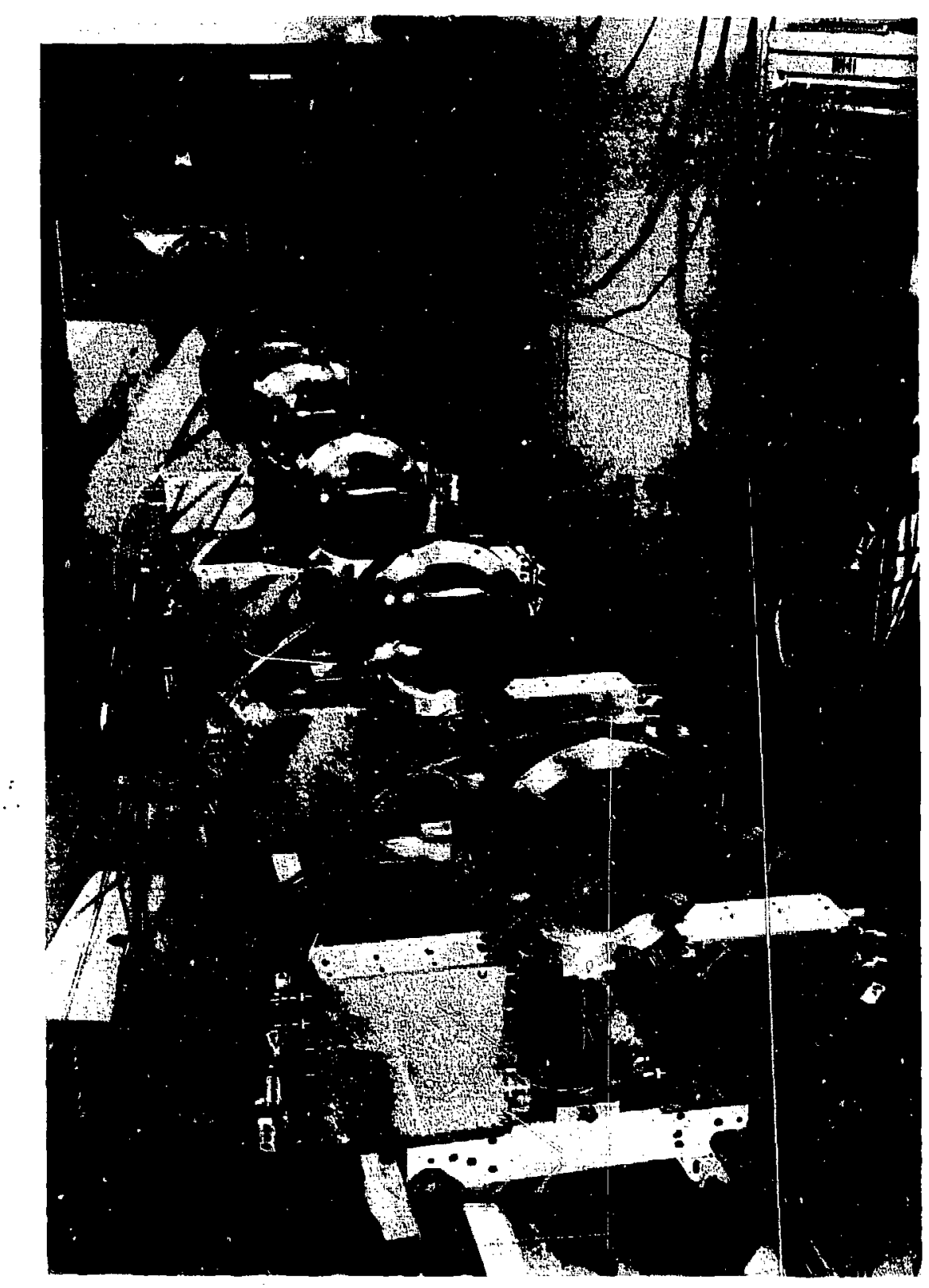

Fig. 2. Photograph of the four detector sets aligned on the optical bench used for the experiment. Vacuum pipes normally inserted between detector sets have been removed for clarity. The beam is deflected by the last bending magnet of the transport system and is incident on the detector array from the top lelt. This type of configuration, with the target removed, is used for recording straight-through events in which the pirticle has not been deflected, which are important for calibration of the detector system.

(CBB 863-1999)

$$
v=r \sin \left(\frac{\pi}{2} \frac{q_{B}-q_{t}}{Q}\right)
$$

Figure 4(a) is a two-dimensional image of a calibration pattern consisting of $1.5 \mathrm{~mm} \times 1.5 \mathrm{~mm}$ square holes, with a center-to-center spacing of
$4.7 \mathrm{~mm}$. The pattern was achieved by placing two bar collimators at right angles to each other directly upstream of detector set 4 . Each collimator consists of a stack of alternating brass bars and spaces. The collimator thickness is well in excess of the beam particle range; nuclear reaction products have been excluded from the pattern. 


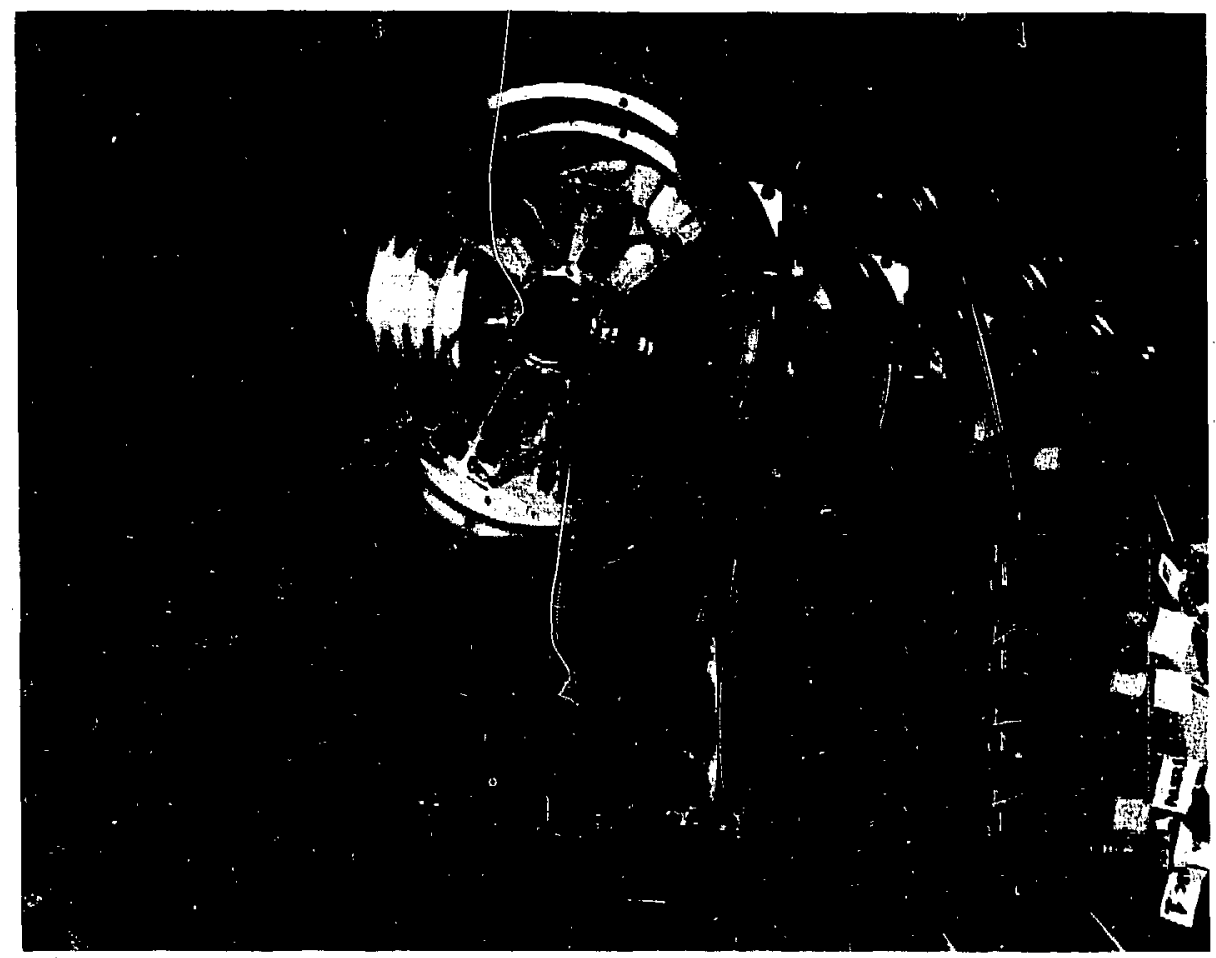

Fig. 3. Photograph shows a close-up view of the position sensitive detectors, their mounting and the associated charge-sensitive preamplifiers. Each set contains two detectors. The front and rear detector of each set can be rotated independently for adjustment of its angular orientation. The entire housing of each set can be translated horizontally and vertically to permit accurate alignment of the detector centers on the optical axis. Two position signals and one energy-deposition signal are fed from each detector to its charge sensitive preamplifier mounted directly on the housing base. A steady flow of dry nitrogen gas is maintained through each detector housing.

(CBB 863-2009)

As may be seen in Fig. 4(a), the image has position-dependent distortion, attribisted to nonuniform resistivity across the detecior surface, In each of the two orthogonal directions, the observed dependence of the distorted coordinate $u$ on the corrected spatial coordinate $x$ in each detector led to the following equation:

$$
x(u, v)=x_{0}+D_{1}(v) u+D_{2}(v) u^{2}+D_{3}(v) u^{3}
$$

where the beam centroid determines $x_{0}$ and where each of the coefficients $D_{i}$ is a function of the perpendicular coordinate $v$ :

$$
D_{i}(v)=A_{i}+B_{i} v+C_{i} v^{2} \quad(i=1,2,3)
$$

A similar set of equations is used for conversion from $v$ to $y$. The nine coefficients for each $x, y$ detector pair are obtained from a two-dimensional least-squares fit to the collimator pattern.

Figure 4(b) shows the two-dimensional image after the corrections described above have been made. The distortion is no longer apparent except near the detector edge. Image test patterns such as these for the four detector sets and equations of the form described above were used to obtain the spatial coordinates $x_{j}$ and $y_{j}(j=1,2,3,4)$ to describe each event for actual multiple scattering data.

Figure 5(a) shows projected angular distributions for the multiple scattering of $650-\mathrm{MeV} / \mathrm{A}$ uranium ions in a $2.7-\mathrm{mm}$ copper target. For each event, the angle through which the incident ion has scatiered has been projected onto the horizontal and vertical planes. The widths of the two distributions agree as expected, (to within $2 \%$ ), since they are two independent measurements of the same quantity. For comparison, Fig. 5(b) shows the same projected distributions measured with the same 'beam but with the copper target removed. Again, the width of the two distributions agree. As can be seen, they are considerably reduced. The measured effective angular resolution of the experiment is 1.2 milliradians. It is composed of the intrinsic 

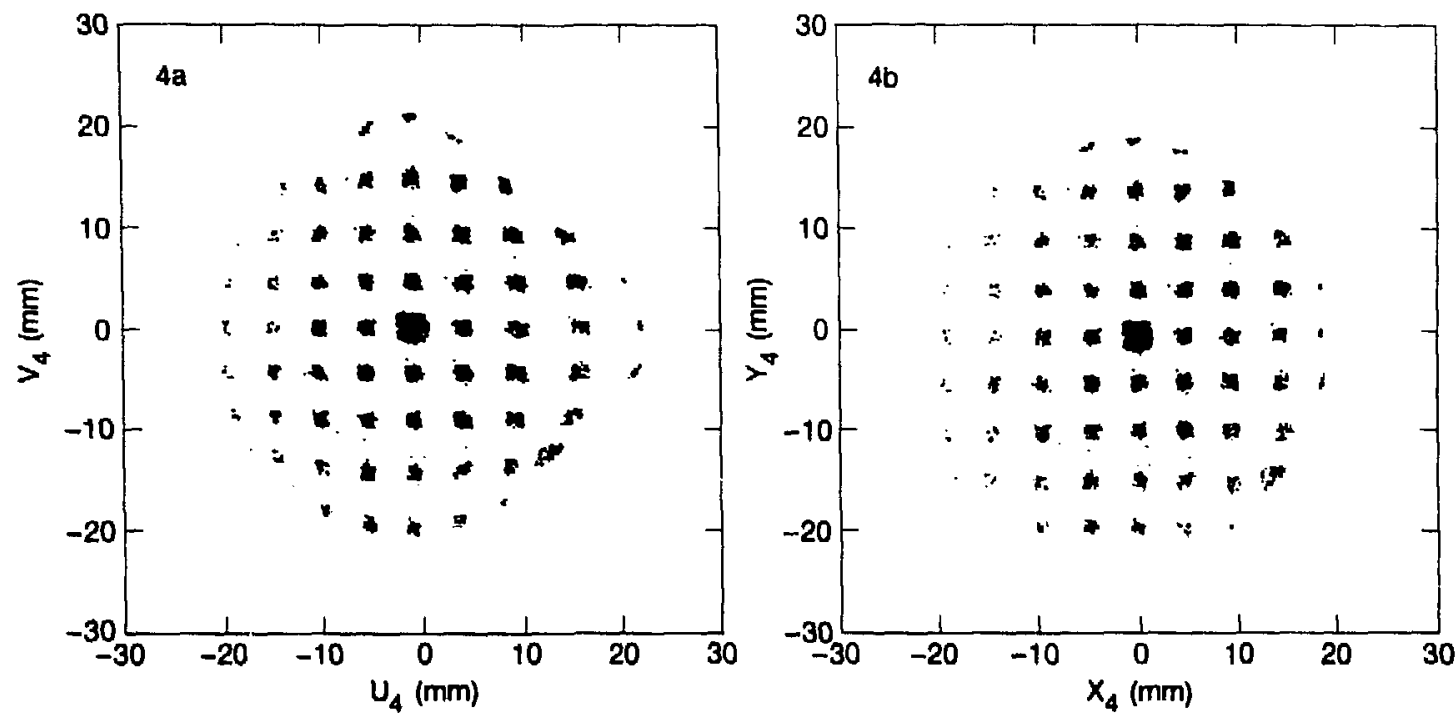

Fig. 4. (Left) Two-dimensional image of rectangular grid collimator pattern obtained using silicon position-sensitive detectors. This pattern was obtained by placing the grid-collimator directly in front of detector set 4 , and exposing it to a $650-\mathrm{MeV} / \mathrm{A}$ uranium-ion beam. The algorithm used for determining detector coordinates $\left(u_{4} v_{4}\right)$ from the charge collected at the three electrodes is discussed in the text. The distortion in the image pattern is attributed to non-uniformity in thickness of the resistive layer across the detector surface. (Right) The two-dimensional image after correcting for distortion. The detector coordinates $u$ and $v$ are converted to the corrected spatial cobrdinates $x$ and $y$ by transformation equations discussed in the text. Nine coefficients are used in the transformation; these are determined from a two-dimensional least squares fit to the collimator pattern.

(XBL 8617-9039)
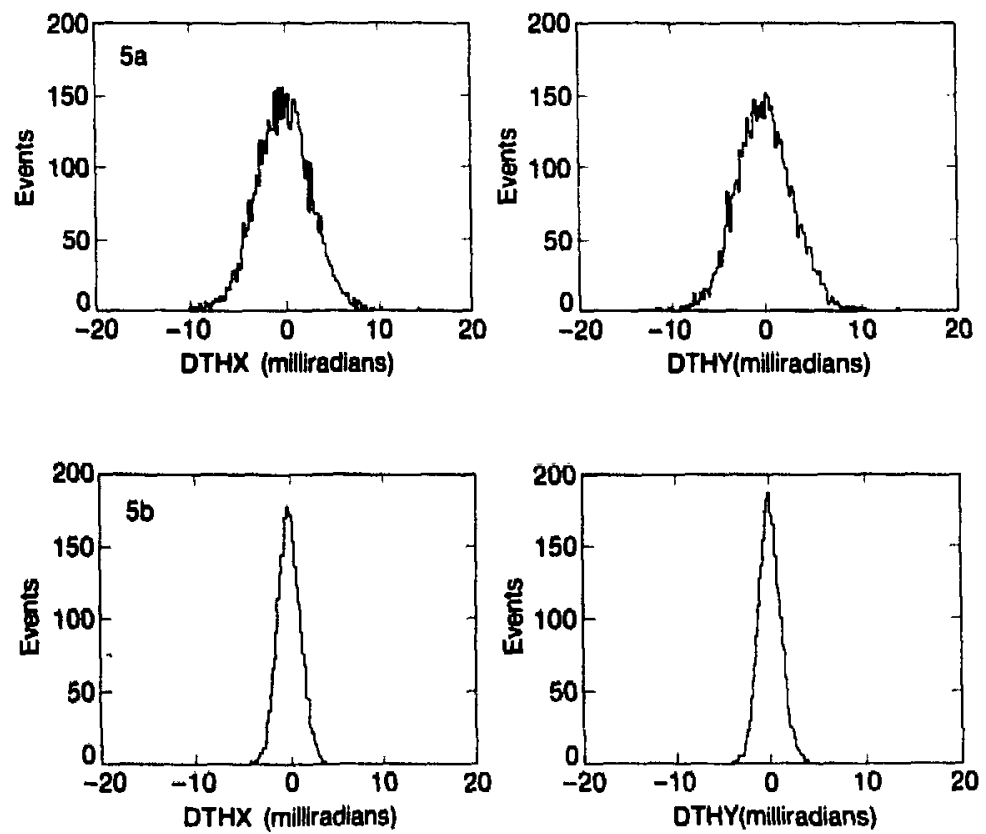

Fig. 5 (a) Measured projected angular distributions of multiple scattering for $650-\mathrm{MeV} / \mathrm{A}$ uranium ions in a 2.7 mm copper target. Left: horizontal projection. Right: vertical projection.

(b) The same distributions for straight-through tracks from $650-\mathrm{MeV} / \mathrm{A}$ uranium lons traversing the detector system. The measured argular resolution is $1.2 \mathrm{mrad}$ in each plane. This represents the overall angular resolution of the system for this beam, made up of intrinsic detector spatial resolution and residual scattering in the detectors. 
spatial resolution of the position-sensitive detectors and of the residual scattering in the material traversed by the beam. The angular resolution will be different for different beams.

Analysis of the full data set summarized in Table 1 and comparison of results obtained with multiple scattering theories are currently in progress.
Table 1 Summary of data collected during 12-month period.

\begin{tabular}{|c|c|c|}
\hline Beam & Energy $(\mathrm{MeV} / \mathrm{A})$ & Target \\
\hline Neon & 585 & $\begin{array}{l}\text { Water column, at seltings } 0,10 \text {, } \\
20,26.5,26.7 \mathrm{~cm}\end{array}$ \\
\hline Iron & 600 & $\begin{array}{l}\mathrm{Al}(2.5 \mathrm{~cm}), \mathrm{Cu}(1.3 \mathrm{~cm}) \\
\mathrm{Pb}(1.2 \mathrm{~cm})\end{array}$ \\
\hline Uranium & 650 & Al $(12.9 \mathrm{~mm}), \mathrm{Cu}(2.7 \mathrm{~mm})$ \\
\hline
\end{tabular}

\title{
COMPARISON OF DOSE LOCALIZATION PROPERTIES OF CARBON AND PROTON BEAMS
}

\author{
Eleanor A. Blakely, William T. Chu," Bernhard A. Ludewigt, Mark H. Phillips, and \\ Cornelius A. Tobias
}

New charged particle accelerators for radiotherapy applications are currently being discussed and designed. Thus, the question of which particle beams are optimal for specific clinical applications needs to be investigated. Dose localization plays an important role in the treatment of ocular melanoma, juxtaspinal tumors, pituitary tumors, and arteriovenous malformations. Precise beam localization greatly improves the dose distributions for treatment of lesions in and near critical structures.

Theoretical considerations based on multiple scattering theories indicate that carbon beams have better dose localization properties than proton beams because heavier charged particles scatter less than lighter ones. Our goal is to establish the size and importance of this effect experimentally.

We have continued our program of measuring dose distributions produced by narrowly collimated carbon and proton beams using an identical experimental setup for both beams, (as shown in Fig. 1), thus allowing a direct comparison. The beam was tuned into the cave as parallel as possible using two wire chambers and then collimated by two apertures. A $3-\mathrm{cm}$ diameter aperture was located at the beam entrance of the cave. Five and a half meters downstream of the beam entrance a smaller aperture defining the beam diameter was positioned next to the water tank. A vacuum pipe was installed between the two apertures to minimize multiple Coulomb scattering. Dose isocontour lines in the water phantom were measured using a diode as a dose-measuring device. The detector was scanned across the beams under computer control with the position and dose values being recorded by the computer.

We have measured dose distributions of 1 - and 2-cm-diameter carbon and proton beams in water and in air looking at multiple scattering and collimation effects. The resulting distributions for $1-\mathrm{cm}$ diameter beams with a range of $15.4 \mathrm{~cm}$ are shown in Fig. 2. It can be clearly seen that even close to the Bragg peak the carbon beam has only slightly broadened, whereas the proton beam shows a significantly wider, almost Gaussian distribution in that region. A second important feature is the much lower peak to plateau ratio of the proton beam compared to the carbon beam. Both features can also be seen in Fig. 3, which shows particle fluence distributions across the carbon and proton beams at different depths in water. In addition, Fig. 3 shows very good agreement between the measured beam profiles and calculated particle fluence distributions. The calculations were based on Moliere's multiple scattering theory.

In summary, we have found significant differences in the dose localization properties of proton and carbon beams, in full agreement with theoretical expectations. 


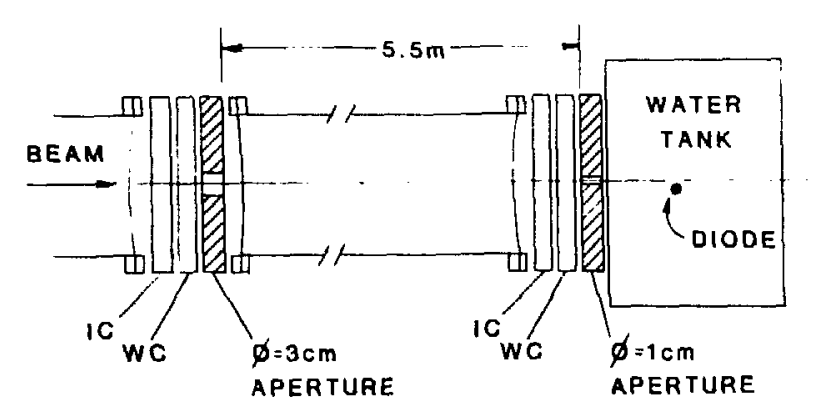

\section{CARBON - PROTON COMPARISON DOSE LOCALIZATION}

Fig. 1. Setup for dose distribution measuremen's in water phantom.

(XBL 864-1588)

Fig. 2. Isocontour lines of two dimensional dose distributions in a plane through the central beam axis.

(XBL 864-1586)
2.0

\section{CARBON}
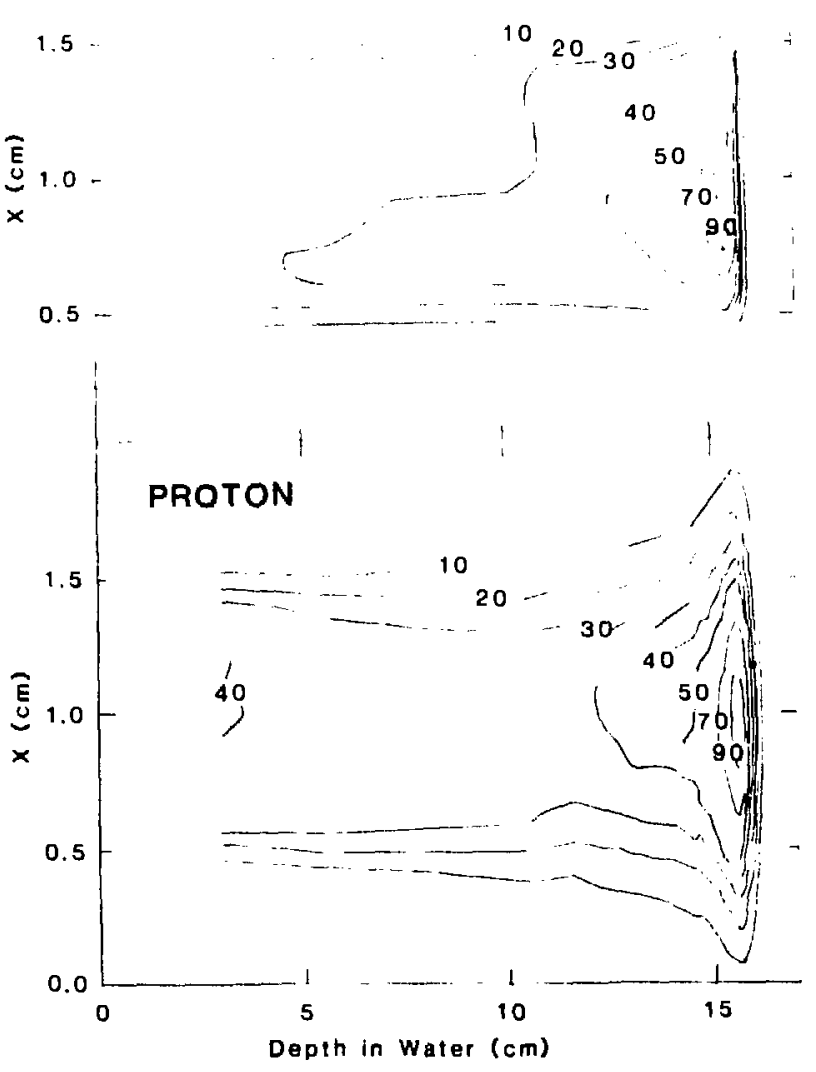
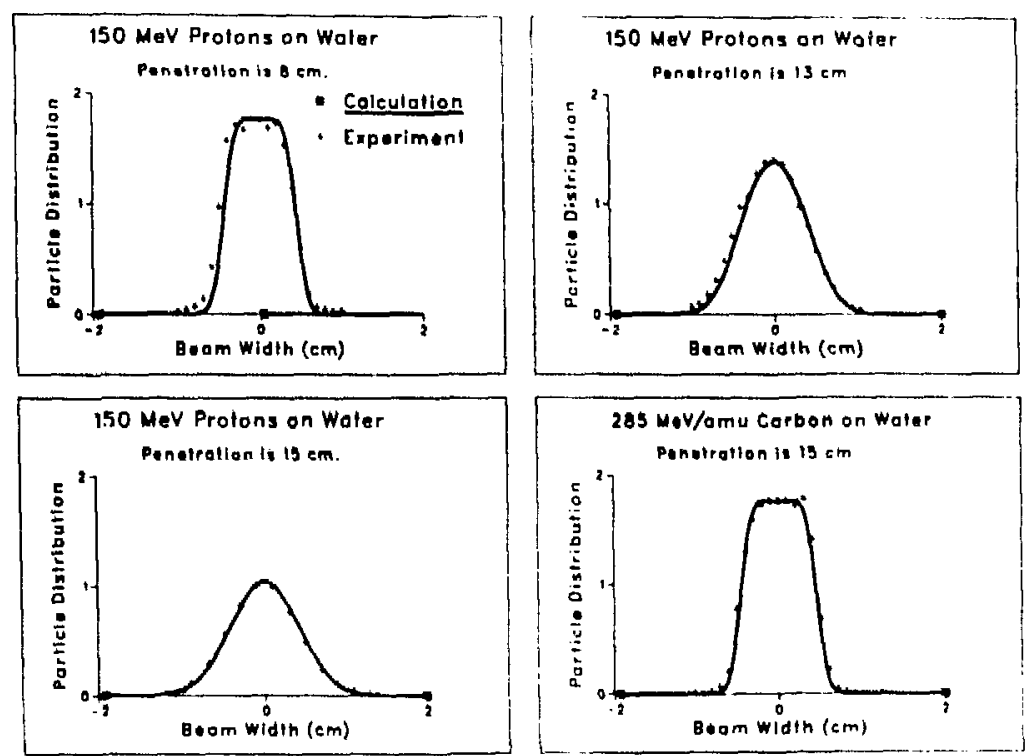

Fig. 3. Comparison of experimental (crosses) and calculated (lines) particle fluence distribulion for carbon and proton beams with a range of $15.4 \mathrm{~cm}$. From upper left: proton beam profile at $8-\mathrm{cm}, 13$. and $15 \mathrm{~cm}$ depth in water; carbon beam profile at $15 . \mathrm{cm}$ depth in water. 


\title{
Molecular and Cellular Radiobiology and Mutagenesis
}

\section{CELL CYCLE DEPENDENT X RAY OER: ROLE OF ENDOGENOUS GLUTATHIONE}

\author{
Eleanor A. Blakely, Ruth J. Roots, Polly Y. Chang, Leora Lommel, Laurie M. Craise, \\ Edwin H. Goodwin, and Elaine Yee*
}

Failures in clinical radiotherapy are thought to be related to the survival of radioresistant tumor cells. Cells are known to be resistant for a number of reasons, some of which are related to the oxygen concentration and to the phase of the cell cycle at the time of irradiation. In general, cells are more resistant when irradiated under hypoxia than under normal oxic conditions. Radiosensitivity is also known to significantly change during the cell division cycle.

There are only a few reports in the literature of experiments designed to measure the oxygen enhancement ratio (OER) in synchronously dividing mammalian cells in vitro using $x$ rays. ${ }^{1.7}$ These are difficult experiments to complete because there are many experimental variables can be hard to control. The published reports are divided between those that report that OER values do change through the cell cycle $e^{1,6,7}$, and those that report that they do not. ${ }^{2-5}$ Comparisons are confounded by experimental differences including the use of drugs in some cases to induce synchrony, the manner in which hypoxia was achieved, and the cell line that was studied.

We have re-examined the question of possible variations in aerobic and hypoxic radiosensitivity during the cell division cycle. Experiments to examine the cell-cycle-dependence of the oxygen effect were completed by synchronizing cells by mitotic selection, allowing them to progress to various ages, and then irradiating them with a fixed single dose or graded doses under aerobic and hypoxic conditions. Our techniques have been previously described. ${ }^{8,9}$

In each experiment, cell progression was monitored by incorporation of tritiated thymidine as described elsewhere. ${ }^{9}$ From the data presented in Fig. 1, the duration of the phases of the cell cycle for the experiments reported here were calculated to be 6.5 hours $\left(G_{1}+M\right)$, 9 hours (S-phase), 4 hours $\left(G_{2}+1 / 2 M\right)$ and a total generation time of 19.5 hours. More than $95 \%$ of the cells incorporated tritiated thymidine 11 hours after synchron-

-Student Employment and Training Program, University of California, Riverside ization, an indication of reproducible high-quality synchrony.

The data from six cell populations irradiated with graded doses between 3 and 17 hours after synchronization are summarized in Fig. 2 . The curves drawn through the data points are computer-assisted best-fit regressions. Replicate experiments were completed for cell populations at $3,8.1$, and 14 hours. Only single experiments were completed at 5,11 , and 17 hours.

Dose-ratio calculations of OER values were made for the pair of curves at each cell age and are presented in Table I. At high dose (e.g., 1\% survival level) the OER values increase from $2.6 \pm 0.1$ in $G_{1}$ /early $S$-phase to $3.0 \pm 0.2$ in late $S / G_{2^{-}}$ phase. At lower doses (e.g., 10\% survival level) the same trend is observed with the OER values again increasing from $2.6 \pm 0.2$ in $G_{1}$-phase cells to $3.2 \pm 0.2$ in late $S-G_{2}$-phase cells. These results are also plotted in Fig. 3. An approximately linear increase in the OER is noted as the cells progressed from early $G_{1}$-phase to late $G_{2}$-phase.

In an attempt to experimentally approach a possible mechanism for the observed cell-cycle-

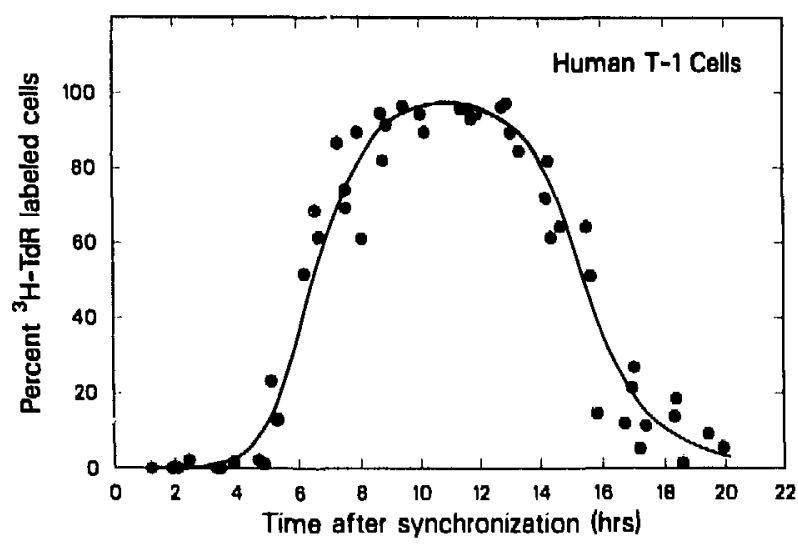

Fig. 1. Reproducibility of DNA synthetic phase in synchronized controls. Composite of DNA labeling patterns from autoradiographs of unirradiated controls pooled from four experiments pulsed with $3 H-T d R$ at various times after synchronization. These control cultures were held at room temperature for a 20 . min period prior to labeling 10 correspond to the gas exchange time in the irradiated samples.

(XCG 869-7409) 


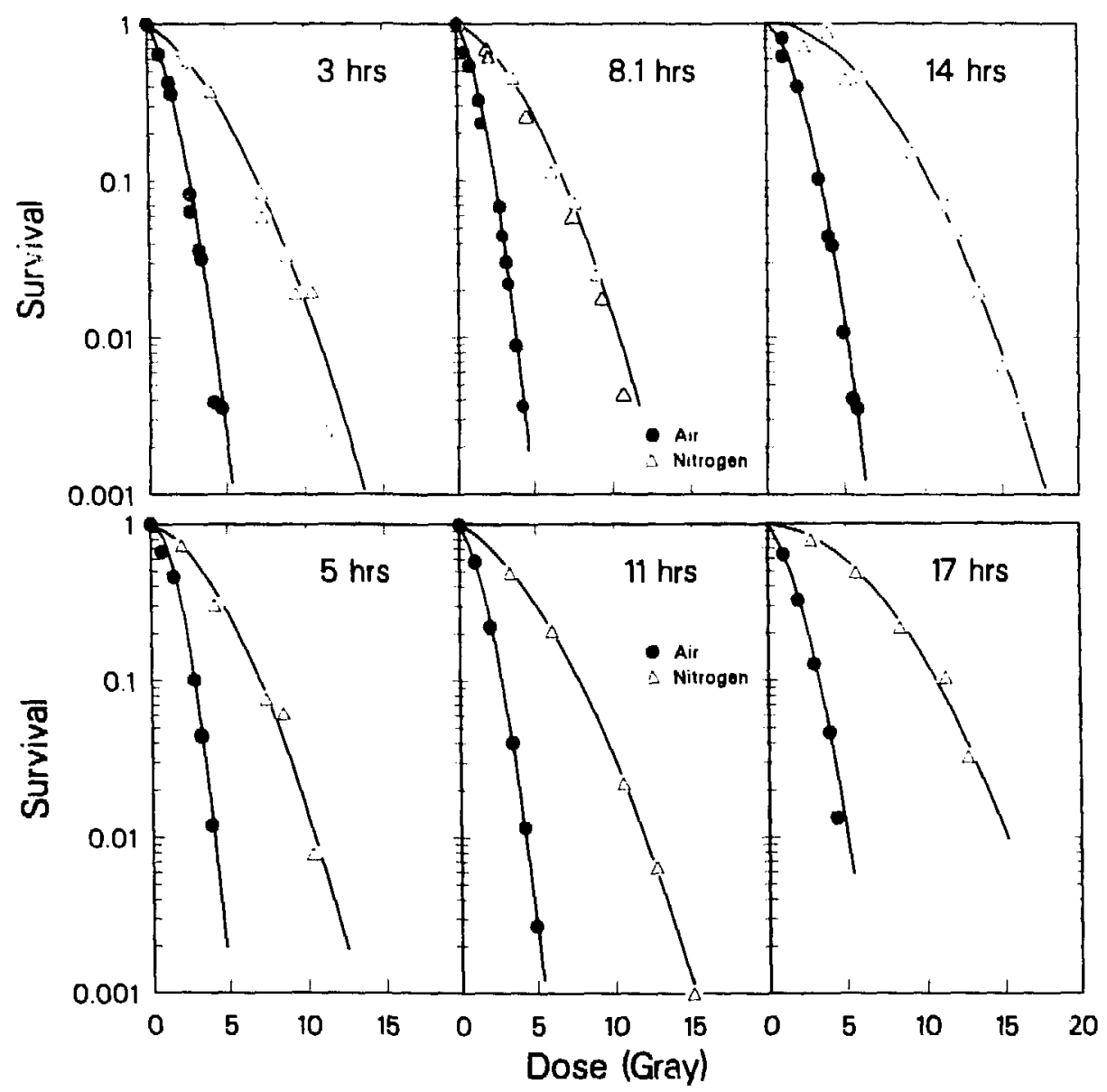

Fig. 2. Complete $x$-ray dose-response curves for cell populations irradiated post-synchronization in air (0) or in nitrogen ( $\Delta$ ) at $3 \mathrm{hr}, 8.1 \mathrm{hr}$, or $14 \mathrm{hr}$ (upper panel) or at $5 \mathrm{hr}, 11 \mathrm{hr}$, or $17 \mathrm{hr}$ (lower panel).

(XCG 869-12126)

dependent increase in the OER, we made some preliminary measurements of nonprotein sulfhydryls at various times during the generation time. This work was done in collaboration with W. Blakely and D. Dodgen in the Radiation Sciences Division at the Armed Forces Radiobiological Research Institute in Bethesda, Maryland. In parallel experiments with synchronized human T-1 cell populations, glu-

Table 1. Oxygen enhancement ratios.

\begin{tabular}{lcccccc}
\hline $\begin{array}{l}\text { Time after synch- } \\
\text { ronization (hr) }\end{array}$ & 3 & 5 & 8.1 & 11 & 14 & 17 \\
\hline OER-10\% survival & 2.58 & 2.47 & 2.75 & 2.78 & 2.98 & 3.22 \\
& $(a)(0.15)$ & $(0.27)$ & $(0.16)$ & $(0.09)$ & $(0.12)$ & $(0.24)$ \\
OER-1\% survival & 2.57 & 2.56 & 2.58 & 2.76 & 2.86 & 2.99 \\
& (a)(0.08) & $(0.13)$ & $(0.08)$ & $(0.04)$ & $(0.06)$ & $(0.15)$ \\
\hline
\end{tabular}

(a) $=( \pm 95 \%$ confidence interval) tathione (GSH) and glutathione disulfide (GSSG) levels were measured by the Tietze's assay, ${ }^{10}$ which were also found to increase steadily over the same period as the cells progressed from $G_{1}$-phase into $G_{2}$-phase.

The increased survival OER in S-phase cells was the result of a greater hypoxic radioresistance compared to that measured with $G_{1}$-phase cells. The mechanisms responsible for the radiation response of mammalian cells involve a number of factors, one of which appears to be GSH levels, especially under conditions of hypoxia. Our data are consistent with the hypothesis that age-dependent fluctuations in GSH content may be correlated with a linear increase in OER during the cell cycle from early $G_{1}$-phase to late $G_{2}$-phase. In addition to providing a potential modification of both the initial damage yields and spectrum, GSH levels, or more relevantly GSH contribution to the redox state, may play an important role in the cells' capacity to 


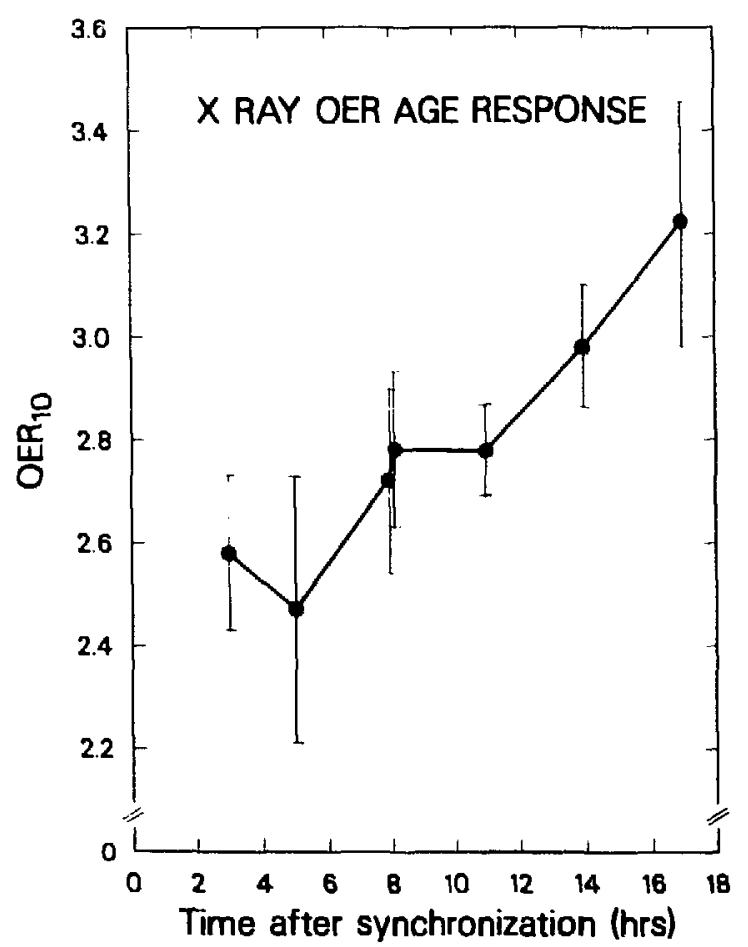

Fig. 3. Summary plot of computer-fitted OER values at $10 \%$ survival from complete aerobic and hypoxic dose-response curves measured from synchronized populations at six cell ages. Data are from five separate experiments.

(XBL 869-3488).

repair radiation injury. Future experiments will specifically examine this point.

\section{REFERENCES}

1. Berry, R.J. A reduced oxygen enhancement ratio for s-ray survival of Hela cells in vitro, after treatment with methotrexate. Nature 208, 1108-1109 (1965).
2. Kruuv, J., and Sinclair, W.K. X-ray sensitivity of synchronized Chinese hamster cells irradiated during hypoxia. Radiat. Res. 36, 45-54 (1968).

3. Legrys, G.A., and Hall, E.J. The oxygen effect and $x$-ray sensitivity in synchronously dividing cultures of Chinese hamster cells. Radiat. Res. 37, 161-172 (1969).

4. Kimler, B.F., Sinclair, W.K., and Elkind, M.M. $N$-ethyl-maleimide sensitization of $x$-irradiated hypoxic Chinese hamster cells. Radiat. Res. 71, 204-213, (1977).

5. Chapman, J.D., Webb, R.G., and Borsa, J. Radiosensitization of mammalian cells by $p$ nitroacetophenone, I. Characterization in asynchronous and synchronous populations. Int. I. Radiat. Biol. 19, 561-573 (1971).

6. Sapozink, M.S. Oxygen enhancement ratios in synchronous HeLa cells exposed to low-LET radiation. Radiat. Kes. 69, 27-39 (1977).

7 Pettersen, E.O., Christensen, T., Bakke, O., and Oftenbro, R. A change in the oxygen effect through the cell cycle of human cells of the line NHIK-3025 cultivated in vitro. Int. J. Radiat. Biol. 31, 171-184 (1977).

8. Blakely, E.A., Tobias, C.A., Yang, T.C.-H., Smith, K.C., and Lyman, J.T. Inactivation of human kidney cells by high energy monoenergetic heavy-ion beams. Radiat. Res. 80, 122 (1979).

9. Blakely, E.A., Chang, P.Y., and Lommel, L. Cell-cycle dependent recovery from heavy-ion damage in $G_{1}$-phase cells. Radiat. Res. 104, S145-S157 (1985).

10. Tietze, F. Enzymic method for quantitative determination of nanogram amounts of total and oxidized glutathione: Applications to mammalian blood and other tissues. Anal. Biochem. 27, 502-522 (1969).

\section{LESIONS IN CHO CELL CHROMATIN PRODUCED BY VERY HIGH LET HEAVY IONS}

\section{Edwin H. Goodwin, Eleanor A. Blakely, and Cornelius A. Tobias}

During the past year we have continued our studies of the breakage of Chinese hamster ovary (CHO) cell chromatin induced by $x$ rays and heavy ions. Irradiated $\mathrm{G}_{1}$-phase $\mathrm{CHO}$ cells are fused to mitotic cells using polyethylene glycol. As the cytoplasms merge, factors from the mitotic cell act upon the $G_{1}$ chromatin, forcing "premature chromosome condensation" (PCC) of the interphase chromatin. ${ }^{1}$ After staining, PCC fragments can be observed by oil immersion microscopy. Irradiation causes a dose-dependent increase in the number of PCC fragments. ${ }^{1,2}$ The PCC method is the only 
technique currently available with the capability of detecting chromatin breaks in individual interphase cells soon after irradiation. In our earlier studies we suspected that some rejoining of chromatin breaks was occurring during the irradiation period and during the PCC procedure. We now do irradiations on ice and pretreat samples with cycloheximide. These modifications inhibit repair processes, leading to a better estimate of the initial damage. ${ }^{3}$

Last year we reported data with $x$ rays and neon-ion beams. ${ }^{4}$ The distribution of chromatin breaks per cell was found to follow Poisson statistics after irradiation with $x$ rays. However, neonion Bragg peak irradiation produced a nonPoissonian break distribution. We were able to successfully model the neon data with the Neyman distribution, 4.5 This investigation has now been extended into the very high LET region using niobium and uranium beams produced at the Bevalac. Results are shown in Fig. 1. As we found before, the $x$-ray data can be fit very well by Poisson statistics. This indicates that chromatin breaks are induced randomly among the cells by $x$ rays. The niobium beam is only slightly more efficient than $x$ rays at producing chromatin breaks, but the distribution of these lesions is remarkably different. After 60 rad of $x$ rays almost all cells have sustained one or more chromatin breaks. Sixty rad of niobium-ion irradiation leaves almost $40 \%$ of the cells with no detectable lesions, while those cells that were hit by an ion tend to be heavily damaged. A major feature of this distribution is a block of cells containing from 6 to 12 lesions. We believe this feature is best understood by assuming that a heavy-ion track intersects chromatin from 6 to 12 times during its passage through the cell nucleus 'the nucleus being thicker in the center than at the edges). The high LET of the niobium ions leads to a high probability of severing chromatin at each intersection. The production of multiple lesions within a nucleus by a single ion has been called "clustering." 5,6 As a result of clustering, the distribution of chromatin breaks per cell induced by heavy ions is not Poissonian but can be modeled by the Neyman distribution. ${ }^{5}$

Despite the much higher LET of the uranium ion beam there is a qualitative similarity between the niobium and uranium-induced lesion distributions. This similarity is primarily due to the biologi- cal structure of the cell nucleus, i.e., the ion crosses chromatin from 6 to 12 times with a high probability of producing a lesion at each crossing. Both the niobium and uranium distributions have a few cells with a small number of lesions (from 1 to 5 per cell). These lightly damaged cells were probably hit by energetic electrons in the penumbra of one or more particle tracks but were spared a direct hit by a more damaging track core. The reason that there are more of these lightly damaged cells in the uranium distribution could be due to the longer range of energetic electrons in the penumbra of tracks produced by the faster moving uranium ions. On a per rad basis the uranium beam is much less effective than the niobium beam in the production of chromatin breaks. This is expected since, at a higher LET, there are fewer uranium particles per rad compared to niobium.

\section{REFERENCES}

1. Waldren, C.A., and Johnson, R.T. Analysis of interphase chromosome damage by means of premature chromosome condensation after $x$ and ultraviolet radiation. Proc. Natl. Acad, Sci. 71, 1137-1141 (1974).

2. Cornforth, M.N., and Bedford, J.S. X-rayinduced breakage and rejoining of human interphase chromosomes. Science 222, 1141-1143 (1973).

3. Hittelman, W.N., and Pollard, M. A comparison of the DNA and chromosome repair kinetics after $\gamma$ irradiation. Radiat. Res. 92, 497-509 (1982).

4. Goodwin, E.H., Blakely, E.A., and Tobias, C.A. Multiple chromatin breaks produced by neon ions. Biology and Medicine Division Annual Report, Lawrence Berkeley Laboratory report LBL-20345, 102-103 (1985).

5. Tobias, C.A., Goodwin, E.H., and Blakely, E.A. Theoretical distributions of DNA lesions in heavy-ion tracks. Thirty-fourth Annual Meeting of the Radiation Research Society, Las Vegas, Nevada, 1986. (Abstract)

6. Neyman, 1., and Puri, S. A hypothetical stochastic mechanism of radiation effects in single cells. Proc. Roy. Soc. (London) B213, 139-160 (1981). 


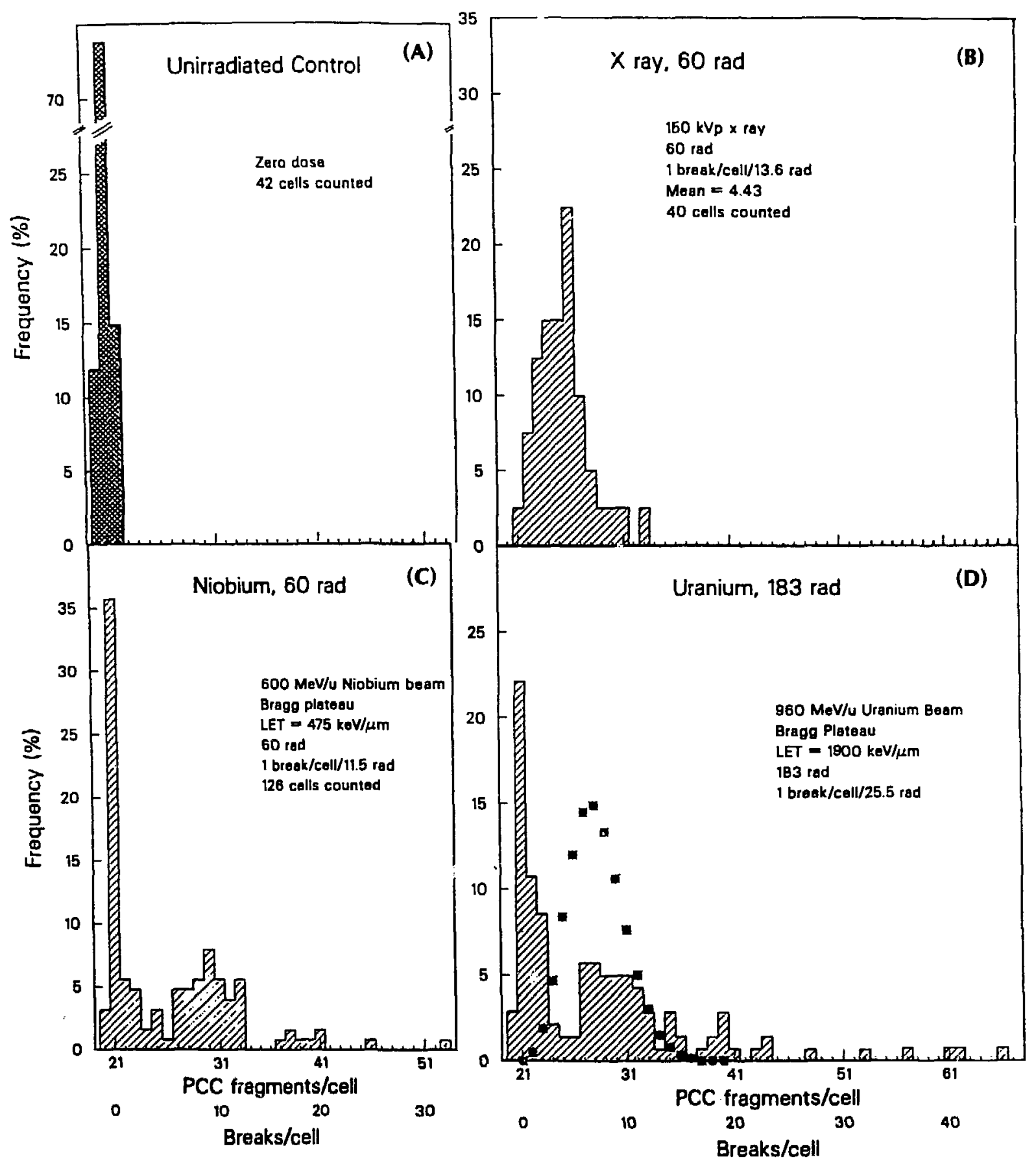

Fig. 1. (A) (upper left) Unirradiated control. The distribution is sharply peaked about zero breaks/call. There are 21 chromosomes in this cell line. (B) (upper right) $60 \mathrm{rad} \times$ ray. (C) (lower left) 60 rad niobium. (D) (lower right) 183 rad uranium. The dark circles represent a Poisson distribution with the same mean as the data. 


\title{
DNA LESIONS IN CELL NUCLEI FOLLOW NEYMAN A DISTRIBUTION
}

\author{
Cornelius A. Tobias, Edwin H. Goodwin, and Eleanor A. Blakely
}

Extremely small amounts of energy from ionizing radiations deposited in the cell nucleus can produce significant biological effects. This is usually explained by assuming that the energy depositions are discrete statistical processes and that an ionization or excitation must occur in a highly specific locality within the cell nucleus. The well known "target" and "hit theories" are based on such considerations. In most of the quantitative models in radiation biology assumptions are made that the lesions are produced in a statistically independent manner and that the targets are homogeneous structures. Kellerer and Rossi, ${ }^{1}$ however, suggested that the distribution of lesions may deviate from the usual Poisson distribution.

We have recently initiated some investigations into the number and distribution of radiolesions produced by $x$ rays and accelerated atomic nuclei in mammalian cells. This is a difficult task because the initial lesions in DNA are usually of atomic dimensioris. During most of the cell division cycle, we cannot observe DNA in the cell nucleus without killing the cells and highly denaturing the genetic material. Goodwin et al. ${ }^{2}$ used a special technique, premature chromosome condensation (PCC) to make chromatin lesions visible. It was found that these lesions are produced in a random Poisson distribution after doses of $x$ rays, but exposures to accelerated heavy ions result in distributions of lesions that were very different from Poisson.

\section{CLUSTER STATISTICS}

To explain such data, we decided to use cluster statistics, as these appear in the theory of contagious distributions. This theory was initially developed in 1939 by the late Jerzy Neyman. Neyman attempted to quantitate the dynamics of spread of contagious diseases. He assumed that initially a small cluster of individuals, located near each other, become inferted; the infection then spreads by diffusion and contact into the rest of the population. In 1943, William Feller demonstrated that random events in nonuniform population densities must be treated in a similar manner. ${ }^{3}$ in 1981, Neyman and Puri proposed a cluster theory for the cellular effects of radiation. They assumed that cells have two types of targets, repairable and irrepairable, as in the model of Kappos and Pohlit. ${ }^{4}$

The initial events in the energy transfer from accelerated heavy nuclei to matter include the formation of clusters of delta rays, and clusters of ionized or excited atoms are left behind. The energy is initially concentrated in a nonuniform .nanner into tracks with a complex radial structure. Chatterjee and Magee have been studying the radial distribution of track structures in homogeneous media. With the passage of time, crucial macromolecular lesions are established as a result of diffusioncontrolled interactions of free radicals and DNA of the chromosomes. Among the DNA lesions are base alterations, strand scissions, and cross-links with other molecules. In normal mammalian cells most of these lesions are very efficiently repaired. The deleterious effects of heavy ions are often associated with their ability to produce doublestranded scissions in DNA, which are difficult and sometimes impossible to repair. For lethal events there are about 50 double-stranded lesions per cell. However, there are some difficulties with assuming that all double-stranded scissions are equally important; there seem to be too many of these per cell, and they are sometimes produced as part of the normal progression of cells through the division cycle. Another view is that chromatin breaks may be more closely related to lethal events than DNA scission. These probably involve not only DNA strand breaks, but also interruption in supporting structures as well. The experiments in our laboratory indicate that chromatin breaks formed in the $\mathrm{G}_{1}$ part of the cell cycle comprise about 10 to $15 \%$ of all double-stranded DNA scissions.

The model is not presented here in mathematical detail. We proceed by calculating the probability that the track will penetrate through the cell nucleus; this is a Poisson process. The number of places that crossing will occur with chromatin, $n$, depends on the amount of DNA in the cell nucleus, its geometrical configuration and on the variations in geometrical structure. Finally, it is important to know the magnitude of the conditional probability, $p$, that a lesion will be established at crossover regions. In Fig. 1 we show theoretical distributions of chromatin breaks per cell at various dose levels for $\mathbf{n}$ crossings and conditional probability $p$. Figure 2 shows how the distribution can vary with the value of $p$; the distribution exhibits 


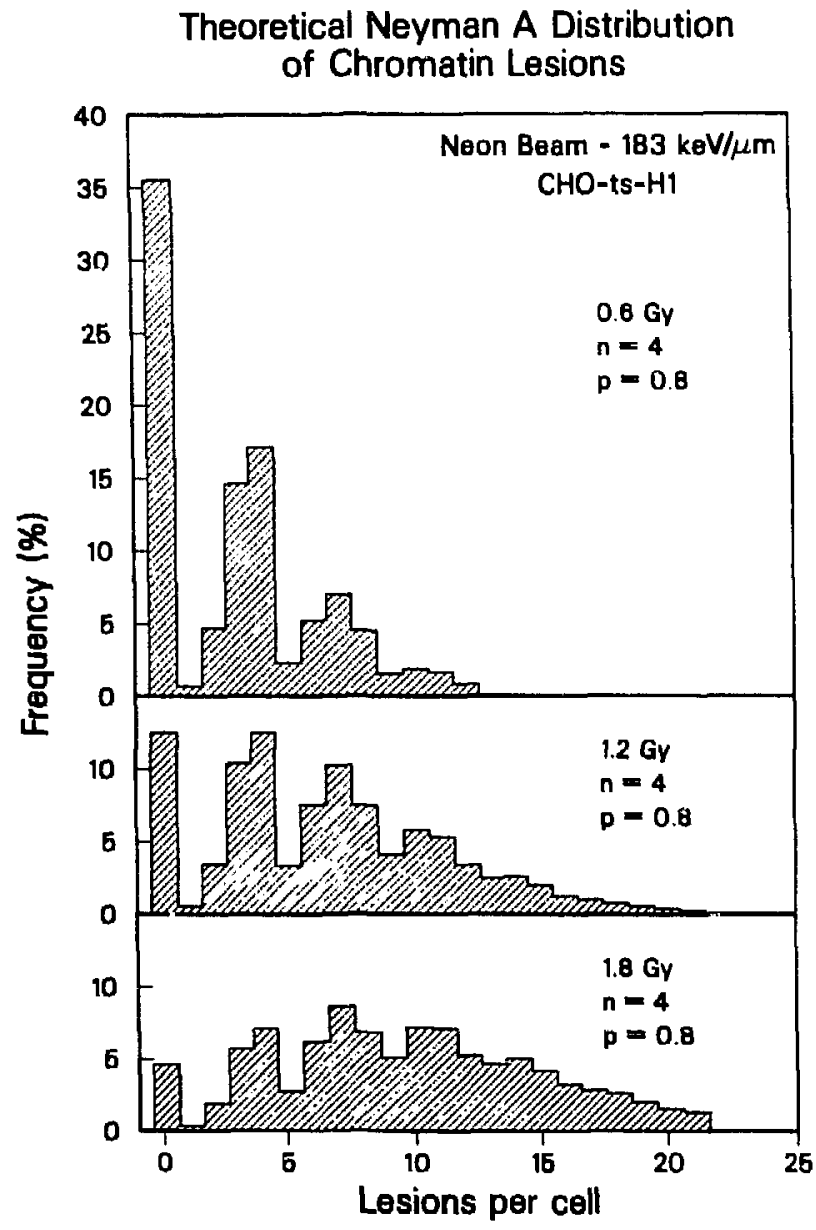

Fig. 1. Theoretical Neyman frequency plots of lesion clusters for three dose levels. Abscissa: number of lesions per cell.

(XCG 864-7192)

discrete peaks if the value of $p$ is about unity. Figure 3 demonstrates that the mean number of lesions produced per cell, the value that one usually obtains from chemical studies, is incorrectly predicted by assuming an ordinary Poisson distribution. When experimental data from experiments with Chinese hamster ovary cells were compared to the cluster model, it became clear that in the case of low-LET radiation, the ordinary Poisson distribution applies, whereas for high-LET neon particles the cluster distribution must be used (Fig. 4). We are engaged in a detailed experimental study of the ability of various heavy ions to produce clusters of chromatin lesions in mammalian cells. A secondary electron from $x$ rays has only about a 1:1000 chance to produce a chromatin lesion. Neon ions produce as many as four chromatin breaks per track, whereas still heavier particles can produce 10

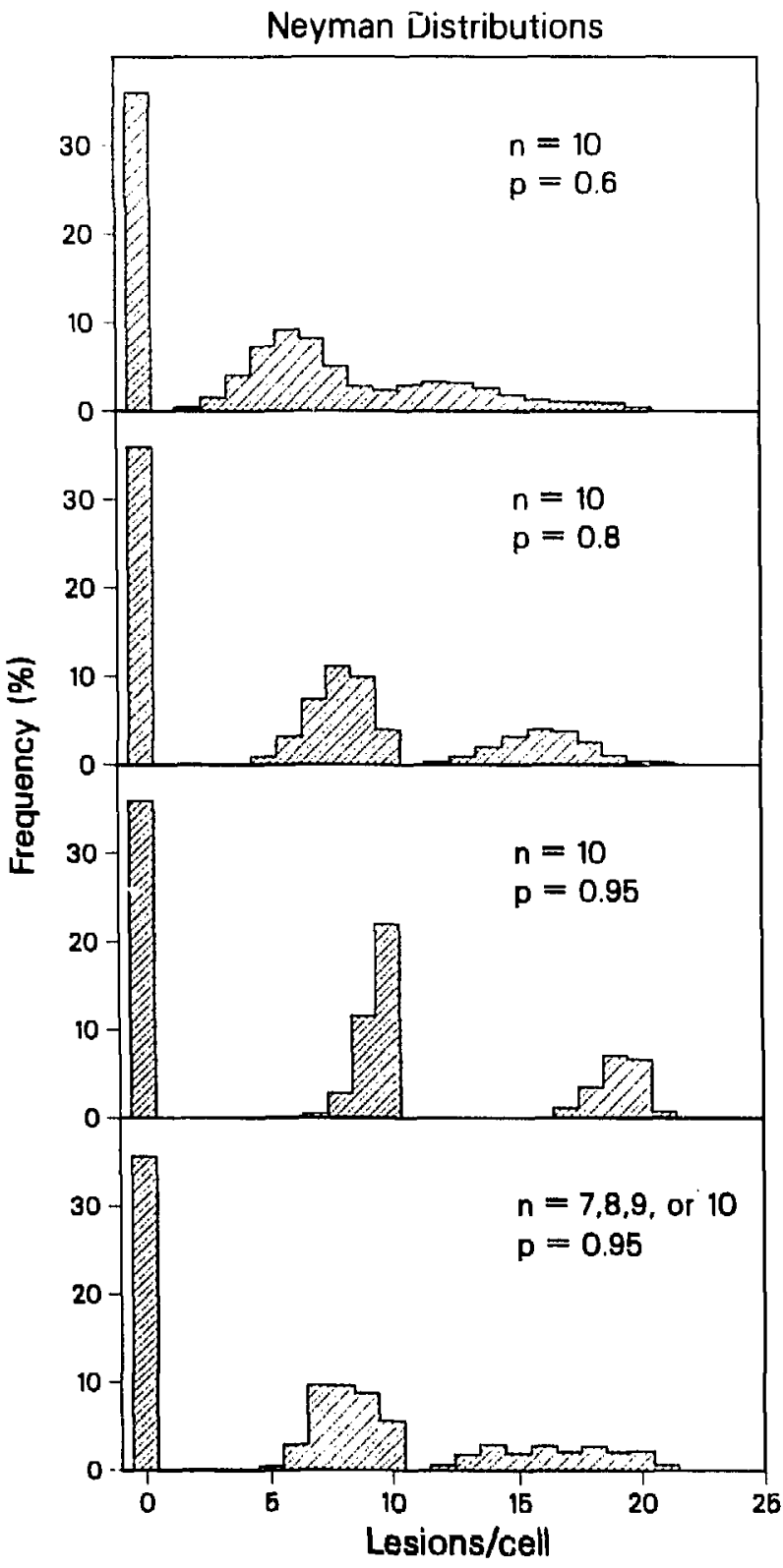

Fig. 2. Lesion frequency distributions for different values of $p$ at the same $n$. Botlom panel: $A$ distribution of $n=7,8,9$, or 10 crossovers with $p=0.94$.

(XCG 864.7183)

breaks. The overall number of breaks per particle is limited by the distribution of DNA in the cell nucleus.

Chromatin breaks can repair by rejoining. Earlier, in the repair-misrepair model, we have suggested that many of the deleterious effects produced by heavy ions are due to misrepair or misrejoining. The mechanisms for rejoining are not understood in detail. The premature chromosome 


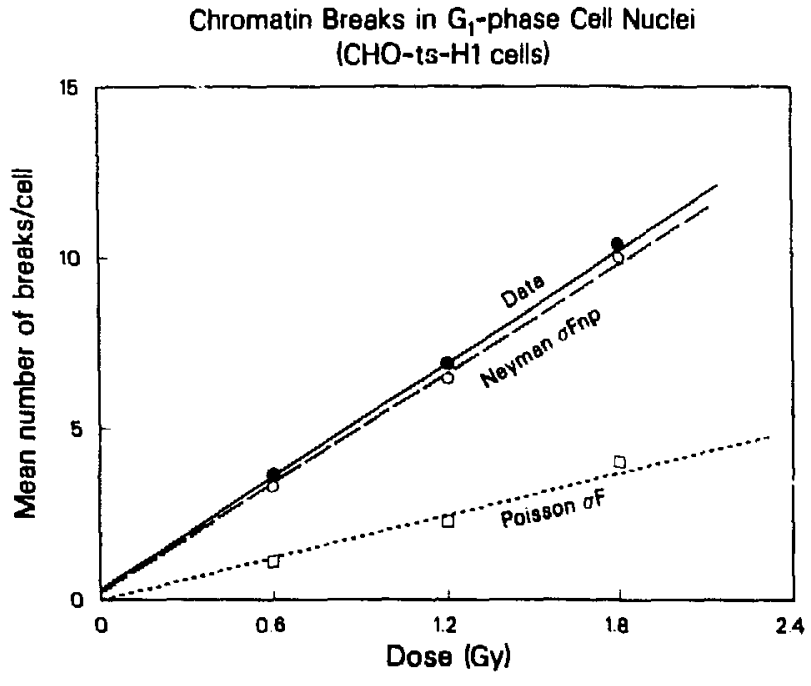

Fig. 3. The mean number of lesions per cell as functions of dose. (O) experimental data (O) Neyman distributions, (ㅁ) Poisson fitted to probability $U(o)$.

(XCG 864-7188)

condensation (PCC) technique is a potentially powerful tool to study how such processes occur. The high biological effectiveness of heavy accelerated ions relates to the multiplicity of lesions each particle can produce. The probability of misrepair rises quadratically with the lesion multiplicity.

\section{REFERENCES}

1. Kellerer, A., and Rossi, H. A generalized form of dual radiation action. Radiat. Res. 75, 471-488 (1978).

2. Goodwin, E., Blakely, E., and Tobias, C.A. Multiple chromatin breaks produced by single heavy ions. Radiation Research Society Annual Meeting, Abstract Ff-1, Las Vegas, NV (1986).

3. Feller, W. On a general class of "contagious"

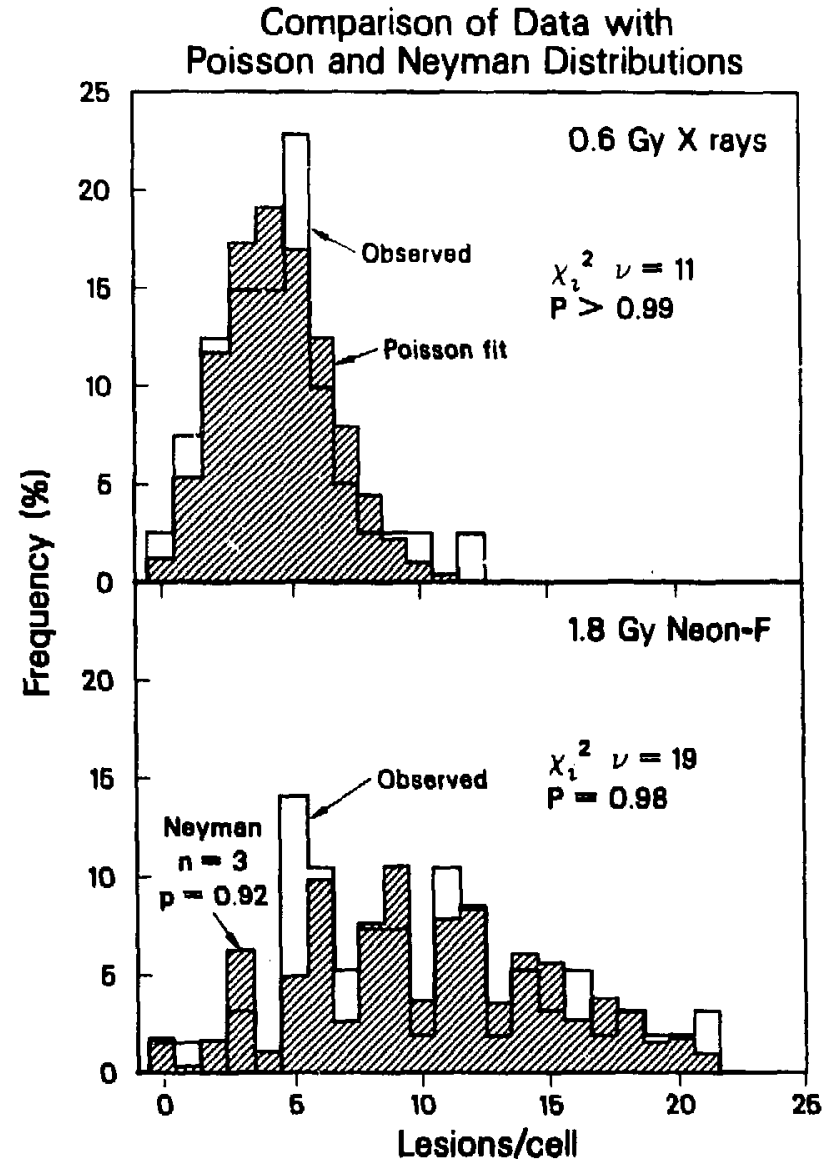

Fig. 4. Top: Poisson distributions fit the $x$-ray-induced rhromatin lesion distributions quite well. Dose $0.6 \mathrm{~Gy}, P>0.99$. Botlom: Neyman distribution fits neon $F$ data at $1.8 \mathrm{~Gy}$ quite well. $P=0.98$.

distributions. Ann. Math. Stat. 14, 389-400 (1943).

4. Kappos, A., and Pohlit, W. A cybernetic model for radiation reactions in living cells. I. Sparsely ionizing radiations; stationary cells. Int. J. Radiat. Biol. 22, 51-65 (1972).

\section{RADIOSENSITIZATION PRODUCED BY IODODEOXYURIDINE IN HEAVY ION BEAMS}

\section{David E. Linstadt, Eleanor A. Blakely, Theodore L. Phillips, Joseph R. Castro, Polly Y. Chang, and Leora Lommel}

There has been a resurgence of interest in the use of iododeoxyuridine (IUDR) as a clinical radiosensitizer. Although it has been studied for over 20 years, the $\in$ xact mechanism of sensitization is unknown. Likewise, little is known about IUDR sensitization using high linear energy transfer (LET) radiation, or the effect of IUDR on repair of sublethal or potentially lethal damage. A series of in 
vitro experiments was conducted at Lawrence Berkeley Laboratory using human T-1 cells grown in the presence of 3.0 micromolar IUDR for 72 hours (approximately 3 doubling times) and subsequently irradiated with either $x$ rays or a variety of heavyion beams. The data obtained show that sensitization decreased as LET increased (Fig. 1) and no significant sensitization was produced with ions having an LET value of $1000 \mathrm{keV} / \mu \mathrm{m}$.

However, significant sensitization occurred with both $x$ rays and in the $585 \mathrm{MeV} / \mathrm{u}$ neon-ion Bragg peak currently used for treating cancer patients. (Sensitization enhancement ratios at $40 \%$ cell survival were 1.7 for $x$ rays, 1.5 for proximal portion of the spread neon Bragg peak, and 1.3 for neon distal peak irradiation.) Control cells show a substantial difference in the level of cell killing between the proximal and distal portions of the 8 -cm Bragg peak of the neon beam. Due to the dependence of sensitization on LET, IUDR-treated cells display near equal levels of cell survival throughout the spread Bragg peak. As expected, IUDR caused a reduction in the size of the shoulder of the cell survival curves. When fitted to the linear quadratic model of cell survival, this reduction was seen to be due to a significant increase in the value of alpha for lower LET radiations. There was no significant change in the value of beta, regardless of LET. Split-dose experiments conducted with both $x$ rays and proximal peak neon ions showed less repair after proximal peak neon ions than after $x$ rays, but no difference in the amount of sublethal damage repair between IUDR treated cells and untreated controls (Fig. 2).

Delayed-plating experiments also were completed after either $x$-ray or neon-ion exposures.

IUOR SET. VS. LET GRAPH

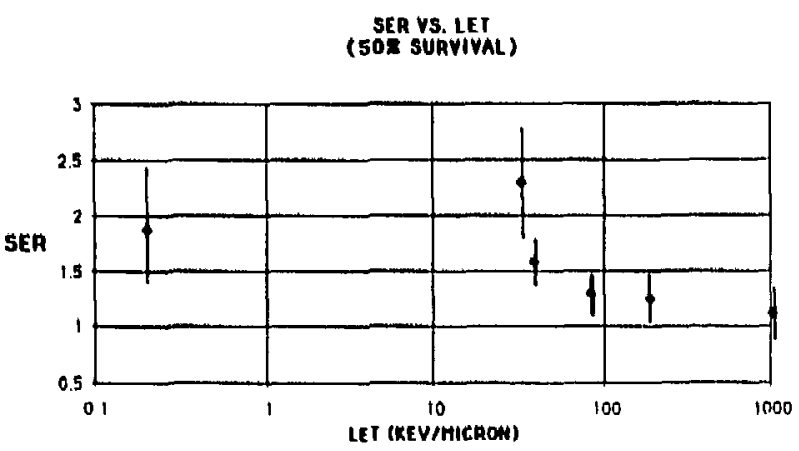

Fig. 1. IUDR sensitization enhancement ratio (SER) vs. LET.

(XBL 868-3095)
XRAY SLDR

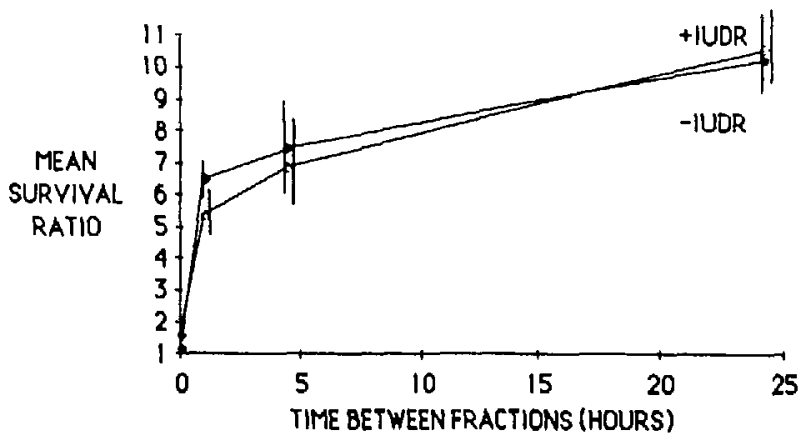

NEPP SLDR

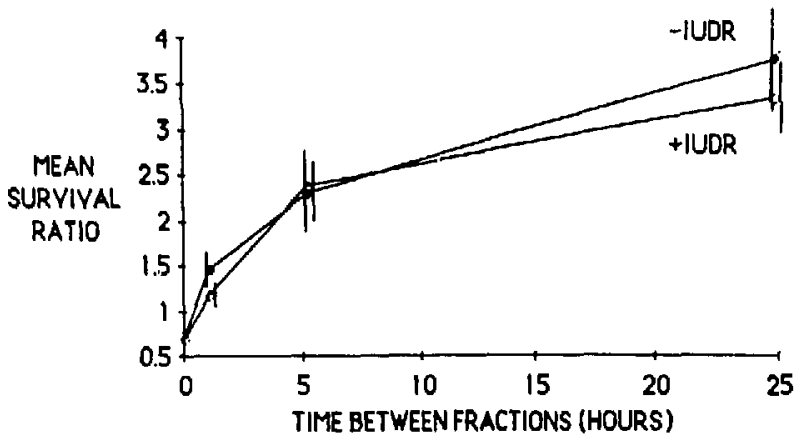

Fig. 2. Sublethal damage repair after $x$ ray or proximal peak neon ion irradiation with/without IUDR.

(XBL 868-3097)

The results showed that repair of potentially lethal damage from neon ions was less than that measured after comparable $x$-ray doses, but there was no difference in the magnitude of potentially lethal damage repair with or without IUDR. These results suggest that IUDR sensitizes primarily by increasing the amount of alpha-type damage ("single hit killing") and agrees with the current hypothesis that sensitization results from the creation of localized regions of heavy DNA damage. These results also suggest that IUDR is likely to function effectively as a sensitizer in fractionated radiotherapy regimens utilizing either photons or clinically useful heavyion beams, particularly where a tumor might exist in a substrate of normal tissues which would have slower turnover than the turnor. Finally, the use of IUDR could possibly simplify design and construction of ridge filters for the neon beam due to the LET dependence of sensitization. 


\title{
THE IMPORTANCE OF TRIGGERING DOSE AND CONDITIONS OF SPLIT DOSE INCUBATION TO THE DEVELOPMENT OF THERMOTOLERANCE
}

\author{
Polly Y. Chang and Eleanor A. Blakely
}

We have observed a lack of development of thermotolerance in $\mathrm{CHO}-\mathrm{TSH} 1$, a protein synthetic mutant hamster cell line, under continuous heat stress treatments of $41.5^{\circ} \mathrm{C}, 42^{\circ} \mathrm{C}$, and $42.5^{\circ} \mathrm{C}$, $^{1,2}$ The parental $\mathrm{CHO}-\mathrm{SC} 1$ line develops thermotolerance under similar conditions. During the past year we have further examined this phenomenon in the mutant by studying the role of the level of the triggering heat dose and the time of administration to the ultimate development of thermotolerance. By increasing the temperature of the triggering dose for a brief interval, followed by time at the permissive temperature, we were able to induce the development of thermotolerance in the proteinsynthetic mutant cell. The wild-type cells were used as controls.

Both cell lines were subjected to a high initial triggering dose of $45^{\circ} \mathrm{C}$ for 15 minutes, incubated at the permissive temperature of $35^{\circ} \mathrm{C}$ for varying times, and then a final test dose of 25 minutes of $45^{\circ} \mathrm{C}$. The experiments were repeated twice and showed marked reproducibility. The survival data from both cell lines are shown in Fig. 1. The thermotolerance curves plotted in Fig. 2 are essentially superimposable and show an initial very fast component of tolerance, a slower but exponential phase, and a final plateau phase which is attained by 12 hours after the first treatment and maintained for more than 12 hours afterwards. The level of maximum tolerance is about $25-30 \%$ survival, which is the survival level attained by the initial test dose of 15 minutes at $45^{\circ} \mathrm{C}$. We have thus confirmed that $\mathrm{TSH} 1$ indeed can develop thermotolerance under permissive conditions, the level of which is dependent on the triggering heat dose. The importance of the triggering dose to the subsequent level of tolerance development is also noted by $\mathrm{Li}$ and Hahn ${ }^{3}$ and Boon-Niermeijer et al. ${ }^{4}$

Another experiment was done where both cell lines were given a triggering dose of 15 minutes at $45^{\circ} \mathrm{C}$, kept at the nonpermissive incubation temperature of $40^{\circ} \mathrm{C}$ for varying periods of time followed by a final test dose of 15 minutes of $45^{\circ} \mathrm{C}$. Preliminary results indicate that there is not only the absence of tolerance in the TSH1 cells as expected, but maybe even a thermal dose potentiation effect. The parent $\mathrm{SC} 1$ line, however, showed an initial increase in survival, indicating tolerance development, but after 1 hour survival rapidly declined, indicating the inability to sustain tolerance.

Development of thermotolerance in Lymnaea is suggested by Boon-Niermeijer et al. ${ }^{4}$ to involve at least two components: the $\alpha$ state, which is the initial rapid response, said to be independent of protein synthesis, and the $\beta$ state, the slower component, only expressed in prolonged heat stresses. The $\beta$ state is said to be accompanied by the appearance of heat shock protein (hsp) and would therefore be dependent on protein synthesis. Our results confirm that there is a fast initial component to the development, as seen by the rapid initial rise in survival. This state, however, must be dependent on protein synthesis since it is not seen in TSH1 under non-permissive incubation after a test dose of $45^{\circ} \mathrm{C}$, but only in SC1, an observation that

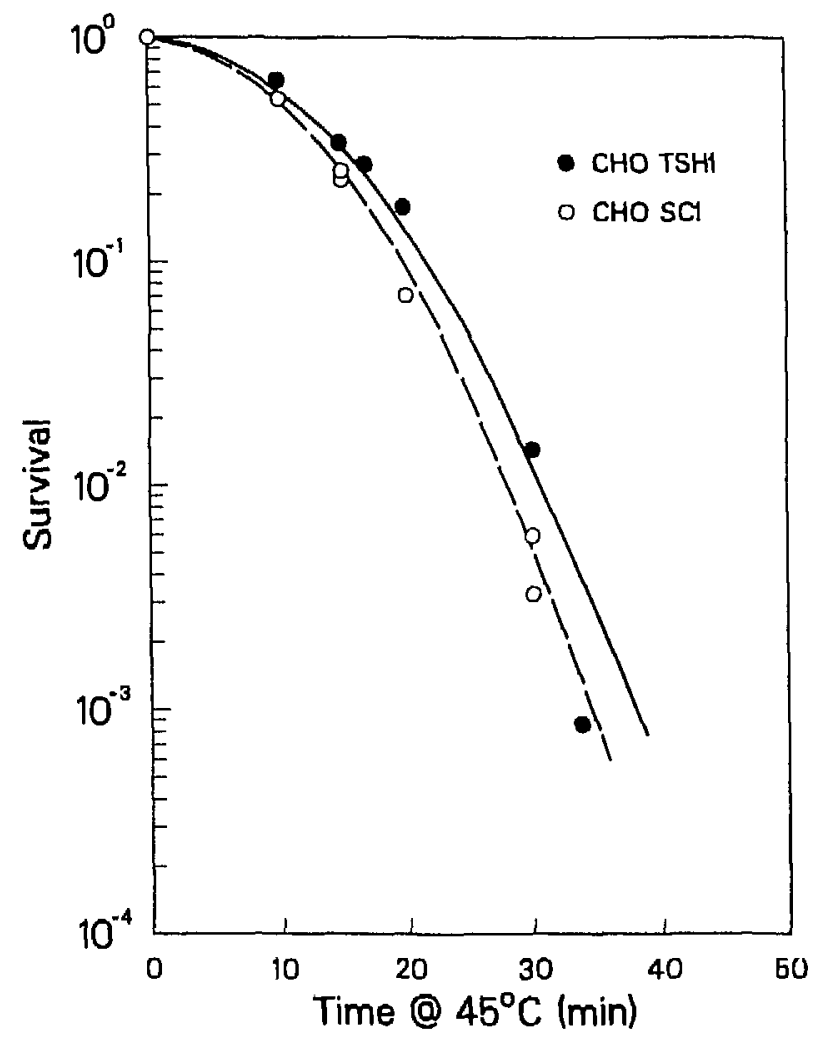

Fig. 1. Survival curves of CHOSCl and CHOTSH1 with continuous heat treatment at $45^{\circ} \mathrm{C}$. 


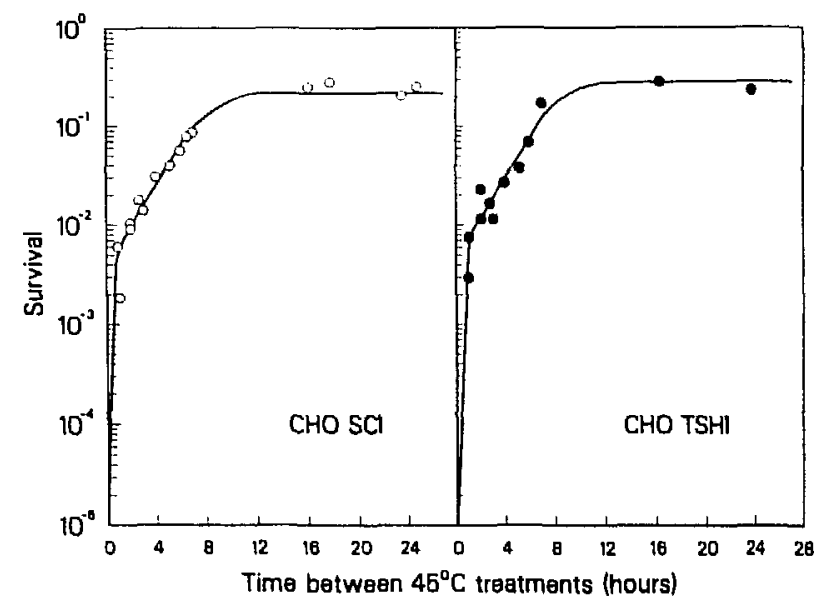

Fig. 2. CHOSCl (panel $A$ ) and CHOTSH1 (panel B) were exposed to 15 minutes of $45^{\circ} \mathrm{C}$, incubated from $0-24 \mathrm{hr}$ at $35^{\circ} \mathrm{C}$ before a second heat dose of 15 minutes at $45^{\circ} \mathrm{C}$. Cells were trypsinized after the second heat treatment and plated for colony formation.

(XCG 8611-12289)

does not support Boon-Niermeijer's theory. Protein kinetic studies are underway to look at the syn- thesis of the various hsp's under the conditions of our experimental protocols.

\section{REFERENCES}

1. Chang, P.Y., Blakely,E.A., and GonzalezFlores, I. Effect of inhibition of protein synthesis on the development of thermotolerance. Lawrence Berkeley Laboratory report LBL-20345 (1985).

2. Chang, P.Y., Gibbs, G.F., and Blakely, E.A. Loss of thermotolerance in a temperaturesensitive protein synthetic mutant. ThirtyThird Annual Meeting of the Radiation Research Society Los Angeles, May 1985 (Abstract).

3. Li, G.C., and Hahn, G.M. A proposed operational model of thermotolerance based on effects of nutrients and the initial treatment temperature. Cancer Research 40, 4501-4508 (1980).

4. Boon-Niermeijer, E.K., Tuyl, M., and Van de Scheur, $H$. Evidence for two states of thermotolerance. Int. J. of Hyperthermia 2, (1) 93-105 (1986).

\section{DOSE PROTRACTION STUDIES WITH LOW AND HIGH LET RADIATIONS ON NEOPLASTIC CELL. TRANSFORMATION IN VITRO}

\section{Tracy Chui-hsu Yang, Laurie M. Craise, Man-tong Mei, and Cornelius A. Tobias}

Usually protracted doses of low-LET radiation are less effective than single acute doses in inducing cancer in vivo. For high-LET radiation there are some data to indicate that protracted doses are more effective than acute doses at inducing tumors.' Recently, in vitro neoplastic cell transformation studies with fission neutrons and gamma rays showed similar results. ${ }^{2}$ At present, there is no conclusive explanation for such findings, although several suggestions have been made. ${ }^{3,4}$

Because human occupational exposure in a space environment and on earth is usually at a low dose rate, it is important to understand the lowdose-rate effects of high-LET radiation for risk assessment. Accelerated heavy ions of different energy and atomic number provide valuable tools for examining the dose-rate effects with various LET and track structure. Using mouse embryonic cells (C3H10T1/2) cultured in vitro, we have earlier investigated the frequency of radiation-induced cell transformation with $x$ rays and energetic heavy ions at high dose rates and found that heavy ions enhanced the frequency of cell transformation more than $x$ rays did. $5,6,7$ The primary objective of the present study is to determine whether low- or high-LET radiations can further enhance the neoplastic cell transformation at low dose rates and to uncover the role of the LET of heavy ions in the enhancement effect.

\section{METHODS AND RESULTS}

For $x$-ray experiments, a Philips $250-k V p$ x-ray unit was used. Physical conditions of irradiation were $225 \mathrm{kVp}, 15 \mathrm{~mA}$, and a half-value layer of 1.1 $\mathrm{mm}$ of copper. A $150-\mathrm{Ci}^{60} \mathrm{Co}$ source was used for low-dose-rate experiments. The dose rates of $x$ rays and gamma rays were measured with a Victoreen condensor $\mathrm{R}$ meter. Corrections were made 
for temperature, atmospheric pressure, and the dosimeter calibration factor. A constant factor of 0.95 was used to convert roentgen into rad.

Heavy-ion beams were generated by the Berkeley Bevalac. Before reaching the biological sample, the beam passed through a lead scatterer, a brass collimator, a dose ionization chamber, a multiwire proportional chamber, a water absorber, and finally, a second ionization chamber. The residual range of the beam was determined before each experiment and was used for calculating the track average LET values for the beam. The variation of radiation dose across the area of sample flask was kept to within $5 \%$. For all experiments we irradiated samples at the plateau region with zero water column and at room temperature. Table 1 shows the physical parameters for various radiations used for present studies. The standard errors (SE) were calculated from the formula $S E=(n) 1 / 2 / N$, where $n$ is the total number of colonies counted, and $N$ is the number of plates used. In all cases, the points as plotted represent the mean value $\pm 1 \mathrm{SE}$. If no bars are shown, one standard error of the mean is smaller than the plotted symbol.

We studied the effects of dose rates and fractionated doses on cell transformation with $x$ and ${ }^{60} \mathrm{Co}$-gamma rays. Figures 1 and 2 show the experimental results. For a given dose, confluent monolayer mouse embryonic cells irradiated by highdose-rate (100 rad/min) gamma rays gave a significantly lower survival and higher transformation frequency than those irradiated by low dose rate (1 $\mathrm{rad} / \mathrm{min}$ ). At low dose rate, the effectiveness of gamma rays for neoplastic cell transformation was reduced by a factor of about 2 to 4 , higher at lower doses, and for cell killing by a factor of about 1.7. The dose-response curves for cell transformation appear to be curvilinear for both high- and lowdose-rate gamma rays.

Figure 2 shows the results of $\mathrm{C} 3 \mathrm{H} 10 \mathrm{~T} 1 / 2$ cells that received $x$ rays either as a single exposure or as two equal fractions separated by one day. It is clear that both the lethal and the transformation effect of $x$ rays decreased significantly when split doses were used. For a dose that gave $10 \%$ survival, the dose-modifying factor was about 1.35 for cell killing and about $\mathbf{1 . 4}$ for cell transformation. These results indicate that mammalian cells can repair some of the $x$-ray-induced sublethal and subtransformation damages. In addition to repair of sublethal and subtransformation lesions, C3H10T1/2 cells can also repair potential lethal and transformation damages. When confluent monolayer $\mathrm{C} 3 \mathrm{H} 10 \mathrm{~T} 1 / 2$ cells were irradiated with $\mathrm{x}$ rays and subsequently incubated at $37^{\circ} \mathrm{C}$ for 1 day before being trypsinized and plated at low density, there was an increase of survival and a decrease of transformation frequency as compared to cells plated right after irradiation (Fig. 3). The kinetics of repairing potentially transformed lesions is shown in Fig. 4. Our results indicate that the repair of potential transformation damages is a relatively slow process and may take many hours for a complete repair.

With confluent cultures of the $\mathrm{C} 3 \mathrm{H} 10 \mathrm{~T} 1 / 2$ cell line, we have initiated some studies on the lowdose-rate effects of high-LET iron particles on cell transformation. Figure 5 shows the results obtained for cell killing and neoplastic cell transformation. The survival curve was exponential, and there was no significant difference between cells irradiated with low- and high-dose-rate iron particles $\$ 600$ $\mathrm{MeV} / \mathrm{u}, 200 \mathrm{keV} / \mu \mathrm{m})$. For cell transformation, however, confluent cells irradiated with $2-\mathrm{rad} / \mathrm{min}$ iron particles gave a significantly higher transformation frequency than those irradiated with 100$\mathrm{rad} / \mathrm{min}$ iron beam. The enhancement effect was greater at lower doses than at higher doses. For example, the transformation frequency for 50 rad of low-dose-rate iron particles was about the same as that for $100 \mathrm{rad}$ of high dose rate. The transformation frequency of dose $175 \mathrm{rad}$ of low dose rate was, however, no higher than that for $250 \mathrm{rad}$ of high dose rate.

Table 1. Physical parameters of various radiations.

\begin{tabular}{|c|c|c|c|c|}
\hline Radiation & Initial energy & $\begin{array}{l}\text { Lead foil } \\
\text { (cm) }\end{array}$ & $\begin{array}{l}\text { Residual range } \\
\text { in water } \\
\text { (cm) }\end{array}$ & $\begin{array}{l}\text { Track average } \\
\text { LET (keV/um) }\end{array}$ \\
\hline$x$ rays & $225 \mathrm{kVp}$ & - & - & 2.6 \\
\hline${ }^{60} \mathrm{Co}$ gamma rays & - & - & - & 0.27 \\
\hline${ }^{40} \mathrm{Ar}$ & $400 \mathrm{MeV} / \mathrm{u}$ & 0.238 & 6.0 & 120.0 \\
\hline${ }^{56} \mathrm{Fe}$ & $600 \mathrm{MeV} / \mathrm{u}$ & 0.238 & 8.5 & 200.0 \\
\hline
\end{tabular}



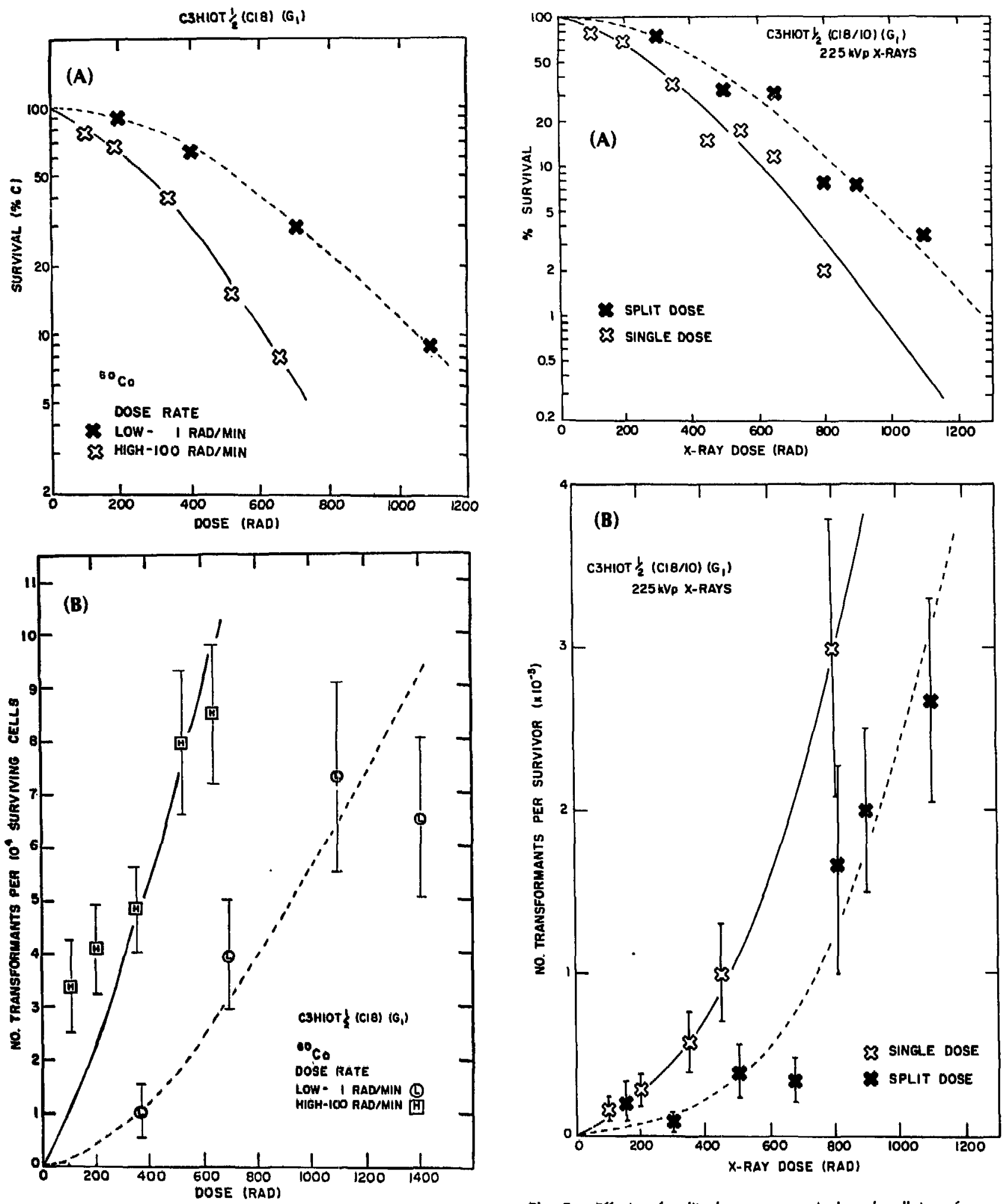

Fig. 1. Confluent monolayer C3H1OT1/2 cells irradiated by high- or low-dose-rate gamma rays. (A) Dose-response curves for survival. (B) Dose-response curves for neoplastic cell transformation. Low dose rate shows a sparing effect for both survival and cell transformation. (XBL 864-1394; XBL 864-1395)

Fig. 2. Effects of split doses on survival and cell transformation. (A) Survival curves for cells irradiated by $x$ rays as single doses or two equally fractionated doses separated by $I$ day. (B) Dose-response curves for cell transformation. X-ray doses were given as single doses or two equal fractions separated by $t$ day.

(XBL 866-2242; XBL 866-2243) 

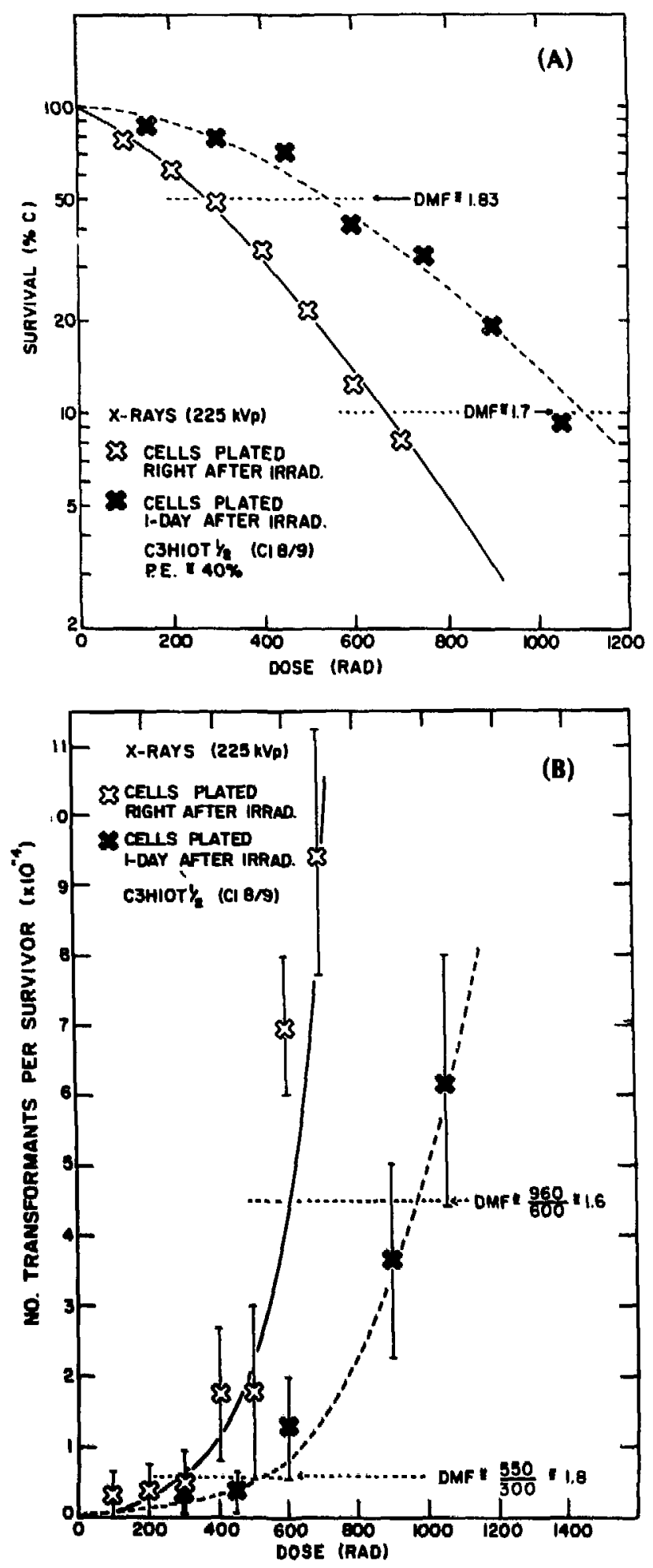

Fig. 3. Repair of potential lesions in C3H1OTI/2 cells. Plateau-phase cells were plated either immedialely or kept at $37^{\circ} \mathrm{C}$ for 1 day after irradiation. (A) Potential lethal damage repair. There is a dose modifying faclor (DMF) about 1.7 to 1.8. (B) Repair of potential transformation damage. The dose modifying factor is about 1.6 to 1.8. (XBL 835-9949; XBL 835-9914)

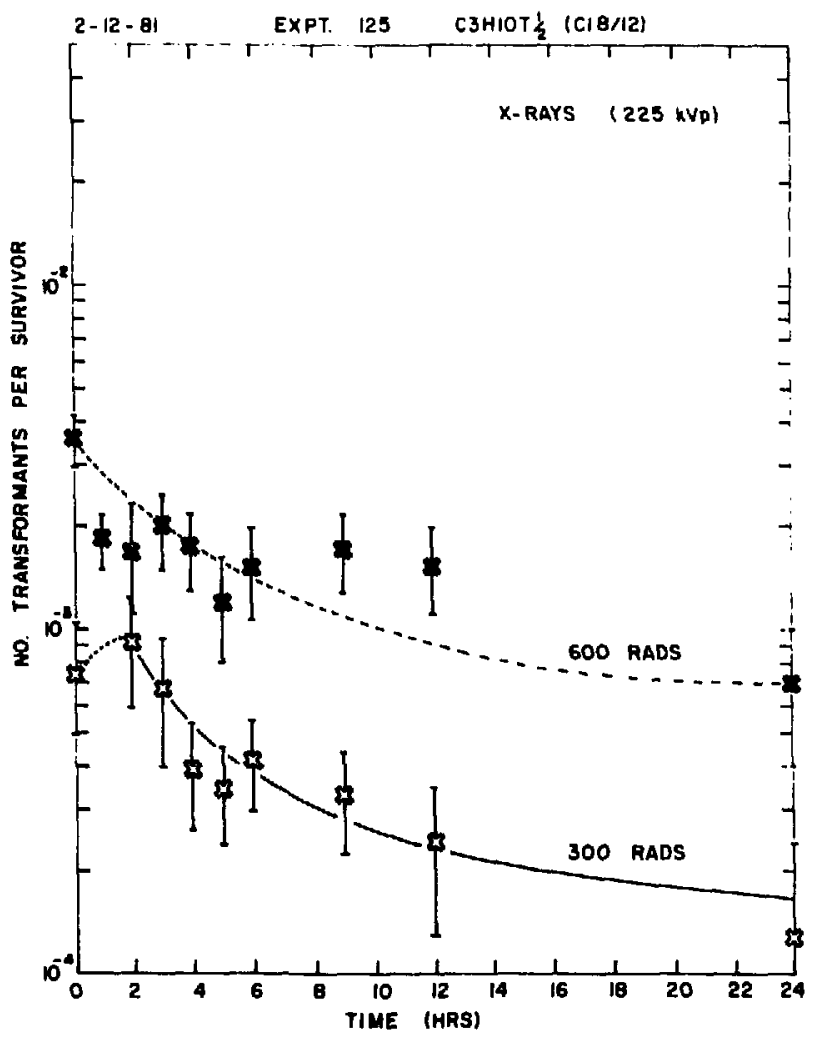

Fig. 4. Kinetics of polential transformation lesion repair in plateau-phase cells. $X$-irradiated cells were plated at various time intervals after irradiation.

(XBL 815-9901)

In general, the doses and dose rates of the heavy-inn beam were measured by an on-line ionization chamber, which was placed right in front of the cell sample. For the low-dose-rate experiment, to double check the dosimetry, we determined the fluence of primary particles with a solid state radiation detector (cellulose nitrate). Figure 6 shows the measured fluence of iron particles for various doses of high- and low-dose-rate iron beams. The fluence increased linearly with dose, and there was no significant difference between $32-\mathrm{rad} / \mathrm{min}$ and 2 $\mathrm{rad} / \mathrm{min}$ iron beams.

In addition to the study with iron particles, we did an experiment with $400-\mathrm{MeV} / \mathrm{u}$ argon ions to determine the effects of various dose rates. Figure 7 displays the results obtained for cell killing and transformation. There was no systematic increase or decrease of cell survival for a wide range of dose rates, from 1.6 to $150 \mathrm{rad} / \mathrm{min}$. Confluent monolayer cells irradiated with low-dose-rate argon particles (less than $2 \mathrm{rad} / \mathrm{min}$ ), however, gave a significantly higher transformation frequency than those irradiated at higher dose rates. 

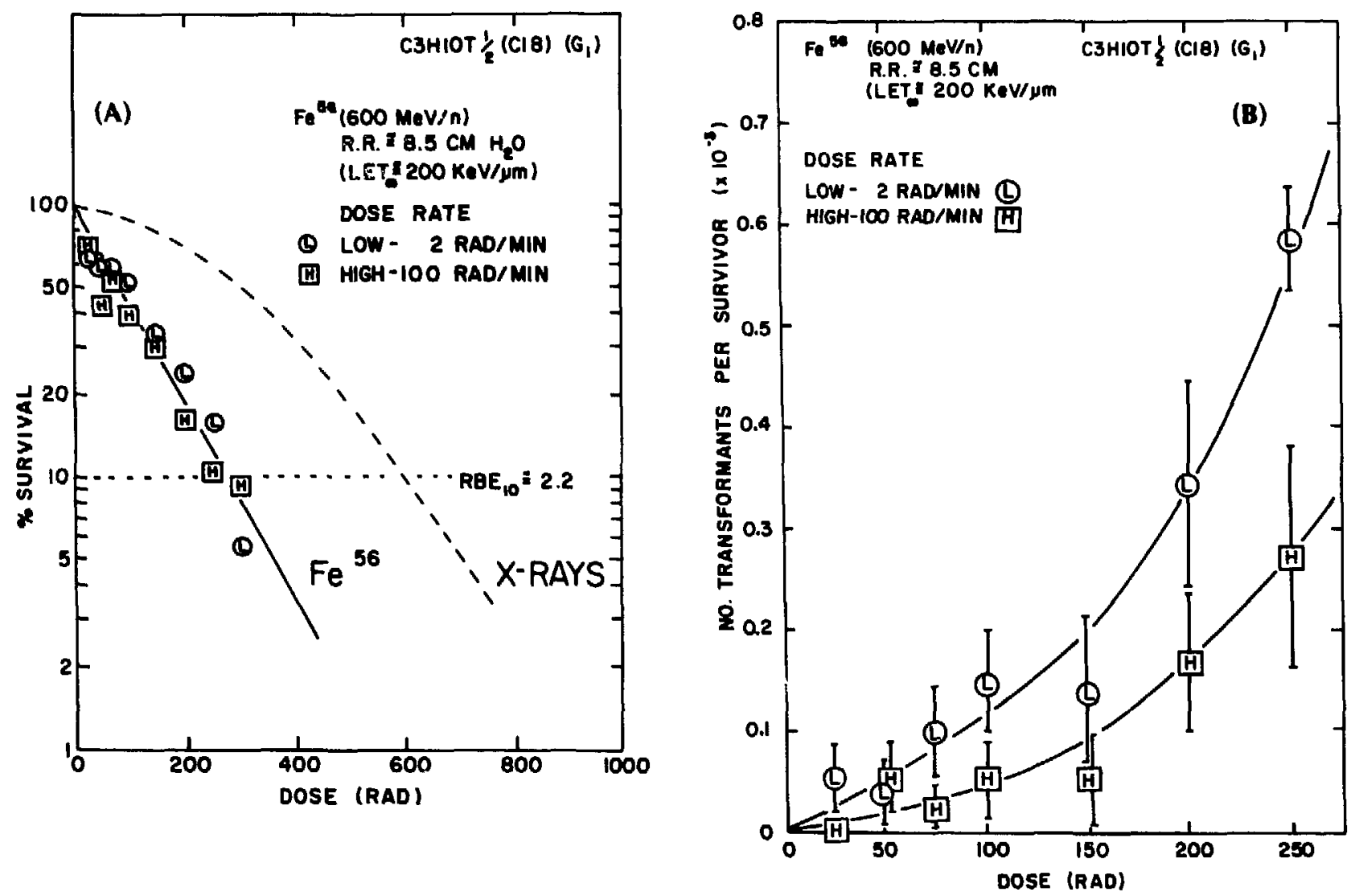

Fig. 5. Effects of low-dose-rate iron particles $(600 \mathrm{MeV} / \mathrm{u}, 200 \mathrm{keV} / \mu \mathrm{m})$ on cell survival and transformation. Plateau-phase cells were irradiated with either $100-\mathrm{rad} / \mathrm{min}$ or $2-\mathrm{rad} / \mathrm{min}$ iron beam. (A) Dose-response curve for cells irradiated by high- or low-dose-rate iron particles. The survival curve is exponential and the same for high and low dose rates. The RBE at 10\% survival level is about 2.2. (B) Dose-response curves for cell transformation. There is an enhancement effect of low dose rate.

(XBL 859-3858; XBL 859-3859)

The dose-response curve for cell killing is exponential and appears to be the same for cells plated either immediately or one day after irradiation, as shown in Fig. 8. These results indicate that $400-\mathrm{MeV} / \mathrm{u}$ argon particles produce mainly irreparable lesions.

\section{DISCUSSION}

In space, radiation is one of the major environmental factors that are potentially hazardous to humans. Space radiation consists of ultraviolet light, energetic electrons, protons, alpha particles, and heavy ions with atomic number up to that of uranium $(Z=92)$. The dose rate of galactic cosmic radiation, in general, is very low, less than 1 $\mathrm{rad} /$ day, although during a solar flare the proton and helium-ion dose can be fairly high, more than $100 \mathrm{rem} / \mathrm{day}^{8}$. For assessing the radiation risk in space, it is, therefore, very important to determine the biological effects of heavy ions at low doses and low dose rates. Detailed studies on the interaction between high- and low-LET radiations and between radiation and other space environmental factors in causing various biological effects are also needed.

An important late effect of radiation is carcinogenesis. Since the initial success in transforming mammalian cells in culture by oncogenic viruses and chemical carcinogens, similar techniques have been used to study the carcinogenic effect of radiation at cellular and molecular levels. Cultured hamster embryo cells, at early passages, and established mouse embryo cell lines, e.g., C3H10T1/2, have been used commonly for radiation celltransformation studies. Although there has been reported success in transforming human cells in culture, a reproducible quantitative assay has yet to be developed. In human cells, altered morphology appears not to be an early expression of neoplastic transformation, but rather the loss of anchorage dependence. No good correlation, however, has 


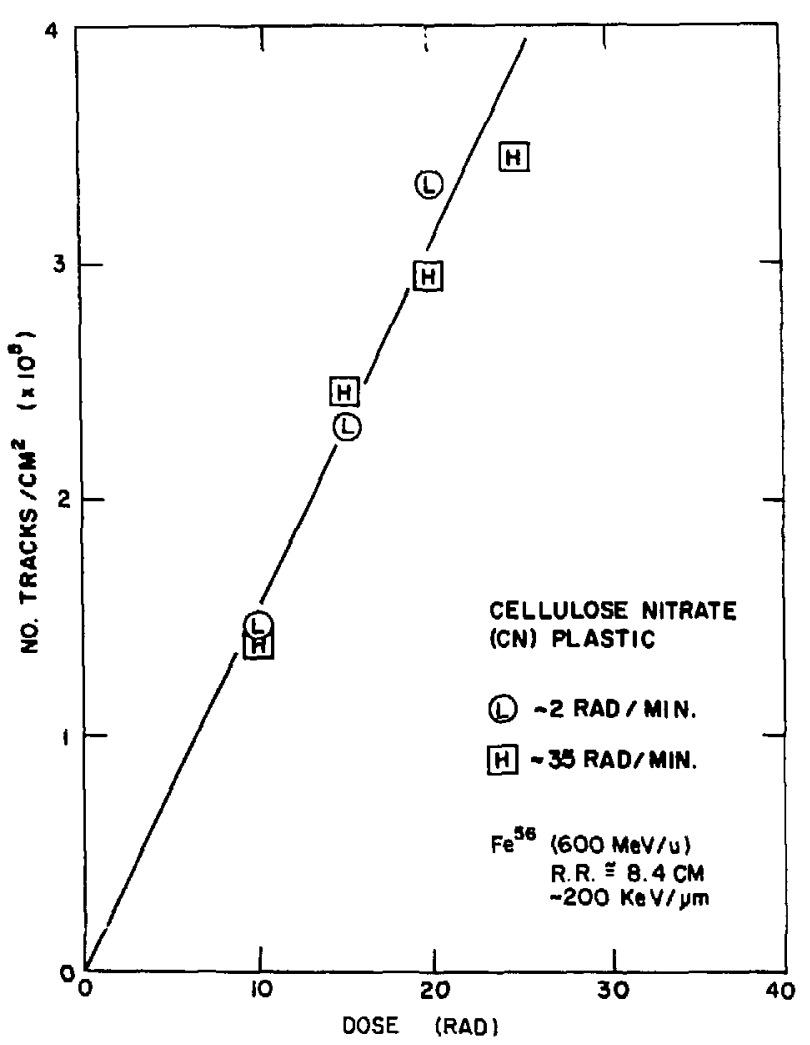

Fig. 6. The fluence of primary iron particles $(600 \mathrm{MeV} / \mathrm{u}, 200$ $\mathrm{keV} / \mathrm{um}$ ), registered in cellulose nitrate plastics, as a function of doses, measured by an ionizaton chamber. The fluence increases linearly with the doses, and there is no significant difference between high- and low-dose-rate iron beams.

(XBL 866-2319)

been found between the loss of anchorage dependence and the tumorigenicity of human cells.

Various investigators have shown that the transformation dose-response relationship for $x$ rays is about the same for primary cultures and for cell lines, and that cells cultured in vitro can be a useful model system for studying the mechanisms of radiation carcinogenesis. $5,7,9,10^{\circ}$ The effects of low doses and dose rates on neoplastic cell transformation have been studied by various groups. It was found that a dose as low as 1 rad of $x$ rays induced neoplastic cell transformation at a frequency of about $0.01 \%$ in hamster embryo cells. ${ }^{11}$ An enhancement of cell transformation was observed when a low dose of $x$ rays was given as two equal fractions separated by several hours. ${ }^{12,13,14}$ However, when a low $x$-ray dose was given in 5 fractions over 4 days, it was observed that the transformation frequency was less than that for a single exposure. ${ }^{15}$ Our results indicate that split doses were less effective than single doses in causing both lethal and transformation effects.
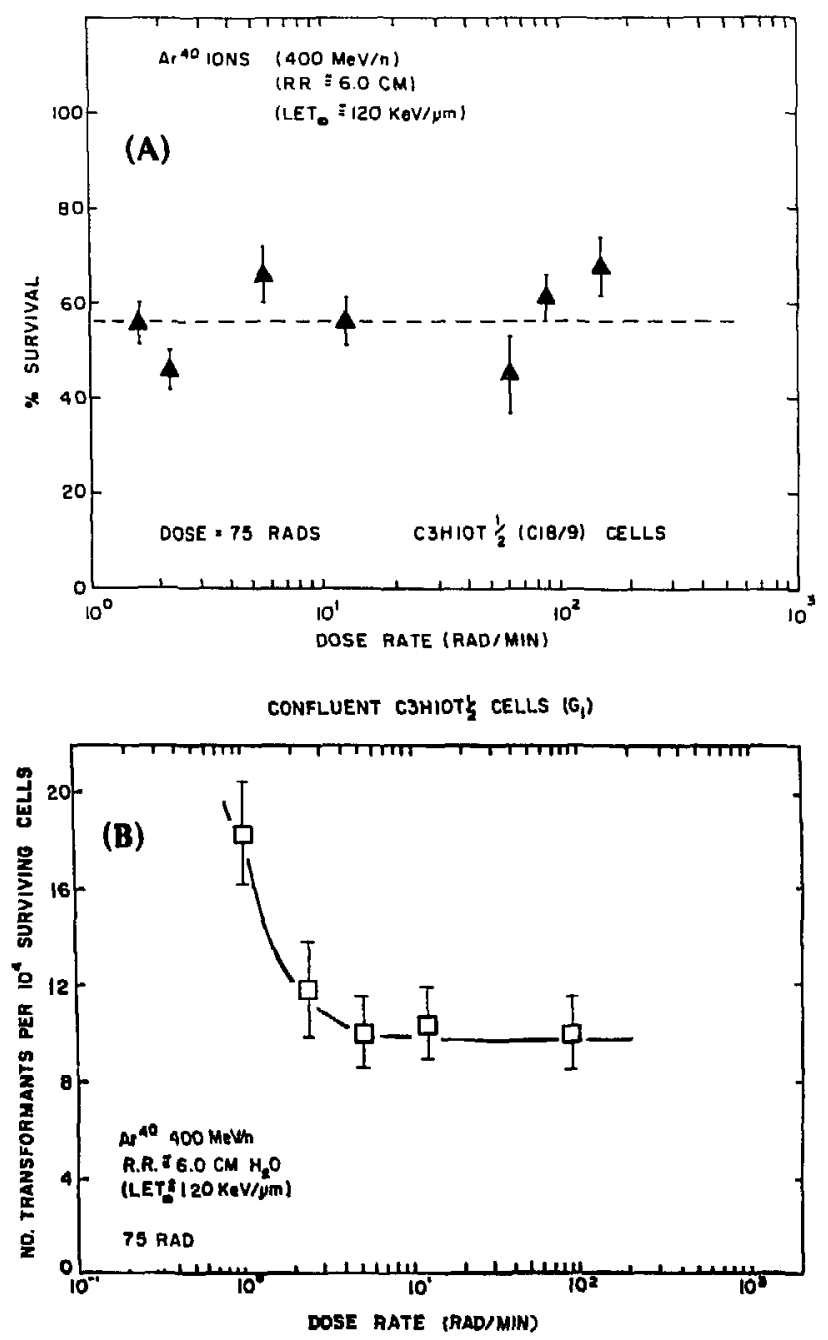

Fig. 7. Effects of various dose rates of $400-\mathrm{MeV} / \mathrm{u}$ argon ions on (A) survival, and (B) cell transformation. The transformation frequency increased significantly at dose rate below $2 \mathrm{rad} / \mathrm{min}$. A dose of 75 rad was used.

(XBL 866-2383; XBL 864-1397)

The effect of radiation on cell transformation has also been found to be dose-rate dependent. The frequency of neoplastic cell transformation for a given dose decreases with a decrease of dose rate of ${ }^{60} \mathrm{Co}$ gamma rays, and at low doses, the transformation frequency per survivor increases linearly with the radiation dose. ${ }^{16,17}$ Experimental data of our dose rate studies show a similar effect of low-dose-rate gamma rays. This decrease of effectiveness of low dose rate in inducing cell transformation may be primarily due to the repair of radiation damages. Our earlier studies have shown that repair enzyme inhibitors, e.g. $\beta$ arabinosyladenosine, can increase the cell transformation frequency significantly. ${ }^{18}$ 


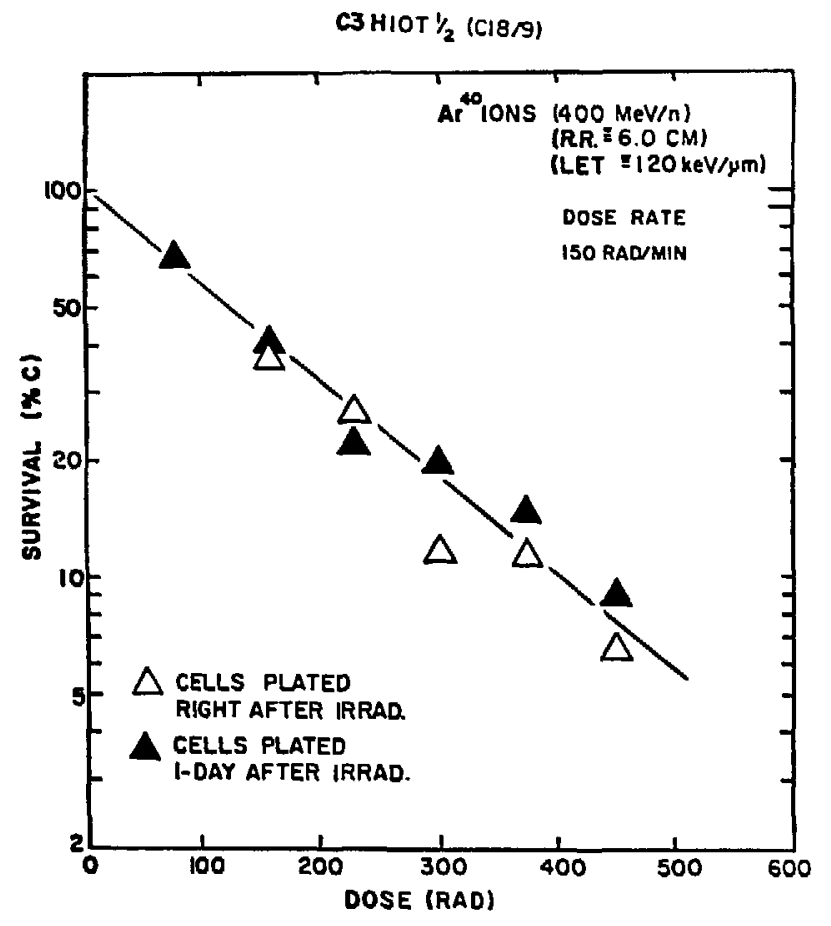

Fig. 8. Dose-response curve for plateau-phase cells irradiated by $400-\mathrm{MeV} / \mathrm{u}$ argon ions. The survival curve is exponential and the same for cells plated either immediately or : day after irradiation.

(XBL 864-1396)

For high-LET radiation, the effect of neutrons on cell transformation has been studied in considerable detail. Using a hamster embryo cloned-cell system, Borek ${ }^{19,20}$ determined the induction of cell transformation in vitro with $430-\mathrm{keV}$ neutrons over a dose range of 0.1 to $150 \mathrm{rad}$ and found the RBE value to be about 20 at low doses. Transformation of mouse C3H10T1/2 cells by single and fractionated doses of fission spectrum neutrons with an average energy of $0.85 \mathrm{MeV}$ was studied by Han and Elkind. ${ }^{15}$ Their results showed that the RBE value for transformation was about 10 in the region of small doses (25 rad), and there was only a small decrease in cell transformation when the fission neutron dose was equally fractionated. In more recent reports, Hill et al. ${ }^{21,22}$ showed that the neoplastic cell transformation was, in contrast to gamma rays, actually enhanced by multiple low doses of high-dose-rate neutrons and by low-doserate neutrons. The dose modifying factor, based on the ratio of the initial linear portions of the induction curves for high and for low dose rates, was the same $(\sim 7.8)$ for both five daily fractions of highdose-rate neutrons and for low-dose-rate neutrons. A summary of the effect of dose protraction on neoplastic cell transformation is given in Table 2; all data are from the literature.

At present, only limited work on cell transformation with high-LET heavy-ions has been reported. Compared with gamma rays, high-LET heavy particles can be many times more effective in causing neoplastic cell transformation. ${ }^{5,7,9}$ Mouse 3T3 cells irradiated with single or split doses of low-energy alpha particles from ${ }^{241} \mathrm{Am}$ showed about the same transformation frequency. ${ }^{23}$ No study on the effect of multiple low doses of heavy ion radiation on cell transformation has been done. When cultured cells were irradiated with heavy ions at dose rates less than $2 \mathrm{rad} / \mathrm{min}$, there was an enhancement of cell transformation, as shown by our results. The enhancement effect of low dose rate, therefore, may be a common phenomenon for high-LET radiation. It is, however, unknown 1) whether the magnitude of enhancement is the same or not for all heavy ions with various LETs and 2) whether there is a limited range of LETs that show enhancement effect of low dose rate.

The enhancement effect of low dose rate neutrons has also been observed in in vivo systems. Studies on the effects of high- and low-dose rato $^{+}$ neutron irradiation on the induction of mamma. adenocarcinomas showed a significant enhancemelı: of the mammary tumorigenic effects of neutrons after single low-dose-rate exposure. ${ }^{1}$ This enhancement was observed even at low doses ( 2.5 to 20 rad). Fractionated or protracted doses at lowdose-rates have been shown to increase life span shortening and advance the appearance time of some tumors. ${ }^{24,25,26}$ The reasons for this enhance. ment are unclear. One possible explanation is that the low-dose-rate enhancement for mammary tumor induction is a result of an enhanced transformation rate as suggested by in vitro cell transformation studies. Alternatively, it is also possible that low-dose-rate neutron irradiation could enhance the expression of the transformational lesion. These two mechanisms are not mutually exclusive. Recent studies with a mouse mammary epithelial cell system suggest that low-dose-rate neutron exposures enhance the probability of progression of radiation-altered cells rather than increase the number of initiated cells. ${ }^{4}$ The molecular mechanism(s) of the enhancement effect, however, is totally unknown at present.

\section{REFERENCES}

1. Ullrich, R.L. Tumor induction in $B A L B / C$ mice after fractionated or protracted exposures to fission-spectrum neutrons. Radiat. Res.97, 587-597 (1984). 
Table 2. Effect of dose protraction on neoplastic cell transformation.

\begin{tabular}{|c|c|c|c|c|}
\hline Radiation & Dose protraction & Cells & Results & References \\
\hline \multicolumn{5}{|l|}{ PHOTONS: } \\
\hline $\begin{array}{l}300-k V p \times \text { rays } \\
(102 \mathrm{rad} / \mathrm{min})\end{array}$ & $\begin{array}{l}\text { Split doses } \\
t=5 \mathrm{hr} \\
<100 \mathrm{rad} \\
>100 \mathrm{rad}\end{array}$ & $\begin{array}{l}\text { Asynchronous } \\
\text { C } 3 \mathrm{H} 10 \mathrm{~T} 1 / 2\end{array}$ & $\begin{array}{l}\text { Enhancement } \\
\text { decrease }\end{array}$ & Miller \& Hall $^{13}$ \\
\hline $300-k \vee p \times$ rays & $\begin{array}{l}\text { Split doses } \\
\mathrm{t}=5 \mathrm{hr} \\
75 \mathrm{rad}\end{array}$ & $\begin{array}{l}\text { Asynchronous } \\
\text { Syrian hamster } \\
\text { embryo cells }\end{array}$ & Enhancement & Borek \& Hall'2 \\
\hline $\begin{array}{r}100-k V p \times \text { rays } \\
(83 \mathrm{rad} / \mathrm{min})\end{array}$ & $\begin{array}{l}\text { Split doses } \\
\mathrm{t}=5 \mathrm{hr} \\
<150 \mathrm{rad} \\
>150 \mathrm{rad}\end{array}$ & $\begin{array}{l}\text { Asynchronous } \\
\text { C } 3 \text { H10T1/2 }\end{array}$ & $\begin{array}{l}\text { Enhancement } \\
\text { decrease }\end{array}$ & Little $^{14}$ \\
\hline${ }^{50} \mathrm{Co}$ gamma rays & $\begin{array}{l}5 \text { fractions } \\
t=1 \text { day } \\
50-150 \mathrm{rad}\end{array}$ & $\begin{array}{l}\text { Asynchronous } \\
\text { C } 3 \mathrm{H} 10 \mathrm{~T} 1 / 2\end{array}$ & Decrease & Hill et al. ${ }^{15}$ \\
\hline $\begin{array}{r}200-k V p \times \text { rays } \\
(50 \mathrm{rad} / \mathrm{min})\end{array}$ & $\begin{array}{l}\text { Split doses } \\
t=3-15 \mathrm{hr} \\
93-372 \mathrm{rad}\end{array}$ & $\begin{array}{l}\text { Plateau- } \\
\text { phase } \\
\text { C3H10T1/2 }\end{array}$ & Decrease & Terasima et al. ${ }^{2 \gamma}$ \\
\hline${ }^{60} \mathrm{Co}$ gamma rays & $\begin{array}{l}\text { Low dose rate } \\
(0.1 \mathrm{rad} / \mathrm{min})\end{array}$ & $\begin{array}{l}\text { Asynchronous } \\
\mathrm{C} 3 \mathrm{H} 10 \mathrm{TI} / 2\end{array}$ & Decrease & Han et $a .^{16}$ \\
\hline \multicolumn{5}{|l|}{ HIGH-LET RADIATION: } \\
\hline $\begin{array}{l}\text { Fission neutrons } \\
\qquad(37.8 \mathrm{rad} / \mathrm{min})\end{array}$ & $\begin{array}{l}\text { Split doses } \\
t=3-24 \mathrm{hr}\end{array}$ & $\begin{array}{l}\text { Asynchronous } \\
\text { C3H10T1/2 }\end{array}$ & $\begin{array}{l}\text { Small } \\
\text { decrease }\end{array}$ & Han \& Elkind ${ }^{28}$ \\
\hline $\begin{array}{l}\text { Fission neutrons } \\
\text { (10 rad/min) } \\
\text { (mean energy: } \\
0.85 \mathrm{MeV})\end{array}$ & $\begin{array}{l}5 \text { fractions } \\
t=1 \text { day }\end{array}$ & $\begin{array}{l}\text { Asynchronous } \\
\text { C3H10T1/2 }\end{array}$ & Enhancement & Hill et al. ${ }^{22}$ \\
\hline Fission neutrons & $\begin{array}{l}\text { Low dose rate } \\
(0.086 \mathrm{rad} / \mathrm{min})\end{array}$ & $\begin{array}{l}\text { Asynchronous } \\
\text { C3H10T1/2 }\end{array}$ & Enhancement & Hill et al. ${ }^{21}$ \\
\hline $\begin{array}{l}{ }^{241} \text { Am alpha } \\
\text { particles }\end{array}$ & $\begin{array}{l}\text { Split doses } \\
t=1 \text { day }\end{array}$ & $\begin{array}{l}\text { Asynchronous } \\
\text { plateau- } \\
\text { phase } 3 T 3\end{array}$ & $\begin{array}{l}\text { Small } \\
\text { decrease }\end{array}$ & Geard et al. ${ }^{23}$ \\
\hline $\begin{array}{l}\text { Heavy ions: } \\
{ }^{40} \mathrm{Ar}(400 \mathrm{MeV} / \mathrm{u}, \\
120 \mathrm{keV} / \mu \mathrm{m}) \text { and } \\
{ }^{36} \mathrm{Fe}(600 \mathrm{MeV} / \mathrm{u}, \\
200 \mathrm{keV} / \mu \mathrm{m})\end{array}$ & $\begin{array}{l}\text { Low dose rate } \\
\leqslant 2 \mathrm{rad} / \mathrm{min}\end{array}$ & $\begin{array}{l}\text { Plateau- } \\
\text { phase } \\
\text { C3H10T1/2 }\end{array}$ & Enhancement & $\begin{array}{l}\text { Yang et al.(1986) } \\
\text { (present study) }\end{array}$ \\
\hline
\end{tabular}

2. Hill, C.K., Han, A., and Elkind, M.M. Fissionspectrum neutrons at low dose rate enhance neoplastic transformation in the linear, low dose region (0-10 cGy). Int. 1. Radiat. Biol. 46, 11-15 (1984).

3. Elkind, M.M., Han, A., and Hill, C.K. Errorfree and error-prone repair in radiationinduced neoplastic cell transformation. In Radiation Carcinogenesis Epidemiology and Biological Significance, I.D. Boice, Ir. and F. Fraumrni, Jr. Eds. Raven Press, New York, pp. 303-318 (1984).

4. Ulltich, R.L. The rate of progression of radiation-transformed mammary epithelial cells is enhanced after low-dose-rate neutron irradiation. Radiat. Res. 105, 68-75 (1986).

5. Yang, T.C., and Tobias, C.A. Neoplastic cell transformation by energetic heavy ions and its modification with chemical agents. Adv. Space Res. 4, 207-218 (1984).

6. Yang, T.C., and Tobias, C.A. Mechanisms of radiation induced neoplastic cell transformation. In Proceedings of the Berkeley Conference in Honor of Jerzy Neyman and Jack Kiefer, L.M. LeCam and R.A. Olshen, Eds. Wadsworth, Inc., California, Vol. 1, pp. 343-371 (1985). 
7. Yang, T.C., Craise, L.M., Mei, M., and Tobias, C.A. Neoplastic cell transformation by heavy charged particles. Radiat. Res. 104, S177-S187 (1985).

8. Tobias, C.A., and Grigor'yev, Y.G. lonizing radiation. In Foundations of Space Biology and Medicine, M. Calvin and O.G. Gazenko, Eds. NASA, Washington D.C., Vol. 2, pp. 473-531 (1975).

9. Yang, T.C., and Tobias, C.A. Radiation and cell transformation in vitro. Advances in Biological and Medical Physics 17, 417-461 (1980).

10. Borek, C. Radiation oncogenesis in cell culture. Advances in Cancer Research 37, 159-232 (1982).

11. Boreki, C., and Hall, E.J. Transformation of mammalian cells in vitro. Nature (London) 243, 450-453 (1973).

12. Borek, C., and Hall, E.J. Effect of split doses of $x$ rays on neoplastic transformation of single cells. Nature (London) 252, 499-501 (1974).

13. Miller, R., and Hall, E.J. X-ray dose fractionation and oncogenic transformation in cultured mouse embryo cells. Nature (London) 272, 58-60 (1978).

14. Little, J.B. Quantitative studies of radiation transformation with the A31-11 mouse Balb/3T3 cell line. Cancer Res. 39, 1474-1480 (1979).

15. Hill, C.K., Han, A., Buonaguro, F., and Elkind, M.M. Multifractionation of ${ }^{60} \mathrm{Co}$ gamma rays reduces neoplastic transformation in vitro. Carcinogenesis 5, 193-197 (1984).

16. Han, A., Hill, C.K., and Elkind, M.M. Repair of neoplastic transformation damage following protracted exposure to ${ }^{60} \mathrm{Co}$ gamma rays.

Br. J. Cancer 49, Suppl. VI, pp. 91-96 (1984).

17. Watanabe, M., Horikawa, M., and Nikaido, $O$. Induction of oncogenic transformation by low doses of $x$ rays and dose-rate effect. Radiat. Res. 98, 274-283 (1984).

18. Yang, T.C., Craise, L.M., Mei, M., and Tobias, C.A. Effect of $\beta$-araA on neoplastic cell transformation by radiation. Int. J. Radiat. Biol. 49, 547 (1986).

19. Borek, C. Cell transformation in vitro by low coses of radiation. In Particle Radiation Therapy, V.P. Smith, Ed. Am. Coll. Radiol.,
Philadelphia, Pennsylvania, pp. 284-301 (1975).

20. Borek, C. In vitro cell transformation by low doses of X-irradiation and neutrons. In Biology of Radiation Carcinogenesis, I.M. Yuhas, R.W. Tennant, and J.D. Regan, Eds. Raven Press, New York, pp. 309-326 (1976).

21. Hill, C.K., Buonagura, F.M., Myers, C.P., Han, A., and Elkind, M.M. Fission-spectrum neutrons at reduced dose rates enhance neoplastic transformation. Nature (London) 298, 67 (1982).

22. Hill, C.K., Carnes, B.A., Han, A., and Elkind, M.M. Neoplastic transformation is enhanced by multiple low doses of fission-spectrum neutrons. Radiat. Res. 102, 404-410 (1985).

23. Geard, C.R., Georgsson, M., and Travisano, $M$. The mouse $3 T 3$ cell transformation system: Mechanistic and split dose studies with high and low LET radiation. Int. J. Radiat. Biol. 49, 518-519 (1986).

24. Ainsworth, E.J., Fry, R.J.M., Grahn, D., Williamson, F.S., Brennan, P.C., Stearner, S.P, .Carrano, A.V., and Rust, J.H. Late effects of neutrons or gamma irradiation in mice. In Biological Effects of Neutron Irradiation, International Atomic Energy Agency, Vienna (ST1/PUB/352) pp. 359-379 (1974).

25. Ainsworth, E.J., Fry, R.J.M., Brennan, P.C., Stearner, S.P., Rust, J.H., and Williamson, F.S. Life shortening, neoplasia, and systematic injuries in mice after single or fractionated doses of neutron or gamma radiation. In Biological and Environmental Effects of Low Level Radiation, International Atomic Energy Agency, Vienna (ST1/PUB/409) Vol. 1, pp. 72-92 (1976).

26. Fry, R.J.M., and Ainsworth, E.J. Radiation injury: Some aspects of the oncogenic effects. Fed. Pro. 36, 1703-1707 (1977).

27. Terasima, T., Yasukawa, M., and Kimura, $M$. Neoplastic transformation of plateau-phase mouse 10T1/2 cells following single and fractionated doses of $x$ rays. Radiation Research 102, 367-377 (1985).

28. Han, A., and Elkind, M.M. Transformation of mouse C3H1OT1/2 cells by single and fractionated doses of $x$-rays and fission-spectrum neutrons. Cancer Research 39, 123-130 (1979). 


\section{INDUCTION OF THYMIDINE-KINASE GENE MUTATION IN MAMMALIAN CELLS BY LOW- AND HIGH-LET RADIATION.}

\section{Tracy Chui-hsu Yang, Carl F. Perez, Man-tong Mei, Laurie M. Craise, and Cornelius A. Tobias}

In our laboratory we have successfully transfected rat embryonic cells (Rat-2) with the plasmid, pTK2, containing the Herpes Simplex virus (HSV) thymidine kinase (TK) gene. The physical map of pTK2 plasmid is shown in Fig. 1. Genomic analysis via Southern blotting and DNA-DNA hybridization showed that cells (H1D), cloned from Rat-2 cultures transfected with pTK2 DNA, contained one single copy of TK gene. Rat-2 cells were thymidine kinase deficient. These H1D cells provided an opportunity to study the mutagenic effect of radiation on a single specific gene of which the size and the DNA sequence were known.

We examined the effectiveness of $x$ rays and heavy ions in inducing $\mathrm{TK}^{-}$-deficient mutants in H1D cells. Figure 2 shows the cell survival curves for $x$ rays and iron particles $(600 \mathrm{MeV} / / \mathrm{u}, \sim 190$ $\mathrm{keV} / \mu \mathrm{m})$. The RBE for iron particles was about 5.2 and 3.0 at $50 \%$ and $10 \%$ survival level respectively. For $x$ rays the $L_{50}$ was about 300 rad, and the $\mathrm{LD}_{10}$ about 600 rad. The dose-response curve for TK- mutation was curvilinear for both $x$ rays and iron beam, as shown in Fig. 3. The RBE for iron

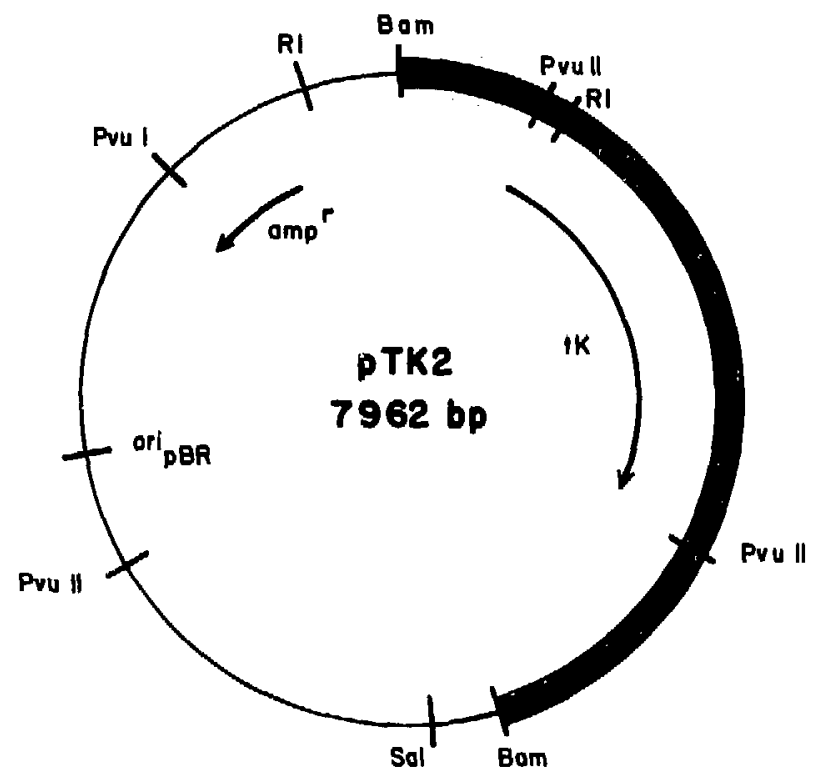

Fig. 1. Physical map of plasmid pTK2.

(XBL 836-10417) particles was about 3 at high doses for mutation. These data suggest that for an equal dose energetic iron particles can be much more effective than $x$ rays in inducing somatic mutation. When the mutation frequency was compared at equal survival level, however, there was no significant difference between $x$ rays and iron beam. The number of mutants per survivor as a function of the logarithm of the survival ratio is shown in Fig. 4, an approximately linear relationship. it appears that the number of lesions involved in the mutagenic effect increases with the lethal effect of radiation.

For understanding the molecular changes involved in the $\mathrm{TK}^{-}$mutation, we cloned many mutants from $x$-irradiated as well as from ironbeam-irradiated H1D cells. Genomic analysis of 10 $x$-ray and 10 iron-beam-induced mutants suggested that the $\mathrm{TK}^{-}$mutation was due to a large deletion of $\mathrm{TK}^{-}$gene in the H1D cells. No $\mathrm{TK}^{-}$gene could

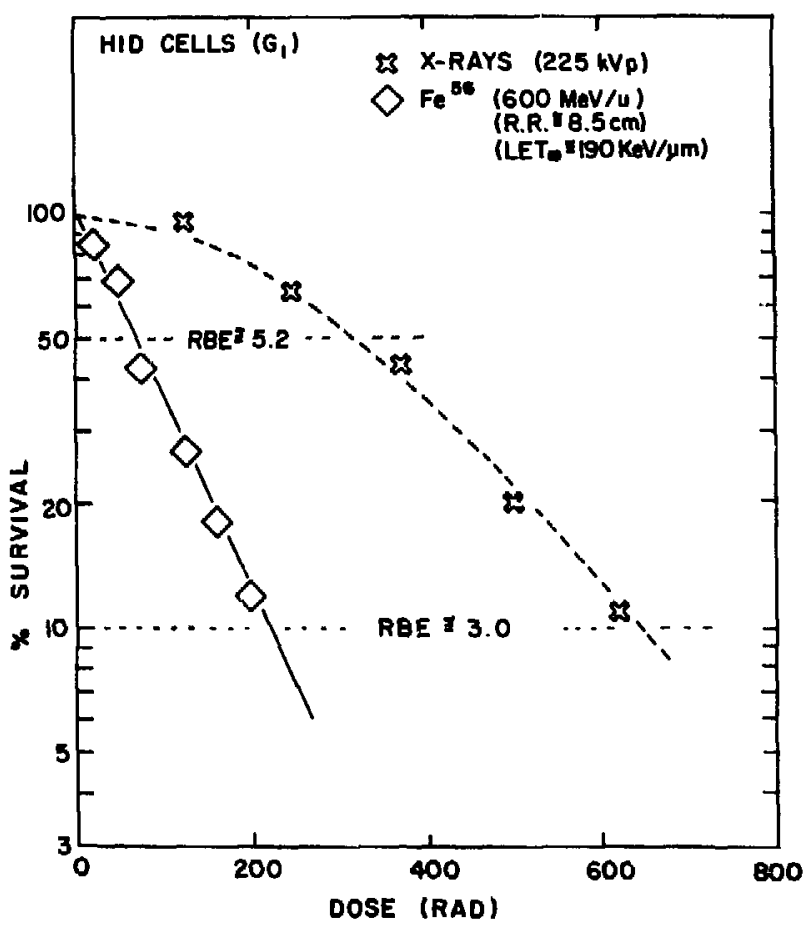

Fig. 2 Survival curves for $H I D$ cells irradiated with $x$ rays or 500-MeV/u iron particles. 


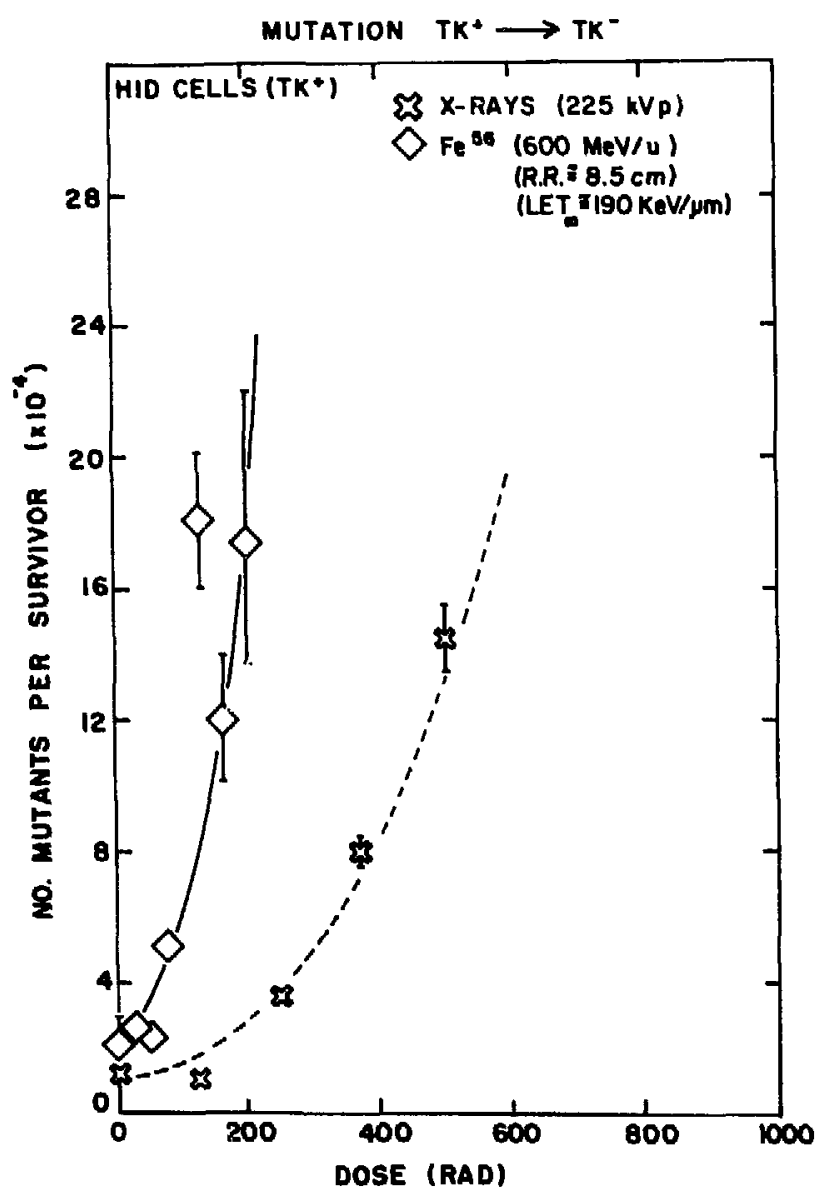

Fig. 3. Dose-response curves for TK mutation.

be detected in any of these isolated mutants. Whether the size of deletion is the same for $x$-ray and iron-particle-induced mutants is unknown at present and should be studied for a better understanding of DNA damage induced by low- and

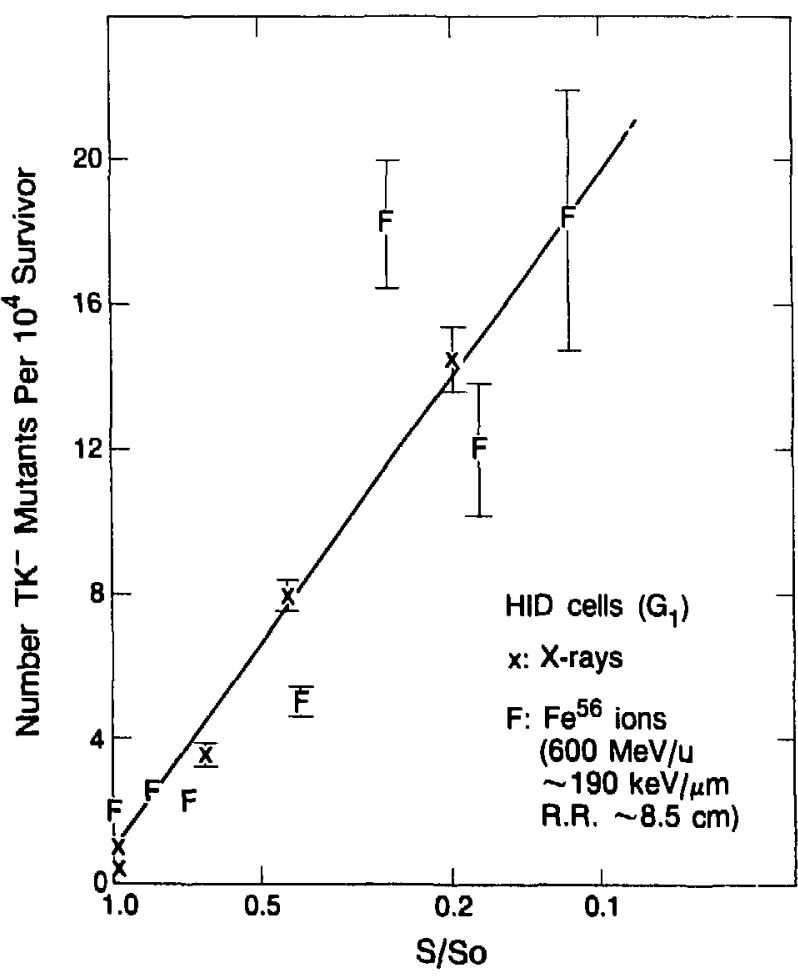

Fig. 4. The mutation frequency per survivor plotted as a function of the logarithm of the survival ratio.

(XBL-8611-6461)

high-LET radiation.

Because ionizing radiation can not directly cause such a large deletion in DNA, the TK- mutation must be a result of misrepair of the primary lesions induced by $x$ rays and heavy ions. The repair processes which produce such large deletion are important to know for a basic understanding of the radiation mutagenesis and should be investigated further.

\section{EFFECTS OF HIGH ENERGY URANIUM PARTICLES ON SOMATIC CELL MUTATION AND DNA TRANSFECTION}

\section{Tracy C. Yang, Laurie M. Craise, Gerhard Kraft, and Cornelius A. Tobias.}

One of the major goals of our research is to understand the potential biological effects of heavy-ion radiation in mammalian cells. Our earlier studies with low energy krypton ions ( $3 \mathrm{MeV} / \mathrm{u}$, L.ET $=6300 \mathrm{keV} / \mu \mathrm{m}$ ) indicated that for lethal effect the inactivation cross-section was about the average nuclear surface area, suggesting high effectiveness of very heavy particles in killing mammalian cells.' For many years, it was assumed that very heavy particles with very high LET would produce mainly 
Expt. \#311

\section{Confluent Rat-2 Cells}

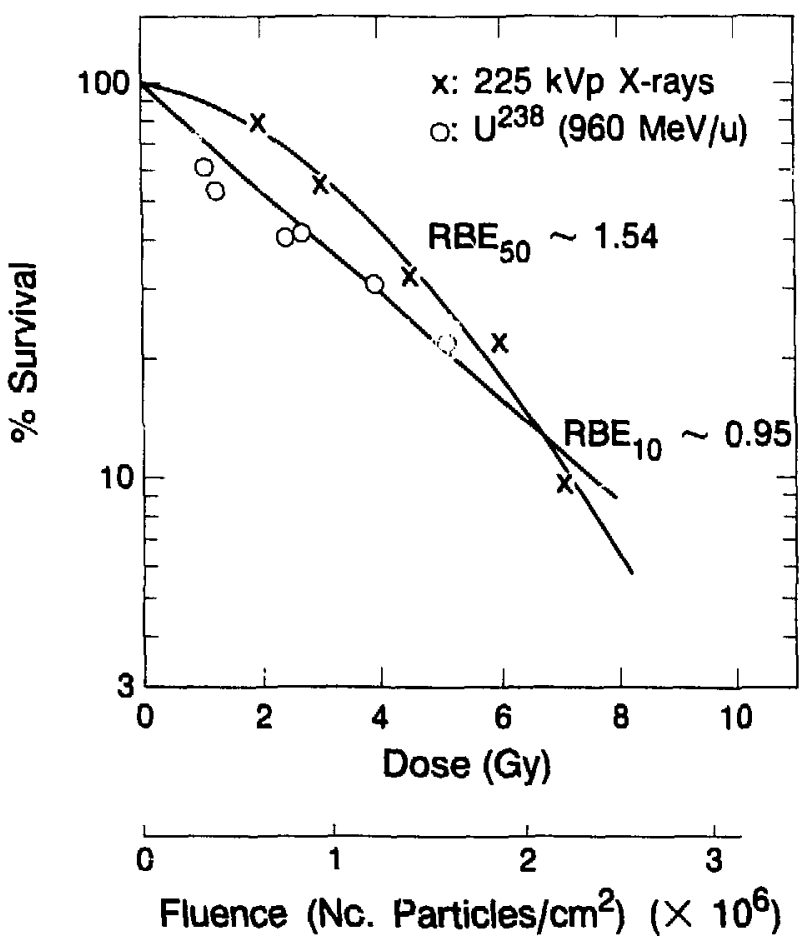

Fig. 1. Survival curves for Rat-2 cells irradialed with $x$ rays or 960-MeV/u uranium particles.

(XBL 8611-9069)

Expt. \#311

Rat-2 Cells

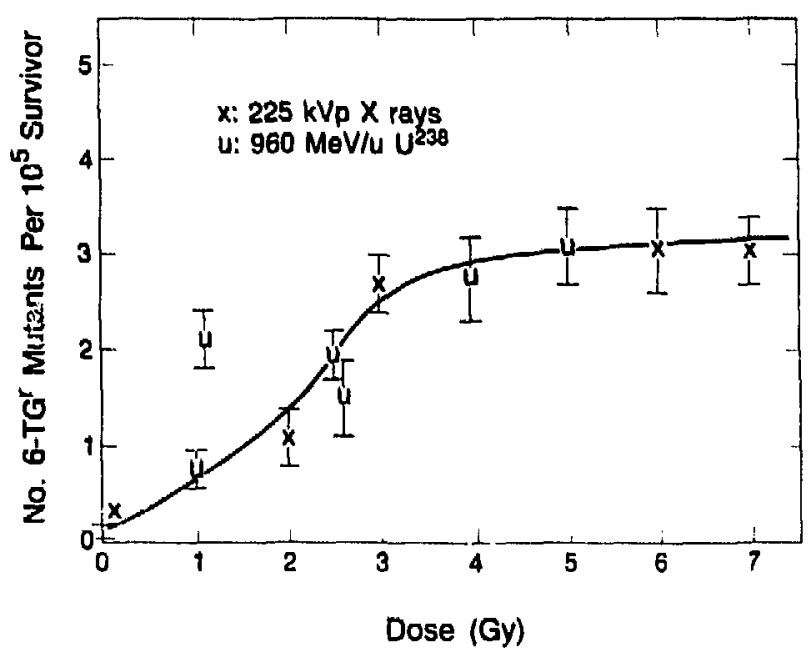

Fig. 2. Dose-response curves for HGPRT mutation in cells irradiated by $x$ rays or $960-\mathrm{MeV} / \mathrm{u}$ uranium ions.

(XBL 8612-9072) lethal effect in cells. Recently, however, experimental data showed that very heavy particles can induce nonlethal lesions. In our laboratory, for example, we have demonstrated that high energy uranium ions $(960 \mathrm{MeV} / \mathrm{u}, \mathrm{LET}=1900 \mathrm{keV} / \mu \mathrm{m})$ can cause neoplastic cell transformation. ${ }^{2}$ During past year, we extended our uranium particle studies to somatic cell mutation and DNA transfection, and found that very heavy particles can cause somatic cell mutation and can enhance the DNA transfection in mammalian cells.

Figure 1 shows the survival curves for mouse Rat- 2 cells irradiated by $x$ rays or $960-\mathrm{MeV} / \mathrm{u}$ uranium beams, accelerated at the Bevalac. The RBEs at $50 \%$ and $10 \%$ survival level were about 1.54 and 0.95 , respectively. The survival curve for uranium particles was exponential, with a $D_{0}$ about 320 cGy, or $1,000,000$ particles per $\mathrm{cm}^{2}$. The cross-section calculated from the $D_{0}$ was about 95 $\mu^{2}$. Since the average nuclear area of Rat-2 cells, as measured, is about $140 \mu \mathrm{m}^{2}$, more than one uranium particle is needed to cause a lethal effect, i.e., a single traversal of a uranium particle through the nucleus of a mammalian cell will not produce cell killing each time. Some uranium ions, when passing through the cell nucleus, can therefore induce various nonlethal lesions, e.g., transformation and mutation damages. Figure 2 shows the dose-response curves for HGPRT $^{-}$mutation for $x$ rays and uranium particles. The mutation frequency increased with the dose up to about 400 cGy and then leveled off. There was no significant difference in mutation frequency between $x$-ray and 960-MeV/u-uranium-particle irradiated cells. Similar results were found with a $325-\mathrm{MeV} / \mathrm{u}$ uranium beam, as shown in Figs. 3 and 4.

In addition to determining the survival and mutation frequencies for cells plated right after irradiation, we investigated the repair of potential lethal and mutation lesions and the importance of indirect effect. Confluent Rat-2 cells, treated with or without $2 \mathrm{M}$ DMSO, were irradiated with 325 $\mathrm{MeV} / \mathrm{u}$ uranium particles (LET $=2600 \mathrm{keV} / \mu \mathrm{m}$ ) and seeded into dishes at low density right after irradiation. For cell killing, DMSO gave a significant protection, and the dose-modifying factor was about 1.5 (Fig. 3). These results indicate that although most lethal damage produced by uranium iuns is by direct hit, some is through indirect action. The lesions induced by uranium ions appear to be irrepairable. Confluent Rat-2 cells, plated either immediately or one day after irradiation, showed no significant difference in response to uranium-ion radiation (Fig. 3). 
Expt. \#312

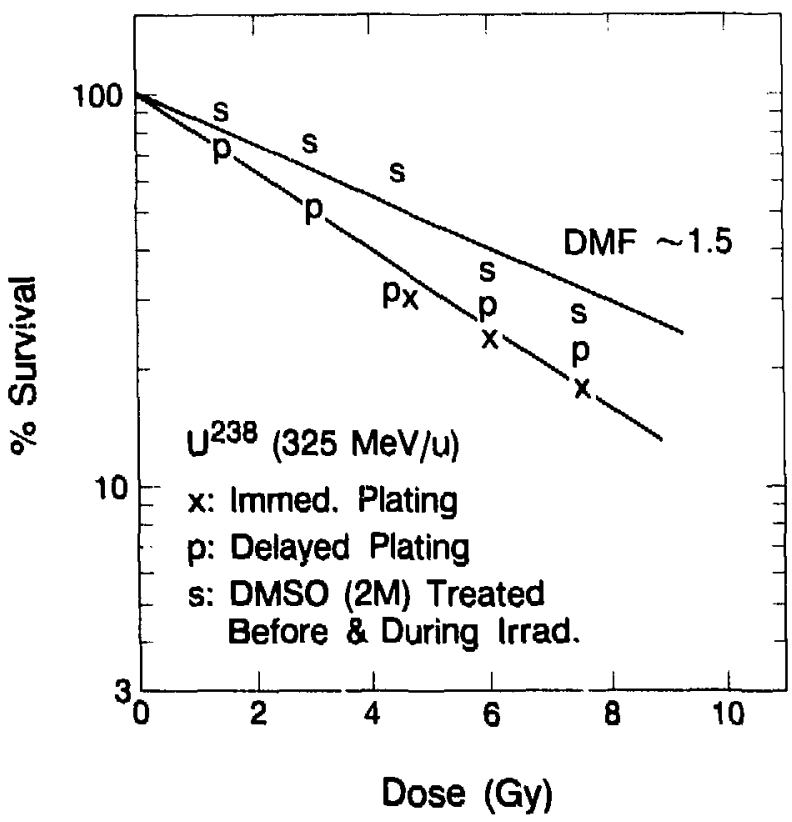

Fig. 3. Survival curves for cells irradiated by $325-\mathrm{MeV} / \mathrm{u}$ uranium particles. DMSO (2M) showed significant protection for cell killing. Confluent cells plated one day after irradiation showed no repair of potential lethal damage. (XBL 8612-9071)

The results of HGPRT $^{-}$mutation are shown in Fig. 4. The dose-response curve for confluent cells plated either right after irradiation or one-day later was about the same. There was no consistent difference in mutation frequency between cells treated with or without $2 \mathrm{M}$ DMSO. Because of the scattering of data points, it is difficult to tell at present whether DMSO is more effective in reducing the formation of lethal damage than mutation injury in heavy-ion-irradiated mammalian cells, and further investigations are needed. Should these observations be true, it implies that direct hit may be more effective in inducing mutation lesions than indirect action. And this may explain why high-LET heavy ions can give higher RBE for HGPRT- mutation than for cell killing, as observed by Cox et al. ${ }^{3}$

The two fundamental processes that determine the final biological effect of radiation are lesion formation and repair. DNA-mediated gene transfer offers an opportunity to study the DNA repair, particularly the misrejoining of DNA. in our laboratory, a recombinant plasmid (pOT-TK) has been
Expt. \#312

Rat-2 Cells (Confluent)

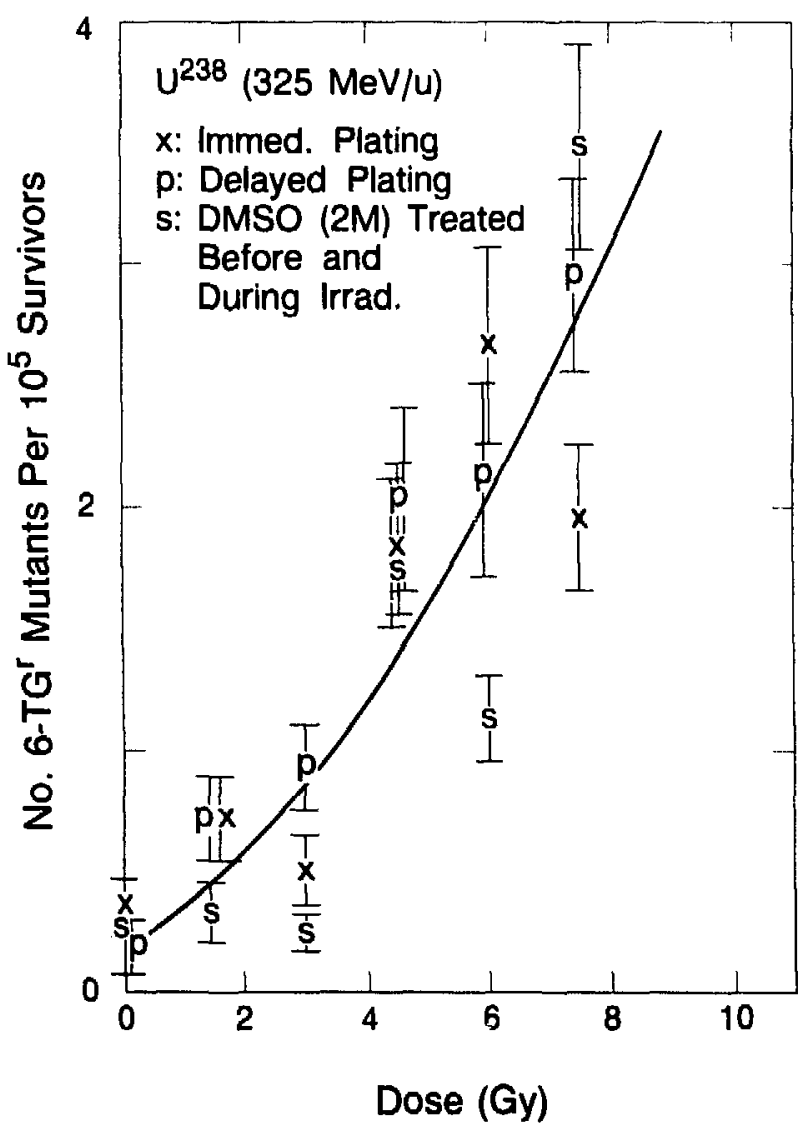

Fig. 4. Dose-response curves for HGPRT- mutation in cells irradiated by 325-MeV/u uranium beam. (XBL 8611-9070)

constructed that contains the A region of the SV40 oncogenic virus and a thymidine kinase gene of the herpes simplex virus. Using this specific DNA plasmid and Rat-2 cells, which are densitydependent, contact-inhibited, and deficient in thymidine kinase activity ( $\mathrm{TK}^{-}$), as a model system, we studied the effects of energetic uranium ions on the efficiency of DNA mediated gene transfer. The results are shown in Fig. 5. Confluent Rat-2 cells were transfected with pOT-TK plasmids after irradiation, seeded at low density and incubated for two days before plating into HAT medium for mutation selection. The number of mutants increased with radiation dose, up to about $3 \mathrm{~Gy}$, suggesting an enhancement effect. At high doses $(\geqslant 6 \mathrm{~Gy})$, there 
Expt. \#312

Rat-2 Cells (confluent) Expressing Time: 13 days

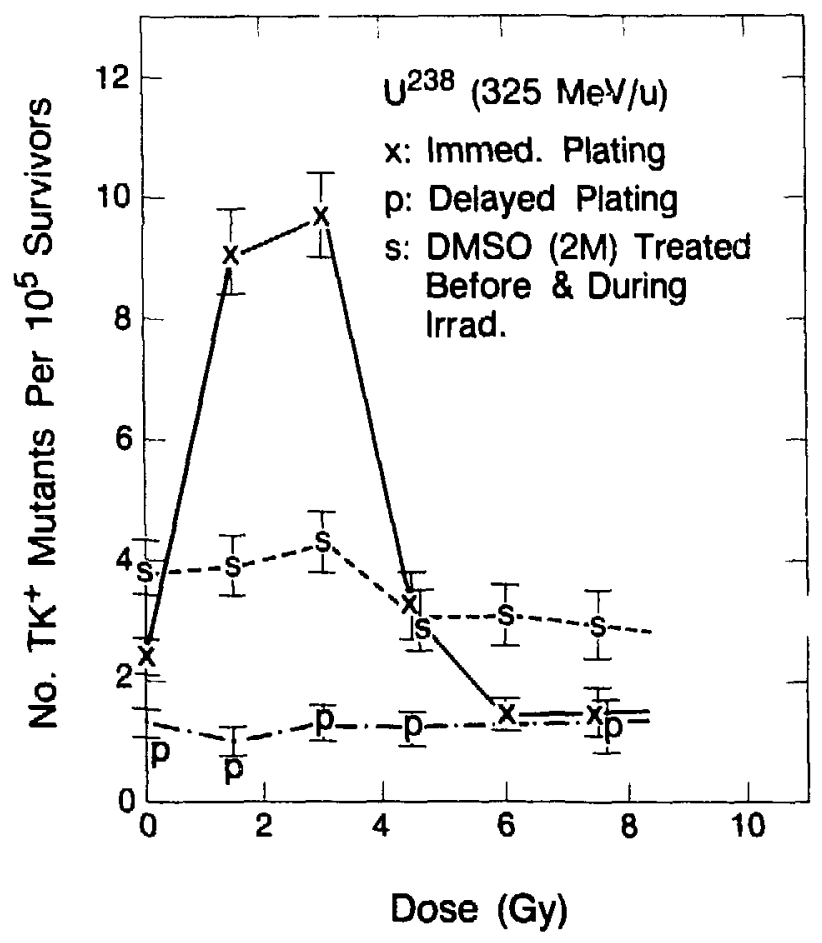

Fig. 5. Dose-response curves for DNA transfection in cells irradiated by $325 \mathrm{MeV} / \mathrm{u}$ uranium beam and plated immediately or one day after irradiation. Rat-2 cells repaired potential enhancemnt lesions effectively. DMSO (2M) showed significant protection effect.

(XBL B611.9678) was no significant difference in mutation frequency between irradiated and nonirradiated cells. When cells were transfected with recombinant plasmids one day after irradiation, however, no enhancement of DNA transfection was observed. The enhancement lesions produced by $325-\mathrm{MeV} / \mathrm{u}$ uranium particles are, therefore, repairable in mammalian cells. The enhancement effect was greatly reduced in cells treated with $2 \mathrm{M}$ DMSO shortly before and during the irradiation. These DMSO results indicate that some enhancement lesions may be formed through indirect effect.

In summary, our results showed that high energy uranium particles induce irrepairable lethal and HGPRT $^{-}$mutation lecions and repairable enhancement damage, and that DMSO can reduce the formation of lethal and enhancement lesions but not HGPRT ${ }^{-}$mutation injury.

\section{REFERENCES}

1. Yang, T.C.H., Blakely, E., Chatterjee, A., Welch, G., and Tobias, C.A. Response of cultured mammalian cells to accelerated krypton particles. Life Sciences and Space Research 15, 169-174 (1977).

2. Yang, T.C.L., Craise, M., Mei, M., and Tobias; C.A. Neoplastic cell transformation by heavy charged particles. Radiat. Res. 104, S-177-S187 (1985).

3. Cox, R., Thacker, J., Goodhead, D.T., and Munson, R.J. Mutation and inactivation of mammalian cells by various ionizing radiation. Nature (London) 267,425-427 (1977).

\section{Tumor and Tissue Effects of Heavy Particles}

\section{THE SANDWICH SYSTEM AS A TUMOR ANALOG}

\section{Lynn Hlatky and Edward L. Alpen}

The sandwich system was developed in our laboratory as an in vitro analog of poorly vascularized tumors. Like spheroids, which are heavily used as tumor analogs, sandwiches are organized diffusion-limited multicellular systems that exhibit a necrotic center and a viable cell border. However, sandwiches are two-dimensional (Fig. 1); they might be thought of as living spheroid cross sections or as monolayers on which diffusion gradients of oxygen and nutrients have been imposed by the consumption of the cells themselves. In this system, cells are grown in a narrow gap between two glass microscope slides (Fig, 1). Because of this sandwiching of cells, oxygen, nutrients, and waste products can move into or out of the local environment of a cell only by diffusing through medium in 
(a)

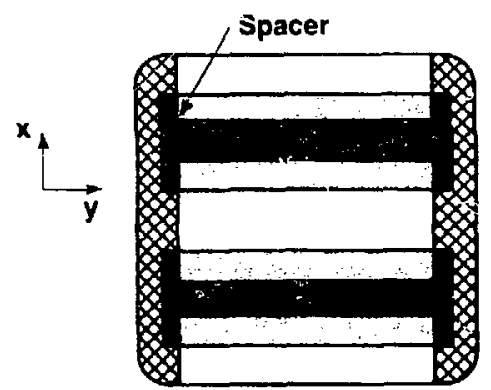

(b)

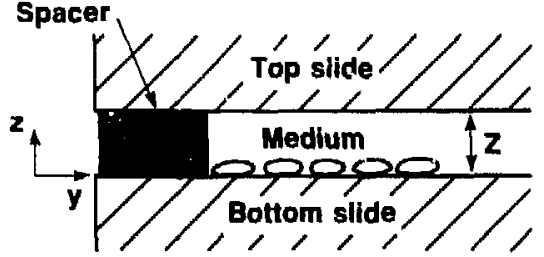

(c)

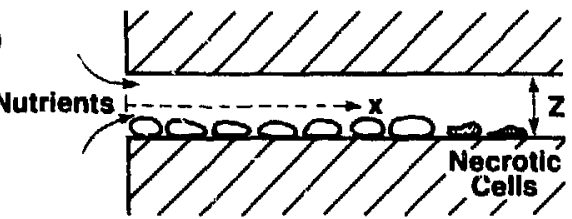

(d)

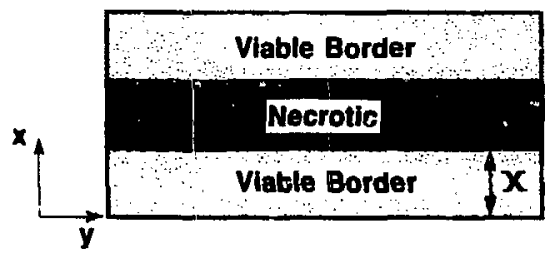

Fig. 1. Sandwich system. (a) Top view of two sandwiches in a Petri dish. (b)-(d) Three views of one sandwich. $x$ is hor. izontal distance from a cell to medium in reservoir and thus to supply to nutrients and oxygen. The $z$ direction is perpendicular to the slides. All quantities are independent of $y . X$ is width of viable border. $Z$, the gap height, was $150 \mu \mathrm{m}$.

(XBL 8611-6470)

the narrow gap between the slides. With the cells actively metabolizing, self-created diffusion gradients develop in the system. The sandwich model preserves many of the crucial features of spheroids, but has important differences and advantages that allow new experimental approaches to questions of diffusion, cell cycle effects, and radiation resistance in tumors.

Our studies using the sandwich system consisted of five main stages: 1) developing the systern as a tumor analogue, 2) characterizing the diffusion limitations and cell kinetics of the system, 3) using the system in radiation experiments, 4) analyzing the effect of varying the oxygen and glucose concentrations in the syster.1, 5) and developing an oxygen-glucose model for growth and necrosis and applying it to the system.

Using sandwiches of the $9 \mathrm{~L}$ cell line, we compared the $x$-ray sensitivity of cells in the inner half of the viable border, adjacent to the necrotic center, with that of cells in the outer half of the viable border, adjacent to the medium. No cells were hypoxic at the time of irradiation. The cells in the inner half of the viable border exhibited an increased radioresistance over cells in the outer half. The effect was dose multiplying, with a multiplying factor of 1.5. In addition to the sandwich studies, the $x$-ray sensitivity of $9 \mathrm{~L}$ plateau monolayer cultures (induced by starvation) was compared to exponentially growing monolayer cultures. The plateau cultures exhibited an increased radioresistance over the exponentially growing cultures. The effect was also dose multiplying, with a multiplying factor of 1.5. These results suggested two main conclusions that may be relevant to poorly vascularized tumors: that the radioresistance is due to the out-of-cycle cells having extra time to repair; and that the increased radioresistance of $9 \mathrm{~L}$ spheroids, which is often attributed to the cell contact effect, may be due, at least partially, to the presence of a more radioresistant subpopulation.

Using sandwiches of the $9 \mathrm{~L}$ and V79 cell lines, we investigated the effects of oxygen and glucose deprivation on the onset and formation of necrosis. Our data indicate that in sandwiches necrosis is a result of a shortage of both substances. We also performed complementary cell monolayer experiments to determine consumption parameters. On the basis of our data we propose a joint oxygenglucose model for necrosis formation. It is assumed a cell dies when oxygen deprivation in conjunction with glucose deprivation lowers the ATP production rate to a value too small for the cell's minimum needs. Interactions of the concentrations and consumptions of oxygen and glucose in sandwiches were analyzed and concentration profiles were obtained by solving coupled nonlinear integral equations numerically. The predicted viable border widths were compared to the observations. Our results suggest that in poorly vascularized tumors, oxygen shortages will almost always be accompanied by glucose shortages. 


\section{TUMOR RADIOBIOLOGY STUDIES WITH HEAVY CHARGED PARTICLE BEAMS}

\section{S.M. Javed Afzal, Thomas S. Tenforde, Kris Kavanau, and Stanley B. Curtis}

The response of a rat rhabdomyosarcoma tumor system to irradiation with heavy charged particles is being evaluated from experiments conducted both in vivo and in vitro. The radiobiological endpoints studied include tumor volume response, cellular survival after in situ tumor irradiation, and cell kinetic parameters measured by flow cytometry. The primary emphasis of our research during the past several years has been in the following areas: 1) tumor repopulation kinetics following in situ irradiation, 2) phase-specific cell survival and cell kinetics following in situ $x$ irradiation; and 3) studies on the immune response to irradiated tumors.

We have previously reported measurements of clonogenic cell survival in $\mathrm{R}-1$ rat rhabdomyosarcoma tumors of the R2C5 subline following in situ irradiation with $225-\mathrm{kVp} \times$ rays or with $557-\mathrm{MeV} / \mathrm{u}$ neon ions in the distal position of a $4-\mathrm{cm}$ extended-peak ionization region. As shown in Figs. 1 and 2, 20 Gy of $x$ rays and 7 Gy of peak neon ions reduced the initial surviving fraction to 0.025 . Significant 5 -fold and 10 -fold decreases in the fraction of clonogenic cells were observed between 3 and 4 days after irradiation with peak

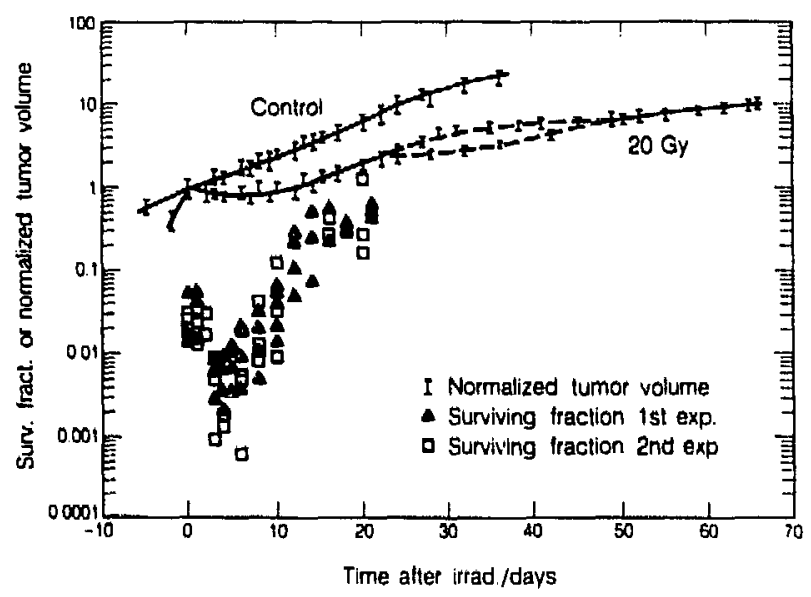

Fig. 1. X-ray data showing average tumor volume (normalized 101 at day 0 , the day of irradiation) as a function of time for the control tumors (upper line) and lumors irradiated with $20 \mathrm{~Gy}$ (lower line; dashed portion represents results of two independent experiments). The solid triangles and open squares denote surviving fractions from individual tumors irradiated with $20 \mathrm{~Gy}$ of $x$ rays oblained in two independent experiments.

$(X B L 845.7124$ neon ions and $x$ rays, respectively. These results represent the first time that a delayed decrease of this magnitude in postirradiation cell survival has been observed for a rat rhabdomyosarcoma tumor line.

Several factors related to tumor-host interactions and the tumor cell envircnment following large doses of radiation have been considered in an attempt to explain significant decrease in clonogenic cell survival. Four of the possible factors are the following:

Diploid Host Cell Infiltration. Our flow cytometry (FCM) studies on R2C5 tumors irradiated with single doses of 10 or $20 \mathrm{~Gy}$ of $225-\mathrm{kVp} x$ rays have demonstrated a significant $20 \%$ increase in the fraction of diploid host cells at $2-3$ days postirradiation. The tumors were excised and enzymatically dissociated with dispase at times ranging from 0 to 16 days postirradiation. The cellular DNA was then stained with a noncytotoxic $5-\mu \mathrm{M}$ concentration of Hoechst 33342 and the relative fractions of tetraploid R2C5 cells and diploid host cells determined by FCM analysis. In nonirradiated tumors and in

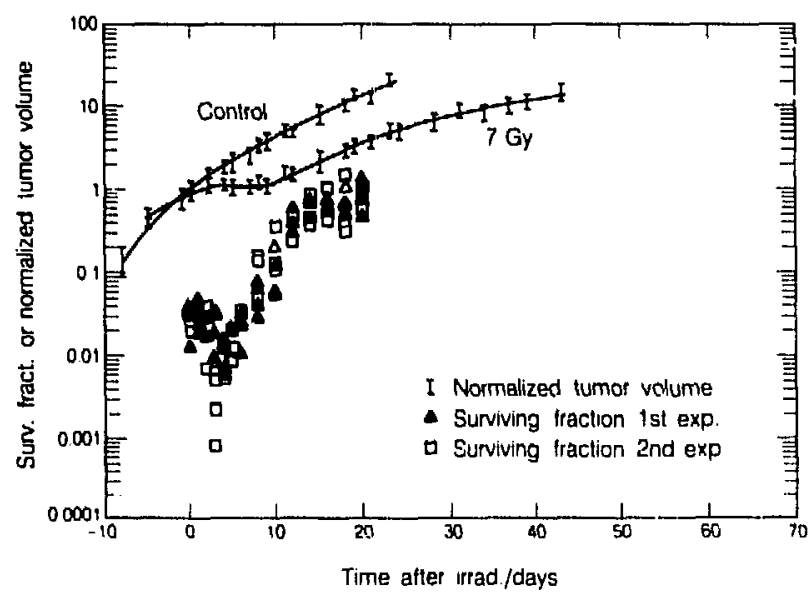

Fig. 2. Neon-ion data showing average tumor volume (normal. ized to $I$ at day 0 , the day of irradiation) as a function of time for the cuntrol lumors (upper line) and lumors irradialed with $7 \mathrm{~Gy}$ of $557 . \mathrm{MeV} / \mathrm{u}$ neon ions in the distal position of a $4 . \mathrm{cm}$ extended-ppak ionization region (lower line). The solid triangles and open squares denole surviving fractions from individual tumors irradiated wilh peak neon ions in two independent experiments.

(XBL 845-7125) 
tumors excised immediately following $x$ irradiation, the fraction of diploid cells in the total cell population was $68 \%$. By 2.5 days following $x$ irradiation, the fraction of diploid cells rose to $86 \%$. As a consequence of this infiltration of diploid cells, the hemocytometer counts of dissociated cells from irradiated tumors could overestimate the actual number of tumor cells. This error, in turn, could lead to an artifactual decrease in the apparent fraction of clonogenic tumor cells assayed in vitro since the diploid cells do not form colonies. An error of this nature, however, would account for at most a $20 \%$ decrease in the surviving fraction of tumor cells at $\mathbf{2 . 5}$ days postirradiation, and not the order of magnitude decrease observed in our experiments. Moreover, a significant error in cell counts is unlikely because of the ease with which the smaller host diploid cells can be distinguished from the tetraploid tumor cells during hemocytometer counts of dispersed cell populations. The diploid cell population is routinely excluded from the tumor cell count that is made prior to plating in vitro for analysis of the surviving cell fraction.

In Vitro "Rescue" of Hypoxic Cells. Based on earlier cell survival studies with irradiated rat rhabdomyosarcoma tumors, it can be calculated that more than $99 \%$ of the cells surviving the large doses of $x$ rays and peak neon ions used in the present experiments were hypoxic at the time of irradiation. In their hypoxic state, these cells would not be expected to actively proliferate. Because of reoxygenation, however, the survival potential of these hypoxic cells may increase during the first 2 days postirradiation. Certainly the sharp decrease in viable cells observed in our experiments would not be expecied. A decrease in survival, however, could be explained by an in vitro "rescule" of the hypoxic cells from tumors that were excised and plated in cell culture during the first 2 days postirradiation. This hypothesis is based on the assumption that hypoxic tumor cells left in situ may have a reduced survival potential, but when removed from the tumor during the first 2 days postirradiation and plated in vitro, the cells are rescued by the pres. ence of a growth medium containing feeder cells, fetal calf serum, and other nutrients. Hypoxic cells left in the tumor for 2 or more days postirradiation may not be capable of rescue, and would therefore exhibit a reduced survival leading to the sharp dip in the fraction of clonogenic cells observed in the present series of experiments.

One test of this hypothesis would be to measure the cellular repopulation kinetics of R2C5 tumors exposed to graded doses of $x$ rays that pro- duce differing survival fractions for oxygenated and hypoxic cells. Initial experiments with these tumors irradiated with 10 Gy of $x$ rays, under which condition approximately $65 \%$ of the surviving cells were hypoxic at the time of irradiation, have demonstrated that a 20 -fold decrease in survival occurs at 2-3 days postirradiation. This decrease is similar in magnitude to that shown here occurring after a 20-Gy dose of $x$ rays. The similarity in tumor repopulation characteristics following $x$ ray doses that leave differing fractions of surviving hypoxic cells argues against the occurrence of in vitro rescue of these cells in the early postirradiation interval.

Cytotoxic Factors Released from Damaged Cells. Another factor contributing to the decrease in survival of the clonogenic cells on the third to fourth day after irradiation could be an environmental one. Clonogenic cell proliferation may depend not only on the inherent characteristics of the cells but also by the environment in which the surviving cells are growing. Therefore, tumor cells that survive a large dose of radiation could nonetheless be killed when left in situ through the gradual release of cytotoxic factors from lethally irradiated cells. If these putative cytotoxic factors reach a high concentration at 3-4 days following irradiation, the survival fraction of cells from excised tumors could exhibit a significant decrease at that time. A large number of factors released from damaged tumor cells have been demonstrated to exert cytotoxic effects, including lactate activated cathepsin B, Ivsosomal enzymes, and nucleoproteins. Indirect effects of these factors on tumor cell survival could also arise because of their effects on the $\mathrm{pH}$ of the extracellular fluid. Experiments to test directly this potential mechanism of delayed tumor cell death following irradiation have been initiated.

Host Immune Response to Irradiated Tumors. The significant infiltration of R2C5 tumors by host cells at 2-3 days following irradiation suggests that an immune response to the irradiated tumor cells may be responsible for the delayed decrease in postirradiation cell survival. Tumor cell survival data analyzed in vitro following FCM and cell sorting procedures performed at 0 and 4 days after in situ irradiation with $10 \mathrm{~Gy}$ of $225-\mathrm{kVp} \times$ rays are presented in Figs. 3 and 4 . Removal of diploid host cells by cell sorting led to a significant increase in postirradiation cell survival of tumor cells. Because an unsorted cell population contains a very large number of diploid host cells, the possibility exists that a large drop in survival may be mediated partly 


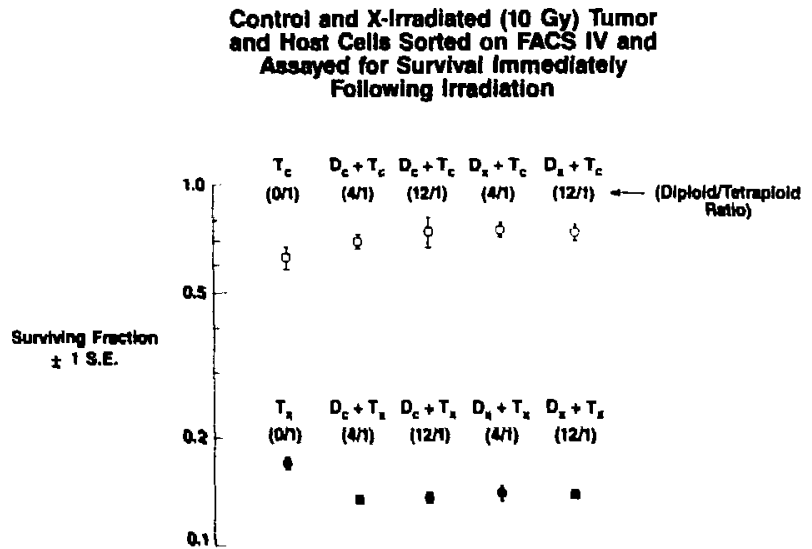

$D_{e}$ : Dtplodd host celle trom unirradiuted contro: tumors

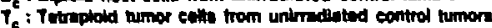

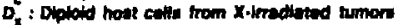

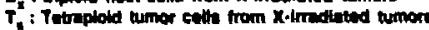

Fig. 3. Survival data are shown for tetraploid tumor cells that were analyzed for DNA content by FCM on a Becton-Dickinson FACS IV unit and isolated by cell sorting. The surviving fraction is displayed for both unirradiated (control) tumor cells and for tumor cells that were isolated immediately following an in situ 10-Gy dose of $225 . \mathrm{kVp} \times$ rays. Diploid host cells from both irradiated and unirradiated tumors were also isolated by cell sorting and admixed with control and irradiated tumor cell populations in $4: 1$ and 12:1 ratios. The survival of the diploid host cells based on an in vitro colony-forming assay was found to be less than 0.0001 . In preparation for $F C M$, the tumors were excised and enzymatically dissociated with dispase (1 $\mathrm{mg} / \mathrm{ml}, 1 \mathrm{hr}, 37^{\circ} \mathrm{C}$ ) to form single-cell suspensions. The cellular DNA in the dispersed cell populations (containing both diploid host cells and tetraploid (umor cells) was stained with Hoechst $33342\left(5 \mu \mathrm{M}, 2 \mathrm{hr}, 37^{\circ} \mathrm{C}\right)$. The diploid and tetraploid cells were then sorted on the basis of DNA fluorescence using a FACS IV unit operated at a UV laser intensity of $150 \mathrm{~mW}$. The survival fractions of unsorted lumor cell populations stained with Hoechst 33342 was nearly identical to that of the sorted tumor + diploid cell populations in both the control and $x$ irradiated experimental conditions.

(XBL 8610.6419)

or wholly by the cytotoxicity of host cells that infiltrate the tumor beginning at 2 to 3 days following irradiation. Experiments were therefore performed to test the specific toxicity of diploid host cells to irradiated tumors. In these experiments, the sorted cells were plated in the following combinations: 1) diploid cells alone, 2) tumor cells alone, and 3) diploid host cells and tetraploid tumor cells in 4:1 and 12:1 ratios. In addition, the following "crossover" platings were made: 4) host diploid cells from unirradiated tumors and tetraploid cells from irradiated tumors in a 4:1 and 12:1 ratio, and 5) host diploid cells from irradiated tumors and tetraploid cells from unirradiated tumors in a $4: 1$ and 12:1 ratio.

Data presented in Figs, 3 and 4 indicate that

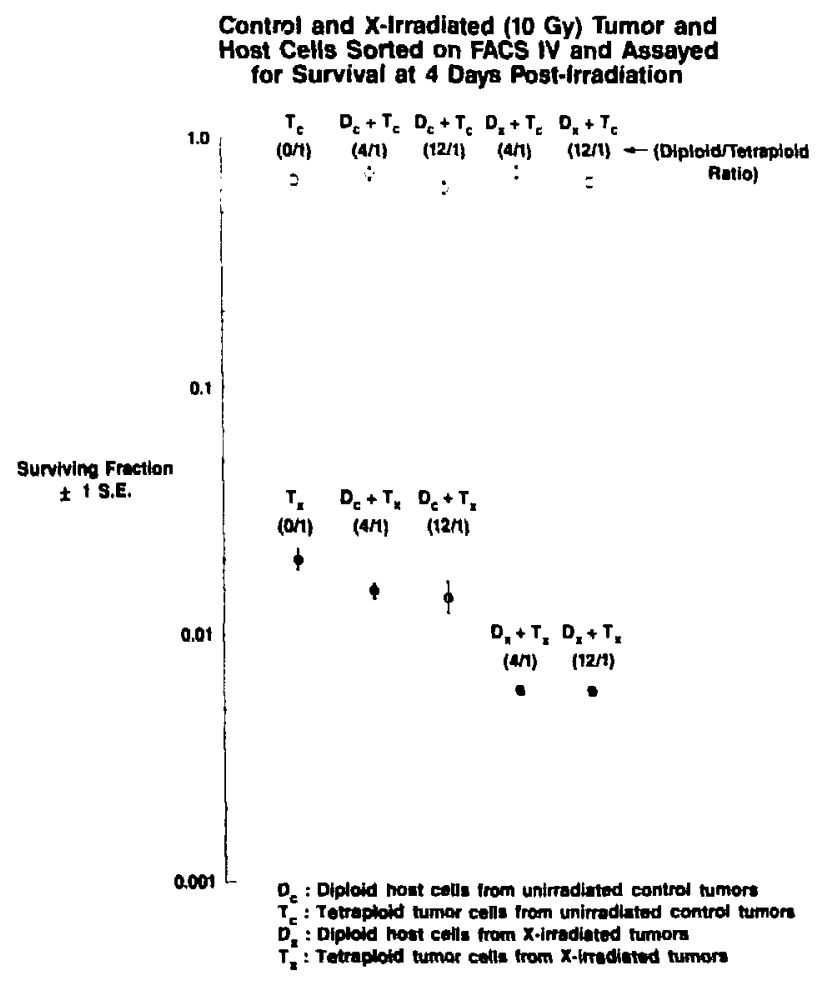

Fig. 4. Survival data are presented for tetraploid cells from both control and irradiated tumors at 4 days following a $10-G y$ dose of $225 . k V p \times$ rays. The experimental conditions and cell sorting procedures were identical to those described in Fig. 3.

(XBL 8610-6421)

diploid cells from irradiated tumors decreased the survival of irradiated tumor cells only marginally on day 0 , but dramatically on day 4 . In contrast, the diploid cells from unirradiated tumors had only a marginal effect on the survival of irradiated tumor cells at both 0 and 4 days following in situ tumor irradiation. The diploid host cells from both irradiated and unirradiated tumors had no effect on the survival of unirradiated tumor cells under any of the conditions that were tested. This observation indicates that the host's immune response is specific for the irradiated tumor cells.

These studies clearly implicate a specific host immune response against irradiated $\mathrm{R} 2 \mathrm{C5}$ tumors as a factor that influences postirradiation tumor cell survival properties. The relative role of this factor and the other factors described above in producing the sharp dip in cell survival at 3-4 days postirradiation is currently under active investigation in our laboratory,

The results of experiments reported here suggest several new treatment regimens that may prove to be highly efficient in achieving tumor 
cure. Because the clonogenic cell survival at 3-4 days postirradiation is dramatically decreased following a single large dose of either $x$ rays or peak necn ions, a top-off dose of radiation administered at that time may be highly efficient in sterilizing the small residual fraction of clonogenic cells. In addition, our results suggest the possible advantages of using monoclonal or polyclonal antibodies directed against tumor antigens that are expressed following irradiation. By combining immunotherapy and radiotherapy, it may be possible to achieve tumor sterilization with radiation doses that are substantially less than the tolerance limit of normal tissue structures within the radiation field.

\section{COMPARISON OF DAMAGE AND RECOVERY IN QUIESCENT RAT LENS CELLS FOLLOWING IRRADIATION WITH HZE PARTICLES AND X RAYS}

\section{Edgar F. Riley," Alice L. Lindgren, ${ }^{\dagger}$ and E. John Ainsworth}

We are using an in vivo stimulus-response test system to quantitatively compare the biological effects of accelerated $\mathrm{Fe}$ ions with those from $x$ rays and to monitor the slow recovery of quiescent $\left(G_{0}\right)$ epithelial cells over an extended period of time.

The $250-k V p \times$ rays are generated using a General Electric Maxitron with 1/4-mm Cu + 1-mm Al filtration, resulting in a half-value layer (HVL) of 1 $\mathrm{mm} \mathrm{Cu}$ and a dose rate of about $70 \mathrm{cGy}$ per minute at $76.2 \mathrm{~cm}$ from the $x$-ray tube target. The accelerated charged ${ }^{56} \mathrm{Fe}$ particles $(600 \mathrm{MeV} / \mathrm{u})$ are from the plateau portion of the Bragg curve and have an LET of about $200 \mathrm{keV} / \mu \mathrm{m}$. During the irradiation procedure anesthetized rats are positioned in Lucite holders for irradiation of the head only.

The quiescent cells exist as a monolayer located in the central zone region of the rat lens epithelium. They can be stimulated to enter the proliferation cycle by the mitogenic stimulus of wounding. A burst of DNA synthetic activity beginning at 14 hours is followed by a burst of mitotic activity beginning at 24 hours.

Irradiation delays and suppresses the proliferative response to wounding. ${ }^{1,2}$ Both the amount of delay and the amount of suppression are directly related to dose level. When increasing the time between irradiation and wounding, we found less delay and suppression of the response. During the burst of proliferative activity, the state of latent damage is also revealed by chromosome abnormalities. Thus, by utilizing this system, we can con-

\footnotetext{
Universily of lowa Radiation Research Laboratory.

'Bemidji State Universily, Bemidji, MN.
}

currently investigate several different features of the responding cells. This is important because the observed relative biological effectiveness (RBE) of radiation often varies depending upon the particular feature studied.

Figure 1 compares the changes in mitotic abnormalities that we observed over a 1 -month period following irradiation with 1.5 Gy of 600$\mathrm{MeV} / \mathrm{u} \mathrm{Fe}$ ions and with $10 \mathrm{~Gy}$ of $250-\mathrm{kVp} \times$ rays. Following stimulation by wounding, tissue samples were taken during the mitotic burst. Whole mounts of the epithelia were prepared and stained. Anaphase and early telophase cells were evaluated microscopically for abnormalities at a magnification

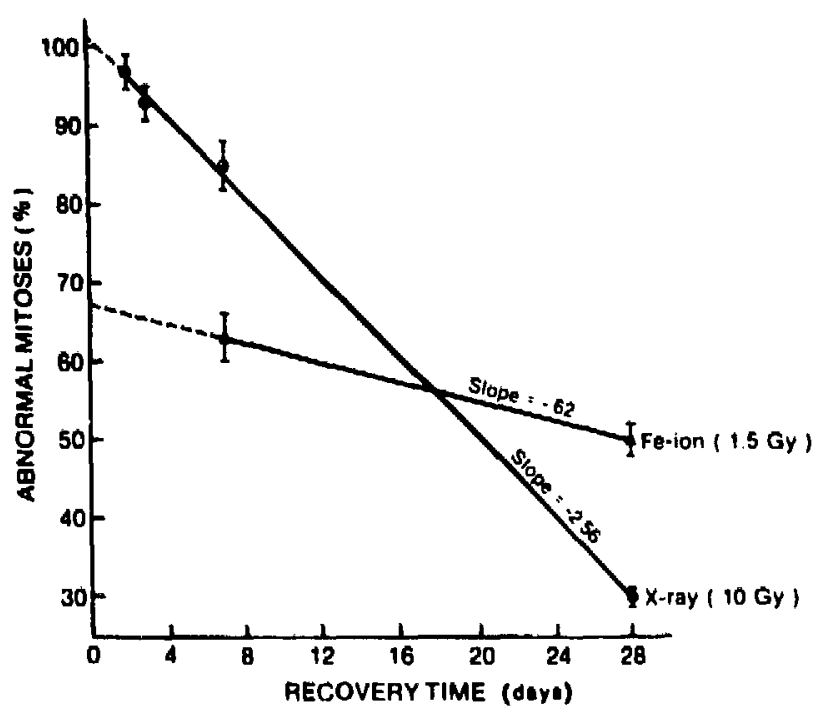

Fig. 1. The frequencies of abnormal mitotic figures as a function of recovery lime following irradiation of rat lens epithelial cells with $10 \mathrm{~Gy}$ of $x$ rays or $1.5 \mathrm{~Gy}$ of Fe ions. (XBL 8610-9014) 
of $430 \times$. The results indicate that, with respect to chromosome aberrations, damage per $G y$ is greater and recovery dramatically less for accelerated for accelerated $\mathrm{Fe}$ ions than for $x$ irradiation. This effect is under investigation for other dose levels of both types of radiations.

Figure 2 compares the recovery of the DNA synthesis response when the cells were maintained in quiescence for 7 days and for 1 month following $1.5 \mathrm{~Gy}$ of $600-\mathrm{MeV} / \mathrm{u} \mathrm{Fe}$ ion irradiation. Following stimulation, cells were pulse labeled with tritiated thymidine at various times during the burst of DNA synthesis and tissue samples were taken 2 hours later. Autoradiographs were made of the mounted epithelia, and percentages of cells that had incorporated ${ }^{3} \mathrm{H}$-TdR into DNA were determined in strips of $100 \mu \mathrm{m}^{2} \times 1200 \mu \mathrm{m}$ radially outward from the wound site. The curves in Fig. 2 depicting the percentages of labeled cells were generated by a computer program that fits a cubic spline curve to the data. ${ }^{3}$ Areas under the curves were calculated ${ }^{4}$ and utilized as measures of the DNA synthesis response. Figure 3 depicts the recovery that we observed in the DNA synthesis response when cells were maintained for various times in quiescence following Fe-ion or $x$ irradiations. These results indicate that $1.5 \mathrm{~Gy}$ of Fe ions is not more damaging than $10 \mathrm{~Gy}$ of $x$ rays. More studies are needed to compare the damage per Gy for the two types of radiations. Figure 3 indicates that the rate of

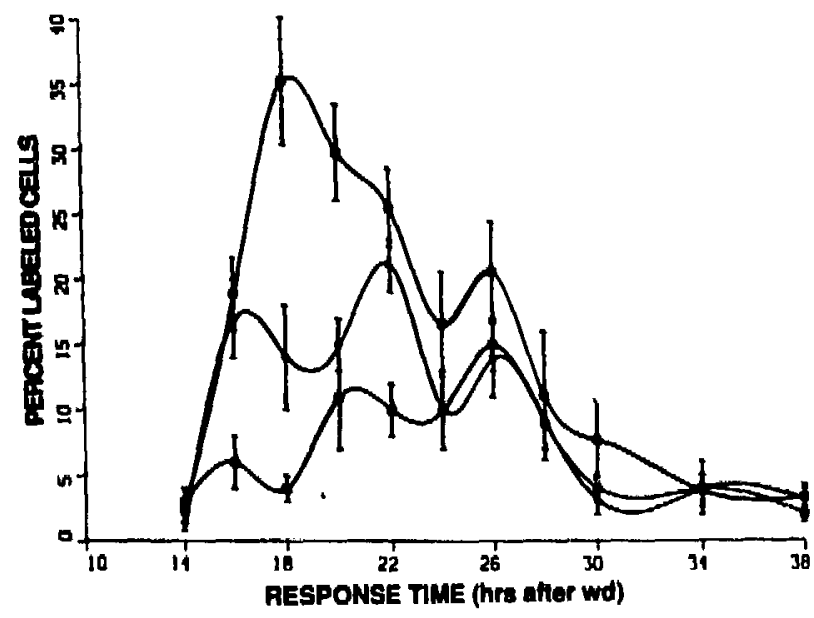

Fig. 2. Recovery of the ${ }^{56} \mathrm{Fe}$-ion-irradiation induced suppression of DNA synthesis in rat lens epithelial cells responding to the mitogenic stimulus of wounding. The percentages of cells labeled with 'H-TdR are shown for unitradiated control epithelia (lop curve), and for epithelia irradiated 7 days (boltom curve) and $t$ month (middle curve) before stimulation.

(XBL 8610.9013)

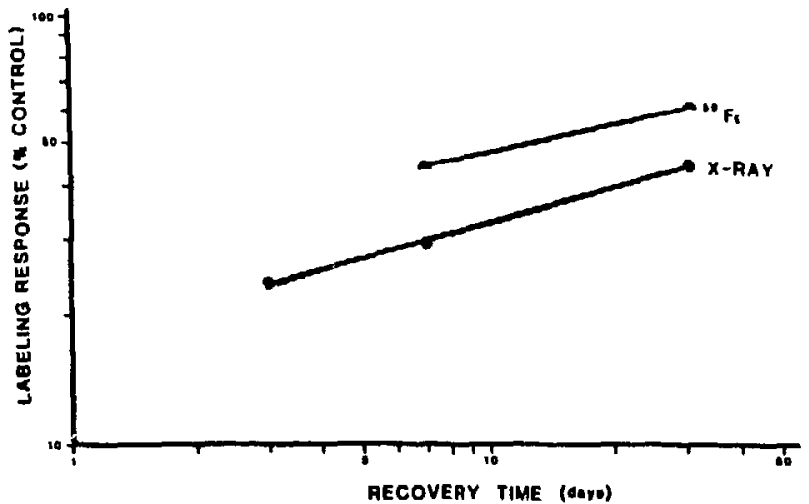

Fig. 3. A comparison of recovery from suppression of the DNA synthesis response to wounding in lens epithelia irradiated with $10 \mathrm{~Gy}$ of $x$ rays and with $1.5 \mathrm{~Gy}$ of ${ }^{56} \mathrm{Fe}$ ions. (XBL 8610-9012)

recovery following Fe-ion irradiation may be similar to that following $x$ irradiation when DNA synthesis is the feature that is investigated.

Intracellular recovery of radiation damage has most typically been studied by fractionation. Movement of cells through the cell cycle has complicated the picture. Since the rat can "incubate" the $G_{0}$ cells in the lens for many months and up to at least 1 year following irradiation, and since the $G_{o}$ cells can be "turned on" by wounding at our discretion, this system is especially suited for long term studies of the latent effect of ionizing radiations. Intracellular long term recovery, uncomplicated by any features involved with the movement of cells through the cell cycle, can be measured.

In addition, by first stimulating the cells and then irradiating at different response times, we are observing and quantitating the impact of radiations on separate events that occur during the emergence of cells from quiescence and their progression through the cell cycle.

We are now investigating the effects of multidose levels of ${ }^{56} \mathrm{Fe}$ particles, fission neutrons, and $x$ rays to get more complete dose-effect curyes and to put their relative damaging effects into perspective.

\section{REFERENCES}

1. Lindgren, A.L., and Riley, E.F. Use of the wound response to demonstrate latent radiation damage and recovery in $x$-irradiated intermitotic cells of the rat lens epithelium. Radiation Research 54, 411-430 (1973).

2. Riley, E.F., Austin, B.T., and Miller, R.C. Use of the wound response to compare recovery of neutron or $x$-irradiated intermitotic $\left(G_{0}\right)$ 
cells of the rat lens epithelium. In Biological Effects of Neutron Irradiation, IAEA, pp. 335-346 (1974).

3. West Point Military Academy Experiment Station. Subroutine USPLIN. In Graphics Com- patibility Systems from the Department of the Army (Vicksburg) (1979).

4. Akima, H. Algorithm No. 433. In Collected Algorithms, Vol. II. Association for Computing Machines, New York (1978).

\section{RESPONSE OF MOUSE MARROW STEM CELLS (CFU-S) TO HEAVY CHARGED PARTICLES}

\section{E. John Ainsworth, John C. Prioleau, and Lynn J. Mahimann}

The mouse marrow stem cell, as measured by the spleen colony technique, is a suitable model system for quantitative studies on early or late effects of ionizing radiations. ${ }^{1}$ Survival may be quantitated accurately in vivo to test hypotheses concerning relationships between cell killing and linear energy transfer (LET) or charged particle track structure, and to determine the extent to which the "normal steady state" is reestablished as a function of time following irradiation., ${ }^{1,2}$ Long-term alterations in hematopoietic regulation have been demonstrated, based on a sustained reduction in femur CFU-S content following single or fractionated doses of photons or high LET fission neutrons. ${ }^{1}$ In addition to incomplete final recovery of the CFU-S compartment, small but statistically significant anemias and thrombocytopenias have been demonstrated, indicating altered regulation in endcell production as well as an alteration or a deficiency in stem cell content. ${ }^{3}$

It was established earlier that CFU-S content and radiosensitivity could be correlated with animal radiosensitivity to hematopoietic death based on $L D_{50 / 30}$ within a single mouse strain when the hematopoietic system had not been previously perturbed. ${ }^{4}$ However, it was also determined that femur CFU-S content did not correlate with $L D_{50 / 30}$ when marrow perturbed by previous irradiation was in a regenerative phase following a single dose of radiation. ${ }^{5}$ This observation indicates that the radiosensitivity of the animal is determined by a more primitive cell than the CFU-S and that CFU-S content does not necessarily reflect the total hematopoietic potential of the animal. Recent studies have stressed the importance of CFU-S subpopula. tions that are assessed indirectly by expression of spleen colonies at times ranging between $B$ and 13 days following marrow transplantation. ${ }^{6}$ An important question concerning radiation-induced altera. tions in hematopoietic regulation concerns the extert to which various subpopulations are affected as a function of time after irradiation. Interest in sustained alterations in hematopoietic control systems relates to the fundamental aspects of regulation of proliferative systems as well as to mechanisms of alterations in relation to neoplastic diseases originating within the hematopoietic system. The status of our current program on CFU-S responses to charged particle radiation is that studies on inactivation as a function of particle charge, velocity and LET are nearly completed, and future studies will focus on triggering CFU-S into cycle, on radioprotective drugs, and on mechanisms by which late alterations in the size of the CFU-S population are produced by ionizing radiations.

The methods of irradiation and measurements of CFU-S survival have been described in detail elsewhere. ${ }^{7}$ In brief, all the results presented here were obtained using hybrid $\mathrm{CB}_{6} \mathrm{~F}_{1}$ mice given total body exposures at between 110 and 140 days of age. Animals were exposed in the plateau portion of the Brags curve, marrow was harvested within 90 minutes, marrow cell suspensions were prepared, and appropriate aliquots were injected into supralethally gamma-irradiated recipients. The recipient mice were sacrificed $\mathbf{B}$ to 10 days following marrow transplantation, and spleens were excised and fixed in alcohol, acetic acid and formalin. Surface colonies greater than $0.5 \mathrm{~mm}$ were counted with the aid of a dissection microscope. Survival curves and associated statistical parameters were computed by standard methods described elsewhere.

Figure 1 summarizes results on CFU-S inactivation as a function of radiation dose (left panel) and particle fluence (right panel). The data from which these curves were derived are presented elsewhere. ${ }^{8}$ When the effect of various charged particles is assessed based on dose, the most effective particles (those producing the steepest survival 

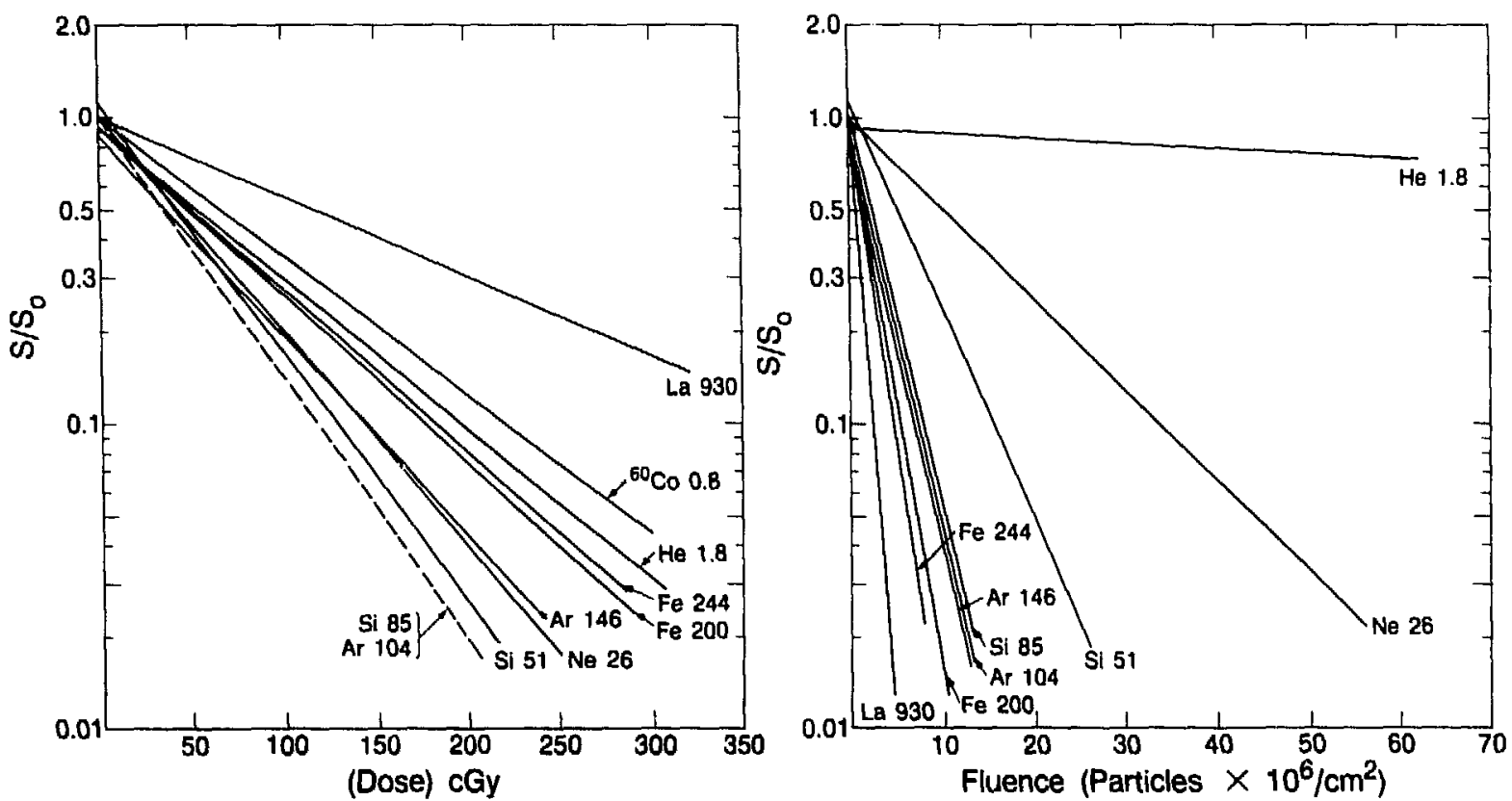

Fig. 1. CFU-S inactivation as a function of dose (left panel) and particle fluence (right panel).

(X8L 861-8635)

curves) are silicon and argon, with lanthanum being the least effective. In contrast, when the effects of charged particles are assessed based upon particle fluence, the relationships between particles are such that the inactivation effect is influenced by $Z$, with the higher $Z$ particles such as lanthanum or iron being appreciably more effective than the lower $\mathbf{Z}$ particles such as neon or helium.

Figure 2 shows the relative biological effectiveness (RBE) as a function of dose-averaged LET for marrow CFU-S compared with human ataxia telangiectasia cells and normal human T-1 cells. Over the full LET range of comparison, the response of mouse marrow CFU-S is similar to that reported by Tobias et al. for human ataxia telangiectasia (AT) cells. ${ }^{9}$ Both CFU-S and AT cells are characterized by a comparatively flat relationship between $R B E$ and LET over the LET range of $50-100 \mathrm{keV} / \mu \mathrm{m}$. The maximum RBEs determined are also similar. Based on the meager repair capacity of both CFU-S and AT cells, the clear inference pointed out earlier by Tobias et al. ${ }^{9}$ is that maximum RBE as well as the curve describing RBE-LET relationship are influenced by repair capacity.

Figure 3 compares inactivation cross sections for mouse marrow CFU-S with the cross sections reported by Wulf et al. for hamster V79 cells. ${ }^{10}$ The high radiosensitivity of CFU-S in comparison with V79 cells is indicated by the higher cross sections at any given LET, especially below $300 \mathrm{keV} / \mu \mathrm{m}$, and the initial slope of the CFU-S curve could also be higher than that for the V79 cells. The LET at which the slope becomes more shallow is at approximately $100 \mathrm{keV} / \mu \mathrm{m}$ for CFU-S and at values

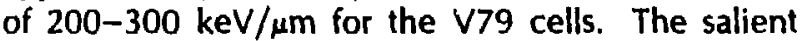
point is that in general, the inactivation kinetics of mouse marrow CFU in vivo and in situ conform generally to expectations based on biophysical prin-

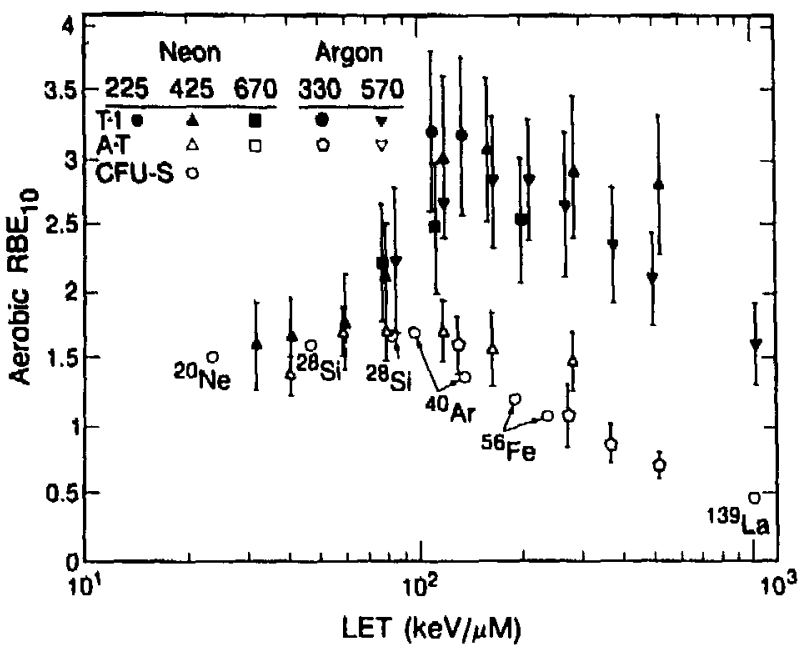

Fig. 2. Relative biological effectiveness (RBE) as a function of dose averaged LET for marrow CFU-S compared with human ataxia telangiectasia cells and normal human T-1 cells.

(XBL 838-3976A) 


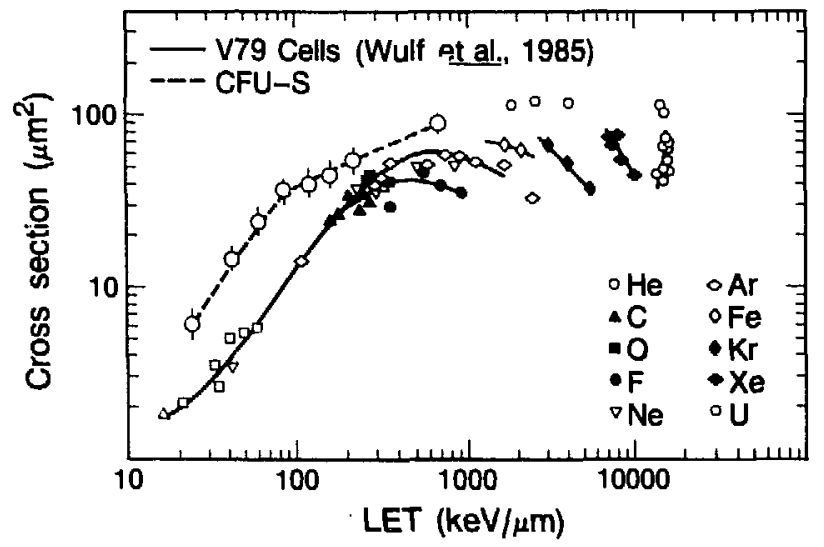

Fig. 3. Comparison of inactivation cross sections for mouse marrow CFU-S with cross sections reported by Wulf, et al. ${ }^{10}$ for hamster V79 cells.

(XBL 865-8793)

ciples inferred from studies with bacterial spores, yeast cells, and mammalian cells cultured in vitro. ${ }^{10,11}$ Because inactivation cross section reflects the effectiveness per particle, inactivation cross sections increase with $Z$ and LET with the highest cross sections being observed with iron and lanthanum particles. The estimated geometrical cross section for mouse marrow CFU-S is of the order of $50 \mu \mathrm{m}^{2}$ based on the assumption that the nuclear diameter of the CFU-S approximates that of a small marrow lymphocyte, namely $6 \mu \mathrm{m}$. Clearly, the inactivation cross section for lanthanum exceeds the geometric cross section, probably significantly, indicating that a nuclear traversal is not necessary for inactivation.

The plan is to conduct a limited extension of these studies to determine the Z-dependence of "hooks" described by Wulf et al. and shown in Fig. 3. Other future experiments with CFU-S will extend work with radioprotectants alone or in combination, such as WR2721 and prostaglandins. These studies will be conducted in collaboration with Javed Afzal and Wayne Hanson. We also plan to conduct limited experiments to determine the extent to which primary particle fragmentation influences charged particle $D_{0}$ in vivo. The basis for the fragmentation study is that results documented elsewhere indicate that a single curve describing RBE-LET relationships could be fitted to data obtained with high energy particles in the plateau portion of the Bragg curve as well as low energy particles in spread Bragg peaks. ${ }^{8}$ The data on spread Bragg peaks indicate that in spite of the primary particle fragmentation, estimates of LET have predictive value for CFU-S inactivation.
Other studies planned include the triggering of CFU-S into DNA synthesis cycle by low fluences of particles and the mechanism by which irradiation of the marrow in one hind limb triggers DNA synthesis and reduces CFU-S content in the contralateral limb that is not irradiated. These latter two studies represent a logical extension of our earlier research on hematopoietic control systems. ${ }^{12,13}$ Studies on triggering CFU-S into cycle will be conducted in collaboration with Michael Hagen.

\section{REFERENCES}

1. Ainsworth, E.J., Fry, R.J.M., Brennan, P.C., Stearner, S.P., Rust, J.H., and Williamson, F.S. Life shortening, neoplasia, and systemic injuries in mice after single or fractionated doses of neutron or gamma radiation. In Biological and Environmental Effects of Low-Level Radiation, Vol. 1, pp. 77-92. International Atomic Energy Agency, Vienna (1976).

2. Ainsworth, E.J. Early and late mammalian responses to heavy charged particles. Adv. Space Res., in press (1986).

3. Ainsworth, E.J., Jordan, D.L., Miller, M., Cooke, E.M., and Hulesch, J.S. Dose rate studies with fission spectrum neutrons. Rad. Res. 67, 30-45 (1976).

4. Ainsworth, E. J., and Larsen, R.M. Colonyforming units and survival of irradiated mice with AET or endotoxin. Rad. Res. 40, 149-176 (1969).

5. Hanks, G.E., and Ainsworth, E.J. Repopulation of colony-forming-units in mice. Nature 215, 20-22 (1967).

6. Hodgson, G.S., Bradley, T.R., and Radley, J.M. The organization of hematopoietic tissue as inferred from the effects of 5 fluorouracil. Exptl. Hematol. 10, 26-35 (1982).

7. Ainsworth, E.J., Kelly, L.S., Mahlmann, L.J., Schooley, J.C., Thomas, R.H., Howard, J., and Alpen, E.L. Response of colony-forming units-spleen to heavy charged particles. Radiat. Res. 96, 180-197 (1983).

8. Ainsworth, E.J. Inactivation of CFU-S by high energy charged particles, in preparation (1986).

9. Tobias, C.A., Blakely, E.A., Chang, P.Y., Lommell, L., and Roots, R. Response of sensitive human ataxia and resitant $\mathrm{T}-1$ cell lines to accelerated heavy ions. Br. J. Cancer 49, Suppl. VI, 175-185 (1984).

10. Wulf, H., Kraft-Weyrather, W., Miltenburger, H.G., Blakely, E.A., Tobias, C.A., and Kraft, G. 
Heavy-ion effects on mammalian cells: Inactivation measurements with different cell lines. Radiat. Res. 104, 122-S134 (1985).

11. Kiefer, J. Cellular and subcellular effects of heavy ions. Int. J. Radiation Biol. 48, 873-892 (1985).

12. Ainsworth, E. J., Mahimann, L.J., and Prioleau, J.C. Response of mouse marrow colony- forming-units-spleen (CFU-S) to heavy charged particles. Biology and Medicine Division Annual Report 1981-1982, Lawrence Berkeley Laboratory report LBL-14986, pp. 68-72, (1983).

13. Hagen, M.P., Holahan, E.V., and Ainsworth, E.J. Effects of heavy ions on cycling stem cells. Adv. Space Res., in press (1986).

\title{
THE RELATIVE BIOLOGICAL EFFECTIVENESS OF $670 \mathrm{MeV} / \mathrm{A}$ NEON AS A FUNCTION OF DEPTH IN WATER FOR A TISSUE MODEL
}

\author{
Walter Schimmerling, Edward L. Alpen, Patricia Powers-Risius, Mervyn Wong, and \\ Marwin Rapkin
}

Heavy ion beams stopping in tissue consist of primary particles and fragments due to nuclear inter.ctions in the materials presented to the beam. These fragments are a significant component of the dose, especially near the Bragg peak and distal volume. Conventional dosimetry does not identify the fluence, charge, or velocity of these components, although biological effects depend on these quantities rather than on dose or mean linear energy transfer (LET) alone. An experimental program to provide a complete characterization of the beams by particle identification and direct measurements of fluence and velocity has been in progress for several years.

A full characterization of the beam requires a three-dimensional description of the radiation field constituted by the incident beam and its nuclear reaction products. For a broad beam spot, however, results obtained on the beam axis (in a socalled good geometry experiment) are likely to be representative of the central radiation field away from the edges. Such central axis measurements have been the first step in our research program; measurements of angular distributions of particie fluence are in progress. We report here the first results of central axis fluence measurements, evaluated in terms of their RBE for testes weight loss, a tissue model for which exterisive RBE data have been developed by Alpen and Powers-Risius. ${ }^{1}$

The testes weight loss model measures the killing effectiveness for early spermatogonia in the mouse testes and provides a precise estimate for the $D_{0}$ for these cells. The cell survival curve is a shoulderless single exponential so that, as an added advantage, the data can be utilized to develop cel- lular inactivation cross sections and a $D_{0}$ expressed in terms of particle fluence.

A comparison of LET/RBE relationships for testes, gastrointestinal crypt cells, and CFU-S shows that data for all these model systems are substantially in agreement. The advantage of the testes system is that data are precise and reproducible, and because of the small volume and superficial location of the mouse testes, little beam degradation occurs in the irradiatéd tissue volume.

Central axis fluence measurements were made at the exit of a water column on which a 670 $\mathrm{MeV} / \mathrm{A}$ neon beam was incident, for 18 settings of the water column thickness. The Bragg curve was measured using an adjustable thickness water column. Individual particles emitted at the exiî of the water column were detected using a particleidentification detector spectrometer, in which the velocity of each particle emitted from the water column was deduced from a measurement of its time of flight (TOF). The finite energy loss in each of three $3-\mathrm{mm}$ thick silicon semiconductor detectors was used in conjunction with TOF for charge identification.

Data on testes weight loss were obtained in separate experiments, using only plateau particles and a minimum of material intervening between the emerging beam and the target tissue. Under these conditions the LET seen by the tissue is a welldefined narrow spectrum. The $D_{0}$ for spermatogonial cell survival was obtained as described by Alpen and Powers-Risius, ${ }^{1}$ from curves fitted to this 28-day weight loss of testes as a iunction of dose. The results are shown as the open circles in Fig. 1, each one of which has been labeled with the parti- 


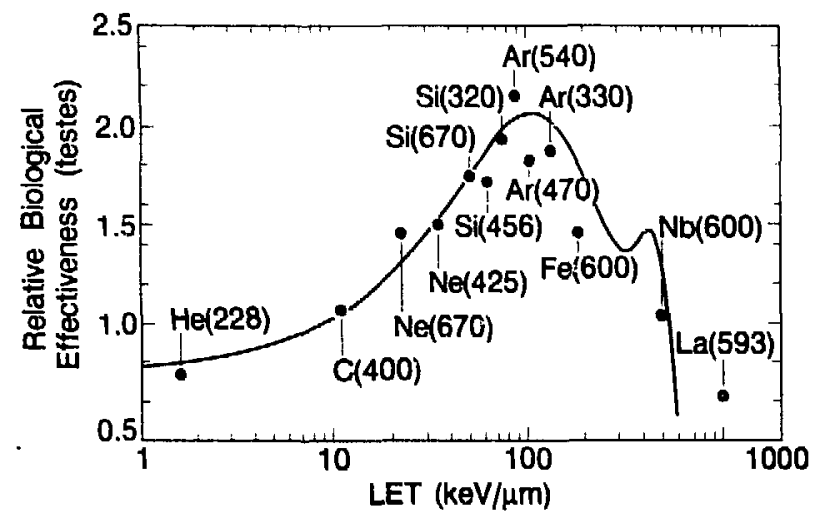

Fig. 1. Relative biological effectiveness for spermatogonial cell survival (open circles, labeled with the particle species and incident energy per nucleon used to obtain each data point) as a function of the plateau LET for the indicated incident beam. A cubic polynomial fit, weighted to approximate the high-LET data, is shown as the full curve.

(XBL 869-8972)

cle species and incident beam energy per nucleon used for each data point.

The RBE rises slowly to a peak value of 2.2 at an LET of $80-100 \mathrm{keV} / \mu \mathrm{m}$. The comparison radiation used in all cases was $250 \mathrm{kVp}$ of x-rays. This is the reason why essentially low LET helium ions have an RBE less than 1.0. If the comparison were made with ${ }^{60} \mathrm{Co}$ as the standard radiation, then the starting RBE for helium ions would be 1.0 and each other point would be respectively elevated. For purposes of the use of these data in this report, the choice of standard radiation is irrelevant, since only the proportional change is important.

From the LET spectrum of each particle at each depth of water, the dose due to each particle species can be calculated as

$$
D(Z)=(1 / A) \int_{0}^{L}[d N(Z, L) / d L] L d L,
$$

where:

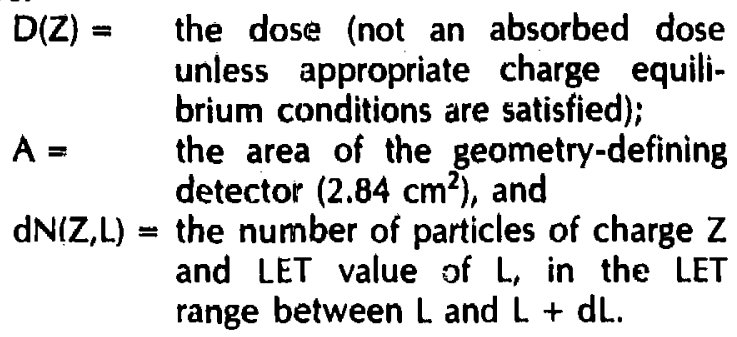

We define a biological "dose equivalent" $D_{e}$ according to

$$
D_{e}(Z)=(1 / A) \int_{0}^{L} \operatorname{RBE}(L)[d N(Z, L) / d L] L d L .
$$

For convenience of interpolation, average points were taken from the RBE data of Fig. 1 and these averaged data points were fitted to a cubic polynomial. Approximation to higher order polynomials did not significantly improve the representation of data from Fig. 1, as was also evident from an F-test; as a matter of fact, a quadratic approximation fit the data of Fig. 1 with a chi-squared of 0.06 (9 degrees of freedom), but showed large discrepancies at high values of LET, as is to be expected. Weighting the high-LET data more resulted in slightly better fits. The various approximations resulted in values of effective RBE that did not differ by more than 0.1 . The cubic polynomial interpolation curve used is shown in Fig. 1. Since all of our LET spectra were below $\sim 400 \mathrm{keV} / \mu \mathrm{m}$, the cut-off at high LET is not relevant to the analysis. For particles with higher values of LET, a better representation of RBE data must be used.

For each one of 12 thicknesses of water in the water column we obtained the value of $\langle R B E\rangle_{2}$ given by

$$
<\text { RBE }>_{\mathrm{z}}=\mathrm{D}_{\mathrm{e}}(\mathrm{Z}) / \mathrm{D}(\mathrm{Z})
$$

for the primary beam ( $Z=10)$ and for each species of fragment with $3<Z<10$. (Isotopes of the primary beam, produced by nuclear fragmentation, were of course indistinguishable from the primary beam in this experiment.) The data for the individual fragments are shown in Fig. 2, where the solid lines join the data points obtained at each water thickness. Data for fragments with $\mathrm{Z}<4(\mathrm{H}$, He and Li) were not obtained because these particles do not deposit sufficient energy to be detected in the present experimental setup. It is clear, however, that their contribution will lower the RBE at every point.

The average value of RBE for the entire radiation field, at each depth of water, was calculated by weighting the value of $\langle R B E\rangle_{2}$ obtained for each particle by the fluence of detected particles of charge Z:

$$
\operatorname{RBE}_{\text {ave }}=\Sigma<R B>_{2} N_{i}(Z) / \Sigma N_{i}(Z) \text {. }
$$

The results are plotted in Fig. 3, which shows $R E_{\text {ave }}$ as a function of depth in water. The maximum RBE is obtained near the Bragg curve (the thickness of the detectors is not negligible at this point, and results in an RBE peak that is slightly displaced) and has a value of 1.66 (errors in this value are estimated to be \pm 0.1 ).

The results can be used to predict the relative biological effectiveness in arbitrary beams, when 


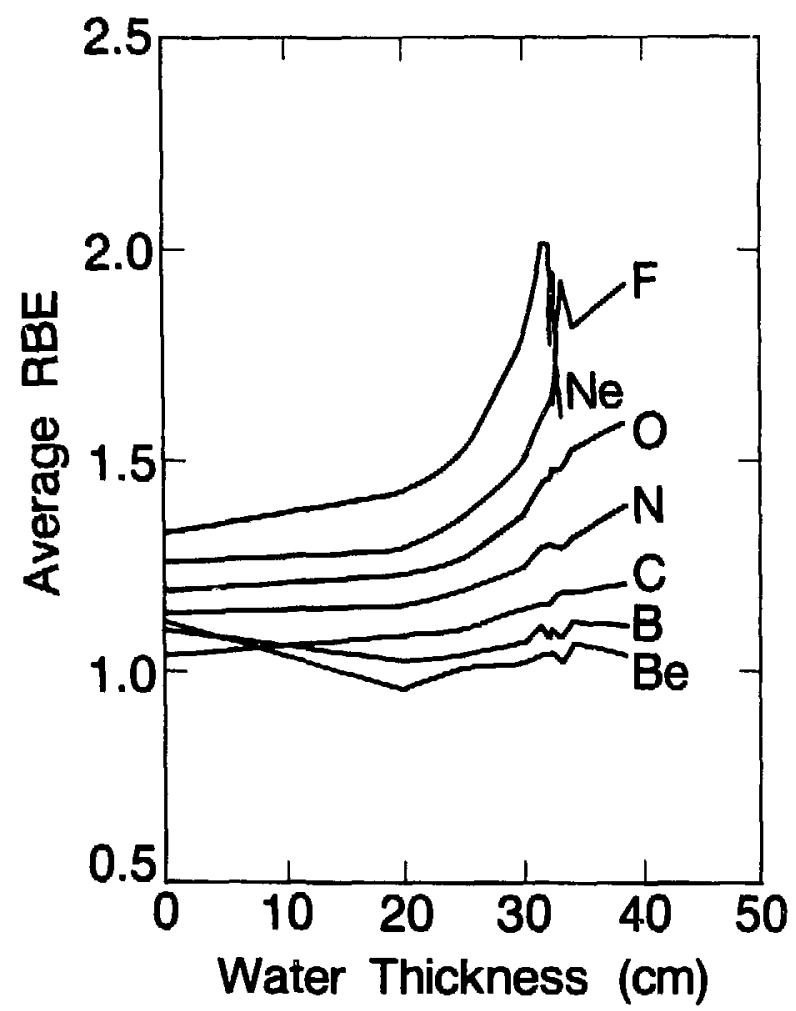

Fig. 2. Average RBE for spermatogonial cell killing calculated for each of the particles species detected in the experiment, as a function of the thickness of the water column used to measure the Bragg curve, for an incident beam of $670-\mathrm{MeV} / \mathrm{A}$ neon.

(XBB 868-8890)

the absolute particle fluences can be calculated, and to interpret the results of radiobiological experiments performed at any value of the water thickness along the Bragg curve of $670-\mathrm{MeV} / \mathrm{A}$ neon

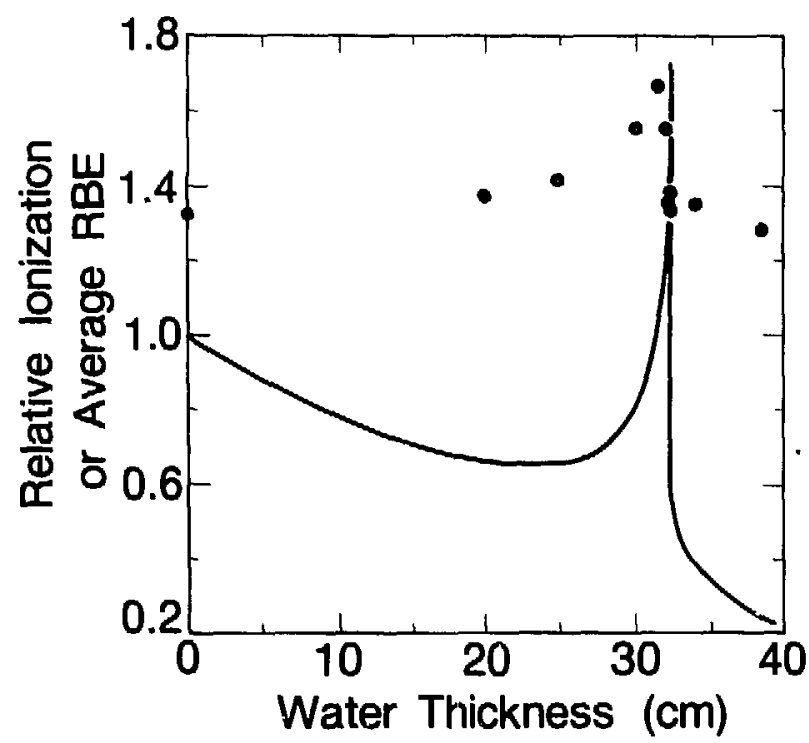

Fig. 3. Bragg curve (relative ionization) for a 670-MeV/A neon beam, and fluence-averaged RBE for the beam, calculated as discussed in the text, as a function of water column thickness.

(XBL 869-8973)

ions used in the last several years of research at the Lawrence Berkeley Laboratory Bevalac Biomedical Facility. The results should also be directly applicable to treatment planning in clinical applications of high energy heavy-ion beams.

\section{REFERENCES}

1. Alpen, E.L., and Powers-Risius, P. The relative biological effect of high-Z, high-LET charged particles for spermatogonial killing. Radiat. Res. 88, 132-143 (1981).

\section{NEON IRRADIATION OF THE RAT SPINAL CORD}

\section{Adrian Rodriguez, Edward L. Alpen, and Randy J. DeGuzman}

The spinal cord represents a site in which serious irreversible injury may be produced if it is within the treatment volume during radiotherapy. Spinal cord studies offer a unique opportunity to examine repair of heavy ion radiation damage in a late responding relatively nonproliferative tissue.

In our present study we will obtain RBE estimates and alpha:beta ratios for the induction of heavy ion-radiation myelopathy in the rat after single doses and multiple fractions. We are examining the hypothesis that the multifraction linearquadratic model predicts a higher relative biological effectiveness (RBE) for late tissue damage and a higher alpha:beta ratio after heavy-ion irradiation. We will also determine the repair potential of the spinal cord after fractionated doses of neon ions $(670 \mathrm{MeV})$ in the plateau region of ionization. 
Helium irradiation results have been used as the reference low-LET radiation for comparison with heavy-ion radiation responses. ${ }^{1-3}$ Irradiation with $670-\mathrm{MeV}$ neon in the plateau region of ionization $(\mathrm{LET}=25 \mathrm{keV} / \mu \mathrm{m})$ was given in single doses or in four or eight fractions in 25 days. Anesthetized male Sprague Dawley rats (10-12 weeks old) were held in restraining holders and irradiated transversely through the thoraco-lumbar region (T12-L1). The field was an $11 \times 16 \mathrm{~mm}$ semieliptical area. The rats were maintained for 300 days postirradiation and observed on a weekly basis for development of hind-limb paralysis or myelopathy. The scoring system to determine paralysis was the same as that used by Leith. ${ }^{2}$ Seven to ten rats were irradiated per dose point. The effective dose that produces myelopathy in $50 \%$ of the rats $\left(E d_{50}\right)$ was estimated from the results by a nonlinear least squares fit of the quantal dose response data to the function:

$$
\begin{gathered}
F=\frac{1}{1+e^{\left(P_{1}-P_{2} \times D\right)}} \\
E D_{50}=e^{\left(P_{1} / P_{2}\right)}
\end{gathered}
$$

\section{RESULTS AND DISCUSSIONS}

Irradiated rats begin to show spinal cord myelopathy within 14 to 20 weeks postirradiation (Fig. 1). The latency of onset of hind-limb paralysis is dose dependent. A minimum latency of approximately 100 days (15 weeks) is observed that is independent of total dose, fractionation scheme, and radiation type. A similar observation was made by van der Kogel and Barendsen ${ }^{4}$ for $300-k e V ~ x$ rays and $15-\mathrm{MeV}$ neutrons. The minimal latency may reflect cell turn-over of the critical cell target in the spinal cord.

Figures 2 and 3 show the plateau helium and neon irradiation results. The proportion of rats with myelopathy or hind-limb impairment after 300 days post-irradiation is plotted versus total dose. It can be seen that dose fractionation has a significant sparing effect. The helium results have been shown to be similar to previously published $x$-ray results. 2,3,5 Neon fractionated irradiation shows a high degree of sparing, although less than for helium. Table 1 shows the response parameters obtained for these data. The $\mathrm{ED}_{50} \mathrm{RBE}$ for neon increases with decreasing dose per fraction (increasing fraction number). The RBE values of single dose (SD), four fractions $(4 F x)$, and eight fractions $(8 \mathrm{Fx})$ are $1.31,1.38$, and 1.41 respectively.

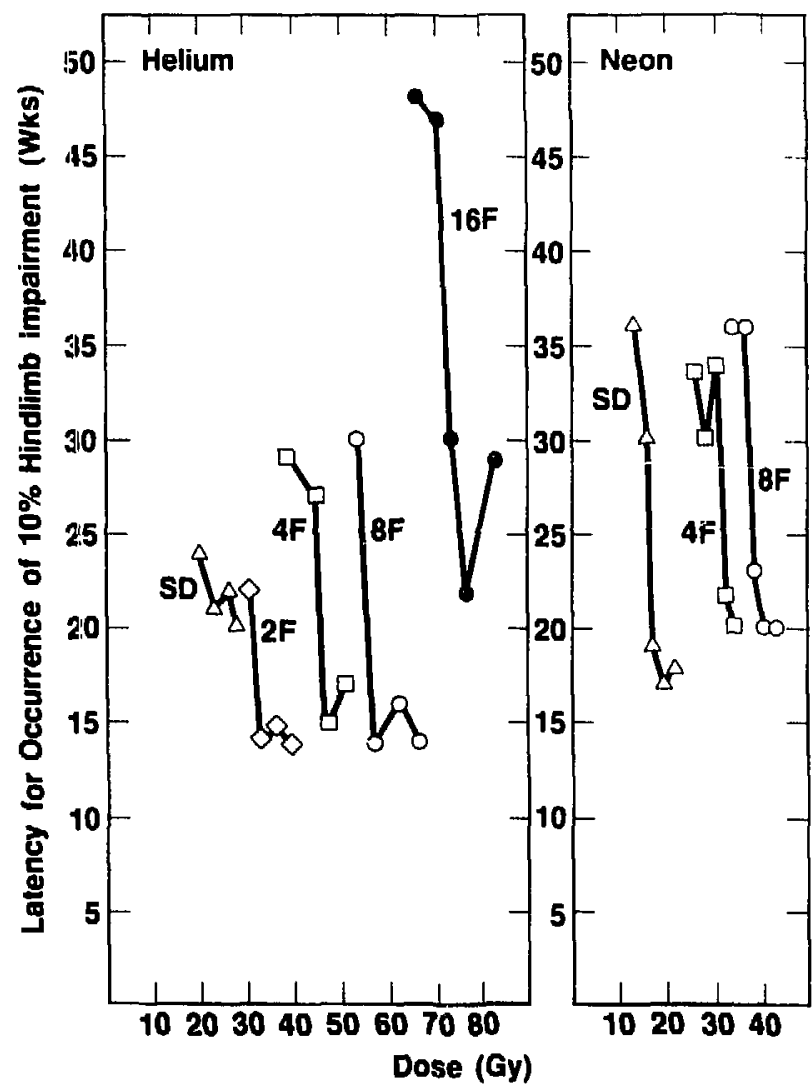

Fig. 1. Relationship between total dose and latent period for the development of partial pardlysis in $10 \%$ of rats after helium and neon irradiation. Single dose $\Delta$, two equal doses $\delta$, four equal doses $\square$, eight equal doses $O$, and sixteen equal doses $O$. (XBL B64-8695)

One of the major aims in studying the effects of heavy ions is to determine the nature of the response when multiple exposures are given. The

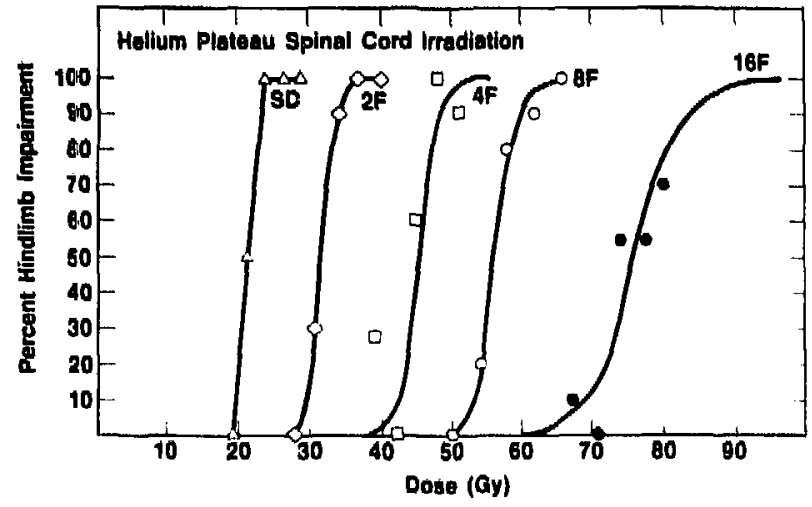

Fig. 2. Dose-effect curves for single dose and 2, 4, 8, and 16 equal doses of helium plateau irradiation in 4 weeks.

(XBL B64-8691) 


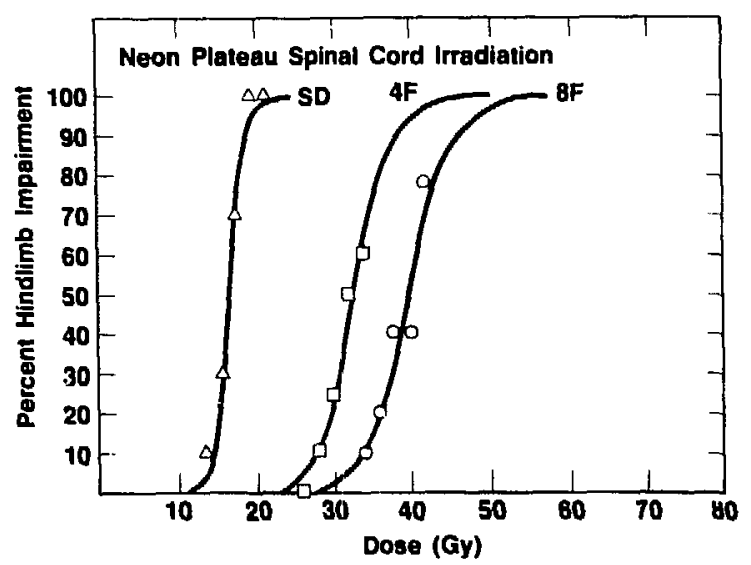

Fig. 3. Dose-effect curves for single dose and 4 and 8 equal doses of neon plateau irradiation in 4 weeks.

(XBL 864-8693)

amount of radiation repair and the RBE are two important parameters to assess as a function of position in the depth dose distribution curve. It is vital that a valid estimate of RBE be made for dose levels that are relevant to the radiotherapist. In order to evaluate RBE and repair, the reciprocal of the $\mathrm{ED}_{50}$ for induction of myelopathy is plotted versus dose per fraction. This approach allows us to generate relative estimates of linear-quadratic parameters for cell inactivation by fitting the data to the transformed multifraction linear quadratic model

$$
\frac{1}{\mathrm{nD}}=\frac{\alpha}{\mathrm{E}}+\frac{\beta}{\mathrm{E}} \mathrm{D} \text {. }
$$

The ratio of the intercept to the slope is an indicator of the nature of the radiation cell-survival curve and of the repair of subeffective radiation damage. This type of plot is useful in comparing responses to different radiation modalities. A linear

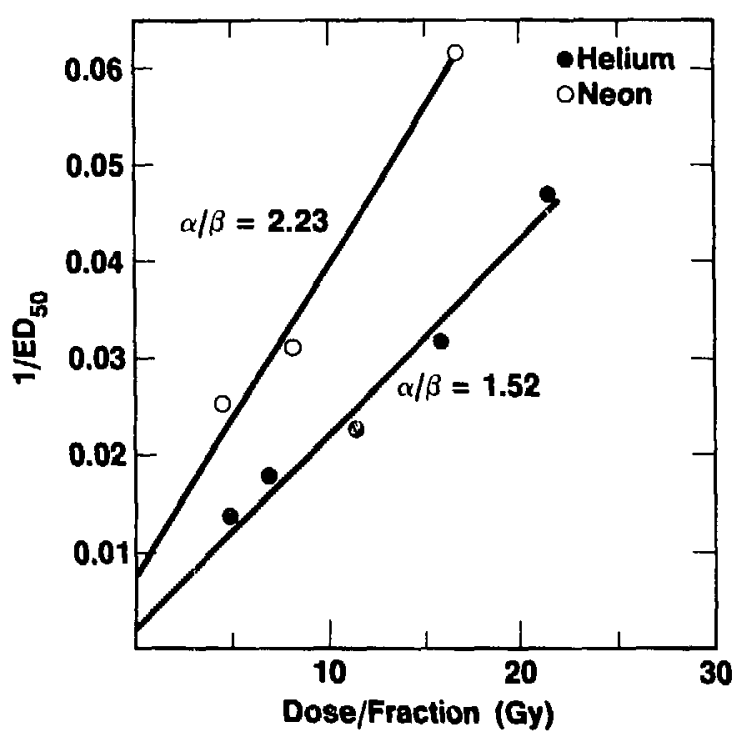

Fig. 4. Plots of reciprocal total dose versus dose per fraction from multifraction isoeffect data of rat spinal cord after helium $(0)$ and neon $(\mathrm{O})$ irradiation in the plateau region of ionization. The ratio of intercept to slope of the best-fit regression line is an estimatic oi $\alpha / \beta$.

(XBL 864-8692)

extrapolation of the multifraction data can be made to obtain RBE estimates for doses relevant to radiotherapy, and the ratio of intercepts will give the maximum possible RBE as a conservative estimate. Figure 4 is the reciprocal plot of the $E D_{50}$ doses versus dose per fraction for the data in Table 1. The data appear to be linear for both helium and neon, and are fitted by least squares regression to the multifraction linear-quadratic model. The ratio of the intercept to the slope (alpha:beta ratio) gives values of $1.52 \mathrm{~Gy}$ and $2.23 \mathrm{~Gy}$ for helium and neon respectively. The increased alpha:beta ratio for neon indicates that the alpha component is

Table 1. Summary of rat spinal cord radiation response parameters.

\begin{tabular}{|c|c|c|c|c|c|c|}
\hline lon & $\begin{array}{c}\text { LET } \\
(\mathrm{KeV} / \mu \mathrm{m})\end{array}$ & Treatment & $E_{50}(G y)$ & $F_{i}$ & RBE" & $\alpha / \beta\langle G y\rangle$ \\
\hline Helium & 1.6 & $\begin{array}{l}5 \mathrm{D} \\
2 \mathrm{Fx} \\
4 \mathrm{Fx} \\
8 \mathrm{Fx} \\
16 \mathrm{Fx}\end{array}$ & $\begin{array}{l}21.5 \pm 0.28 \\
31.5 \pm 0.08 \\
45.0 \pm 11.21 \\
57.0 \pm 1.40 \\
75.9 \pm 2.86\end{array}$ & $\begin{array}{l}0.65 \\
0.69 \\
0.70 \\
0.76\end{array}$ & $\begin{array}{l}- \\
- \\
-\end{array}$ & $1.51 \pm 1.28$ \\
\hline Neon & 25 & $\begin{array}{l}5 D \\
4 \mathrm{Fx} \\
8 \mathrm{Fx}\end{array}$ & $\begin{array}{l}16.4 \pm 0.38 \\
32.6 \pm 0.64 \\
39.7 \pm 1.26\end{array}$ & $\begin{array}{l}0.66 \\
0.67\end{array}$ & $\begin{array}{l}1.31 \\
1.38 \\
1.44\end{array}$ & $2.23 \pm 1.42$ \\
\hline
\end{tabular}

- RBE is relative to helium. 
greater for cell killing and there is less repair of subeffective damage than for helium. However, the alpha:beta ratio for neon is small compared to estimates obtained for $15-\mathrm{MeV}$ neutrons of approximately $77 .{ }^{4}$ This value indizates a very large alpha or single hit inactivation component and little sparing due to fractionation. From Fig. 4, one can obtain an estimate of RBE at 2 Gy per fraction of 2.06. The ratio of the reciprocal of the intercepts yields an RBE of 2.64 and represents a theoretical value based on an infinite number of small doses per fraction.

\section{REFERENCES}

1. Rodriguez, A., Alpen, E.L., and DeGuzman, R.D. Helium irradiation of the rat spinal cord. Annual Report: Biology and Medicine Divi- sion, Lawrence Berkeley Laboratory (1985).

2. Leith, I.T., Lewinsky. 8.L., Woodruff, K.H., Schilling, W.A., and Lyman, J.T. Tolerance of the spinal cord of rats to irradiation with cyclotron accelerated helium ions. Cancer 35, 1692 (1975).

3. Leith, J.T., Ainsworth, E.J., and Alpen, E.L. Heavy-ion radiobiology: Normal tissue studies. Advances in Radiation Biology 10, 191-235 (1983).

4. Van der Kogel, A.j., and Barendsen, G.W. Late effects of spinal cord irradiation with 300 keV x-rays and $15 \mathrm{MeV}$ neutrons. Br. J. Radiol. 47, 393-389 (1974).

5. White, $A$., and $F$ 'ornsey, S. Radiation damage to the rat spinal cord: The effect of single and fractionated doses of x-rays. Br. J. Radiol. $55,515-523$ (1978).

\title{
INDUCTION OF HARDERIAN GLAND TUMORS IN MICE BY HEAVY ION IRRADIATION
}

\author{
Edward L. Alpen, Patti Powers-Risius, R.f. Michael Fry, John Ainsworth, \\ Randy J. De Guzman, Virginia Havens, and Kris Kavanau
}

Several years ago we began to investigate the carcinogenic effects of heavy charged particles generated at the Berkeley Bevalac. Using the mouse Harderian gland system, we have accumulated data on the relative biological effectiveness (RBE) for tumor induction by cobalt, helium, carbon, neon, argon, and iron beams. Risk estimates for tumor induction by heavy ions have become important with the advent of heavy-ion radiotherapy and also with the likelihood of an increase in the number of persons in space where exposures to heavy ions can occur. Equally important is the value of these data in setting RBE values in carcinogenesis for high-linear-energy-transfer (LET) radiations in general.

A description of the Harderian gland system and data from the early experiments was presented in a previous LBL annual report. ${ }^{1}$ Eriefly, pituitaries from isogenic mice are implanted into the spleen of $B 6 C F_{1} / A N L$ female mice prior to irradiation. The pituitary isografts enhance the expression of radiation-induced tumors and advance the time of

"Biology Division, Oak Ridge National Laboratory, Oak Ridge, TN 37831 . their appearance. The increase in prolactin as a result of the pituitary implant stimulates the mammary gland tissue; the implant can be macroscopically observed at the termination of an experiment and is indicative of an active pituitary implant. The mice are killed 16 months after irradiation, the Harderian glands are processed histologically and the tumors are microscopically enumerated.

Our goal has been to determine the carcinogenic effect of heavy ions representing a wide range of LET values. During the first few years of experiments, it was necessary to irradiate the mice in the distal portion of the spread Bragg peak to obtain a high-LET beam. in recent years Bevalac improvements have resulted in the availability of high-LET plateau beams that are more homogeneoLis and that have fewer fragments than beams with spread Bragg peaks. LET values can be more precisely determined for plateau than for spread Bragg-peak beams, making for a more accurate assessment of the RBE-LET relationship for tumorigenesis. Table 1 lists the radiations we have used and the number of mice for each dose group. Although we have irradiated about 6500 mice, only those mice with confirmed successful pituitary isografts that survive to sacrifice date are considered 
Table 1. Number of mice irradiated for Harderian tumor induction. Txperiments (Hgl \#1-41)

\begin{tabular}{|c|c|c|c|c|c|c|c|c|c|c|c|c|c|c|}
\hline \multirow[b]{2}{*}{ Irrad. } & \multirow{2}{*}{$\begin{array}{c}\text { Energy } \\
\text { (MeV/amu) }\end{array}$} & \multirow{2}{*}{$\begin{array}{c}\text { Bragg- } \\
\text { peak } \\
\text { position }\end{array}$} & \multicolumn{12}{|c|}{ Dose (Gy) } \\
\hline & & & 0.05 & 0.1 & 0.15 & 0.2 & 0.4 & 0.6 & 0.8 & 1.2 & 1.6 & 2.4 & 3.2 & 7.0 \\
\hline Cobalt & & & & & & & 292 & - & 280 & - & 180 & - & 181 & 90 \\
\hline Helium & 228 & Distal & - & - & - & 211 & 201 & - & 250 & - & 275 & - & 210 & 40 \\
\hline Helium & 228 & Plat. & - & - & - & - & - & 134 & - & 88 & - & 90 & - & - \\
\hline Carbon & 400 & Distal & 143 & 100 & - & - & 194 & - & 112 & - & 109 & - & 63 & - \\
\hline Neon & 425 & Distal & 226 & 190 & 97 & 195 & 121 & - & 144 & - & 111 & - & 65 & - \\
\hline Neon & 670 & Plat. & 80 & 50 & - & 44 & 45 & - & 44 & - & - & - & - & - \\
\hline Argon & 570 & Distal & 168 & 120 & 80 & 126 & 102 & - & 154 & - & 151 & - & - & - \\
\hline Iron & 600 & Plat. & 217 & 193 & 113 & 124 & 74 & - & - & - & 95 & - & - & - \\
\hline Iron & 350 & Plat. & 52 & 54 & - & 40 & - & - & - & - & - & -- & - & - \\
\hline
\end{tabular}

Unirradiated implant controls: 310

to be at risk. Preliminary results have been published. 2,3

The most recent tumor data is shown in Fig. 1. The prevalence of Harderian gland tumors at 16 months is plotted as a function of dose in the range of 0.05 to 1.6 gray. It can be seen that the tumorigenic effects for all charged particles, including helium, are greater than for the low-LET ${ }^{60}$ cobalt reference radiation. There is a general trend of an increase in the initial slopes of the curves with increasing LET. The LET values in units of $\mathrm{keV} / \mu \mathrm{m}$ are: helium distal peak, 6; neon plateau, 25; carbon distal peak, 80; neon distal peak, 150; iron plateau, 190; argon distal peak, 650. The difference in prevalence of tumors induced by heavy ions becomes less with increasing dose. The data are

Harderian Gland Tumors

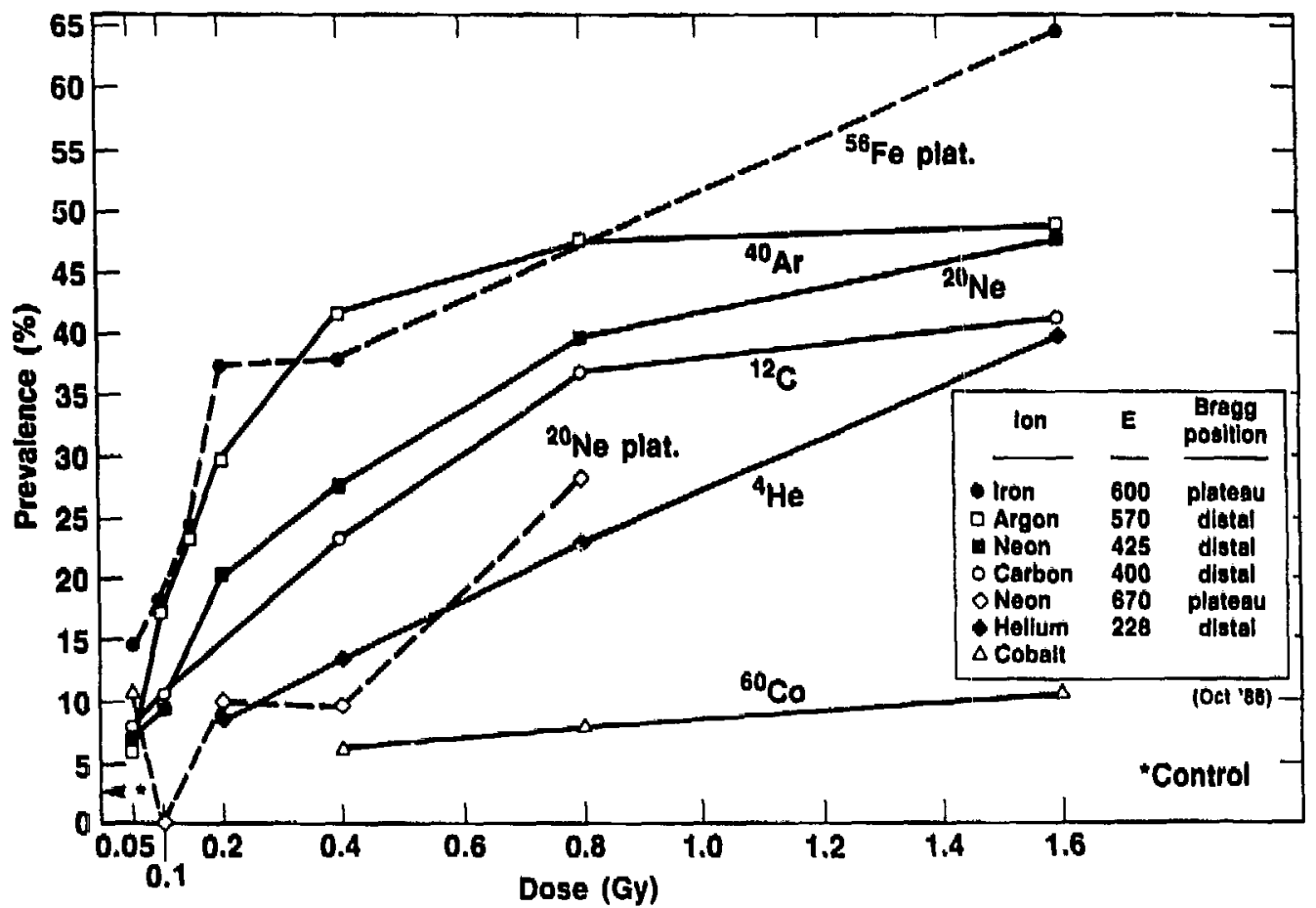

Fig. 1. Prevalence of Harderian gland tumors as a funrtion of dose of cobalt gamma rays and various heavy ions. The energy and position in the Bragg peak for each ion is shown in the inset.

(XBL 869-8974) 
not shown here, but after exposure to 7 Gy of cobalt garrima rays the percent prevalence reached about the same level as the higher LET radiations, $35-45 \%$. The number of cells with a potential for tumor production must decrease with an increase in dose due to cell killing. Such a cell loss may cause the dose-response curve for tumor production to bend over as the effect per dose of radiation decreases. However, the tumor prevalences from heavy-ion radiation remain at the same level over a wide range of doses. In general, it is believed that the level at which the tumor response reaches a plateau may be influenced by such factors as dose distribution, cell transformation, cell killing, and the susceptibility of the mouse population to the tumor under study. Satisfactory interpretation of the shape of the heavy-ion doseresponse curve is still being investigated.

The data in Fig. 2 are the same as in Fig. 1, but without the most recent iron and neon plateau results. The dose scale is simply expanded to allow the reader to sense the quality of the data in the region of the initial slope. The initial slope is used for the determination of the RBE. From these data, using linear regression analysis, the initial slope of each ion is determined and the RBE evaluated as

\section{Harderian Gland Tumore}

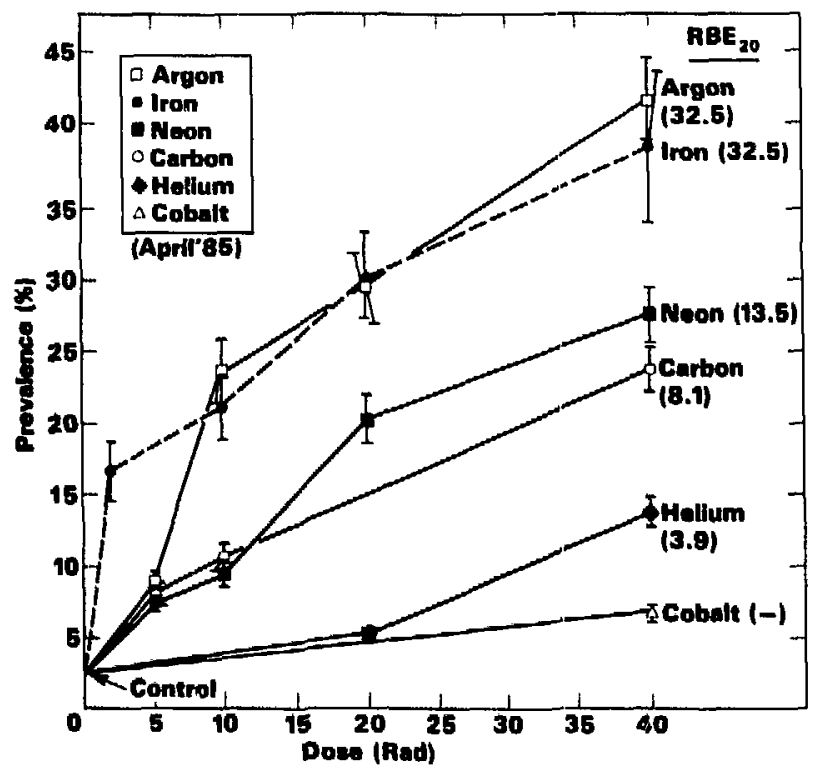

Fig. 2. Prevalence of Harderian gland turnors over the dose range of 0.5 to $0.40 \mathrm{~Gy}$. The RBEs based on the ratio of the initial slopes are noted for each ion. the ratio of the slopes. The RBEs so obtained are as follows:

\begin{tabular}{lccr}
\hline Radiation & $\begin{array}{c}\text { Energy } \\
(\mathrm{MeV} / \mathrm{amu})\end{array}$ & $\begin{array}{c}\text { LET } \\
(\mathrm{keV} / \mu \mathrm{m})\end{array}$ & RBE \\
\hline Helium-4 & 228 & 6 & 5 \\
Carbon-12 & 400 & 80 & 12 \\
Neon-20 & 425 & 150 & 18 \\
Argon-40 & 570 & 650 & 27 \\
Iron-56 & 600 & 190 & 27 \\
\hline
\end{tabular}

From these data several issues arise. Firstly, all of the initial slope RBEs depend entirely upon the precision of the low-dose cobalt data, which are limited to a single data point at $0.40 \mathrm{~Gy}$. Secondly, we have not reached an LET value at which carcinogenic potential is decreased. Cell killing and other normal tissue end points for radiation damage have peak efficiency related to LET at values $100-150 \mathrm{keV} / \mu \mathrm{m}$. This value is clearly not appropriate for heavy-ion carcinogenesis. Experiments in progress are designed to clarify the initial slopes and to more precisely evaluate the peak LET for Harderian gland tumor induction by using monoenergetic beams with minimum fragmentation.

It is interesting that the maximum $\mathrm{RBE}$ values found in these studies are quite similar to the RBEs of 25 to 30 found for carcinogenesis with other high-LET systems. ${ }^{4}$ Furthermore, our Harderian RBEs are equally close to the recently proposed value of 20 for human high-LET carcinogenesis set by the ICRP.

\section{REFERENCES}

1. Alpen, E.L., Powers-Risius, P., Fry, R.J.M., Ainsworth, E.J., De Guzman, R.J., Harrison, L.H., and Havens, V. Biology and Medicine Division Annual Report 1981-1982, Lawrence Berkeley Laboratory report LBL-14986 (1983), p.65.

2. Fry, R.J.M., Powers-Risius, P., Alpen, E.L., Ainsworth, E.J., and Ullrich, R.L. High-LET radiation carcinogenesis. Adv. Space. Res 3, 241-248 (1983).

3. Fry, R.J.M., Powers-Risius, P., E.L. Alpen, E.J. Ainsworth. High-LET radiation carcinogenesis. Radiat. Res. 104, S-188-195 (1985).

4. International Commission on Radiation Units and Measurements. Task Group on Radiation Protection Quantities. The Quality Factors in Radiation Protection: ICRU Report 40 (1986). 


\title{
LIFE SHORTENING EFFECTS OF HEAVY CHARGED PARTICLES AND PHOTONS
}

\author{
E. John Ainsworth, John C. Prioleau, and Lynn J. Mahlmann
}

Large scale rodent studies on radiation carcinogenesis date back to the 1940s, focusing on the effects of reactor-produced neutrons as compared with photons.' Typically, life-span studies have been conducted under circumstances in which animals were given total-body irradiation, placed in a microbially controlled environment, and permitted to live out their life spans. Autopsies were performed on the decedents, and neoplastic and nonneoplastic diseases were diagnosed histopathologically. ${ }^{2,3}$ While the primary objective of most lifespan studies has been the collection of tumor data suitable for determination of tumor dose-response relationships, the shortening of average life span in relation to radiation dose has also provided very useful information.

Several years are often required for collection and complete analysis of autopsy data that yield tumor dose-response results after all animals have died. Estimates of life shortening are based simply on reduction of mean survival time among irradiated groups in comparison with controls; consequently, it is the life-shortening data from life-span studies that are published first. The shortening of life span by radiation is corsidered to reflect the total excess burden of neoplastic diseases that are either induced by or promoted by radiation. While no clear inferences are possible concerning doseresponse relationships for a particular tumor type based on life shortening, significant life shortening heralds the presence of one or more tumor types, or conceivably other pathological processes, attributable to the radiation dose administered. Extensive data on life shortening in mice after total body doses of fission spectrum neutrons from reactors or of gamma radiation have been published, and the results analyzed carefully for purposes of predictive modeling. The life shortening results have figured prominently in recent deliberations leading to recommendations that the quality factor $(Q)$ for fission neutrons be increased by a factor of the order of 2.0 since the earlier estimates of $Q$ probably significantly underestimated the neutron radiation hazard. ${ }^{4}$

The carcinogenic effects of charged particles are of fundamental importance for man in space because risk of radiation-induced cancer impacts upon the duration of missions as well as the design specifications for shielding spacecraft to manage the dose to personnel. Additionally, animal studies on the carcinogenic effects of particles are expected to yield important new basic information relating charged particle track structure or linear energy transfer (LET) to the induction or promotion of tumors in vivo, and to provide required information that will assist in the assignment of quality factors appropriate for estimation of radiation risk in space. An additional issue that has received little attention is the extent to which microgravity in space may result in physiological alterations that may alter radiation responses and thereby influence the biological effects of particles in a space radiation environment.

Because of the dearth of information concerning, carcinogenic effects of particles, a workshop was convened at the Lawrence Berkeley Laboratory in 1978 to consider what experiments were needed in support of charged-particle carcinogenesis research. A group of experts concluded that, in addition to animal studies, a comprehensive program was required that should include experiments on radiation damage and repair at the molecular and cellular levels. Such experiments would be complemented by studies on in vitro transformation and in vivo animal carcinogenesis. The animal studies should utilize unique tumor model systems (such as Harderian gland and mammary tumors) as well as life-span studies that would yield information on tumor dose-response relationships as well as data on life-span shortening for various charged particles.

Support for the program was not sufficient for large scale mouse experiments or to provide the staffing necessary for histopathology, so the decision was made to mount a series of "range finding" experiments with various particles where the singular endpoint would be life-span shortening. Comparisons with life-shortening data published earlier for fission-spectrum neutrons would yield significant new information regarding the risk of carcinogenesis after exposure to heavy particles and provide at least some limited insight regarding $Q$ values. Additionally, pilot life-span studies over a range of LETs would provide information essential for the design of fature low-dose studies in which collection of tumor dose-response data would be the primary goal. Collection of tumor dose-response data, together with life-shortening information, is expen- 
sive and time consuning. Such an effort can be mounted best on a cost-effective basis when results from pilot life-span studies indicate the range of doses and sample sizes most appropriate for tumorigenesis studies.

A series of experiments was initiated in 1978 to explore the life-shortening effects of single or fractionated doses of various charged particles or photons. The photons represent an essential control so that estimates of relative biological effectiveness (RBE) can be made. Hybrid $\mathrm{CB}_{6} \mathrm{~F}_{1}$ male mice purchased from the lackson Laboratory were given total body exposures to various charged particles or to ${ }^{60} \mathrm{Co}$ gamma rays at $110-150$ days of age. Following irradiation, the mice were placed in a "geriatric ward," and provided acidified ( $\mathrm{pH} 2.0)$ tap water and standard laboratory chow ad libidum. Death checks were performed 5 to 7 times per week, and mortality data were entered in computer files at the University of California, San Francisco, using a filing and analysis system designed by the late Sylvanus Tyler. Sham irradiated controls were entered into the experiment with each shipment of mice that was assigned to irradiation status. Estimates of life shortening are based on the difference in mean survival time between sham irradiated controls and animals that received doses of particle or photon radiation; estimates of life-span shortening are based either on percentage change or absolute number of days of life span lost.

The hypotheses tested were trat 1) $\mathrm{LE}:$ has predictive value and that the life-shortening eifects of stopping carbon particles would be approximately equal to fission spectrum neutrons since the LETs are about equal, 2) dose fractionation with stopping carbon particles would produce entanced life shortening as do fission spectrum nitstrons, 3) the maximum RBE for life shortening wauld occur at the same LET for the RBE for cell-killiwe peaks, and 4) RBE-LET relationships for life shortersing after single or fractionated exposures can be predicted based on results from neoplastic transformation studies conducted in vitro.

Current estimates of life shortening expressed as days of life span lost after single or fractionated doses, are summarized in Figs. 1 and 2. In general, the life shortening produced by most of the charged particles used in these pilot experiments was appreciably less than expected, because the RBE was overestimated. It was assumed in the original planning that dose-averaged LET would be an adequate predictor of life shortening and carcinogenic effects. This appears not to be the case over the full range of LETs for which data have been collected. It was assumed that low energy carbon

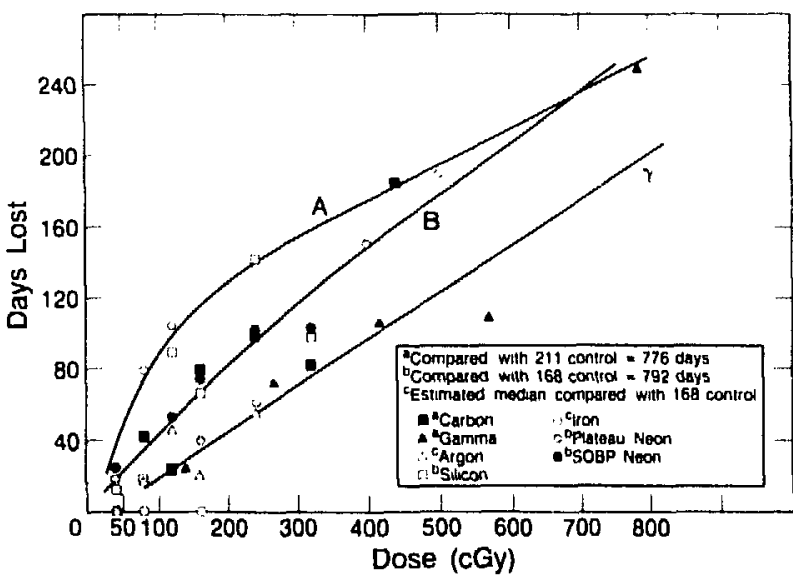

Fig. 1. Comparison of days of life lost after single doses of radiation from gamma rays, various charged particles, and fission-spectrum neutrons.

(XBL 8511-8579)

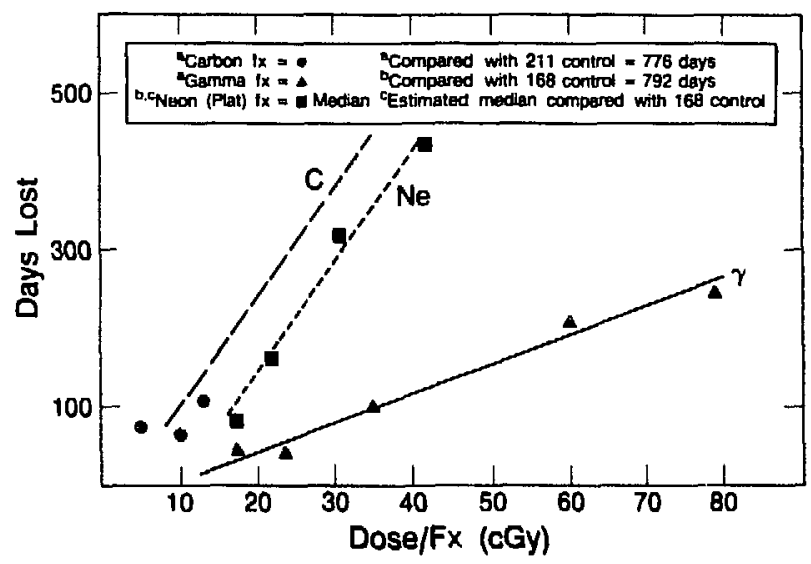

Fig. 2. Life-shortening results following 24 weekly fractionated doses of low energy carbon particles, high energy neon ions, and gamma radiation.

(XBL 8511-8578)

particles would produce a life-shortening effect similar to that observed previously for fissionspectrum neutrons, because estimates of doseaveraged LET are in the range of $70-80 \mathrm{keV} / \mu \mathrm{m}$.

Some of the estimates presented here are derived from control and irradiated populations in which mortality is complete, but others are based only on estimates of median survival time in groups where mortality is incomplete. Many unirradiated control animals remain alive.

The present preliminary evaluation of lifeshortening results involves two different control groups. These two control estimates for mean survival time do not differ significantly, and they will probably be pooled ultimately. Mean survival time 
is marked from time after irradiation or from entry of control mice into experimental status at about 100-150 days of age. For the purpose of the interim analysis presented here, the two control groups had been considered separately, and estimates of life-span shortening for irradiated animals are based on comparison with the controls that were most closely associated in time with the particular irradiated groups.

Figure 1 compares days of life lost after single doses of radiation from gamma rays, various charged particles, and fission-spectrum neutrons. The fission-spectrum neutron results are from experiments reported by Thomson et al. ${ }^{5,6}$ Direct comparison of the neutron and charged particle results based on days of life lost is tenuous because of differences in mean survival times among unirradiated control groups in the neutron and heavy ion studies. Nevertheless, the single-dose results presented in Fig. 1 are consistent with the interpretation that single doses of charged particles are appreciably less effective than fission spectrum neutrons for production of life-span shortening. The use of percent life shortening, rather than days lost, leads to the same interpretation. Because the sample sizes are small in the charged particle studies, the $95 \%$ confidence limits are sufficiently broad that a variety of curves could be drawn through the results for any particular charged particle or radiation quality, and significant differences between the various particles are not apparent. Note that a consistent trend of increased life shortening with increasing dose was not always observed. Because final data analysis is not yet complete, only qualitative trends will be considered here. The first point of interest concerns the overall shape of the dose-response curves, such as they are. While a single more or less linear function (curve B) could represent an adequate fit to all the data collected, another possibility is that the initial slope at doses below about $240 \mathrm{cGy}$ is higher than at higher doses, at least for some particles (curve A). The resemblance of curve $A$ to the neutron results is clear, and one possible interpretation of these results is that high energy neon and silicon particles could be more effective than low energy carbon or neon particles. Also, note that no life shortening was detected among animals exposed to iron particles over the dose range studied $(40,80$, or $160 \mathrm{rad}$ ). Assuming that curves $A$ and $B$ have relevance to the charged particle results, the worst case and more conservative estimates may be offered for RBE. At 50 days of life shortening, the RBEs inferred from curves $A$ and $B$ would be about 3.8 and 2.1 , respectively. $A$ value of about 9 is associated with the neutron curve based on the previously published gamma-radiation results. ${ }^{5}$

Life shortening following 24 weekly fractionated doses of low energy carbon particles, high energy neon ions, and gamma radiation are summarized in Fig. 2. The results for high energy neon particles are based on median survival time, because mortality in the population is not yet complete. The eye-fitted curves for the gamma and neon results appear adequately dose-dependent, but the curve drawn for low energy carbon particles is incomplete, because only the three highest doses produced life shortening in excess of 50 days. The curve for carbon particles is drawn parallel to the neon curve and represents only an estimate for life shortening, based on incomplete results. The limited data available for carbon ions are consistent with the interpretation that high energy neon particles are probably less effective than low energy carbon particles. Estimates of RBE for life shortening, based on these incomplete results are of the order of 3.5 to 4 for low energy carbon particles and about 2 for high energy neon particles. The RBE inferred from previously published results is of the order of $20 .^{6}$

Inferences pertaining to the sparing effect of dose fractionation are possible from the results with low energy carbon and high energy neon particles, because single doses were administered over the same time frame when animals assigned to fractionated dose groups entered experimental status. With gamma rays and neon ions this sparing effect of fractionation was clear; for example, a fractionated gamma dose of $840 \mathrm{cGy}$ produced 101 days of life shortening, while a single dose of only 417 cGy produced 107 days of life shortening. Likewise for neon ions, a fractionated dose of $408 \mathrm{cGy}$ resulted in 89 days lost, while a single dose of $80 \mathrm{cGy}$ produced 79 days of life shortening. With low energy carbon ions, the results are less clear regarding sparing effects of fractionation. For example, at $320 \mathrm{cGy}$, single and fractionated doses produced 81 and 109 days lost, respectively; and at $120 \mathrm{cGy}$, single and fractionated doses resulted in 25 and 74 days lost, respectively. At 160 and $240 \mathrm{cGy}$, fractionation decreased the life shortoning by 35-80 days. Larger sample sizes are necestary to quantitate sparing or enhancement with carbon ions.

Several tentative conclusions have been drawn from the range finding experiments that have been completed so far. Tentative estimates of RBE have been generated for single and fractionated exposures, and we have identified the dose ranges appropriate for future low dose carcinogenesis 
experiments. After single doses of carbon and silicon particles where the LET is $70-80 \mathrm{keV} / \mu \mathrm{m}$, less life shortening is produced than for fission spectrum neutrons. Thus, the hypothesis is rejected that dose-averaged LET has broad predictive value. Although the LETs were similar, biologically significant differences in track structure must be important. Iron particles at about $190 \mathrm{keV} / \mu \mathrm{m}$ are over the peak of effectiveness for life shortening, whereas, iron particles are highly effective for induction of Harderian gland tumors and for production of neoplastic transformation in vitro. ${ }^{7.8}$ Thus, differing model systems yield different inferences that must be resol:/ed. A clearer LET dependence and distinction between particles is not clear after single doses of particles over the LET range from about 30 to $140 \mathrm{keV} / \mu \mathrm{m}$.

In summary, single linear dose-response curves will probably not fit all the single-dose data, and a convex upward curve for neon and silicon particles is a possibility. After 24 weekly fractionated doses, little or no sparing effect occurs with carbon particles at $70-80 \mathrm{keV} / \mu \mathrm{m}$, although the RBE for life shortening is low as compared with fission neutrons. A sparing effect occurs with neon particles but the effect is appreciably less than for gamma rays.

We will extend these studies by continuing to collect mortality data from existing groups where mortality is incomplete, namely, some unirradiated controls and animals exposed to gamma rays, argon, iron, and neon particles. We have also proposed to supplement the mortality data base by addition of higher single doses of argon and iron particles and by the addition of higher fractionated doses of carbon particles. Supplementation of the mortality data is essential to estimate RBE for the particles specified. We also plan to initiate a comprehensive life-span study in the future in which histopathology data will be collected for the purpose of determining dose-response relationships for various tumor types. This study will be conducted using the particle deemed maximally effective based on our range-finding results, and the particle will probably be silicon at an LET of the order of $80 \mathrm{keV} / \mu \mathrm{m}$. The emphasis will be on a range of low-radiation doses, both single and fractionated, to determine the initial slopes of dose-response curves and to determine the extent to which dose fractionation produces a sparing effect or, alternatively, an enhancement of life-span shortening and tumorigenesis. We hope also to initiate pilot studies on a myeloid leukemia model system in the $\mathrm{CBA} / \mathrm{H}$ mouse and to explore the extent to which corticosteroid promotion of expression of the disease will increase sensitivity over a range of low radiation doses. The $\mathrm{CBA} / \mathrm{H}$ model has been used in earlier studies by Mole and Davids, and Major and Mole. ${ }^{9,10}$ The limited data available after single radiation doses indicate that RBE for induction of myeloid leukemia in this strain is of the order of 3.0 based on the initial slopes of the dose-response curves (Fig. 3). An important question is the extent to which fractionation of charged particle doses or low dose rates influences the initial slopes of the dose-response curve for myeloid leukemia. We hope to conduct fractionation experiments with gamma rays and silicon particles. Serial studies of the alterations in bone marrow colony forming units-spleen (CFU-S) and other indices of marrow activity should yield significant new information on the natural history of the development of myeloid leukemia with and without promotion by corticosteroids.

In the absence of extensive human data on the effects of high-LET radiations, including charged particles and neutrons, the results from life-span experiments, studies with specific tumor model systems such as myeloid leukemia or mammary tumors, and information on induction and promotion of tumors obtained by in vivo transplantation of known numbers of irradiated clonogens, together with additional information using in vitro transformation, will all play an important role in establishing the comprehensive data base from which $Q$ can be estimated for heavy ion carcinogenesis.

\section{REFERENCES}

1. Neary, G.J., Munson, R.J. and Mole R.H. Chronic Radiation Hazards, Pergamon, New York (1957).

2. Storer, J.B., Serrano, L.J., Darden, E.B., Jr., Jernigan, M.C. and Ulirich, R.L. Life shortening in RFM and BALB/C mice as a function of radiation quality, dose and dose rate. Radiat. Res. 78, 122-161 (1979).

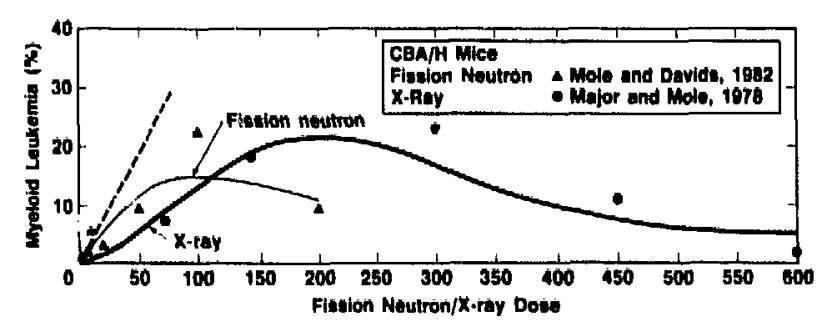

Fij. 3. Dose-response curves for induction of myeloid leukemia after single radiation doses.

(XBL 863-8670) 
3. Ullrich, R.L. Tumor induction in BALB/C mice after fractionated or protracted exposures to fission-spectrum neutrons. Radiat. Res. 97, 587-594 (1984).

4. The quality factor in radiation protection. Report of a joint task group of the ICRP and the ICRU to the ICRP and ICRU. ICRU Report No. 40 (April 4, 1986).

5. Thomson, J.R., Williamson, F.S., Grahn, D., and Ainsworth, E.J. Life shortening in mice exposed to fission neutrons and gamma rays. 1. Single and short-term fractionated exposures. Radiat. Res. 86, 559-572 (1981).

6. Thomson, J.R., Williamson, F.S., Grahn, D., and Ainsworth, E.J. II. Duration-of-Life and long-term fractionated exposures. Radiat. Res. 86, 573-579 (1981).
7. Fry, R.j.M., Powers-Risius, P., Alpen, E.L., Ainsworth, E.J., and Ullrich, R.L. High LETradiation carcinogenesis. Radiat. Res., 104, S188-Si95 (1985).

8. Yang, C., and Tobias, C.A. Neoplastic cell transformation by energetic heavy ions and its modification with chemical agents. $A d v$. Space Res. Vol. 4, No. 10, 207-218 (1984).

9. Mole, R.H. and Davids, J.A.G. Induction of myeloid leukemia and other tumors in mice by irradiation with fission neutrons. In Radiation Protection: Neutron Carcinogenesis, pp. 31-34. Commission of the European Communities, Luxembourg [EUR-8084-EN] (1982).

10. Major, I.R., and Mole, R.H. Myeloid leukemia in x-ray irradiated CBA mice. Nature, 272, 455-456 (1978).

\section{Heavy Ion Therapy, Radiosurgery, and Therapy Physics}

\section{BEAM DELIVERY SYSTEMS FOR HEAVY CHARGED PARTICLE THERAPY AT THE BEVALAC}

Edward L. Alpen, William T. Chu," Bernhard A. Ludewigt, Maurice B. McEvoy, ${ }^{\dagger}$

Mark A. Nyman, ${ }^{\dagger}$ Timothy Renner," and Rajinder P. Singh ${ }^{\dagger}$

\section{WOBBLER BEAM DELIVERY SYSTEM}

The wobbler beam delivery system consists of two dipole magnets that are powered in such a way as to "paint" a ring dose distribution at the target (see Fig. 1). By superimposing several such dose distributions of the appropriate size and fluence a uniform dose distribution up to $30 \mathrm{~cm}$ in diameter can be achieved (Fig. 2).

The wobbler system has now been used for one year in treating over 25 patients. In the last five months of full-time operation 18 patients have been treated for a total of 255 treatments. Its reliability and consistency have been comparable to that of the double-scattering system previously used for patient treatment. Neon ions of an energy per nucleon of 456 and $585 \mathrm{MeV}$, corresponding to clinically usable ranges at treatment isocenters of 18.6 and $27.6 \mathrm{~cm}$ of water equivalent thicknesses, respectively, have been developed. The system is

-Acclerator and Fusion Research Division (Accelerator Operations Group), LBL.

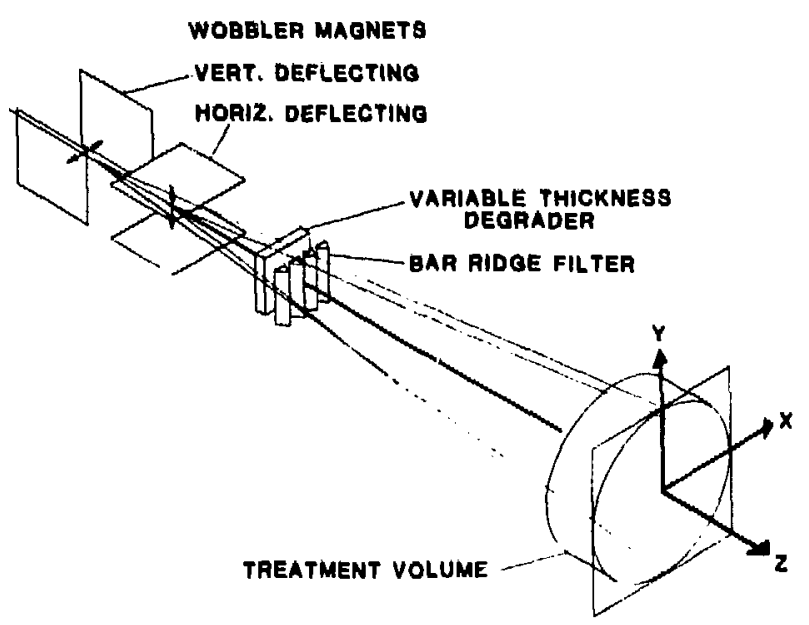

WOBBLER BEAM DELIVERY SYSTEM

Fig. 1. Schematic diagram of wobbler beam delivery system. (XBL 868-2853) 

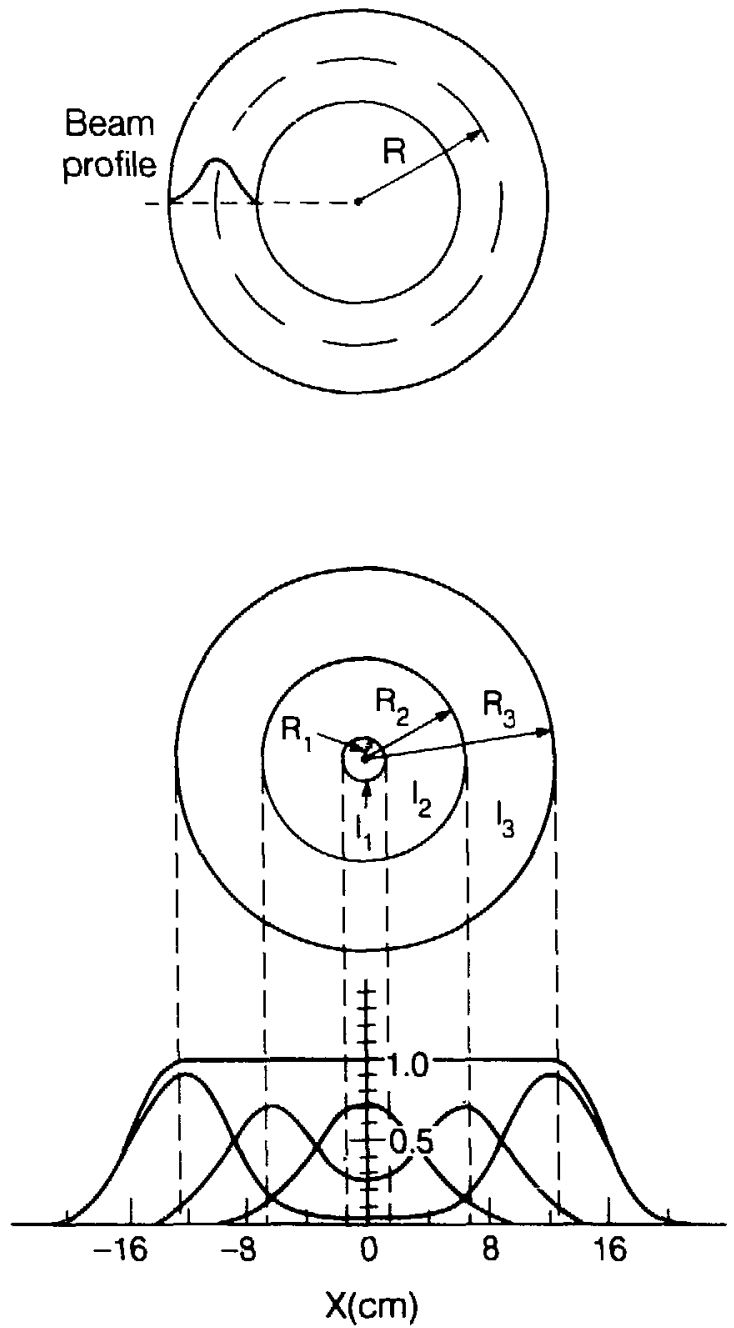

Fig. 2. Illustration for producing a flat field using wobbles of three different radii.

(XBL 854-9820)

currently set up to provide four field sizes, of 8-, 17-, 25-, and 30-cm diameter. The ability to change field sizes as appropriate to the patient port has reduced treatment time and improved beam utilization.

\section{DEVELOPMENT OF RASTER SCANNER SYSTEM}

A raster scanner currently being built will be a more versatile beam delivery system than the wobbler system. Its magnets are designed to spread high-energy heavy-ion beams with a maximum magnetic rigidity of 9.0 tesla-meters to deliver uniform doses into large rectangular areas, up to $40 \mathrm{~cm}$ by $40 \mathrm{~cm}$, at a distance of 6 meters. The raster scanner consists of two dipole magnets: the fast magnet with a gap of $5.7-\mathrm{cm}$ height and 10.2$\mathrm{cm}$ width, a frequency of $40 \mathrm{~Hz}$, and a maximum field of $0.27 \mathrm{~T}$; and the slow magnet with a gap of $15.2-\mathrm{cm}$ height and $8.9-\mathrm{cm}$ width, a frequency of $0.5 \mathrm{~Hz}$, and a field of $0.34 \mathrm{~T}$. The fabrication of the fast magnet has been completed and the slow magnet is under construction. The power supplies are designed to drive the magnets at a maximum sweep rate at the target of $1200 \mathrm{~cm} / \mathrm{sec}$ and 40 $\mathrm{cm} / \mathrm{sec}$, respectively. The fabrication of the power supplies is under way.

For better beam diagnostics a 144-element ionization chamber has been built (Fig. 3). The elements are ordered in a $12 \times 12$ matrix with collecting areas of $2.5 \times 2.5 \mathrm{~cm}$ each. They cover a total area of $30 \times 30 \mathrm{~cm}$. For the readout new current-to-frequency converters have been fabricated and tested. Their linearity is better than $1 \%$ for input currents ranging from $10^{-10}$ to $5 \times 10^{-6}$ amperes. The new ionization chamber is now being used for the verification of the dose uniformity of wobbler-generated fields.

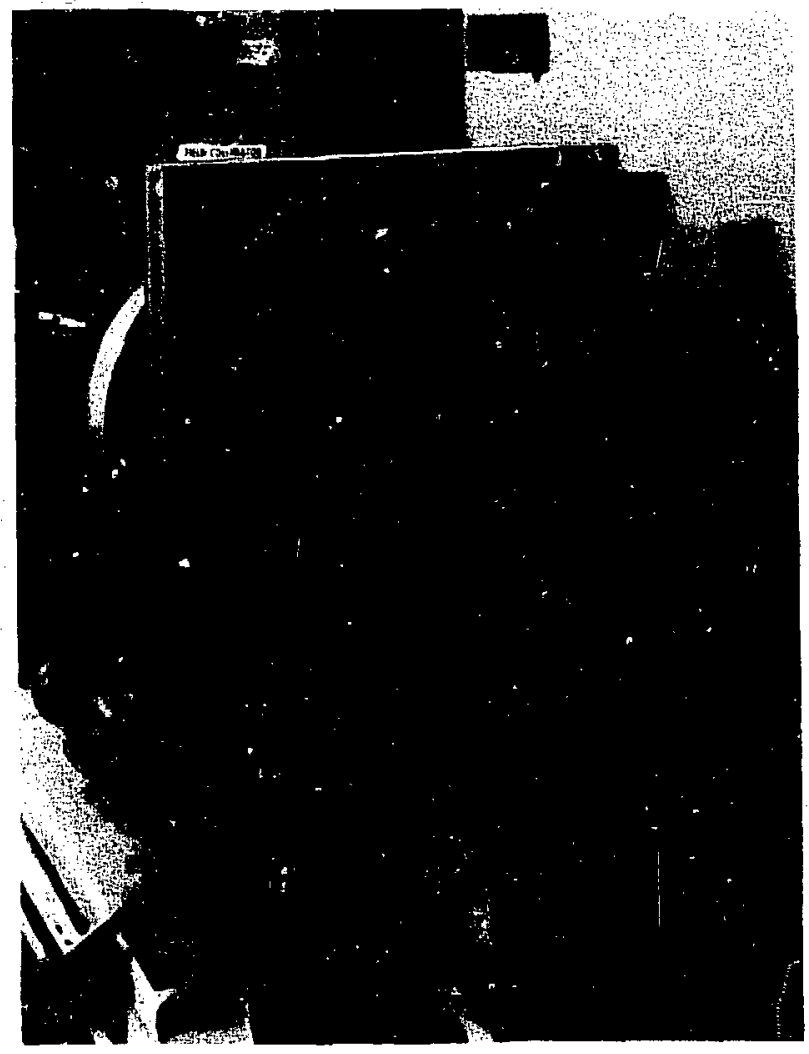

Fig. 3. Ionization chamber with 144 elements. (BBC 864-2667) 


\section{RADIATION THERAPY TOLERANCE DOSES FOR TREATMENT PLANNING}

John T. Lyman

To adequately plan acceptable dose distributions for radiation therapy treatments it is necessary to ensure that normal structures do not receive unacceptable doses. Acceptable doses are generally those that are below a stated tolerance dose for development of some level of complication. To support the work sponsored by the National Cancer Institute, data for the tolerance of normal tissues or organs to (low-LET) radiation has been compiled from a number of sources. ${ }^{1-8}$ These tolerance dose data are ostensibly for uniform irradiation of all or part of an organ, and are for either $5 \%\left(T_{5}\right)$ or $50 \%\left(\mathrm{TD}_{50}\right)$ complication probability. The "size" of the irradiated organ is variously stated in terms of the absolute volume or the fraction of the organ volume irradiated, or the area or the length of the treatment field. The accuracy of these data is questionable. Much of the data represent doses that one or several experienced therapists have estimated could be safely given (i.e., data from Rubin and data from the Treatment Planning Intercomparison Survey) rather than quantitative analyses of clinical observations. Because these data have been obtained from multiple sources with possible different criteria for the definition of a complication, there are sometimes different values for what is apparently the same end point. The data from some sources show a tendency to be quantized in 5-Gy increments. This reflects the size of possible round off errors. It is believed that all these data have been accumulated without the benefit of three-dimensional dose distributions and therefore the estimates of the size of the volume and/or the uniformity of the irradiation may be less accurate than is now possible. The data in the table are for "conventional" fractionation schedules (1.8-2.0 Gy per fraction and 5 fractions per week) that assume relatively normal physiologic function before irradiation, and assume there to be no adjuvant therapy. The extrapolation to other fractionation schedules ${ }^{9}$ and the modification of these tolerances when there is compromised function or when adjuvant therapy is given are important problems that need to be addressed.

These data have two potential applications: First, and most simply, as a set of tolerance doses that can be referred to when drawing up a protocol for treatment of a particular disease - in which case the table values can be interpreted as upper limits on dose which the planner should attempt to respect. Second and more quantitatively, one would like to be able to estimate a complication probability for each normal tissue and end point of interest-in which case the table values can be considered to be data to which any model of normal tissue complication probability should conform.

\section{INTERPOLATION (AND EXTRAPOLATION) OF THE DATA}

How may these data be extended to predict the complication probability when the dose to a (uniformly) irradiated volume is different from the few cases tabulated? Obviously, to do this, some model of the variation of normal tissue complication probability with both volume and dose must be implicitly or explicitly assumed. (Even an "eyeball" interpolation between two points implicitly assumes some smoothness criterion.) Many authors $^{10-14}$ have suggested a simple model for the dependence on irradiated volume (or other size parameter) of the dose to achieve a specified complication, namely a power-law relationship of the form:

$$
T D(v)=T D(1) \times v^{-n}
$$

where TD stands for tolerance dose, $v$ is the fraction of the volume (or area or length) of an organ irradiated (or the ratio of the size of irradiated tissue to some reference size) and $n$ is a size dependence parameter whose value is determined by the particular organ and the size dimension (volume, area or length) of concern.

While an areal or linear characterization of the irradiated tissue is functionally appropriate for some tissues, such as esophagus, skin, or spinal cord for example, it is much simpler to make an analysis for a treatment plan based on the irradiated volume; for many geomeiries the other size parameters tend to scale with volume. Therefore, except for skin, all organ and tissue size dependences are considered to be convertable to volume dependences. The tolerance doses relate to situations where the irradiated normal tissue is uniformly irradiated. The data were probably derived from the most commonly used treatment plans which are assumed to be either parallel opposed port or four-field box plans using photon radiation. For small field sizes 
the irradiated volume can be significantly different depending upon the treatment plan; this can lead to possible errors in assigning a value to the parameter $\mathrm{n}$.

The dependence of complication probability on dose (for fixed volume) is considered by many authors to have a sigmoidal shape. ${ }^{3,14-17}$ This can be represented with sufficient accuracy by one of a number of two-parameter expressions, of which the most common are the logistic function and the error function. The two free parameters for such curves, namely the slope (or standard deviation) and $\mathrm{TD}_{50}$ (dose to achieve $50 \%$ complication), can be fit by two data points, such as the doses to achieve $5 \%$ and $50 \%$ complication.

A three-parameter model that connects the three variables of interest (complication probability $P$, dose $D$ and volume $V$ ) has been developed. The expression used is as follows:

$$
P=(2 \pi)^{-1 / 2} \int_{-\infty}^{t_{\text {mex }}} e^{t^{2} / 2} d t
$$

where $\left.t=\left[D-T D_{50}(V)\right] / \sigma(V)\right)$ and $\sigma(V)=m \times$ $\mathrm{TD}_{50}(\mathrm{~V})$. Equation (1) is used to obtain $T \mathrm{~T}_{50}(\mathrm{~V})$ and $m$ is the third free parameter, which is to be determined by the data.

This formula is a combination of the power law dose-volume relationship (Eq. 1) and an error function representation of the complication-dose relationship, and it gives back these equations if complication probability or volume, respectively, are held constant. Figure 1 shows a perspective display of the three-dimensional surface described by Eq. (2) for the heart.

Selected data have been fit by choosing values for the three parameters of Eq. (2). In many

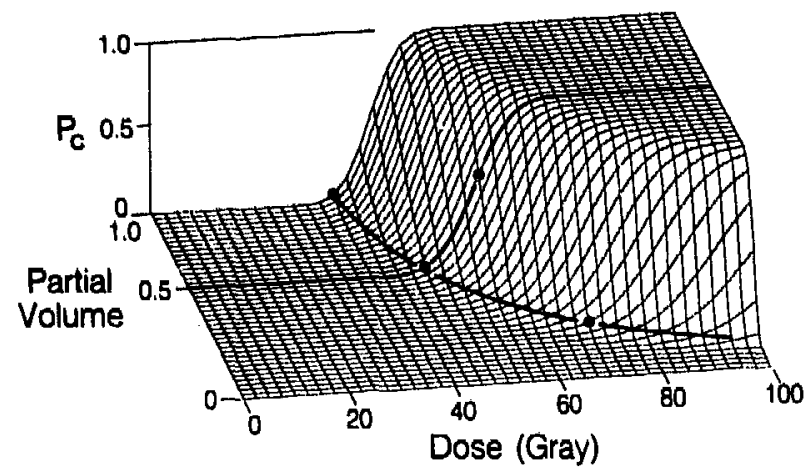

Fig. 1. A perspective display of the three-dimensional surface described by Eq. (2) for the heart using the parameters given in Table 1. instances there are insufficient data to determine the three parameters at all, or with reasonable accuracy, therefore some simplifying assumptions have been made and the fitting has been biased toward the $\mathrm{TD}_{5}$ values.

Table 1 lists the selected values of the three parameters of Eq. (2). A number of tissues have data at only a single volume; in these cases the parameter $\mathbf{n}$ was chosen to have a low value (i.e., small volume dependence). This is a conservative approach since it is expected to predict a tolerance dose smaller than the true value for the smaller volumes. The parameter $n$ for skin is meant to be applied, not to a partial volume, but to the size of the equivalent square in decimeters. This is numeri-

Table 1.

\begin{tabular}{lllll}
\hline \hline \multicolumn{1}{c}{$\begin{array}{c}\text { Organ } \\
\text { or tissue }\end{array}$} & TD & $n$ & $m$ & Note \\
\hline Bladder & 72 & 0.10 & 0.1 & \\
Bone & 70 & 0.05 & 0.1 & a \\
Brain & 64 & 0.20 & 0.1 & \\
Brain Stem & 64 & 0.05 & 0.1 & a \\
Cauda equina & 57.5 & 0.10 & 0.1 & b \\
Esophagus & 66 & 0.15 & 0.1 & c,d \\
Eye & 60 & 0.05 & 0.1 & a \\
Femoral Head & 62 & 0.05 & 0.1 & a \\
Heart & 41.9 & 0.50 & 0.1 & \\
Intestine & 55 & 0.10 & 0.1 & \\
Kidney & 29 & 0.15 & 0.1 & \\
Liver & 35 & 0.40 & 0.1 & \\
Lung & 22 & 0.65 & 0.1 & \\
Mandible & 77 & 0.05 & 0.1 & \\
Optic Nerve & 65 & 0.05 & 0.1 & a \\
Paratid & 72 & .05 & 0.1 & a \\
Pituitary & 54 & 0.05 & 0.1 & a \\
Rectum & 75 & .10 & 0.1 & e \\
Skin & 68 & 0.30 & 0.1 & f \\
Spinal Cord & 50 & .10 & 0.1 & c \\
Stomach & 55 & 0.35 & 0.1 & \\
\hline \hline & & & & \\
\hline
\end{tabular}

" Very small volume dependence (0.05) assumed; no supporting data.

b Assumed to have a radiosensitivity 1.15 times that of cord.

c This structure is assumed to have a cylindrical shape with a length of $25 \mathrm{~cm}$. Partial volumes are then related to this length; partial irradiation of the cross-section is not addressed.

d Moderate volume dependence (0.15) assumed; no supporting data.

- Small volume dependence (0.10) assumed; no supporting data.

' Dependence is for the side of an equivalent square relative to $10 \mathrm{~cm}$. All values in this table are preliminary and should not be taken as definitive. The purpose of this table is to provide a set of parameters that can be used in conjunction with Eq. (2) to model the late response of the selected normal tissues to radiation for treatment planning intercomparisons only. 
cally the same as the ratio of the size of the equivalent square to the Reference size of $10 \mathrm{~cm}$ $\left(100 \mathrm{~cm}^{2}\right)$. The value for $\mathrm{n}$ was chosen as a compromise value based on values from Refs. 12-14.

While all these approximations may be a little troubling, they do not hinder the use of the data, provided the crude nature of the fit is kept in mind. The parameters of Table 1 are just one set from several that could have been obtained, depending upon which of the compiled tolerance values were of the most concern. In comparing two or more treatment plans, their ranking, with respect to their relative probabilities of damage to a normal structure, will be relatively insensitive to the choice of parameters if the same set of parameters is used for all the plans.

Obviously, these fitting procedures are an ad hoc and relatively crude way of establishing the parameters of Eq. (2). The justification of the selected values must lie primarily in the accuracy with which the resulting model represents the compiled data. When comparing the calculated data to the fitted data, there are no instances where the difference between a calculated value and the corresponding data point was more than $5 \mathrm{~Gy}$. The quality of the data probably does not warrant efforts to use more or different parameters or different models.

Clearly, Eq. (2) must be used with extreme caution when extrapolating to conditions that are significantly outside those for which data are available, particularly when considering small volumes.

\section{NON-UNIFORM IRRADIATION}

The estimation of normal tissue complication when the tissue or organ is non-uniformly irradiated is both essential to the task of evaluating any practical treatment plan, and takes one well out of the range of quantitative clinical data. One approach to collect the needed data is to compare dosevolume histograms of patients with and without complications. ${ }^{18}$ Another way to make such an estimation is through a model. Such a model has been developed, using dose-volume histograms to characterize the dose distribution. Details of this model are presented elsewhere. ${ }^{19,20}$

\section{REFERENCES}

1. Lyman, J.T. Tolerance Doses for Treatment Planning. Lawrence Berkeley Laboratory report LBL-22416, (1985).
2. Cohen, L. Biophysical Models in Radiation Oncology. CRC Press, Inc, Boca Raton, FL (1983).

3. Rubin, P. Clinical Oncology for Medical Students and Physicians. American Cancer Society (1983).

4. Rubin, P., Cooper, R.A., and Phillips, T.L., (Eds.), Radiation Biology and Radiation Pathology Syllabus, Set R.T.1: Radiation Oncology, pp. 2-7. American. College of Radiology, Chicago, 1975.

5. ICRP. Nonstochastic Effects of lonizing Radiation. ICRP Publication 41. Annals of ICRP 14, No. 3 (1984).

6. Private communications, Treatment Plarıning Intercomparison Survey and Questionnaires (October 1983-March 1984).

7. Morrish, R.B., Chan, E., Silverman, S., Meyer, J., Fu, K.K., and Greenspan, D. Osteonecrosis in patients irradiated for head and neck carcinoma. Cancer 47, 1980-1983 (1981).

8. Marks, J.E., Davis, C.C., Gottsman, V.L., Purdy, J.E., and Lee, F. The effects of radiation on parotid salivary function. Int. J. Radiat. Oncol. Biol. Phys. 7, 1013-1019 (1981).

9. Barendsen, G.W. Dose fractionation, dose rate and iso-effect relationships for normal tissue responses. Int. J. Radiat. Oncol. Biol. Phys, 8, 1981-1997 (1982).

10. Withers, H.R., Thames, H.D., and Peters, L.). Dose fractionation and volume effects in normal tissues and tumors. Cancer Treatment Symposia 1, 75-83 (1984).

11. Schultheiss, T.E., Orton, C.G., and Peck, R.A. Models in radiotherapy: Volume effects. Med. Phys. 10, 410-415 (1983).

12. von Essen, C.F. A spatial model of time-area relationships in radiation therapy. Radiol., 81, 881-883, (1963).

13. Prasad, S.C. Relation between tolerance dose and treatment field size in radiation therapy. Med. Phys. 5, 431-433 (1978).

14 Cohen, $L$. The tissue factor in radiation oncology. Int. J. Radiat. Oncol. Biol. Phys. 8, 1771-1774 (1982).

15. Goitein, $M$. The utility of computed tomography in radiation therapy: An estimate of outcome. Int. J. Radiat. Oncol. Biol. Phys. 5, 1799-1807 (1979).

16. Marks, J.E. Computed tomography and dose optimization: Brain tumors. In Computed Tomography in Radiation Therapy, C.C. Ling, C.C. Rogers, and R.J. Morton (Eds.) Raven Press, New York, pp 81-87 (1983). 
17. Porter, E.H. The statistics of dose/cure relationships for irradiated tumors. Part 1. Brit. I. Radiol. 53, 210-227 (1980).

18. Mizoe, J.E., and von Essen, C.F. Complication probability analysis for late rectal reactions of patients treated with negative pions. In SIN Medical Newsletter Number 6, 1984, $\mathrm{H}$. Blattman, (Ed.). Villigen, pp. 23-25 (1985).
19. Lyman, J.T. Complication probabilities as assessed from dose-volume histograms. Radiat. Res. Suppl 8, 5-13-S-19 (1985).

20. Lyman, J.T., and Wolbarst, A.B. Optimization of radiation therapy, III: A method of assessing complications from dose-volume histograms. Int. J. Rad. Oncol. Biol. Phys. (in press, 1987).

\section{RADIOTHERAPY PHYSICS}

\section{Michael Collier, Sheri D. Henderson, Samuel Pitluck," Paula Petti, Marc Kessler, and Kari Baken}

The Radiotherapy Physics Group continues the development and improvement of treatment planning, dosimetry, and other technical aspects of heavy charged particle radiotherapy. In addition the Group provides the treatment planning for patients undergoing radiotherapy together with creation of new iechniques for that therapy. The past year has seen significant improvements in the neon particle delivery system at the Bevatron and increased regular application of the PEBA in vivo dosimetry system to the clinical situation in both phantom and patient work. Highlights of the Group's activities are detailed below.

\section{BEVATRON NEON BEAM DOSIMETRY}

During the past year three new elements have been introduced into the beam line at the Bevatron to improve patient treatment. These are the wobbler magnets, the bar-ridged filters, and the binary absorber. The wobbler consists of a pair of orthogonal dipole magnets that sweep the beam in concentric circles up to $30 \mathrm{~cm}$ in diameter. This device replaces the lead scattering-foil system for spreading the beam laterally. This change allows the reduction of the beam energy from 670 $\mathrm{MeV} / \mathrm{amu}$ to $585 \mathrm{MeV} / \mathrm{amu}$ while maintaining the same depth of penetration in the patient.

The binary absorber is a set of copper and aluminum plates of binary increments in thickness. Like the variable thickness water column, which it replaces, the binary range absorber sets the residual range of the particles as they enter the final patient compensating filter.

\footnotetext{
"Engineering Division , LBL.
}

These improvements have forced the creation of new devices for spreading the Bragg peak over the thickness of the target volume, resulting in the bar-riclged filters. Eleven of these have been mai ufactured for spread peaks from 4 to $14 \mathrm{~cm}$ in $1-\mathrm{cm}$ steps. There are in fact two filters used simultaneously; one actually spreads the peak the desired amount, and the second, with grooves the size of the steps of the first, smooths the depthdose curve.

In order to dosimetrically characterize the treatment beams produced by these new elements, two methods have been utilized. For central axis depth-dose measurements a $1 \mathrm{~cm}^{3}$ EGG ionization chamber was placed in a large water phantom that has stepping motors capable of translating the chamber in three dimensions under computer control. For maximum and practical minimum depths of penetration into the phantom for each ridged filter used, the phantom was positioned so that the mid-peak fell in the isocenter of the room. This simulated treatment geometry. Information on the transverse character of the beam was taken using therapy verification $X V$ film and the more sensitive XTL film. These were sandwiched in between slabs of polyethylene.

To date data have been taken for about half of the current ridged filters, both at the maximum penetration of the beam and and a penetration 3 $\mathrm{cm}$ greater than the spread of the peak. Examples of depth-dose curves are shown in Fig. 1 for the 10-cm spread peak. The curve for the shallower penetration of $13 \mathrm{~cm}$ ( $A$ in the figure) has been translated so that the peak lies on top of the peak from the curve with maximum penetration ( $B$ in the 


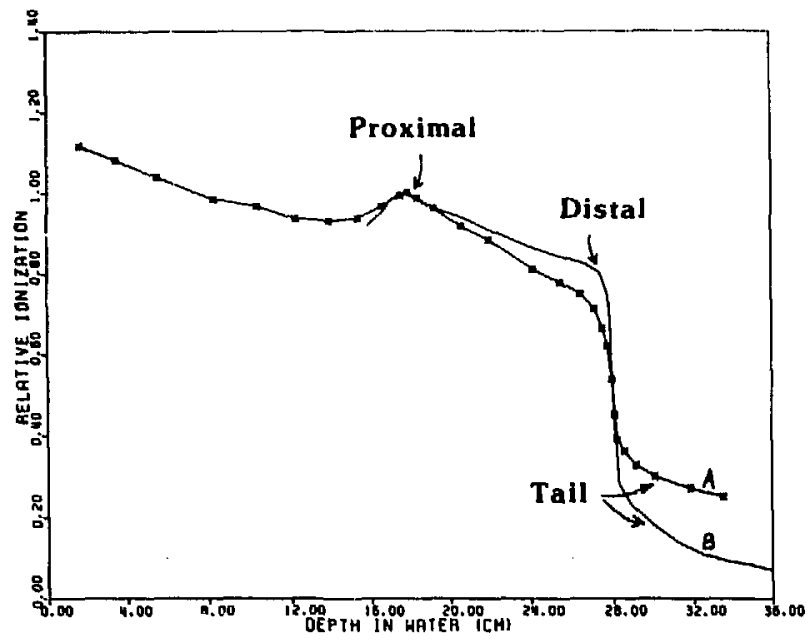

Fig. 1. Central axis depth-dose curve for the $10 \mathrm{~cm}$ spread Bragg peak for residual ranges of $27.6 \mathrm{~cm}$ and $13 \mathrm{~cm}$. The data for the latter have been translated so that the Bragg peaks are superimposed for comparison.

(XBL 869-3395)

figure). The two curves have been normalized to the proximal peak value.

From these data it can be seen that the slope of the spread peak is less for the shallower penetration and the tail dose is also reduced. The slope change occurs because for the lesser penetration the lower energy particles emanating from the ridged filter far upstream of the phantom have larger scattering angles and consequently fewer actually enter the phantom. The lack of these particles in the proximal peak reduces the dose in that region as compared with the deeper distal peak. The tail dose is reduced because for the lower penetration peak water of the phantom is replaced by copper of the binary absorber. The copper produces fewer fragments than water. It is these fragments that give the tail dose.

The values of tail dose at the proximal point, and the distal peak dose as percentages of the proximal peak dose for the six spread peaks are recorded in Table 1 . For the maximum depth the distal peak dose and tail dose both decrease as the spread peak is increased. No comparable conclusion can be drawn for the shallower depths since they are all different. When the maximum depth is compared with the lesser depths, it is seen that the distal peak dose remains the sarne or increases but the tail dose decreases in every case.

\section{IMAGE CORRELATION}

Image correlation is the process by which volumetric imaging data information is translated
Table 1. A comparison of doses at the distal peak and at the most proximal point in the tail. The dose values are given as percent of the proximal peak dose.

\begin{tabular}{cccc}
\hline $\begin{array}{c}\text { Spread peak } \\
(\mathrm{cm})\end{array}$ & $\begin{array}{c}\text { Residual range } \\
(\mathrm{cm})\end{array}$ & $\begin{array}{c}\text { Distal peak } \\
(\%)\end{array}$ & $\begin{array}{c}\text { Tail } \\
(\%)\end{array}$ \\
\hline 4 & 27.6 & 96 & 46 \\
4 & 7.0 & 95 & 14 \\
6 & 27.6 & 89 & 42 \\
6 & 9.0 & 100 & 19 \\
8 & 27.6 & 82 & 37 \\
8 & 11.0 & 90 & 25 \\
10 & 27.6 & 71 & 34 \\
10 & 13.0 & 80 & 26 \\
12 & 27.6 & 67 & 33 \\
12 & 15.0 & 77 & 27 \\
14 & 27.6 & 60 & 32 \\
14 & 17.0 & 68 & 27 \\
\hline
\end{tabular}

and displayed from one imaging modality to another. $^{2}$ In diagnostic radiology and radiation therapy, volumetric imaging data are acquired and analyzed to establish the extent of disease, to plan the optimal therapy, and to follow the patient posttreatment. Obviously, image correlation becomes useful in clinical situations where it is necessary or desired to accurately correlate regions or volumes of interest defined in one imaging study to another. For example, the ability to map tumor and critical organ locations as visualized on the MRI study to the $x$-ray $C T$ study for treatment planning is invaluable to the physician when the lesion is visible only on the MRI scan. ${ }^{3}$ Often a patient is scanned more than once for treatment planning purposes (e.g., photon treatment followed by charged particle treatment). The ability to correlate the images and produce a composite treatment plan is extremely useful in estimating doses to critical structures for the combined treatment. Finally, correlating functional imaging modalities such as positron emission tomography (PET), allows one to analyze conditions of radiation necrosis and tumor recurrence in patient follow-up.

Methods are currently being developed that allow one to carry out the process of image correlation in a quantitative manner. ${ }^{4}$ The general technique involves accurately determining the transformation matrix relating the three-dimensional geometry of the various studies. In cases where a one-to-one correspondence between anatomical landmarks or fiducials from different studies can be found, the transformation is calculated using a least squares approach. When this is not possible, a surface description of a particular structure |e.g., the skull cap| visible in both studies is constructed. 
Iterative search algorithms are used to calculate the mapping of the surface as described on one study to the other, producing the necessary transformation. Such techniques also allow one to "synthesize" images with particular features by mapping certain data from various studies onto a new "composite" three-dimensional volume. One example of this is mapping bony structures defined clearly on CT onto an NMR study in which bone and air provide no signal (Fig. 2).

Work in the area of image correlation is relatively new and its potential is not yet determined. We expect our future investigations to aid in discovering its useful clinical applications and to contribute to its further development and analysis. Image correlation requires a reasonable amount of computer hardware and sophistication. The programs thus far have been run on a VAX 11-780 and use image displays such as the Lexidata 3400 and the Ramtek 9465.

\section{BRAGG PEAK LOCALIZATION WITH RADIOACTIVE BEAMS}

A significant number of patients are referred for heavy charged particle radiotherapy because of tumors adjacent to critical radiosensitive organs such as the spinal cord. In this important group of patients, an error of 2 to $3 \mathrm{~mm}$ in the stopping point of the beam could lead to a severe overdose to the critical organ, or to underdosing a part of the tumor. The most commonly used technique for determining the range or depth to the distal edge of the target volume in treatment planning is based on the utilization of quantitative data from computerized tomographic (CT) scans. With this method, the $C T$ number of each pixel in the beam path is converted to water-equivalent length by using an experimentally derived calibration curve. In most clinical situations this method is thought to predict the range with a typical error of about 3\%. For a beam with a required penetration of $20 \mathrm{~cm}$ to deliver Bragg-peak dose on a lumor volume in a patient, this error amounts to an uncertainty of 6 $\mathrm{mm}$ in the stopping point of the bearn. An alternate approach is offered with the technique developed by Chatterjee et al. $^{5}$ for localization of the Bragg peak with radioactive beams ( ${ }^{11} \mathrm{C}$ or ${ }^{19} \mathrm{Ne}$ ).

Radioactive beams are produced as secondary beams from the Bevalac. In order to obtain ${ }^{19} \mathrm{Ne}$ (a positron emitter; half-life $17 \mathrm{sec}$ ), one accelerates ${ }^{20} \mathrm{Ne}$ (a stable isotope for therapy beam) particles at sufficient flux. The radioactive particles are then produced as secondary beams from peripheral nuclear fragmentation reactions. One can then magnetically separate the desired secondary beam. Yields of these particles are approximately 1 part in 750 and can be obtained without significant contamination from other fragments. For increased efficiency in detection, it is better to select those radioactive beams that decay by positron annihilation. This physical process generates two detectable $511-\mathrm{keV}$ gamma rays in time coincidence at a $180^{\circ}$ angle. Because of these physical properties, a collimator is not necessary and the effective solid angle is quite large. In order to form an image of the stopping point of the radioactive particles, a positron camera has been developed and used in clinical trials. ${ }^{6}$ This camera is called a positron emitting beam analyzer (PEBA). It consists of 128 detectors arranged in two $8 \times 8$ arrays, with the entrance plane parallel to the image plane. Each detector consists of one BGO crystal of dimensions $1.25 \times 1.25 \times 3 \mathrm{~cm}$ and a one-half-inch photomultiplier. The detectors present a square cross section to the incoming gamma rays.

Image processing is done on-line using programs adapted from simulation experiments. A certain time slice is selected from a run, the desired events are histogrammed, back-projected and filtered, resulting in a $48 \times 48$ image that is linearly interpolated into a $128 \times 128$ matrix for display. $A$ PDP-11/34 computer with a 32K-word fast array processor and a RAMTEK color video display are used with the camera. Data acquisition is carried out through a CAMAC interface. Reconstruction time using the present demonstration programs is approximately 1 minute.

Thus far 12 patients have undergone diagnostic procedures for Bragg-peak localization using ${ }^{19} \mathrm{Ne}$ beams. The majority of patients presented with lesions in the brain or in the base of the skull. In most patients, the PEBA measurements were in gocid agreement with the calculations of the water equivalent thicknesses by the standard CT techniques. However, in a few cases the differences were as much as $7 \mathrm{~mm}$. These differences are being analyzed. The depth of beam penetration as determined by the localization of the stoppingpoint beam can be overlaid on a precisely registered $C T$ slice covering the beam entrance point. Such a procedure graphically yields the distal edge of the beam relative to internal anatomical structures, internal organs, or target volume (Fig. 3). The depth of penetration may also be related to a lateral radiograph. The total time required for each measurement (beam injection, count time, and decay of residual activity) is about 1 minute, resulting in about 10 point measurements 

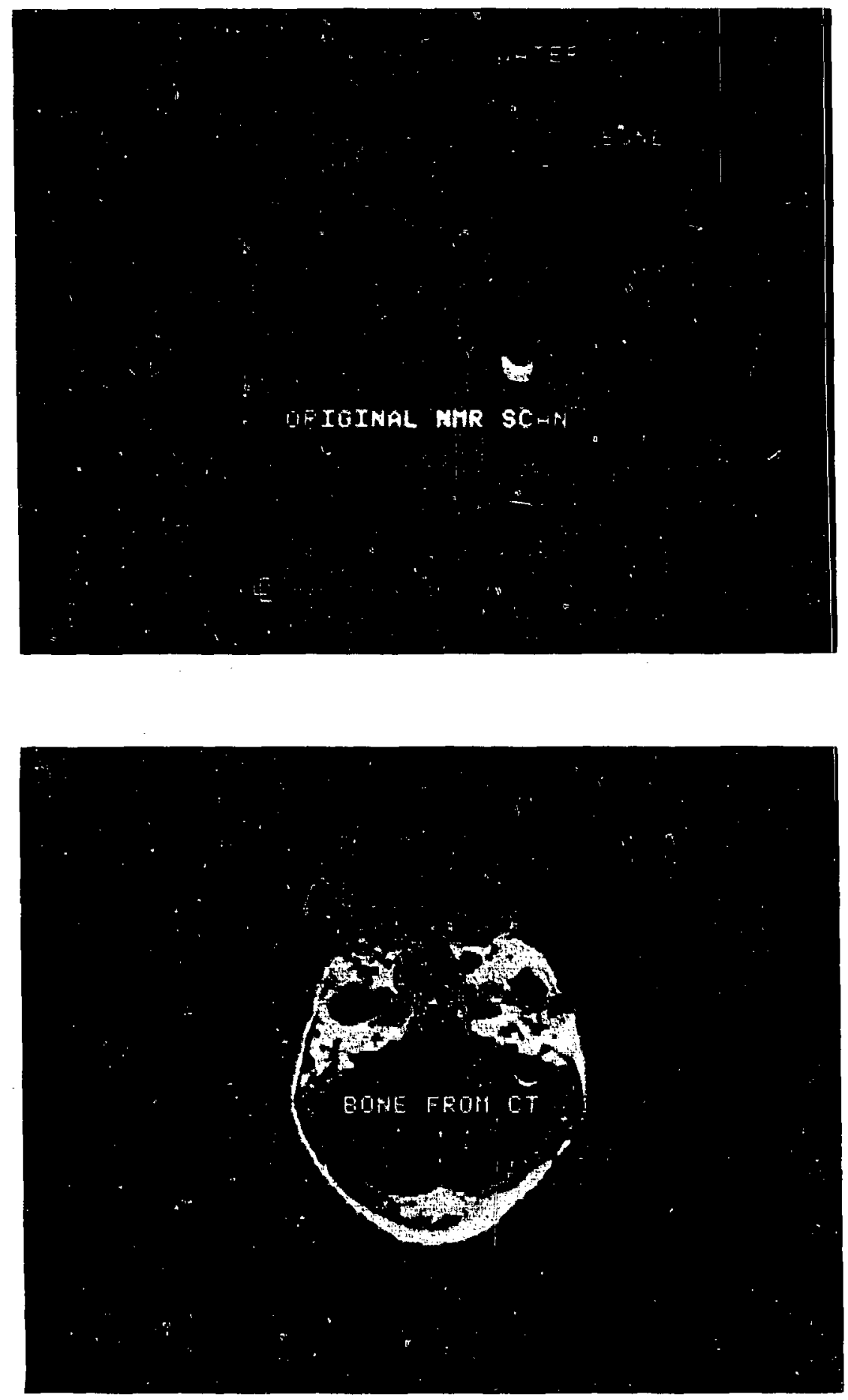

Fig. 2. a) NMR scan of a bone/water phantom from IBM-LBL-MIT Imager. Note the absence of signal from bony structures. b) Synthetic image consisting of original NMR scan and the bony structures as imaged by from $C T$. 


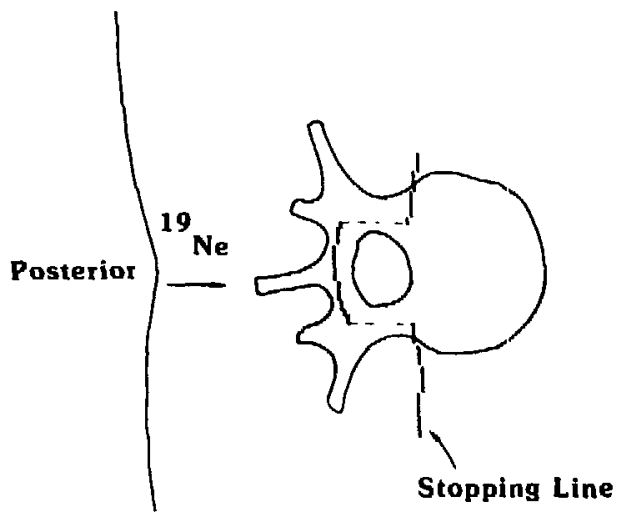

Fig. 3. Graphic illustration of spread PEBA beami stopping line in a clinical situation. The stopping points of the beam indicate that the compensator is properly contouring the distal edge of the therapy beam to be located posterior to the spinal cord.

(XiBL 869-3413)

of depth in a clinically acceptable period of about 10 minutes.

Direct dose verification of a treatment plan for a patient is normally not possible. PEBA is therefore a significant and exciting diagnostic modality for charged particle therapy in-vivo verification of treatment beams. The radioactive beam technique will also complement and improve on the use of CT in the assessment of tissue densities derived from $C T$ in charged particle radiotherapy treatment planning. As this is the data set on which dose distributions and range calculations are based, information produced in this analysis will be of significant value. Further clinical analysis is still needed, as well as a few upgrades in technique to achieve general application. Collaboration will continue with the Accelerator Division at LBL toward improvement of radioactive beam characteristics. These improvements will include beam line modifications and software developments. Similarly, work will be performed in conjunction with Chatterjee and his group on the gathering of more information to determine the clinical utility of radioactive beams for diagnosis.

\section{COMPUTER DEVELOPMENTS}

During the past year additional memory has been added to the VAX 11-780, bringing the total memory up to 32 megabytes. This added memory has allowed the working set size for users to be increased, thereby reducing the number of disk accesses. A second Unibus adapter was also installed, which should also improve system performance by balancing the input/output load resulting from all of our peripheral devices. Countering the trend of easing the $1 / O$ load, an additional eight terminal lines have been added to support more users. A fourth Ramtek display system has been added to the VAX display system. The new Ramtek is a model 9465 with 12 planes of memory and a resolution of $1280 \times 1024$.

\section{REFERENCES}

1. Collier, M., Renner, T., Kessler, M., Pitluck, S., and Henderson, $S$. Characterization of the new neon radiotherapy beams at the Lawrence Berkeley Laboratory. Med. Phys. 13, 601 (1986).

2. Chen, G.T.Y., Kessler, M., Pitluck, S. Structure transfer in three dimensional medical imaging studies. Proc. Natl. Computer Graphic Assoc. Meeting, pp. 171-177 (1985).

3. Chen, G.T.Y., Collier, M., Endo, M., Kessler, M., Pitluck, S., Capra-Young, D., Zink, S. Radiotherapy physics. Biology and Medicine Division Annual Report,

1983-84, Lawrence Berkeley Laboratory Report LBL-18393, (1985).

4. Kessler, M.L., Chen, G.T.Y., Pitluck, S. Computer techniques for 3-D image spatial correlation. Med. Phys. 12, 520 (1985).

5. Chatterjee, A., Alpen, E.L., Llacer, J., Alonso, J.R., Tobias, C.A. High energy beams of radioactive nuclei and their biomedical applications. Int. J. Radiat. Oncol. Biol. Phys. 7, 503-507 (1981).

6. Lacer, J., Chatterjee, A., Alpen, E.L., Saunders, W., Andreae, S., Jackson , H.C. Imaging by injection of accelerated radioactive particle beams. IEEE Transactions on Medical Imaging, Vol. MI-3 (2), 80-90 (1984). 


\section{HEAVY CHARGED PARTICLE RADIOTHERAPY}

Joseph R. Castro, Theodore L. Phillips, David Linstadt, Anthony Berson, Kay H. Woodruff, Grant E. Gauger, Philip H. Gutin," I. Michael Collier,

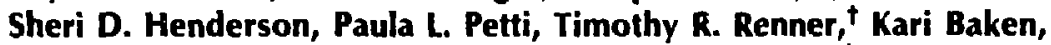
Robert E. Walton, ${ }^{\ddagger}$ Niedra Dodson, Charles R. Pascale, ${ }^{\ddagger}$ Elizabeth C. Williamson, Marc Kessler, Monica Reimers, James Judnick, Jacquelyn J. Iler

The heavy charged particle radiotherapy trial accrued 96 patients in 1985, with an additional 15 patients receiving control irradiation. The total number of patients treated through October 1986 was 921 with another 60 patients treated as lowLET controls. All patients have been treated on Phase I, II and III clinical research protocols.

\section{PHASE I-II STUDIES WITH HELIUM, CARBON, NEON AND SILICON IONS}

Patients with subcutaneous nodules or lung metastases from various tumors have been irradiated in Phase I studies to study skin RBE values for various ions. Control irradiations have been low-LET electrons and helium ions (after experience confirmed the accuracy of helium-ion skin RBE values).

Phase I-II studies have been done using the neon particle for a number of different anatomical regions and tumors: glioma of brain, head/neck tumors, esophagus, pancreas, stomach, lung, prostate, soft tissue and bone sarcoma, and a few miscellaneous sites.

Neon RBE values, relative to megayoltage radiotherapy, have been clinically studied in skin and mucosa and range from about 2.0 to 3.0 (relative to megavoltage $x$ ray) depending on particle beam energy, position in the beam path (entrance or spread out Bragg peak), dose size, technique of spreading the beam, amount of fragmentation, etc.

The Phase I-II neon studies have suggested several potential sites worthy of Phase III evaluation including prostate, lung, selected head and neck tumors, and bone/soft-tissue sarcoma. Phase III studies have been established for lung and prostate and a Phase II study is being designed for soft tissue and bone tumors. For head and neck tumors we envision a future prospective study following completion of Phase I-II studies with neon and silicon ions.

\footnotetext{
* University of California San Francisco.

'Accelerator and Fusion Research Division, LBL.

'Engineering Division, LBL.
}

Silicon RBE studies on patients with advanced or metastatic nodules in skin, subcutaneous tissues or lymph nodes have been started. Comparative studies have suggested the RBE for silicon skin reactions may be relatively high (about 3-4) depending on LET and dose fraction size.

We anticipate careful further Phase I studies of skin and other normal tissues before a Phase II clinical trial can be designed for silicon irradiation.

\section{DOSE LOCALIZATION STUDIES •}

The common attribute of this selected group of "dose localization" patients is the use of the biophysical parameters of heavy charged-particle beams to deliver a high dose to a iarget volume adjoining a critical structure.

Through December 1985, a toial of 59 patients received heavy charged particle irradiation for chordoma, chondrosarcoma, meningioma, or neurilemmoma of the base of skull or juxtaspinal area. The mean tumor dose was 68 gray-equivalent (GyE), ranging from 26 to $80 \mathrm{GyE}$. Control within the irradiated area was obtained in 41 of 59 patients $(70 \%)$.

The mean follow up in all 59 patients is 30 months with a range from 3 to 106 months. Moderate to major complications were seen in 9 of the 59 patients, with cranial nerve injury in 5, spinal cord damage in 1, and brain injury in 3 patients.

We believe that there are additional tumor sites that will ultimately show long-term benefit from precision treatment with heavy charged particles, and we plan to continue exploration of these sites, including selected childhood tumors, in order to minimize disturbances in growth and development.

\section{BONE AND SOFT TISSUE SARCOMA}

A Phase I study of heavy charged-particle radiation in soft tissue and bone tumors has been started to evaluate the potential high-LET and doselocalization advantages of heavy charged particles.

Through December 1985, 41 patients with soft tissue and bone sarcoma have received a minimum 
of $50 \mathrm{GyE}$, with a maximum of 78 , and a mean tumor dose of $65 \mathrm{GyE}$. Most patients had some gross residual tumor postsurgery or biopsy, although the extent of residual disease varied considerably.

There were 28 of 41 patients who had local control of disease in the charged-particle irradiation volume, with follow up ranging from 4 to 75 months. The actuarial local control rate (KaplanMeier) is $70 \%$ at 2 years posttreatment, which appears very promising in comparison to historical data.

Serious complications were encountered in 6 of the 41 patients, 4 in the CNS, 1 in bone and 1 in small bowel. These types of complications are expected to decrease with further experience. This exciting siudy will be extended to Phase II and III prospective trials.

\section{SELECTED LOCALLY ADVANCED HEAD AND NECK TUMORS}

Through December 1985, 47 patients with locally advanced tumors of the head and neck area including the upper aero-digestive tract, paranasal sinuses, salivary glands, thyroid gland, and neck have been irradiated using heavy charged particles, chiefly neon or helium ions.

In 14 patients treated solely or partially with helium ions, 7 have had local control. The mean survival time was 14 months.

In 33 patients treated solely or in part with heavier ions, either carbon (4 patients) or neon (29 patients), 13 had local control. The mean survival was 6 months for the carbon patients and 16 months for the neon patients.

Toxicity has been within acceptable limits, with generally moderate skin and mucosal reactions. Two patients treated with neon ions for paranasal sinus tumors have developed CNS complications.

In view of the local control rate (about $45 \%$ ) in these patients with quite difficult and advanced tumors, the use of heavy charged particles in the treatment of head and neck tumors appears to be a potentially fruitful area. We will continue this study while evaluating future combinations with other modalities such as chemical modifiers of radiation. In conjunction with the Radiation Biophysics Group, we have started cellular studies on the interaction of heavy charged particles and halogenated pyrimidines such as IUDR, and we will extend these to cisplatinum in the future, as a prelude to possible Phase I combined modality trials.

\section{CARCINOMA OF THE LUNG}

A Phase I-1I study of the use of heavy charged particles in treatment of advanced unresectable non-small cell cancei of the lung has accrued 14 patients with locally advanced surgically unresectable primary lung tumors.

A prospective randomized Phase III trial (NCOG 2N84/RTOG 85-20) has been opened to define whether neon heavy charged particles can offer an improvement over low-LET irradiation of unresectable lung cancer.

\section{LOCALLY ADVANCED CARCINOMA OF THE PROSTATE}

A small group of pilot patients have completed treatments using neon heavy charged particles as the "boost" portion of their therapy after low-LET photon irradiation of the pelvis to $4500-5000$ rads. A prospective controlled study (NCOG 4P85/RTOG 85-21) has been opened to compare low-Let irradiation to neon ions.

\section{MALIGNANT GLIOMA OF BRAIN}

Forty-seven patients with malignant glioma of the brain have been irradiated with heavy charged particles. Twenty-four patients had glioblastoma while 23 had anaplastic astrocytoma (14 patients) or lower grade tumors (9 patients).

The actuarial median survival in the glioblastoma group is 13 months while in the anaplastic astrocytoma patients it is $\mathbf{1 2}$ months. Most patients have died with tumor persistence, except two patients who died without tumor being demonstrated at autopsy, one with focal gliosis, and another with a brain abscess secondary to prolonged steroid administration.

A longer survival (median: 56 months) has been achieved in the lower grade tumors, suggesting that slower growing glioma might be a logical tumor in which to use high-LET heavy charged particles.

\section{CARCINOMA OF THE PANCREAS}

Since 1975 we have irradiated 163 patients with locally advanced carcinoma of the pancreas in Phase I, II, and III trials to doses of at least $48 \mathrm{GyE}$.

With helium ions there was approximately a $10-20 \%$ level of local control within the irradiated volume as judged by strict criteria including follow up clinical examinations, CT scanning, and histological confirmation whenever available. In a study of 
22 helium-treated pancreas patients who had autopsies, our study pathologist, Kay Woodruff, found a local control rate of $27 \%$.

The follow up in all 163 patients treated with helium or heavier ions (carbon or neon) ranged from 2 to 74 months with an actuarial survival of 11.7 months.

Overall, the addition of any amount of $5 \mathrm{FU}$ or FAM chemotherapy improved the actuarial survival from 8.2 to 12.6 months although the local control rate was not increased.

We continue to look for new approaches to provide local and regional control of this disease as well as control of occult metastases.

Intraoperative radiotherapy plus external beam heavy charged particle therapy and chemotherapy is under consideration. A Phase I trial may also be started using neon heavy charged particles and infusional 5FU-Methotrexate chemotherapy.

\section{UVEAL MELANOMA}

The overall results in the dose searching Phase I-Il study of helium ion irradiation in 208 patients with uveal melanoma were excellent.

A local tumor control rate of $95 \%$ with follow up from 3 to 90 months (mean: 26 months) was achieved. Eight patients with local failure of initial helium-ion treatment were salvaged with enucleation (5 patients) or re-irradiation ( 3 patients).

Complications have included neovascular glaucoma, perforated sclera, severe keratitis, and painful or dry eye; 14 patients required enucleation for complications, in addition to the 5 patients with tumor progression.

Overall, about $55 \%$ of patients have retained vision of 20/400 or better, more often in patients with tumors more than $3 \mathrm{~mm}$ from the fovea or optic nerve and less than $10 \mathrm{~mm}$ in greatest dimension.

While the results are quite good, we are uncertain that for some tumor locations and sizes radioactive plaque therapy might not be as good as charged particles. Therefore we are randomizing patients with lesions up to $10 \mathrm{~mm}$ in height and 15 $\mathrm{mm}$ in diameter to either helium-ion therapy or iodine $\left[{ }^{125} \mid\right]$ plaque treatment. For this randomized Phase III study, we deliver 70 GyE in 5 fractions over 7 to 12 days as the optimal current helium technique. Over 70 patients have been accrued in this study (NCOG 7085), with about 200 additional patients being needed.

\section{FUTURE PLANS}

Planned areas of research in the next five years include further testing of neon charged particles in several tumor sites such as head and neck, bone and soft tistises, lung, prostate, pancreas, and any histologica! lumor demonstating slow growth kinetics. We hope to develop assays that might reveal details of iumor growth parameters or other factors that will helik to predict those tumors that can be advantageousiy treated with high-LET charged particles.

Evaluation of MRI, PET scanning, or other techniques to study effects of heavy charged particles on tumors and normal tissues will be pursued in conjunction with the Research Medicine Group.

Evaluation of possible combined modality treatment, especially with some classes of radiation sensitizers will be studied in collaboration with E.A. Blakely and C.A. Tobias of the Radiation Biophysics Group.

Further optimization of radiation therapy treatment planning and delivery will be studied as improved beam delivery techniques are developed. A special effort will be made to assess which ion might be best for dose localization. The clinical trials (eye and para-CNS) in dose localization will be continued with helium ions for the present, to optimize treatment methods, provide data on long term results and explore new sites in which to apply these techniques. 


\title{
STEREOTACTIC HEAVY CHARGED PARTICLE BRAGG PEAK RADIOSURGERY
}

\author{
Jacob I. Fabrikant, Kenneth A. Frankel, Mark H. Phillips, John T. Lyman,
}

Edward L. Alpen, Neela B. Manley, tichard P. Levy, Eng Lo, Myrtle L. Foster, George T. Hampton," Frederick W. Yeater," Leal Kantstein," Dresden Van Deusen, Barbara Modlinski (in collaboration with Gerald D. Silverberg, ${ }^{\dagger}$ John W. Hanbery, ${ }^{\dagger}$ Charles B. Wilson, ${ }^{\ddagger}$ Yoshio Hosobuchi, ${ }^{\ddagger}$ William H. Marshall, ${ }^{\dagger}$ Dieter Enzmann, ${ }^{\dagger}$ Thomas H. Newton, ${ }^{\ddagger}$ and David Norman ${ }^{\ddagger}$ )

\section{CLINICAL RESEARCH}

Deep and surgically-inaccessible intracranial arteriovenous malformations (AVMs) have not been treated until the last two decades, primarily because of their variable symptoms, changeable pathoanatomical relationships, and inaccessibility to vascular brain suigery. Recently developed neuroradiological imaging and microsurgical techniques are now making certain of the deep AVMs potentially amenable to treatment. However, serious limitations to neurovascular surgical or interventional reuroradiological procedures may exist in the case of deep AVMs, because of location, size, patient condition, or other factors. Conventional radiotherapy as a method of induced thrombosis has been attempted in the past, but has been generally abandoned as unsuccessful. However, stereotactic heavy charged-particle Bragg-peak radiosurgery currently being developed holds considerable promise for the treatment of those liie-threatening intracranial vascular disorders that are surgically inaccessible. Evaluation and treatment with stereotactically directed narrow beams of heavy ions in approximately 225 patients in our laboratory, plus extensive clinical and neuroradiological follow up, indicates that heavy charged particle radiosurgery obliterates intracranial arteriovenous malformations or protects against rebleeding with considerable reduction in morbidity and mortality.

Clinical research objectives are to achieve changes in the intracerebral hemodynamic flow conditions, which result in a decrease in frequency of hemorrhages, and in neurological deficits. Results in the largest majority of patients thus far treated indicate that these objectives are being achieved. Radiological objectives are to achieve hemodynamic alterations, e.g., decrease in blood flow through the AVM associated with decrease in size of the pathological cluster of vessels until par-

\footnotetext{
"Accelerator and Fusion Research Division, LBL

'Stanford University School of Medicine

University of California San Francisco
}

tial obliteration or total disappearance occurs (Fig. 1). These hemodynamic changes occur progressively, and are usually present before morphological vascular alterations can be demonstrated. Following radiosurgery, there is a significant reduction in the rate of rebleeding in vulnerable patients, indicating that the procedure affords considerable protection against future hemorrhage; this is apparent in $85 \%$ of patients. In some $25 \%$ of patients, the AVMs are obliterated (Fig. 1), and in an additional $65 \%$, there is evidence of reduction in size and in rate of blood flow in the abnormal shunting vessels. There have been 7 cases of spontaneous intracranial hemorrhage occurring following radiosurgery and only 1 after 15 months. Three of these patients recovered completely and were cured of their AVMs, and four died as a result of hemorrhage. Approximately $2 \%$ of patients experience strokes as recovery ensues; these are usually minor and transient in nature, but in two patients profound neurological deficits have resulted.

Certain early conclusions can be drawn from these initial clinical research trials. 1) Stereotactically directed heavy charged-particle Bragg-peak radiosurgery appears more advantageous than the use of $x$-ray beams, gamma-ray beams or proton beams because of improved spatial definition and dose-distribution in the AVM target volume within the brain, 2) Initial therapeutic results demonstrate that heavy-ion focal irradiation of the intracranial AVM can induce hemodynamic and neurovascular changes associated with reduction in size and flow, partial or complete obliteration of the vascular abnormality, and protection against future rebleeding. 3) The advantages of the procedure are a) for certain selected cases of surgically inaccessible abnormalities within the brain, it is a relatively low-risk, noninvasive procedure without any blood loss; b) in patients under threat of hemorrhage from inoperable AVMs, successful treatment can be achieved where neurosurgery is unavailable; c) pro- 

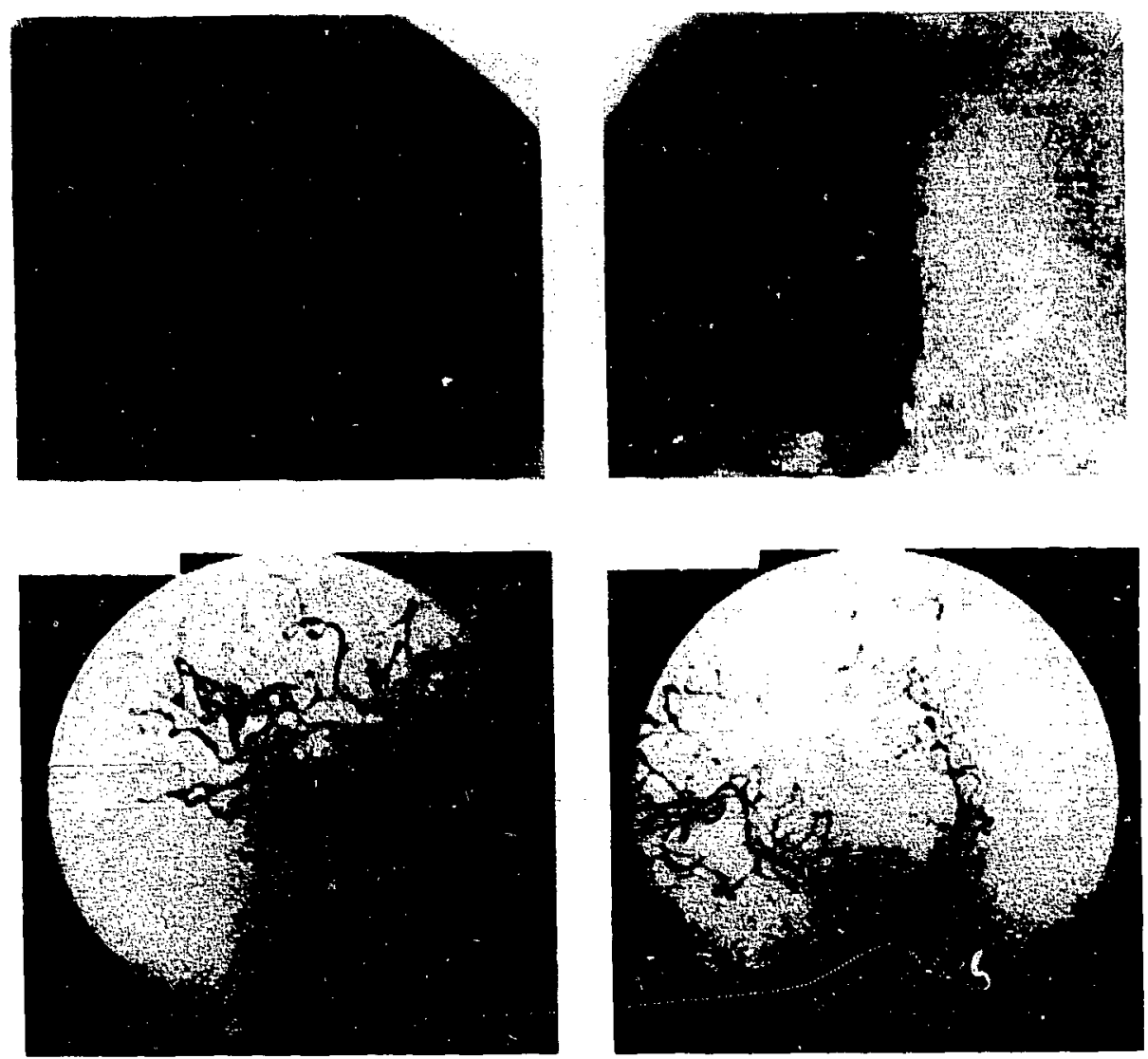

Fig. 1. The patient is a ten year old boy with a large surgically inaccessible arteriovenous malformation in the frontoparietal region of the right cerebral hemisphere. The upper panels illustrate the neuroradiological images of the stereotactic cerebral angiogram that demonstrate the location and size of the large arteriovenous malformation arising primarily from branches of the anterior cerebral artery circulation. The patient is immobilized in the stereotactic head mask and frame. The lower panels illustrate the cerebral angiograms obtained one year after stereotac. tic heavy changed particle beam radiosurgery; there has been complete obliteration of the arterial feeders and the shunting vessels of the AVM with restoration of the normal cerebral blood how. The boy is in excellent health.

(XBB $871-79)$

longed hospitalization in not required-patients are treated on an ambulatory basis in one or two days, thereby decreasing the attendant costs, 4) The disadvantages of the method are that a) evidence of radiation-induced change within the AVM does not appear for some 6 months, and partial or complete obliteration requires 9 to 18 months or more; thus patients are not protected and are under threat of hemorrhage for a long time; b) time, dose, volume, and fractionation relationships for heavy charged particle focal irradiation of brain and other CNS tissue are not fully understood, and these must be known, since there exists the danger of serious brain damage and neurological deficits when large doses of radiation in large radiation fields must be applied.
The clinical research program on stereotactic heavy charged-particle Bragg-peak radiosurgery for intracranial deep-seated neurovascular disorders in patients is designed 1) to introduce technological modifications and improvements of the radiosurgical technique-including stereotactic neuroradiological studies for quantitating radiation changes in the brain in vivo, computer-based interactive treatment planning, heavy-ion beam delivery and dosedistribution-to achieve a safe, reliable, and efficacious therapeutic procedure; 2) to evaluate the long-term clinical trial results in patients treated with accelerated helium ion beams for AVMs of the brain and spinal cord; 3) to develop the method using accelerated heavy ions (Bragg-peak beams) at the Bevalac, for all patients, to obtain improved 
biological and physical effects using carbon ions or possibly heavier ions. The advantages of heavier ions over helium ions and protons include narrow beams with less range straggling and less multiple scattering for the same residual range in tissues, and improved dose-distribution in the Bragg peak, with very sharp lateral and distal borders, and with greater sparing of critical structures in adjacent CNS tissues in the brain and spinal cord. The carbon and neon ion beams also provide radioactive beams that are currently being investigated for precise localization of the stopping point of the beam inside the brain; and 4) to perfect the method at the Bevalac for application at the clinical level in neurological medicine when lighi-ion medical accelerators are more generally available throughout the world.

\section{PHYSICS RESEARCH}

Stereotactic heavy charged-particle treatment planning has undergone continuous development to perfect stereotactic systems for delivery of narrow heavy-ion beams to intracranial targets. The procer. - $\checkmark$ involves the fabrication of a thermoplastic $h_{\mathrm{L}}$ nolder adapted to a modified Leksell stereotactic frame for immobilization of the patient during stereotactic cerebral angiography, stereotactic $x$-ray computerized tomography (CT) scanning, stereotactic nuclear magnetic resonance (NMR) scanning, and stereotactic heavy charged-particle Bragg-peak radiosurgery. Stereotactic cerebral angiography and stereotactic CT brain scans provide data that are transfeired to a VAX-11/780 computer system for interactive charged particle treatment planning and provide the basis for stereotactically directed charged-particle beams to AVM target contours. Entry angles and beam ports are chosen to confine the high-dose Bragg-peak region to the defined target volume while carefully protecting adjacent normal brain structures. Stereotactic radiosurgical treatment planning programs use the $\mathrm{CT}$ data on a pixel-by-pixel basis to a) design a collimator aperture for each entry portal, b) select the appropriate spread Bragg peak for irradiation, c) design appropriate compensators to contour the stopping region of the heavy charged-particle beams, d) generate isoeffect and physical dose distributions with CT image composites for adaptation to stereotactic radiosurgical treatment, and e) develop optimal non-coplanar treatment planning procedures.

The central AVM dose, the aperture size, the number of ports, the angulation of the delivered beams, and the spread Bragg peak all determine the isodose contour. Total doses of 25 grey- equivalent are now delivered to treatment volumes of over $25,000 \mathrm{~mm}^{3}$; the smallest volumes treated with the unmodified Bragg peak are about 100 $\mathrm{mm}^{3}$, and the average volumes treated in most patients range from 1500 to $5000 \mathrm{~mm}^{3}$. Treatment occurs through three to six entry portals, delivered daily for 1 or 2 days depending on the location of the AVM within the brain, the treatment volume, and the volume of normal brain tissue traversed by the heavy-ion beams.

Noninvasive imaging research procedures are used for diagnostic localization and treatment planning, and for examination of the temporal response to heavy charged-particle irradiation, hemodynamic function and regional cerebrai blood flow, and alterations in brain tissue structure and function following treatment. Cerebral angiography, x-ray CT scanning, and certain newly developed specialized neuroradiological investigative studies, including nuclear magnetic resonance (NMR) imaging, xenon-enhanced computerized tomography, positron emission tomography (PET), and cine computerized tomography are being carried out or planned for all patients prior to stereotactic radiosurgery and at intervals following treatment. The following changes, if present, are being quantitated. a) Hemodynamic changes, e.g., decrease in blood volume and flow through the AVM with decrease in size of the feeder arteries and shunting vessels and draining veins. These change, may best be quantitated with PET and/or NMR scans, which measure blood flow changes inore accurately. (b) Anatomicopathological changes, e.g., progressive decrease in the size of the AVM, potential transient or permanent disturbances in myelinatation processes leading to demyelination, and early and late delayed radiation-induced injury in the brain following stereotactically directed radiosurgical treatment. Initial studies on selected patients using positren emission tomography and nuclear magnetic resonance imaging demonstrate considerable promise for elucidating mechanism of cell and tissue response of the central nervous system to irradiation with narrow beams of heavy charged particles; these collaborative research studies are being carried out in the LBL Research Medicine Laboratory of Thomas F. Budinger, and under the direction of Peter Valk.

Significant progress has been achieved in utilizing and incorporating the fine anatomical and pathological detail provided by improved resolution of NMR imaging into stereotactic sadiosurgical treatment planning as well as for clinical response follow up in all patients after treatment. The recent advances in NMR imaging have introduced valuable 
methods for mapping of the deep structures of the brain that are complementary to currently available imaging procedures; NMR imaging permits identification and measurement of the internal structure of the AVM, including size, shape, location, and relationships involving adjacent normal and displaced critical structures in the brain that is essential for treatment planning for precision beam-delivery and dose-distribution. Studies are in progress, in collaboration with Stanford University Medical Center, that correlate cerebrai angiographic and NMR structural anatomy of the AVM in selected patients, and current observations indicate that for defining the irradiation target of the nidus of large arteriovenous malformations, NMR may prove to be superior. In addition, NMR imaging is now essential for precise treatment planning and for comparing NMR target volumes with angiographically defined volumes; studies are in progress, in collaboration with the LBL Radiation Oncology Section of Joseph R. Castro, that apply image correlation techniques to NMR, x-ray CT, and cerebral angiographic images, introducing for the first time a reliable method of applying the angiographically derived target contour to th: NMR image. The method significantly extends the capabilities of the developed treatment planning protocols based on the quantitative data derived and transferred from stereotactic cerebral angiography and stereotactic CT scanning (Fig. 2). The technique involves contouring anatomical structures on correlation $x$-ray CT scans, transformation to NMR image space, and display on the corresponding NMR image for correctness of the transformation matrix. Such comparisons defining the precise anatomical size, shape and location of the AVM in multiple planes, as imaged in NMR reconstructions, provide accurate displays of the neuro-anatomical relationships to the critical structures in the brain (Fig.3), thereby improving doselocalization and distribution, and minimizing the potential for radiation-induced brain injury.

NMR imaging is becoming invaluable in assessing the temporal response of the brain tissues and the pathogenesis of induced changes in the AVM and in the adjacent brain following stereotactic radiosurgery. The current methods of cerebral
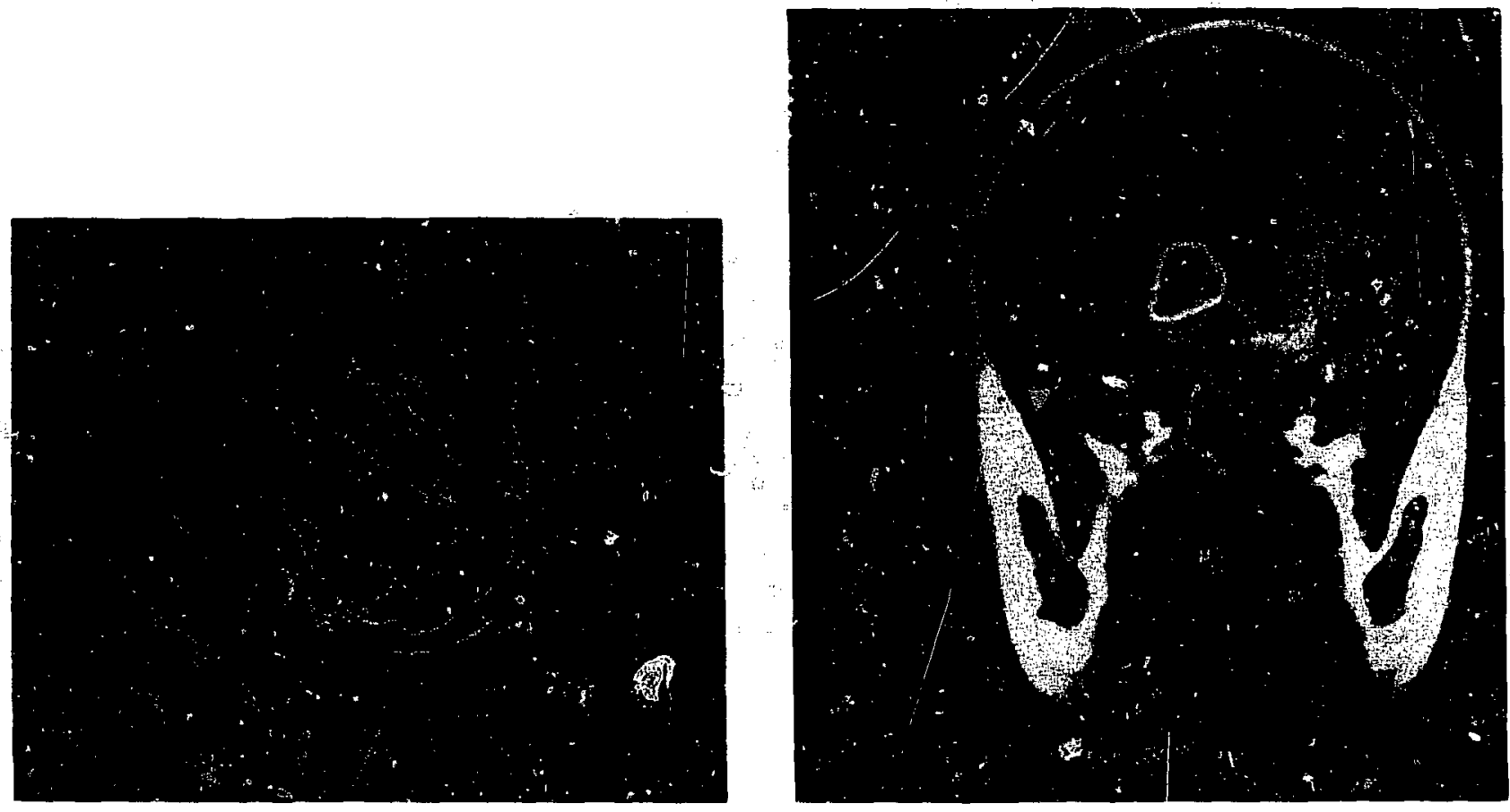

Fij. 2. NMR images of the head of a patient with an intracranial AVM. In the left panel (axial viaw) the inncr table of the skull has been contoured on correlation $x$-ray CT scans, and transformed to NMR image space for

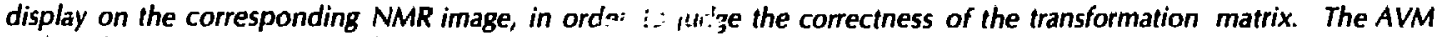
within the brain is precisely localized. In the ight and (coronal view) the boundaries of the AVM, transferred from the stereotactic cerebral angiographic study, have been transformed to $x$-ray and NMR image spaces, respectively, and the image demonstrates the precision of the quantitative correlation of the angiographic and NMR procedures as regards the size, shape, and location of the AVM.

(XBB $871-80)$

(XBB 871-81) 

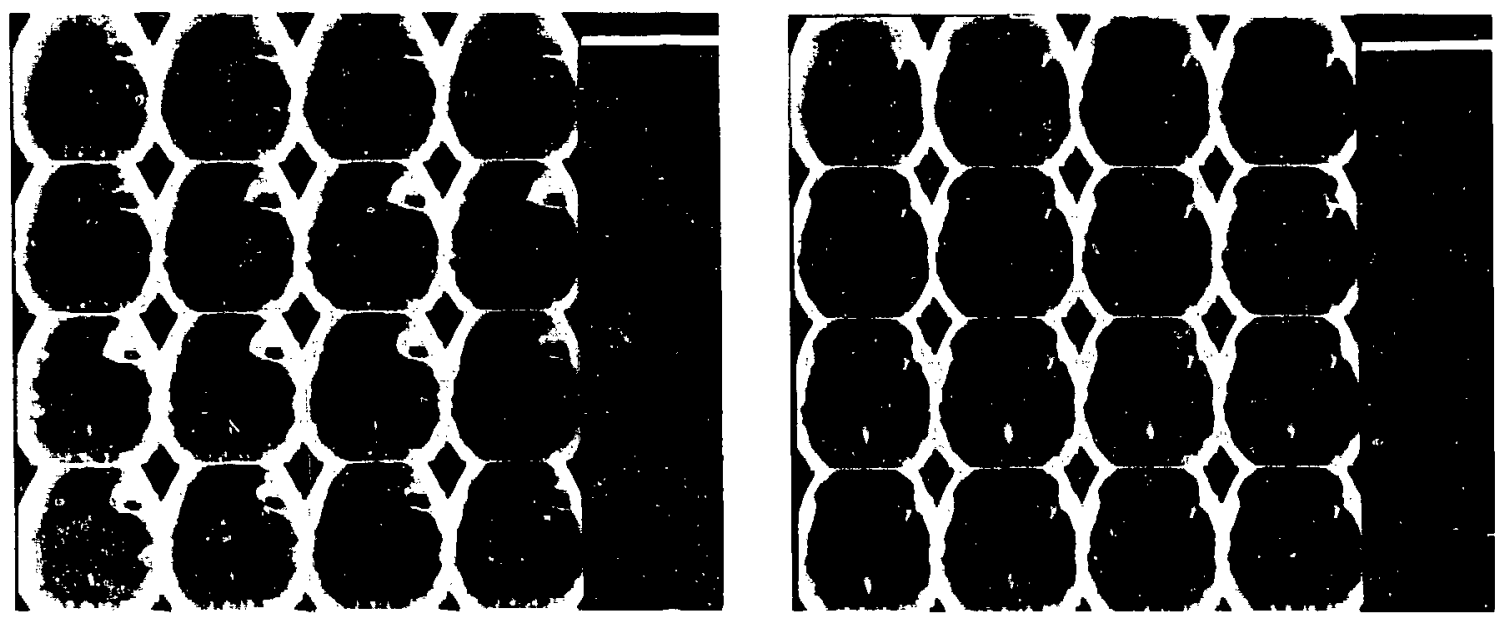

Fi.. 3. Cine-CT study in a patient with an intracranial arteriovenous malformation. Correlation is made between cerebral blood flow dynamics and such quantities as the percentage of the cardiac output diverted through the high-volume, high-flow arteriovenous malformation.

(XBB 852-1032)

angiography and x-ray CT scanning cannot characterize subtle tissue changes with accuracy; only gross alterations such as hemmorhage or advanced delayed injury can be identified. NMR is noninvasive, can be used in most patients, and demonstrates alterations in tissues (regional edema, disturbances of myelination processes, minimal intraparenchymal bleeding, temporal changes in AVM architecture, etc.) that provide insights into mechanisms of the neurovascular response to stereotactic heavy charged-particle Bragg-peak radiosurgery.

Thus far, over 30 patients have been followed for periods of over 12 to 18 months after stereotactic radiosurgery using NMR imaging. Intracranial AVMs were irradiated at doses of $45,35,25$, and $20 \mathrm{GyE}$, depending on a number of factors, with the Bragg peak helium beam at the LBL 184-Inch Synchrocyclotron. Thus far, there is usually no NMR evidence of brain tissue response for the initial six month latent interval; this interval is most frequently followed by a decrease in the volume of the AVM, occasionally associated with a sharplydefined narrow band of increased signal in the immediately adjacent brain tissue, and only in regions in which the dose to brain tissue was greater thin 15 CyE. In almost all of these patients. ssymptomatic recovery period without incidence or progressive or fixed neurological deficit suggests that these NMR changes, when they occur, may be transient in nature, and either due to persistent regional cerebral edema or possibly transient perturbation of the myelination processes.
These important long-term clinical research studies are continuing.

Studies are continuing on the analyses of cineCT scanning investigations of cerebral blood flow dynamics in selected patie is with intracranial arteriovenous malformation. Following the onesecond scan sequence, the relationships of arterial cerebral blood flow dynamics, cardiac output, and various regions of the brain can be correlated (Fig. 3). We are presently assessing these data as a measure of temporal response of regional cerebral blood flow through AVM structures following stereotactic radiosurgery. The studies of xenonenhanced $C T$ imaging, in collaboration with Stanford University Medical Center, are providing a detailed mapping for quantifying altered regional cerebral perfusion in brain tissues adjacent to and remote from the intracranial AVM. Over 50 patients have thus far been examined with this dynamic imaging procedure. Initial analyses of temporal sequences over the prolonged latent interval follnwing stereotactic radiosurgery suggest that following partial or complete vascular obliteration of the AVM, regional cerebral blood flow is decreased, whereas previously diminished blood flow in adjacent and remote brain tissue is gradually characterized by a return to normal cerebral perfusion. This investigation is leading to a better understanding of the mechanisms of the vascular steal phenomenon which deprives the adjacent brain of normal regional perfusion, and may provide a progno ic index of the potential for reversing the progression of the attendant neurolngical syndrome. 


\section{BIOPHYSICS RESEARCH}

Physics studies continue, in collaboration with the Radiation Biophysics Group of Cornelius A. Tobias, on characterization of the accelerated heavy charged-particle beams applied to stereotactic radiosurgery and radiation oncology, and particularly multiple Coulomb scattering. We have developed computer programs that calculate the effect of multiple scattering on the propagation of heavy- and light-ion beams as they pass through matter. This new information is of importance in the irradiation of brain tissue, and thus, one aim is to incorporate these findings to improved treatment planning, dose-localization, and distribution for stereotactic radiosurgery. In investigations of the multiple Coulomb-scattering effects of proton and carbon beams at the Bevalac, measurements of beam profiles in water have demonstrated results that agree quite well with computer calculations. In parallel studies, high resolution nuclear detectors are being used to measure individuc' chargedparticle trajectories; analysis of these data are in progress. We have demonstrated that for narrow beams of accelerated charged particles delivered deep within the brain, considerable limitations exist for proton as compared with helium and carbon beams. The lighter the ion, the greater the extent of multiple Coulomb scattering during propagation of the beam, with a resulting significant degradation of the Bragg peak to plateau radiation dose; this applies to narrow beams of the order of $1 \mathrm{~cm}$ or less in diameter at depths of $12 \mathrm{~cm}$ or more (Fig. 4). Furthermore, the light ions do not main-
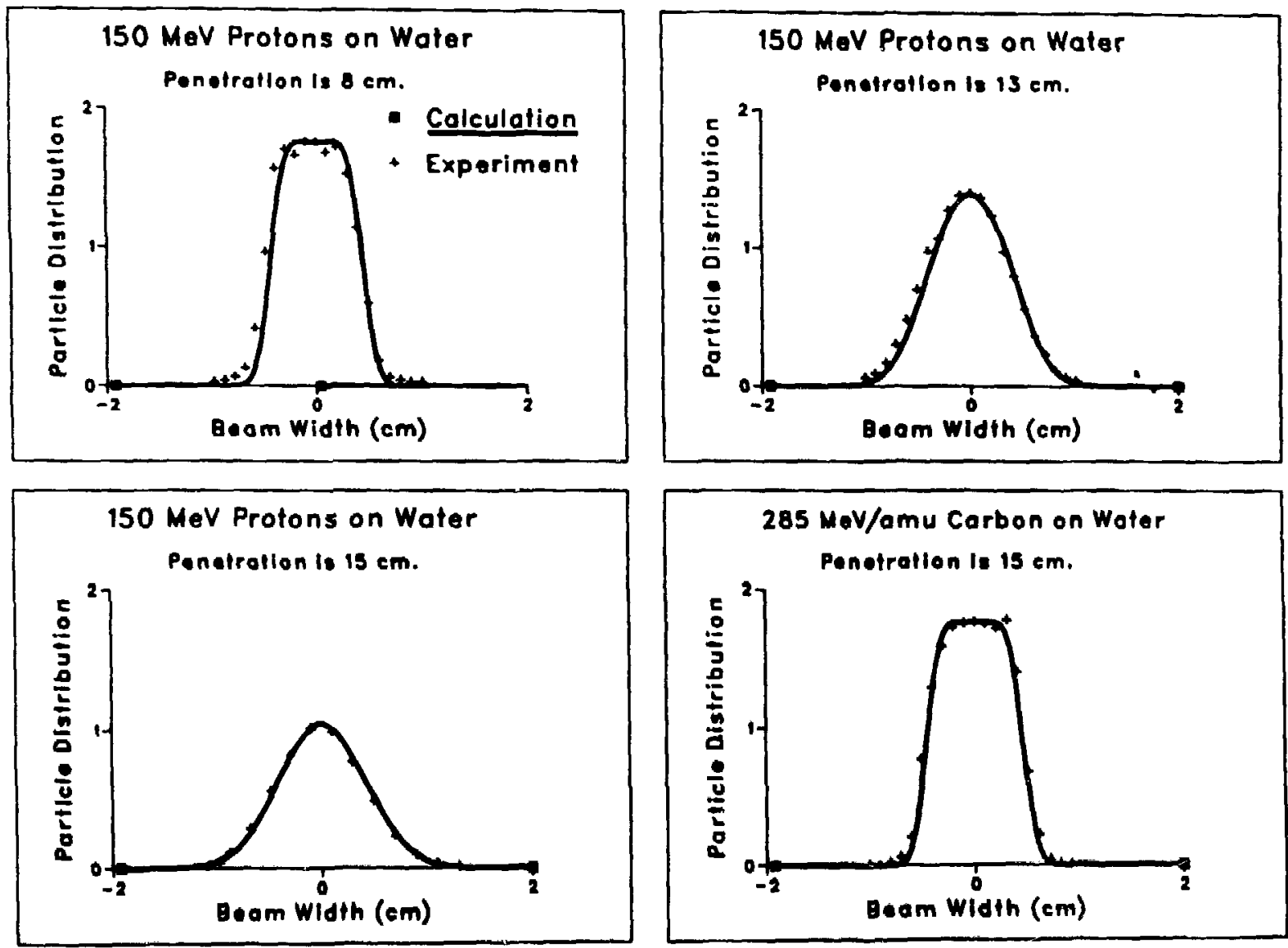

fig. 4. The effect of multiple Coulomb scallering on the propagation of charged particle beams as they pass through water; the particle distributions of $150 . \mathrm{MeV}$ protons and $285-\mathrm{MeV} / \mathrm{amu}$ carbon ions are correlated with beam width at various penetrations. The four experiments demonstrate good agreement between theorofical calculations and measured beam profiles for $15-\mathrm{cm}$ range proton and carbon beams. The proton beam, measured at three different depths, demonstrates spreading of the beams, and considerable decrease in the Bragg peak to plateau huence. The carbon beam maintains extremely sharp edges and an elevaled peak to plateau fluence at the end of its $15 . \mathrm{cm}$ range.

(XBL 871.42$)$ 
tain the sharply defined beam profile essential for precision stereotactic radiosurgery, since this results in an increased radiation dose delivered to brain tissue outside the desired narrow beam path.

Calculation of neutron production in heavy-ien collisions provides further information on the characterization of heavy-ion beams. We have analyzed the data of Schimmerling and his colleagues (in collaboration with the Radiation Biophysics Group) for 337-MeV/amu neon + uranium $\rightarrow$ neutrons $+X$, using a cascade code to generate a primordial neutron distribution, calculating the primordial deuteron distribution, and thereby deriving the calculated neutron distribution. With the restriction that the calculated deuteron distribution is less than $40 \%$ of the primordial neutrons, excellent agreement between calculated and experimental data results (Fig. 5). However, this fails when the calculated deuteron distribution exceeds $40 \%$ due primarily to the contribution of heavier mass composites.

A method using the electro-optical effect to measure conformation changes in phage DNA has been developed to measure the yield of DNA strand breaks in SV40 genome, irradiated extracellularly. Decay curve coefficients are being determined as a function of radiation dose for SV40 strand breaks for $250-k V p \times$ rays and for heavy ions; this information yields the dose applied and may be used to compare the RBE for radiations of

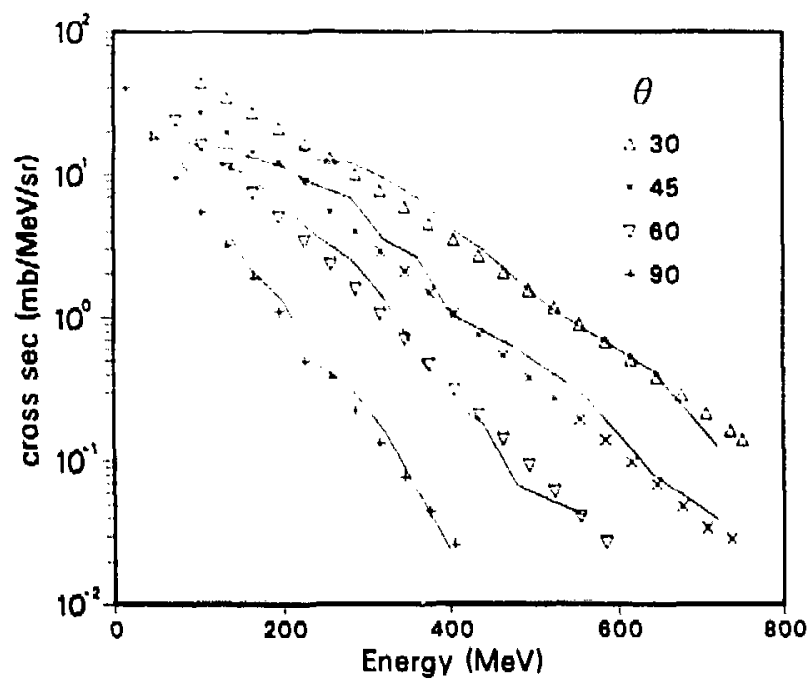

Fig. 5. Comparison of experimental data (symbols) with theoretical calculations (solid lines) for neutron production resulting from the nuclear reaction $337-\mathrm{MeV} / \mathrm{amu} \mathrm{Ne}+\mathrm{U} \rightarrow>$ neutrons $+X$. The symbols are the experimental data taken at 30, 45, 60, and 90 degrees: the solid lines are the calculated distributions.

(XBL 871.34) different quality. The method has proven to be reliable in the dose ranges used in stereotatic radiosurgery, and may be applied to the study of kinetics of radiation-induced DNA damage and repair and biological dosimetry in isolated mammalian neuronal and glial cells.

Mechanisms of early delayed radiation effects in brain appear to be neuronal in origin and associated with processes of myelination and myelin maintenance. Those of late delayed injury are considered vascular in origin and associated with vascular endothelial injury and functional disturbances, leading to ischemia, cell death and necrosis. We are examining the induction and repair of DNA damage in single glial and capillary endothelial cells from rat and mouse brain following selected dose increments of accelerated heavy-ion beams, characterizing dose-response and time-dependent relationships (in collaboration with Dr. Kirsten Rosander, Swedish National Defense Research Institute). The induction of DNA strand breaks is a very sensitive index of early cellular radiation injury; our method uses a fluorescent dye intensity staining of isolated cells evaluated by microscope photometry. The intensity of fluorescence from single-stranded DNA relative to fluorescence from double-stranded DNA is used as a measure of DNA strand breaks. We have examined the effects of beams of 153 $\mathrm{MeV} / \mathrm{amu}$ helium (doses, 5 to $15 \mathrm{~Gy}$ ) and 425 . $\mathrm{MeV} / \mathrm{amu}$ neon (doses, 1.8 to $6 \mathrm{~Gy}$ ). The experimental data thus far indicate a general increase in DNA damage with neon (425 MeV/amu) radiation dose, and the dose-dependent effect is much greater for glial cells than for endothelial cells. Following exposure to 2 Gy of neon-ion irradiation, DNA damage in vivo is repaired rapidly in both glial and endothelial cells, and the greatest amount of repair occurs within thirty minutes following exposure, whereas the remaining in vivo repair occurs over a longer period of time (Fig. 6).

The process of myelin maintenance is being examined in isolated, mammalian oligodendroglia. The method for preparation, isolation, and maintenance involves trypsinization and dissociation of white matter and preparation of single-cell suspensions by separation by density gradient centrifigation. Maintained oligodendrocytes can actively incorporate tritiated galactose into cerebroside, a major component of myelin, indicating that cells remain viable in culture. These purified glial cell populations obtained in vivo can be maintained in vitro for periods of up to 96 hours and more and used for examining cell-specific metabolic processes involved in heavy-ion-induced late delayed radia- 

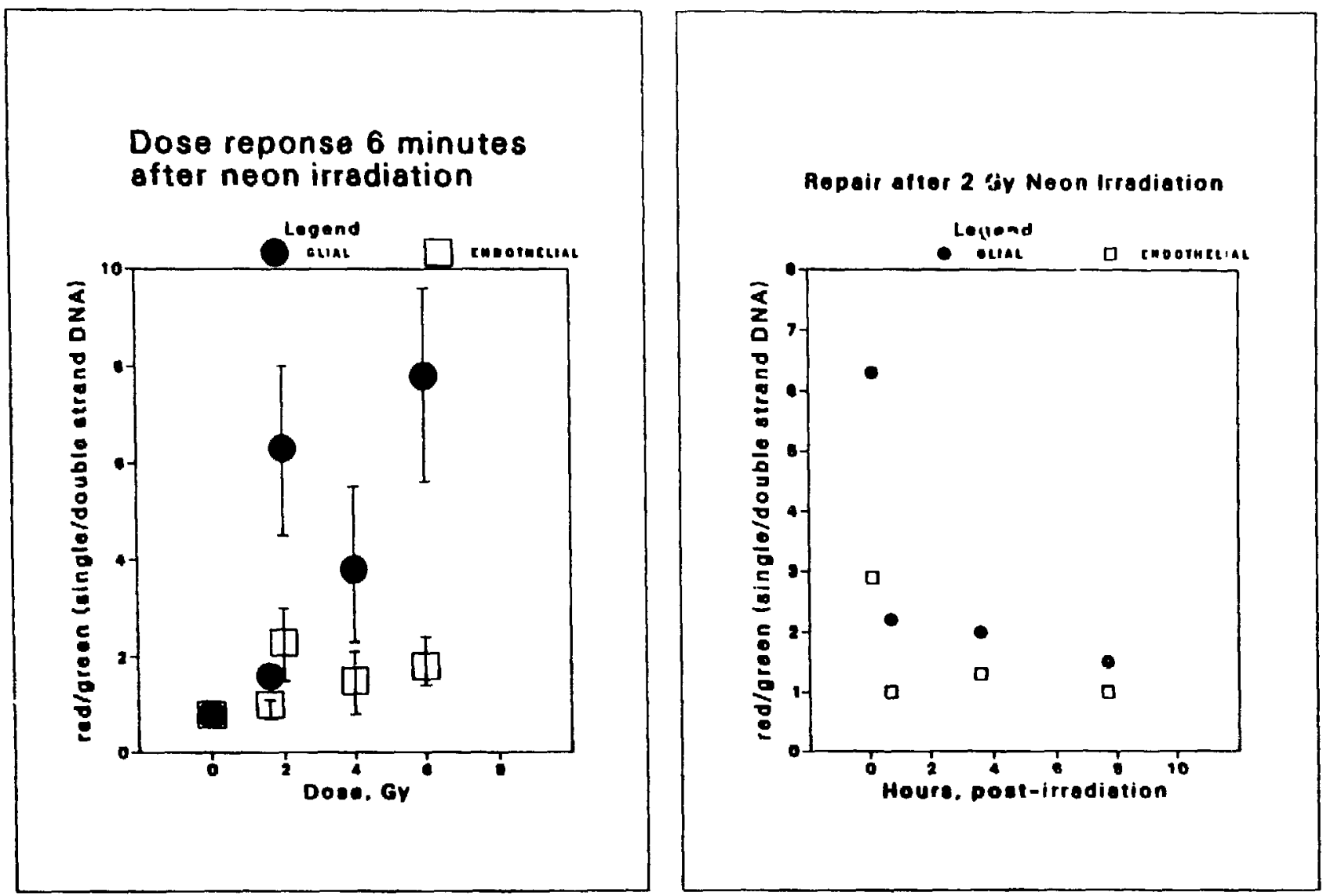

Fig. 6. Left panel. Dose-response relationships for induction of DNA strand breaks in rat brain glial and vascular endothelial cells following neon-ion (425 MeV/amu) irradiation. An alkali solution is applied to cells to cause DNA unwinding: the cells are stained with the fluorescent dye, acridine orange, and the ratio of red fluorescence from single-stranded DNA to green fluorescence from double-stranded DNA provides a measure of the number of strand breaks. There is a dose-dependent response that is more marked in the glial cell populations. Right panel. Timedependent relationships of in vivo DNA repair in rat brain glial and vascular endothelial cells following exposure to 2.Gy neon-ion (425 MeV/amu) irradiation. Most of the DNA repair in both cell populations occurs within the first 30 minutes, while the remaining repair of DNA damage may occur over a longer period.

(XBL 871-35)

tion effects in mammalian brain and for studying attempts at therapy.

The brain cell populations consist of neurons, neuroglia, and vascular endothelium. In the adult brain, the neurons are highly differentiated cells that are closely associated with and functionally related to the glial cells. The neuroglial cells consist of astrocytes that serve as bridges between neurons and capillaries, oligodendroglia that are involved in the process of myelin production, and microglia that serve primarily as phagocytes. For some cell types, cell kinetic studies indicate that cell renewal takes place in the normal adult brain - in the cells of the subependymal layer, endothelial cells, olgodendrocytes, and microglia. Our studies demonstrate that the normal cell cycle
20 hours; the duration of DNA synthesis is about 7 hours for neuroglial, and about 10 hours for endothelial cells. The growth fraction for oligodendroglia is about $0.4 \%$. Following mitosis, some $20 \%$ of the neuroglial celis become pyknotic; due to cell loss, one division results in about 1.67 proliferative daughter cells. In the mammalian brain, the cells of the subependymal layer of the ventricles consist of populations of dark and light cells; the small dark cells are considered the glial stem cell population. They have a relatively high tritiated thymidine labeling index and are the most rapidly renewing brain cell population in the young adult mammal. The cellularity of the subependymal plate is rapidly depressed following $x$ irradiation, but the effect is more pronounced after exposure to $153-\mathrm{MeV} / \mathrm{amu}$ helium-ion and 425 - 
$\mathrm{MeV} / \mathrm{amu}$ neon-ion beams. The response is rapid and dose-dependent. Following irradiation with both helium and neon ions, the sinall dark glial cells disappear rapidly, while the light cells persist. The response is dose-dependent as well, but much more rapid and sustained for a longer interval following neon irradiation. Furthermore, the population doubling times of the subependymal cells following exposure to $153-\mathrm{MeV} / \mathrm{amu}$ heliurn ions is markedly reduced after $45 \mathrm{~Gy}$, from a normal value of about 140 hours to about 45 hours in the irradiated cortex, and to about 55 hours in the unirradiated cortex of irradiated mice. The glial cell kinetic data in normal and irradiated mouse brain are con- sistent with the conclusions that in the subependymal plate the light cells remaining after radiation represent a resistant stem cell population with a reiatively slow rate of renewal. The dark cells comprise a rapidly proliferating stem cell population, which is thus relatively radiosensitive, and it is this stem cell pool that is responsible for recovery following heavy charged-particle irradiation. This appears to be effected by an increase in glial cell proliferation rates associated with decreased cell loss, an increase in size of the growth fraction associated with a decrease in the cell cycle time, and recruitment of undamaged glial cells into the proliferative pool.

\title{
THE FUTURE FOR RESEARCH WITH ACCELERATED NUCLEI: BUG AND LIBRA
}

\author{
The LIBRA Study Group: Cornelius A. Tobias, Richard A. Gough,* \\ David A. Shirley,t Gerd M. Rosenblatt,t Paul H. Silverman, Klaus H. Berkner," \\ Thomas F. Budinger, Joseph R. Castro, and Jacob I. Fabrikant
}

There has been a continuously rising interest in basic research with accelerated nuclei and in the therapeutic and diagnostic applications of heavycharged-particle: beams. There is a likelihood that the new technologies developed with support of the Department of Energy and the National Cancer Institute can be successfully transferred to the private sector for wider applications.

On a world wide basis, there are already eight proton accelerators, either in operation, or in various stages of construction, with energies sufficient for biomedical applications. These are located at Harvard University, at Uppsala, Sweden, in Japan, three in the Soviet Union, and one in the Republic of South Africa (Groote Schuur Hospital near Cape(own). In addition, a new cryogenic cyclotron is being built for medical uses at the Radiation Oncology Center in Detroit, Michigan. For heavy-ion accelerators, two ambitious construction programs exist. One at the GSI laboratories at Darmstadt, West Germany and another at the National Institute for Radiological Sciences at Chiba, Japan. However, the Berkeley Bevalac is still unique in its ability to accelerate all of the nuclei in the periodic table and with its comprehensive biomedical

*Accelerator and Fusion Research Division, Lawrence Berkeley Laboratory

† Director's Office. Lawrence Berkeley Laboratory research program. In order to stay abreast of the demands of the future, the laboratory is in a period of intense planning. A change in terminology has been made. We shall refer to "light-ion beams," when the atomic number is less than or equal to that of neon $(Z=10)$ and to "heavy-ion beams" when the nuclei are heavier than neon.

According to a joint proposal of the nuclear and biomedical users of the Bevalac, it will be upgraded, beginning about 1992. An entirely new synchrotron ring will be built. Other changes contemplated include increases in the intensities of the heavy beams, and increased uses of secondary beams formed when the primary beams undergo fragmentation. For the biomedical program, the Bevalac Upgrade (BUG) will allow more effective utilization of the heavier beams for therapy research, the detailed exploration of biological effects produced by space radiations and much broader biomedical studies with radioactive secondary beams than are possible at present.

Recently a consortium was formed for future investigations with accelerated light ions. Initial members comprise the Board of the Merritt Peralta hospital complex in Oakland, the University of California Medical School in San Francisco and the staff of the Lawrence Berkeley Laboratory, particularly the Biology and Medicine and Accelerator and Fusion Research Divisions. Plans are being 
developed for a new synchrotron, to be located at the Merritt-Peralta site. The beams of the Light-Ion Biomedical Research Accelerator (LIBRA) include protons, helium and carbon ions and other light ions up to and including neon. The range of the carbon ions will be at least $34 \mathrm{~cm}$ in tissue so that any part of the human body will be accessible. Some of the physical specifications for the particle beams are given in Table 1 .

LIBRA may be the first biomedical accelerator that will be designed to take full advantage of radioactive secondary beams. These are formed from the primary beam, when the particles are passed through a thin slab of matter, (usually beryllium). The secondary radioactive particles are magnetically separated from the particles in the primary beam and are focused in a separate beam. Table 2 lists the radioactive beam species that can be produced by LIBRA.

The machine will use highly automated computerized control systems so that its operation will be simple and the reliability of its operation should approach that of conventional $x$-ray or electron equipment used in medicine. The beam intensity of the carbon beams will be about $3 \times 10^{-10-11}$ particles per minute, ample for most applications.

A portion of the LBL biomedical research program will be carried out at the LIBRA facility after it is completed. The capacity of the machine will also allow a large increase in medical applications. External users with appropriate research projects will be also admitted. We envisage thas the LIBRA program will have several components. These include

Table 1. Some specificalions for LIBRA (Light-Ion Biomedical Research Accelerator) at nominal energy $500 \mathrm{Mel} /$ nucleon.

\begin{tabular}{llll}
\hline Particles & $\begin{array}{c}\text { Range } \\
\text { (cm in water) }\end{array}$ & $\begin{array}{c}\text { Intensity } \\
\text { (particles/min) }\end{array}$ \\
\hline Proton & ${ }_{1}^{1} \mathrm{H}$ & $38^{4}$ & \\
Helium & ${ }_{2}^{4} \mathrm{He}$ & $38^{4}$ & $4 \times 10^{11}$ \\
Carbon & ${ }_{6}^{12} \mathrm{C}$ & 38 & $4 \times 10^{11}$ \\
Nitrogen $\quad 7^{14} \mathrm{~N}$ & 33 & $2 \times 10^{11}$ \\
Oxygen $\quad{ }_{8}^{16} \mathrm{O}$ & 29 & $1 \times 10^{11}$ \\
Neon & ${ }_{10}^{20} \mathrm{Ne}$ & 23 & $4 \times 10^{10}$ \\
Plateau dose rate for expanded beam \\
Cross section: $400 \mathrm{~cm}^{2}$ \\
Carbon: $165 \mathrm{~Gy} / \mathrm{min}$ \\
Neon: $0.46 \mathrm{~Gy} / \mathrm{min}$ \\
=
\end{tabular}

- Longer range available.
Table 2. LIBRA radioactive beam species.

\begin{tabular}{cccc} 
& \multicolumn{2}{c}{ Radioactive secondary beams } \\
\cline { 2 - 3 } Primary beam & Main species & Mode of decay & Half-life \\
\hline${ }_{1}^{1} \mathrm{H}$ & None & & \\
${ }_{2}^{4} \mathrm{He}$ & None & & \\
${ }_{6}^{12} \mathrm{C}$ & ${ }_{6}^{11} \mathrm{C}$ & $\beta, \mathrm{EC}^{2}$ & $20.5 \mathrm{~min}$ \\
${ }_{6}^{12} \mathrm{C}$ & ${ }_{6}^{10} \mathrm{C}$ & $\beta, \gamma$ & $19 \mathrm{sec}$ \\
${ }_{7}^{14} \mathrm{~N}$ & ${ }_{7}^{13} \mathrm{~N}$ & $\beta$ & $10 \mathrm{~min}$ \\
${ }_{8}^{16} \mathrm{O}$ & ${ }_{8}^{15} \mathrm{O}$ & $\beta$ & $2.05 \mathrm{~min}$ \\
${ }_{10}^{20} \mathrm{Ne}$ & ${ }_{10}^{19} \mathrm{Ne}$ & $\beta$ & $19 \mathrm{sec}$ \\
${ }_{10}^{20} \mathrm{Ne}$ & ${ }_{9}^{18} \mathrm{~F}$ & $\beta, \mathrm{EC}$ & $109.7 \mathrm{~min}$ \\
\hline \hline
\end{tabular}

2 EC $=$ electron capture

- Therapy for tumor sites where light ions have demonstrated usefulness.

- Controlled cancer therapy research programs.

- Research on intraoperative uses of particle beams.

- Research in cancer diagnosis and localization.

- Research in the applications of localiced particle beams to abnormal brain and spinal cord.

- Research on treatment verification with radioactive beams.

- Nuclear medicine research with radioactive beams.

- Radiation biology research with light particles.

- Cell transformation and mutation.

- Carcinogenesis.

- Radiological physics and chemistry.

Design engineering groups are working on plans for LIBRA. We expect to optimize the design by going through various schemes. It appears that initially the machine may have four separate treatment rooms on two different levels. The beams will be magnetically switched from one room to another. Figure 1 is a schematic of the lower level of a proposed building that will house LIBRA including the main control room and the central radiotherapy facilities. Three exposure rooms would be located on this floor. A "room $A$ " may be designed for scanned beams where tumors of any size can be treated. A "room B" could have special facilities for radioactive beams and for precision treatment of regions in brain and spinal cord. A "room C" may be available for vertical beams and possibly for intraoperative applications of reduced-energy 


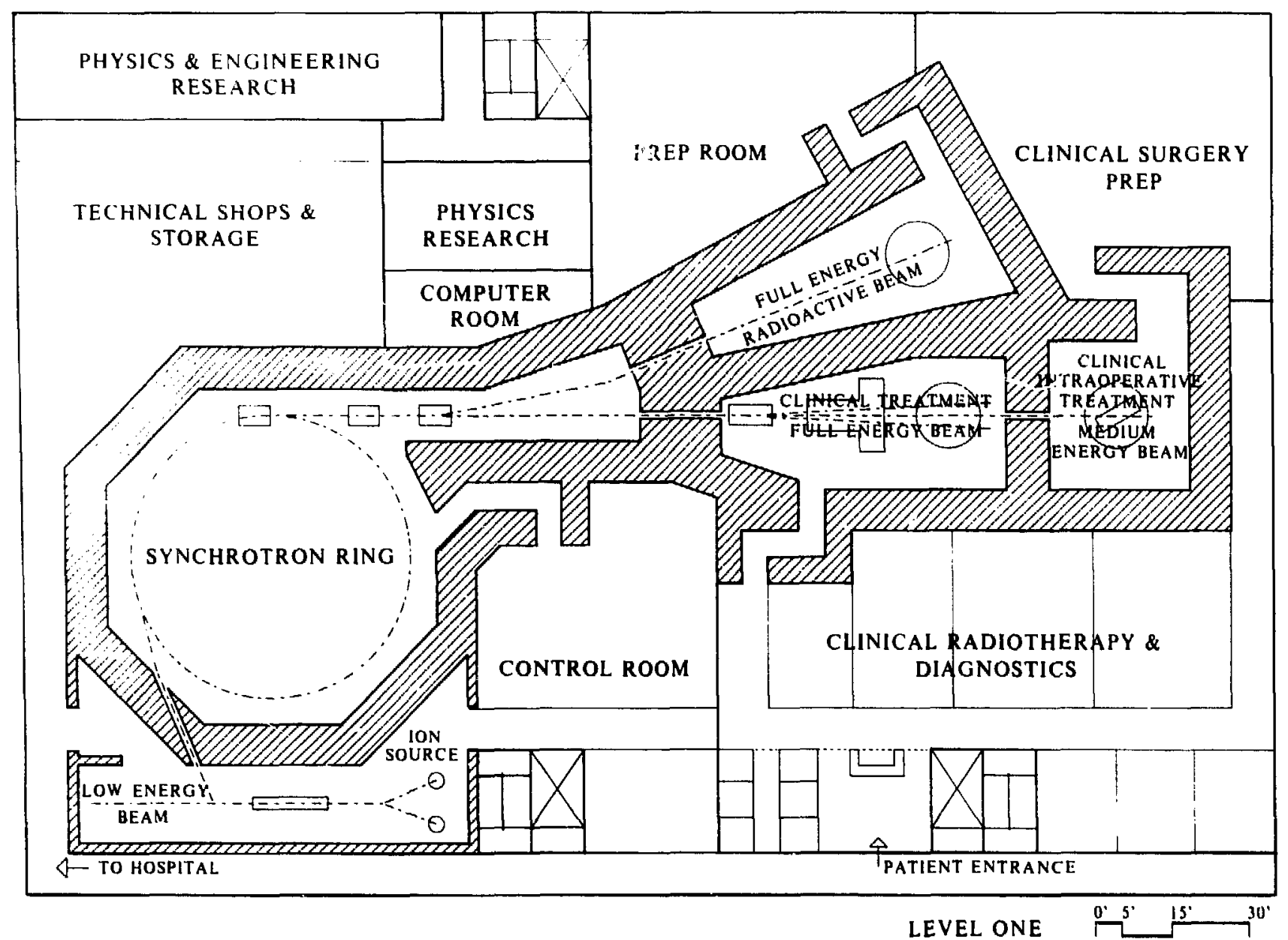

Fig. 1. Light-ion biomedical research accelerator.

$(X B L 8611-4310 A)$

beams. A second level could contain two exposure rooms. One would be specially equipped for the treatment of ocular tumors and other lesions near the surface of the body, utilizing relatively lowenergy beams. Another room may be specially reserved for biological and physical research investigations.

LIBRA will have ample capacity to channel beams into several exposure rooms. If medical applications require it, the facilities can be expanded to include a second beam line, three additional treatment rooms, and additional clinical space. Design and construction of LIBRA would' take approximately three years. The machine could be operating late in 1990, before the Bevalac is scheduled for a shutdown. While the Bevalac upgrade is in progress (approximately in 1992), the entire LBL heavy-ion biomedical research program would be carried out at LIBRA.

There are a number of biomedical acceleratorrelated research projects in various countries. The European Economic Community is also interested in building a light-ion accelerator, to be located in Nice, France. This machine, EULIMA, (European Light-Ion Medical Accelerator) is being designed at the CERN laboratories in Geneva. LIBRA represents an ambitious technology transfer program between a national laboratory and the private sector. In order to make it a success, strong support is needed from the communities in the San Francisco Bay Area, and particularly from the community of Oakland. 


\section{SECTION 4. LIPOPROTEIN AND STRUCTURAL BIOLOGY}

\section{INTRODUCTION}

The major focus of this group is to use biophysical approaches to elucidate functional aspects of biologically important macromolecules or cell structures including DNA, membrane channels, receptors, lipoproteins, and alveolar structure. In addition to purely physical techniques, emphasis is also being placed on the genetic regulation of biological structures, especially structural changes in specific lipoprotein subclasses.

\section{LIPOPROTEINS}

Lipoprotein research is multidisciplinary in its approach and uses not only in vitro model systems but also isolated cells and the whole organism as a means to study the overall metabolism of lipoproteins and their roles in hyperlipidemia. The investigators in lipoprotein metabolism have been instrumental in developing and applying new techniques that measure and define lipoprotein levels and subclasses and provide new insights into lipoprotein metabolism in health and disease. Moreover, the genetic contribution to regulation of lipoprotein subclasses is also an area that is showing promising results. The summaries in the lipoprotein section indicate the breadth of research, which ranges from structural analysis of the lipoprotein protein (apolipoprotein) to complex analysis of the inheritability of a genetic trait that appears to increase the odds of myocardial infarction by threefold.

Two apolipoproteins, apoB and apoE, have been directly linked to the process of atherogenesis. ApoB is extremely large and hydrophobic, thus leading to difficulties in its isolation and characterization. To determine the size of the apoB moiety, Frank Lindgren and his associate Talwinder Kahlon carried out very precise elemental analysis and sedimentation equilibrium studies on highly purified subfractions of human plasma LDL. The molecular weight for apoB was 579 kilodaltons, which is similar to that reported recently for apoB determined by cDNA analysis. Lindgren's independent meásurement using purely physical-chemical approaches therefoie supports the molecular weight of 555 kilodaltons deduced from amino-acid analysis. ApoE is an apolipoprotein that in normal subjects is present in small quantities; however, in certain hyperlipidemias this protein is elevated.
Investigators at the Gladstone Foundation in San Francisco have recently been able to isolate large quantities of this protein and have been studying its structural properties. In collaboration with Frank Lindgren, they have been investigating whether the iwo proteolytic fragments formed by thrombin cleavage of apoE are able to self-associate. The problem was resolved by Lindgren, who showed by sedimentation equilibrium that only the 1012 kilodalton fragment can self-associate.

Opposite to apoB and apoE, apoAl and apoAll, which are carried on high density lipoproteins $(\mathrm{HDL})$, have a protective role in cardiovascular disease. The plasma HDL are extremely heterogeneous both in size, density, and protein composition. We still do not fully understand how this complex array of particles arises or what role they play in the overall metabolism of lipoproteins. Elaine Gong and Alex Nichols and their associates have been forming in vitro model complexes of apoAl and apoAll in order to elucidate the origin and fate of specific HDL subclasses. Adult normal females have a high proportion of large, less dense $\mathrm{HDL}$, called $\mathrm{HDL}_{2 \mathrm{~b}}$, which probably are functionally significant in decreasing women's risk to coronary artery disease (CHD). In this report Elaine Gong and her colleagues demonstrate that it is possible to form $\mathrm{HDL}_{2 \mathrm{~b}}$ model precursors that contain four apoAls per particle and that these precursors are converted to plasma-like $\mathrm{HDL}_{2 b}$ by incubation with free cholesterol and the lecithin-cholesterol acyltransferase.

Yet another protective role for HDL has been described by Tom Musliner and Ron Krauss. They observed that when very low density lipoproteins (VLDL) undergoes lipolysis in the presence of LDL, a large VLDL-LDL complex is formed. Since in vivo lipolysis of VLDL takes place on the surfaces of capillary endothelium, the formation of large lipid aggregates could be deleterious. However, these investigators noted that $\mathrm{HDL}$ added to the incubation mixture prevent aggregate formation. These observations are the basis for yet another antiatherogenic role for $\mathrm{HDL}$, i.e., they may act as a sink for excess free fatty acids, thus preventing the formation of large, possibly thrombogenic lipoprotein aggregates. 
The Lipoprotein Group of the Biomedical Division is well known for its development of new methods for determining LDL and HDL heterogeneity. Using these basic technologies, Melissa Austin and Ron Krauss have linked the appearance of a specific LDL subclass with a geneic predisposition to CHD. They have recently described two LDL subclass patterns by means of gradient gel electrophoresis; one, pattern $A$, has large, less dense LDL, the other, pattern B, consists of sinall, dense LDL. To test how these patterns are related to $\mathrm{CHD}$, these investigators examined 109 patients (part of the Boston Area Health Study) under the age of 65 who survived a myocardial infarction. Analysis of gradient gel patterns indicate that the odds for having a myocardial infarction are threefold greater in subjects with pattern B. This atherogenic-prone pattern is also associated with elevated plasma triglyceride and decreased plasma HDL cholesterol. This line of research using the pattern of LDL metabolism may be genetically determined.

In order to investigate the contribution of genes versus environment and diet in lipoprotein metabolism and the onset of premature atherosclerosis, it is useful to have a stable genetic animal model in which to carry out the analysis. The work reported by Wendy Fitch and her colleagues using inbred pigs at the University of Wisconsin may play an important role in our understanding of the complex events that lead to CHD. Fitch has described mutant pigs that have abnormal intermediate density lipoproteins and LDL. The aberrant LDL are catabolized $30 \%$ more slowly than normal LDL even in control pigs, thus indicating an abnormality in LDL particles themselves. This may well be the first study to show a genetically determined lesion affecting LDL structure.

Relationships between lipoproteins and cardiovascular disease have clearly been established in these research approaches, yet we still know relatively little about the synthesis and assembly of lipoproteins at the cellular level. This important aspect of lipoprotein research is being probed in human liver and intestinal cell lines as described in the reports by Trudy Forte and her colleagues. Mark McCall, using the hepatoma-derived cell line Hep $\widetilde{G}$, has shown that the liver produces several distinct HDL subclasses. The physical properties of each $\mathrm{HDL}$ subclass are unique, which would suggest that the metabolism of each is substantially different. These isolated HDL subclasses will be valuable tools for future studies to elucidate HDL metabolism and its protective role in the atherogenic process. A new cell line (caco2) that is the intesti- nal counterpart for the Hep G2 cell is also described by Trudy Forte. The caco 2 cells appear to secrete plasma-like lipoproteins, and hence offer the possibility to study regulation of lipoproteins in intestinal cells by dietary factors.

\section{STRUCTURAL BIOLOGY}

Investigators focusing on the molecular structure of biological macromolecules use a wide armament of techniques including spectroscopic measurements, electron diffraction and high voltage electron microscopy. Application of these new approaches has provided new and significant insights in important structures such as condensed DNA, membrane proteins, and lung capillaries.

The possibility of using electron crystallographic techniques to determine molecular structures is one of the most exciting developments in structural biology. Bob Glaeser and his associates have a long-term commitment to develop and apply electron crystallography for molecular structural determination of membrane proteins. Their unique contributions in this field have led to the award of a Program Project Grant in the past year. This commitment is clearly demonstrated by reports presented by Ken Downing, Joe Taylor, and Bob Glaeser. Downing and Glaeser have shown that beam-induced movement during electron irradiation is a major limiting factor in obtaining high resolution images and they have demonstrated that reducing the area of electron irradiation using a small-spot illumination technique enhances the contrast of the high resolution information in the image. This newly developed technique has great promise for routine application in obtaining high resolution images for three-dimension structural determination at the molecular level. In conjunction with the small-spot illumination technique, techniques to obtain robust specimen support films that serve to increase the stability of specimens against beam-induced movement have also been developed by Taylor and Glaeser.

A major breakthrough in reconstituting an outer membrane protein, PhoE porin, with phospholipids forming two-dimensional crystals diffracting to high resolution has been made by Bing Jap. This opens the door for electron crystallography as a unique technique to obtain the structures of membrane proteins that are not naturally organized in periodic arrays. Bing Jap has completed the threedimensional reconstruction of PhoE porin negatively stained with uranyl acetate. The reconstruction reveals that trimeric channels of PhoE form a basic unit and that the trimeric channels converge but do not merge as they traverse the membrane. 
Jacob Bastacky and Tom Hayes together with their associates have continually made progress in the use of low-temperature scanning electron microscopy for structural studies of lung in the frozen hydrated state. With this method, alveolar and airway surfaces in the lung in the native state can be observed. The group of Bastacky and Hayes have also used high-voltage electron microscopy to study human lung alveolar wall in thick sections in order to observe spatial relationships of its surface and internal structure.

The application of spectroscopy to study the structure of biological macromolecules is a major area of interest to the Structural Biology Group. Marcos Maestre, Cynthia Phillips, and their associates employ circular dichroism (CD) spectroscopies to study the DNA-protein condensates using DNAbinding dyes as reporters. This technique is a promising way to study the dynamics of DNA condensates in biological processes.

\section{Lipoproteins}

\section{ANALYTIC ULTRACENTRIFUGATION OF LIPOPROTEINS: SOME CURRENT COLLABORATIONS}

\section{Frank $\mathrm{T}$. Lindgren}

During this past year there have been several collaborative studies involving analytic ultracentrifugation of lipoproteins. We are presenting brief reports concerning three of these projects.

\section{HETEROGENEITY OF LOW DENSITY LIPOPROTEINS (LDL) IN THE CYNOMOLGUS MONKEY}

Collaborative study with 1.). Goldberg, W.V. Brown, et al., Columbia University, New York.

Studies were performed to establish whether there was kinetic heterogeneity in the metabolism of subclasses of low density lipoproteins (LDL) of the cynomolgus monkey. Previous studies of the effects of inhibition of hepatic triglyceride lipase in this species had shown an increase in the mass of lighter LDL (Sf $>$ 9) and a decrease in the mass of denser LDL. LDL (d 1.019-1.063) were subdivided into two subfractions, $\mathrm{LDL}_{1}(1.019<\mathrm{d}<1.035)$ and $\mathrm{LDL}_{2}(1.035<\mathrm{d}<1.063)$ by ultra entrifugation.

The lipoproteins in these two fractions were shown to have different flotation rates by analytic and different densities by isopycnic ultracentrifugation. When tracer amounts of homologous 125 labeled very low density lipoproteins (VLDL) were injected into chow-fed cynomolgus monkeys, apo-B radioactivity appeared in $\mathrm{LDL}_{1}$ prior to its appearance in $\mathrm{LDL}_{2}$. $\quad{ }^{125} \mid \mathrm{LDL} \mathrm{L}_{1}$ injected into the monkey was removed from the $\mathrm{LDL}_{1}$ density subclass with a half-life of 5.5-10.3 hr. Much of the radioactivity injecteu as $L^{\prime} L_{1}$ was converted to denser $L D L$ $\left(\mathrm{LDL}_{2}\right)$. Labeled $\mathrm{LDL}_{2}$ injected into the monkey was not converted to $L D L_{1}$. Thus, at least two kinetically distinct subpopulations of LDL circulate in the plasma of this species. The lighter $L L_{1}$ is to a large extent a metabolic precursor of the more dense $\mathrm{LDL}_{2}$.

\section{DOMAIN NATURE OF APOLIPOPROTEIN E-3}

Collaborative study with L.P. Aggerback and I.R. Wetterau, Gladstone Foundation, San Francisco.

These studies revealed the domain nature of the important atherogenic apolipoprotein E-3. This arginine-rich peptide is associated mainly with intermediate density lipoproteins (IDL) $(1.0063<\mathrm{d}<$ $1.019 \mathrm{~g} / \mathrm{ml}$ ) and with the low density lipoprotein (LDL) subfractions $(1.019<d<1.063 \mathrm{~g} / \mathrm{ml})$, as well as with the larger high density lipoproteins (HDL) during phases of fat absorbtion. The IDL have been identified as the lipoproteins most significantly related to cororiary heart disease progression, as evaluated by angiography. ${ }^{1,2}$ Apolipoprotein $\mathrm{E}-3$ is a $34-\mathrm{kDa}$ peptide that associates physically and chemica!ly in solution as a tetramer as evaluated by analytic and sedimentation eqr:librium ultracentrifugation. Moreover, thrombin proteolytic fragments of 22,12 , and $10 \mathrm{kDa}$ have been characterized by sedimentation velocity, diffusion, and 
sedimentation equilibrium. The $22-\mathrm{kDa} \mathrm{N}$ terminal region of 1-191 amino acids is a monomer and does not self-associate. However, the region from 192-299 amino acids that also contains the carbohydrate moiety (containing a $10-$ or $12-\mathrm{kDa}$ fragment including the carboxy terminal) self-associates as a tetramer. The characterization of these fragments indicates unique and different physicalchemical properties. For example, the $22-\mathrm{kDa}$ fragment amino acid number 140-150 from the $\mathrm{N}$ terminal contains an LDL-related receptor, essential for cell uptake of the apoE-3- and apoB-containing lipoproteins. On the other hand, the 10- to $12-\mathrm{kDa}$ fragments contain a structure and conformation that allows association into tetramers, both for the fragments and for the intact total $35-\mathrm{kDa}$ apolipoprotein $\mathrm{E}-3$.

THE MOLECULAR WEIGHT OF aPoB-100 IN LOW DENSITY LIPOPROTEIN SUBFRACTIONS IN

NORMAL MALES

Collaborative study with Talwinder S. Kahlon, guest scientist at LBL.

Low density lipoprotein (LDL) subfractions $(d=$ $1.025-1.047 \mathrm{~g} / \mathrm{ml}$ ) were obtained in healthy normolipoproteinemic male subjects $(n=4)$. Molecular weights of five LDL subfractions were determined by sedimentation equilibrium using true $\bar{v}$ (by sixth place densities and elemental mass analyses). Protein wt\% content of LDL subfractions was determined by $\mathrm{CHN}$ mass analyses of individual subfractions with corrections for phospholipid nitrogen. Molecular weights were $2.92 \pm 0.26,2.94 \pm 0.12$, $2.68 \pm 0.09,2.68 \pm 0.28$, and $2.23 \pm 0.22$ (mean \pm SEM) million daltons for subfractions $1,2,3,4$, and 5, respectively. Protein content for the respective subfractions was $21.05 \pm 0.24,21.04 \pm 0.71$, $22.05 \pm 0.63,23.60 \pm 0.70$, and $29.10 \pm 1.19 \%$ (assuming $28.5 \%$ phospholipid). This resulted in molecular weights for total LDL peptide of $614 \pm$ $53,621 \pm 45,588 \pm 9,637 \pm 83$, and $645 \pm 62$ thousand daltons for LDL subfractions $1,2,3,4$, and 5, respectively.

LDL subfractions of the above density range contain variable amounts of apolipoprotein (apo) $E$, apoC-1II, as well as the major apoB- $100 .^{3}$ However, the major LDL component of $d=1.036 \mathrm{~g} / \mathrm{ml}$ contains the least amount of apoE and apoC-III, approximately $1 \%$ each. Several combinations of apolipoproteins in this LDL fraction are possible, for example, minute amounts of lipoproteins containing all apoC-III, all apoE (approximately 1\% each), and $98 \%$ of all apoB-100. In this case, the molecular weight of apoB-100 must equal 588 daltons. Since the molecular weights of apoE and apoC-III are 34 and $9 \mathrm{kDa}$, no lipoprotein molecule of this subfraction can contain apoB plus a single apoE. Considering the error of measurement, one apoB and one apoC-III is possible. If the former is the case, apoB-100 has a molecular weight of $588 \mathrm{kDa}$; if the latter with one small apoC-III plus one apoB-100, the molecular weight of apoB-100 would be $579 \mathrm{kDa}$. Either value is consistent with determinations and structure by recombinant DNA gene cloning. ${ }^{4}$ The amino acid sequence of 4,536 amino acids gives a molecular weight of $514 \mathrm{kDa}$, which does not include approximately $10 \%$ by weight carbohydrate. Our data confirms this value within the error of measurements of our sedimentation equilibrium and elemental $\mathrm{C}, \mathrm{H}$, and $\mathrm{N}$ analyses.

\section{REFERENCES}

1. Levy, R.I., Brensike, I.F., Epstein, S.E., Kelsey, S.F., Passamani, E.R., Richardson, J.M., Loh, I.K., Stone, N.J., Aldrich, R.F., Battaglini, J.W., Moriarty, D.J., Fisher, M.L., Friedman, L.F., Friedewald, W., Anderson, D.W., Lindgren, F.T., and Detre, K.M. Circulation 69, 325-337 (1984).

2. Krauss, R.M., Lindgren, F.T., Williams, P.T, Kelsey, S.F., Prensike, J., Detre, K.M., and Levy, R.I., submitted to Circulation, 1986.

3. Lee, D. and Alaupovic, P., Biochim. Biophys. Acta, in press, (1986).

4. Knott, T.J., Pease, R.J., Powell, L.M., Wallis, S.C., Rall, S.C. Ir., Innerarity, T.L., Blackhart, B., Taylor, W.R., Lusis, A.J., McCarthy, B.J., Mahley, R.W., Levy-Wilson, B., and Scott, I. Nature 23, 6090 (1986). 


\section{LARGE HIGH DENSITY LIPOPROTEINS: THEIR APOLIPOPROTEIN SPECIFICITY AND ORIGINS}

Elaine L. Gong, Alex V. Nichols, Trudy M. Forte, and Patricia J. Blanche

Studies elucidating the origins and functions of high density lipoproteins (HDL) continue to be of majur importance due to their role in transport of cholesterol out of cells and because of the inverse correlation of HDL levels with coronary heart disease in humans. The HDL class of lipoproteins is characterized by its unique apolipoprotein content (apolipoprotein $\mathrm{Al}[\mathrm{apoAI}$ and apolipoprotein AII [apoAII]) and its density upon ultracentrifugal isolation. Ultracentrifugal analysis of total $\mathrm{HDL}$ (density $1.063-1.20 \mathrm{~g} / \mathrm{ml}$ ) shows two major subclasses of $\mathrm{HDL}$, namely $\mathrm{HDL}_{2}$ (mean hydrated density $1.090 \mathrm{~g} / \mathrm{ml}$ ) and $\mathrm{HDL}_{3}$ (mean hydrated density $1.145 \mathrm{~g} / \mathrm{ml}$ ). Ultracentrifugal methods of analysis have also shown a significant difference in the HDL profiles between normal human males and females. The average male HDL profile shows a predominance of smaller more dense particles that form the $\mathrm{HDL}_{3}$ subclass, while the average female profile exhibits a greater content of larger less dense particles that comprise the $\mathrm{HDL}_{2}$ subclass. This particle size distribution difference coupled with the lower incidence of coronary heart disease in females has led to increased interest in the origins and furctions of $\mathrm{HDL}_{2}$.

\section{PARTICLE SIZE AND APOLIPOPROTEIN HETEROGENEITY OF HDL PARTICLES}

The particle size profiles of total HDL obtained by gradient gel electrophoresis (GGE) from plasma of normal males and females reinforce the observation that females generally have relatively more of the larger $\mathrm{HDL}_{2}$ subclass than males. Furthermore, in analysis of plasma HDL by GGE in 193 subjects, we have established the occurrence of subpopulations within both the $\mathrm{HDL}_{2}$ and $\mathrm{HDL}_{3}$ subclasses. $\mathrm{HDL}_{2}$ contains two subpopulations, $\left(\mathrm{HDL}_{2 \mathrm{~b}}\right)_{\text {gge }}$ and $\left(\mathrm{HDL}_{2 \mathrm{a}}\right)_{\text {ggee }}$ and $\mathrm{HDL}_{3}$ contains three subpopulations, $\left(\mathrm{HDL}_{3 \mathrm{a}}\right)_{\mathrm{gge}}\left(\mathrm{HDL}_{36}\right)_{\mathrm{gge}}$ and $\left(\mathrm{HDL}_{3 \mathrm{c}}\right)_{\mathrm{gge}}{ }^{\prime}$ Figure 1(a) shows a representative GGE pattern from a normal human male subject, which is enriched in $\left(\mathrm{HDL}_{3 \mathrm{a}}\right)_{\text {gge }}$ and $\left(\mathrm{HDL}_{3 \mathrm{~b}}\right)_{\mathrm{gge}}$ particles. The GGE pattern from a female subject [Fig. 1(b)] shows a greater proportion of $\mathrm{HOL}_{2}$ particles, most of which are in the $\left(\mathrm{HDL}_{2 \mathrm{~b}}\right)_{\text {gre }}$ size range.

By means of immunoaffinity chromatography $(\mathrm{ACC})^{2}$, we have shown that $\left(\mathrm{HDL}_{2 \mathrm{~b}}\right)_{\mathrm{gge}}$ are predominantly $\mathrm{HDL}$ particles that contain apoAl but no
apoAll. Thus, Fig. 2 shows the GGE patterns of (a) total HDL, (b) HDL particles containing apoAl without apoAll (Al without AlI), and (c) HDL particles containing apoAl with apoAll (Al with All), isolated by IAC. The above profiles show that the population of particles containing apoAl without apoAll consists not only of the $\left(\mathrm{HDL}_{2 \mathrm{~b}}\right)_{\mathrm{gge}}$ subpopulation, but also includes a subpopulation within the $\left(\mathrm{HDL}_{3 a}\right)_{\text {gge }}$ particle size interval. The particle size profile of the HDL population containing apoAI with apoAll indicates the presence of at least three subpopulations that fall within the size intervals, designated $\left(\mathrm{HDL}_{2 \mathrm{a}}\right)_{\text {gger }}\left(\mathrm{HDL}_{3 \mathrm{a}}\right)_{\mathrm{gge}}$ and $\left(\mathrm{HiL}_{\mathrm{i}} \mathrm{L}_{3 \mathrm{~b}}\right)_{\mathrm{gge}}$. The above findings demonstrate the presence of both particle size and apolipoprotein heterogeneity within the total HDL distribution. All of these subpopulations of HDL may have separate or combined contribution to the inverse correlation with coronary heart disease.
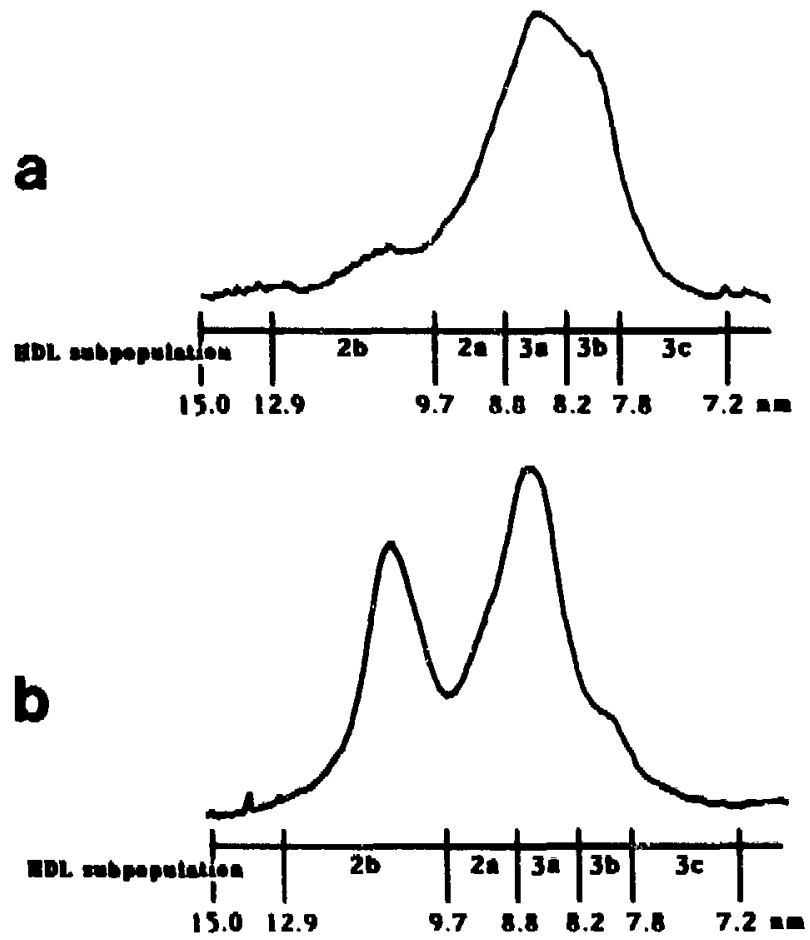

Fig. 1. Densifiometric scans showing the rof crofites of itiL from plasma of a normal (a) male and (b) female. The scale at the bottom of each profile indicates the size ranges and desig. nations of each of the HDL subpopulations. 

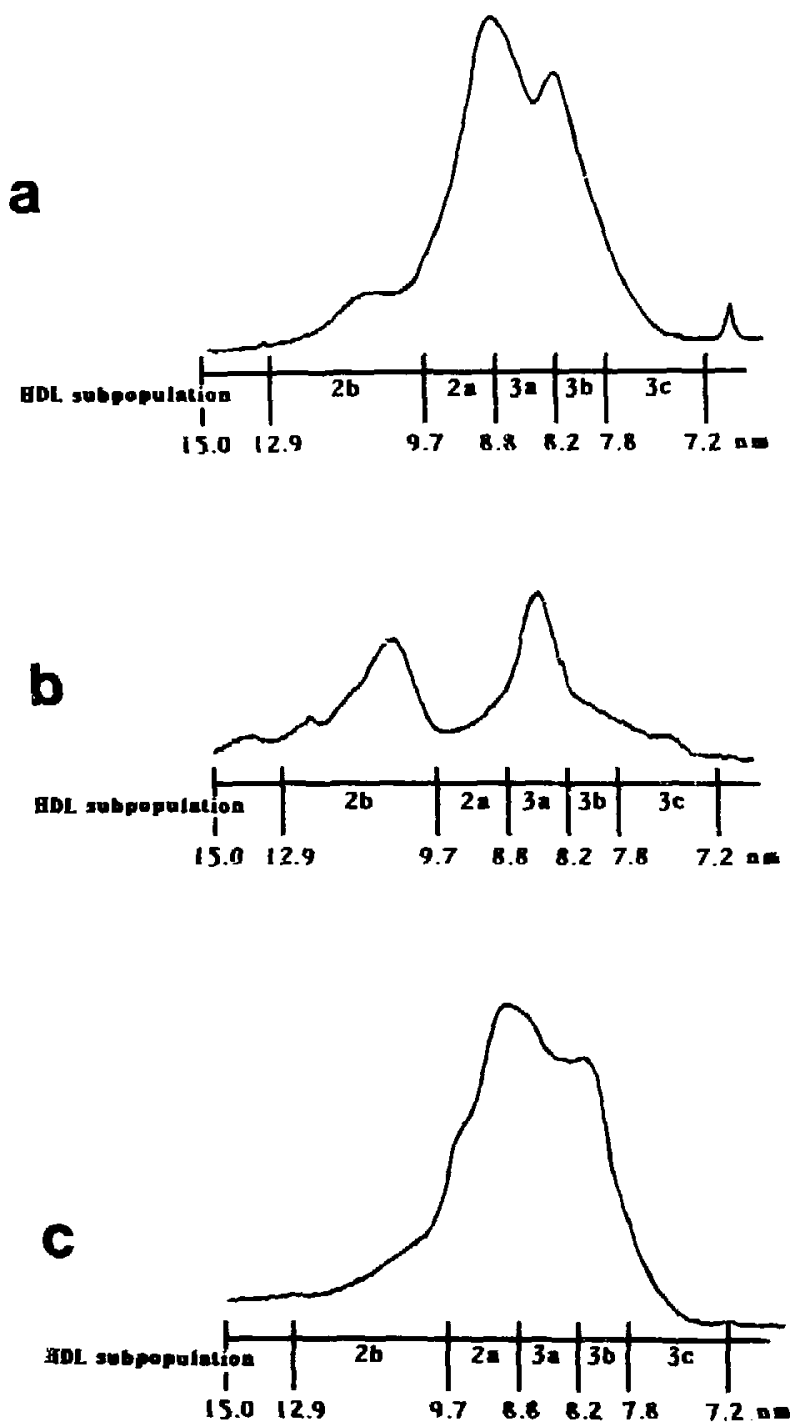

Fig. 2. GGE profiles of (a) total HOL prior to immunoaffinity chromatography; (b) (Al without $A l l$ ) fraction following $I A C$, showing peaks in the $\left(H D L_{2 b}\right)_{g e}$ and $\left(H D L_{3 a}\right)_{\text {gge }}$ regions; (c) (AI with $A I I)$ fraction, indicating its heterogeneity and the absence of a peak in the $\left(H D L_{2 b}\right)_{\text {gre }}$ region. (XBL 8611-9065)

\section{ORIGINS OF HDL SUBPOPULATIONS CONTAINING AI WITHOUT AII}

Our studies investigating the origins and functions of $\left(\mathrm{HDL}_{2 \mathrm{~b}}\right)_{\mathrm{gge}}$ are based on the general idea that plasma HDL are formed by transformation and remodeling of precursor or nascent HDL particles. Thus, HDL are not secreted as mature particles from the liver, but as nascent discoidal structures and small spherical particles that are transformed by lecithin:cholesterol acyltransferase (LCAT) and remodeled, to a greater or lesser extent, by lipid transfer proteins and lipases to spherical structures with cholesteryl ester and triglyceride cores. Potential precursors to mature HDL have been isolated from the plasma of patients with LCAT-deficiency. Upon GGE of the isolated $d 1.063-1.20 \mathrm{~g} / \mathrm{ml}$ fraction from these patients, a reproducible multicomponent pattern is observed [Fig. 3(a)]. Examination by electron inicroscopy shows large particles in the size range of 9.5 to $25 \mathrm{~nm}$ that are discoidal in shape, and small spherical apo Al-containing particles. The discoidal particles correspond to the multiple peaks in the $9.5-25 \mathrm{~nm}$ size range, while the spherical particles represent the peak in the $\left(\mathrm{HDL}_{3 b}\right)_{\mathrm{gge}}$ and $\left(\mathrm{HDL}_{3 \mathrm{c}}\right)_{\mathrm{gge}}$ region.

Precursors to the $\left(\mathrm{HDL}_{2 b}\right)_{\mathrm{gge}}$ subpopulation could include either or both of these types of nascent particles. We have demonstrated that model compilexes of apolipoprotein and lipid can be formed that are similar in size, shape and apolipoprotein composition to native $\mathrm{HDL}$ precursors ${ }^{3}$. We have investigated two possible pathways leading to the formation of $\left(\mathrm{HDL}_{2 \mathrm{~b}}\right)_{\mathrm{gge}}$ using complexes containing exclusively apoAl as the protein component. The first pathway involved transformation of small model HDL particles. Their incubation with LCAT and a source of unesterified cholesterol (low density lipoproteins [LDL] or very low density lipoproteins), is shown in Fig. 3. The initial small model complexes [Fig. 3(b)], whose major peak is in the $\left(\mathrm{HDL}_{3 \mathrm{c}}\right)_{\mathrm{gge}}$ size range, are transformed into spherical particles in the $\left(\mathrm{HDL}_{3 a}\right)_{\mathrm{gge}}$ size range which contain a cholesteryl ester core [Fig. 3(d)]. Attempts to further transform this product into the size range of the $\left(\mathrm{HDL}_{2 \mathrm{~b}}\right)_{\mathrm{gge}}$ by addition of unesterified cholesterol and incubation with LCAT did not yield a larger product. This observation indicates that the transformation of the small spherical precursors may reach an endpoint in this system with a product in the $\left(\mathrm{HDL}_{3 a}\right)_{g g e}$ size range. This final product has a migration position that is nearly identical to that of the major peak of the smaller of the two subpopulations found in the (Al without AlI) fraction isolated by IAC [Fig. 3(e)]. We found the initial model complexes to be comprised of two apoAi molecules per particle, based on using dimethylsuberimidate cross-linking. The final product following incubation with LCAT and a source of unesterified cholesterol contains three apoAl molecules per particle. This transformation is identical to that which occurs when the small spherical particles from plasma of LCAT-deficient patients are incubated with LCAT in the presence of additional unesterified cholesterol. ${ }^{4}$ The transformation of the model complexes appears to result from fusion of two of the small precursor particles to form a larger 

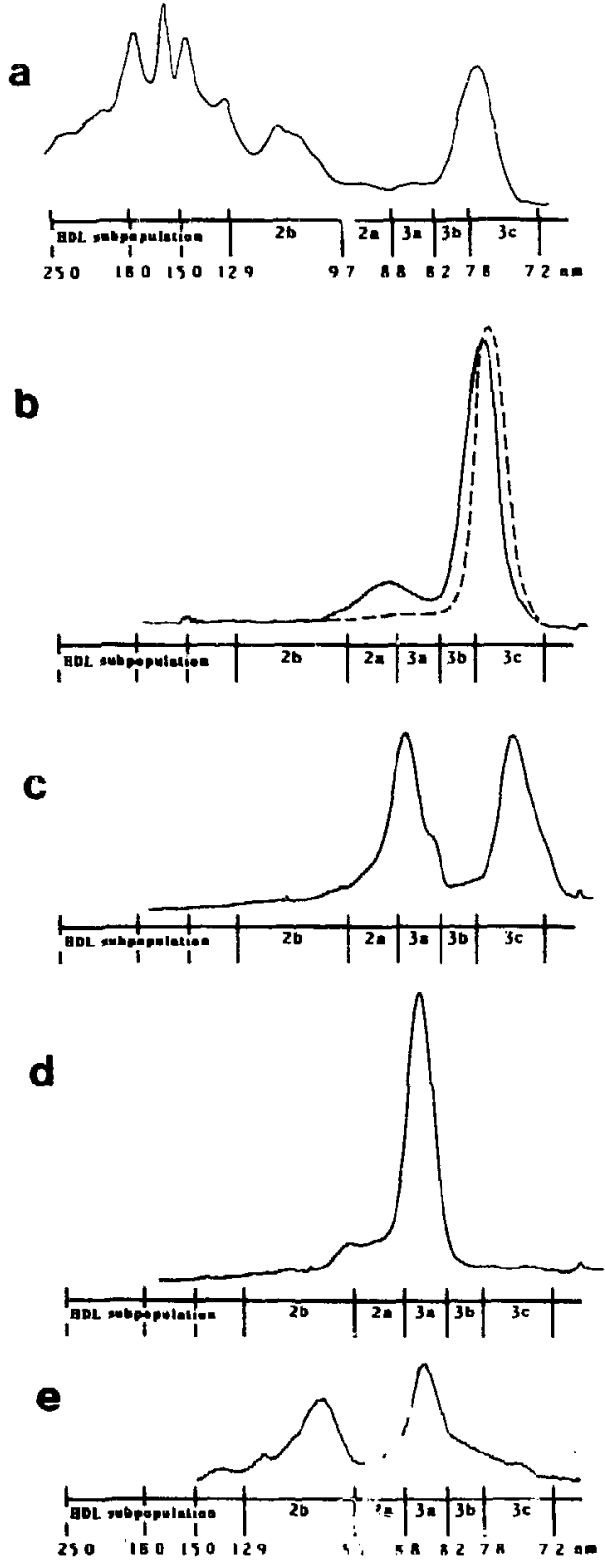

Fig. 3. Effect of $37^{\circ} \mathrm{C}$ incubation of small spherical particles with a source of LCAT and LDL: GGE profile of (a) isolated HOL fraction from LCAT-deficient patient (not incubated); (b) small spherical particles before (_) and after (-.-) gel filtration chromatography; (c) 6-hour incubation product; (d) 24-hour incubation product, (e) (Al' without All) fraction $1 \cdot \mathrm{m}$ IAC.

(XBL 8611-9 -)

product with the resulting loss of one apoAl molecule per particle.

Based on the above evidence, we considered a second pathway in which the formation of the larger $\left(\mathrm{HDL}_{2 \mathrm{~b}}\right)_{\mathrm{gge}}$ subpopulation may not involve fusion of smaller particles, but may result from direct core build-up in large discoidal particles such as those seen in the plasma of LCAT-deficient patients [Fig. 4(a)]. We have established methods for preparation and isolation of large model discoidal complexes, in the size range of discoidal particles detected in LCAT-deficiency. The large discoidal complexes contain four apoAl molecules per particle and are in the size range of 15-18 nm [Fig. 4(b)]. When inculoated with LCAT and a source of unesterified cholesterol (LDL), they are transformed to large spherical particles with size falling within the size range of the $\left(\mathrm{HDL}_{2 b}\right)_{\text {gge }}$ interval. The resulting product [Fig. 4(c)] contains four molecules of apoAl per particle and has a cholesteryl ester core. The size of the transformation product is somewhat larger than that of the $\left(\mathrm{HDL}_{2 \mathrm{~b}}\right)_{\text {gge }}$ subpopulation observed in the particle size distribution of the (A! without All) population in normal plasma HDL [Fig. +id)].

The progression of change in size and shape of the large precursor particles throughout the transformation process was followed by electron microscopy. The precursor discoidal complex particles (Fig. 5, $0 \mathrm{hr}$ ) show a mean. long dimension of $18.3 \mathrm{~nm}$. Incubation with LCAT and LDL for 2.5 and 6 hours resulted in rectangular particles that picked together in linear arrays. The 24-hour product (Fig. 5, $24 \mathrm{hr}$ ) appears spherical with a particle size $(11.7 \mathrm{~nm})$ that agrees with the size of the major peak as measured by GGE. The rectangular particles seen at the intermediate times are

Fig. 4. Effects of $37^{\circ} \mathrm{C}$ incubation of the large discoidal complex preparation with LCAT an LDL: GGE profiles of (a) isolated $H D L$ fraction from LCAT-deficient patient; (b) large discoidal complex preparation; (c) 24-hour incubation product; (d) (AI without AlI) fraction from IAC. 


\section{Time Sequence of Transformation of}

\section{Class 6 Complexes}

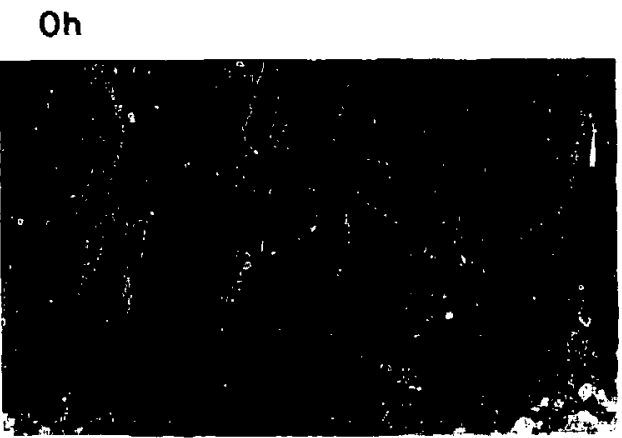

6h

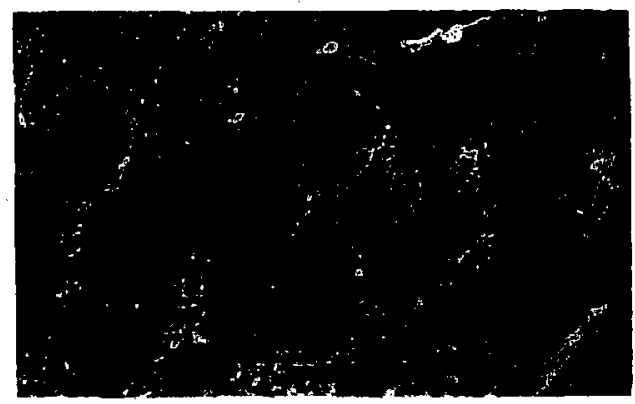

$2.5 h$

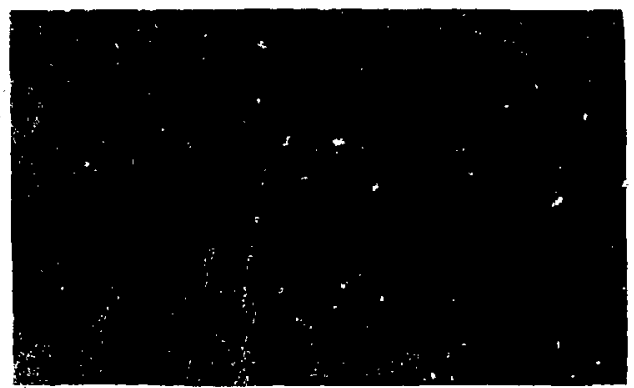

24h

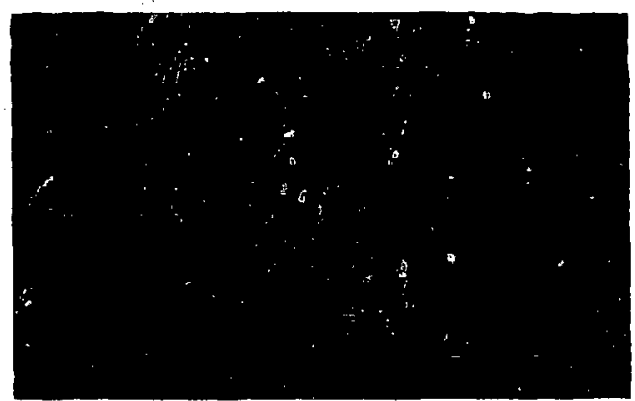

Fig. 5. Time course of transformation of large discoidal complex (see Fig. 4) followed by electron microscopy. Bar marker in 24-hour micrograph represents $100 \mathrm{~nm}$.

(XBB 869-7639)

phospholipid-rich and similar in size and shape to particles seen in lymph and interstitial fluid. ${ }^{5}$ In the body, such particles have been considered to result from enrichment of lymph HDL with phospholipid from lipoprotein or cellular suurces. However, our studies indicate that these angular products most likely arise during core build-up of large phospholipid-rich discoidal particles secreted into the lymph, and that the imbalance between surface and core content of the particles is normalized during progression of core build-up cholesteryl esters. The angularity of the intermediate products appears to arise during negative staining for electron microscopy.

Our studies thus indicate possible origins of large deformable HDL in lymph and interstitial fluid, and clearly indicate that large HDL can arise directly from discoidal precursors rather than from progressive build-up of small precursor species. Since the properties of the products observed in the present studies are somewhat different from those of native $\left(\mathrm{HDL}_{2 b}\right)_{8 g e}$, we plan to investigate the remodeling of these products to the native
$\left(\mathrm{HDL}_{2 b}\right)_{8 g e}$ by use of physiological processes involving plasma lipid-transfer proteins and lipases. The possibility that other precursor species and apolipoproteins may be involved in the genesis of the native $\left(\mathrm{HDL}_{2 b}\right)_{\text {gee }}$ is currently under investigation.

\section{REFERENCES}

1. Nichols, A.V., Blanche, P.J., and Gong, E.L. In CRC Handbook of Electrophoresis. Vol. III. L. Lewis and J.Opplt (Eds.). CRC Press, Boca Raton, FL pp. 29-47.

2. Cheung, M., and Albers, 1. I. Biol. Chem. 259, 12201-12209 (1984).

3. Nichols, A.V., Gong, E.L., Blanche, P.J., and Forte, T.M. Biochim. Biophys. Acta 750, 353-364 (1983).

4. Glomset, J.A., Mitchell, C.D., King, W.C., Applegate, K.R., Forte, T.M., Norum, K.R., and Gjone, E. Ann. N.Y. Acad. Sci. 348, 224-243 (1980).

5. Reichl, D., Forte, T.M., Hong, I-L., Rudra, D.N., and Pflug, J. J. Lipid Research 26, 1399-1411 (1985). 


\section{LIPOPROTEIN COMPLEX FORMATION}

Thomas A. Musliner and Ronald $\mathbf{M}$. Krauss

Transfers of lipids and proteins between different lipoproteins are known to occur in the course of their metabolism. It is likely that these transfers take place during transient physical associations between lipoprotein particles, but the nature and chemical basis for such interactions are poorly understood. The fact that lipid and apolipoprotein movements are particularly prevalent during the intravascular lipolysis of triglyceride-rich lipoproteins suggested to us that lipolysis products accumulating on these particles might promote physical binding with other lipoproteins. To test this hypothesis, we recently studied interactions between very lowdensity (VLDL), low-density (LDL), and high-density (HDL) lipoproteins in the setting of partial lipolysis by bovine milk lipoprotein lipase in the presence of limited unesterified fatty acid acceptor.

LDL that had been radiolabeled with ${ }^{125}$ | were incubated with VLDL or hypertriglyceridemic plasma in the presence of bovine milk lipoprotein lipase. The size distribution of the ${ }^{125}$ /-LDL was then analyzed by electrophoresis on $2-16 \%$ polyacrylamide gradient gels, followed by electrophoretic blotting onto nitrocellulose paper and visualization of labeled lipoproteins by autoradiography. With partial VLDL degradation, association of ${ }^{125}$ LDL with VLDL remnants or larger aggregates of VLDL-density was readily demonstrated (Fig.1). The observed high-molecular-weight complexes were stable under conditions of high ionic strength and could be separated from residual LDL by density gradient ultracentrifugation, as well as by molecular sieve chromatography. These findings suggested that lipid products generated by lipolysis might be responsible for VLDL-LDL complex formation. To investigate this possibility, lipids were extracted from unlipolyzed VLDL and from VLDL that had been preincubated with lipoprotein lipase. When the extracted lipids were subsequently incubated with VLDL and LDL in the absence of lipase, marked stimulation of complex formation /measured as ${ }^{125} \mathrm{I}$-apolipoprotein (apo) $B$ recovered in the $d<$ $1.012 \mathrm{~g} / \mathrm{ml}$ fraction was observed with lipids from lipolyzed compared with unlipolyzed VLDL (Table 1). Lipid fractions containing free fatty acids were most active, and complex formation could also be reproduced by incubation with purified palmitic acid. Complex formation was inhibited by addition of increasing amounts of albumin as free fatty acid acceptor, but could be detected at molar ratios of free fatty acids to albumin that occur in vivo.

When increasing amounts of $\mathrm{HDL}$ were included in incubations of VLDL, LDL, and lipase, progressive inhibition of high-molecular-weight complex formation was observed. At a ratio of 2:1 HDL:VLDL protein or greater, VLDL-LDL binding was no longer seen. When HDL radiolabeled with 125I was used, however, association of HDL-derived apoproteins with both VLDL and LDL was observed by gradient gel electrophoretic analysis followed by autoradiography (Fig. 2). SDS-gradient gel electrophoresis showed that the transferred apoprotein consisted primarily of apoAl, which under appropriate conditions comprised up to $15 \%$ of VLDL and $12 \%$ of LDL total protein. Total LDL mass was increased, along with an increase in LDL particle diameter of 15-20 A, as measured by gradient gel electrophoresis (Fig. 2). The HDL also increased in particle diameter by up to $10 \AA$, and on chemical composition analysis was found to be considerably enriched in triglyceride. Transfer of apoAl from HDL to VLDL or LDL under these conditions, like VLDL-LDL complex formation in the absence of HDL, could be reproduced in the absence of lipase upon incubation with extracted lipolysis products or purified free fatty acids, and was inhibited by increasing amounts of fatty-acid-free albumin.

These findings have led us to the following working hypothesis. In the course of lipolysis at the capillary endothelial surface, lipolytic products accumulate on VLDL particles. The distribution of these products within the microenvironment in which lipolysis takes place is competitive between the lipoprotein surface, possible binding sites on the endothelial surface, albumin, and other lipoproteins in proximity. Lipolysis products on lipoproteins mediate complex formation with nearby apoB-containing lipoproteins, as well as binding of apoAl, possibly as the free apoprotein or possibly as an apoAl-enriched subspecies of HDL. Physical complex formation between the VLDL remnant and other lipoprotein particles, in turn, fosters the transfer of lipid and protein components. Lipid transfer reactions, which may include the transfer of fatty acids and other lipolysis products, influence the binding of apoAl (and probably other non-B apoproteins) to the lipoproteins involved. These 


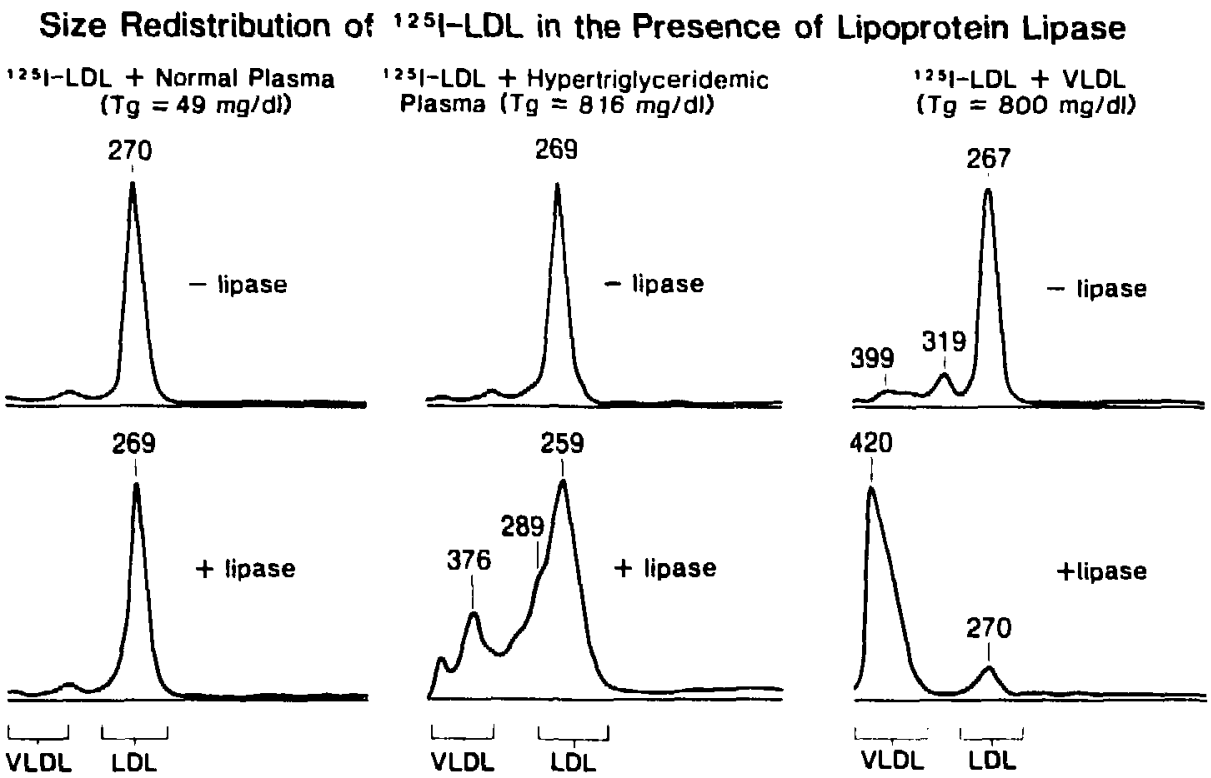

Fig. 1. Densitometric scans of autoradiograms of nitrocellulose blots of 2-16\% polyacrylamide gradient gels. The samples consisted of mixtures of ${ }^{125} /-L D L\left(2 \mu g\right.$ protein) incubated for $1 \mathrm{hr}$ at $37^{\circ} \mathrm{C}$ in a final volume of $100 \mathrm{\mu l}$ in the presence or absence of lipoprotein lipase (B units) with: (A) normal plasma (triglyceride $49 \mathrm{mg} / \mathrm{dl}$ ), (B) hypertriglyceridemic plasma (triglyceride $816 \mathrm{mg} / \mathrm{dl}$ ), and (C) hypertriglyceridemic VLDL (800 $\mu \mathrm{g}$ triglyceride). Particle diameters are shown in angstroms.

(XBL 864-8710)

\section{Redistribution of 125 -HDL with Lipolysis}

$2-16 \%$ gradient gels

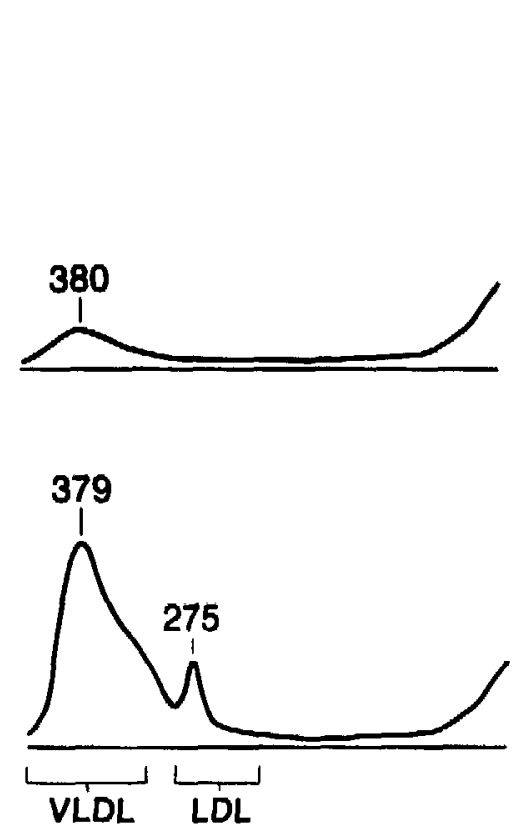

\section{$4-30 \%$ gradient gels}
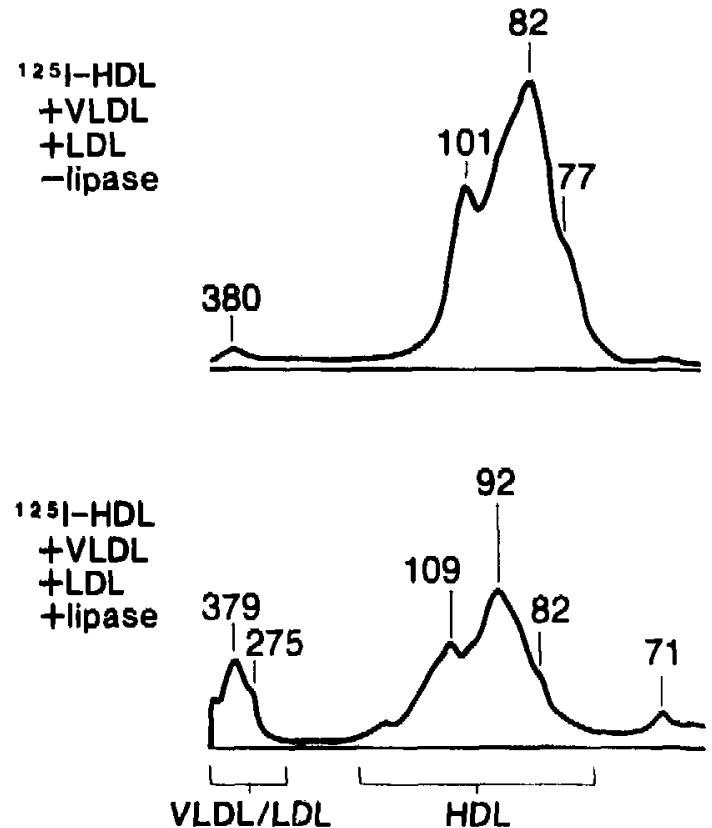

Fis. 2. Redistribution of ${ }^{125} / . H D L$ with lipolysis. Densitometric scans of autoradiograms of nitrocellulose blots of 2-16\% and 4-30\% polyacrylamide gradient gels of incubation mixtures containing $125 /-H D L$ (15 $\mu \mathrm{g}$ prolein), and normal LDL (4 48 protein), in the absence ( $A$ and $C$ ) or presence ( $B$ and $D$ ) of bovine milk lipoprotein lipase. Incubations were for $2 \mathrm{hr}$ at $37^{\circ} \mathrm{C}$. Particle diameters are shown in angstroms.

(XBL 864-8711A) 
Table 1: Distribution of $125 /-L D L$ following incubation with $V L O l$ and extracted lipids from native and lipolyzed VLOL.

\begin{tabular}{lcc}
\hline & \multicolumn{2}{c}{$\begin{array}{c}\text { \% total }{ }^{125} \text {-apoB recovered in density } \\
\text { fractions }\end{array}$} \\
\cline { 2 - 3 } Lipid extract & $\mathrm{d}<1.012 \mathrm{~g} / \mathrm{ml}$ & $\mathrm{d}=1.020-1.050 \mathrm{~g} / \mathrm{ml}$ \\
\hline None & 6.8 & 92.7 \\
Unlipolyzed VLDL (nonacidic) & 5.0 & 94.1 \\
Unlipolyzed VLDL (acidic) & 8.5 & 90.4 \\
Lipolyzed VLDL (nonacidic) & 12.9 & 86.4 \\
Lipolyzed VLDL (acidic) & 79.7 & 18.1 \\
\hline \hline
\end{tabular}

apoproteins, in turn, may themselves influence other metabolic processes to which these particles are subject. Complex formation between apoBcontaining lipoproteins may be disrupted by reequilibration of lipolysis products with other bind- ing sites (e.g., albumin, other lipoproteins), or by interactions with HDL or apoAl. Consequently, the existence of these complexes in vivo may be a short-lived phenomenon possibly localized primarily to the capillary endothelial surface.

\section{LOW DENSITY LIPOPROTEIN SUBCLASSES AND RISK OF MYOCARDIAL INFARCTION}

\section{Melissa A. Austin and Ronald M. Krauss}

Low density lipoprotein (LDL) cholesterol is a major coronary heart disease (CHD) risk factor, as established by epidemiologic studies, clinical trials, and experimental studies. In contrast, high derisity lipoprotein (HDL) cholesterol appears to protect against the development of CHD. It has also been proposed that plasma levels of the apoproteins associated with LDL and HDL particles may actually be stronger risk factors than lipid levels. Thus, understanding the mechanisms underlying variations in both lipoprotein and apoprotein levels among individuals is essential to elucidating the etiology of coronary heart disease in the general population.

Heterogeneity within the major lipoprotein classes, including LDL and $\mathrm{HDL}$, has been recognized for many years. Four distinct LDL subclasses have been defined and measured in our laboratory using the techniques of density gradient ultracentrifugation and gradient gel electrophoresis (GGE). We have hypothesized that the heterogeneity within the LDL range of particles could reflect genetic influences on lipoprotein metabolism, and preliminary studies of healthy families support this hypothesis. Using GGE results, we have identified two LDL subclass patterns, $A$ and $B$. Pattern $A$ is characterized by a predominance of large buoyant LDL and a minor amount of small dense LDL. In contrast, pattern B has a predominance of small dense LDL and less of the large buoyant LDL subclasses. LDL subclass pattern B occurred in approximately $17 \%$ of the unrelated family study participants. Among all the subjects, those with pattern B consistently had increased levels of triglyceride, intermediate density lipoprotein (IDL), and apoprotein B, and decreased levels of HDL cholesterol and apoprotein A-I. This profile strongly suggests increased CHD risk for subjects with LDL subclass pattern B.

Recently, we performed LDL subclass analyses as part of the Boston Area Health Study to assess whether the LDL subclass patterns are risk factors for myocardial infarction. The Boston Area Health Study was a case-control study in which myocardial infarction survivors, under age 65, from hospitals in the Boston area were identified and studied. Controls were selected from the neighborhoods where the cases lived, and were matched for age and sex. Plasma samples for 230 of the subjects, including 109 cases and 121 controls, were shipped to our laboratory for lipoprotein analyses. GGE was per- 
formed on each sample, and the LDL subclass pattern was determined without knowledge of the case/control status of the subject. This data set has then provided an opportunity to investigate any direct relationship between the LDL subclass patterns and coronary heart disease in a large population-based study.

The relationship between LDL subclass pattern and case/control status is shown in Table 1, stratified by sex. For males, $54 \%$ of the cases had $L D L$ subclass pattern B and $31 \%$ of the controls had pattern $B$, for an odds ratio of 2.70 . That is, the odds of being a myocardial infarction case were 2.7 times higher for a subject with pattern B compared to a subject with pattern $A$. This result was highly statistically significant (chi-square $=10.97 ; p=$ 0.0009 ), as shown by the $95 \%$ confidence interval of 1.48 to 4.82 . For females, the odds ratio was 9.17. However, the association was only marginally significant (Fisher's exact test $p=0.06$ ) due to the small sample size. Thus, based on the subjects in this study, LDL subclass pattern B appears to substantially increase CHD risk.

The associations found in the studies of healthy families between LDL subclass patterns and lipid, apoprotein, and lipoprotein levels were examined in this data set among male controls. The average age was 52 years, and females were not considered because of the small sample size. The results are shown in Table 2. Triglyceride and IDL levels were both significantly higher among the subjects with LDL subclass pattern B compared to those with pattern A. $\mathrm{HDL}$ cholesterol and $\mathrm{HDL}_{2}$ cholesterol were both significantly lower in pattern B subjects. Although the differences in apoprotein $A-I$ and apoprotein B levels were not significant in the two groups, the mean apoprotein A-I level was lower and the mean apoprotein $B$ level was higher in the

Table 1. Frequency distribution of $L D L$ subclass patterns by case/control stalus.

\begin{tabular}{|c|c|c|c|c|c|c|}
\hline \multirow{2}{*}{$\begin{array}{c}\text { LDL } \\
\text { subclass } \\
\text { pattern }\end{array}$} & \multicolumn{2}{|c|}{ Males } & \multicolumn{2}{|c|}{ Females } & \multicolumn{2}{|c|}{ All subjects } \\
\hline & Cases (\%) & Controls $(\%)$ & Cases (\%) & Controls $(\%)$ & Cases (\%) & Controls (\%) \\
\hline B & $50(54)$ & $30(31)$ & $5(29)$ & $1(4)$ & $55(50)$ & $31(26)$ \\
\hline A & $42(46)$ & $68(69)$ & $12(71)$ & $22(96)$ & $54(50)$ & $90(74)$ \\
\hline Total & $92(100)$ & $98(100)$ & $17(100)$ & $23(100)$ & $109(100)$ & $121(100)$ \\
\hline Odds ratio & \multicolumn{2}{|c|}{2.70} & \multicolumn{2}{|c|}{9.17} & \multicolumn{2}{|c|}{2.96} \\
\hline $\begin{array}{l}95 \% \text { confidence } \\
\text { interval }\end{array}$ & \multicolumn{2}{|l|}{$(1.48,4.82)$} & \multicolumn{2}{|l|}{$(0.95,45.62)$} & \multicolumn{2}{|l|}{$(1.69,5.08)$} \\
\hline
\end{tabular}

Table 2. Comparison of plasma lipid levels, plasma apoprotein levels, and lipoprotein measurements by $L D L$ subclass pattern among male controls.

\begin{tabular}{|c|c|c|c|c|}
\hline & \multicolumn{2}{|c|}{ Pattern A (n=6B) } & \multicolumn{2}{|c|}{ Pattem B $(n=30)$} \\
\hline & Mean & SD & Mean & SD \\
\hline Total cholesterol $(\mathrm{mg} / \mathrm{dl})$ & 210.1 & 40.0 & 220.5 & 39.5 \\
\hline Triglycerides* (mg/dl) & 121.4 & 68.1 & 232.2 & 81.0 \\
\hline $\mathrm{HDL}$ cholesterol $\left(\mathrm{m}_{\mathrm{g}} / \mathrm{dl}\right)$ & 42.2 & 11.0 & 34.3 & 7.1 \\
\hline Apoprolein A-I (mg/dl) & 97.8 & 94.2 & 94.2 & 27.9 \\
\hline Apoprotein B (mg/dl) & 93.3 & 22.3 & 100.8 & 22.1 \\
\hline VLDL Sf $200 \cdot 400^{\circ}$ (mg/dl) & 84.7 & 62.1 & 193.4 & 80.9 \\
\hline IDL Sf $12-20^{b}(\mathrm{mg} / \mathrm{dl})$ & 35.9 & 13.2 & 45.4 & 19.7 \\
\hline LDL Sf $0.7^{\prime}(\mathrm{mg} / \mathrm{dl})$ (small) & 193.4 & 70.3 & 248.7 & 60.7 \\
\hline Sf $7-12^{\star}(\mathrm{mg} / \mathrm{dl})$ (large) & 147.3 & 52.9 & 79.4 & 21.3 \\
\hline Peak SP & 6.2 & 0.7 & 4.5 & 0.6 \\
\hline $\mathrm{HDL}_{3}$ cholesterol $\left(\mathrm{mg}_{\mathrm{g}} / \mathrm{dl}\right)$ & 25.4 & 7.2 & 23.4 & 6.8 \\
\hline $\mathrm{HDL}_{2}$ cholesterol (mg/dl) & 16.5 & 8.8 & 10.9 & 5.3 \\
\hline
\end{tabular}

- $p<0.01$ for differences in means between patterns based on t-test.

b $p<0.05$ 
pattern B subjects. Thus, the high-risk lipid and lipoprotein profile of subjects with LDL subclass pattern B was confirmed in this study.

These initial results from the Boston Area Health Study indicate that LDL subclass pattern B, characterized by a predominance of small dense LDL particles, is associated with increased risk of myocardial infarction. The mechanism for this association could be through the associated changes in lipid and lipoprotein profiles, which in turn could be the result of pleiotropic effecis of an underlying gene. It is also possible that environmental factors, such as diet and exercise, could influence LDL subclass patterns and lead to potential intervention strategies. Understanding the underlying metabolic, genetic, and environmental influences on LDL subclasses and their relationship to coronary heart disease will be a continuing focus of our research efforts.

\section{A NEW ANIMAL MODEL FOR GENETIC HYPERCHOLESTEROLEMIA}

\section{Wendy L. Fitch, Ronald M. Krauss, William J. Checovich," and Alan D. Attie*}

We have investigated a new animal model of a genetic defect affecting intermediate density lipoproteins (IDL) and low density lipoproteins (LDL) that leads to hypercholesterolemia, premature atherosclerosis, and death in a strain of inbred pigs developed at the University of Wisconsin. We have characterized the LDL particles in controls and mutants according to flotation rate, particle size, and distribution of mass across the density range of IDL and LDL.

Pigs with a specific mutation involving apolipoprotein $B^{\prime}$ have very high plasma cholesterol and die of atherosclerosis by 3 years of age, even though normal lifespan of the pig is 15 years. We obtained plasma from five such mutants and from ten control pigs, isolated the IDL and LDL, and performed characterization studies.

LDL, IDL, and very low density lipoprotein (VLDL) were analyzed by analytic ultracentrifugation to measure characteristic flotation rates $\left(S^{8}\right)^{2}{ }^{2}$ From the resulting schlieren patterns, we calculated lipoprotein mass in the ranges of $S P$ 0-12, 12-20, and 20-400, corresponding to $L D L, I D L$, and VLDL, respectively. We isolated nine or ten LDL subfractions by subjecting the whole LDL to discontinuous density gradient ultracentrifugation. ${ }^{3}$ Particle size of the major particle in each subfraction was determined by polyacrylamide 'gradient gel electrophoresis, and protein concentration in each fraction was measured.

There was a striking difference in mass and distribution of lipoproteins of $S_{i}^{p} 0-20$ (corresponding

University of Wisconsin, Madison to IDL and LDL) between mutant and control pigs as demonstrated by analytic ultracentrifugation (Fig. 1). Dramatic differences in lipoprotein mass are shown in Table 1 . It is clear that the mutant pigs have considerably more $\mathrm{LDL}$ and IDL mass present and that the peak amount of this mass occurs in a more buoyant density range than is seen in control pigs. The mutants have a predominance of a different LDL subspecies than do controls.

Gradient gel electrophoresis revealed multiple bands within the LDL for both mutants and controls. Representative gel scans are shown in Fig. 2.

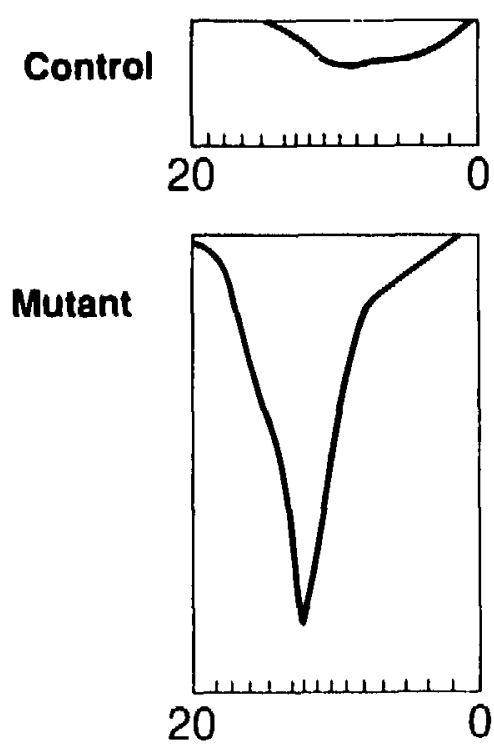

Fig. 1. Schlieren patterns of $L D L$ and $I D L\left(S_{f}^{0} 0-20\right)$ from (a) a control pig and (b) a mutant pig.

(XBL. $8611-9021)$ 

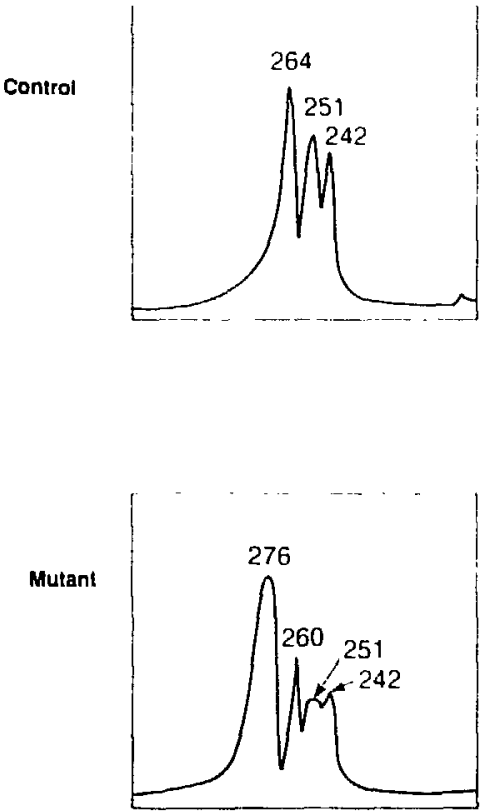

Fig. 2. Gradient gel scans of whole LDL from (a) a control pig, $8 \mu \mathrm{l}$, and (b) a mutant pig, $2 \mu \mathrm{l}$. Particle diameters are shown above the major peaks.

(XBL 8611-6503)

Discrete peaks can be seen in the region from 240-275 A, with maxima for the affected animals occurring at $275,260,251$, and $242 \mathrm{~A}$, shown in Fig. 2(a), designated LDL-I, LDL-II, LDL-IIIA, and LDL-IIIB, respectively. Mutants have a marked preponderance of L.DL-1, while unaffected animals [Fig. 2(b)] showed predominance of LDL-II and LDL-III with barely detectable amounts of LDL-I.

The distribution of LDL mass was further quantified by the concentration of protein in the LDL subfractions and is shown in Fig. 3. The mutant pigs have much greater amounts of total LDL protein, with the major increase occurring in the more buoyant LDL range, including overlap with the density range of IDL-2. The abundance of these buoyant lipoprotein subspecies may indicate overproduction of IDL and LDL-I and/or a partial blockage in further metabolism of these species.

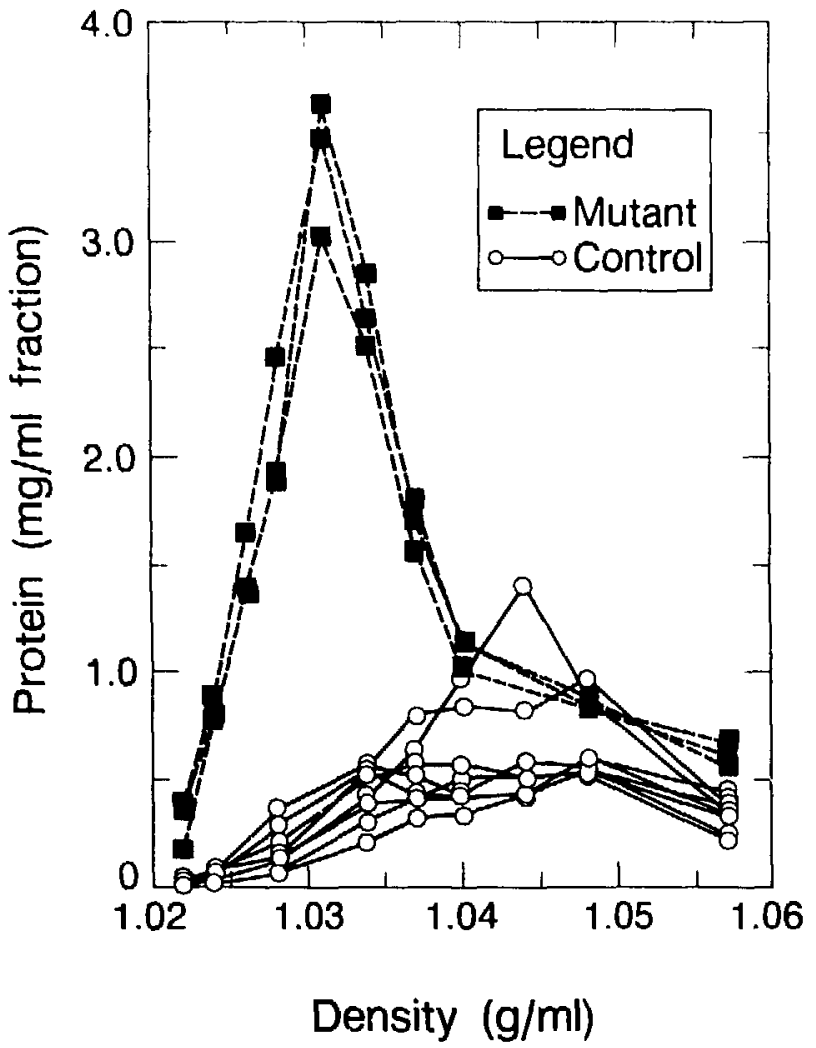

Fig. 3. Protein content of pig LDL subfractions.

(XBL 8611-6502)

LDL receptors in the affected animals appear to be normal. ${ }^{4}$ Turnover studies with normal and mutant LDL revealed that affected animals have a fractional catabolic rate that is one-half that of normal pigs, whether they are tested with mutant or normal LDL. The pool size in the mutants, however, is twice that of the normal animals. Thus, they catabolize LDL at a normal rate, but the fractional catabolic rate is decreased. In addition, it was found that normal pigs catabolize mutant LDL $30 \%$ slower than normal LDL, confirming tilat the abnormality lies in the lipoprotein particles and not in the recef tors. ${ }^{4}$

Table 1. Peak flotation rates and mass in $S_{f}^{0} 0-400$ in control and mutant pigs.

\begin{tabular}{lcccc}
\hline & & \multicolumn{3}{c}{ Mass $(\mathrm{mg} / \mathrm{d}$ ) } \\
\cline { 3 - 5 } & Peak $s_{f}^{0}$ & $S_{f}^{0} 0-12$ & $s_{f}^{0} 12-20$ & $s_{f}^{0} 20-400$ \\
\hline Controls & $5.64 \pm 2.07^{2}$ & $115 \pm 24$ & $0.8 \pm 1.2$ & $0.8 \pm 1.2$ \\
Mutants & $7.43 \pm 0.51$ & $400 \pm 55$ & $62 \pm 23$ & $17 \pm 21$ \\
\hline \hline
\end{tabular}

a Mean \pm S.D. 
The data suggest that IDL and the largest, most buoyant LDL particles are either overproduced or altered in the mutants such that they are not metabolized normally, leading to their accumulation in the circulation. Other evidence from our laboratory has implicated these lipoprotein species in the development of coronary disease in humans. These findings are the first to indicate that a genetically determined lesion affecting IDL and LDL structure may be a direct cause of atherosclerosis.

\section{REFERENCES}

1. Rapacz, J., Hasler-Rapacz, I., Taylor, .K.M.,
Checovich, W.I., and Attie, A.D. Science, in press (1986).

2. Lindgren, F.T., Jensen, L.C., and Hatch, F.T. In Blood Lipids and Lipoproteins, Nelson, G.J., ed. New York: John Wiley Interscience, pp. 181-274 (1972).

3. Shen, M.M.S., Krauss, R.M., Lindgren, F.T., and Forte, T.M. J. Lipid Res. 22, 236-244 (1981).

4. Checovich, W.J., Fitch, W.L., Smith, M.P., Krauss, R.M., Rapacz, J., Hasler-Rapacz, J., Smith, C.L., and Attie, A.D. Manuscript in preparation.

\title{
SYNTHESIS OF LECITHIN:CHOLESTEROL ACYLTRANSFERASE BY THE HUMAN LIVER CELL LINE
}

\author{
Trudy M. Forte, Richard T. Thrift, Barbara E. Cahoon, Ching-Hong Chen," and \\ John J. Albers*
}

The enzyme lecithin:cholesterol acyltransferase (LCAT) is a key enzyme in the metabolism of plasma lipoproteins. This enzyme is responsible for the esterification of cholesterol by the following reaction: LCAT phospholipid + unesterified cholesterol $\stackrel{\text { LCAT }}{\longrightarrow}$ lysolecithin + cholesteryl ester. The cholesteryl ester formed by this reaction is ultimately catabolized by the liver; hence, it is thought that LCAT plays an essential role in removal of excess cholesterol from plasma.

It has long been thought that the liver is the chief site of synthesis of LCAT, since plasma LCAT is low in patients with severe liver disease, ${ }^{1}$ and LCAT has been detected in rat liver perfusates. ${ }^{2}$ The synthesis and secretion of this enzyme by human liver parenchymal cells has yet to be unequivocally demonstrated; thus we set out to determine whether this important enzyme is secreted by the human liver cell line, Hep G2. We had previously shown that these cells possessed numerous morphological and functional features of the in situ human liver parenchymal cells. ${ }^{3}$

Hep G2 cells were grown to confluency in Eagle's minimal essential medium (MEM) plus $10 \%$ fetal bovine serum. When confluent, the spent medium was removed and fresh serum-free medium added. Cells were incubated for $6,12,24$,

"University of Washington, Seattle and $48 \mathrm{hr}$, and at each time point, medium was removed, concentrated, and assayed for LCAT mass by radioimmunoassay and LCAT activity. The latter was determined by means of an artificial substrate consisting of phosphatidylcholine and isotopically labeled $\left({ }^{14} \mathrm{C}\right)$ cholesterol liposomes containing apolipoprotein Al (apo Al) (an activator for LCAT). The conversion of labeled cholesterol to cholesteryl ester was determined and expressed as pmol cholesteryl ester formed per $\mathrm{hr}$ per $\mathrm{ml}$ medium. Table 1 shows that LCAT is secreted into the medium and that the rate of secretion is more or

Table 1. Lecithin-cholesterol acyltransferase mass. Cholesterol esterification rate and apolipoprotein Al mass in human hepatoma Hep $G 2$ cell culture medium.

\begin{tabular}{cccc}
\hline $\begin{array}{c}\text { Culture } \\
\text { time } \\
\text { (hr) }\end{array}$ & $\begin{array}{c}\text { LCAT } \\
\text { mass } \\
(\mathrm{ng} / \mathrm{ml})\end{array}$ & $\begin{array}{c}\text { LCAT } \\
\text { activity } \\
\text { (pmol/hr per ml) }\end{array}$ & $\begin{array}{c}\text { Apo Al } \\
\text { mass } \\
(\mathrm{ng} / \mathrm{ml})\end{array}$ \\
\hline 6 & $1.7^{\mathrm{a}}$ & $85^{\mathrm{a}}$ & $305^{\mathrm{b}}$ \\
12 & 4.1 & 170 & 720 \\
24 & 7.9 & 315 & 2400 \\
48 & 13.7 & 402 & 3940 \\
\hline
\end{tabular}

- Each value given is the mean of three determinations on the same experiment with an average error less than $6 \%$.

b Each value given is the mean of three determinations on the same experiment with an average error less than $9 \%$. 
less linear over a 24 -hr period. LCAT activity as expected is also almost linear over the 24-hr period. There is a decline in both mass and activity over a 48-hr period, which may reflect a starved cell situation since cells were not re-fed after $24 \mathrm{hr}$. Because apo $\mathrm{Al}$ is a cofactor in the reaction, we also determined whether this protein is secreted by the cells. Apo $\mathrm{Al}$ is secreted in fairly suistantial amounts as indicated in Table 1.

Although apo $\mathrm{Al}$ is the prime apolipoprotein cofactor for activation of LCAT, it has recently been demoristrated in plasma that other apolipoproteins can serve as cofactors, although they are less potent activators. In order to determine whether Hep G2 LCAT functionally responds to other cofactors similar to normal plasma LCAT, the newly secreted enzyme was challenged with liposomes containing other apolipoproteins as activators. These included apo E2, apo E3, and apo AIV, all known to elicit a response in plasma LCAT. In Table 2, we summarize the response of Hep G2 LCAT in the presence of other cofactors as compared with apo $\mathrm{Al}$, and contrast the response to that of plasma LCAT activity. It is evident that apos $E 2$, E3, and AIV can act as cofactors, but the response is only $20-25 \%$ that of apo Al. However, Hep G2 LCAT activation by other apolipoprotein cofactors is not dissimilar from the response of normal plasma LCAT.

These experiments strongly suggest that LCAT with functional characteristics similar to plasma LCAT is secreted by the human liver cell line, Hep
Table 2. Comparison of apolipoproteins Al, E2, E3, and AIV on the activation of Hep C2-secreted lecithin:cholesterol acyltransferase and purified human plasma lecithin:cholesterol acyltransferase.

\begin{tabular}{|c|c|c|}
\hline \multirow{2}{*}{ Apolipoproteins } & \multicolumn{2}{|c|}{$\begin{array}{c}\text { Source of enzyme } \\
\text { LCAT activity } \\
\text { (pmol cholesterol esterified/hr } \\
\text { per ng LCAT mass) }\end{array}$} \\
\hline & $\begin{array}{c}\text { Hep G2-secreted } \\
\text { LCAT }\end{array}$ & $\begin{array}{l}\text { Purified human plasma } \\
\text { LCAT }\end{array}$ \\
\hline $\mathrm{Al}$ & $25.6 \pm 1.5^{a}$ & $32.4 \pm 1.8^{2}$ \\
\hline E2 & $8.2 \pm 0.4$ & $12.0 \pm 0.7$ \\
\hline E3 & $7.4 \pm 0.5$ & $9.7 \pm 0.5$ \\
\hline AIV & $6.1 \pm 0.3$ & $8.4 \pm 0.5$ \\
\hline
\end{tabular}

a Mean \pm S.D., $n=4$ (four separate experiments).

G2. These cells can potentially serve as an important cell model in which to study regulation of LCAT synthesis and secretion.

\section{REFERENCES}

1. Sabesin, S.M., Hawkins, H.L., Kuiken, L., and Ragland, J.B. Gastroenterology 72, 510-518 (1977).

2. Hamilton, R.L., Williams, M.C., Fielding, C.J., and Havel, R.J. J. Clin. Invest. 58, 657-680 (1976).

3. Thrift, R., Forte, T.M., Cahoon, B.E., and Shore, V.G. J. Lipid Res. 27, 236-250 (1986).

\section{CHARACTERIZATION OF HIGH DENSITY LIPOPROTEIN SUBCLASSES ISOLATED FROM THE HUMAN HEPATOMA DERIVED CELL LINE, HEP G2}

\section{Mark R. McCall, Trudy M. Forte, and Virgie C. Shore"}

The study of lipoprotein synthesis and catabolism is complicated by the dynamic nature of these processes. Nascent lipoproteins from hepatic or intestinal synthetic sites undergo a variety of extremely rapid enzymatic and exchange processes when released into the extracellular milieu. Interstitial fluid, lymph, or plasma lipoproteins may therefore not be very representative of lipoproteins as released from the cell. Intracellular lipoproteins, although visible with an electron microscope, are

"Lawrence Livermore Laboratory, Livermore, CA. difficult to isolate for study due to their close association with cellular membranes.

The isolation by Knowles, Howe, and Aden ' of a human liver ceil line (Hep G2, hepatoma-derived) that retained well-differentiated function may prove to be of considerable benefit in the study of lipoprotein metabolism. The use of a tissue-culture system permits easy isolation of lipoproteins that have not been exposed to lymph or plasma and also makes it possible to differentiate between dietary and hormonal factors affecting lipoprotein production. 
Recent work with the Hep G2 cell line in this laboratory has focused on the characterization of the major lipoprotein classes produced under basal defined conditions. ${ }^{2}$ It was evident in these studies that the high density lipoprotein (HDL) class (density between 1.063 and $1.21 \mathrm{~g} / \mathrm{ml}$ ) was heterogeneous with respect to particle size and structure. Our current efforts are focused on characterizing this heterogeneity. The lipoproteins found in Hep G2 $\mathrm{HDL}$ appear to share some similarities with $\mathrm{HDL}$ isolated from other nascent lipoprotein model systems and from humans with LCAT deficiency. Characterization of the observed heterogeneity may help to elucidate some of the metabolic interconversions that occur within the human HDL fraction. In this report we present our preliminary Hep G2 HDL fractionation results.

Twenty-four-hour-conditioned serum-free medium was collected from $54175-\mathrm{cm}^{2}$ confluent cultures over a two-week period. The medium was collecied on ice, then spun to remove detached cells and debris, and the following preservatives and protease inhibitors were added: gentamicin sulfate, $K_{2}$-EDTA, phenylmethylsulfonyl fluoride, $p$ hydroxymercuriphenylsulfonic acid, pepstatin A, and leupeptin. The conditioned medium was subsequently concentrated, then two lipoprotein fractions were isolated $(d<1.063$ and $d ; 03-1.25)$ using standard sequential ultracentrifugation techniques. The lipoproteins isolated in the HDL density range were further fractionated by density gradient ultracentrifugation. Twenty-one $1 / 2-\mathrm{ml}$ fractions were aspirated following the 48-hr (SW 41 rotor, $15^{\circ} \mathrm{C}, 40 \mathrm{~K}$ ) density gradient spin. Each fraction was electrophoresed using nondenaturing polyacrylamide gradient gels $(4-30 \%)$. Fractions were subsequently pooled based on their electrophoretic mobility.

Hep $G 2$ lipoproteins isolated in the HDL density range usually form four distinct bands when run on 4-30\% nondenaturing polyacrylamide gels and stained with Coomassie 6250 . Although minor differences from one gel to another are observed, the major bands correspond to standard proteins having Stokes' diameters of 13.3, 12.0, 9.5, and $7.5 \mathrm{~nm}$. The comparison of gradient-gel electrophoretic patterns obtained from Hep G2 HDL with patterns obtained from the various $\mathrm{HDL}$ density gradient fractions permitted pooling of fractions with electrophoretic mobilities characteristic of the major HDL bands. The success of this procedure in isolating the characteristic Hep G2 HDL subclasses is pictorially displayed in Fig. 1. Pools 4 and
5 appear to have fewer contaminants than pools 2 and 3. The estimated Stokes' diameter of the major lipoprotein peak from each of the four major pools is presented in Table 1 . The mean isolation density for each pool has also been included.

Electron micrographs of the negatively stained lipoproteins within eacil pool are shown in

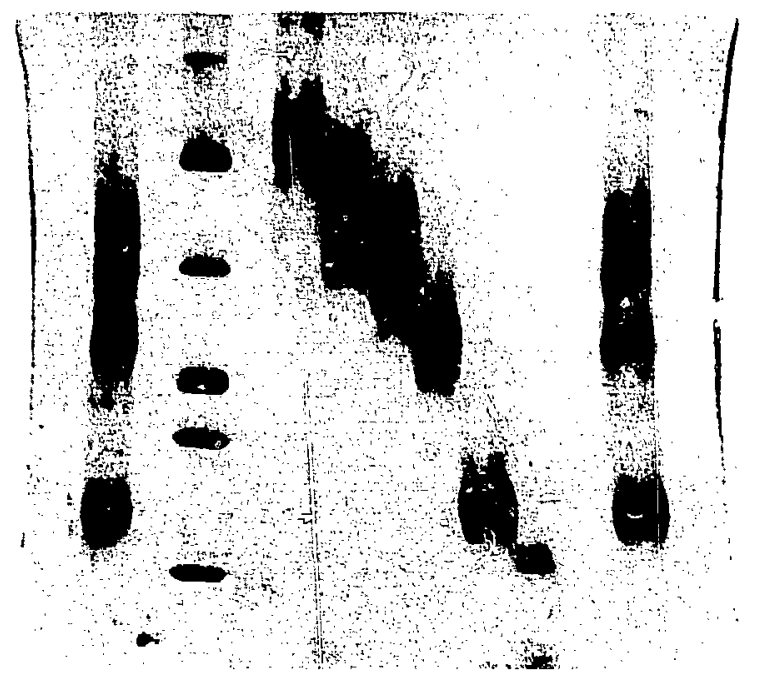

Fig. 1. A Coomassie G250-stained 4-30\% polyacrylamide gradient gel of Hep G2 HDL and the various density gradient pools. Standard proveins were run in lane 4. The bands (from top to bottom) correspond to thyroglobulin, ferritin, calalase, lactate dehydrogenase, and albumin. Lanes 2 and 13 contain Hep G2 HDL. The apparent Stokes' diameters of the four major bands in this gel are (from top to boltom) 13.5, $11.7,9.8$, and $7.5 \mathrm{~nm}$. Pools 2, 3, 4, and 5 were run in lanes $7,8,9$, and 10, respeclively.

(XBB 860-9569)

Table 1. Characterization of the major pooled Hep G2 HDL density gradient fractions.

\begin{tabular}{lrcc}
\hline \hline Pool & $\begin{array}{c}\text { Fiactions } \\
\text { pooled }\end{array}$ & $\begin{array}{c}\text { Mean density } \\
(\mathrm{g} / \mathrm{ml})\end{array}$ & $\begin{array}{c}\text { Major peak position } \\
\text { Stokes' }\end{array}$ \\
\hline 2 & 3,4 & 1.076 & 13.4 \\
3 & 5,6 & 1.087 & 11.9 \\
4 & 8,9 & 1.105 & 9.8 \\
5 & $13-17$ & 1.162 & 7.4 \\
\hline
\end{tabular}

- Determined using refractometry.

b Size estimates based on gradient-gel electrophoresis assume spherical geometry. The particles isolated in pools 2 through 4 were discoidal; therefore their peak positions can only be used as a relative size determinant. 

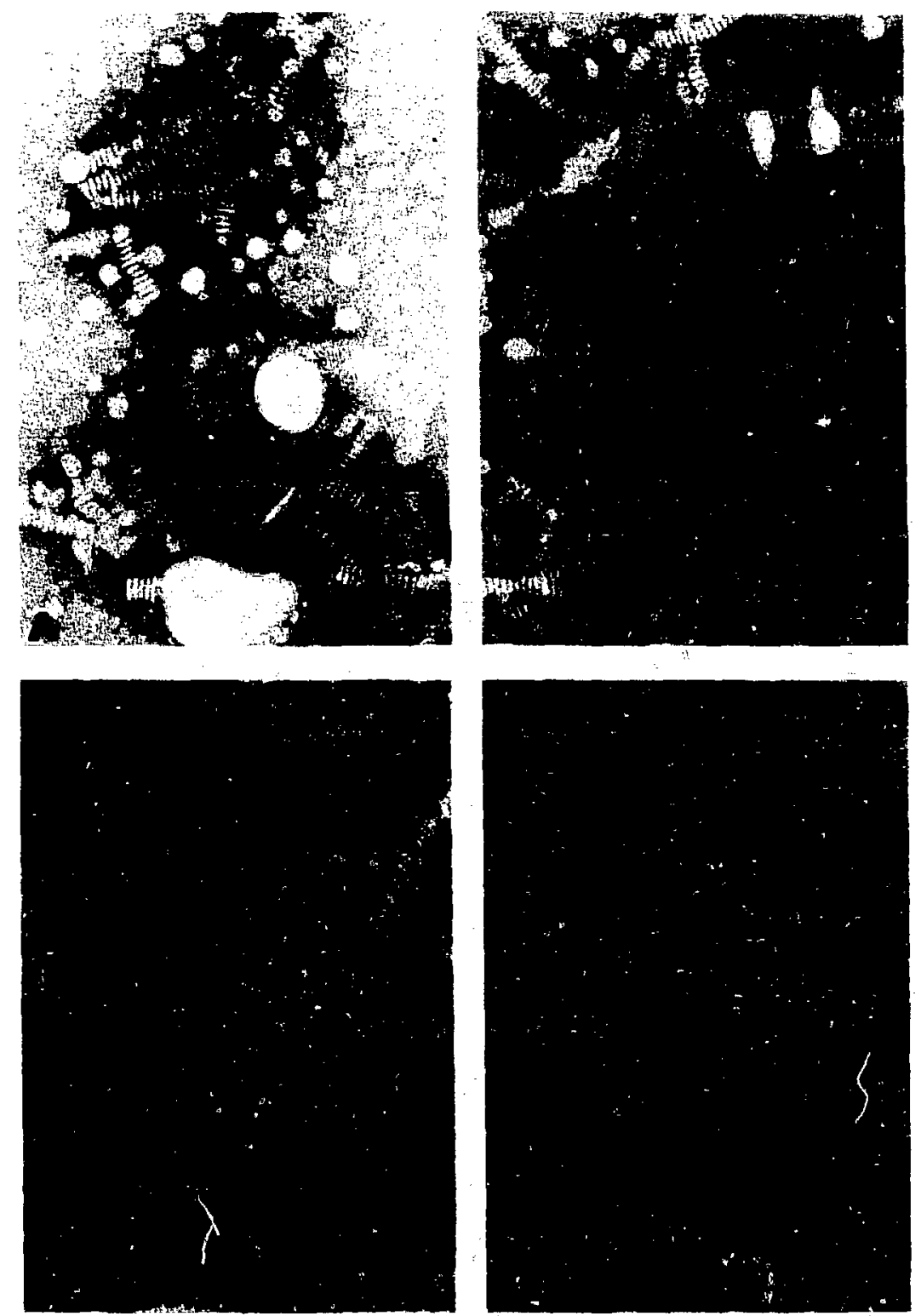

Fig. 2. Electron micrographs of negatively stained lipoproteins: (A) pool 2; (B) pool 3; (C) pool 4; and (D) pool 5.

(XBB 860-9570)

Figs. 2(A) through (D). Mean particle sizes estimated from the micrographs are as follows: Pool 2, $20.0 \mathrm{~nm}$; Pool 3, $19.1 \mathrm{~nm}$; Pool 4, $13.1 \mathrm{~nm}$; Pool $5,7.7 \mathrm{~nm}$ (each value represents the mean of 400 measurements). The discrepancy between the sizing of particles by gradient-gel electrophoresis and electron microscopy relates to the nonspherical nature of the lipoproteins isolated in fractions 2 through 4; gradient gel electrophoresis assumes spherical geometry. The two methods were in better agreement with the spherical particles isolated in fraction 5 .

\section{REFERENCES}

1. Knowles, B.B., Howe, C.C., and Aden, D.P. Human hepatocellular carcinoma cell lines secrete the major plasma proteins and hepatitis B surface antigen. Science 2.19, 497-499 (1980).

2. Thrift, R., Forte, T.M., Cahoon, B.E., and Shore, V.G. Characterization of lipoproteins produced by the human liver cell line, Hep G2, under defined conditions. /. Lipid Res. 27, 236-250 (1986). 


\title{
CACO-2 CELLS: A HUMAN INTESTINAL CELL LINE WITH THE CAPACITY FOR SECRETING LIPOPROTEINS
}

\author{
Trudy M. Forte, Thomas E. Hughes," W. Vodek Sasok," Jose M. Ordovas," \\ Stefania Lamon-Fava* and Ernst J. Schaefer*
}

In man, lipoproteins are secreted by parenchymal liver cells and small intestine absorptive cells. The challenge in the lipoprotein field has been to develop cell models in which to study differentiation and regulation of lipoprotein secretory processes. We have recently shown that the human liver cell line, Hep G2, is an important model in which to study low density and high density lipoprotein secretion. ${ }^{\prime}$ The Hep G2 cell has been well defined and is functionally well differentiated, thus making it a favorite cell not only for lipoprotein synthesis, but also for investigation in receptor activity and bile secretion. An intestinal counterpart to the Hep C2 cell has recently been developed by Pinto et al.? This cell was derived from colonic carcinoma but has many functional aspects of absorptive cells of the small intestine.

We have been involved in a collaborative study with Dr. Schaefer and his colleagues at Tufts University, in an effort to determine whether the Caco- 2 cells might be a reasonable model in which to study lipoprotein synthesis and regulation.

The Caco-2 cells ultrastructurally have many features in common with in situ intestinal cells is shown in Fig. 1. The cells are columnar and their apical surfaces possess uniform microvilli. The fact that the cells form sheets of columnar cells indicate that they are polarized in a fashion similar to in vivo cells.

To identify lipoproteins, Caco-2 cells were grown to conflueicy in DMEM plus fetal bovine serum (FBS) at which time FBS was removed and cells were grown in serum-free defined medium. In some experiments, ${ }^{35} \mathrm{~S}$-methionine was included in the medium to look for de novo synthesis of apolipoproteins and distribution of label in lipoprotein classes. Lipoprotein fractions were also examined by electro: microscopy to determine whether identifiable structures were secreted by the cells.

Table 1 shows the total acid-insoluble ${ }^{35} \mathrm{~S}$ methionine in the medium and its lipoprotein fractions. Very low density lipoproteins (VLDL) are isolated at $\mathrm{d} \leqslant 1.006 \mathrm{~g} / \mathrm{ml}$, low density lipoproteins (LDL) are isolated at $\mathrm{d} 1.006-1.063 \mathrm{~g} / \mathrm{ml}$ and high density lipoproteins (HDL) at d 1.063-1.21 g/ml;

-Tufts University, Boston, MA. the $\mathrm{d} \geqslant 1.21 \mathrm{~g} / \mathrm{ml}$ fraction represents nonlipoprotein protein. All three classes of lipoproteins contained labeled amino acid with $\mathrm{HDL}$ as the major de novo synthesized particle.

Particles of $\mathrm{d} \leqslant 1.063 \mathrm{~g} / \mathrm{ml}$ and $\mathrm{d} 1.063-1.21$ $\mathrm{g} / \mathrm{ml}$ (HDL) were characterized by electron microscopy. The former density contains VLDL and LDL; a representative micrograph of these particles are seen in Fig. 2(A). The particles are heterogeneous in size with $25 \%$ in the VLDL size range (28-90 $\mathrm{nm})$ and $54 \%$ in the LDL (20-28 nm) range; the remainder $(21 \%)$ were particles smaller than LDL. The particle distribution of the Caco-2 $d \leqslant$ $1.063 \mathrm{~g} / \mathrm{ml}$ is different from Hep G2 in that the

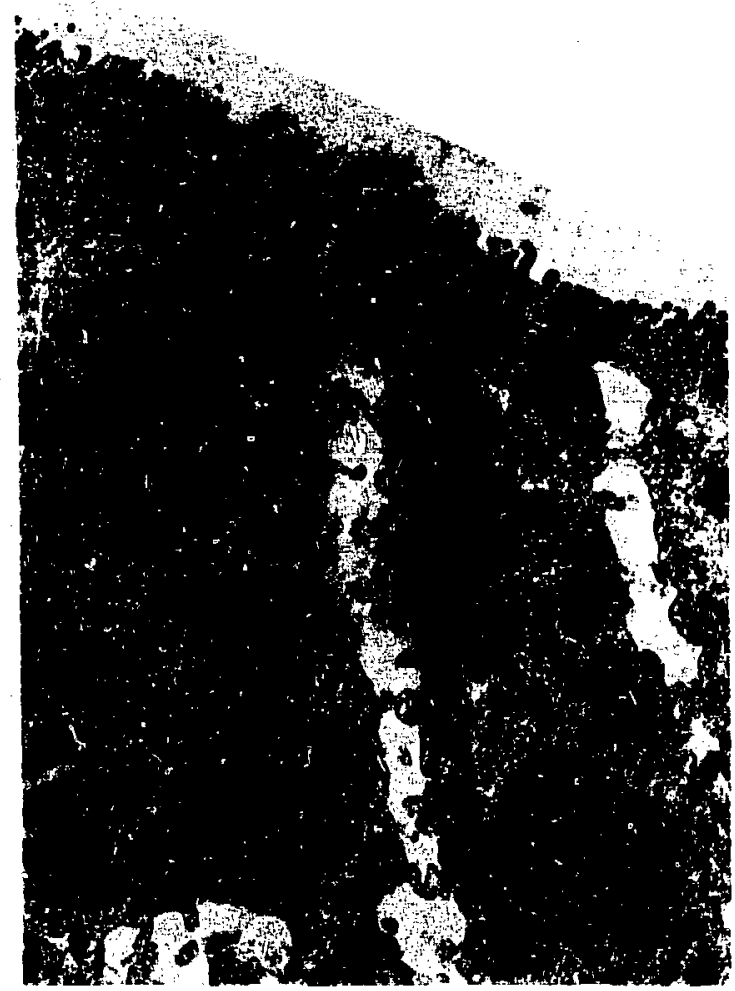

Fig. 1 Electron micrograph of a thin section of fixed Caco-2 monolayer sectioned perpendicular to plane of culture flask. Caco-2 cells are columnar cells capped with uniform short microvilli (MV). The overall morphology of the cells is similar to in situ small intestinal cells.
(XBB 860-8639) 
Table 1. Distribution of radioactivity among lipoprotein fractions of $\left.\right|^{35} \mathrm{~S} \mid \mathrm{methionine-labeled}$ conditioned medium from Caco-2 cells. Lipoprotein fractions were isolated by sequential ultracentrifugation of conditioned medium collected after a 24 hour incubation of 14 day post-confluent $\mathrm{Caco}-2$ cells in $100_{-\mu} \mathrm{Ci} / \mathrm{m} /\left.\right|^{35} \mathrm{~S} \mid$ methionine. Trichloroacetic acid insoluble radioactivity was determined in isolated lipoprotein fractions.

\begin{tabular}{ccc}
\hline & $\begin{array}{c}\text { [55|methionine } \\
\text { \% medium } \\
\text { acid-insoluble } \\
\text { activity }\end{array}$ & $\begin{array}{c}\text { \% total } \\
\text { lipoprotein } \\
\text { acid-insoluble } \\
\text { activity }\end{array}$ \\
\hline Lipoproteins & $27.52(1.31)$ & \\
VLDL & $2.75(0.52)$ & 10.0 \\
LDL & $3.79(0.30)$ & 13.8 \\
HDL & $20.97(2.09)$ & 76.2 \\
d > 1.21 & $49.70(5.64)$ & \\
Total & $72.21^{*}$ & 100.0 \\
\hline \hline
\end{tabular}

- The remaining $27.79 \%$ of the medium radioactivity was not recovered after ultracentrifugation.

latter cells produce little or no identifiable VLDL. The HDL secreted by Caco-2, Fig. 2(B), are very similar in morphology to Hep G2 secreted HDL. The cells secrete both discoidal particles and the more plasma-like spherical particles.

These preliminary studies suggest that the Caco-2 cell line is a potential valuable model in which to study intestinal assembly of lipoproteins and the role of nutritional factors and hormones in regulating lipoprotein synthesis.

\section{REFERENCES}

1. Thrift, R.N., Forte, T.M., Cahoon, B.E., and Shore, V.G. Characterization of lipoproteins produced by the human liver cell line, Hep G2, under defined conditions. /. Lipid Res. 27, 236 (198f).

2. Pinto, M., Robirie-Leon, S., Appay, M-D., Ked-
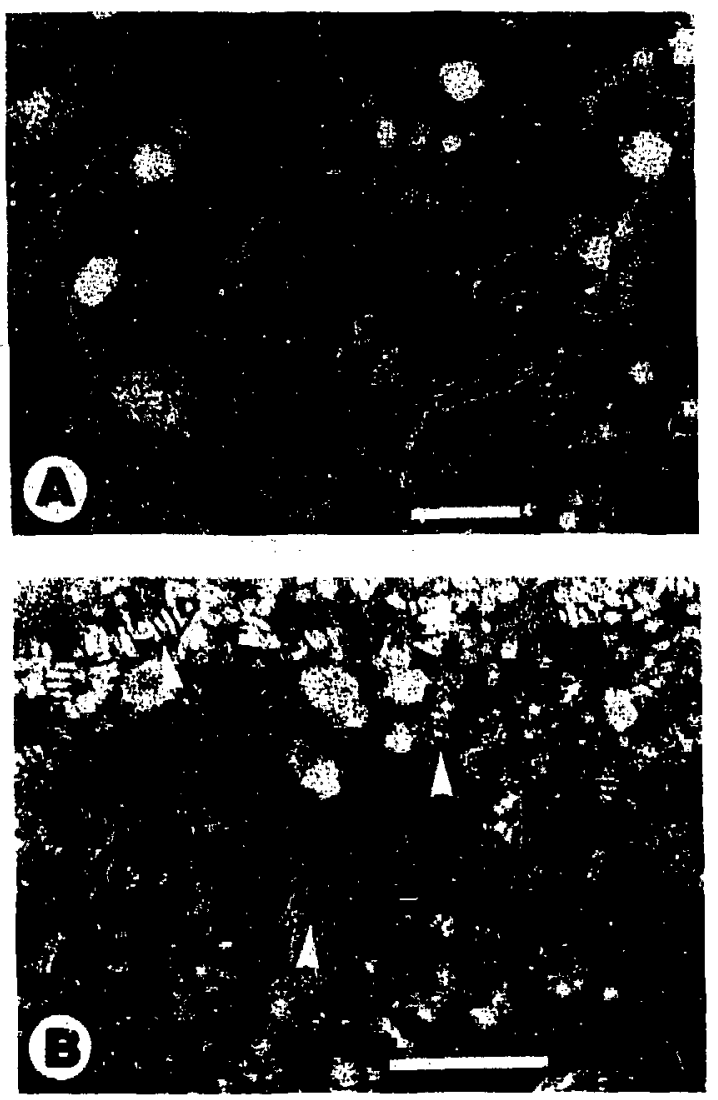

Fig. 2 Characterization of isolated lipoprotein particles by negative- staining electron microscopy. (A) Micrograph of $d \leqslant$ $1.063 \mathrm{~g} / \mathrm{ml}$ (VLDL and $L D L$ ) particles. (B) Micrograph of $d$ 1.063-1.21 g/m/ (HDL) particles; note discoidal particles (arrowheads). Bars represent $100 \mathrm{~nm}$.

(XBB 860-8640)

inger, M., Triadou, N., Dussaulx, E., Lacroix, B., Simon-Assmann, P., Haffen, K., Fogh, J., and Zweibaum, A. Enterocyte-like differentiation and polarization of the human colon cell line Caco-2 in culture. Biol. Cell 47, 323-330' (1983). 


\title{
Structural Biology
}

\section{IMPROVEMENT IN HIGH RESOLUTION IMAGE QUALITY OF RADIATION SENSITIVE SPECIMENS ACHIEVED WITH REDUCED SPOT SIZE OF THE ELECTRON BEAM}

\author{
Kenneth H. Downing and Robert M. Glaeser
}

Although electron diffraction patterns extending to better than $3 \AA$ can be obtained from a number of thin ("two-dimensional") protein crystals, the resolution in images of these crystals has seldom reached beyond even $5 \mathrm{~A}$. It is now evident that beam-induced specimen motion is a major factor in limiting resolution. Long-range as well as shortrange motion must occur in specimens as a result of the stress and local distortion around sites of defects produced in the specimen by radiation damage. This motion should be greatly reduced by restricting the area of the specimen that is illuminated at any one time. Not only should the total stress on the specimen be reduced in proportion to the area illuminated, but the area that is illuminated should be quite highly constrained by the unirradiated, surrounding area.

We have developed methodology to allow efficient recording of images using illumination spot sizes as small as 500 A. Lattice images of monolamellar paraffin crystals have been recorded under a variety of conditions to test the effect of reducing the illumination spot size. Images recorded with spots as large as 2000 A show preservation of both contrast and symmetry that are significantly greater than found in images recorded with more conventional flood illumination. The same improvements have also been observed in images of purple membrane. To allow efficient utilization of the specimen and photographic film, the beam can be stepped through a two-dimensional raster of points, under control of a microcomputer that is interfaced to the beam deflection system. The computer provides flexible control of the number of points in the raster, their spacing, and exposure time at each spot.

Paraffin was used as a test specimen in evaluating the small spot scan system. Large, monolayer crystals of paraffin were prepared by evaporation from dilute solutions of tetratetracontane $\left(\mathrm{C}_{44} \mathrm{H}_{90}\right)$ in heptane. The primary lattice spacings, 3.7 and $4.2 \mathrm{~A}$, are in the particular resolution range of interest, where the image contrast is severely degraded in conventional images. The constant and reproducible crystal thickness of monolamellar paraffin crystals is particularly useful in quantitative analysis of image contrast.

The quality of the paraffin micrographs was initially judged by optical diffraction. The differences between images recorded with flood illumination and with a small spot were quite evident in the optical transforms. Transforms of fairly large areas ( 5000-10,000 A) of micrographs recorded with flood illumination often showed the pseudohexagonal pattern of the electron diffraction patterns, including the (110) spots at $4.2 \AA$ and the (200) spots at $3.7 \AA$. When smaller areas ( 2000 A) were examined, however, at least one of the three pairs of spots was usually missing. The orientation of the missing spots often changed over distances of a few thousand angstroms.

In contrast to the results for flood-beam illumination, transforms of images recorded with the small-spot illumination almost always contained all six spots even when the laser beam was as small as the individually exposed areas on the film. In addition, the diffraction spots were significantly brighter than those in the transforms of the conventional images.

Figure 1 is a set of optical transforms that present a typical comparison of images recorded with flood- or small-beam illumination. All transforms are from areas of $10-\mathrm{mm}$ diameter on the micrograph (2000 $\AA$ on the specimen). Figure 1(a) is the transform of an image recorded with an electron beam of $3-\mu \mathrm{m}$ diameter. The specimen was selected on the basis of the symmetry of its electron diffraction pattern, and examination of the diffraction pattern after the image exposure showed that the crystal orientation had not changed. However, the image transform shows large differences in the spot intensities. The transform in Fig. 1(b) is from a micrograph of the same crystal, but recorded with a beam diameter of $2000 \mathrm{~A}$. The spots are quite clearly brighter than those of Fig. 1(a), in addition to being of much more uniform intensity. Note that the $(020)$ spots, corresponding to a lattice spacing of $2.5 \AA$, are also visible in this transform. 

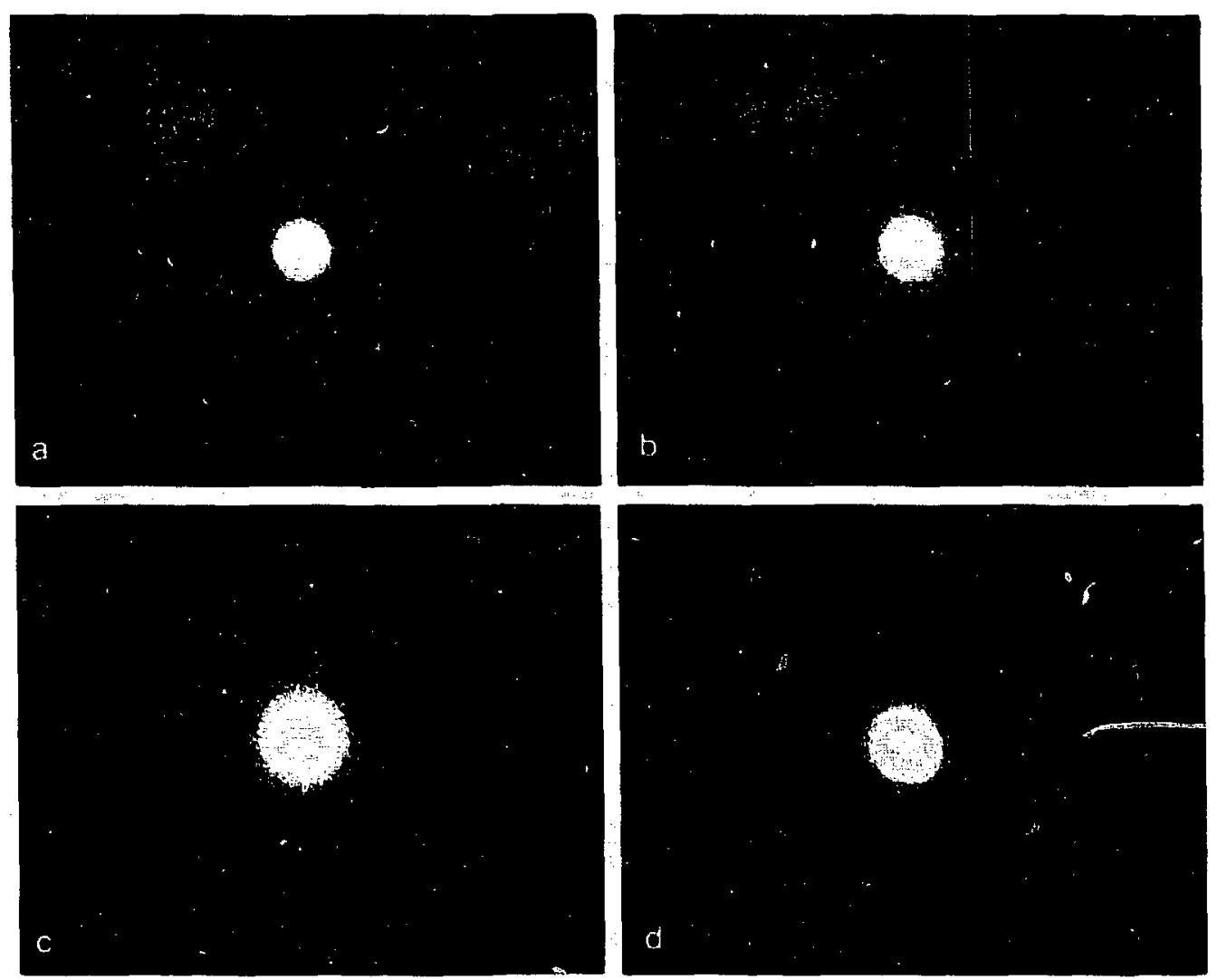

Fig. 1. Optical transforms from 10-mm-diameter areas of paraffin lattice images recorded at 50,000 magnification, with flood illumination, (a) and (c) and with a 2000-A-diameter beam (b) and (d). The transforms in (c) and (d) have been defocused by shifting the beam crossover to a point beyond the plane of the recording film.

(XBB 866-4964)

The intensity of the diffraction spots can be more accurately judged by slightly defocusing the optical transform. Figures $1(\mathrm{c})$ and $1(\mathrm{~d})$ are defocused optical transforms of the same areas as in Figs. 1 (a) and 1(b), respectively. Here, with each diffraction spot spread out into a type of dark-field image, the differences in spot intensities are particularly clear. In addition, it can be seen that there exists a non-uniform distribution of intensity, and therefore of contrast, within the image obtained with the 2000-A diameter beam.

Quantitative measurements of the contrast, or $F(g) / F(0)$ ratio, were made for a number of micrographs obtained both with flood illumination and with small-spot illumination. It was found that reducing the illumination spot to a diameter of around $2000 \AA$ improved the image contrast by a factor of 3 to 5 . The $F(g) / F(0)$ in the paraffin lattice images recorded with flood illumination is at best around 0.01 , or $5 \%$ of the contrast in an "ideal" image. When images were recorded with smallbeam illumination, areas with $F(g) / F(0)$ around 0.04 were frequently found.

Under some conditions, the lattice image contrast uniformly fills the entire exposed area. More often, however, the image appears to be nonisoplanatic, as seen in Fig. 1(d). To further investigate this effect, the optical diffractometer was configured as a reconstruction system that could image larger areas ( $40 \mathrm{~mm}$ diam) of the micrograph. By placing an aperture at the diffraction plane of this system, and masking out all but one of the six diffraction spots in the image transform, dark field images of the electron micrographs could be obtained to display the regions of the illuminated areas in which the lattice was imaged with good contrast.

Figure 2 presents an analysis of a micrograph of a paraffin crystal which was recorded with a beam diameter of $2000 \AA$. Figure 2(a) is a bright-field 

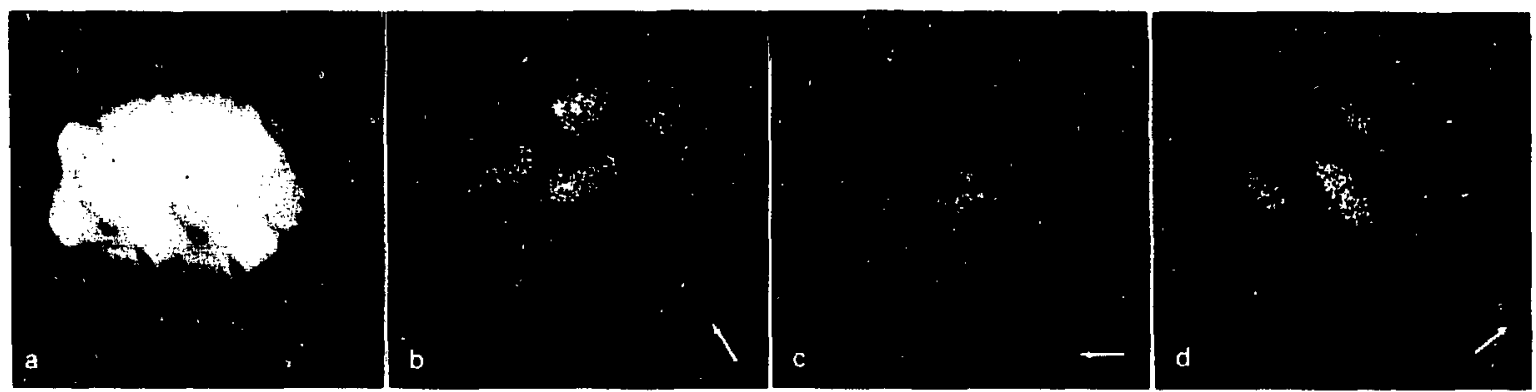

Fig. 2. Optical analysis of contrast variation of paraffin lattice images, within small exposed areas. In this example the illumination spot size had a diameter of $2000 \mathrm{~A}$. (a) Bright-field image of the electron micrograph obtained on a coherent-optical reconstruction bench. $(b, c, d)$ Dark-field images of the micrograph obtained by masking out all but one of the diffraction spots in the optical transform of the micrograph. Arrows indicate the direction of the reciprocal lattice vector associated with the diffraction spot used to form each image.

(XBB 866-4965)

image obtained on the optical reconstruction system, and shows the pattern of illumination of the specimen. The pattern of intensity within the beam is caused by the Fresnel fringes around the edge of the spot, and by a $60-\mathrm{Hz}$ deflection of the beam position on the specimen. Figures 2(b), (c) and (d) are corresponding dark-field images that were obtained with each one of the three independent diffraction spots. The lower intensity of the images in Fig. 2(b), corresponding to lower contrast of the corresponding lattice line image, may be caused by nonoptimum defocus or by slight specimen drift. It is clear that the contrast of the lattice image can vary within each of the exposed areas. In addition, it can be observed that the contrast associated with each of the three sets of Bragg planes can have its strongest values in quite different areas of the image. Often, each lattice component is well resolved in a band that runs through the illuminated area in a direction that is parallel to the lattice lines.

The pattern of contrast within the illuminated area is quite constant from one illuminated spot to another within each micrograph, but may be quite different from one micrograph to another. The most frequently observed pattern of lattice image contrast is consistent with that to be expected if the specimen were to undergo some radial movement during exposure to the beam, while remaining essentially stationary at the center of the spot. Such motion would be consistent with a change in the projected area per molecule as the crystal accumulates defects. In the case of paraffin, radiationinduced kinks in the alkane chains may lead to an increase in molecule area, which could cause the specimen to bulge up into a dome ai each site of exposure. This motion would be a specific form of the more random wrinkling and warping of specimens exposed to a flood beam. Evidence for this sort of distortion has been found in experiments currently in progress.

Preliminary images of purple membrane have also been recorded with the small spot technique. Even though the image contrast was again not uniform across the exposed areas, a clear improvement in quality is already seen in optical transforms of these images. Figure 3 shows a comparison of optical transforms of purple membrane images obtained with conventional and small spot illumination. Figure $3(\mathrm{a})$ is the transform of a $30-\mathrm{mm}$ diameter area of an image recorded at a magnification of 50,000 with a beam of about $5-\mu \mathrm{m}$ diameter. This transform is representative of "good" purple membrane images. The density of spots is much greater than in transforms of paraffin images, although diffraction spots are visible only to a resolution of around 7-8 A. Figure $3(\mathrm{~b})$ is a transform of the same micrograph, but with the laser beam masked down to a diameter of $7 \mathrm{~mm}$, correspond-. ing to $1400 \AA$ on the specimen. Not only is the signal to noise ratio so bad in this transform that most of the spots are lost in the noise, but the spots show up primarily only in one direction. As the micrograph is scanned over the $7-\mathrm{mm}$ laser beam, the "good" direction of the transform changes, as was seen in paraffin images, again indicating the occurrence of beam-induced differential drift. Figure $3(\mathrm{c})$ is the transform of a $7-\mathrm{mm}$ diameter area from an image recorded with a beam diameter of $1500 \mathrm{~A}$. The signal to noise ratio in this "small spot" image is not only as good as that obtained with a much larger beam of a conven- 

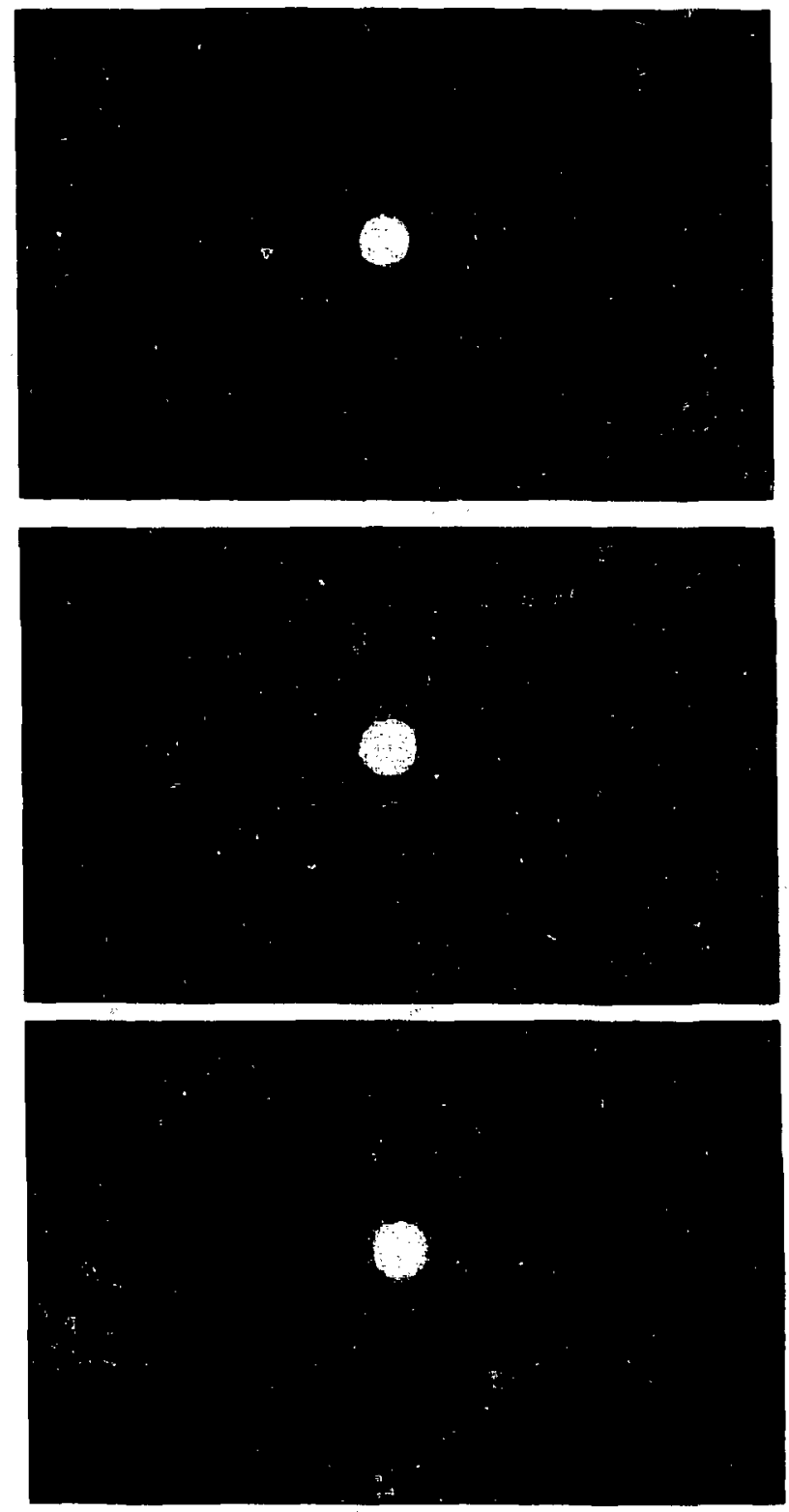

tional micrograph, but the spots are seen uniformly in all directions.

The suggestion that higher image contrast might be achieved with radiation sensitive organic crystals by the use of very small illumination spots has now been found experimentally to be valid. The results reported here thus give additional confirmation that beam-induced movements in sensitive organic materials have been a major factor in causing the image contrast to be much less than it theoretically ought to be. The practical exploitation of smallspot illumination remains flawed, however, by the fact that strong contrast variation can still be observed within images where the illuminated area is only $1000 \AA$ or $2000 \AA$ in diameter. The physical basis for the observed contrast variation remains still to be identified and brought under control, if possible. The results at this point are nevertheless very encouraging, and suggest that the use of coherent, isoplanatic conditions of small spot illumination can be an important tool in extending the low-dose imaging technique to achieve routine image resolutions of $4 \AA$ or better with beamsensitive, crystalline organic materials.

Fig. 3. Optical Iransforms of images of purple membrane. (a) Transform of a 30-mm-diameter area of a micrograph that was recorded with flood illumination. (b) Transform of the same area as in (a), but with the laser beam masked down lo a diameter of $7 \mathrm{~mm}$. (c) Transform of a $7 . \mathrm{mm}$-diameler area of a micrograph that was recorded with an electron beam of $1400 \mathrm{~A}$ (7 $\mathrm{mm}$ at the image plane).

(XBB-863-1958)

\section{IMPROVED SUPPORT FILMS FOR ELECTRON MICROSCOPY OF BEAM SENSITIVE SPECIMENS}

\section{Joseph R. Taylor and Robert M. Glaeser}

In electron crystallography, the molecular structure of biological macromolecules is determined by combining information obtained from electron diffraction patterns with phase data derived from low-dose images. The two types of data can be obtained from crystals that range from 1 to $10 \mu \mathrm{m}$ in diameter, while their thickness may be less than 100 angstroms. In order to examine such miniature crystals in the electron microscope, it is necessary to place the specimen, which consists of a disper- 
sion of the microscopic crystals, on a very thin but self-supporting electron-transparent support film (usually comprised of quasi-amorphous carbon that itself is supported on a conventional 400-mesh metal grid). Since the imaging process would magnify any motion of the specimen that might occur during exposure by the same factor as it magnifies the structure of the molecule, the siability of the support film and of the specimen itself is extremely critical if high-resolution structural information is to be preserved in the imaging process.

Diffraction data are currently available from some specimens to better than 3 angstroms resolution, but the best images of the same beamsensitive biological materials only contain structural information to a resolution of 7 angstroms. At the present time, what seems to be preventing the imaging of these materials at the resolution of available diffraction data is the mechanical stability of the specimen itself. We now believe that absorption of energy from the electron beam due to inelastic electron scattering causes sufficient specimen motion to occur during imaging to account for most of the loss of information that we observe in the images themselves.

It was not until the work of Henderson and Glaeser $^{1}$ that the problem of beam-iriduced movement was fully recognized to be a major limiting factor in the technique of electron crystallography. Since then we have been exploring methods in our laboratory to deal with the problem. Recent work by Downing and Glaeser, ${ }^{2}$ which involves exposure of the specimen by a small illumination spot that is scanned in a raster over the specimen, has resulted in an improvement relative to the quality of images obtained by means of conventional flood-beam illumination. Further improvement is required, however, and we have therefore been simultaneously developing techniques to produce more robust specimen-support films that might serve to better stabilize the thin crystalline specimens against beam-induced movement.

\section{METHODS}

This work has included the development of new techniques in both carbon film deposition and in the production of ultra fine mesh metal supports (often called microgrids) to underlie and stabilize the carbon specimen-support film.

\section{Carbon Films}

A novel method of carbon-film production by vacuum evaporation in a reducing (hydrogen) environment has been developed. This new tech- nique produces films having noticeably more consistent properties when compared with conventionally produced films, and it has made possible the evaluation of newly developed specimen-support techniques by quantitative comparison of their effectiveness in the imaging of paraffin test specimens at 4.6 angstroms.

\section{Microgrids}

Microgrids are fabricated by first producing a highly perforated or "fenestrated" polymer film which is then reinforced and made electrically conductive with carbon or metal by means of vacuum evaporation. A number of methods of producing the fenestrations in the polymer have been described in the past. The most commonly practiced of these is the method first described by Fukami and Adachi $^{3}$ in which a thin polymer film is deposited from solution on a glass substrate on which a fog of microscopic water droplets has previously been condensed. We have experimented extensively with numerous methods of producing fenestrated plastic films and have most recently settled on the procedure shown schematically in Fig. 1. This method, which we deem most suitable
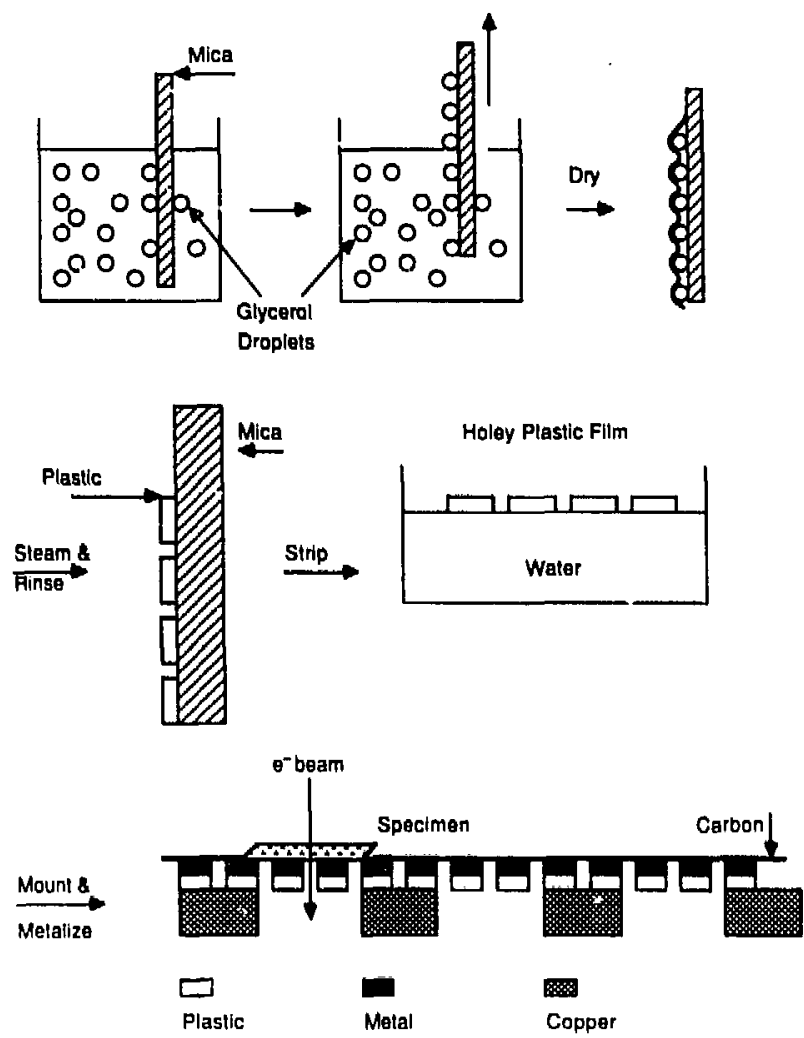

Fig. 1. Microgrid fabrication

(XBL 8611-9056) 
for producing substrates for high-resolution imaging, involves the emulsification of aqueous glycerol in a dilute nonpolar solution containing cellulose nitrate. The detergent Brij 58 is included in the novel protocol to facilitate the complete emulsification of the glycerol. This is an important innovation developed by Lunsdorf and Spiess ${ }^{4}$ that greatly improves the uniformity of the emulsion and thereby produces films having much more uniform hole size and a superior and more reproducible distribution of holes.

\section{RESULTS}

Despite the development of improved carbon films and microgrids having optimized properties, preliminary results of imaging experiments in which carbon films on microgrids were used to support the specimen showed no improvement in the signal present in high-resolution images, and therefore no evidence that beam-induced movement had been reduced. As a result of these experiments two different areas of concern have been identified that may indicate that specimen-support interaction has yet to be completely optimized. Present efforts to develop better support film technology are primarily being concentrated in the two related areas of carbon-film to microgrid bonding and overall microgrid flatness (where there is probably the most potential for improvement).
In order to improve bonding of the carbon film to the grid, a technique has been developed to bond the two together. A polymer residue is deposited between the carbon film and the metal supporting grid. A second thin carbon film is then applied to the back side of the grid thereby presumably forming a conducting bond between the carbon film and its support. Preliminary experiments with this method using conventional 400mesh grids have resulted in an apparent improvement in the regularity with which high-resolution images can be obtained, although no improvement in the level of signal present in the images has yet been observed. Work in this area is now being extended with microgrids produced by a new technique described hereinafter which may improve the overall flatness of the microgrid support.

In order to improve the procedure that is used to reinforce microgrids, a new metalization apparatus (Fig. 2) has been constructed. This device provides the capability to evaporate from an array of metal sources so that the polymer film can be symmetrically sandwiched between metal films of approximately equal thickness. The purpose of this is to minimize the tendency of the multilamellar microgrid assembly to undergo bimetal distortion during the metalization procedure, which unavoidably involves exposure of the substrate to radiation and concomitant heating. In addition, the

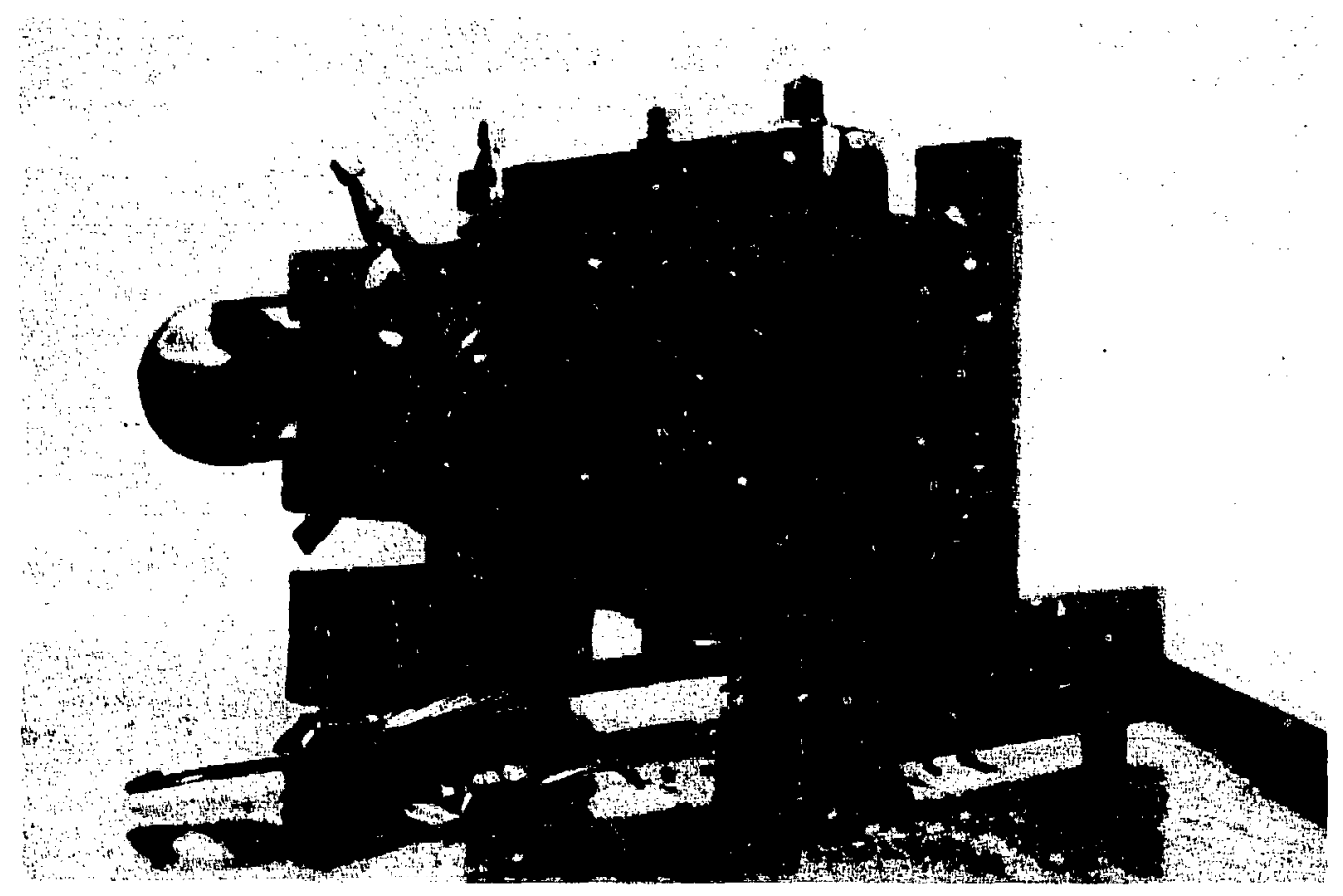

Fig. 2. Metalization apparatus.

(X88 860-10317) 
apparatus makes provision for heating to be minimized by shielding the grids from the hot metalvapor sources except during the relatively brief final stage of the procedure when the metal is actually being deposited. Together with these advantages, this device also employs a novel filament design that provides for improved control of the evaporation procedure, which should contribute to the ultimate goal of producing flatter films having more uniform and reproducible characteristics.

\section{SUMMARY}

Preliminary results indicate that technical innovations can address the problem of beam-induced movement and provide improved prospects for high resolution imaging of beam-sensitive specimens. Second-generation experiments with microgrid supports are in progress with efforts focusing on the objectives of maximizing the contact between the carbon film and its microgrid support and on improving the flatness of microgrids. When more robust support films are available, they will be used in conjunction with small spot illumination

\section{THE MOLECULAR DESIGN OF MEMBRANE CHANNELS}

\section{Bing K. Jap}

PhoE porin is a member of a family of related pore-forming proteins found in the outer membrane of Escherichia coli. It is expressed under growth conditions involving phosphate-starvation. PhoE porin, similar to other members of the family, such as $\mathrm{OmpF}$ and $\mathrm{OmpC}$, possesses an aqueous channel that allows small solutes to cross the membrane. In addition, PhoE facilitates the diffusion of phosphate-containing solutes and other negatively charged compounds.

We have reconstituted purified PhoE with phospholipids, forming two-dimensional crystals. The unit cell dimensions of the crystal are $a=150 \mathrm{~A}$ and $b=130 \AA$. The are four trimers of PhoE in $a$ rectangular unit cell. The crystals diffract to a resolution better than $6 \mathrm{~A}$. This is the first case that a membrane protein, which does not naturally form two-dimensional crystalline arrays, has been reconstituted to form crystalline patches giving high reso- to reduce the effects of beam-induced movement to as low a level as possible.

\section{REFERENCES}

1. Henderson, R., and Glaeser, R.M. Quantitative analysis of image contrast in electron micrographs of beam-sensitive crystals. Ultramicroscopy 16, 139 (1985).

2. Downing, K.H., and Glaeser, R.M. Improvement in high resolution image quality of radiation-sensitive specimens achieved with reduced spot-size of the electron beam. Ultramicroscopy 20, 269 (1986).

3. Fukami, A., and Adachi, K. A new method of preparation of a self-perforated micro plastic grid and its application. Journal of Electron Microscopy 14, 112 (1965).

4. Lunsdorf, H., and Spiess, E. A rapid method to prepare perforated supporting foils for thin carbon films used in high resolution transmission electron microscopy. Journal of Microscopy 144, 211 (1986). lution diffraction information. The diffraction pattern normal to the membrane plane (Fig. 1) shows high intensities at a region corresponding to a resolution of about $10 \AA$. Since the secondary structure of PhoE is predominantly $\beta$-sheet, these high intensities suggest that the plane of the $\beta$-sheets are oriented perpendicular to the plane of the membrane.

We have also obtained the three-dimensional reconstruction of PhoE porin negatively stained with uranyl acetate. The three-dimensional map was obtained from images having tilt angles ranging \pm $60^{\circ}$. The resolution of our map is currently limited to $\sim 20 \mathrm{~A}$. The map (Fig. 2) shows that trimeric stain-filled channels form a basic unit. The threefold symmetry axis of the trimeric channel is somewhat tilted away from the perpendicular to the membrane plane. The center to center distance between channels within a trimer decreases as they 


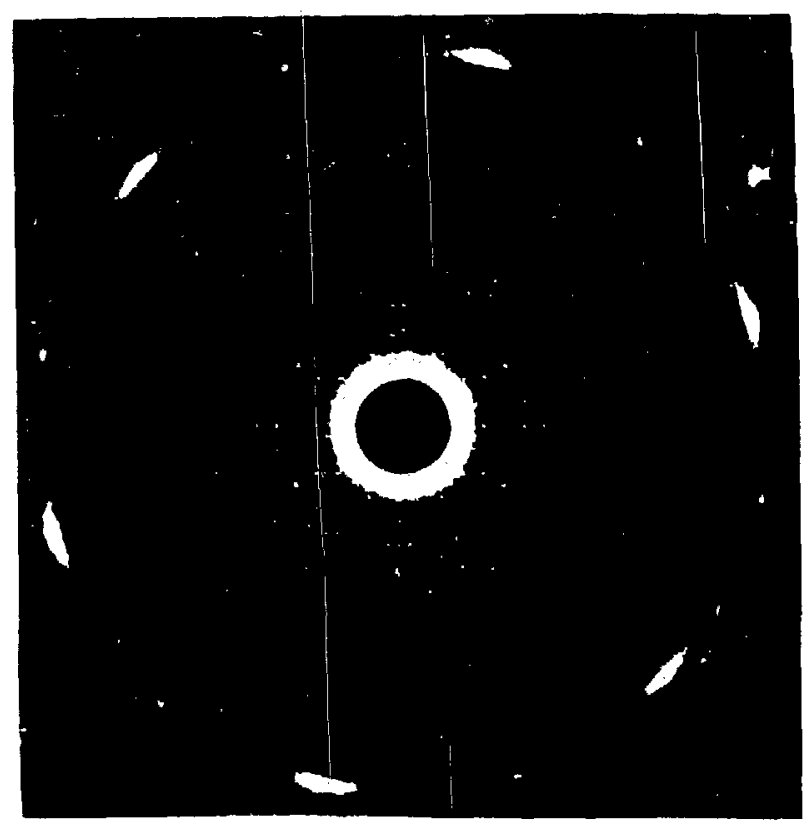

Fi. 1. High resolution diffraction pattem of our PhoE crystal. The diffraction was obtained with the electron beam normal to membrane plane. The specimen was embedded in glucose and was frozen at about $-120^{\circ} \mathrm{C}$. The diffaction pattern shows high intensities at a region corresponding to resolution of $\sim 10$ A. This indicates that the $\beta$ sheets of the PhoE secondary structure are oriented normal to the membrane plane.

(XBB 860-10256) traverse across the membrane, but, the channels do not merge.

In the three-dimensional map, the dimensions of the stain-filled channels are $\sim 35 \AA$ long and $\sim 20 \AA$ wide. Adjacent trimeric stain-filled channels are displaced with respect to each other in the direction perpendicular to the plane of the membrane. This suggests that the channels may have an extended narrower segment that is not accessible to our stained molecules. This narrower segment of the channels may play an important role in both the selectivity and gating mechanism. A high resolution map of PhoE should be able to show whether such a segment does indeed exist. Work is underway to obtain the high resolution threedimensional map of PhoE in unstained glucoseembedded samples. High resolution structural information about PhoE will provide a structural basis for understanding the regulation of passive diffusion, the selectivity towards various solutes, and the role of this protein as receptor for bacteriophages. It is possible that an understanding of the structure and function of this protein may also provide us with information concerning more effective use of antibiotics since these compounds are believed to penetrate the outer membrane by means of the aqueous channel of the porins.

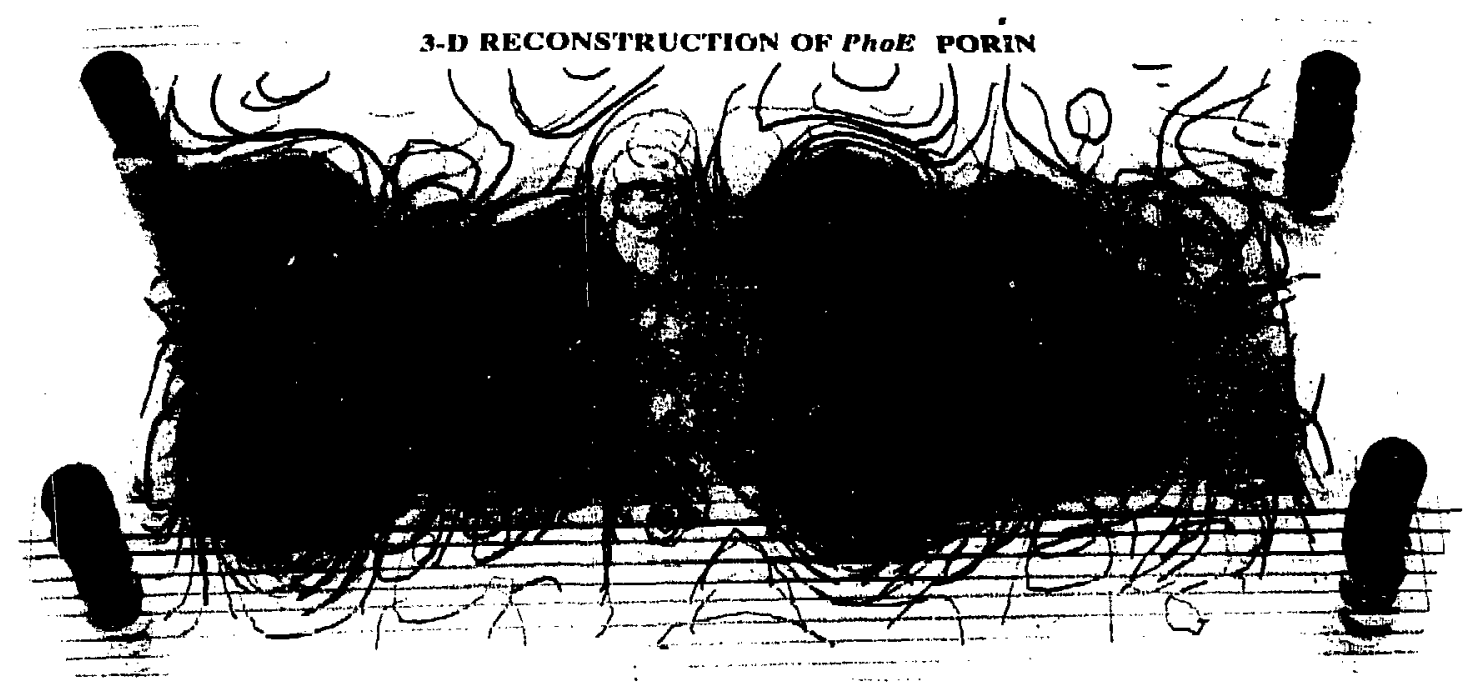

Fig. 2. Three-dimensional reconstruction of PhoE porin negatively stained with uranyl acetate. The stain-filled regions (holes) are shaded and are also indicated by dashed contour lines. Two trimeric PhoE porins are shown here. The orientation of one trimer is related to that of the adjacent one by $180^{\circ}$ rotation around an axis parallel to membrane plane. The trimeric channels of PhoE within a single trimer do not merge as they traverse the membrane.

(CBB 860-10222) 


\title{
LOW TEMPERATURE SCANNING ELECTRON MICROSCOPY OF FROZEN HYDRATED LUNG
}

\author{
Jacob Bastacky, Gregory R. Hook, Gregory L. Finch, ${ }^{\dagger}$ Jon Goerke, \\ John Clements, ${ }^{\ddagger}$ John Gilbert, ${ }^{\ddagger}$ Rob Conhaim, \\ Josephine Barr, and Thomas L. Hayes
}

The air-liquid interface of the airways and alveoli in the lung produces functionally significant surface forces. The configuration of this interface, however, is in question because electron microscope sample preparation methods generally remove tissue water. We have developed techniques to study the normal structure of mammalian lung in the frozen hydrated state with water preserved as ice, using the low-temperature scanning electron microscope (LTSEM) of Pawley and Norton. ${ }^{1}$

The lungs from anaesthetized mice are deflated to allow wide excision of the chest wall and en bloc removal of trachea, lungs and heart. The lungs are inflated and maintained at $20 \mathrm{~cm}$ water pressure with a large flow-by of room air, are plunged into liquid/solid nitrogen at $-213^{\circ} \mathrm{C}$ and stored under liquid nitrogen. Blocks of lung $5 \times 5$ $\times 10 \mathrm{~mm}$ are cut under liquid nitrogen with a tiny circular saw in a dental handpiece, mounted on a special low-temperature vise-stub and transferred to the sample preparation cryochamber (Biochamber, AMRay, Bedford, MA) integral to the SEM. ${ }^{2}$ The uncoated sample can be moved to the microscope column without breaking vacuum and examined in the secondary electron mode at $1-10 \mathrm{kV}$. To reduce charging effects and produce the pictures shown here, the lung is transferred back to the cryochamber and gold coated on a double-axis rotating cold block. The lung may be examined with a reflected-light microscope, refractured with a pivoting microtome, radiant-etched, and re-coated all within the vacuum. ${ }^{3}$ The Joule-Thomson refrigerator built into the microscope stage maintains the lung at $-180^{\circ} \mathrm{C}$ and the sample may be

\footnotetext{
"Surgical Neurology Branch, NINCDS, National Institutes of Health, Bethesda, MD.

'Inhalation Toxicology Research Institute, Lovelace Biomedical and Environmental Research Institute, Inc., Albuquerque, NM.

tCardiovascular Research Institute, University of California Medical Center, San Francisco.

'Department of Preventive Medicine, University of Wisconsin, Madison, WI.

"Mechanical Engineering Department, University of California, Berkeley, CA.
}

screened for several hours without sublimation of ice at the submicrometer level.

The pleural surface, parenchyma, bronchus, and branching airway can be seen at low magnification Fig. 1(A). At higher magnification the terminal bronchiole, alveolar duct, and alveoli continue out to the pleura in Fig. 1(B). An alveolus along this alveolar duct, Fig. $1(C)$, is cross-fractured perpendicular to its mouth. The alveolar wall, Fig. 1(D), is fully hydrated and the alveolus contains surface ice and bubbles in the aqueous subphase of the alveolar lining.

LTSEM preserves water as ice in the frozenhydrated state. Cell and tissue dimensions, configuration, and ion distribution are maintained (within the limits imposed by freezing) without the shrinkage, distortion, and ion movement of drying. ${ }^{4}$ Intraluminal cells, particles, and mucus are preserved in situ. LTSEM allows direct visualization of alveolar and airway surfaces in the lung with nothing removed. This method should be useful in elucidating the configuration of the air-liquid interface in the lung.

\section{REFERENCES}

1. Pawley, J. B. and Noton, J. T. J. Microsc. 112, 169-182 (1978).

2. Bastacky, J., et al. Am. Rev. Respir. Dis. 128, S7-S13 (1983).

3. Hook, G., et al. Proc. Ann EMSA Meeting 40, 372-373 (1982).

4. Bastacky, J., et al. Scanning $7,134-140$ (1985). 


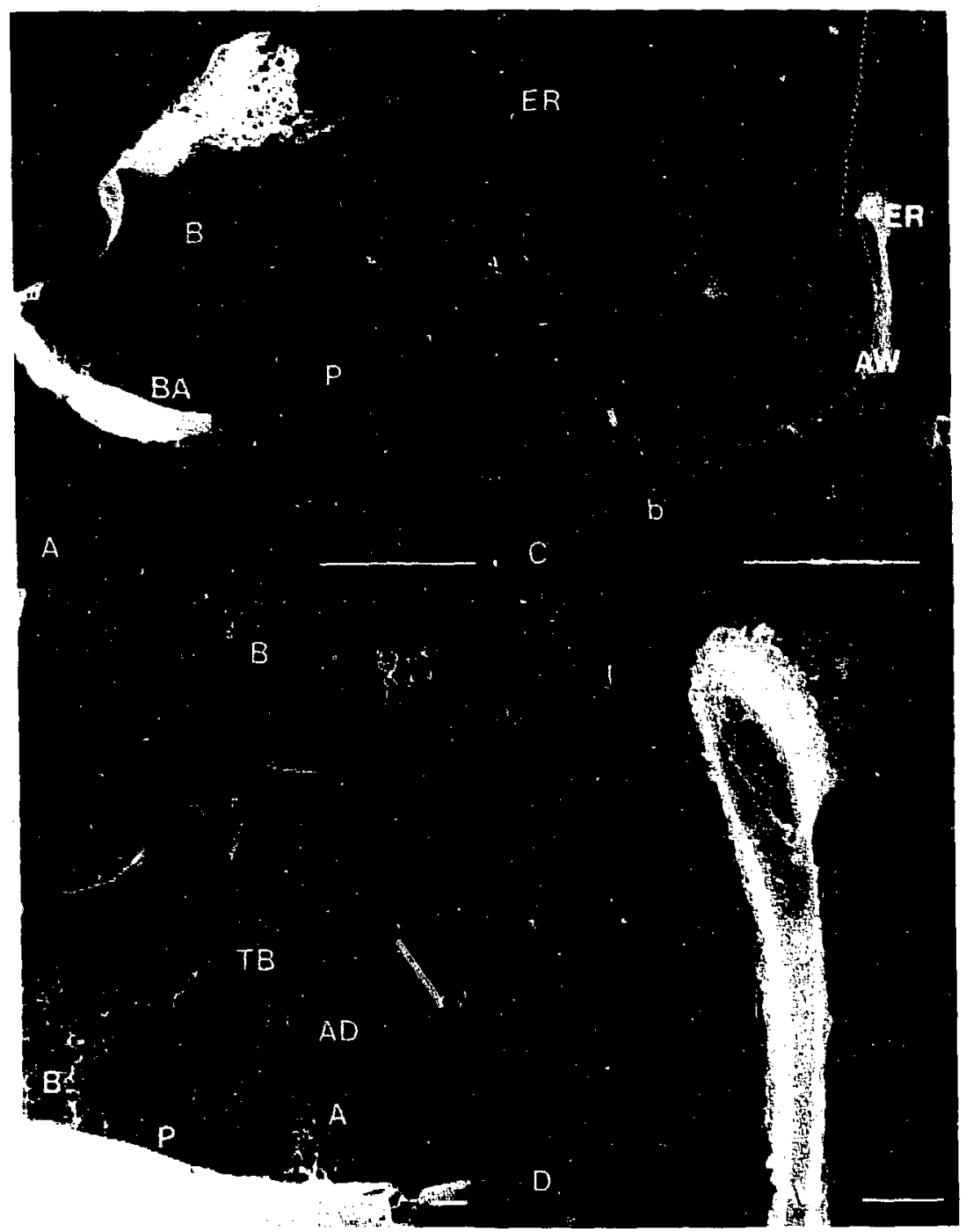

Fig. 1. Frozen-hydrated mouse lung LSEM magnification series at $10 \mathrm{kV}$.

(A) Left lung cut transversely with pleural surface (P), bronchus (B) and branching unit airway (BA). Bar $=2 \mathrm{~mm}$.

(B) Branching airway with terminal bronchiole $(T B)$ alveolar duct $(A D)$ and alveoli $(A)$. Bar $=100 \mu \mathrm{m}$.

(C) Alveolus (A) from Fig. 2 fractured perpendicular to its entrance ring showing alveolar wall (AW), entrance ring

$(E R)$ and bubbles in aqueous subphase (b). Bar $=50 \mu \mathrm{m}$.

(D) Tip of alveolar wall at entrance ring cross-fractured demonstrating glassy appearance of fully-hydrated frozen tissue. Cubic and spherical ice (I) on surface. Bar $=10 \mu \mathrm{m}$.

(XBB 867-5993A) 


\title{
HIGH VOLTAGE ELECTRON MICROSCOPY OF UNSECTIONED HUMAN LUNG ALVEOLAR WALLS
}

\author{
Jacob Bastacky, Thomas L. Hayes, David W. Ackland," Saundra Parra, ${ }^{\dagger}$ and
} Timothy Takarot

The lung presents a very thin tissue barrier $(0.2$ $\mu \mathrm{m})$ to gas exchange with alveolar walls on the order of $10 \mu \mathrm{m}$ thick. We.found the highly energetic (1.5 MeV) beam of the high voltage electron microscope (HVEM) able to penetrate whole-mount unsectioned alveolar walls. Thus, it is possible to simultaneously image the two surfaces of the wall and the internal contents of the wall. The result resembles a high-resolution scanning , electron microscope (SEM) surface image with a superimposed transmission electron microscope (TEM) image of internal structure. The composite image resembles a freeze-fracture image; however, this technique has the advantage that the specimen is still present.

Samples are prepared by routine SEM preparation protocol. Human lungs from surgery are inflated with $2.3 \%$ glutaraldehyde in $0.05 \mathrm{M}$ sodium cacodylate $(360 \pm 5 \mathrm{mOsm}$ total) to $20 \mathrm{~cm}$ water pressure and stored in fixative for a minimum of $24 \mathrm{hr}$. Blocks of lung are cut, post-fixed in 1\% $\mathrm{OsO}_{4}$ for $90 \mathrm{~min}$, rinsed in distilled water for $45 \mathrm{~min}$, post-stained in aqueous uranyl acetate for $90 \mathrm{~min}$, dehydrated through ethanol over $2 \mathrm{hr}$ and critical point dried from bone dry $\mathrm{CO}_{2}$ over $2 \mathrm{hr}$. Mounted blocks of lung are dissected, coated with platinum and examined in the SEM. Individual alveolar walls are selected, excised by hand, ${ }^{1}$ then mounted on $50 \times 50$ mesh folding TEM grids without Formvar or carbon. Septa are coated with platinum on both sides to a total of 5-40 nm coat thickness on each side in a sputter coater and imaged in the Kratos 1500 HVEM at the National Center for Electron Microscopy, Lawrence Berkeley Laboratory. Exposures of $1-20 \mathrm{sec}\left(1 \times 10^{-8}\right.$ ampere seconds) with a $750 \mu \mathrm{m}$ condenser aperture on Kodak 50163 film are developed in lowcontrast developer (D76/Dektol (1:1)). In such preparations, microvilli on the surface of type II alveolar epithelial cells can be seen in relation to the nucleus of the same cells (Fig. 1), In a broader

"Center for Electron Microscopy, (MMRD) Lawrence Berkeley Laboratory, Berkeley, CA.

'Veterans Administration Medical Center, Asheville, NC. field, the distribution of red blood cells in the capillaries of the whole-mount alveolar walls can be determined (Fig. 2).

Other investigators have used the HVEM to exarnine whole-mount, ${ }^{2-4}$ detergent extracted, ${ }^{5}$ or sectioned $^{6}$ preparations of single-cell monolayers. Sections for HVEM are generally limited to a thickness of $0.5 \mu \mathrm{m}$ to allow sorting out of nanometerlevel information throughout the section. However, if larger structures (such as vesicles, nuclei, collagen) and their spatial relations to nanometer-level surface structures are of primary interest, then considerably thicker whole-mount tissue samples can be imaged, as is demonstrated by the accompanying micrographs. With this procedure, surface resolution exceeds conventional SEM and equals highresolution SEM; internal structure resolution is less than conventional TEM.

We are interested in the biomecnanical consequences of normal alveolar structure and the earliest stages of structural damage to the alveolar wall as well as the mechanisms of surfactant production, secretion, and spreading. This HVEM technique is useful in correlating surface and internal structures of the alveolar wall.

\section{ACKNOWLEDGEMENT}

Use of the HVEM at the National Center for Electron Microscopy, Lawrence Berkeley Laboratory, supported by the U.S. Department of Energy, is gratefully acknowledged.

\section{REFERENCES}

1. Bastacky, J. et al. Am. Rev. Respir. Dis. 128, (1983) S7-S13.

2. Wolosewick, J.J. and Porter, K.R. Am. I. Anat. 147, (1976) 303.

3. Hama, K. 38th Proc. Ann EMSA Meeting (1980) 802-805.

4. Allen, C. 38th Proc. Ann EMSA Meeting (1980) 808-811.

5. Schliwa, M. 38th Proc. Ann EMSA Meeting (1980) 814-817.

6. Ris, H. 37th Proc, Ann EMSA Meeting (1979) 166-167. 


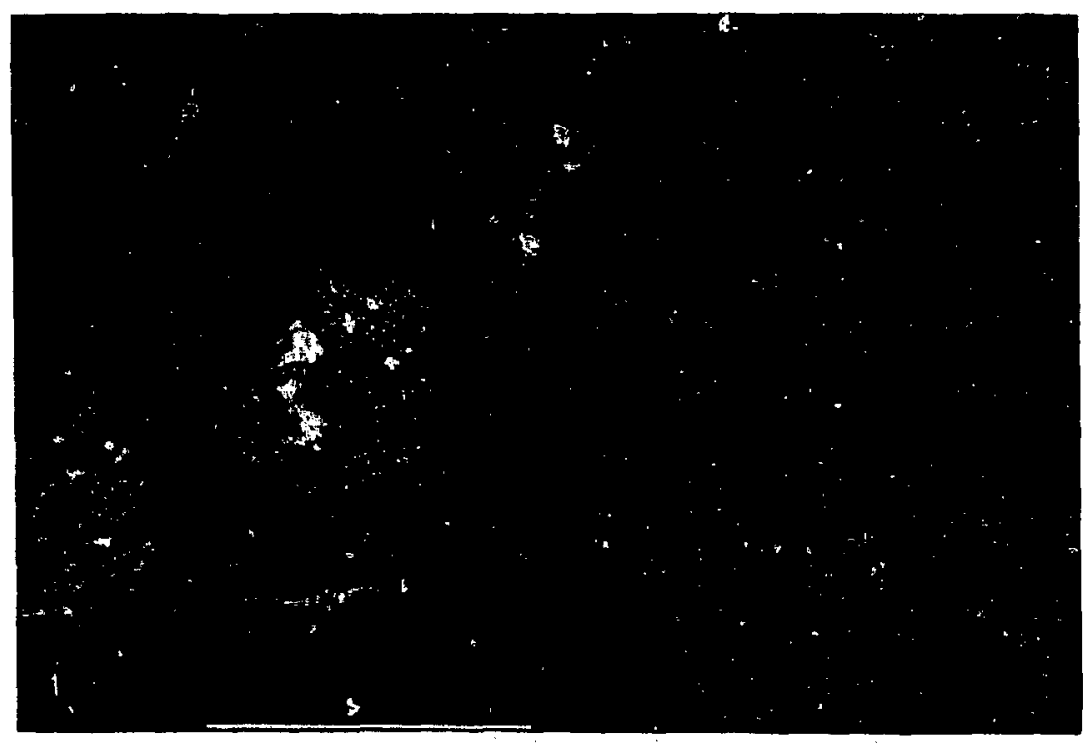

Fig. 1. HVEM stereopair of whole-mount unsectioned alveolar walls from human lung. Type II alveolar epithelial cell on surface of alveolar wall has microvilli of uniform length over its surface. Nucleus is seen within cell, red blood cell $(R)$ in capillary within alveolar wall. Epithelial surface detail of far-side of alveolar wall (arrow). Tilt \pm $3^{\circ}$ Bar $=6 \mu \mathrm{m}$.

(XBB 8611-10003)

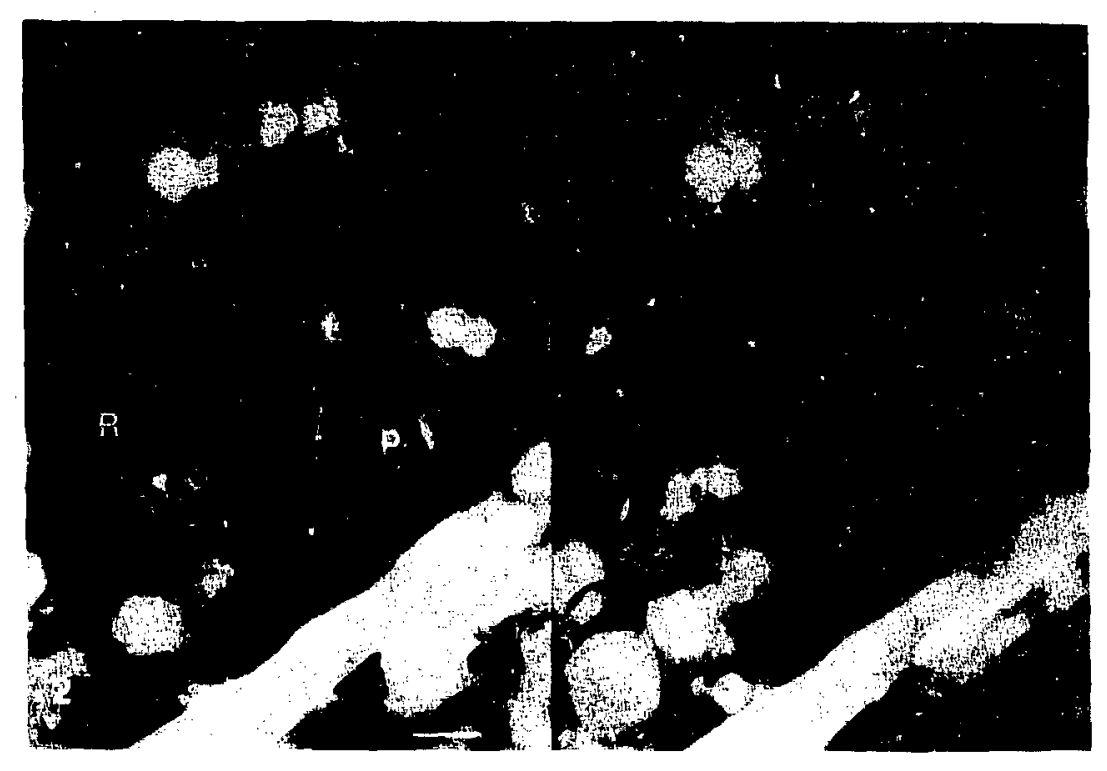

Fig. 2 HVEA stereopair of alveolar wall at lower magnification than Fig. 1, showing capillary network containing red blood ceils $(R)$, polymorphonuclear leukocyte $(P)$, lymphocyte $(L)$, and endothelial cell nucleus (e). Tilt $\pm 10^{\circ}$. Bar $=6 \mu \mathrm{m}$.

(XBB 8611-10004) 


\title{
CIRCULAR DIFFERENTIAL SCATTERING AND CIRCULAR DIFFERENTIAL ABSORPTION OF DNA PROTEIN CONDENSATES AND OF DYES BOUND TO DNA PROTEIN CONDENSATES
}

\author{
Cynthia L. Phillips," William Mickols," Marcos F. Maestre, and Ignacio Tinoco, Ir."
}

Condensed forms of DNA are found in many kinds of life, from viruses to cysts of bacteria to eukaryotes. Two of the most obvious places where condensed DNA in higher eukaryotes is observed are in chromosomes and in sperm. Several proposed model systems have been studied by various physical chemistry methods. One spectroscopically well defined form of condensed DNA is the Psi form.

Psi condensates are characterized by very large circular dichroism (CD) extinction coefficients. Light scattering is an important part of the total absorption spectrum of Psi condensates and is also an important contribution to the $C D$. $C D$ depends both on circular differential absorption and circular differential scattering. Several experimental methods have been developed to correct for circular differential scattering in $C D$ spectra in order to see primarily circular differential absorption. The scatter-corrected CD spectra of Psi DNA condensates do not look like classic uncondensed DNA $C D$ spectra. The magnitude and shape of the $C D$ spectra (composed of both circular differential absorption and circular differential scattering) and the magnitude and shape of the circular differential scattering can be independent of each other. They are often dependent on the preparation conditions. The conditions to produce Psi-type spectra include nonaqueous solvents, high salt concentration, the presence of polymers, and gelation into fibers. Small changes in preparation cause large changes in the spectra. Differences in $C D$ extinction coefficient are found from researcher to researcher and from preparation to preparation. These variable results do not impiy poor technique, but rather that the range of effects obtainable within a small range of experimental conditions is large. These results also imply that a Psi preparation is a continuum of closely related structures.

$X$-ray diffraction of fibers pulled from solutions of Psi DNA condensates show the B conformation for the secondary structure of DNA. This has lead to the proposal that the anomalous $C D$ spectra of DNA condensates are due to liquid-crystal-like

Department of Ehemistry, UCB, and Laboratory of Chemical Biodynamics, LBL packing of the DNA. Evdokimov has shown that the $C D$ spectrum of the DNA-binding drug daunomycin is altered when bound to Psi condensates. ${ }^{1}$ High concentrations of daunomycin inverts the Psi CD spectra of both the DNA and the dye. Low concentrations of daunomycin does not invert the DNA $C D$, but at all concentrations the dye mimics the DNA Psi-type spectra. Moreover the induced $C D$ of aclacinomycin-A mimics that of the Psi-type DNA spectra. However, Evdokinov also reports on several other strong intercalators that do not have Psi-type $C D$ spectra in the dye bands.

We have studied Psi condensates prepared by condensing DNA with either polylysine or poly(lysine-alanine) to produce large Psi-type uv CD spectra. Dyes with no intrinsic $C D$, and no $C D$ induced by binding to polylysine or poly(lysinealanine), were bound to the Psi condensates and found to take on the Psi spectral characteristics of the DNA. Scattering-corrected CD spectra were generated to approximate circular differential absorption spectra by using fluorscat cuvettes or by altering the acceptance angles of the detector. The intercalative dyes used (ethidium, proflavine, $\mathrm{H}_{2}$ TMpyP-4, Cu(II)TMpyP-4, and Ni(II)TMpyP-4) have different $C D$ spectra when bound to uncondensed DNA, but all have a similar $C D$ spectra when bound to Psi DNA condensates. The circular differential scattering and scattering-corrected $C D$ spectra of the intercalative dyes bound to Psi condensates mimic the circular differential scattering and scattering-corrected $C D$ spectra in the DNA bands of the condensed DNA with and without dye bound to it. Two dyes that do not intercalate in DNA do not show the mimiching $C D$ patterns when bound to DNA. The groove bindirg dye Hoechst 33342 has inverted Psi-type circular differential scattering and absorption spectra, and the groove binding dye $\mathrm{Mn}(\mathrm{III}) \mathrm{TM}$ MpyP-4 has little or no Psi-type CD spectra. Our measurements show that it is not the short-range structure (nearest-neighbor or other near-neighbor interactions) that controls the CD spectra of the dyes in Psi condensates, but rather is the long range chiral order and the relative orientation of the transition dipoles to those of the DNA. For this reason, a small amount of condensed DNA with Psi-type characteristics in a larger background of non-Psi DNA can be detected using 
several dyes and checking to see that the visible circular differential scattering and absorption spectra of the bound dyes are similar.

Further results of these studies show the Psitype $C D$ spectra are due to the condensed nucleic acid, not the peptide chromophores. Thus, condensen. ${ }^{r}$ is DNA could be detected in a complex cellular environment from the Psi-type CD spectra in the visible region that is induced by bound dyes. By the appropriate choice of dyes, this type of induced $C D$ will allow separation of a DNA signal from the RNA and protein contributions. This is important in such complex systems as the Psi conderised DNA seen in mitotic Chinese hamster ovary cells.

\section{THE PSI TYPE SIGNAL}

Our results show Psi condensates have anomalous scattering-corrected $\mathrm{CD}$ and circular differential scattering signals because of the long range chiral structure of the DNA. Our interpretation of the $C D$ behavior of the Psi condensates bound with dyes is related to recent theoretical models proposed by Keller et al. $^{2}$ (on circular differential scattering), and Kim et al. ${ }^{3}$ (on circular differential absorption). The theoretical treatment of Keller, et al. successfully predicts the circular differential scattering from a microscopic helix, and from the stacked chiral array found in cholesteric liquid crystals. The calculations are based on a finite number of polarizable groups possessing only one electronic transition between 200 and $320 \mathrm{~nm}$. Kim et al. similarly predict Psi-type circular differential absorption signals resulting from threedimensional, dense, chirally arranged pointpolarizable groups. Although a Psi condensate is considerably more complex than a simple set of point-polarizable groups, much of the theory appears to account for the spectroscopic phenomena we see.

We interpret the mimicking pattern seen in the dye band of intercalators bound to the Psi condensates as a resonance phenomenon of aligned transition dipole moments. ${ }^{3}$ Proflavine, ethidium, $\mathrm{H}_{2}$ TMpyP-4, Ni(II)TMpyP-4, and Cu(II)TMpyP-4 have transition dipole moments in approximately the same set of planes as those of the DNA bases, and are expected to occupy similar sites in any long range interaction as the bases themselves. We believe the Psi condensates have long range structural periodic repeats within an order of magnitude of the wavelength of uv or visible light. ${ }^{3}$ When the large chiral repeat structure interacts with the chiral light, it behaves as an antenna, and there is a reso- nance phenomenon at the induced dye band resulting in the large circular differential absorption and circular differential scattering. The resonance phenomenon is thought to arise in the visible spectrum due to electronic coupling of the dyes over a long range, and is not due solely to nearestneighbor or next to nearest-neighbor effects. Figure 1 is a possible model of a Psi condensate (similar to a model proposed by Schellman and Parthasarathy ${ }^{4}$ for DNA condensed with polyamines) to which intercalative dyes are bound at sites similar to those the DNA bases occupy.

The $C D$ spectra of the two ionintercalating dyes, Hoechst 33342 and Mn(III)TMpyP-4, when bound to Psi condensates, can also be explained in terms of a long range chiral structure. When the Hoechst 33342 binds in the minor groove of the Psi DNA, it has an average transition dipole well out of the plane of the DNA base pairs, so it is not expected to have a CD signal like that of the bases or intercalators. However, the Hoechst 33342 is still binding at specific sites on the DNA in the long range chirally ordered Psi condensate, and the $C D$ signals of the bound dye should reflect that long range chiral order. The bound Hoechst 33342 does report that long range chiral structure and has $C D$ spectra inverted to those observed for intrecalative dyes. Figure 1 shows how a minor groove specific dye that binds uniformly could report the long range chiral structure of a Psi condensate. We pro-

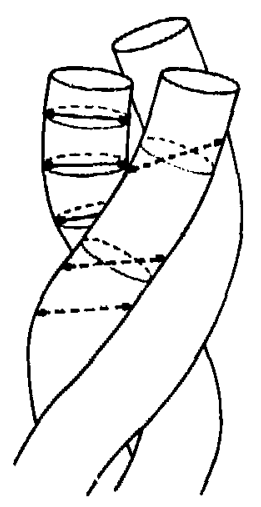

Fig. 1. Hypothetical model of a Psi condensate with bound dyes. Many strands of these DNA and polylysime or poly(lysine-alanine) groups may be closely packed in a chiral arrangement such as a helix. Planar intercalators are bound with their planes in the same set of planes as the DNA bases (line arrows represent intercalator planes). Minor groove bind. ing dyes are bound with their long axis or average transition dipole moment at an angle oul of the DNA base planes, aligned in the DNA minor groove (dashed arrows represent dipoles of minor groove binding dyes).

(XBL 866-2522) 
pose two possible explanations for why the Mn(II)TMpyP-4 has negligible CD when bound to Psi condensates. Although the dye has been shown to bind in the minor groove of DNA at the ratios we used, ${ }^{5}$ it may bind non-uniformly within the minor groove, with respect to the DNA bases, such that it cannot report a long range chiral repeat unit. This is equivalent to saying the effective polarizability tensor in the model of Kim et al. ${ }^{3}$ is spherical. Mn(II)TMpyP-4 may in fact have a specific orientation with respect to the DNA bases, but the orientation may simply give very small circular differential absorption and circular differential scattering. If there are dye orientations with respect to the DNA bases in the Psi condensates that give mimicking $C D$ spectra (such as those of intercalators), and there are dye orientations that result in inverted Psi-type spectra in the dye band, it follows that there may be dye orientations where there is no reporting of the Psi-type signal in the dye band.

\section{APPLICATIONS}

Using information on how the transition dipole moments of the bound dyes are aligned with those of the DNA bases, it may be possible to distinguish between different types of dye binding by comparing the visible $C D$ signals of dyes bound to Psi condensates with the uv CD signals of the Psi condensates. At the present, distinguishing between groove binding dyes, intercalators, and nonspecific dyes typically involves temperature jump experiments or DNA restriction fragment footprinting (in the case of some groove binders, Harshman and Dervan), ${ }^{6}$ or $x$-ray diffraction (which is conclusive, but requires crystals). With an estimate of the direction of a dye transition dipole moment, binding a dye of unknown specificity to positive and negative Psi condensates gives information on dye binding orientation. A dye that followed the $C D$ mimicking patterns of an intercalator or the inverse $C D$ patterns of a groove binder when bound to both positive and negative Psi condensates would likely be an intercalator or groove binder, respectively.

\section{REFERENCES}

1. Evdokimov, Y.M., Salyanov, V.I., Dembo, A.T., and Berg, H. Biomed. Biochim. Acta 42, 855-866 (1983).

2. Keller, D., Bustamente, C., Maestre, M. F., and Tinoco, Ir., I. Biopolymers 24, 783-797 (1985).

3. Kim, N., Ulibarri, L., Keller, D., Maestre, M. F., and Bustamente, C. J. Chem. Phys. 84(6), 2981-2989 (1986).

4. Schellman, I.A. and Parhasarathy, N. J. Mol. Biol. 175, 313-329 (1984).

5. Dabrowiak, I. Biophysical Society Conference, Lecture, Abstract \#M-PM-C3 (1986).

6. Harshman, K.D. and Dervan, P.B. Nucl. Acids Res. 13, 4825-4835 (1985). 


\section{CELLULAR AND MOLECULAR BIOLOGY}

\section{INTRODUCTION}

The Cellular and Molecular Biology Group was formed in 1983 in response to the need to bring focus to the research on carcinogenesis and mutagenesis in the Division of Biology and Medicine. The Group's major research effort continues to be the study of all aspects of carcinogenesis and mutagenesis at the cellular and molecular levels. To provide coherence to this program the Group has been divided into the four interactive subgroups shown in Table 1 with the investigators participating in each subgroup. The interactive nature of these subgroups is demonstrated by the fact that many investigators participate in more than one subgroup.

\section{REPLICATION AND RECOMBINATION}

The goal of the research program in DNA replication and recombination is to understand the processes that maintain fidelity of the genome during replication of the genome and recoinbination of the strands; for example, the mechanisms for enhancing proper alignment of strands, pairing of the bases, and recognition of mistakes, incongruities, and modifications to the template. These mechanisms involve proteins that interact, with some specificity, with single-stranded or doublestranded DNA.

Junko Hosoda, a recognized pioneer in the field of DNA binding proteins in bacteriophage, has extended her research perimeter to include eukaryotic cells, namely yeast. The work here provides evidence that a protein with affinity for single-stranded DNA possesses other activities indicative of a role in DNA replication in yeast. Because of their apparent importance, these studies are being actively pursued under the field task proposal in DNA Repair. Michael Esposito and his co-workers, who collaborate with Junko Hosoda, have utilized mutants of yeast defective in recombinant activity to identify genes involved in recombinational events. The current report identifies a gene that modulates mitotic chromosomal integrity and is essential for normal meiosis. Libby Holbrook, a postdoctoral trainee with Esposito, has been responsible for the program's efforts to purify DNA resolvase, the enzyme suggested as catalyzing the final recombinational step. Her work on this newly identified mutant indicates that deficient resolvase activity is not the explanation for the recombinational defective mutant.

A research program consisting of yeast geneticists, who can isolate and biologically characterize DNA recombination and repair defective mutants, and of protein biochemists, who can isolate and characterize the gene products chemically, has a high potential for developing new information regarding both the genes and their protein products involved in DNA transactions and the mechanisms of action.

Table t. Research affinities in the Cellular and Molecular Biology Group.

\begin{tabular}{llll}
\hline $\begin{array}{l}\text { DNA Replication } \\
\text { and Recombination }\end{array}$ & DNA Repair & Carcinogenesis & Differentiation \\
\hline P. Cooper & P. Cooper & B. Ames & J. Bartley \\
M. Esposito & R. Goth-Goldstein & J. Bartley & M. Bissell \\
I. Hosoda & R. Mortimer & M. Bissell & G. Parry \\
R. Mortimer & T. Leadon & L. Gold & M. Stampfer \\
& B. Singer & T. Leadon & R. Schwarz \\
& & R. Schwarz & \\
& & B. Singer & \\
\hline
\end{tabular}




\section{DNA REPAIR}

The goal of research in DNA repair is to understand on a molecular level the interaction between the DNA repair processes that promote survival after damage and those repair processes that enhance mutation following DNA damage. Understanding these processes is ultimately necessary for assessing the potential for carcinogenesis after long-term exposure to low levels of DNA damaging agents. The mutagenic or lethal consequences of any particular lesion in DNA depend on cellular processing of that lesion, and often these responses are facets of regulatory networks of genes induced by the damage itself. The fact that many carcinogens are mutagens, together with evidence suggesting that mutations inat activate oncogenes can result directly from DNA damage, lend impetus to these studies and ties them to the basic research in carcinogenesis that is another focus of the Group's research effort. The broad objective of the DNA studies is to understand the biochemical and molecular basis for the processing of damaged DNA and the genetic control of these repair processes.

Robert Mortimer and his co-workers have contributed greatly to our understanding of the genetic control of DNA repair by identifying and characterizing repair deficient mutants in yeast. For example, they have identified a series of yeast strains deficient in repairing irradiation-induced DNA damage, the genes involved appearing to depend upon the type of damage induced. When appropriate crosses are made between these mutants to yield a yeast strain totally deficient in DNA repair, a threesecond exposure to sunlight is lethal - a dramatic example of the everyday importance of DNA repair in eukaryotic cells. Another aspect of the work in Mortimer's laboratory is the exploitation of orthogonal field alternating gel electrophoresis (OFAGE) in support of many aspects of their work, including monitoring DNA damage and repair. The current work describes an imaginative means of using OFAGE separation of DNA from a yeast carrying one linear and one circular copy of a gene to follow the kinetics of recounbination during meiosis. These studies are producing unique insights into the intermediates produced during recombination. In their second report, Robert Mortimer and his associates provide an update on their work on yeast genes responsible for repair of double-strand breaks in DNA. Cloning of these genes followed by genetically engineered insertions between promoter sequences and the structural gene is providing basic information on the regulation of the damage inducible DNA repair process in eukaryotic cells.
Priscilla Cooper is internationally recognized for her work on the damage induced repair of DNA in $E$. coli. The induced response results in repair sequences exceeding 1500 nucleotides (long patch repair) and correlates with the recovery of DNA replication. This latter finding led to the hypothesis that long patch repair is induced in lesions at or near DNA replication forks. Cooper, who described her technique for resolving DNA fragments containing growing forks in last year's report, presents results here using this technique in confirmation of her hypothesis.

Cooper, who chose $E$. coli with its wealth of relevant mutants as a model cell system to study these responses, is currently applying the techniques, ideas and approaches developed with this system to studies with human cells, in collaboration with Tony Leadon. Tony Leadon's research in the area of DNA repair is also concerned with the relationship between the location of the damage and its repair, but from a different standpoint than that of Cooper's research. Leadon is testing the theory that factors, such as chromosomal location of damage or the transcription activity of a genome, might affect the processing of DNA damage in mammalian cells. These studies depend on molecular techniques for isolation of specific DNA sequences and on immunological isolation of the repaired sequences. Results from two different experimental approaches support Leadon's hypothesis that there is preferential repair in the more transcriptionally active regions of the genome.

\section{CARCINOGENESIS}

The nature of the events that occur when a normal cell becomes malignant, and the factors that may influence such transformations, are major areas of research in carcinogenesis by members of the Group. A broad range of questions in this area is being addressed - from investigations into basic molecular mechanisms accompanying carcinogenesis to studies that may lead to the development of practical assays for detecting potential environmental carcinogens. The different skills of the investigators in this area, as well as their close interaction, provide them and those in their laboratories with the variety of research backgrounds necessary to approach these questions; these backgrounds include organic chemistry, biochemistry, cell biology, and molecular biology.

Bea Singer's research in the area of carcinogenesis is recognized as providing a sound chemical basis for understanding the damage inflicted in DNA by carcinogens and the implications of that damage on DNA processing. The report here 
describes using polynucleotides with specific modified bases as accurate and defined models of DNA damage to study the mechanisms for recognizing and repairing specific chemical lesions in DNA. A major current effort is on the modifications induced in polynucleotides by the metabolites of vinyl chloride, a documented carcinogen in humans. The research effort of Regine Goth-Goldstein is related to that of Bea Singer in that her goal is to understand the biological sequences of alkylation damage at certain sites on the bases of DNA. Her current results indicate that the lethal effects of monofunctional alkylating agents are not necessarily related to the persistence of lesions in DNA. Goth-Goldstein's current objective is to determine the molecular and/or biochemical bases for the hypersensitivity to the effects of these chemicals in certain strains of human cells. The conclusions from these studies will likely increase our understanding of the mechanism for chemical induction of lethal versus mutagenic/carcinogenic damage.

Mina Bissell was the first to suggest and document that viral carcinogenesis is a multistep process not unlike that proposed for chemical carcinogenesis. The three reports here are parts of a cohesive effort to understand these stages and their interactions at the molecular level. These reports indicate that the differentiated state of a cell infected with a carcinogenic virus can modulate transformation of that cell. The basis for this modulation may be the expression of specific cellular gene products that are substrates for viral enzymes. Research such as this contributes greatly to a basic understanding of carcinogenesis and provides insights into means of intervening in the carcinogenic process.

In contrast to other major cancers in humans, our understanding of breast cancer is not sufficient to recommend changes in life style, diet, smoking, or sexual practices that are likely to lead to a reduced risk. Martha Stampfer addressed this need by developing a means of growing normal human breast epithelial cells in culture. Her success, in collaboration with lack Bartley, led to the first evidence that these cells actively convert environmental carcinogens to the form that modifies DNA and to the first demonstration that carcinogen treatment in culture can produce transformed, immortal cells. This cell system provides an appropriate basis for the many types of studies needed to better understand induction of breast cancer, and, to this end, cells are being provided to laboratories all over the world. Stampfer's current efforts are focused on the role of perturbations in normal cellular regula- tion on the carcinogenic process and, in this report, on a possible role of regulatory genes shown to be homologous to viral oncogenes.

One of the questions remaining unanswered in breast cancer is the causative factors. Because of the general consensus that carcinogenesis involves modifications in DNA, Tony Leadon, in collaboration with Jack Bartley and Martha Stampfer, initiated studies on a possible role of oxidative damage to DNA from chemical carcinogens and, in collaboration with Tracy Yang, from irradiation. The results demonstrate for the first time the quantitative importance of oxidative damage in DNA of breast epithelial cells treated with chemical carcinogens. The use of radical scavenges and of chemicals that produce oxidative damage but not DNA adducts will help clarify which DNA lesion is related to carcinogenesis. A precise relationship between DNA modification and carcinogenesis awaits development of a rapid transformation assay so that the carcinogenic potential of various DNA lesions and the protective effect of various chemicals can be quantified. Development of such an assay is the objective of Stampfer's field task proposal and provides the impetus, in part, for the studies reported by Bartley and co-workers on the identification of markers for the differentiated state of mammary epithelial cells. The rationale is that some normal markers might be expressed in transformed cells under nonpermissive conditions.

\section{DIFFERENTIATION}

Studies with many cell types have indicated that malignant transformation involves perturbations in normal cellular function and in the pathway of cellular maturation and terminal differentiation. The unifying concept of the work in this area is to determine how changes in expression of normal cell differentiation and maturation are related to the carcinogenic process. Special emphasis is being placed on the role of cell-matrix interactions, generation of polarity in epithelial cells, and hormonal regulation in organ and tissue specific gene expression. Where pos.ible, the regulation of function in culture is compared and contrasted to regulation in the organ or tissue of origin in vivo. The cell systems in use are mouse and human mammary epithelial cells and primary avian tendon cells.

Jack Bartley's research, in collaboration with Martha Stampfer, with human mammary epithelial cells in culture has demonstrated that the expression of differentiated function in these cells can be modulated by the cul:ture medium to a state 
apparently comparable to that of early prelactation; and that, in contrast to rodent mammary cell systems, this modulation takes place on plastic without the addition of extracellular matrix material. We have preliminary evidence that cells in a totally optimized serum-free medium lay down sufficient extracellular matrix to allow expression of lactational genes when the cells are fed with the medium that induces prelactation. These studies on gene expression in normal cells are being carried out in parallel in the transformed cell lines in an attempt to test for a relationship between transformation and differentiation in the human mammary epithelial cells.

In his studies reported here on regulation of mammary gland differentiation, Gordon Parry is using a set of monoclonal antibodies to milk-fat globule membrane to follow kinetically the assembly of milk-fat globules and, thereby, of apical surface membrane in rodent mammary cells in culture. These studies will not only supply basic information with respect to normal regulation of membrane assembly but also provide a groundwork for understanding the expression of membrane components on the surface of malignant cells.

Mina Bissell, in a review with Gordon Parry and Glenn Hall, first coined the term dynamic reciprocity to describe accurately and graphically the relationship between a cell and its extracellular matrix. Here she and her current associates focus on the influence of specific extracellular components on the regulation of gene expression in mammary epithelial cells. They demonstrate not only that this reciprocity functions in vivo but also that the modulation occurs at the level of translation. Their subsequent aim is to dissect the mechanism(s) by which information is transmitted between the cell and the extraceilular matrix.

Rick Schwarz, like Martha Stampfer, Gordon Parry, Mina Bissell and Jack Bartley, is also interested in the relationship between normal differentiation and carcinogenesis, but in primary avian tendon cells. These cells provide an excellent model in that their differentiated function is directed toward synthesis of a single protein, collagen I, and they can be virally transformed. His studies on regulation of differentiation have shown that ascorbate induces PAT celis to increase their procollagen production 6-fold. This ability of a reducing agent to specifically stimulate procollagen synthesis is possible only because most steps (secretion, translating, transcription) in the pathway for collagen are tightly coupled beginning with secretion. In contrast, a high concentration of serum in the growth medium decreases collagen syrithesis and does so, as shown here, by disrupting this tight coupling. Serum does not affect the effect of ascorbate on secretion but does influence the rate of translation of DNA into RNA. Understanding the mechanisms for modulating collagen synthesis in tendon cells ultimately has implications in such areas as normal growth and development, sports medicine, and aging (the procollagen output from a tendon cell drops 50-fold with aging).

The Group has been active in areas other than research but nonetheless important to the vitality of the research effort in life sciences at LBL. Martha Stampfer is serving on the Panel to Explore New Directions (PEND) and is chairing the subpanel for Life Sciences. The subpanel's work was a factor in bringing about the highly successful Life Sciences Retreat in Napa last Spring. Their report and subsequent activities are likely to have an impact on the research initiatives and programs in life sciences at LBL for years to come. Group members have been major contributors to the development of programs in biotechnology and bioprocessing. Priscilla Cooper has coordinated the efforts to develop a training program under the auspices of the University of California Biotechnology Research and Education Program. Jack Bartley assisted Alex Quintanilhis of the Applied Science Division in preparing a proposal to the same UC program for a workshop on bioprocessing. This proposal was funded, and participants in the workshop included representatives from several of the biotechnology companies, most of the UC campuses, the LBL Divisions of Applied Science, Engineering, Chemical Biodynamics, and Biology and Medicine. Almost all the members of the Group participated in this program and were instrumental in developing the report of the workshop. A more detailed and coherent program is being developed from the information and concepts presented at the workshop. The next step is to develop sufficient funding for this expanded program. 


\title{
DNA Replication and Recombination
}

\section{THE CRO1 GENE OF SACCHAROMYCES CEREVISIAE CONTROLS MITOTIC CROSSING OVER, CHROMOSOMAL STABILITY AND SPORULATION}

\author{
Michael S. Esposito, Dimitrios T. Maleas, Kathleen A. Bjornstad, and \\ Libby L. Holbrook
}

In this report we describe the properties of a novel temperature-sensitive recombinationdefective mutant of Saccharomyces cerevisiae, cro1-1. The cro1-1 mutant is the first instance of a rec mutation that reduces drastically the rates of spontaneous mitotic crossing-over events but not those of gene conversional events. The crol-1 mutation thus provides evidence that mitotic crossing-over is dependent upon gene products that are not essential for gene conversional events. The cro1-1 mutation also results in enhanced mitotic chromosomal instability and MATa/MATa cro11/cro1-1 mutants are sporulation deficient. These phenotypes indicate that the $C R O 1$ gene modulates mitotic chromosomal integrity and is essential for normal meiosis. The cro 1-1 mutant possesses Holliday junction resolvase activity, hence its recombinational defect does not involve failure to execute this putative final recombinational step.

\section{PROPERTIES OF THE cro1-1 MUTANT}

The cro1-1 mutant was recovered among survivors of ultraviolet-light-induced mutagenesis of an $n+1$ disomic strain, LBL1 (Table 1), ${ }^{1,2}$ This strain permits simultaneous monitoring of spontaneous gene conversion (e.g., $\mathrm{CYH}{ }^{5}$ to $c y h 2^{r}$ ), intergenic mitotic crossing-over (e.g., in the interval $\mathrm{CrH}$ TRP5) and chromosomal stability (i.e., restitution of haploidy due to chromosomal breakage and loss, or mitotic nondisjunction). The temperature sensitive recombinational defect of the crot-1 strain is demonstrated in Table 2. At $36^{\circ} \mathrm{C}$ the cro1-1 strain (Rec490) exhibits a tenfold reduced level of mitotic intergenic recombinants when compared with the LBL1 parental control strain and has little effect upon conversional events. During growth at $36^{\circ} \mathrm{C}$ the cro1-1 strain also exhibits a tenfold higher level of restitution of haploidy, i.e., enhanced chromosomal instability (Table 3 ).

MITOTIC RECOMBINATION IN cro 1-1/cro1-1 MATa/MATa HYBRIDS

CRO1/CRO1, CRO1/cro1-1, and crol-1/crol-1 hybrids (Table 1) having chromosome VII genotypes that allow one to distinguish red $\mathrm{Cyh}^{r}$ mitotic gene convertants from white Leu ${ }^{+} \mathrm{Trp}^{+} \mathrm{Cyh}^{\mathrm{r}}$ mitotic intergenic recombinants were analyzed further. The hybrids were also heteroallelic at the MET13 (chro-

Table 1. Principal strains employed."

\begin{tabular}{|c|c|c|}
\hline \multirow{2}{*}{$\frac{\text { Strain }}{\text { LBLt }}$} & \multicolumn{2}{|r|}{ Genotype } \\
\hline & MATAa & ade5 metr3-c cyhr trp5 LEUT ade6 cly8 \\
\hline & & ADE5 met13-d CYH2' TRP5 leul ADE6 CLY8 \\
\hline & & ade2-1 ura3-1 CANI \\
\hline \multirow[t]{2}{*}{ NLBLt } & MATa & ade5 metli-c cyh2 trp5 LEUI ade6 clyB \\
\hline & & ade2-1 ura3-1 CANI \\
\hline \multirow[t]{2}{*}{ NLBL3 } & $M A T \alpha$ & ADE5 met13-d CYH2 TRP5 leul ADE6 CLYB \\
\hline & & his $7-2$ lyrl-1 lys2-1 \\
\hline
\end{tabular}

- The Rec490 strain carrying the crol-1 mutation has the same residual genotype as LBLI. CROI/CROI, CROI/Crol-1, and crol-1/crol-1 diploid hybrids have the residual genotype obtained by intercrossing NLBLI and NLBL3. 
Table 2. Conversion and intergenic recombination in MATa $n+1$ chromosome VII disomics at $24^{\circ} \mathrm{C}$ and $36^{\circ} \mathrm{C}$.

\begin{tabular}{|c|c|c|c|c|c|}
\hline \multirow[b]{2}{*}{ Strain } & \multirow[b]{2}{*}{ Temp. } & \multicolumn{2}{|c|}{ Leu ${ }^{*} \operatorname{Trp}^{*}$ Cyh $^{\prime}$} & \multirow{2}{*}{$\begin{array}{l}\text { Total } \\
\text { Cyh' }\end{array}$} & \multirow{2}{*}{$\begin{array}{l}\text { Number of } \\
\text { Experiments }\end{array}$} \\
\hline & & Red Convert. & White Inter. Rec. & & \\
\hline LBL1 & $24^{\circ} \mathrm{C}$ & $70 \times 10^{-7}$ & $138 \times 10^{-7}$ & $159 \times 10^{-6}$ & 25 \\
\hline $\operatorname{Rec} 490$ & $24^{\circ} \mathrm{C}$ & $116 \times 10^{-7}$ & $57 \times 10^{-7}$ & $548 \times 10^{-6}$ & 5 \\
\hline LBLI & $36^{\circ} \mathrm{C}$ & $186 \times 10^{-7}$ & $998 \times 10^{-7}$ & $463 \times 10^{-6}$ & 5 \\
\hline $\operatorname{Rec} 490$ & $36^{\circ} \mathrm{C}$ & $97 \times 10^{-7}$ & $149 \times 10^{-7}$ & $59 \times 10^{-6}$ & 5 \\
\hline
\end{tabular}

- Median Frequencies.

b Total Cyh' segregants at $24^{\circ} \mathrm{C}$ include convertants, intergenic recombinants, and non-recombinant resistant haploids. Total Cyh' segregants at $36^{\circ} \mathrm{C}$ do not include non-recombinant haploids because these carry cly 8 and do not grow at $36^{\circ} \mathrm{C}$.

Table 3. Restitution of monosomy for chromosome VII during growth of MATa $n+1$ chromosome VII disomics at $36^{\circ} \mathrm{C}$.

\begin{tabular}{lccccc}
\hline Strain & $\begin{array}{c}\text { No. cultures } \\
\text { tested }\end{array}$ & $\begin{array}{c}\text { Total no. haploids } \\
\text { recovered }\end{array}$ & $\begin{array}{c}\text { Total no. } \\
\text { colonies tested }\end{array}$ & $\begin{array}{c}\text { Average frequency } \\
\text { of haploids }\end{array}$ & $\begin{array}{c}\text { Median frequency } \\
\text { of haploids }\end{array}$ \\
\hline LBL & 5 & $4^{\mathrm{b}}$ & 1053 & $3.80 \times 10^{-3}$ & $<0.50 \times 10^{-2}$ \\
Rec490 & 5 & $16^{\mathrm{c}}$ & 333 & $4.80 \times 10^{-2}$ & $6.10 \times 10^{-2}$ \\
\hline \hline
\end{tabular}

- Five cultures of each strain grown at $36^{\circ} \mathrm{C}$ in glucose nutrient were diluted and plated on synthetic complete medium and incubated at $36^{\circ} \mathrm{C}$. Haploid segregants (ADE5 met13-d CYH2 TRP5 leu1 ADE6 $C L Y B$ ) initially detected as Red Leu- segregants were crossed to test strains to confirm their haploid status. The segregants reflect loss of the cly 8 bearing copy of chromosome VII. Loss of the CLYB bearing chromosome during growth at $36^{\circ} \mathrm{C}$ cannot be detected because $c / y 8$ segregants fail to grow at $36^{\circ} \mathrm{C}$.

b All recovered from the same culture.

c Recovered from four of five cultures.

mosome VII), LYS2, TYRI, and HIS7 loci (chromosome II) and heterozygous at the CANt locus (chromosome V). Heteroallelic recombination at the MET13, LYS2, TYR1, and HIS7 loci results in $\mathrm{Met}^{+}, \mathrm{Lys}^{+}, \mathrm{Tyr}^{+}$and $\mathrm{His}^{+}$prototrophs that arise almost exclusively ${ }^{3,4}$ by gene conversional events. Can' segregants result principally from mitotic crossing-over, chromosomal loss and gene conversion. The results summarized in Table 4 demonstrate that at $36^{\circ} \mathrm{C}$ mitotic crossing-over is drastically diminished in the crol-1/crol-1 strain while conversional events are virtually unaffected.

The cro1-1 mutation confers sporulation (meiosis plus ascospore formation) deficiency at $36^{\circ} \mathrm{C}$ implicating the CRO1 gene in some aspect of meiotic chromosomal dynamics (Table 5).

\section{IN VITRO RESOLUTION OF HOLLIDAY JUNCTIONS}

The crol-1 mutant is defective in in vitro recombination at $36^{\circ} \mathrm{C}^{5}$ We tested cro $1-1$ mutant cells grown at $36^{\circ} \mathrm{C}$ for presence of Holliday junction resolution activity ${ }^{6,7}$ since this resolvase activity may be the final step in production of cross-overs. The cro1-1 strain exhibits resolvase activity (Fig.1) thus its recombinational defect is not ascribable to inability to cleave Holliday junctions.

\section{REFERENCES}

1. Esposito, M.S., Maleas, D.T., Bjornstad, K.A., and Bruschi, C.V. Simultaneous detection of changes in chromosome number, gene 
Table 5. Temperature sensitive sporulation of MATa/MATa crol-1/cro1-1 diploids.

\begin{tabular}{|c|c|c|c|c|}
\hline Genotype & $\begin{array}{l}\text { Temper- } \\
\text { ature }\end{array}$ & $\begin{array}{c}\% \text { four- } \\
\text { and } \\
\text { three-spored } \\
\text { asci }\end{array}$ & $\begin{array}{c}\% \text { two- } \\
\text { and } \\
\text { one-spored } \\
\text { asci }\end{array}$ & $\begin{array}{c}\% \text { Total } \\
\text { asci }\end{array}$ \\
\hline CROI/CRO1 & $\begin{array}{l}24^{\circ} \mathrm{C} \\
36^{\circ} \mathrm{C}\end{array}$ & $\begin{array}{l}72.2 \\
61.8\end{array}$ & $\begin{array}{l}19.5 \\
21.6\end{array}$ & $\begin{array}{l}91.7 \\
83.4\end{array}$ \\
\hline CRO1/crol-1 & $\begin{array}{l}24^{\circ} \mathrm{C} \\
36^{\circ} \mathrm{C}\end{array}$ & $\begin{array}{l}84.0 \\
67.2\end{array}$ & $\begin{array}{r}5.6 \\
15.4\end{array}$ & $\begin{array}{l}89.6 \\
82.6\end{array}$ \\
\hline crol-1/crol-1 & $\begin{array}{l}24^{\circ} \mathrm{C} \\
36^{\circ} \mathrm{C}\end{array}$ & $\begin{array}{r}70.0 \\
0.3\end{array}$ & $\begin{array}{r}20.2 \\
0.3\end{array}$ & $\begin{array}{r}92.2 \\
0.6\end{array}$ \\
\hline
\end{tabular}

Fig* 1. Resolvase Activity in LBLI and Rec490 strains grown at $36^{\circ} \mathrm{C}$. Cell-free extracts were prepared and assayed, as described by Holbrook, L.L., et al., ${ }^{6}$ from $L B L 1$ and Rec490 strains grown at $36^{\circ} \mathrm{C}$. Lane 1 - ColEI supercoiled DNA. Lane 2 - ColEI DNA linearized by Eco R1. Lane 3-ColEI supercoiled DNA treated with LBLI extract. Lane 4 - ColEl supercoiled DNA treated with Rec490 extract. Resolvase cleaves the Holliday junction present in the cruciform structure extruded by supercoiled ColEt circular plasmids. The product of this reaction is a linearized monomer, i.e., L. OC = open circular DNA; $L=$ linear monomer DNA; $S C=$ supercoiled circular DNA.

(XBB 860-8351)

conversion and intergenic recombination during mitosis of Saccharomyces cerevisiae: Spontaneous and ultraviolet light induced events. Curr Genet. 6, 5-11 (1982). 
2. Esposito, M.S., Hosoda, J., Golin, J., Moise, H., Bjornstad, K.A., and Maleas D.T. Recombination in Saccharomyces cerevisiae: RECgene mutants and DNA-binding proteins. Cold Spring Harbor Symp. Quant. Biol. 49, 41-48 (1984).

3. Esposito, M.S. Molecular mechanisms of recombination in Saccharomyces cerevisiae: Testing mitotic and meiotic models by analysis of hypo-rec and hyper-rec mutations. In: Controlling Events in Meiosis, (Eds. C.W. Evans, H.G. Dickinson). Symp. Soc. Exp. Biol. The Company of Biologists Ltd., Scarborough, North Yorkshire, 38, 123-159 (?984).

4. Esposito, M.S., Maleas, D.T., Bjornstad, K.A., and Holbrook, L.L. The REC46 gene of Saccharomyces cerevisiae controls mitotic chromosomal stability, recombination and sporulation: Cell-type and life cycle stage-specific expression of the rec46-7 mutation. Curr Genet. 10, 425-433 (1986).

5. Symington, L.S., Morrison, P.T., and Kolodner,
R. Genetic recombination catalyzed by cellfree extracts of Saccharomyces cerevisiae. Cold Spring Harbor Symp. Quant. Biol. 49, 805-814 (1984).

6. Holbrook, L.L., Bjornstad, K.A., Maleas, D.T., and Esposito, M.S. In vitro resolution of $\mathrm{Hol}-$ liday junctions by cell-free protein extracts of Saccharomyces cerevisiae: Resolvase activity of haploid and diploid cells. In Biology and Medicine Annual Report 1985, 189-194, Lawrence Berkeley Labortory report LBL20345 (1986).

7. Hosoda, J., Holbrook, L.L., Moise, H., Bjornstad, K., Maleas, D., and Esposito, M.S. Fractionation of DNA metabolic proteins of Saccharomyces cerevisiae by DNA cellulose chromatography: SSB-1, SS-DNA dependent ATPase, DNA polymerase, DNA primase, topoisomerase 1 , and resolvase. In The Upjohn/Labatt Symposium on the Biochemistry and Molecular Biology of Industrial Yeasts (eds. G. Stuart and R. Klein). CRC Press, Boca-Raton, Florida (in press).

\section{PARTIAL PURIFICATION OF HOLLIDAY JUNCTION RESOLVASE ACTIVITY FROM SACCHAROMYCES CEREVISIAE}

Libby Litzenberger Holbrook, Kathleen A. Bjornstad, Dimitrios T. Maleas, and Michael S. Esposito

Toward the goal of understanding genetic recombination on a molecular level, we are focusing on the essential final step, resolution of the Holliday junction intermediate. This report describes progress in the purification and characterization of this activity from the yeast Saccharomyces cerevisiae.

Resolvase activity is assayed by conversion of cruciform-containing supercoiled PBR322 dimer DNA or cruciform-containing supercoiled ColE1 DNA to linear forms by cleavage at the base of the cruciform. (Maps of these plasmids are given in Fig. 1.) The cruciform is topologically analogous to the Holliday junction formed during genetic recombination. Resolvase activity, which can be detected in cell-free extracts prepared from haploid and diploid mitotic cells, is dependent on the presence of magnesium, is independent of ATP, and the reaction is inhibited by concentrations of sodium or potassium chloride of $100 \mathrm{mM}$ or greater." Linear DNA is not a substrate for this activity (Fig. 2).
For development of a purification protocol, we used the protease-deficient haploid strain B/2168 (MATa prb1 pep4 prc1) constructed by Dr. Elizabeth $W$. Jones of Carnegie-Mellon University. The strain was grown in a fermentor at $30^{\circ} \mathrm{C}$ to a cell density of $8 \times 10^{7} / \mathrm{ml}$. Cells were harvested and stored in Buffer $A(50 \mathrm{mM}$ Tris- $\mathrm{HCl}, \mathrm{pH}$ $7.5,1 \mathrm{mM}$ EDTA, $10 \%$ sucrose) at $-70^{\circ} \mathrm{C}$. Protease inhibitors $(1 \mu \mathrm{g} / \mathrm{ml}$ antipain, $1 \mu \mathrm{g} / \mathrm{ml}$ chymostatin, $1 \mu \mathrm{g} / \mathrm{ml}$ leupeptin, $2 \mu \mathrm{g} / \mathrm{ml}$ pepstatin $\mathrm{A}, 2$ $\mu \mathrm{g} / \mathrm{ml}$ soybean trypsin inhibitor, $1 \mathrm{mM}$ benzamidine, $1 \mathrm{mM}$ sodium bisulfite) and dithiothreitol (1 $\mathrm{mM})$ were added and a $25-\mathrm{ml}$ aliquot $(12.5 \mathrm{~g})$ of frozen cells was disrupted in a modified Eaton press under 8000 psi. All subsequent steps were performed at $4^{\circ} \mathrm{C}$. Cell debris was pelleted $(14,000$ rpm, $45 \mathrm{~min}$, SS34 rotor) and nucleic acids were precipitated by treatment of the cleared extract with $0.8 \%$ Polymin $\mathrm{P}(\mathrm{pH} 8.1$ ) for 30 minutes followed by centrifugation $(10,000 \mathrm{rpm}, 20 \mathrm{~min}, \mathrm{SS} 34$ rotor). The supernatant fluid was slowly brought to 


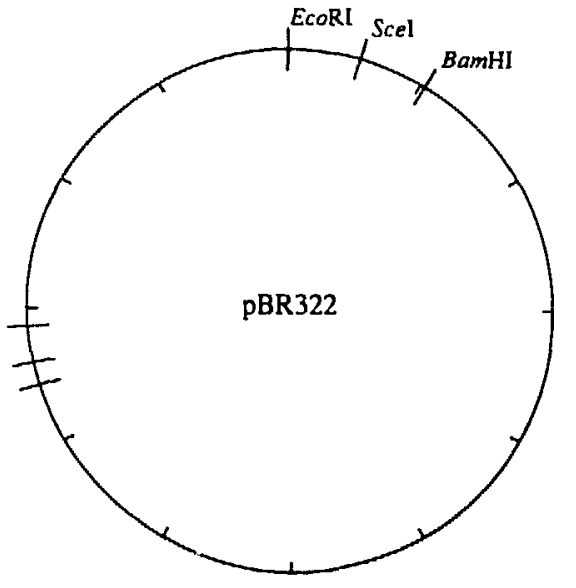

a

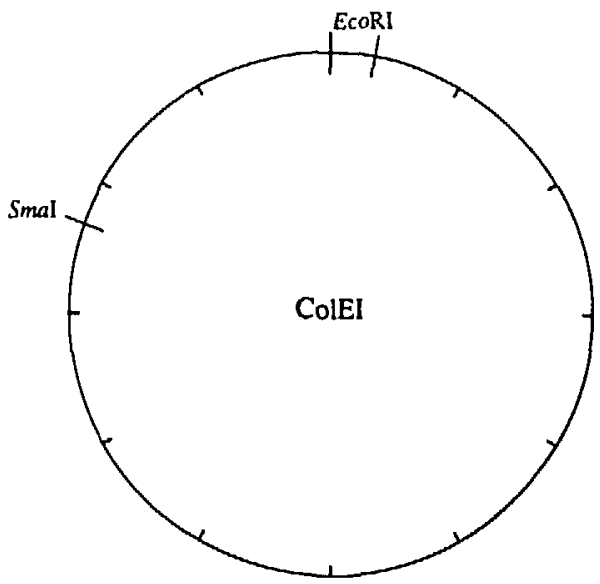

b

Fig. 1. Plasmids used. (a) Map of pBR322 (4362 bp) showing sites of cleavage by EcoRI (0), Scel (146), BamHI (375) and sites of three inverted repeats that are sensitive to 51 nuclease $(3065,3123,3221)$ and presumably to resolvase when extruded to form cruciforms. Restriction sites are based on the DNA sequence from Sutcliffe." (b) Map of ColEl (6646 bp) showing sites of cleavage by EcoRI (O) and Smal (5353) and the site of the inverted repeat that is sensitive to $S 1$ nuclease ${ }^{3}(100)$ and presumably to resolvase when extruded to form a cruciform. Restriction sites are based on the DNA sequence from Chan et al.s

(XBL 8610-9015)

2.4 $\mathrm{M}$ ammonium sulfate and stirred for 1 hour. The pellet was collected by centrifugation $(10,000$ rpm, $30 \mathrm{~min}, \mathrm{~S} S 34$ rotor), resuspended in buffer $B$ (20 mM Tris-HCl, pH 7.5, $1 \mathrm{mM}$ EDTA, 10\% glycerol) containing protease inhibitors and dithiothreitol as above, dialyzed versus the same, and applied to a column containing phosphocellulose pre-equilibrated in the same buffer. The column was washed with 4 volumes of buffer and eluted with a linear gradient of $O$ to $1 \mathrm{M} \mathrm{NaCl}$. Resolvase activity eluted with $0.3 \mathrm{M} \mathrm{NaCl}$. (A double strand restriction enzyme, most likely $\left.S c e\right|^{2}$ eluted with $0.9 \mathrm{M} \mathrm{NaCl}$ shown in Fig. 3.) The active fractions were pooled, concentrated by precipitation with $3 \mathrm{M}$ ammonium sulfate, resuspended in and dialyzed versus buffer B containing $1.2 \mathrm{M}$ ammonium sulfate and $1 \mathrm{mM}$ mercaptoethanol, and applied to a column containing octyl sepharose pre-equilibrated in the same buffer. The column was washed with starting buffer and eluted with a linear gradient of 1.2 to $0 \mathrm{M}$ ammonium sulfate. Resolvase activity eluted at approximately $50 \mathrm{mM}$ ammonium sulfate. Active fractions were pooled, concentrated to $400 \mu \mathrm{l}$ by vacuum dialysis, and stored at $-70^{\circ} \mathrm{C}$.

The partially purified enzyme was tested for activity toward several DNA substrates in Buffer $C$ (10 $\mathrm{mM}$ Tris- $\mathrm{HCl}, \mathrm{pH} 7.5,10 \mathrm{mM} \mathrm{MgCl}, 1 \mathrm{mM}$ dithiothreitol, $0.1 \mathrm{mg} / \mathrm{ml}$ bovine serum albumin). The fraction is active on pBR322 supercoiled dimer DNA producing linear dimer DNA (Fig. 4). Once resolvase cleaves at a cruciform, the DNA becomes relaxed, other cruciforms are no longer stabilized by supercoiling, and no further cutting by resolvase will occur. On the other hand, a sequence specific restriction enzyme will cut supercoiled or linear dimer to linear monomer. No linear monomer DNA is produced by resolvase, indicating the preparation is free of restriction enzyme activity. The resolvase preparation also cleaves ColE1 supercoiled DNA to linear DNA (Fig, 5). Resolvase presumably cuts each DNA at the site of an extruded cruciform independent of its sequence.

Nuclease S1 also cleaves at cruciform structures, but at the single strand loop. Therefore, it is important to demonstrate the lack of S1-type activity in a preparation of resolvase. No single strand endonuclease activity was detected toward $\phi \times 174$ virion DNA (Fig. 6), indicating the preparation is free of nuclease S1-type activity.

A double digest with 5 mal was done in order to map the site of cleavage on ColE1 by resolvase. The Smal site is $1.4 \mathrm{~kb}$ from the site of the extruded cruciform. ${ }^{3}$ When ColE1 is cleaved by nuclease $\mathrm{S} 1\left(37^{\circ} \mathrm{C}, \mathrm{pH} 4.6\right)$ followed by $S$ mal, the expected fragments of sizes $1.4 \mathrm{~kb}$ and $5.25 \mathrm{~kb}$ are 


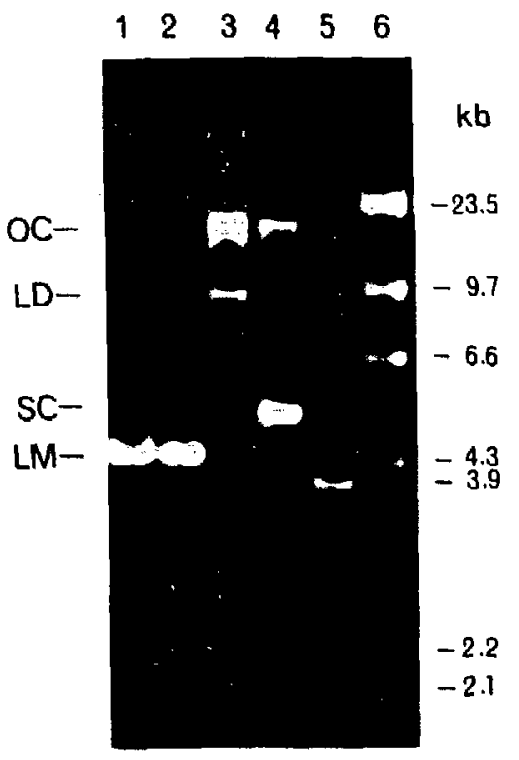

Fig. 2. Endonucleolytic activity in cell-free extracts. Whereas extracts show resolvase activity toward supercoiled DNA producing linear DNA, no double strand endonuclease or exonuclease activity is detected toward linear DNA. Lane 1 - A cellfree extract prepared essentially as described previously 1 was incubated with $0.5 \mu \mathrm{g}$ BamHl linearized PBR322 DNA for 1 hour at $30^{\circ} \mathrm{C}$ in Buffer $\mathrm{C}(10 \mathrm{mM}$ Tris- $\mathrm{HCl}, \mathrm{pH} 7.5,10 \mathrm{mM} \mathrm{MgCl}, 1$ $\mathrm{mM}$ dithiothreitol, $0.1 \mathrm{mg} / \mathrm{ml}$ bovine serum albumin). Lane 2 . $0.5 \mu g$ BamHI linearized pBR322 DNA incubated in Buffer $C$ without yeast extract. Lane 3 - extract incubated with $0.5 \mu g$ supercoiled pBR322 dimer DNA in Buffer $C$ for 1 hour at $30^{\circ} \mathrm{C}$. Lane 4 - $0.5 \mu \mathrm{g}$ supercoiled pBR322 DNA incubated in Buffer $C$ without extract. All reactions were stopped by addition of 20 mM EDTA and DNA species were separated by electrophoresis through a $0.8 \%$ agarose gel in TAE buffer $(40 \mathrm{mM}$ Tris-acetate, $2 \mathrm{mM}$ EDTA, $\mathrm{pH} 8$ ) at 20 volts for 18 hours. DNA bands were visualized by uv transillumination of ethidium bromide stained gels. Lane 5 shows a control cleavage reaction of the BamHI linearized DNA by EcoRl resulting in fragments sized $3.9 \mathrm{~kb}$ and $0.4 \mathrm{~kb}$ (the small fragment is not seen on this gel). Lane 6 shows linear DNA standards generated by Hindlll digestion of lambda DNA. Sizes are indicaled in kilobases (kb). Abbreviafions: $O C$, open circular DNA; $L D$, linear dimer DNA $(8.7 \mathrm{~kb})$; $S C$, supercoiled DNA; LM, linear monomer DNA (4.36 kb).

(XBB 860-8697)

observed (not shown). When the experiment was done using resolvase $\left(30^{\circ} \mathrm{C}, \mathrm{pH} 7.5\right)$ instead of $\mathrm{S1}$, the pattern shown in Fig. 7 resulted. Resolvase cut supercoiled ColE1 DNA to the linear form. Several new bands appear upon subsequent digestion with $S$ mal, including one approximately $5.2 \mathrm{~kb}$, indicating the resolvase fraction cleaved the DNA at several locations. This result most likely means that the resolvase activity recognizes and cleaves other smaller cruciforms that may be present in supercoiled ColE1 DNA as well as the major one. This idea is currently being tested by performing the

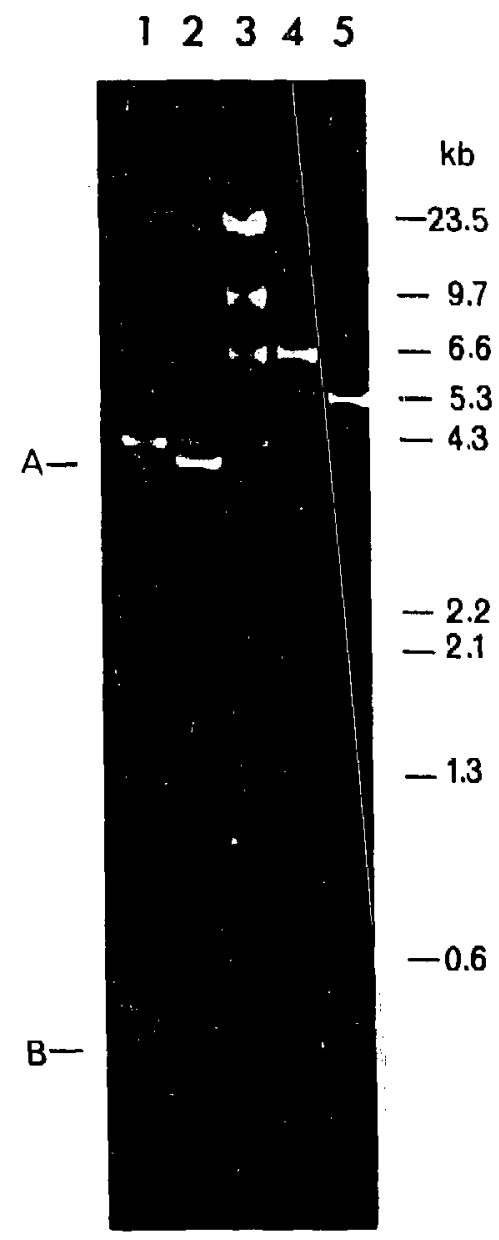

Fig. 3. Detection of a double stranded endonuclease activity eluted from phosphocellulose. Electrophoresis was as in Fig. 1 using $1 \%$ agarose. Lane 1 - BamHl linearized pBR322 DNA 10.2

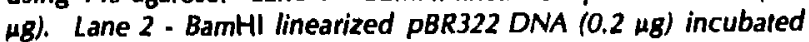
in Buffer $\mathrm{C}$ for 1 hour at $30^{\circ} \mathrm{C}$ with the $0.9 \mathrm{M} \mathrm{NaCl}$ fraction eluted from phosphocellulose. Two bands, A (approximately 4 $\mathrm{kb}$ ) and $B$ (approximately $0.3 \mathrm{~kb}$ ) are produced. Digestion of pBR322 with BamHI and Scel will yield two fragments of sizes $4.1 \mathrm{~kb}$ and $0.2 \mathrm{~kb}^{2}$. Lane 3 = linear DNA standards as in Fig. 1. Lane 4 - Linear ColE1 DNA standard $(6.6 \mathrm{~kb})$. Lane 5 - Linear DNA standards $(5.3,1.3 \mathrm{~kb})$ produced by Smal and EcoRI digestion of ColE1 DNA.

(XBB B60-8695)

reaction under a range of temperatures and yrobing the DNA with mung bean nuclease which is active under reaction conditions similar to those of resolvase.

Figure 8 shows denaturing polyacrylamide gel electrophoresis (SDS-PAGE) of partially purified resolvase. Two major polypeptide species of molecular weights $100 \mathrm{kDa}$ and $50 \mathrm{kDa}$ (indicated by arrows) and several minor bands are observed. It is not known at this time which of these represents the resolvase polypeptide(s). 
$\begin{array}{llll}1 & 2 & 3 & 4\end{array}$

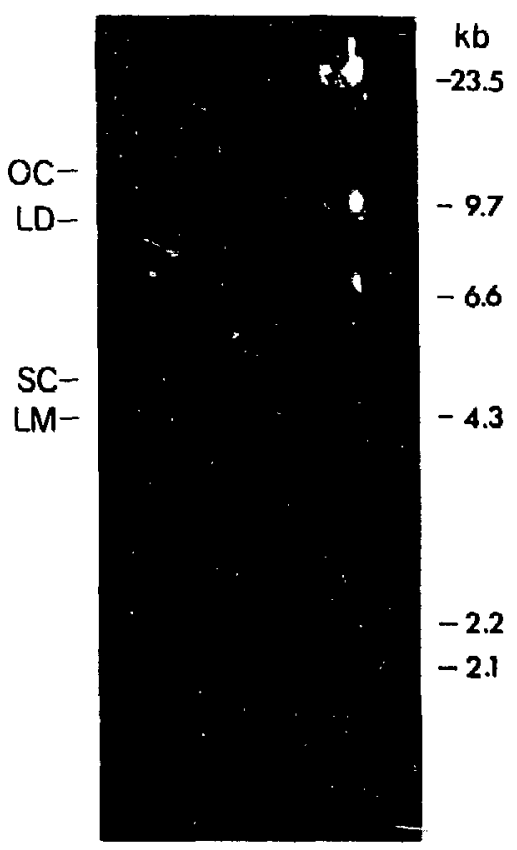

Fig. 4. Resolvase cleavage of cruciform-containing supercoiled pBR322 dimer DNA. Five (Lane 1) or 10 (Lane 2) $\mu$ l of partially purified resolvase was incubated with $0.1 \mu \mathrm{g}$ of supercoiled pBR322 dimer DNA at $30^{\circ} \mathrm{C}$ for 1 hour. Linear dimer DNA is produced, but linear monomer DNA is not. Lane 3 - pBR322 DNA incubated without resolvase. All reactions were stopped and analyzed as in Fig. 1. Lane 4 - standards as in Fig. 1. Abbreviations: $O C$, open circular DNA; $L D$, linear dimer DNA $(8.7 \mathrm{~kb})$; $S C$, supercoiled DNA; $L M$, linear monamer DNA (4.36 kb).

(XBB 960-8696)

Fig. 6. Resolvase does not cleave single strand DNA. The partially purified resolvase fraction $(5 \mu l)$ was incubated with $0.3 \mu \mathrm{g}$ single strand $\phi \times 174$ virion DNA for 0 (Lane 1), 30 (Lane 2) or 90 (Lane 3) minutes at $37^{\circ} \mathrm{C}$ in Buffer $\mathrm{C}$. Reactions were stopped and analyzed as in Fig. 1 using $1 \%$ agarose. Lane 4 shows linear DNA fragments as in Fig. $t$.

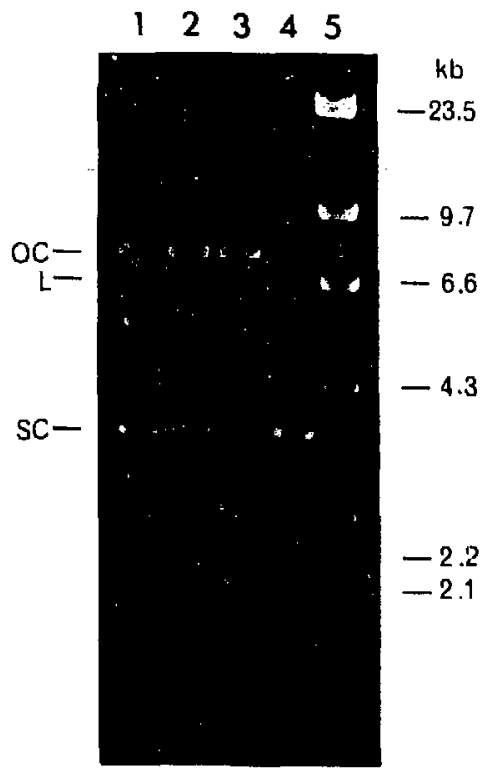

Fig. 5. Resolvase cleavage of cruciform-containing supercoiled ColE1 DNA. Partially purified resolvase $(5 \mu l)$ was incubated with $0.1 \mu \mathrm{g}$ supercoiled ColE1 DNA at $30^{\circ} \mathrm{C}$ for 1 (Lane 1), 3 (Lane 2), or 5 (Lane 3) hours in Buffer C. Lane 4 - $0.1 \mu g$ ColE1 DNA incubated in buffer alone. Reactions were stopped and analyzed as in Fig. 1. Lane 5 - standards as in Fig. 1 Abbreviations: $O C$, open circular DNA; $L$, linear DNA $(6.6 \mathrm{~kb})$; $S C$, supercoiled DNAA.

(XBB 860-8700)

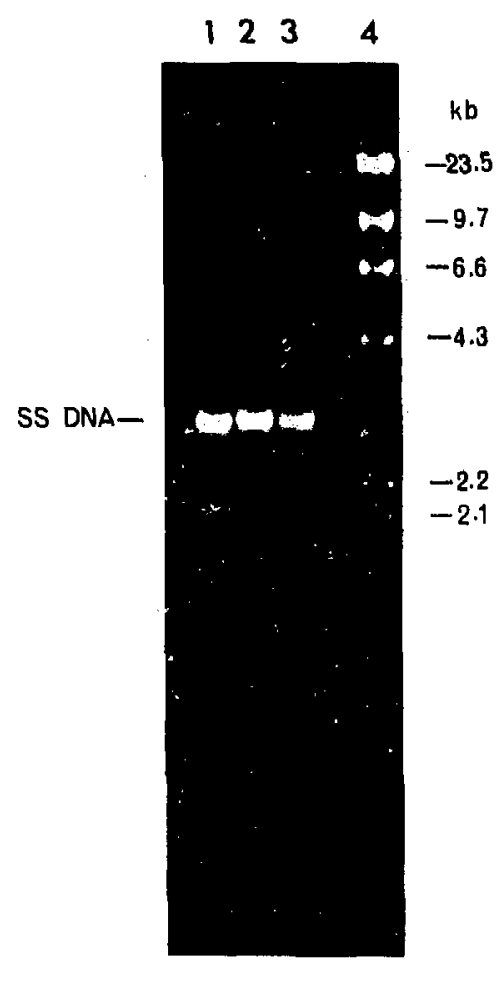


12345

67

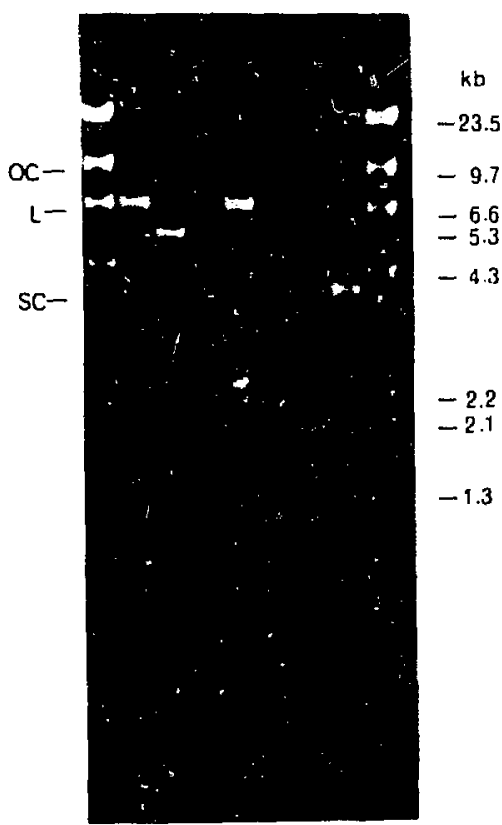

Fig. 7. Digestion of ColE1 DNA with resolvase followed by Smal. Partially purified resolvase $(10 \mu \mathrm{l})$ was incubated with 0.5 ug supercoiled ColEI DNA for 12 hours at $30^{\circ} \mathrm{C}$ in Buffer $C$. The DNA was subjected to phenol-chloroform extraction and ethanol precipitation. $0.4 \mu g$ was then treated with Smal for 2 hours at $25^{\circ} \mathrm{C}$. Reaction products were analyzed as described in Fig. 1 with $1 \%$ agarose. Lanes 1 and 7 - linear DNA standards as in Fig. 1. Lane 2 - linear ColEI DNA $(0.1 \mu \mathrm{g})$ produced by treatment with Smal (2 hours, $25^{\circ} \mathrm{C}$ ). Lane 3 - ColE1 DNA $(0.1 \mu \mathrm{g})$ treated first with $\mathrm{Smal}\left(2\right.$ hours, $\left.25^{\circ} \mathrm{C}\right)$ and then with EcoRl (90 minutes, $37^{\circ} \mathrm{C}$ ). Fragments of sizes $5.3 \mathrm{~kb}$ and $1.3 \mathrm{~kb}$ result. Lane 4 - ColE1 DNA $(0.1 \mathrm{\mu g})$ treated with resolvase. Supercoiled DNA is converted to the linear form $(6.6 \mathrm{~kb})$. (Compare with Lane 6). Lane 5 - ColEt DNA $(0.4 \mu \mathrm{g})$ treated with resolvase followed by Smal. The linear DNA is converted to several discrete DNA bands. The open circular form is converted to the linear form. Lane 6 - supercoiled and open circular forms of ColEI. Lane 7 - standard's as in Fig. 1. Abbreviations: $O C$, open circular DNA; L, linear DNA $(6.6 \mathrm{~kb}) ; S C$, supercoiled DNA.

(XBB 860-8698)

Further purification of this activity from both mitotic and meiotic cells is in progress. Pure material will allow physical and kinetic characterization of the enzyme and will facilitate the study of its expression through the yeast life cycle by immunological assays.

\section{REFERENCES}

1. Holbrook, L.L., Bjornstad, K.A., Maleas, D.T., and Esposito, M.S. In vitro resolution of $\mathrm{Hol}$ liday junctions by cell-free protein extracts of Saccharomyces cerevisiae. Resolvase activity of haploid and diploid cells. In Biology and
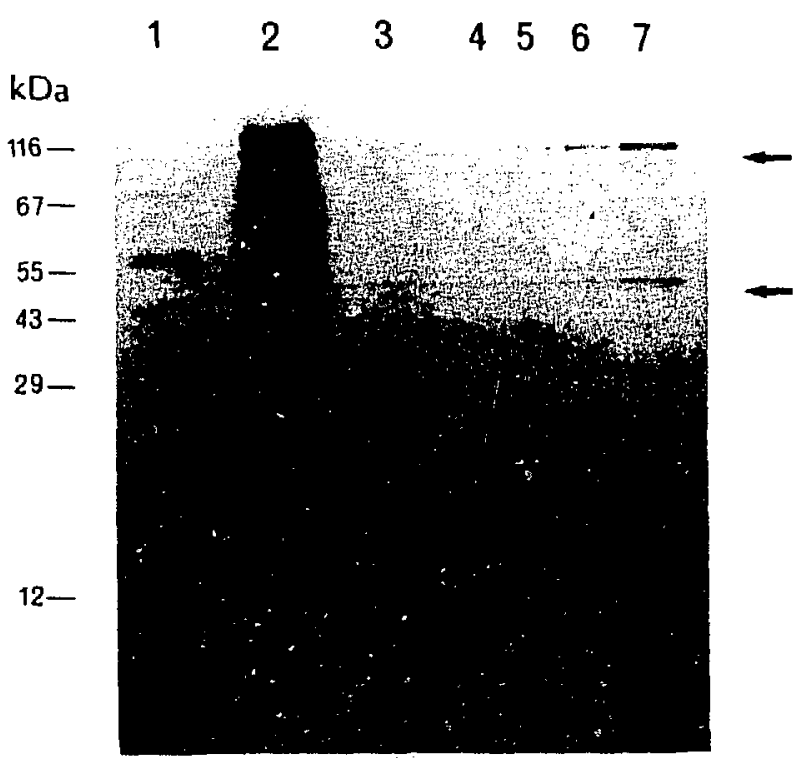

Fig. 8. Polyacrylamide gel analysis (SDS-PAGE) of resolvase fractions purified by chromatography on phosphocellulose and octyl sepharose. Samples ivere elecirophoresed through a $12.5 \%$ polyacrylamide separating gel and a $4.5 \%$ stacking gel using the LaemmlP system at $15 \mathrm{~mA}$ for 3 hours. Polypepli-ies were visualized by staining with Coomassie blue dye. Lane 1 maiker proteins; molecular weights are indicated in kilodaltons $(k D a)$. Lane 2 - aliquot of resolvase activity eluted from phosphocellulose and applied to octyl sepharose. Lane $3 \cdot 10 \mu /$ of resolvase activity eluted from octyl sepharose. Lanes 4-7 - 1, 2, 4, and $10 \mathrm{\mu l}$ of concentrated resolvase fraction used in the assays described in Figs. 3-7. Arrows indicate polypeptide species of molecular weights $100 \mathrm{kDa}$ and $50 \mathrm{kDa}$.

(XBB 860-8703)

Medicine Annual Report 1985, 189-194, Lawrence Berkeley Laboratory report LBL20345 (1986).

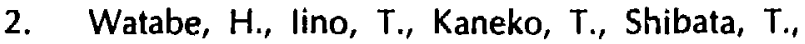
and Ando, T. A new class of site-specific endodeoxyribonuclease. J. Biol. Chem. 258, 4663-4665 (1983).

3. Lilley, D.M.J. The inverted repeat as a recognizable structural feature in supercoiled DNA molecules. Proc. Natl. Acad. Sci. USA 77, 6468-6472 (1980).

4. Sutcliffe, J.G. Complete nucleotide sequence of the Escherichia coli plasmid pBR322. Cold Spring Harbor Symp. Quant. Biol. 43, 77-90 (1979).

5. Chan, P.T., Ohmori, H., Tomizawa, J., and Lebowitz, I. Nucleotide sequence and gene organization of ColE1 DNA. J. Biol. Chem. 260, 8925-8935 (1985).

6. Laemmli, U.K. Cleavage of structural proteins during the assembly of the head of bacteriophage T4. Nature 227, 680-685 (1970). 


\title{
SINGIE STRAND SPECIFIC DNA BINDING PROTEINS OF SACCHAROMYCES CEREVISIAE
}

\author{
Junko Hosoda, Maren Bell, Kathleen A. Bjornstad, Midori M. Hosobuchi, \\ Herbert W. Moise, and Michael S. Esposito
}

We previously described the purification of a single-strand specific-DNA-binding protein, SSB-1, from mitotic cells of a protease-deficient mutant of Saccharomyces cerevisiae and showed that the protein stimulated yeast DNA polymerase I activity, indicating that this protein may have some roles in yeast DNA replication. ${ }^{1}$ To elucidate the physiological significance of SSB-1, we surveyed various activities known to be associated with prokaryotic SSB proteins, $E$. coli SSB and T4 gp32: destabilization of poly $[\mathrm{d}(A-T)]$, and DNA renaturation. Proteins with strong selective affinity for singlestranded (ss) DNA and have inherent capacity to destabilize the double-stranded helix to singlestranded coil-melting transition. Thus they are called helix-destabilizing proteins (HDP), DNA melting proteins or DNA unwinding proteins (UP). Upon binding to ssDNA, HDP melts intrastrand hairpin helices and holds the DNA strand in a conformation in which bases are exposed to the outside of the complex. In the case of T4 gp32, the disruption of the hairpins on the template strands is known to be one of the mechanisms by which it facilitates DNA synthesis by the homologous DNA polymerase. For the most recent review of SSB proteins, see Ret. 2.

HDPs are also implicated in recombination. Homologous molecules of DNA can pair in two ways: Complementary single strands can join to form double stranded (ds) DNA, or a linear singlestrand cari pair with a complementary sequence in superhelical duplex DNA to form a triple-stranded structure called a D-loop. Both reactions are pertinent to current models of genetic recombination. In the absence of protein, both reactions occur very slowly at $37^{\circ} \mathrm{C}$. The first reaction, the pairing of complementary single-strands, is greatly facilitated by the HDPs, E. coli SSB and T4 gp32. The disruption of intrastrand helix and exposure of the bases by these HDPs as described above facilitates pairing of complementary strands, the ratedetermining step of DNA renaturation.

\section{MELTING OF POLY[d(A-T)]}

In contrast to the helix destabilizing proteins of bacteriophage T4 (gp32) and E. coli (SSB), the presence of yeast SSB-1 stabilized rather than destabilized the poly $[\mathrm{d}(\mathrm{A}-\mathrm{T})]$ double strand. We monitored the melting of poly $[\mathrm{d}(A-T)]$ at a $\mathrm{NaCl}$ concentration of $25 \mathrm{mM}$ by optical absorbance at $260 \mathrm{~nm}$. Hyperchromicity is observed when the helix conformation is disrupted. Figure 1 (curve 1) demonstrates that the melting of $\operatorname{poly}[\mathrm{d}(\mathrm{A}-\mathrm{T})]$ in the absence of SSB-1 occurred with a $T_{m}$ (temperature at midpoint of melting) of $48^{\circ} \mathrm{C}$. At a nucleotide/protein molecule ratio of 20 to 1 (curve $2)$, the $T_{m}$ in the presence of SSB-1 increased to $50.5^{\circ} \mathrm{C}$ compared to $48^{\circ} \mathrm{C}$ in the blank. The final absorbance reached with SSB-1 is lower than the absorbance plateau reached in the blank. At a nucleotide/protein ratio of 10 to 1 (curve 3), the melting temperature was even higher $\left(T_{m}=52^{\circ} \mathrm{C}\right)$ and the final absorbance even less than that obtained with the 20 to 1 ratio. This apparent stabilization of the poly[d(A-T)]-SSB-1 complex does not, however, exclude SSB-1 from potential involvement in DNA replication. These phenomena were also observed with adenovirus DNA binding protein, AdDBP, which is essential for DNA replication. ${ }^{2}$

\section{DNA RENATURATION}

SSB-1 was assayed for renaturation activity according to the inethod of Bryant and Lehmann. ${ }^{3}$ The presence of magnesium ions is essential for gp32-catalyzed DNA renaturation, while $E$. coli SSB prefers spermidine. We therefore examined yeast SSB-1 for renaturation activity in the presence of

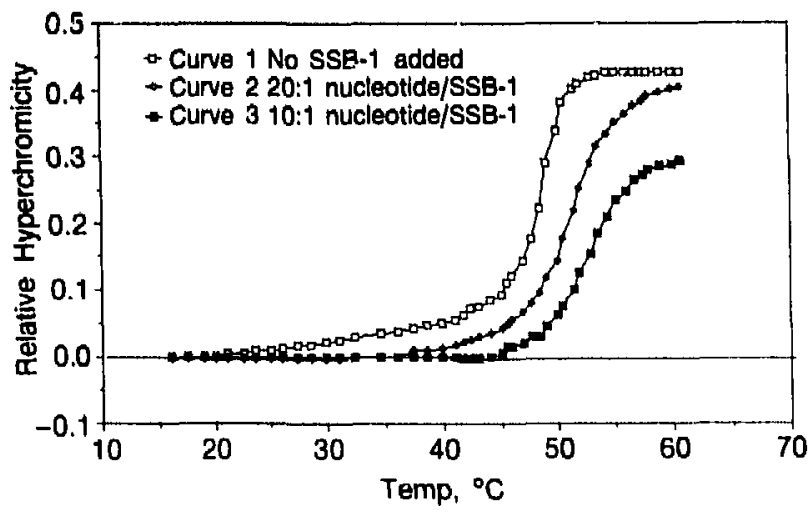

Fig. 1. Thermal melting of poly|d(a-T) in the presence and absence of $550 \% 1$. 
either $10 \mathrm{mM} \mathrm{Mg}{ }^{2+}$ (Fig. 2) or $2 \mathrm{mM}$ spermidine (Fig. 3). Assay time points were taken at 0, 5, 30 and 60 minutes for the reactions containing yeast SSB-1. Time points of 0 and 60 minutes were taken for the protein minus controls. DNA renaturation in the presence of SSB-1 and $10-\mathrm{mM} \mathrm{Mg}^{2+}$ (curve 2) was indistinguishable from that in the absence of SSB-1 (curve 3). In contrast, in the presence of $\mathrm{gp} 3280 \%$ of the total ssDNA initially added was renatured within $60 \mathrm{~min}$ (curve 1). Concentrations of $50 \mu \mathrm{g} / \mathrm{ml}$ (curve 4) and $100 \mu \mathrm{g} / \mathrm{ml}$ (curve 5) of SSB-1 in the presence of spermidine failed to cause any marked renaturation when compared to the protein minus control (Fig. 3).

\section{YEAST SSBS THAT STIMULATE DNA RENATURATION}

As described in the previous section, the DNA renaturation activity of HDPs are attributed to their

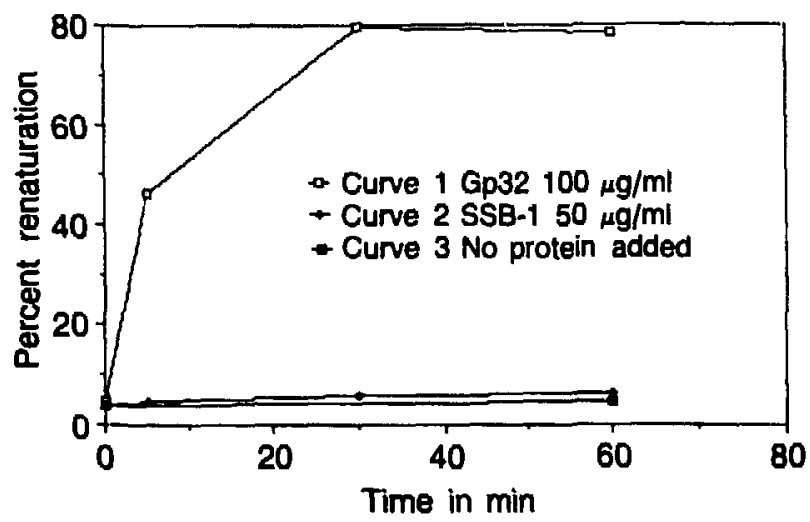

Fig. 2. Renaturation activity of gp32 and SSB-1.

(XBL $8611-9044)$

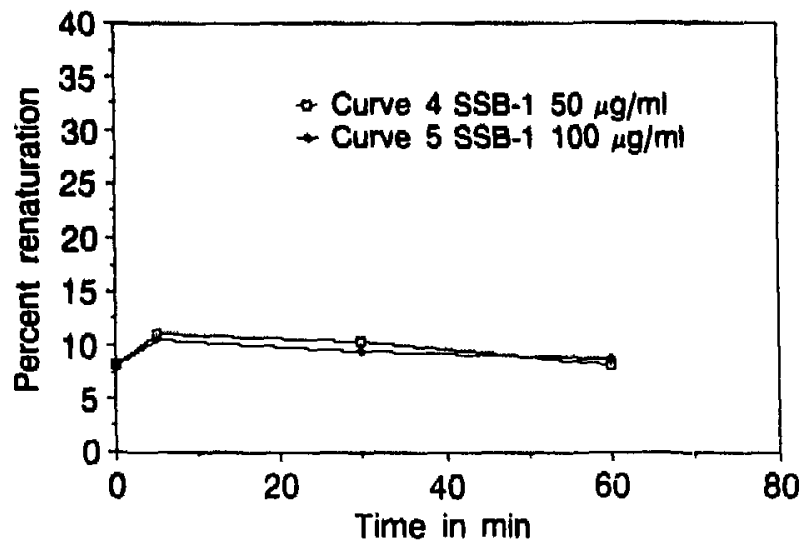

Fig. 3. Renaturation activity of $5 S B-1$ with 2-mM spermidine. (XBL 8611 -9045) ability to open up the intrastrand helices. This DNA renaturation activity may be a better assay for uncovering physiologically important HDPs than the poly $[\mathrm{d}(\mathrm{A}-\mathrm{T})]$ melting assay because the former is associated with the prokaryotic proteins with proven physiological functions, while the latter activity was also found with proteins whose roles in DNA processing are negative or questionable such as RNAase or calf thymus UP-1. We were also interested in isolating yeast SSB with functional roles in recombination and repair processes. For these reasons, fractions from the ssDNA cellulose column that eluted at lower $\mathrm{NaCl}$ concentrations than SSB-1 were scanned for DNA renaturation activity. Two fractions, ss3 and ss7, that were eluted from the ssDNA cellulose column at respective $\mathrm{NaCl}$ concentrations of 0.2 and $0.36 \mathrm{M}$, showed significantly higher activity after 15 minutes incubation $(15.6 \%$ and $27.7 \%$ renaturation, respectively) than the other fractions (Fig. 4). The assay was repeated using the two peak fractions ss 3 and ss7, and ss8 (which also had significantly high activity) in the presence and absence of ATP (Fig. 5). Under these conditions, fractions 557 and s58 showed no marked ATP dependence, while fraction ss3 displayed a significant inhibition of activity with ATP, decreasing its maximum renaturation by more than $50 \%$. While the ss 3 fraction had a strong ATPase activity the $5 s 8$ fraction had a low ATPase activity. Both were stimulated by the presence of ssDNA. In contrast, the ss7 fraction had no detectable ATPase activity. ${ }^{1}$ RecA-like enzymes and helicase, both of which can catalyze DNA renaturation are known to have DNA stimulated ATPase activity. The renaturation activity of fraction 5s7, which has no ATPase activity, may be due to a new SSB and was thus chosen for further purification.

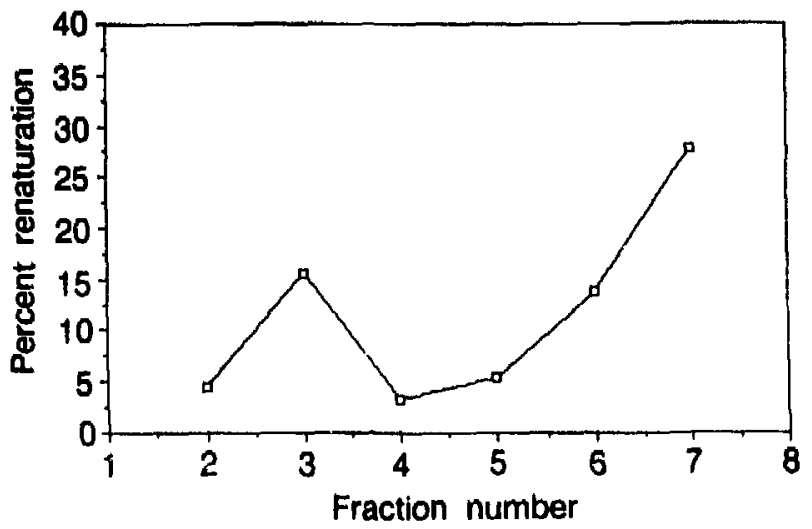

Fig. 4. 5can of renaturation activity from $550 N A$ cellulose chromalography fractions. 


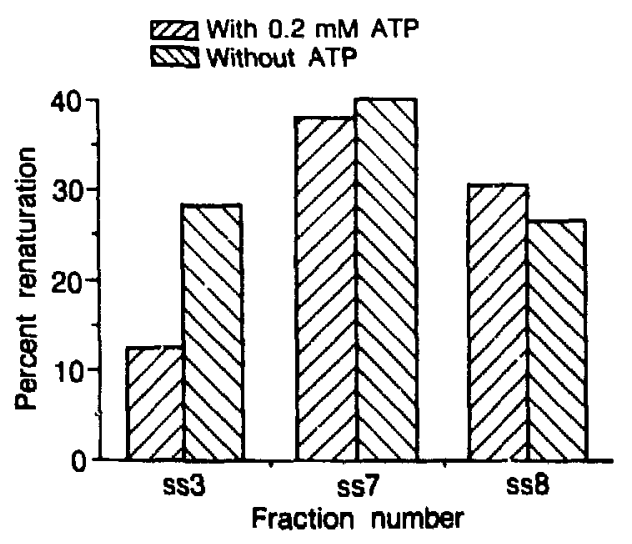

Fig. 5. Renaturation activity of s5DNA cellulose chromatography fractions in the presence and absence of ATP.

(XBL 8611-9047)

The ss7 fraction was subjected to DEAE cellulose anion exchange chromatography, and the proteins in the fraction were eluted with a 3-ml linear gradient of buffered $\mathrm{NaCl}$ from $0.1 \mathrm{M}$ to $0.6 \mathrm{M}$. Most of the activity did not bind to the column, but passed through the column into the breakthrough fractions, which were pooled and applied to a $0.5-\mathrm{ml}$ cellulose phosphate column. The renaturation activity was adsorbed by the cellulose phosphate and could not be eluted, even with 2.0$\mathrm{M} \mathrm{NaCl}$. The column was stripped with Laemmli's buffer containing $2 \%$ sodium dodecyl sulfate, and the resulting eluate was analyzed by onedimensional SDS polyacrylamidegel electrophoresis. The two major bands present in the column strip fractions displayed molecular masses of approximately 24,000 and 17,000 daltons.

\section{REFERENCES}

1. Hosoda, J., Holbrook, L., Moise, H., Maleas, D., and Esposito, M.S. Fractionation of DNA Metabolic Proteins of Saccharomyces cerevisiae by DNA Cellulose Chromatography: SSB-1, SS-DNA Dependent ATPase, DNA Polymerase, DNA Primase, Topoisomerase I, and Resolvase. In The Biochemistry and Molecular Biology of Industrial Yeast, Upjohn/Labatt Symposium, in press (1986).

2. Chase, J., and Williams, K. Single-stranded DNA binding proteins required for DNA replication. Ann. Rev. Biochem. 55, 103 (1986).

3. Bryant, F., and Lehman, I. On the mechanism of renaturation of complementary DNA strands by the RecA protein of Escherichia coli. PNAS 82, 297 (1985).

\title{
DNA Repair
}

\section{GENETIC AND PHYSICAL STUDIES OF REPAIR AND RECOMBINATION IN YEAST}

\author{
John C. Game, Robert K. Mortimer, David Schild, Vincent Cook, and \\ Shirley Sutton
}

We have continued to study DNA repair and recombination in yeast using genetic and biophysical methods. Progress has been made in several areas, especially in $x$-ray repair and meiosis. We have examined the role of the excision-repair genes RAD1 and RAD3, known to be important in the repair of thymine dimers after ultraviolet radiation, in $x$-ray repair. We find that these genes can play an important role in $x$-ray repair, but that this role is only exposed when other pathways of $x$-ray repair are blocked. Double mutants blocked in the RAD5t and RAD18 pathways are significantly less $x$-ray sensitive than triple mutants blocked in these pathways but also mutant in either the RAD1 or $R A D 3$ genes. This finding was demonstrated in two sets of strains, one involving the RAD51, RAD18 and $R A D t$ genes, and the other involving the $R A D 54, R A D 6$ and $R A D 3$ genes. RADI and $R A D 3$ mutants have little or no effect by themselves or when only one of the other pathways is blocked. We believe that this implies that a type of $x$-ray damage exists that can be repaired by any of the three pathways, so that it is not a significant cause of lethality unless all pathways are blocked. Nevertheless, the cell's ability to survive depends on being able to repair this type of damage as well 
as the types of damage repaired specifically by the RAD 51 and RAD18 pathways.

In a related experiment, we decided to test the importance of DNA repair in nature by determining the sensitivity to natural unfiltered sunlight of a strain lacking all known DNA repair pathways. We crossed a triple-mutant strain used in our $x$-ray repair studies with a very closely related strain that was mutant in the PHR1 gene, which controls a fourth type of DNA repair, photoreactivation, which is dependent on visible light. We were able to isolate a strain in this way that was mutant in all four known DNA repair processes. We found that this strain was so sensitive to sunlight that less than three seconds exposure would cause an average of one iethal hit per cell, and survival was less than 2\% after 10 seconds exposure (see Fig.1). Sensitivity near sea level was compared with sensitivity to sunlight at an elevation of 8,000 feet in the Sierra Nevada, where a small increase was observed. Wild-type yeast at sea level showed no killing after 30 minutes, and a subsequent decline

Time of Exposure in Minutes

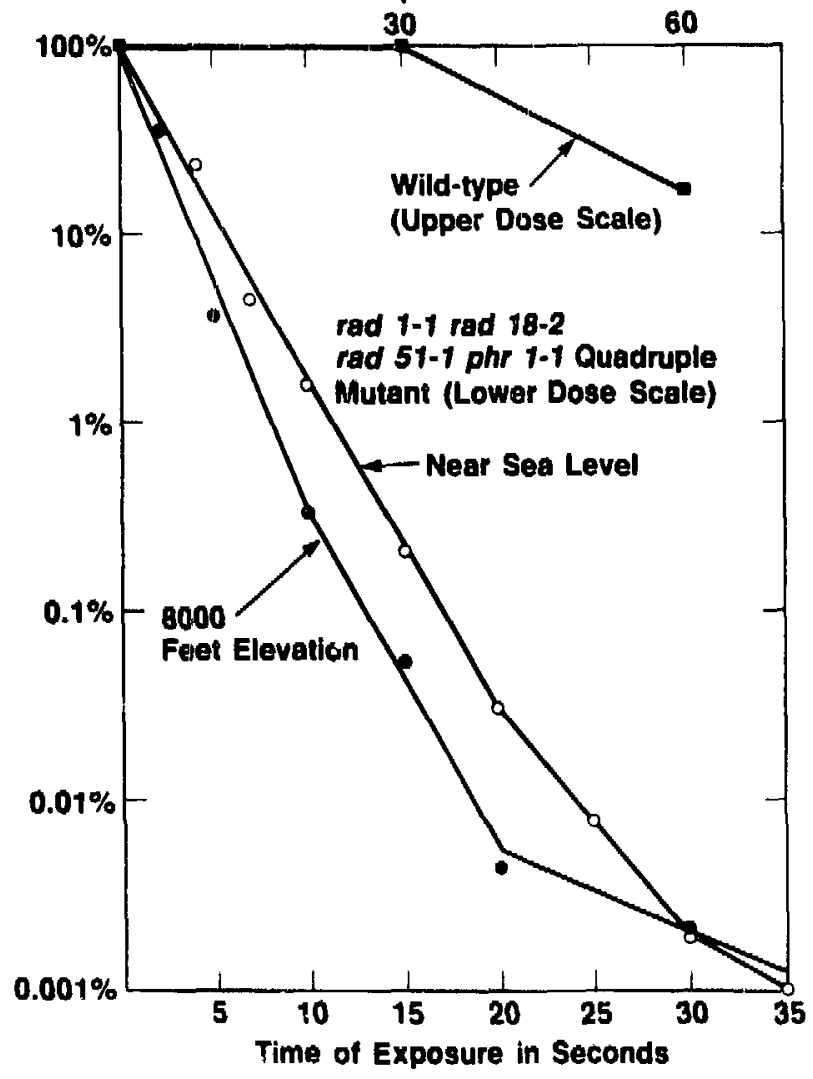

fig. 1. Yeast sensitivity to natural sunlight.

(XBL 3610-9002) to $15 \%$ survival at 60 minutes may have been at least partially due to thermal effects rather than ultraviolet-radiation damage. Thus, the quadruple mutant is approximately 1000 times more sensitive to surilight than the related wild type, and demonstrates the extreme lethality of sunlight in the absence of DNA repair. The strain may be useful in assessing the possible biological hazards of artificial sources of radiation, such as near-uv lamps or fluorescent lighting, because of its high sensitivity.

In a separate project, we have been using the OFAGE gel system described by Carle and Olson in $1984^{\prime}$ to study meiotic recombination in yeast. We wanted a simple system to monitor the time of appearance and amounts of a chromosomal recombinant DNA species during meiosis. This required a system such that recombination anywhere within a given chromosomal interval would lead to recombinant molecules of one or more new, and uniform, size classes. The system we have developed involves the use of diploids carrying one linear and one circular copy of Chr. III. (A strain carrying a circular derivative of Chr. III was initially obtained from A. Klar. ${ }^{2}$ ) Single recombination events between the circular and linear molecules anywhere along their length should lead to a new size class of linear dicentric molecules equal in length to the circular plus the linear molecule. Our results indicate that 1) circular molecules the size of Chr. III will not enter the OFACE gel, 2) it is necessary to probe blots of OFAGE gels by Southern hybridization with a marker from Chr. III to visualize recombinant bands, 3) when this is done, two fainter new bands of approxirnately equal intensity are seen, one approximately twice and one approximately three times the size of Chr. III, and 4) normal chromosomal DNA is not seen to be sheared during meiosis and nothing that might correspond to "bivalents" or other recombination intermediates is seen on our OFAGE gels. We interpret our two new bands as probably representing the single recombination events between one circular and one linear chromatid, and the three-strand double events involving two circular and one linear chromatids, respectively.

We believe this system will provide a powerful method of studying molecular recombination in meiotic populations simultaneously being monitored for other end-points such as commitment to recombination (scored biologically) or DNA synthesis, and it can be used to characterize meiotic mutants for recombination at the molecular level. We hope to develop the system to monitor double-strand breaks and sister-chromatid exchange, as well as the homologous recombination currently detecled. 


\section{REFERENCES}

1. Carle, G.F., and Olson, M.V. Nucl. Acids Res. 12 , 5647-5664 (1984).
2. Klar, A.J.5., Strathern, J.N., Hicks, J.B., and Prudente, D. Mol. Cell. Biol. 3, 803-810 (1983).

\title{
MOLECULAR ANALYSES OF YEAST RECOMBINATION-REPAIR GENES
}

\author{
Robert K. Mortimer, David Schild, Mari Aker, Gary Cole, John Game, \\ Susan Lovett, and Karen Sitney
}

Genes in the recombinational repair pathway in the yeast Saccharomyces cerevisiae confer resistance to $x$-ray irradiation. Mutations in these genes disrupt the repair of double-stranded DNA breaks with multiple phenotypic effects such as abnormalities in meiosis and recombination. This epistasis group includes the genes RAD50 to RAD57. As discussed in previous annual reports, we have cloned many of these genes and are using the clones for molecular analyses of these genes. During the past year we have continued these studies, with particular emphasis on the transcriptional regulation of RAD51, RAD52 and RAD54, and the sequencing of RAD51. In addition we have initiated molecular research on $R A D 24$, a gene involved in several DNA repair pathways in yeast.

\section{TRANSCRIPTIONAL PATTERNS AND NUCLEOTIDE SEQUENCE OF RAD5 AND ITS FLANKING REGIONS}

RAD51 was previously cloned and characterized by Calderon et al. ${ }^{1}$ We have now sequenced the entire gene as well as a 1.4 kilobase (kb) region upstream to the gene. We identified the open reading frame that corresponds to the RAD51 gene by deletion and complementation analysis of subclones of the original isolate (Fig. 1). From these data, we predict the RAD51 protein is 336 amino acids, with a calculated molecular weight of 42,000 daltons. The length of the open reading frame1008 nucleotides-is consistent with our finding by Northern analysis of an mRNA of approximately 1300 nucleotides (nt). We have mapped the transcript initiation site by primer extension to a single location-36 base-pairs (bp) upstream from the putative translation start. A TATA element is present at position -139 .

An insertion of a $2.1-\mathrm{kb}$ LEU2 fragment at position -86 separates the RAD51 promoter from its structural gene and prevents expression. We have transplaced the fragment containing the insertion into a haploid strain and since this haploid is still viable it shows that RAD51 does not code for an essential function. This disrupted mutant shows $x$ ray survival comparable to that of a strain with the mutation rad51-1, ${ }^{2}$ We will determine if a disruption mutant with a deletion of part of the coding sequence of the $R A D 51$ gene will display a more severe phenotype of increased sensitivity to $x$ rays.

Notably, there is a three- to four-fold increase in the level of the RAD51 transcript following x-ray irradiation. The induced transcripts are the same size as the noninduced copies and appear to have the identical start site. Other yeast repair genes$R A D 54, R A D 2$, and $C D C 9^{3-5}$-are also inducible by DNA-damaging agents. Whether a common regulatory mechanism can explain these similarities remains to be investigated. To identify particular sequences required for induction of the RAD51 gene, we are constructing deletions within the 200-bp region upstream of the transcript start site.

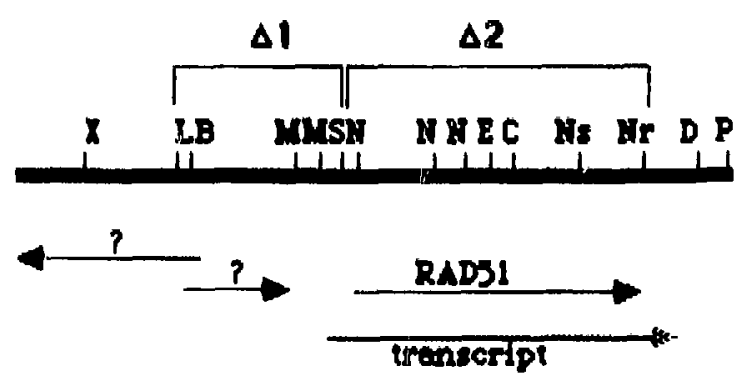

$$
\begin{aligned}
& \Delta 1 \text { - lethal } \\
& \Delta 2 \text { - Rod- }
\end{aligned}
$$

Fig. 1. Restriction and transcription maps of RAD51 and surrounding chromosomal region. Deletion of region $l(\Delta l)$ is lethal, while deletion of region $2(\Delta 2)$ leads to a rad51 $x$-ray sensitive phenotype. 
There are several unidentified open reading frames (ORFs) within the sequenced $3.0 \mathrm{~kb}$ region. One ORF, nonoverlapping and starting 720 bp upstream from RAD51, could encode a protein of 104 amino acids, with a molecular weight of approximately 13,000 daltons. We have determined that a fragment containing a deletion of this ORF is transformable only into diploid cells. The diploid cells give rise to two spore-surviving tetrads. The deletion is therefore lethal to the haploid spores containing it. The ORF may encode an essential gene, with a protein product necessary for germination of the spore. We currently have no evidence for a role of this gene in DNA repair or metabolism except its location directly adjacent to RAD51.

\section{REGULATION OF RAD54- AND RAD52-lacZ GENE FUSIONS}

Following observations in this laboratory that $R A D 54$ message levels increase in response to $x$-ray irradiation, and that $R A D 52$ levels remain constant ${ }^{3}$, we constructed gene fusions of the $5^{\prime}$ sequences of these genes to the structural gene $\beta$-galactosidase of $E$. coli in order to more completely characterize the regulation of these genes. We observed that the RAD54-lacZ gene fusion is inducible 3- to 12 fold by a number of DNA damaging agents, including $x$ rays, ultraviolet irradiation, and methyl methanesulfonate, but not in response to heat shock. Induction by these agents is dosedependent. In addition, there is no cell-cycledependent signal in response to DNA damage, as assayed in cells blocked by $\alpha$-factor. Double-strand breaks alone are sufficient for RAD54-lac Z induction, as determined using a yeast plasmid containing the ECoRI restriction enzyme structural gene under the yeast GAL1 promoter. ${ }^{6}$ The $R A D 52-$ lac gene fusion was not inducible in response to $x$ rays.

Both the RAD54 and the RAD52 construct are induced during meiosis at levels comparable to RAD54 induction in response to DNA damage, about 3- to 7-fold. In addition, the RAD54 construct shows a peak in expression immediately prior to the highest levels of recombination during meiosis, as determined by interrupted meiosis assays using his 1 heteroalleles.

We are currently conducting 5 ' deletions of the RAD54 fusion to determine what sequences are necessary for the response to DNA damage and meiosis. We have succeeded in isolating the constitutive and induction signals, and preliminary data suggest that the mode of regulation of RAD54 is via a negative inducible system. Further deletions and mutations in this region are being made.
CLONING AND CHARACTERIZATION OF A GENE REQUIRED FOR BOTH EXCISIONAL AND RECOMBINATIONAL REPAIR IN YEAST

The rad24-1 mutation (previously designated $r_{1}^{s}$ ) is sensitive to both $x$ - and uv-irradiation. On the basis of its double-mutant phenotypes with members of both the RAD3 and RAD51 epistasis groups, it is thought to be involved in both the excision and recombinational repair pathways. ${ }^{7}$

RAD24 had been mapped to chromosome V, 1 to $2 \mathrm{cM}$ from $R A D 3,{ }^{7}$ We have determined, through a three-point cross involving rad4-2, rad3-2 and rad24-1, that $R A D 24$ is the most centromeredistal marker on chromosome $V$. The tight genetic linkage between $R A D 3$ and $R A D 24$, in conjunction with their similar phenotypes and epistasis with respect to uv sensitivity, raised the possibility that $R A D 3$ and $R A D 24$ might be under coordinate control. However, this is probably not the case, as evidenced by the molecular characterization of RAD24.

We have cloned the RAD24 gene by complementation of the $x$-ray sensitive phenotype of a rad3-2 rad24-1 double mutant. The synergistically increased $x$-ray sensitivity seen in the double mutant ${ }^{7}$ was useful in this experiment to reduce the high background of survivors seen with the moderately sensitive rad24-1 single-mutant strain. A single clone, an $11-k b$ insert in a YCp50 yeast genomic library, conferred wild-type levels of $x$-ray survival on the rad24-1 mutant. Subcloning, in combination with preliminary Northern analysis, indicates that the gene itself is on the order of $3 \mathrm{~kb}$.

A cloned RAD3 gene was obtained from $L$. Naumovski. Coordinate control of RAD3 and RAD24 can probably be ruled out by failure of each clone to complement the other mutation, and by Southern hybridization experiments, which indicate that the two inserts share no common sequences.

A genomic disruption of RAD24 has been made, and is haplolethal. This indicates that the RAD24 gene has an essential cellular function. We are currently isolating temperature-sensitive alleles of RAD24 in order to determine what this function is. The RAD24 gene is now being sequenced, and cloning of the mutant allele is in progress.

\section{REFERENCES}

1. Calderon, I.L., Contopoulou, C.R., and Mortimer, R.K. Isolation and characterization of yeast DNA repair genes, II. Isolation of plasmids that complement the mutations 
rad50-1, rad51-1 rad54-3, and rad55-3. Current Genetics, 7, 93-100 (1983).

2. Game, J.C., and Mortimer, R.K. A genetic study of $x$-ray sensitive mutants in yeast. Mutation Research, 24, 281-292 (1974).

3. Emery, H., Schild, D., and Mortimer, R.K. Xrays induce accumulation of the transcript of $R A D 54$, a yeast recombinational-repair gene. Submitted to Mol. Cell. Biol.

4. Robinson, G.W., Nicolet, C.M., Kalainov, D., and Friedberg, E.C. A yeast excision-repair gene is inducible by DNA damaging agents. Proc. Nat'l. Acad. Sci. USA, 83, 1842-1846 (1986).
5. Peterson, T.A., Prakash, L., Prakash, S., Osley, M.A., and Reed, S.R. Regulation of CDC9, the Saccharomyces cerevisiae gene that encodes DNA ligase. Molecular and Cellular Biology, 5, 226-235 (1985).

6. Barnes, G., and Rine, I. Regulated expression of endonuclease EcoRI in Saccharomyces cerevisiae: nuclear entry and biological consequences. Proc. Nat'l. Acad. Sci. USA, 82, 1354-1358 (1985).

7. Eckardt-Schupp, F., Siede, W., and Game, J.C. The RAD24 (=R1) gene product of $S$. cerevisiae participates in two different pathways of DNA repair. Genetics, (1986) in press.

\section{INDUCED EXCISION REPAIR IS REQUIRED FOR REPAIR OF LESIONS IN THE VICINITY OF DNA REPLICATION FORKS}

\section{Priscilla K. Cooper, Sherry Gee, and Vincent ling}

The release of a normal mammalian cell from the restraint of differentiation, control of cell division, and a finite life-span produces a cancer cell. The succession of steps that cause the transformation of a normal cell to a cancerous one is not well understood. However, the characterization of many DNA-damaging agents as mutagens and carcinogens suggests a requirement for repair of DNA damage in prevention of carcinogenesis. The extreme cancer-proneness of patients suffering from human genetic repair-deficiency diseases is further evidence of the link between unrepaired DNA damage and carcinogenesis. Moreover, many of these diseases are characterized by severe developmental, neurologic, and immunologic abnormalities, suggesting a fundamental requirement for DNA repair processes for normal growth and development. Such findings lend impetus to basic studies of cellular responses to DNA-damaging agents, and studies of the effects of carcinogens in bacteria (which have the distinct advantage of availability of relevant mutants and extensive genetic and biochemical characterization) continue to be essential in providing models for analyzing the effects of carcinogens in mammalian cells. Our research program utilizes Escherichia coli as a model system for understanding on a molecular level the cellular processes that determine the consequences for both mutation and cell survival of damage of DNA.

In recent years evidence has accumulated establishing that cells possess a large (and frequently overlapping) array of responses to DNA damage that are induced by the presence of the damage itself. The best understood (and perhaps the most general) of these is the SOS response of $E$. coli, a regulatory network consisting of a set of at least 18 chromosomal genes whose expression is induced by treatments that damage DNA or otherwise interfere with DNA replication. The diverse physiological responses attributable to induction of this set of "SOS genes" include increased resistance to the cytotoxic effects of DNA damage and induced mutagenesis in response to the damage. It is now apparent that these two responses are separable, although jointly regulated, and are the result of different processes. In particular, induced resistance to cell killing by ultraviolet (uv) irradiation has been shown by us and others to be primarily dependent on an excision repair process. Moreo:!er, we have shown that this induced resistance to killing is accompanied by a dramatic increase in the ability to continue DNA replication after irradiation, whereas no such effect accompanies SOS induction in mutants that are unable to perform excision repair. Involvement of an induced excision repair process in resistance to the lethal and replication-blocking effects of DNA damage is suggested by these observations. Such an SOS-regulated induced repair process, long patch excision repair, has been documented and charac- 
terized by us and shown to be of general importance in resistance to DNA damage, including that introduced by treatment with alkylating agents.

In contrast to the constitutive excision repair process, which inserts patches of 20-30 nucleotides at the majority of lesions, the induced process produces very much longer patches of at least 1500 nucleotides at approximately $1 \%$ of the damage sites following uv irradiation. The two processes are temporally distinct, with short patch synthesis being initiated immediately after irradiation but long patches appearing only midway through the repair period, presumably because of the requirement of the latter process for new protein synthesis. The low frequency of long patches is not simply explained by this delay in their appearance due to the requirement for induction, however, and available evidence suggests that instead the long patches occur at a particular small subclass of lesions that are refractory to repair by the constitutive process. The correlation of long patch repair with a more rapid recovery of DNA replication after damage suggested that one requirement for the induced long patch process might be in repair of lesions introduced in the vicinity of DNA replication forks, which would in any case be expected to pose special problems for the constitutive excision repair process because of the unwinding and separation of the complementary strands. During the past year we have obtained evidence supporting this hypothesis.

As described in last year's Annual Report, we have recently developed a technique for resolving DNA fragments containing replication forks from linear DNA fragments by two-dimensional agarose gel electrophoresis. The technique is based on the altered mobility in agarose of branched structures relative to linear double-stranded molecules as a function of gel concentration and voltage. When pulse-labeled DNA is isolated, purified, fragmented by digestion with restriction nucleases, and run in the two-dimensional gel system, the bulk of the DNA migrates in a single arc visible by staining with ethidium bromide. However, when autoradiograms are prepared from the gels, it can be seen that the nascent DNA, represented by the radioactive pulse label, is contained in a second distinct arc. We have shown by a variety of criteria that the nascent DNA migrating in this minor arc has properties consistent with replication fork structures. We have now applied this technique to testing the hypothesis that long patch repair occurs at lesions in the vicinity of DNA replication forks.

The experimental approach we are using is dependent on our ability to specifically radioac- tively label only constitutive (short patch) or only induced (long patch) repair events in the absence of any appreciable replicative incorporation of label. We have previously defined conditions that allow differential labeling of only short patches (during the first 10 minutes after a single uv dose) or only long patches (between 20 and 45 minutes after the irradiation). Normal semiconservative replication can be blocked by performing the experiment in a strain carrying a $d n a B$ mutation that renders replication fork progression thermosensitive. Thus, when a $d n a B$ mutant is shifted to the restrictive temperature, replication ceases immediately but can resume when the culture is returned to the permissive temperature. We have previously shown that excision repair proceeds normally in this mutant at the restrictive temperature. The experimental protocol thus calls for shifting an exponentially growing culture of the $d n a B$ strain to the restrictive temperature prior to irradiation, exposing the cells to $60 \mathrm{~J} / \mathrm{m}^{2}$ uv light, and incubating the cells at the restrictive temperature during the entire 60 minutes needed for the completion of excision repair. Radioactive label is added either during the first 10 minutes to label only the constitutive short repair patches or between 20 and 45 minutes after irradiation to label only the induced long patches. In either case, the radioactivity is removed at the end of the labeling period and incubation is continued at the restrictive temperature until the end of the 60-minute repair period. At this time the cells are harvested, DNA is purified from them, and agarose gel electrophoresis in two dimensions of the restricted DNA is performed as described.

The model would predict that lesions introduced into the vicinity of replication forks that existed at the time of irradiation will be repaired only by the induced process. Since these forks will not advance during the repair period because of inactivation of the essential dna $B$ protein, the radioactive label incorporated into long repair patches should preferentially appear in the minor arc of DNA containing the otherwise-unlabeled replication forks when the two-dimensional gel analysis is performed. In contrast, the short patches are expected to be randomly distributed throughout the genome, reflecting repair of the vast majority of the uv-induced lesions, and hence the short patch label should appear in the major arc. The results of such an experiment are shown in the autoradiograms of Fig. 1. In support of the hypothesis, a significant portion of the radioactivity incorporated during long patch repair appears in the minor arc of DNA that contains replication forks [Fig. 1(B)], whereas essentially none of the short patch label is 

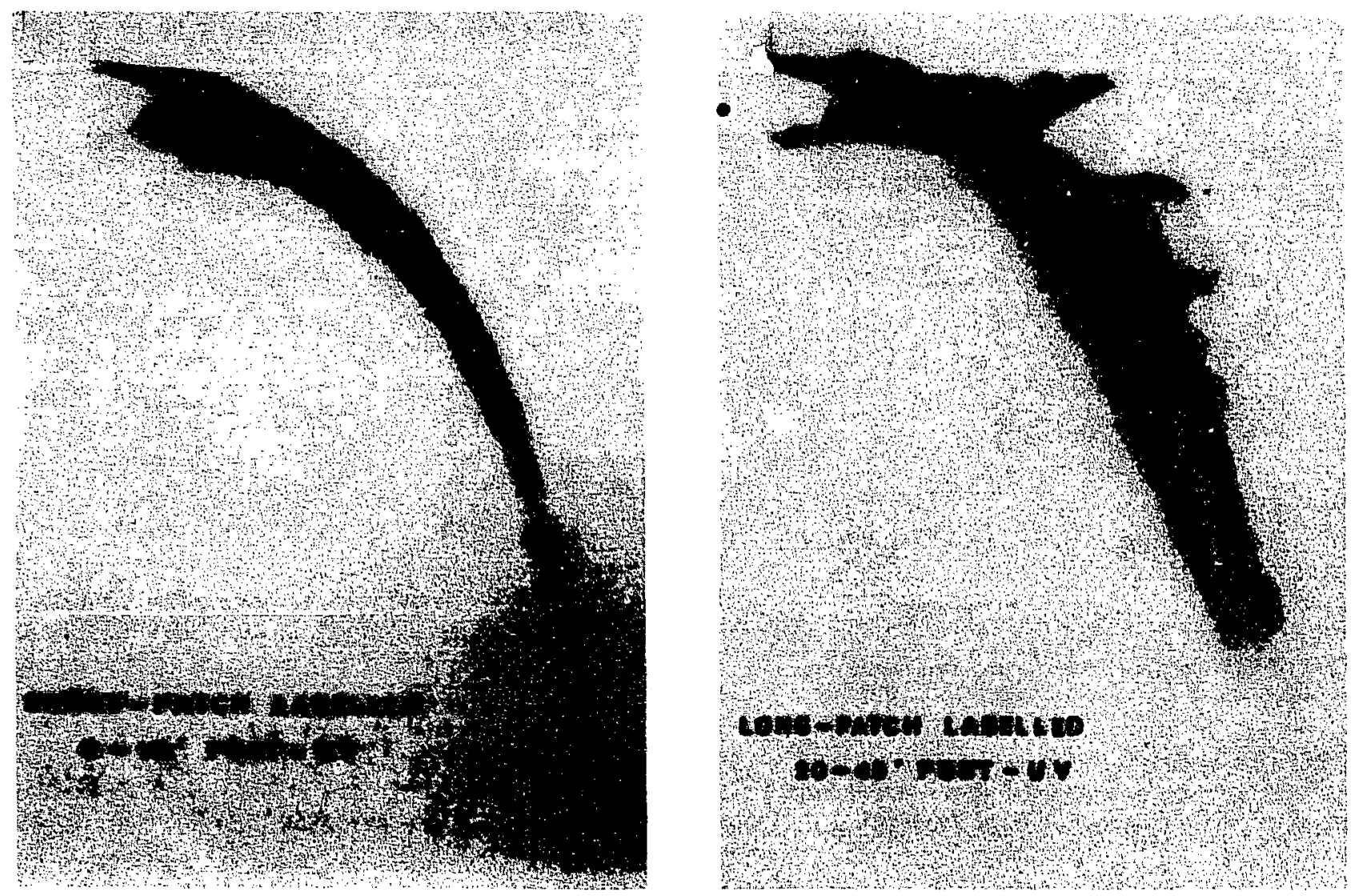

Fig. 1. Autoradiograms prepared after two-dimensional agarose gel electrophoresis of restriction digests of repairlabeled DNA purified from uv-irradiated $\mathrm{E}$. coli as described in the text. ' $\mathrm{H}$-thymidine was incorporated during' the first 10 minutes of incubation after exposure of cells to $60 \mathrm{~J} / \mathrm{m}^{2}$ uv (A) or between 20 and 45 minutes after exposure $(B)$.

(A XBB 860-10543 - B XBB 860-10544)

in this position [Fig. 1(A)]. Control experiments have shown no appreciable incorporation of label into the minor arc in DNA from unirradiated cells incubated under identical conditions, and other important controls have verified that replication forks labeled under permissive conditions can be shown to persist as electrophoretically distinguishable structures during an hour at the restrictive temperature. Since the position of the restriction sites is random relative both to the location of the replication forks and to the sites of repair, and since the induced patches are several thousand nucleotides in length, it might be anticipated that cuts would frequently occur within the long patches when the DNA is restricted for the analysis, thus dividing the long patch label between linear and forked DNA molecules. It is therefore not surprising that radioactivity incorporated into long patches would appear in both arcs even if all long patches occur near replication forks. Hence the results of this experiment cannot distinguish whether long patches might also occur in other regions of the genome where excision repair might be structurally constrained, but they do support the interpretation that uv-induced lesions in the vicinity of DNA replication forks are repaired by the induced process.

Indirect evidence that methyl adducts introduced into DNA in the vicinity of replication forks by treatment of cells with alkylating agents might also be repaired by the SOS-induced long patch process has come from a very different kind of 
experiment. We have recently begun to investigate the effects of SOS induction on mutagenesis by alkylating agents. Most of the mutagenesis resulting from treatment with $\mathrm{N}$-methyl- $\mathrm{N}^{\prime}$-nitro- $\mathrm{N}$ nitrosoguanidine (MNNG) can be attributed to direct mispairing by a tautomeric form of the $\mathrm{O}^{6}$ methylguanine adduct during replication, and MNNG mutagenesis is thus largely independent of error-prone processing by the SOS system. We therefore anticipated that SOS induction prior to MNNG treatment would have little effect on mutation frequency, although (as we had previously shown) cell survival is increased by such a treatment. However, as shown in Fig. 2, reversion of a his - mutation by MNNG is essentially abolished by the prior SOS induction. A possible explanation of this surprising observation invokes the fact that MNNG mutagenesis in bacteria and yeast occurs primarily at the location of the replication forks at the time of damage, presumably because the methyl transferase activity that repairs $O^{6}$. methylguanine adducts rapidly removes the premutagenic lesions elsewhere while those near the fork are immediately "fixed" by replication. Decreased mutagenesis in SOS-induced cells would thus be in keeping with and lend support to our hypothesis that SOS-induced resistance is achieved at least in part through induced ability to repair lesions near replication forks. Testing this intriguing explanation will be a subject for continued investigation.

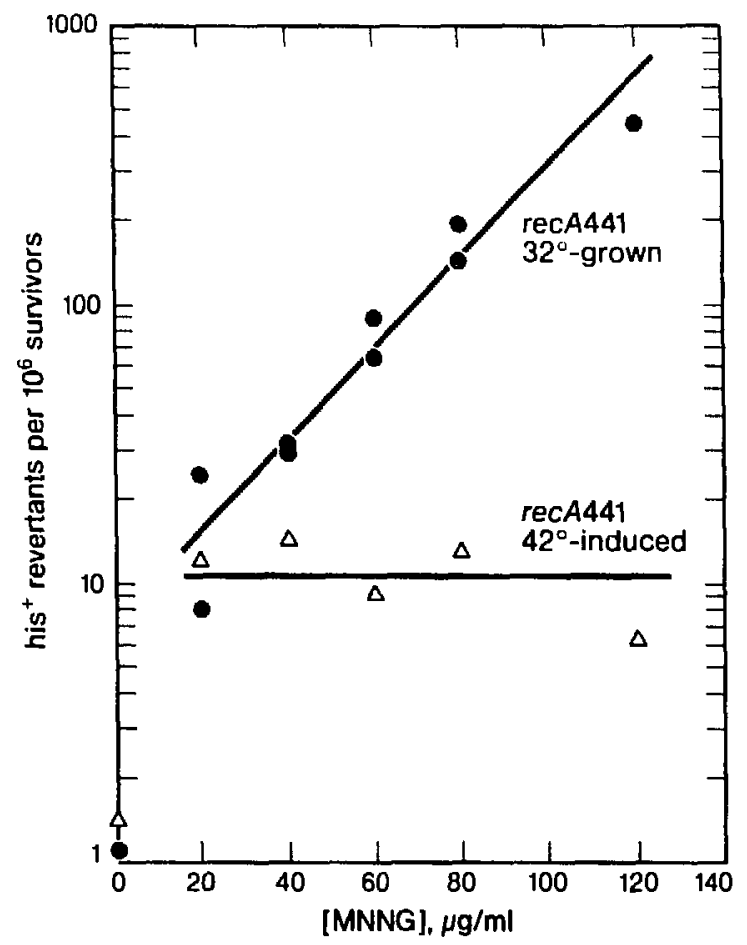

Fig. 2. Frequency to mutation to his ${ }^{\star}$ of an E. coli strain carrying the recA1441 mutation (which renders SOS functions inducible by a period of growth at $42^{\circ} \mathrm{C}$ ) as a function of MNNG concentration in a 10-minute treatment with or without induction of $5 O S$ functions prior to treatment. In both cases exposure to the alkylating agent was at $32^{\circ} \mathrm{C}$, and all assay plates were incubated at $32^{\circ} \mathrm{C}$.

(XBL 8612-9073)

\section{REPAIR OF DNA DAMAGE IN THE HUMAN METALLOTHIONEIN GENE FAMILY}

\section{Steven A. Leadon and Margaret M. Snowden}

The long-term objective of this research program is to better understand the relationship between the processing of DNA damage and the location of that damage in various regions of the genome. Although the general pathways for excision repair of various classes of structural defects in mammalian cells have been worked out, the "average" response of the entire genome has been assayed in most repair studies. Therefore, it is important to understand in detail how the excision repair responses might reflect unique features or regions of the genome, such as chromosomal location or status of expression of structural genes. The overall experimental strategy is to simplify the analysis by using defined DNA sequences so that processing of damage can be analyzed at the molecular level.

We have developed an immunological method for the measurement of repair using a monoclonal antibody to bromouracil-containing DNA to separate DNA fragments containing repair patches from unrepaired DNA. ${ }^{1}$ The presence of specific sequences in the repaired and unrepaired DNA fragments are detected by using nick-translated probes for the sequences of interest. Unlike methods that rely upon the loss of a labeled adduct or of an endonuclease-sensitive site from a sequence, this technique sensitively detects the repair synthesis event itself and therefore allows direct comparison of repair in specific sequences of 
a variety of types of DNA damage. We initially applied this method to the question of whether the repair of damage in a region of the genome that contains an integrated and expressed foreign DNA was different from that in the genome overall. A comparison was made between the overall rate of repair synthesis following uv irradiation or treatment with the carcinogen $A F B_{1}$ and the rate of repair in regions of the monkey genome containing the integrated and transcribed gpt gene of $E$. coli (Ecogpt). A more rapid repair of both uv and $A F B_{1}$ damage was observed in DNA fragments containing the Ecogpt gene than in the genome overall. We also employed the immunological method to measure repair in DNA fragments containing the alpha DNA sequence, since repair in this sequence in monkey cells has been extensively characterized. Consistent with previous observations measuring repair synthesis and direct adduct removal, repair of uv damage in alpha DNA occurred at the same rate as that in the genome overall, while repair of $A F B_{1}$ damage was deficient in alpha DNA. The rapid repair of damage in the Ecogpt gene may be related to the functional state of the sequence and/or to alterations produced in the chromatin conformation by the integration of plasmid sequences carrying the gene.

In order to distinguish enhanced repair of a sequence due to its transcriptional activity from enhanced repair due to chromatin alterations brought about by integration of a sequence into the genome, we are currently investigating the repair of damage both in endogenous genes and in cell lines that contain an integrated gene with an inducible promoter. The endogenous genes we are studying are the metallothioneins (MTs), a family of low molecular weight heavy-metal-binding proteins, unique in their cysteine content. MTs specifically bind heavy metals, such as zinc, cadmium, copper, and mercury. These proteins are inducible in experimental animals and cultured cells by exposure to heavy metal ions or glucocorticoid hormone. In all mammals studied, MTs exist in two electrophoretically distinguishable forms, designated MT1 and MT2.

In man, MTs comprise a multigene family consisting of about 10-12 members. However, only half of these genes appear to be expressed. Most of the human MT genes have been isolated and the nucleotide sequence of the functional MT1A and the MT2A genes and a processed pseudogene, MT2B, have been deterrnined. When human genomic DNA is digested with Eco RI, fractionated by electrophoresis on agarose gels and transferred to nitrocellulose filters, at least 11 different bands hybridizing to a probe containing a full-length cDNA clone of human MT2 mRNA can be detected. Three of these bands have been identified: MT1A (at $10 \mathrm{~kb}$ ), MT2A (at $5.7 \mathrm{~kb}$ ) and MT2B (at $4.8 \mathrm{~kb}$ ). The other restriction bands remain unassigned. Therefore, because of the complexity of this gene family, the initial experiments have focused on repair in these three genes.

Cultured human cells were exposed to $10-\mathrm{J} / \mathrm{m}^{2}$ uv light and allowed to repair in the presence of bromodeoxyuridine. The DNA was then isolated, digested with Eco RI, and fully hybrid density DNA made by semiconservative synthesis was separated from unreplicated DNA by centrifugation in $\mathrm{CsCl}$ density gradients. Unreplicated, parental-density DNA was then reacted with a monoclonal antibody against bromouracil and the DNA bound by the antibody was precipitated in $50 \%$ ammonium sulfate and separated from free DNA by centrifugation. Equal amounts of DNA from the bound and free fractions were electrophoresed on agarose gels and transferred to nitrocellulose. The presence of the MT genes was detected by using a ${ }^{32} \mathrm{p}$-labeled cDNA probe from MT2 mRNA. The autoradiogram in Fig. 1 shows that for the same amount of DNA loaded into each lane of the gel $(2 \mu \mathrm{g})$, more hybridization to the MT1A and MT2A genes occurs in the fraction bound by the antibody than in the free fractions, while similar amounts of hybridization to the MT2B pseudogene were found in both fractions. For sequences with a high degree of homology, the intensity of probe hybridized to a genomic restriction fragment relects the amount of that sequence present. The intensity of hybridization was quantitated by densitometry and the percentage of each gene in the bound fraction when compared to the percentage of total DNA bound by the antibody (Table 1). A 2- to 3-fold enhancement was found in the percentages of MT1A and MT2A in the bound fraction when compared to total DNA bound by the antibcdy. This indicates that there are more repair patches in those DNA fragments which contain these genes. In contrast, the same percentage of the MT2B gene was found in the bound fraction as in the total amount of DNA bound by the antibody. This indicates that there are the same number of repair patches in this pseudogene as in the rest of the genome. These findings support the hypothesis that there is a preferential repair of uv damage in transcriptionally active regions of the genome. Examination of the autoradiogram in Fig. 1 indicates that other members of the MT gene family also show preferential repair. As the functional state of the genes in these fragments becomes known, we should be 


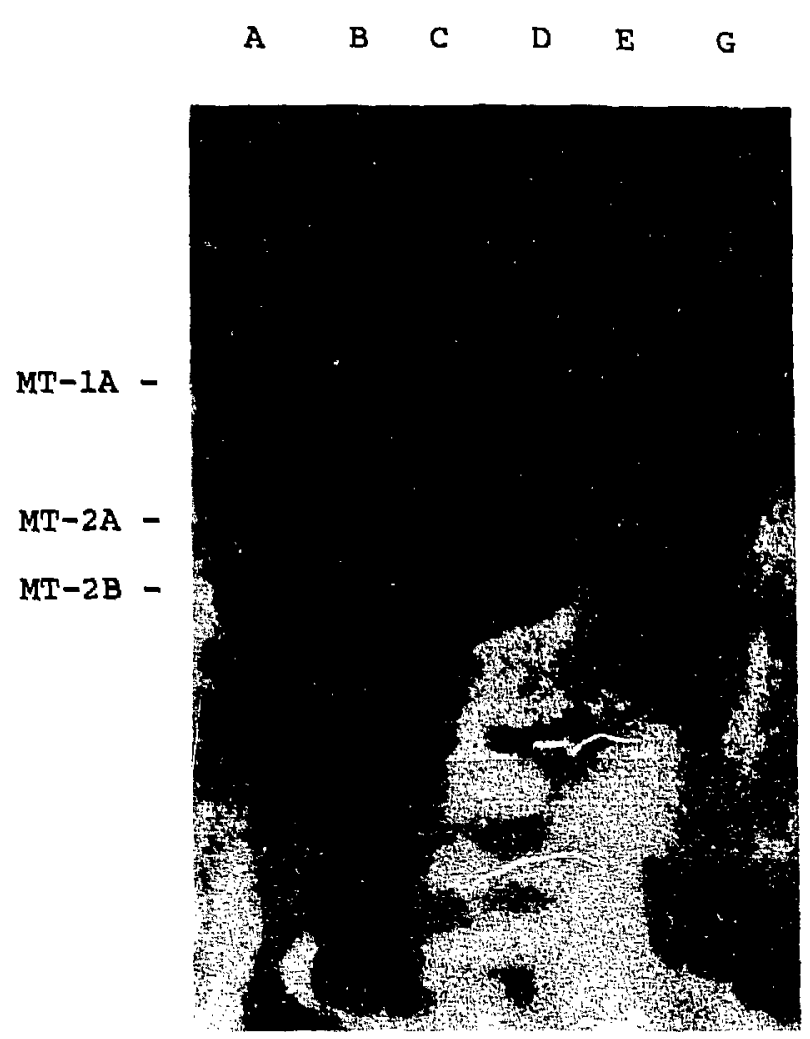

Fig. 1. Detection of the human MT genes in repaired DNA. Cell cultures were irradiated with $10 \mathrm{I} / \mathrm{m}^{2}$ uv and incubated for various times in the presence of $50 \mu \mathrm{M}$ BrdUrd. Genomic DNA, digested with Eco RI, was reacted with the antibody to BrdUrd. DNA bound by the antibody was precipitated with $50 \%$ ammonium sulphate and $2 \mu g$ from the bound and free fractions were electrophoresed on a $0.7 \%$ agarose gel. The DNA was transferred to nitrocellulase and probed with a "2p-nicktranslated cDNA from MT2 mRNA. Lane (A), O hr lotal DNA; (B) $3 \mathrm{hr}$ bound DNA; (C) $3 \mathrm{hr}$ free DNA; (D) $6 \mathrm{hr}$ bound; (E) $6 \mathrm{hr}$ free; (F) Hind III digested lambda DNA molecular weight markers. The location of 3 MT genes are also indicated.

(XBB 860-9579)

able to obtain additional information concerning differential repair within the genome.

We are also currently investigating what effect
Table 1. The percentages of the MT genes present in DNA fragments bound by the antibody during a post-uv incubation.

\begin{tabular}{llr}
\hline & \multicolumn{2}{c}{ Percentage in bound fraction } \\
\cline { 2 - 3 } & $3 \mathrm{hr}$ & $6 \mathrm{hr}$ \\
\hline Total DNA & $2.5 \%$ & $4.0 \%$ \\
MT1A & $8.4 \%$ & $13.9 \%$ \\
MT2A & $6.8 \%$ & $12.7 \%$ \\
MT2B & $2.7 \%$ & $4.3 \%$ \\
\hline
\end{tabular}

induction of transcription of the MT genes has on the efficiency of repair. The MTIA and MT2A genes show different induction responses to heavy metals and glucocorticoids. The induction of the MT-1A gene occurs only in the presence of $\mathrm{Cd}^{++}$ but not in the presence of $\mathrm{Zn}^{++}$or dexamethasome. Therefore, repair will be studied under conditions where one or both of the genes are induced by incubation heavy metals or dexamethasome. In addition, we will analyze the effects of DNA damage and repair upon the transcriptional activity of the genes.

The studies on the repair of DNA damage in regions of the genome that contain an integrated plasmid will be extended by using a pSV2-gpt vector that carries a 4-kb Eco RI mouse genomic fragment containing the mouse MT1 gene (pMT-GPT). This vector has been transfected into human cells and clones have been isolated that contain a single copy of the plasmid integrated into their genome. Studies, complementing those described for the endogenous human MT genes, will be carried out using these clones.

\section{REFERENCES}

1. Leadon, S.A. Differential repair of DNA damage in specific nucleotide sequences in monkey cells. Nucleic Acids Res. 14, 8979-8995 (1986). 


\section{Carcinogenesis}

\section{ALKYLATION OF POLYNUCLEOTIDES IN VITRO AND IN VIVO}

\section{Bea A. Singer, Sylvia J. Spengler, Frank Chavez, Heinz Fraenkel-Conrat, and Jarek T. Kusmierek}

$\mathrm{O}^{4}$-alkyl thymine DNA adducts have been implicated as causative lesions in chemical mutagenesis and carcinogenesis induced by $\mathrm{N}$ nitrosamine and $\mathrm{N}$-nitrosourea. The kinetics of incorporation of $\mathrm{O}^{4}$-alkyl dTTPs as analogues of dTTP indicate that they are recognized by a variety of polymerases but, in their presence, synthesis from synthetic polymers or DNA templates terminates within a short time. Relatively more synthesis, compared to that observed with dTTP, is seen as the temperature of polymerization is decreased. ${ }^{1}$ Our interpretation of the termination of synthesis is that the primer end with $\mathrm{O}^{4}$-alkyl dT did not form hydrogen bonds of sufficient stability to permit utilization of the $3^{\prime}-\mathrm{OH}$ by polymerases, as contrasted to possible proofreading activity or idling. To discriminate between these explanations, we studied the ability of the $3^{\prime} \rightarrow 5^{\prime}$ exonuclease activities of $E$. coli DNA polymerase $I$ (Pol I) and the $T_{4}$ DNA polymerase, both known to degrade poly $d(A-T)$ in the absence of dNTPs, to digest a similar polymer containing $\mathrm{O}^{4}$-methyl $d T\left(m^{4} \mathrm{~T}\right)$ as well as dT. Contrary to expectation, the polymers containing $\mathrm{m}^{4} \mathrm{~T}$ were completely resistant to the $3^{\prime} \rightarrow 5^{\prime}$ exonuclease activity of the two polymerases. $^{2}$

In vivo studies have focused on assessment of the mutagenic potential of $\mathrm{O}^{4}$-methyl thymidine. ${ }^{3}$ Pol I was used in vitro to incorporate a single $\mathrm{m}^{4} \mathrm{~T}$ residue at the 3 '-terminus of an oligonucleotide primer opposite the adenine residue of the amber codon in $\phi \times 174$ am3 DNA. After extension with unmodified nucleotides, the partial duplex product was transfected into $E$. coli spheroplasts (Fig. 1). Replication of the $\mathrm{m}^{4} \mathrm{~T}$ DNA in (ada) cells, which lack the $\mathrm{m}^{4} \mathrm{~T}$-DNA methyltransferase, yielded 10 times the mutant progeny phage as the control. In repair-proficient strains (ada) ${ }^{+}$, no increase in mutation frequency was observed. All 20 independently isolated mutant plaques contained an $A: T \rightarrow G: C$ transition at the site of $\mathrm{m}^{4} \mathrm{~T}$ incorporation, indicating that $\mathrm{m}^{4} \mathrm{~T}$ is mutagenic in $E$. coli and directly implicating such adducts in mutagenesis by methylating agents, confirming our in vitro results. ${ }^{4}$ In addition, the enzymatic method for site-specific mutagenesis provides an alternative to the chemical synthesis of oligonucleotides containing altered bases.

Another area of research involves studies on the products of alkylaton of nucleic acides by chloroacetaldehyde (CAA) and chloroethylene oxide

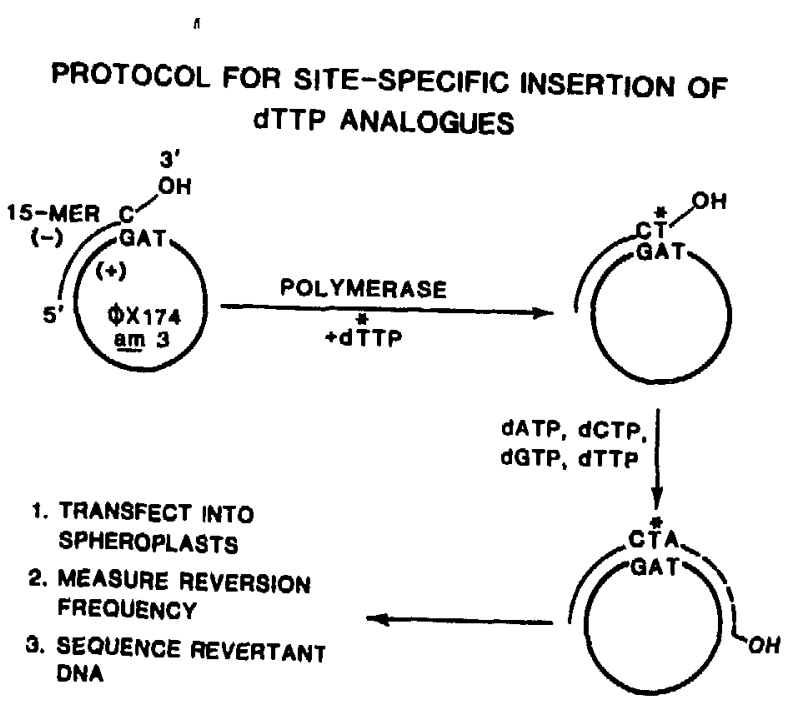

Fig. 4. Effects of $37^{\circ} \mathrm{C}$ incubation of the large discoidal complex preparation with LCAT an LDL: GCE profiles of (a) isolated HDL fraction from LCAT-deficient palient; (b) large discoidal complex preparation; (c) 24-hour incubation product; (d) (AI without AlI) fraction from IAC.

(XBL 8611-9067)
Fig. 1. Assay for the site-specific incorporation and mulagenicity of thymidine analogues ( $T^{*}=$ thymidine analogue).

(XEL 869-8967) 
(CEO), active metabolites of vinyl chloride, a human carcinogen. We have synthesized angular $\mathrm{N}^{2}$,3-ethenoguanine and the riboside (angeG), prepared the riboside diphosphate, and incorporated the adduct into ribopolymers. Using reverse transcriptase (AMV DNA polymerase), we have made accurate DNA copies to measure the type and amount of misincorporation directed by this derivative, previously deterted after treatment of nucleic acids with CAA. Incorporation of $\mathrm{T}$ and $C$ are significantly above background, indicating that ang $E$ is read by the polymerase as $G$ or $A$ when in a polymer. The additional ring can readily be accommodated in a canonical $\mathrm{G} \cdot \mathrm{C}$ base pair, with the loss of a single hydrogen bond. Similarly, ang $\in \mathrm{G}$ can base pair with $T$ with a minimum of helical distortion (Fig. 2). We are currently atiempting to synthesize the deoxytriphosphate for additional studies. However, the use of reverse transcriptase has permitted us to utilize the more stable riboside diphosphate to determine the probable mutagenic consequences of vinyl chloride exposure.

Our other preliminary study in the mechanism of vinyl chloride carcinogenesis involves the reaction of a model compound, N-1-oxo-adenosine, with the vinyl chloride metabolite, chloroacetaldehyde. This is a bifunctional alkylating agent that has the possibility of introducing crosslinks in the DNA. Lacking the possibility of alkylation at $\mathrm{N}-3$, $\mathrm{N}$-1-oxo-adenosine could yield a model crosslink. The CAA produces significant amounts of modification (at least five products) under physiological conditions. We are using reverse phase and cation exchange HPLC as well as other chromatography techniques to purify these products for further characterization.
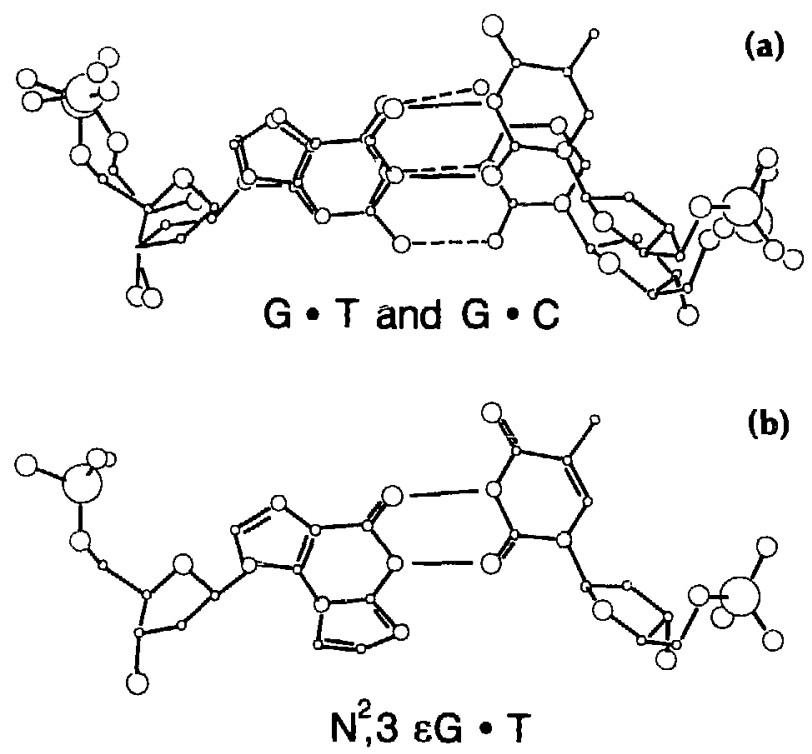

Fig. 2. Base pairing by $G$ and ang $N^{2}, 3$ etheno $G$. (a) The canonical G.C base pair and the wobble base pair, $G \cdot T$ superimposed as in a helix. (b) Angular $\in G$ base pair with $T$ with no further helix distortion than the wobble G.T pair.

(XBL 869-8966)

\section{REFERENCES}

1. Singer, B., Chavez, F., and Spengler, S.J. Biochemistry 25, 1201-1205 (1986).

2. Singer, B. Nucleic Acids Res. 14, 6735-6743 (1986).

3. Preston, B.D., Singer, B., and Loeb, L. Proc. Nat'l. Acad. Sci. USA 83, 8501-8505 (1986).

4. Singer, B., Spengler, S.J., Fraenkel-Conrat, H., and Kusmierek, J.T. Proc. Nat'l. Acad. Sci. USA 83, 28-32 (1986).

\section{EFFECTS OF ALKYLATING CARCINOGENS ON HUMAN TUMOR CELLS IN CULTURE}

\section{Regine Goth-Goldstein and Mildred Hughes}

A number of alkylating agents are powerful carcinogens and mutagens. They exert their biological effects primarily through alkylation of DNA. None of these agents form only a single product on reaction with DNA, and all react with DNA at various sites, the relative amounts of addiucts formed being characteristic for each agent. Because alkylating agents have been well characterized in respect to their reaction with DNA, one can now investigate the biological relevance of alkylation at certain sites of DNA bases. Alkylation at N7 of guanine, the major site of reaction with all methylating agents, has no apparent biological consequences. There is much evidence that alkylation of DNA bases at exocyclic oxygens can lead to mutations and malignant transformation. The formation, accumulation and persistence of these adducts in DNA of animal tissues correlates with the tissue- or species-specific 
tumorigenicity of alkylating agents. Much less is known about which lesions formed by alkylating agents are responsible for cell killing. In Escherichia coli 3-methyladenine and 3-methylguanine could be identified as lethal lesions, since two types of alkylating agent-sensitive mutants were deficient in repair of either of these lesions. Characterization of such mutants is an important tool to analyze certain repair pathways and to understand the biological role of the lesions repaired. Even though there is no reason to believe that 3methyladenine and 3-methylguanine are not potentially lethal lesions in mammalian cells as in bacteria, this question could not yet be addressed, because no cells with a defect in repair of these alkylated bases have been identified. Alkylationsensitive human celi lines do exist. These are the tumor cell lines of the complex Mer- phenotype. Originally, the $\mathrm{Mer}^{-}$phenotype was defined by the inability of cells to support growth of adenovirus treated with the alkylating agent $\mathrm{N}$-methyl- $\mathrm{N}^{\prime}$-nitro$\mathrm{N}$-nitrosoguanidine (MNNG). Ail $\mathrm{Mer}^{-}$cells examined were also hypersensitive to killing by MNNG and other alkylating agents, and failed to repair $0^{6}$ methylguanine. Since $\mathrm{Mer}^{-}$cells seem as efficient as $\mathrm{Mer}^{+}$in repair of all other alkylated DNA bases, it has been concluded that $0^{6}$-methylguanine, which has been implicated in $E$. coli solely as a potentially mutagenic lesion, is also a potentially lethal lesion in mammalian cells, responsible for the increased sensitivity of $\mathrm{Mer}^{-}$cells.

The widely studied HeLa $\$ 3$ cell line, which originated from a human carcinoma, has the $\mathrm{Mer}^{+}$ phenotype, but a $\mathrm{Mer}^{-}$variant (HeLa MR) has arisen in one laboratory. This offers the possibility to study $\mathrm{Mer}^{-}$and $\mathrm{Mer}^{+}$cells of otherwise similar genetic background. We are using these two variants to analyze the $\mathrm{Mer}^{-}$phenotype further. In previous studies we had found that there exists a mechanism whereby Chinese hamster ovary cells that survive a toxic dose of the alkylating agent MNNG are permanently more resistant to its killing effects. Last year we reported that this phenomenon of acquired resistance occurs also in HeLa cells but only in those of the $\mathrm{Mer}^{-}$phenotype. When HeLa S3 and HeLa MR were treated with a highly toxic dose of MNNG, and the surviving population exposed to a second dose of MNNG 2-3 weeks later, HeLa $\$ 3\left(\mathrm{Mer}^{+}\right)$cells were equally or even slightly more sensitive to a second exposure of MNNG, whereas the surviving Hela MR $\left(\mathrm{Mer}^{-}\right)$population was much more resistant to MNNG, the level or survival being close to that of HeLa S3. The increased resistance of HeLa MR cells following MNNG-treatment has been studied further during the last year.

To ascertain that this dramatically increased resistance of pretreated MR cells was not simply due to selection of a preexisting resistant subpopulation in this $M R$ line, clones derived from single cells of the $M R$ line were grown to mass-culture, then treated with MNNG and after cells had recovered, the response of nonpretreated and pretreated cells to MNNG was compared. Of six clones tested the ratio of survival of pretreated versus nonpretreated cells after a certain MNNGdose $(0.2 \mu \mathrm{g} / \mathrm{ml})$ was $10,18,27,67,240,395$, that is, in each case MNNG-pretreatment resulted in an increased resistance of the surviving population, although the absolute increase varied between the clones.

So far the sensitivit : of the total surviving population was always measured, and it seemed quite likely that this population was in fact heterogeneous, consisting of cells of different sensitivity. To estimate the composition of a pretreated HeLa MR population several clones derived from single cells of a pretreated population were isolated and their dose-response to MNNG determined. Of eight clones isolated five had a dose-response like HeLa $\mathrm{Mer}^{+}$cells, and three showed an intermediate sensitivity to MNNG. So all clones were more resistant to MNNG than the parent line MR. One of the resistant clones, named $\mathrm{Cl} 4$, was chosen for further characterization. $\mathrm{Cl} 4$ had the same dose response as $\mathrm{Mer}^{+}$cells to all monofunctional alkylating agents tested, but $\mathrm{Cl} 4$ cells were as sensitive as the untreated MR cells to the cross-linking agent chloroethylnitrosourea (CNU) (Fig. 1). Since the formation of DNA cross-links, the lethal lesions of CNU is prevented by the protein that repairs $0^{6}$. methylguanine $\left(0^{6}-\mathrm{MG}\right)$, the finding that $\mathrm{Cl} 4$ cells are still sensitive to $\mathrm{CNU}$, indicated that these cells are lacking this protein.

To determine the repair capacity in the various Hela variants for $0^{6}-\mathrm{MG}$ the persistence of $0^{6}-\mathrm{MG}$ in DNA of cells treated with radioactively labeled methylnitrosourea (MINU) was measured. (MNU forms the same spectrum of methylated bases as MNNG and has several advantages for use in these experiments.) DNA of cells treated with $3 \mathrm{H}-\mathrm{MNU}$ was isolated at different times after posttreatment incubation of cells, and methylated purine bases were analyzed by HPLC chromatography. The amounts of methylated purine bases measured are summarized in Table 1 . The overall methylation of DNA as judged by the amount of 7-methylguanine formed was the same in the three lines (5.1-6.1 

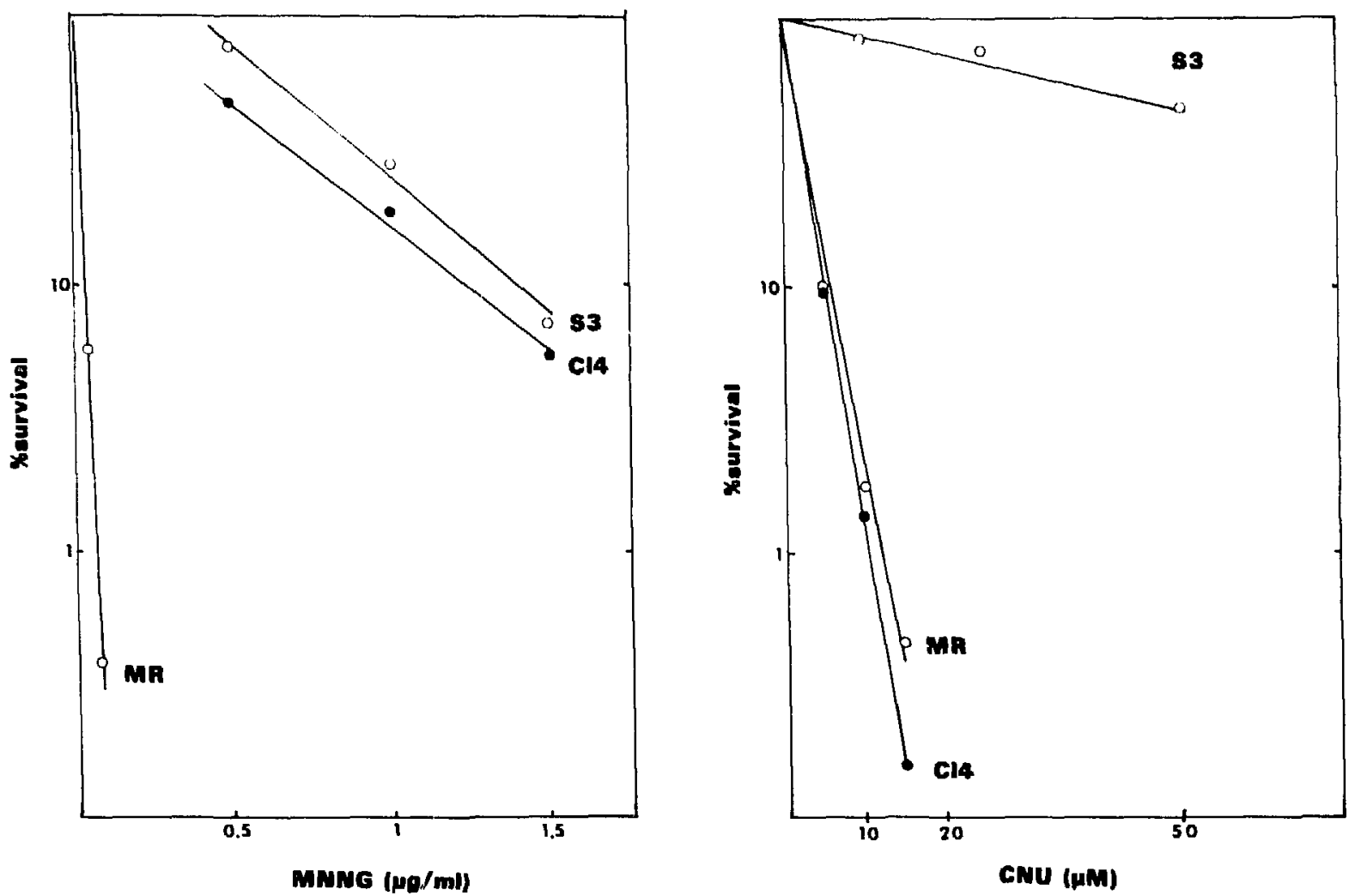

Fig. 1. Cell survival of HeLa Mr (Mer-), Cl 4 (isolated from MNNG-ireated HeLa MR culture) and Hela S3 $\left(\right.$ Mer $^{+}$) after a 1-hr treatment with MNNG (left panel) or with the cross-linking agent CNU (right panel).

(XBL 8671-9037)

Table 1. Methylation of HeLa DNA by ${ }^{3} H-M N U$ and repair of methylated bases during posttreatment incubation.

\begin{tabular}{|c|c|c|c|c|}
\hline $\begin{array}{l}\text { Hela } \\
\text { line }\end{array}$ & $\begin{array}{l}\text { Posttreatment } \\
\text { incubation }|\mathrm{h}|\end{array}$ & $\begin{array}{l}\text { N7-methyl- } \\
\text { guanine }\end{array}$ & $\begin{array}{l}\text { N3-methyl- } \\
\text { adenine' }\end{array}$ & $\begin{array}{l}0^{6} \text {-methyl- } \\
\text { guanine }\end{array}$ \\
\hline \multirow[t]{3}{*}{ MR } & 0 & 5.85 & 0.34 & 0.58 \\
\hline & 6 & 5.49 & 0.05 & 0.5 \\
\hline & $21^{b}$ & 4.04 & $-c$ & 0.52 \\
\hline \multirow[t]{3}{*}{ S3 } & 0 & 5.11 & 0.26 & 0.18 \\
\hline & 4 & 4.33 & 0.07 & 0.12 \\
\hline & $21^{b}$ & 3.77 & $-^{c}$ & $(0.05)^{c}$ \\
\hline \multirow[t]{3}{*}{$\mathrm{Cl} 4$} & 0 & 6.14 & 0.51 & 0.66 \\
\hline & 4 & 5.89 & 0.13 & 0.61 \\
\hline & $20^{b}$ & 4.38 & $-c$ & 0.58 \\
\hline
\end{tabular}

- f mol/rg DNA

b These values were corrected for dilution of label by cell population

- Too low to be evaluated $\mathrm{fm} / \mu \mathrm{g}$ DNA). Also the loss of this base and 3methyladenine was similar in all variants. The initial amount of $0^{6}-\mathrm{MG}$ in the DNA of HeLa S3 was only a third of that in $\mathrm{MR}$ and $\mathrm{Cl} 4$. This is due to the instant reaction of the repair protein with $0^{6}-M G$, which occurs already during the short incubation with $3 \mathrm{H}-\mathrm{MNU}$. The amount of $0^{6}-\mathrm{MG}$ in HeLa MR and $\mathrm{Cl} 4$ was not reduced over 20-h interval.

Clearly, $\mathrm{Cl} 4$ cells, just like the MR cells, lack the protein which repairs $0^{6}-M G$, that is they have the $\mathrm{Mer}^{-}$phenotype in this respect, whereas they display the $\mathrm{Mer}^{+}$phenotype in respect to sensitivity to monofunctional alkylating agents.

In conclusion, MNNG-treatment of HeLa $\mathrm{Mer}^{-}$ cells leads to partial phenotypic reversion to the $\mathrm{Mer}^{+}$phenotype in that the revertants become resistant to MNNG. The results suggest 1) that the two characteristics of the Mer- phenotype, lack of $0^{6}-M G$ repair and hypersensitivity to MNNG though 
usually coupled, can be uncoupled, 2) that sensitivity of $\mathrm{Mer}^{-}$cells to monofunctional and bifunctional alkylating agents has a different basis, 3) that only sensitivity to bifunctional alkylating agents is related to lack of $0^{6}-\mathrm{MG}$ repair protein, and 4) that therefore $0^{6}-M G$ is not a lethal lesion.

\section{CARCINOGENIC POTENCY}

Lois Swirsky Gold, Bruce N. Ames, Renae I. Magaw, Georganne M. Backman, Thomas H. Slone, Joan Schwalbe," Maria Da Costa, Susan Eisenberg, Paul Chous, and Veronica Cabras

The Carcinogenic Potency Database is an attempt to quantify and standardize the animal bioassay literature and to organize it systematically. In the past, efforts to use animal bioassays in the evaluation of the potential health risk of chemicals to humans were hampered by the lack of a standardized method of comparing experimental results. Experimental protocols as well as the type of information reported in the literature are quite diverse. Moreover, quantitative estimates of carcinogenic potency had not been made for large numbers of chemical carcinogens.

\section{TD $50:$ A NUMERICAL INDEX OF CARCINOGENIC POTENCY}

Our group has proposed as an index of carcinogenic potency, the $T D_{50}$, or tumorigenic dose rate for $50 \%$ of the test animals. Briefly, the TD 50 is defined as the chronic dose rate to induce tumors in half the test animals (in the absence of tumors in the control group). $T D_{50}$ can be calculated for any single category of neoplasm or any combination of neoplasms. This standardized numerical index permits comparisons among tests and also improves past efforts to estimate carcinogenic potency by taking into account whatever spontaneous tumors occur in control animals. In addition, where data are available about the time of death and tumor incidence for each animal, we estimate a $T D_{50}$ using this information. This is important because animals given high doses of a chemical frequently die early due to chemical toxicity rather than to tumors, and failure to account for this early mortality sometimes leads to underestimation of the true potency. A full statistical description of $\mathrm{TD}_{50}$ is given in Sawyer et al. ${ }^{1}$ and

"Department of Biochemistry, University of California Berkeley
Peto et al. ${ }^{2}$ A comparison of resuits obtained from estimation of $T D_{50}$ using lifetable data vs. summary incidence data appears in Ref. 3.

\section{THE CARCINOGENIC POTENCY DATABASE}

The database of test results reported in the literature prior to July 1981 was published in a plot format in 1984, ${ }^{4}$ and a chronological supplement through December 1982 appeared in the same journal this year. $^{5}$ The two published databases together contain results of approximately 3,200 experiments on 835 chemicals.

The database is readily accessible for qualitative and quantitative analysis. All positive and negative experiments that fit a set of standard criteria based on suitability for estimation of potency have been included in the database, namely, tests in which a single compound was administered to the animals, the exposure was chronic and lasted at least six months in rodents, the route of administration was likely to result in the whole body being exposed, the experiment length was at least one year in rodents, and a control group was used.

We have included information in the database about a variety of factors that are important in interpreting bioassays, such as the $T D_{50}$ and its statistical significance, the species and strains that have been tested chronically, the route and duration of compound administration, the tumor types, the proportion of animals with specific types of tumors in dosed and control groups, the shape of the dose-response curve, and the author's opinion about carcinogenicity.

During the past year we have continued to update the database with experimental results published through December 1984. We have also added several tests that were published in earlier years but were only identified recently in our ongoing literature search. 


\section{RANGE OF CARCINOGENIC POTENCY $\left(T^{50}\right)$}

We have found that the range of $T D_{50}$ is more than 10 millionfold for male and female rats and for male and female mice. In Fig. 1 we present the most potent $\mathrm{TD}_{50}$ value for several substances chosen from among a much larger group that were evaluated by the authors of published papers as tumorigens in female mice. For each chemical we indicate the value of the most potent $T D_{50}$ for a target-site(s) identified by the author and for which the $T D_{50}$ is statistically significant $(p<0.01)$. The most potent compound is TCDD $(2,3,7,8$,tetrachlorodibenzo-p-dioxin) with a value of 530 ng. The least potent is di(2-ethylhexyl)phthalate with a value of $3.40 \mathrm{~g}$.

In eariier analyses we obseryed a very good correlation of carcinogenic potency between rats and mice over large numbers of test chemicals that are positive in both species. ${ }^{6}$ We demonstrated that the potency of a carcinogen $\left(T D_{50}\right)$ is restricted to an approximately thirtyfold range, about the maximum dose tested in the experiment in the absence of $100 \%$ tumor incidence in treated animals. An incidence of $100 \%$ is only rarely observed in animal bioassays. The maximally tolerated doses (MTDs) of chemicals tested in chronic animal bioassays span a range of seven orders of magnitude, and these doses are highly correlated between rats and

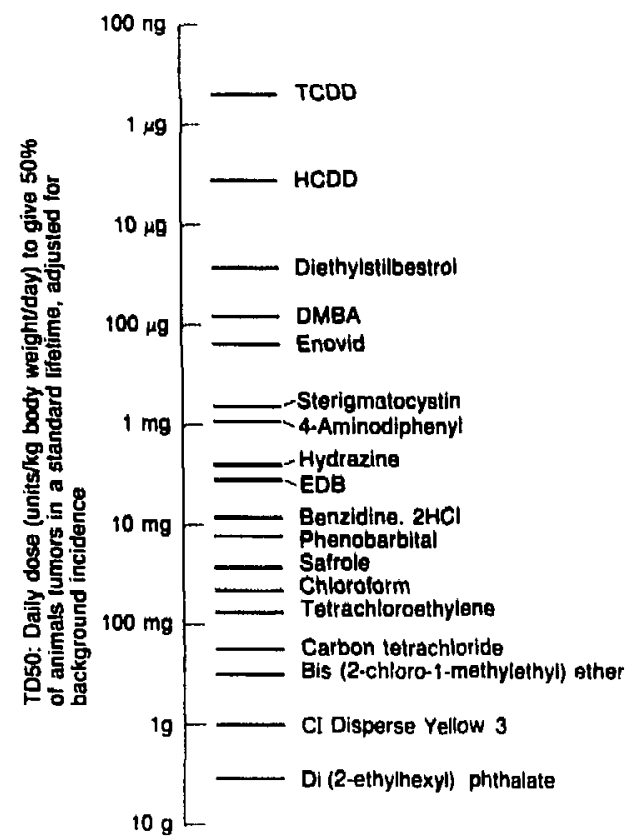

Fig. 1. Range of carcinogenic potency in female mice.

(XBL 8610-6450) mice. These facts, together with the restricted range of potency about the MTD, 1) account for high correlations in carcinogenic potency between rats and mice, and 2) provide a statistical basis for the relationship between potency values and MTDs. One would expect to observe a similar relationship between carcinogenic potency $\left(T_{50}\right)$ and $L D_{50}$, assuming that the acute and the chronic toxicity are related. Biologically it may indeed be the case that $T D_{50}$ and the MTD are closely related. Tissue damage with cell killing and consequent cell proliferation has been shown to be important in the promotion of liver tumors and possibly other tumors as well. Therefore, a single mutagenic compound given at tissue-damaging doses (near the MTD) can act as its own promoter as well as initiator. $^{6,7}$

\section{REPRODUCIBILITY OF RESULTS IN “NEAR- REPLICATE" CARCINOGENESIS BIOASSAYS}

Two major assumptions underlie the use of animal bioassay data to assess possible carcinogenic hazards to humans: first, that the biological processes involved in the development of cancer are similar in rodents and humans, and second, that the results of animal bioassays are reproducible. During the past year our group has examined the question of reproducibility using results reported in the Carcinogenic Potency Database. We consider two or more "near-replicate" experiments of the same chemical as reproducible if three outcomes are similar, 1) whether or not the test agent is carcinogenic in both experiments, 2) the particular sites are the same, and 3) whether the estimates of carcinogenic potency are similar. We define two experiments conducted at different times as nearreplicates if they test the same chemical agent in the same strain and sex of rat, mouse, or hamster by the same route of administration. We investigated reproducibility of results in 70 near-replicate comparisons of 38 chemicals and have found good reproducibility of positivity, target site, and carcinogenic potency in hamsters, mice, and rats. Tine published authors' opinions about whether the test was positive disagreed in only 9 of the $70 \mathrm{com}$ parisons $(13 \%)$. Among the 35 comparisons in which all tests of the chemical were positive, 33 of the near-replicates had at least one identical target site $(94 \%)$. The carcinogenic potency values estimated from near-replicate tests in these $35 \mathrm{com}$ parisons were within a factor of 2 of each other in $40 \%$ of the comparisons, within a factor of 5 in $80 \%$, and within a factor of 10 in $90 \%$. 
We have tried to account for thie discordant findings on carcinogenicity in the nine cases, and have found that the differences between the protocols of the near-replicate experiments were not greater among the discrepant comparisons than among the concordant ones. In terms of experiment length, exposure duration, proportion of the animal's life that dosing lasted, and maximum total doses, the discordant tests were as similar to one anuther as the concordant ones.

We also have examined whether the individual experiments in the discrepant cases may have shared characteristics of length or dose that might tend to produce inconsistent results. We hypothesized that if experiments were of short duration, then a carcinogenic response might be detected in some cases but not in others, since tumors generally appear late in life. Our analysis indicates that in mice the discrepant cases were more often below the median experiment length than the concordant-positive cases (Fisher exact test, two-sided $p<0.01$ ); however, in rats the opposite was true-the discrepant tests tended to be longer.

With respect to dose we have found that the highest daily dose level as well as the total dose of the chemicals in the discrepant cases are generally larger than the dose levels in the concordantpositive cases, for both mice and rats (Fisher exact test, two-sided $p<0.01$ ). Therefore, the chemicals tested in these discordant comparisons tended to be weakly active (i.e., chemicals which are toxic only at higher doses).

\section{COMPARISON OF CARCINOGENICITY IN RATS AND MICE}

In a related analysis we focused on the 303 chemicals in the database that have been tested in both rats and mice. By examining how well tests in rats and mice predict one another we hope to gain insight into the feasibility of interspecies extrapolation between rodents and man. Of the 303 chemicals tested in both rats and mice, $34 \%$ are positive in both, $42 \%$ are negative in both, $9 \%$ are positive only in the rat, and $15 \%$ are positive only in the mouse. The predictive value of positive results in one species for carcinogenicity in the second is as follows: $78 \%$ of rat carcinogens $(103 / 132)$ were positive in the mouse and $70 \%(103 / 148)$ of mouse carcinogens were positive in the rat. Only $26 \%$ $(45 / 171)$ of chemicals negative in the rat were positive in the mouse and $19 \%(29 / 155)$ of chemicals negative in the mouse were positive in the rat.

To gain some perspective on the finding that
$70-78 \%$ of compounds positive in one species are positive in the other, we made a similar comparison between the sexes. Within each species, $81-87 \%$ of the chemicals positive in one sex are positive in the other. Thus, the predictive values of species and sex are similar, although prediction between the sexes is slightly better.

A corollary to the prediction of positivity in one species from positivity in another is the predictive value of individual target sites. We analyzed the target organs of the 177 chemicals that were tested in both rats and mice and were positive in at least one of the species.

In Table 1 we first show the total number of chemicals positive at each target site separately for rats and mice; second we report the number of chemicals positive at each site in each species that are also positive at some site in the second species. For example, 50 chemicals were positive in the rat liver and 47 of these (94\%) were also positive at some site in the mouse. Among the 89 chemicals that induced tumors in the mouse liver, $55(62 \%)$ were positive at some site in the rat. Although the numbers here are not large, most target sites in each species are excellent predictors of positivity in the other species. If the target organ in the rat is the liver, lung, mammary gland, ear/Zymbal's gland, or vascular system, there is at least an $88 \%$ chance that it will be positive at some site in the mouse. If a chemical is positive in the mouse in the lung, mammary gland, hematopoietic system, stomach, kidney, urinary bladder, Zymbal's gland, or skin, there is at least an $85 \%$ chance that it will be positive at some site in the rat. The poorest predictors in the rat are the urinary bladder $(43 \%)$ and the hematopoietic system $(64 \%)$; the poorest predictors in the mouse are the liver $(62 \%)$ and the vascular system $(71 \%)$. We note that while there has been considerable debate about the mouse liver, the liver is no worse a predictor of interspecies carcinogenicity than these other sites. We conclude that overall prediction of carcinogenicity from one rodent species to the other is reasunably good, and that positivity in the second species can be predicted from most common target sites in the first species.

\section{OTHER ANALYSES}

We have also proposed a method for using TD 50 to rank the potential hazards to humans from various chemical exposures, and we have applied this method to exposures in the workplace. ${ }^{8,9}$ We propose a rough index, the Exposure-Potency Index (EPI), that compares human exposure levels (in 
Table 1. Predictive value of target sites in one species for carcinogenicity in a second species: rats and mice

\begin{tabular}{|c|c|c|c|c|}
\hline \multirow[b]{3}{*}{ Target site } & \multicolumn{4}{|c|}{$\begin{array}{c}\text { Chemicals tested in both rats and mice } \\
\text { and evaluated as carcinogenic in at least } \\
\text { one experiment }\end{array}$} \\
\hline & \multicolumn{2}{|c|}{ Rats } & \multicolumn{2}{|c|}{ Mice } \\
\hline & $\begin{array}{l}\text { Total number } \\
\text { positive at } \\
\text { site } \\
\text { Number }^{b}\end{array}$ & $\begin{array}{l}\text { Number positive } \\
\text { at site in rats } \\
\text { that are also } \\
\text { positive at some } \\
\text { site in mice } \\
\text { Number }(\%)\end{array}$ & $\begin{array}{c}\text { Total number } \\
\text { positive at } \\
\text { site } \\
\text { Number }^{b}\end{array}$ & $\begin{array}{l}\text { Number positive } \\
\text { at site in mice } \\
\text { that are also } \\
\text { positive at some } \\
\text { site in rats } \\
\text { Number (\%) }\end{array}$ \\
\hline Liver & 50 & $47(94 \%)$ & 89 & $55 \quad(62 \%)$ \\
\hline Lung & 11 & $10(91 \%)$ & 35 & $30(86 \%)$ \\
\hline Mammary gland & 31 & $28(90 \%)$ & 7 & $7(100 \%)$ \\
\hline Hematopoietic system & 14 & $9(64 \%)$ & 26 & $22(85 \%)$ \\
\hline Vascular system & 11 & $9(82 \%)$ & 21 & $15(71 \%)$ \\
\hline Stomach & 16 & $13(81 \%)$ & 14 & $12(86 \%)$ \\
\hline Kidney & 16 & $12(75 \%)$ & 7 & $6(86 \%)$ \\
\hline Urinary bladder & 14 & $6(43 \%)$ & 5 & $5(100 \%)$ \\
\hline Zymbal's gland/ear & 13 & 12 (92\%) & 1 & 1 (100\%) \\
\hline Skin & 10 & $8(80 \%)$ & 1 & $1(100 \%)$ \\
\hline Any site & 132 & $103(78 \%)$ & 148 & $103(70 \% .0)$ \\
\hline
\end{tabular}

- Target sites are those affected by at least 10 chemicals in one species.

b Numbers add to more than total for "any site" because there is often more than one target site per chemical per species.

$\mathrm{mg} / \mathrm{kg} /$ day) to the dose (in $\mathrm{mg} / \mathrm{kg} /$ day) that induces tumors in $50 \%$ of test animals. By calculating EPI values for a variety of chemical exposures, it is possible to rank-order exposures according to possible hazard. One benefit of this approach is that it orients us among many human exposures; this improves the chances that policy-making efforts will be directed first toward those substances that may be most hazardous and away from substances that may present only minor hazards.

In our examination of nine industrial chemical carcinogens, we have found that for some of these substances the Permissible Exposure Levels (PEL's) set by the U.S. Occupational Safety and Health Administration (OSHA) allow workers to be exposed to doses that are close to the doses that induce tumors in rodents. Moreover, the margin of protection provided by the PEL for different chemicals varies over 1000-fold.

In earlier work, our group also examined the association between tumor pathology and carcinogenic potency. ${ }^{10}$ Currently we are expanding our work on the EPI to include human exposures in air, water, and food. We are also examining the shape of the dose-response in experiments with multiple doses and the correlation of mutagenic and carcinogenic potencies.

\section{REFERENCES}

1. Sawyer, C., Peto, R., Bernstein, L., and Pike, M.C. Calculation of carcinogenic potency from long-term animal carcinogenesis experiments. Biometrics 40, 17-40 (1984).

2. Peto, R., Pike, M.C., Bernstein, L., Gold, L.S., and Ames, B.N. The $T D_{50}:$ A proposed general cunvention for the numerical description of the carcinogenic potency of chemicals in chronic-exposure animal experiments. Environmental Health Perspectives 58, 1-8 (1984).

3. Gold, L.S., Bernstein, L., Kaldor, J., Backman, G., and Hoel, D. An empirical comparison of methods used to estimate carcinogenic potency in long-term animal bioassays: Lifet- 
able vs. summary incidence data. Fundam. Appl. Toxicol. 6, 263-269 (1986).

4. Gold, L.S., Sawyer, C.B., Magaw, R., Backman, G.M., de Veciana, M., Levinson, R., Hooper, N.K., Havender, W.R., Bernstein, L., Peto, R., Pike, M.C., and Ames, B.N. A carcinogenic potency database of the standardized results of animal bioassays. Environ. Health Perspect. 58, 9-319 (1984).

5. Gold, L.S., de Veciana, M., Backman, G.M., Magaw, R., Lopipero, P. Smith, M., Blumenthal, M., Levinson, R., Bernstein, L., and Ames, B.N. Chronological supplement to the carcinogenic potency database; Standardized results of animal bioassays published through December 1982. Environ. Health Perspect. 67, 161-200 (1986).

6. Bernstein, L., Gold, L.S., Ames, B.N., Pike, M.C., and Hoel, D.C. Some tautologous aspects of the comparison of carcinogenic potency in rats and mice. Fundam. Appl. Toxicol. 5, 263-269 (1985).

7. Bernstein, L., Gold, L.S., Ames, B.N., Pike,
M.C., and Hoel, D.G. Letter to the Editor: Toxicity and carcinogenic potency. Risk Analysis, Vol. 5, No. 4, 263-264 (1985).

8. Hooper, K., and Gold, L.S. The exposurepotency index (EPI): ranking the carcinogenic hazards of volatile industrial chemicals. $C$. Becker (Ed.), Cancer Prevention: Strategies in the workplace, Hemisphere Publishing, Washington, D.C., pp. 1-11 (1985).

9. Hooper, K. and Gold, L.S. Ranking the carcinogenic hazards of occupational exposures: Exposure-potency index (EPI) values for nine volatile industrial chemicals. $M$. Sorsa and $H$. Norppa (Eds.), Monitoring of Occupational Genotoxicants: Proceedings of a satellite symposium to the fourth international conference on environmental mutagens, Alan R. Liss, Inc., New York, N.Y., pp. 217-228 (1986).

10. Gold, L.S., Ward, J.M., Bernstein, L. and Stern, B. Association between carcinogenic potency and tumor pathology in rodent carcinogenesis bioassays. Fundam. Appl. Toxicol. 6, 677-690 (1986).

\title{
TISSUE TROPISM AND TEMPORAL EXPRESSION OF ROUS SARCOMA VIRUIS IN EMBRYONIC AVIAN LIMB IN VIVO AND AFTER TRANSFER TO CELL CULTURE
}

\author{
Anthony R. Howlett, Betsey L. Cullen, Mark D. Hertle, and Mina J. Bissell
}

Embryonic avian tissue has been shown to be resistent to the transforming potential of Rous sarcoma virus (RSV) in ovo during the first semester of development; the transformed phenotype is expressed following dissociation of infected tissues and seeding into tissue culture. This suggests that host factors, associated with the in vivo environment of the developing embryo can influence the tumourigenicity of RSV, perhaps by regulating synthesis of inhibitory components (e.g., tyrosine specific phosphatases) or endogenous tumor promoters (e.g. growth factors or hormones). Alternatively, the temporal and spatial sequence of the expression of viral and cellular factors within the dynamic developing field of the limb could in some way determine the outcome of host-viral interactions and thereby regulate susceptibility to expression and/or infection (tissue tropism) and competence for transformation. In order to examine the pattern of interactions between virus and host tissues in the chick embryo, monoclonal antibodies specific for the viral structural protein P19gag and host tissue determinants, myosin and collagen types I and II were used to identify the early cellular targets for RSV infection and the kinetics of viral spreading in the developing limb bud in vivo and in cultured limb bud cells. We showed previously that the spreading of RSV in infected tissues follows two distinguishable patterns in ovo and in culture. Viral transmission in ovo was characterized by a saltatory kinetic with two rapid bursts of infectious spread with intervening periods of stasis. In culture, the percentage of infected cells from early (day 5-6) embryos did not increase appreciably within 7 days, whereas the number of infected cells in cultures from day 8-11 embryos approached $100 \%$ at a rate that increased with increasing embryo age (see Howlett et al. in 1985 Annual Report). Morphological examination of frozen sections made from limbs at days 6,8 , and 10 and stained by Mallory.Heidenhain's trichrome method showed that the RSV-infected limb buds developed 
normally as compared to control (uninfected) limbs. However, by day 10 hemorrhagic lesions, characterized by large edematous spaces surrounded by tissue of low cohesivity, were apparent in some infected specimens as described previously.' Analysis of the source of RSV-infected cells by immunolocalization showed that RSV was located primarily in the skeletal muscle of infected limbs.
This tissue tropism was apparent by day 6 of development and persisted through to day 10 , although by day 10 evidence of viral spreading was demonstrable in some nonmyogenic tissues, notably vascular and connective tissue.

At day 6, muscle tissue could be visualized as condensed dorsal and ventral masses of myosinpositive cells [Fig. 1(B)]. In some cases splitting of
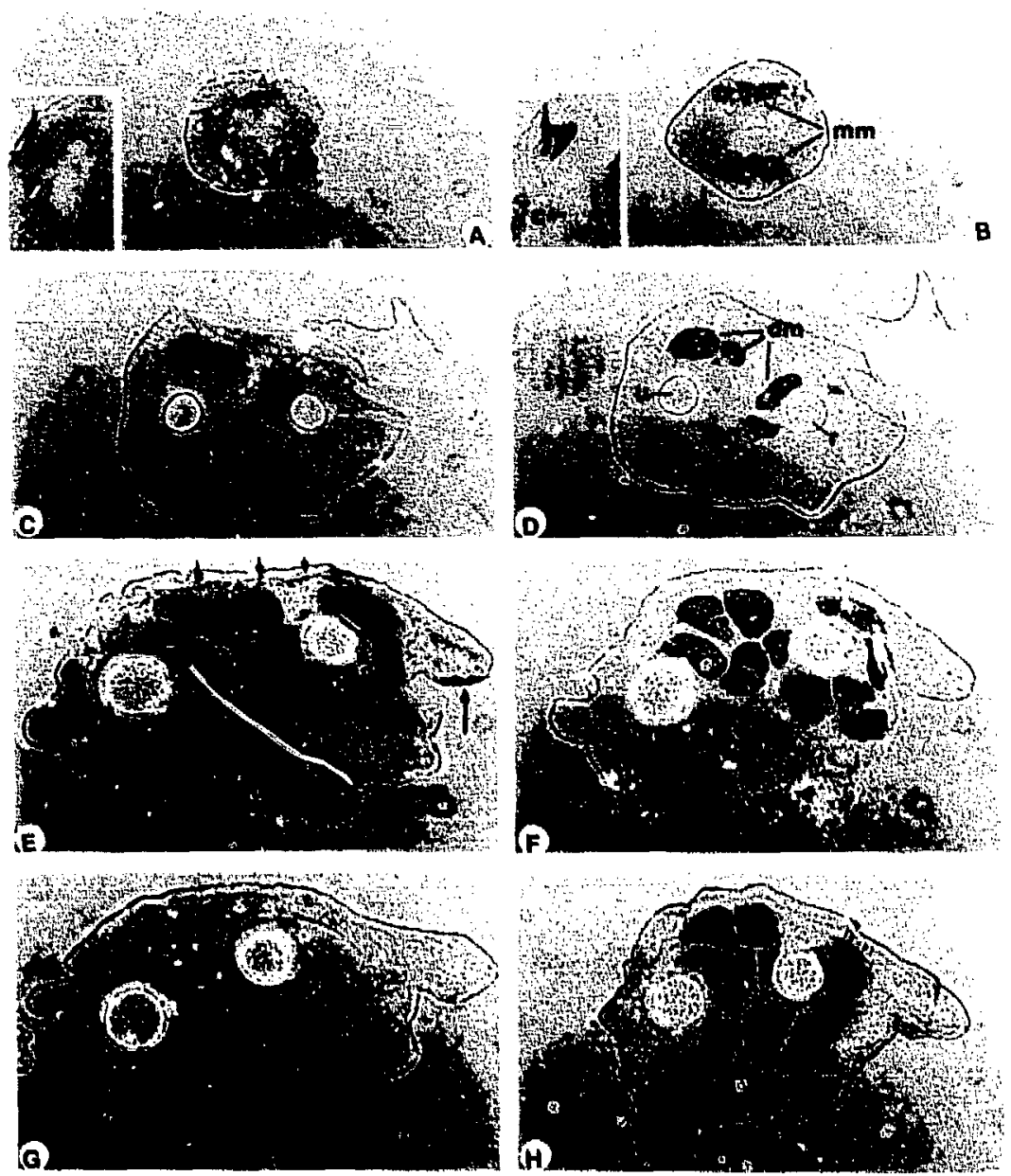

Fis. I Immunohistochemical localization of P19 and myosin in 6-um frozen sections taken from RSV-infected embryos at days $6(A, B), 8(C, D)$ and $10(E, F)$ of development, and from normal embryos at day $10(G, H)$. Tissues were sectioned at the approximate mid-point of the limb at day 6 and at the proximal to mid-radius-ulna at days 8 and 10. Sections were stained with anti-P19 (left panel) and anti-myosin (right panel) antibodies using an indirect biotin-streptavidin alkaline phosphatase detection procedure, At day 6 myosin positive cells are detected in transverse sections as dorsal and ventral premuscle masses, $\mathrm{mm}$, as shown in (B). (A) shows a similar area stained with anti-P19. Insets of $(A)$ and (B) show PI9 and myosin localization in longitudinal sections. The arrows show the correspondence of P19 and myosin in an area above the central core of cartilage, c, lowards the dorsal limb surface. At day 8 P19 is clearly localized in the various blocks of skeletal muscle that have split from the premuscle masses as shown in $(C)$ and $(D)$. (D) shows myosin staining in the dorsal, dm, and ventral, vm, muscles above and below the ulna, $u$, and radius, $r$. At day 10 P19 is localized in all the blocks of skeletal muscle and is now also expressed in nonmyogenic tissues as shown in (E) and (F). The arrows show P19 staining in myosin negative loose connective tissue, subdermal biood vessels perichondrium and the epidermis. (E) shows P19, (F) shows myosin. (G) and $(H)$ show PI9 $(G)$ and myosin $(H)$ in control normal embryos. |X23.5|

(XBB 860-8763) 
these premuscle masses had begun. Stage 26 (ca. day 5$)^{2}$ is the approximate first point at which the fusion of myoblasts and the organization of multinucleate myotubes can be identified morphologically ${ }^{3}$ and when myosin synthesis can be detected biochemically. ${ }^{4}$ Infected cells visualized with antiP19 antibodies were only found after recourse to longitudinal sectioning. The staining was weak and was localized to a small group of myosin-positive cells near the limb surface at the level of the distal humerous. P19 was not localized in chondrogenic tissue, nonmyogenic mesenchyme or ectoderm [Fig. 1(C), (D)]. At day 8 viral localization was more widespread than found at day 6 and showed clear tropism for the limb musculature. Splitting of the premuscle masses was complete, segregating the primary generation fibers into the characteristic pattern that acts as the blueprint for future adult mus- cle organization. ${ }^{4}$ P19 was found in all muscle blocks of the infected day- 8 limb but not in the many other differentiating limb structures, with the exception of some limited staining found in nonmyogenic mesenchymal connective tissue [Fig. $1(\mathrm{E}) ;(\mathrm{F})$. At day 10 large areas of the limb were infected with virus [Fig. I(G)]. Although the greatest proportion was concentrated in the numerous blocks of skeletal muscle [Fig. $\mathrm{l}(\mathrm{H})$ ], other tissues now expressed viral antigens and these included myosin-negative mesenchymal cells, blood vessels, perichondrium, and epidermis. Viral expression in nonmyogenic tissue was variable, rather limited and contrasted to the invariate expression in muscle (Fig. 2). Preliminary data from embryos infected with a transformation-defective TD mutant of RSV (which lacks the src gene), showed that by day 10 there was no evidence of
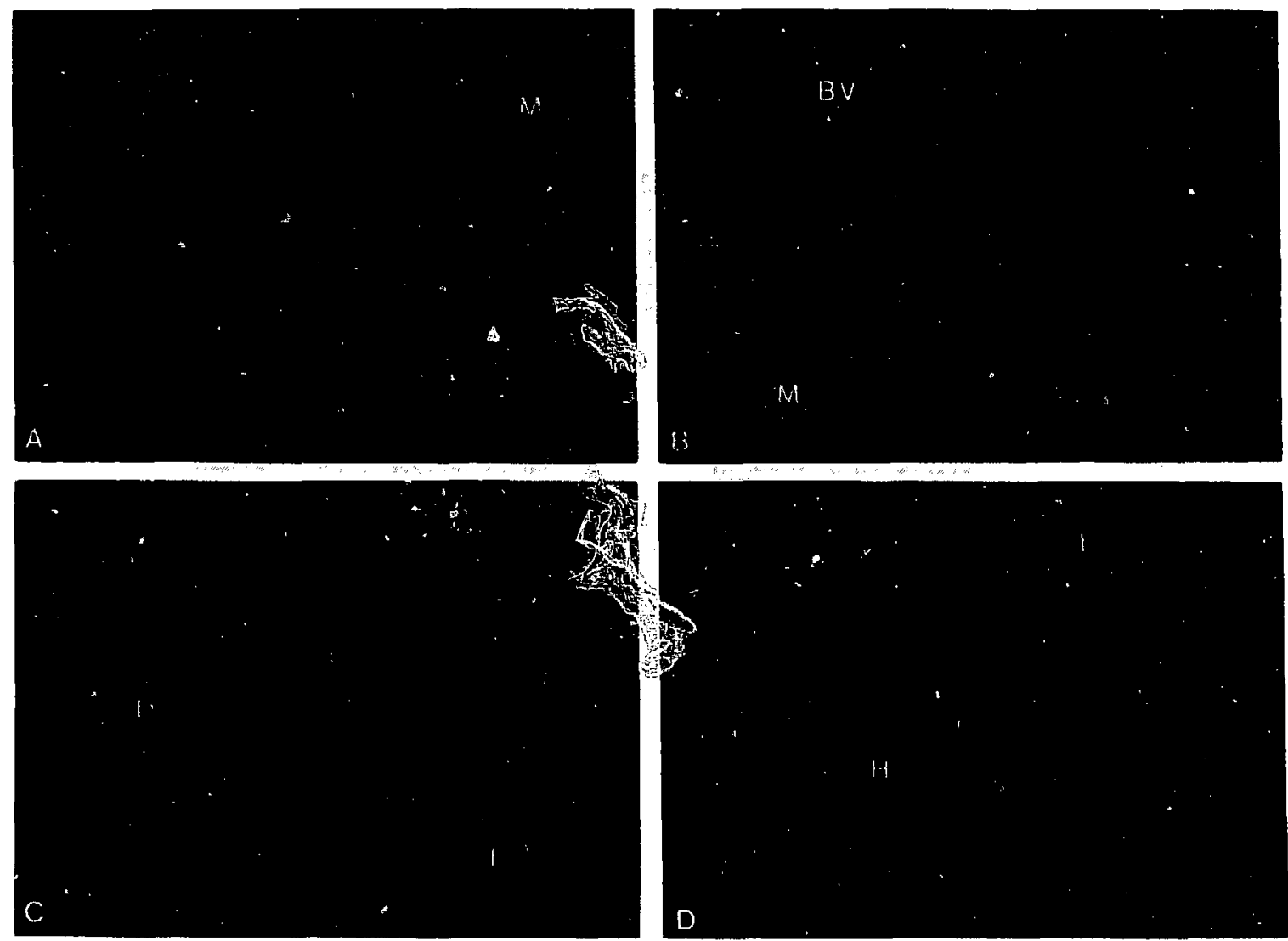

Fib. 2 Immunoftuorescent localization of P19 fluorescein in transverse sections of RSV infected day-10 embryo limbs taken at the level of the proximal to mid-radius-ulna. (A) shows P19 localized in a block of skeletal muscle, $m$. (B) shows $P 19$ in an infected muscle, $m$, and in an area corresponding to blood vessel, bV, development directly above. $(C)$ shows the superficial regions of the limb and the absence of P19 in this portion of the epidermis, $e$, and dermis, $d$, but the presence of P19 in infected subdermal tissues beneath. (D) shows a large hemorrhaghic lesion, $h$, bounded by a thin zone of P19-positive infected cells. $|\times 800|$

(XBB 860-8762) 
gross hemhorraghic disease. Immunoloca'zation of anti-P19 in frozen sections of day-10 limbs infected with TD virus showed that the tissue tropism of the virus was similar to that found for wild type RSV. The results of P19 immunolocalization in frozen sections is summarized in Table 1.

To examine P19-positive cells other than myeloblasts, cells were recovered from dissociated limbs at each day post-microinjection. The numbers of myogenic, chondrogenic, and fibroblastic cells established in culture were analyzed after 2 hours using anti-myosin and anti-type $\|$ and 1 collagen antibodies respectively. Less than $1 \%$ myosin or type II collagen-positive cells were recovered between days 5-11 inclusively. However the majority of the population ( $>90 \%$ ) expressed type 1 collagen.

The data on the kinetics of virus spreading in vivo together with the tissue section data suggest that the early infectious targets are myogenic in origin with muscle tissue remaining the principal source of infected cells as development continues. The participation of nonmyogenic cells in the infection becomes increasingly apparent in the older embryos, supporting a hypothesis that muscle tissue may act as a sink of virus that can be transmitted to other cell types as they acquire susceptibility. The delayed susceptibility of nonmyogenic tissue to infection may be linked to the specific program of differentiation of the various tissue com- ponents of the limb. Part of the differentiation program of these cells may involve the expression of proteins that act as substrates for the $p p 60^{v-s i c}$ kinase, e.g., the $36-\mathrm{kDa}$ protein substrate p36 (recently named calpactin 1), or vinculin. By regulating the expression of such substrates the cells may regulate competence for transformation by avian sarcoma viruses. Such a regulation may account in part for the inability of RSV to cause sarcomas in the avian embryo. These possibilities have been investigated and are reported elsewhere (see Carter et al., in this Annual Report).

\section{REFERENCES}

1. Dolberg, D.S., and Bissell, M.J. Inability of Rous sarcoma virus to cause sarcomas in the avian embryo. Nature 309, 552-556 (1984).

2. Hamburger, V., and Hamilton, H.L. A series of normal stages in the development of the chick embryo. J. Morphol. 88, 49-62 (1951).

3. Hilfer, S.R., Searls, R.L., and Fonte, V.G. An ultrastructural study of early myogenesis in the chick wing bud. Develop. Biol. 30, 374-391 (1972).

4. Crow, M.T., and Stockdale, F.E. Myosin expression and specialization amongst the earliest muscle fibers of the developing avian limb. Developmental Biology 113, 238-254 (1986).

Table 1. Summary of viral immunolocalization in frozen sections.

\begin{tabular}{cccccccccc}
\hline \hline $\begin{array}{c}\text { Embryo } \\
\text { age } \\
\text { (days) }\end{array}$ & $\begin{array}{c}\text { Number } \\
\text { of } \\
\text { expts. }\end{array}$ & $\begin{array}{c}\text { Skeletal } \\
\text { muscle }\end{array}$ & $\begin{array}{c}\text { Loose } \\
\text { conn. } \\
\text { liss. }\end{array}$ & $\begin{array}{c}\text { Cart- } \\
\text { ilage }\end{array}$ & $\begin{array}{c}\text { Peri- } \\
\text { chond. }\end{array}$ & Fascia & $\begin{array}{c}\text { Blood } \\
\text { vess. }\end{array}$ & Dermis & Epidermis \\
\hline 6 & 5 & $1 \pm$ & 0 & 0 & - & 0 & 0 & - & 0 \\
8 & 3 & 3 & 1 & 0 & 0 & - & 0 & 0 & 0 \\
10 & 8 & 8 & $8 \pm$ & 0 & $2 \pm$ & 0 & 4 & $4 \pm$ & $3 \pm$ \\
\hline \hline
\end{tabular}

\pm denotes limited or weak staining 


\title{
QUANTITATIVE IMMUNOCYTOCHEMICAL ASSAY FOR AVIAN RETROVIRUSES
}

\author{
Andrew W. Stoker and Mina I. Bissell
}

Much of our work revolves around the use and study of the avian RNA tumor virus (retrovirus) Rous sarcoma virus (RSV) and its transformationdefective derivatives (tdRSV). The major emphasis is upon the analysis of consequences of virus infection and expression in the early avian embryo.' Throughout this work, it is necessary to obtain and monitor the integrity of high titer stocks of our viruses for the critical work in the embryo. One problem is the maintenance of virus with low titers of tdRSV (which arise spontaneously at high rates in RSV populations), these being direct competitors of RSV for cell infection in vivo and in culture. A second and related problem is posed by the lack of a good assay for such tdRSV, or in fact, any nontransforming avian retrovirus. A commonly used assay quantitates the amount of the viral enzyme, reverse transcriptase (the enzyme that copies the viral RNA genome into a DNA copy in the infected cell) in a viral stock. However this assay lacks high sensitivity, and cannot distinguish between RSV and tdRSV viruses. A novel detection system has therefore been developed in our laboratory that utilizes immunocytochemical staining of infected cells in culture to accurately quantitate both RSV and tdRSV.

The cells used are secondary chick embryo fibroblasts, plated initially at $8 \times 10^{5}$ cells per 60 $\mathrm{mm}$ plate. These cells are infected overnight with the required innoculum of virus, and the next day overlaid with $0.8 \%$ agar. The agar overlay is a standard procedure used in the virus focus forming assays, ${ }^{2,3}$ in order to prevent virus dispersion upon secondary virus production from infected cells. After a period of 7-10 days, the agar overlay is removed and the cells are fixed in $2 \%$ paraformaldehyde for 20 minutes. To detect the proteins expressed by the virus, three sequential affinity layers are applied to the fixed cell population. The first layer is a monoclonal antibody directed against the viral p198ag protein ( kindly supplied by Dr. T. Pawson, University of Toronto), and the second layer is a biotinylated goat anti-mouse $F(a b)_{2}$ reagent (Amersham Corporation). The final component is a streptavidin-linked alkaline-phosphatase complex (Enzo-Biochem), which, when provided with the correct substrates (Nitroblue tetrazolium and 5-bromo-4-chloro-3-indoyl phosphate), gives a blue-purple precipitate in cells positive for the p1983 protein. This detection system utilizes very efficient amplification steps, providing high sensitivity with low background.

Analyses of RSV-infected CEF using this assay revealed that under the above conditions it provided a very effective means by which to quantitate virally infected foci of cells. The assay has been named the EFU assay, as the units of virus are enumerated in expression focus units (efu's). The foci of p19gag expression are clearly visible both microscopically and macroscopically, and can be counted by either means (Figs. 1 and 2). The assay has been shown to give a linear response upon serial dilution of the infecting virus, in accordance with the single-hit kinetics of infection by this virus (Fig.2). ${ }^{2}$ An important finding is that tdRSV can be enumerated in exactly the same manner as RSV, thus providing a straightforward and sensitive assay for these nontransforming virus types [Fig. 1(b)].

The assay is now in routine use in our laboratory, where its sensitivity and ability to quantitate both RSV and toRSV is of considerable value. We hope that it will gain wider acceptance as an additional means by which to quantitate retroviruses, both avian and mammalian.

\section{REFERENCE}

1. Dolberg, D.S., and Bissell, M.J. Nature 309, 552-556 (1984).

2. Temin, H.M., and Rubin, $H$. Virology 6 , 669-688 (1958).

3. Vogt, P.K. In Fundamental Techniques in Virology, K. Habel and N.P. Salzman, Eds. Academic Press, New York. 198-211 (1969). 
A
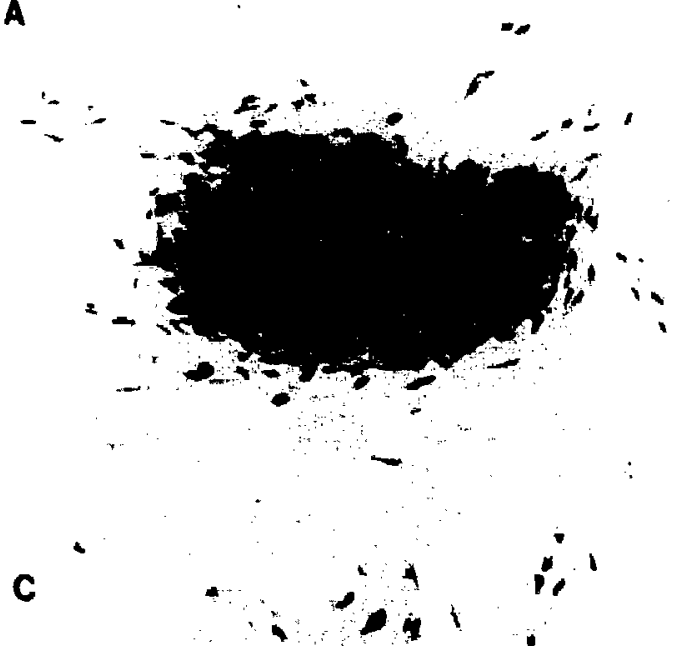

$\cdot \mathbf{B}$

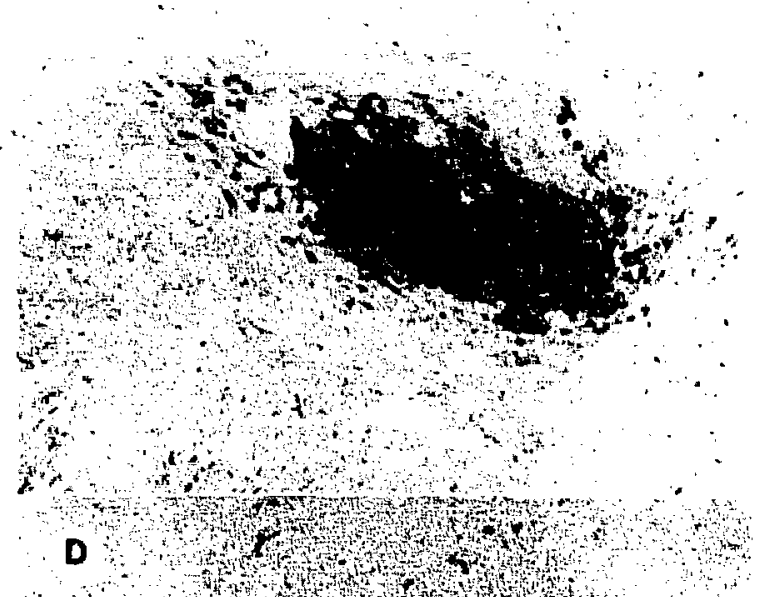

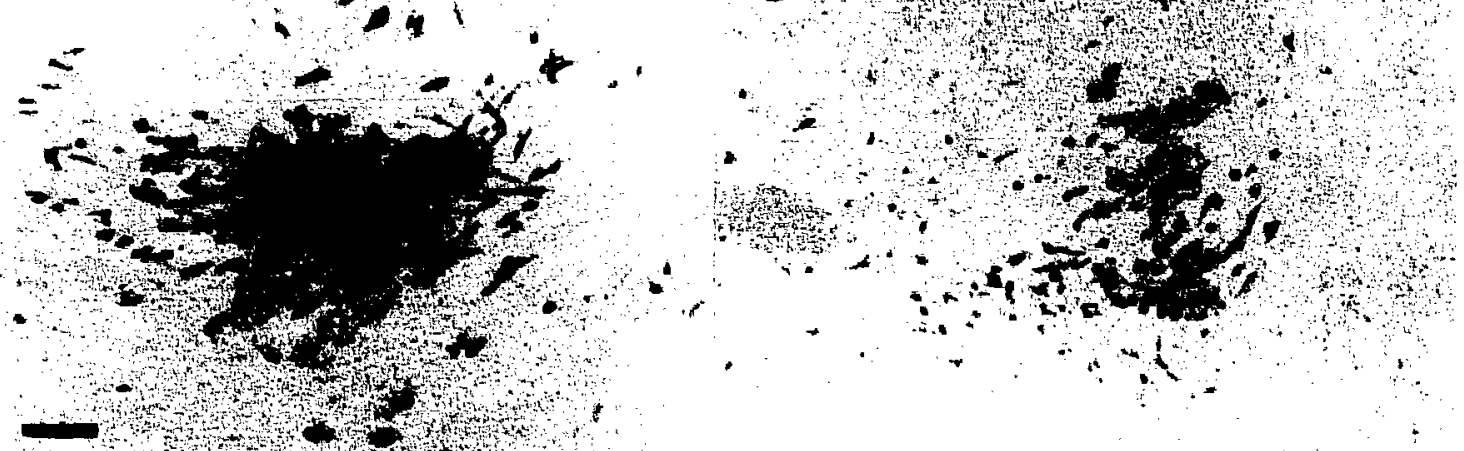

Fig. 1. Nonphase photomicrographs of alkaline-phosphatase-stained foci of plgevexpressing virus-infected chickembryo fibroblasts. The clear surrounding areas are confluent layers of uninfected cells. (a) RSV infected cell focus. (b) tdRSV infected cell focus. Bar $=0.25 \mathrm{~mm}$.

(XBB 8610-8760)
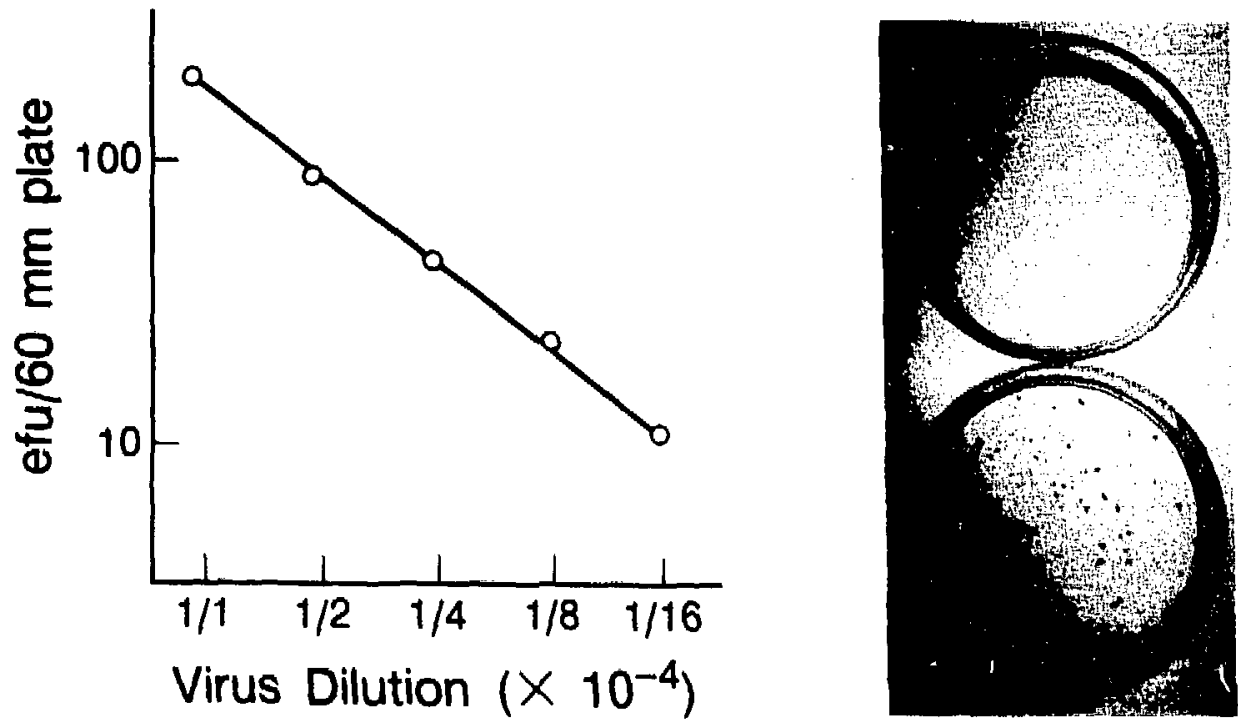

Fig. 2. Dilution analysis of efu versus serial two-fold dilution of infecting virus. The pair of $60-\mathrm{mm}$ plates presented (right) show the macroscopic appearance of stained-infected (lower) and control-uninfected (upper) plates of CEF (0.6 X mag). 


\title{
DEVELOPMENTAL REGULATION OF TYROSINE PHOSPHORYLATION SUBSTRATES CALPACTIN 1 AND VINCULIN IN EMBRYONIC AVIAN LIMB AND EXPRESSION IN CULTURED LIMB CELLS
}

\author{
V. Celeste Carter," Anthony R. Howlett, G. Steven Martin,* and Mina J. Bissell
}

Phosphotyrosine-containing proteins are minor components of normal cells that, in certain instances, are associated with the regulation of cellular metabolism and growth. An increase in the phosphotyrosine content of cellular proteins is observed following tansformation by several avian sarcoma viruses. Two of the most prominent cellular substrates are a $36-\mathrm{kDa}$ protein, p36 (recently named calpactin 1) and vinculin. Both proteins are phosphorylated at tyrosine following transformation by retroviruses.' Biochemical fractionation of cells and immunofluorescence microscopy indicate that both proteins are located on the cytoplasmic face of the plasma membrane, possibly functioning as structural proteins, and recently calpactin 1 has also been postulated to be involved in membrane cytoskeletal interactions, membrane traffic and the regulation of phospholipase $A_{2}$ : The internal face of the plasma membrane is also the location of the tyrosine-specific phosphokinase $p p 60^{v-s r c}$ coded by Rous sarcoma virus (RSV). Phosphorylations occurring on substrates like vinculin and calpactin may play a role in malignant transformation by avian sarcoma viruses. Because the embryonic avian limb has been shown to support the replication of RSV but to be resistant to transformation in ovo, ${ }^{2}$ we have examined the expression of calpactin 1 and vinculin in developing chick limb tissue in ovo and in culture using biochemical and immunocytochemical techniques.

To determine the sites of calpactin and vinculin synthesis frozen sections were prepared and stained with anti-calpactin and anti-vinculin serum. At day 5 in vivo, calpactin is selectively localized in the ectodermal layers of the limb bud [Fig. 1(A)]. The mesenchymal cells of the limb interior are negative, with the exception of rare foci of cells that may represent part of the endothelial lining of the limb vasculature [Fig. 1(D)]. At day 9, the epidermal derivatives of the ectoderm express calpactin [Fig. 1(B)] and expression is now detectable in some mesenchymal derived limb structures including cartilage, $C$, perichondrium, P, [Fig. 1(E)] and blood vessels, BV, |Fig 1(B)]. The protein is absent from loose connective tissue including dermis and from muscle tissue. At day 14, the distribution of calpactin is essentially similar to that at day 9, although the intensity of staining appears greater. The epidermis [Fig. 1(C)], cartilage, C, [Fig. 1(F)], and perichondrium, $\mathrm{P},[\mathrm{Fig} .1(\mathrm{~F})]$ are all positive and additional structures including the dermis [Fig. 1(C)] and the fascia, $F$, separating the large blocks of skeletal muscle, $M$, are now also expressing calpactin [Fig. 1(G)].

Vinculin appears to be generally distributed in the ectodermal and mesenchymal cells of the day- 6 limb bud. By day 8, vinculin synthesis appears to be selectively switched off, with expression detected in the epidermis, cartilage, perichondrium, and blood vessels only. At day 10 this tissue distribution remains essentially unchanged and resembles closely the pattern obtained for calpactin. Both proteins are conspicuously absent from the limb musculature. It has been shown previously that in infected limbs, muscle tissue is the primary source of infection by RSV (see Howlett et al, in this Annual Report). The shared localization pattern of vinculin and calpactin and the distinction of this pattern from that expressed by RSV has been confirmed by dual label immunofluorescence (Fig. 2).

To determine the pattern of expression of vinculin and calpactin in cultured limb cells the synthesis and accumulation of the two proteins was analyzed by immunofluorescence, immunoprecipitation of ${ }^{35} \mathrm{~S}$-labeled proteins, and immunoblotting. Both proteins were found to be rapidly induced in culture and were detected by immunofluorescence in all cells by two hours. By biochemical analysis calpactin expression could be detected as early as 30 minutes after seeding into culture (Fig. 3).

The results indicate that both calpactin and vinculin are developmentally regulated in the avian limb in ovo and are induced rapidly when limb bud cells are dissociated and plated into tissue culture. In culture the expression of the two proteins becomes coincident with the expression of viral determinants in an environment that has been shown to be permissive for the expression of the 

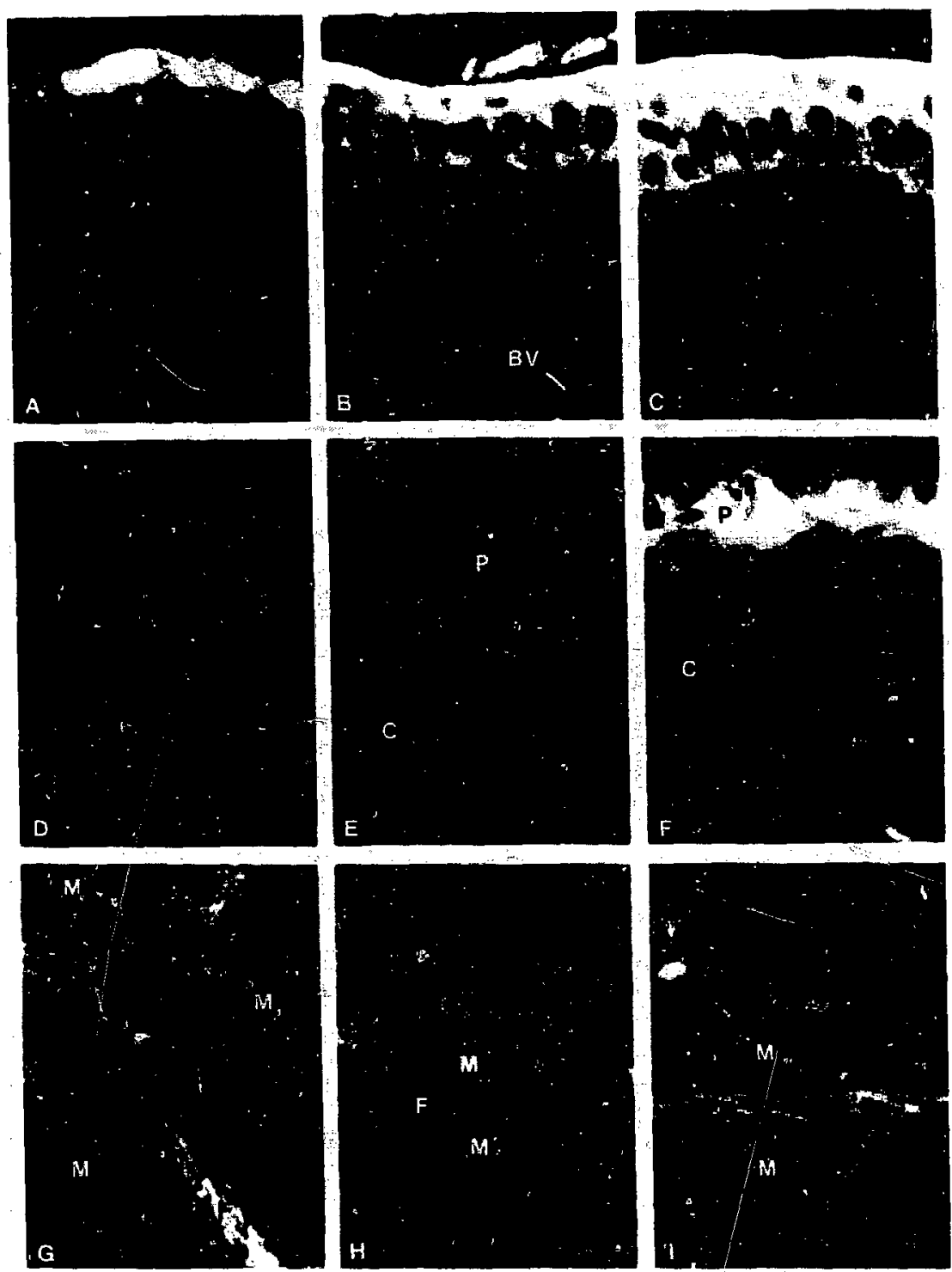

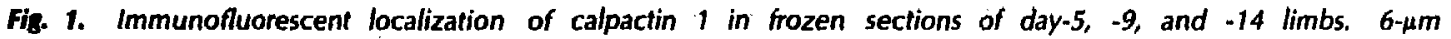
transverse sections were cut at the approximate midpoint of the day-5 limb bud and at the proximal radius-ulna at days 9 and 14. Sections were stained with anti-calpactin antiserum $(A-G, I)$ or antimyosin antibody (H). (A-C) show the superficial regions and (D-F) the internal regions of the limb at days 5 ( $A$ and $D), 9(B$ and $E$ ), and 14 (C and $F$ ). At day 5 calpactin is present in the ectoderm but not the underlying mesenchyme $(A)$ with the exception of foci of cells in the limb interior (D). At day 9, calpactin is expressed in dirferentiating epidermis, blood vessels, BV, but not dermis, (B), cartilage $C$, and perichondrium $P,(E)$. At day 14 calpactin is present in both the epidermis and dermis (C), cartilage and perichondrium (F). (G-l) shows calpactin expression in the connective tissue fascia $F$, associated with skelefal muscle $M$. (H and I) show the lack of calpactin expression in muscle tissue by dual immunofluorescence. Two blocks of muscle $M$, stained with anti-myosin/rhodamine $(H)$ are separated by a band of connective tissue fascia $F$, stained with anti-calpactin/fluorescein (I). (× 500).

(XBB 863-1567) 

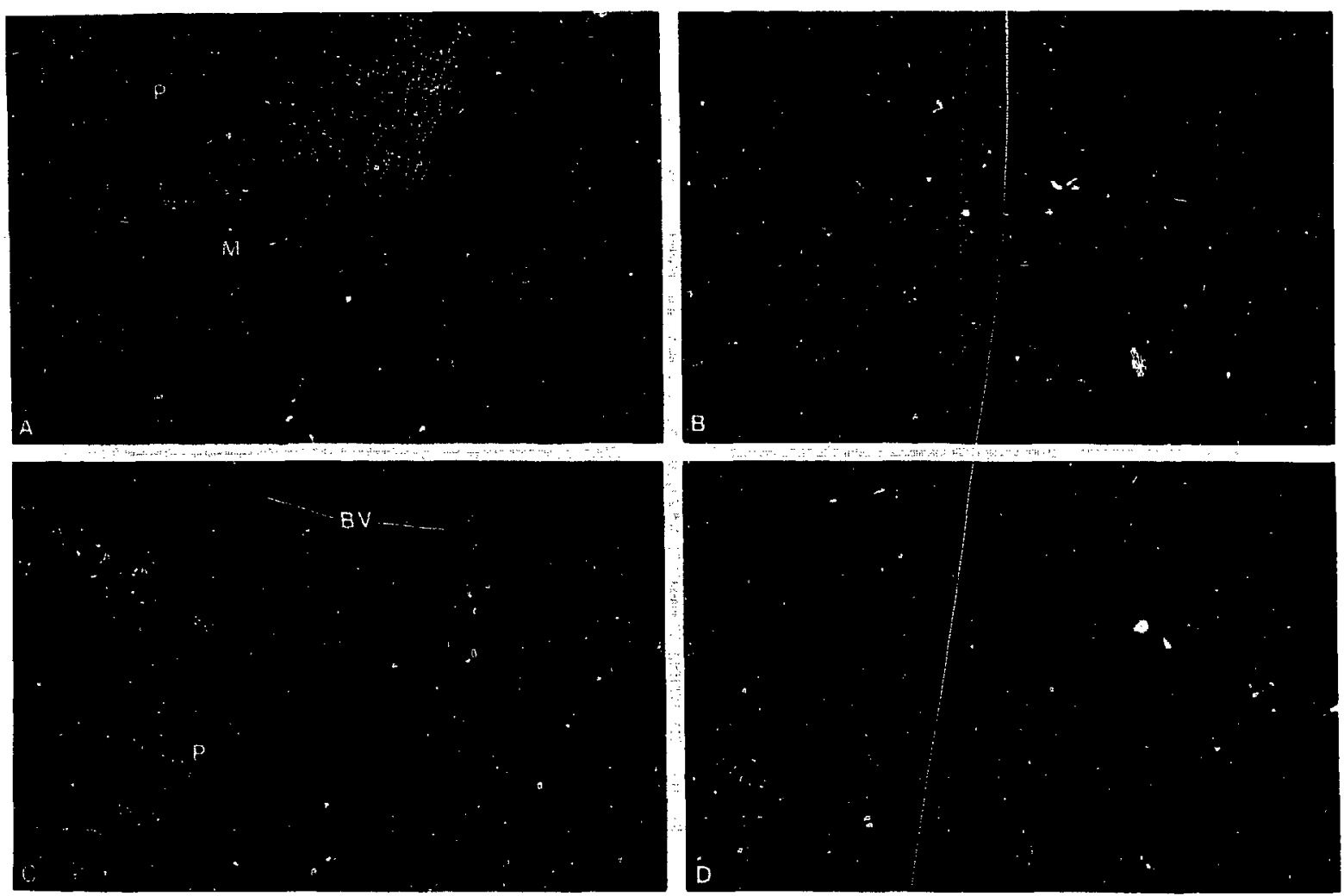

Fig. 2. Immunofluorescent localization of calpactin, vinculin and P19 in day-10 RSV-infected limb tissues, by dual label immunofluorescence. $(A, B)$ show a sheath of fibrous connective tissue, the perimysium, $P$, stained with anticalpactin fluorescein (A) encapsulating a block of skeletal muscle, $M$, stained with antibody specific to the viral structural determinant P19ms rhodamine as shown in (B). (C and D) show blood vessels, $B V$ and perimysium, $P$, stained with vinculin fluorescein $(C)$ and an area of tissue expressing viral antigens, stained with P19 rhodamine (B). $|\times 460|$.

(XBB 8610-8764)

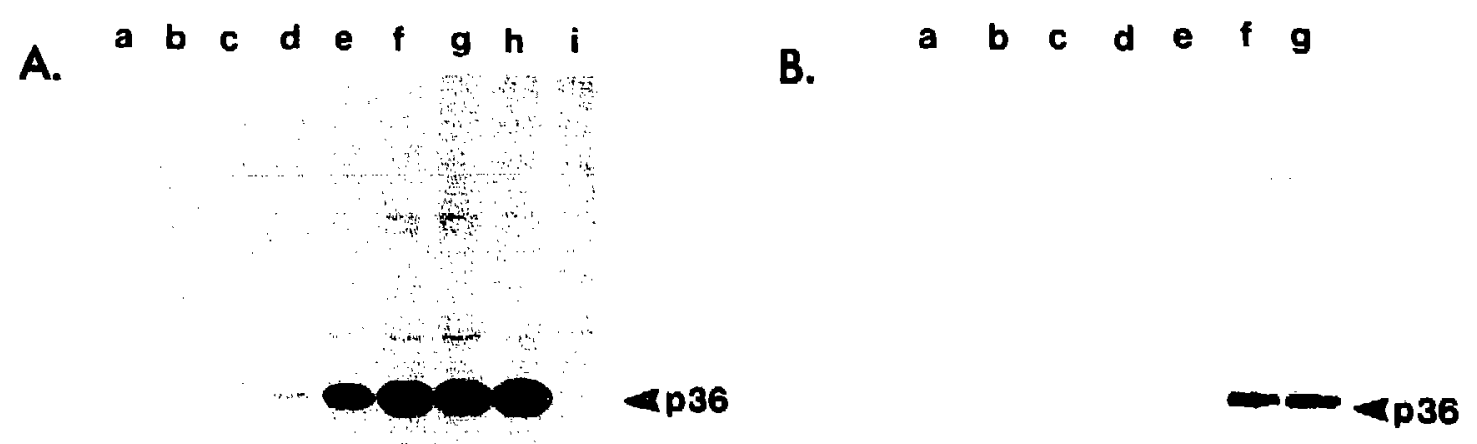

Fig. 3. Time course of induction (A) and accumulation (B) of calpactin in day-5 limb cells plated in culture. (A) Tissues were ${ }^{35} \mathrm{~S}$ methionine labeled from 0-30 $\mathrm{min}$, (a), 30-60 min, (b), 60-90 min, (c), 1.5-2 hr, (d), 3.5-4 hr, (e), 5.5-6 hr, (f), 23.5-24 hr, (g), and 47.5-48 hr, (h and i). Cells were lysed and equivalent numbers of counts of cell Iysate were immunoprecipitated with equal amount of preimmune (i) or anti-calpactin serum (a-h). Imminoprecipitates were resolved by SDS PAGE and detected by autoradiography. (B) Equivalent amounts of total cell protein (50 g) were resolved by SDS PAGE, iransferred to nitrocellulose, and analyzed by immunoblotting (a) 60 min, (b) 90 min, (c) $2 \mathrm{hr}$, (d) $4 \mathrm{hr}$, (e) $6 \mathrm{hr}$, (l) $24 \mathrm{hr}$ and (g) $48 \mathrm{hr}$ cell lysates.

(XBB $865-3790)$ 
transformed phenotype. The absence of calpactin and vinculin in muscle tissue in ovo raises the possibility that the resistance of embryonic tissue to transformation by RSV in ovo may result from developmental regulation of the cellular substrates for tyrosine phosphorylation. However, the question of whether other kinase substrates are also subject to developmental regulation remains to be established. In addition, the question of whether the $\mathrm{pp}^{\mathrm{sic}}$ kinase is active in ovo and capable of phosphorylating substrate proteins if, or when, they

\section{HUMAN MAMMARY EPITHELIAL CELL CULTURE}

\section{Martha Stampfer and Jack Bartley}

Our laboratory has developed culture systems that permit long term active growth of human mammary epithelial cells (HMEC) derived from both normal tissues (reduction mammoplasties) and mastectomies. Additionally, we have created the first non-tumor-virus transformed immortalized cell lines from normal human epithelial cells. The availability of large numbers of these cells allows biochemical, immunological, and molecular investigations of the normal and abnormal mechanisms involved in human epithelial cell growth control, differentiation, and carcinogenesis. The development of serum-free medium for the growth of these HMEC has further enhanced their usefulness.

Our laboratory has a bank of primary HMEC tissue from over 150 individual donors. Of these, HMEC from about 23 different individuals have been grown in the serum-free medium, MCDB170, with large pools of cells frozen down for future use. Two immortalized cell lines have been isolated after exposure of normal cells in culture to the chemical carcinogen benzo(a)pyrene (normal cells from one individual, specimen 184: normal reduction mammoplasty tissue from a 21 -year old). These lines (184A1 and 184B5) display differences from their normal parental cells in morphology, karyotype, response to growth factors, expression of antigens and differentiated properties. Yet they are not tumorigenic in immunosuppressed mice, and show little or no anchorage-independent growth (properties associated with malignancy). Thus, they likely represent a premalignant type, and are useful for studies to determine what factors can confer malignancy. Their relatively stable karyotype, com- are expressed cannot be discounted. These considerations are currently under investigation.

\section{REFERENCES}

1. Hunter, T., and Cooper, J.A. Protein-tyrosine kinases. Ann. Rev. Biochem. 54, 897-930 (1985).

2. Dolberg, D.S., and Bissell, M.J. Inability of Rous sarcoma virus to cause sarcomas in the avian embryo. Nature 309, 552-556 (1984).

pared to most human epithelial cell lines (which are obtained from tumor sperimens or tumor virus transformation), provides for more uniform experimentation. Besides having these co-genic normal and immortal cell substrates, we also have cells from specimen 184 that show an extended (but still finite) life as a consequence of benzo(a)pyrene exposure, and we have malignantly transformed cells from the immortal cell lines as a consequence of their exposure to oncogenes.

These cell substrates are now being widely utilized in our laboratory, as well as in many others around the country, to further our understanding of human cellular physiology and carcinogenesis. We are examining what factors can promote or inhibit the different stages in the progression from normal to malignant cells (e.g., chemical carcinogens, irradiation, factors effecting the level of oxidative damage, and nutritional components). The biological consequences of transformation are being explored in terms of expression and response to growth factors (estradiol, EGF, TGF- $\alpha_{t}$ TGF- $\beta$ ), antigens (human mammary milk-fat globule antigens, possible differentiation or tumor-specific antigens), and differentiated properties (caseins, $\alpha$-lactalbumin, cellmatrix components, glucose metabolites, thioesterase II, apo-lipoproteins). Cell fusion studies have been started to investigate the possible existence of tumor suppressor genes, and the nature of the gene(s) involved in immortalization and tumorigenicity. Our laboratory has developed the necessary cell bank and computerization of the cell bank inventory to provide interested investigators with the appropriate HMEC cell substrates and expertise. 


\title{
EXPRESSION OF KNOWN ONCOGENES IN HUMAN MAMMARY EPITHELIAL CELLS
}

\author{
Martha Stampfer, Ming Li, Kari Koszdin, and Mina Bissell
}

While viral oncogenes ( $v$-onc) have been identified due to their potential to cause unregulated growth and tumor formation, many of the cellular homologues (proto-oncogenes) of these viral genes, found in the DNA of normal cells, have been shown to be involved in the normal regulation of cell growth and differentiation, coding for growthfactors, or growth factor receptors. We have begun studies with our human mammary epithelial cell (HMEC) cultures to determine the possible role of known oncogenes and proto-oncogenes in normal and aberrant growth control in vitro.

In initial studies (in collaboration with Paul Arnstein, Frank McCormick, and Robin Clark), we examined the effect of introduction of viral oncogenes to the HMEC cell lines. Infection of 184B5 with the Kirsten ras sarcoma virus (K-RSV) rendered these cells tumorigenic in all nude mice tested; however the cells did not gain anchorage independent growth (AIG). In contrast, 184A1 infected with K-RSV or Harvey-RSV showed a large increase in AIG, but the cells showed weak tumorigenicity. Infection with the H-RSV after introduction of either the SV40T oncogene, or the mos oncogene was required for producing tumorigenicity in the 184A1 cells.

We have now begun screening the different HMEC specimens for presence and expression of various oncogenes. Cell harvests from different cultures were lysed and their DNA and RNA isolated. The organization of DNA was examined by Southern transfer and hybridization to various oncogene probes. The degree of gene expression was determined by RNA dot blots. The oncogene probes utilized thus far are human c-Ki-ras, human c-Ha-ras, human c-mos, human c-myc, mouse int-1, mouse int-2, v-src, v-sis, v-Ki-ras, and v-erb-B. The cell specimens examined are 184, 184A1N4 (a subclone of 184A1), 184B5, 184A1N4V (K-RSV infected), 184B5KT (K-RSV infected cells taken from a nude mouse tumor), and 184 breast fibroblast cells.

Southern transfer analysis has shown no differences in the cells thus far except in the case of 184B5KT hybridized to v-Ki-ras, where several EcoRI fragments containing virus sequences were detected, possibly indicating multiple sites of viral integration. The absence of detectable bands in the 184AIN4V cells may be due to the low multi- plicity of infection with the K-RSV; we are now testing isolated anchorage-independent 184A1N4V cells to see if they show V-Ki-ras integration sites.

The results thus far of the RNA dot blot analyses are summarized below in Table 1 .

Table 1.

Probe 184 184A1N4 184B5 184A1N4V 184B5KT

\begin{tabular}{llllll}
\hline c-Ki-ras ++++ & ++++ & ++++ & ++++ & ++++ \\
c-Ha-ras ++ & ++ & ++ & ++ & ++ \\
c-myc & ++ & ++ & ++ & ++ & ++ \\
c-mos & -- & -- & -- & -- & -- \\
int-1 & ++ & + & + & + & + \\
int-2 & + & + & + & + & + \\
v-src & ++ & -- & -- & -- & $+/-$ \\
v-sis & -- & -- & -- & -- & -- \\
v-erbB & -- & -- & -- & -- & -- \\
\hline
\end{tabular}

The c-Ki-ras RNA is the most abundantly expressed of these oncogenes/proto-oncogenes, with all of the examined HMEC exhibiting the same extent of synthesis. It is possible that expression of this gene (and/or the c-Ha-ras gene) is important for HMEC viability or growth; preliminary observations by other collaborators that microinjection of antibodies to the ras-coded p21 protein causes inhibition of DNA synthesis in nearly $100 \%$ of normal HMEC support this hypothesis. The mechanism of ras-gene effects in these cells is still completely unknown. We have thus far seen little difference in the level of expression of these genes in the different cell types, with the exception of $v$ src. The nature of this increased expression of the $v$-src oncogene in the normal HMEC compared to the transformed HMEC is currently being investigated. Further studies are being planned to elucidate the possible role of these protooncogenes/oncogenes in the normal growth control of these cells. Additionally, we are collaborating with several other investigators to examine the biological consequences of transfection of a variety of oncogenes into the normal as well as transformed HMEC cells. 


\title{
PRODUCTION OF OXIDATIVE BASE DAMAGE BY BENZO(a)PYRENE METABOLISM AND IONIZING RADIATION IN HUMAN MAMMARY EPITHELIAL CELLS
}

\author{
Steven A. Leadon, Martha Stampfer, Tracy Yang, and Jack Bartley
}

There is increasing interest in the potential role of active oxygen species and free radicals as intermediates in carcinogenesis. For certain chemical carcinogens there is good correlation between the formation of active oxygen, convertibility to free radicals, and carcinogenicity. Active oxygens and the ensuing lipid peroxidations could affect carcinogenic processes in at least two ways: by causing chromosomal damage and rearrangements, and by modulating cell growth and differentiation through epigenetic nechanisms.

Certain chemical carcinogens, such as the active metabolites of 2-naphthylamine, can exhibit several types of interactions with cellular DNA: direct interaction leading to adducts with various bases, and indirect effects through the generation of active oxygen species. Damage to DNA by active oxygen species can result in single- and double-strand breaks, apurinic and apyrimidinic sites, and ring-saturated thymine derivatives, such as 5,6-dihydroxydihydrothymine (thymine glycol). In order to understand the role of indirect action produced by chemicals or radiation in carcinogenesis, it is important to identify the primary DNA lesions and ascertain how these lesions are processed at the molecular level.

A monoclonal antibody that recognizes thymine glycols in DNA has been developed and used in a sensitive immunoassay. The sensitivity of this assay is sufficient to detect 1 thymine glycol per $8.5 \times 10^{5}$ bases in DNA. The essential features of this assay are as follows: a constant amount of immobilized $\mathrm{OsO}_{4}$-modified DNA and various amounts of a competitor DNA in solution are reacted with a fixed amount of antibody. The amount of antibody bound to the immobilized DNA is then compared to the amount bound in the absence of the competitor. $\mathrm{OsO}_{4}$-modified DNA is used in the assays because $\mathrm{OsO}_{4}$ selectively oxidizes the 5,6-double bond of thymine to produce the glycol but reacts very little or not at all with other bases. The enzyme-linked immunosorbent assay (ELISA) was calibrated using serial dilutions of radioactively labeled DNA modified by $\mathrm{OsO}_{4}$ as the standard. The thymine glycol content of the standard DNA was determined chromatographically.

We have recently shown that thymine glycols are produced in DNA following treatment of cul- tured human fibroblasts with N-hydroxy-2naphthylamine (NOH-2-NA), a metabolite of the bladder carcinogen 2-naphthylamine.' This damage is produced by the autoxidation of NOH-2-NA, which generates hydroxyl radicals and superoxide anions. However, as with ionizing radiation, the formation of free radicals by $\mathrm{NOH}-2-\mathrm{NA}$ is a chemical reaction in solution, not part of a metabolic process. We tested the hypothesis that during metabolic activation of a carcinogen, oxidative damage might also be produced. Human mammary epithelial cells (HMEC) have been shown to efficiently metabolize the carcinogen benzo(a)pyrene $(B(a) P)$. B(a)P can be converted to a very reactive electrophile, which forms covalent DNA adducts, as well as other metabolites. These metabolites can give rise to free radical intermediates and reactive reduced oxygen species as a result of autoxidative processes and oxygen-reduction cycles. These cycles are coupled with molecular oxygen to form reactive oxygen species such as superoxide anions and hydrogen peroxide. HMEC cultures were exposed to various doses of the carcinogen for 4 hours and the presence in the DNA of thymine glycols, as determined by the ELISA, and covalent adducts, as determined chromatographically, were measured. Both thymine glycols and covalent adducts were produced in the DNA in a dose dependent manner (Fig, 1). At a concentration of $1 \mu \mathrm{g} / \mathrm{ml} \mathrm{B}(\mathrm{a}) \mathrm{P}$, the frequency of thymine glycol formation was 4 per $10^{6}$ bases compared with 3.3 covalent $B(a) P$ adducts per $10^{6}$ bases. Since thymine glycols are only one type of DNA-lesion produced by active oxygen, the damage produced by the indirect action represents a large fraction of the total damage induced by metabolism of $\mathrm{B}(\mathrm{a}) \mathrm{P}$ in HMEC.

Hydroxyl radicals are responsible for the majority of the biological effects of ionizing radiation on DNA. Since thymine glycols are produced by ionizing radiation, a comparison was made between their formation in HMEC following exposure to either B(a)P or gamma rays. At equitoxic doses of both agents (30-40\% survival), the efficiency of thymine glycol formation was approximately 35-fold greater in HMEC cultures exposed to $\mathrm{B}(\mathrm{a}) \mathrm{P}$ than to gamma rays (Table 1). Therefore, even though the damage produced by indirect action during the 


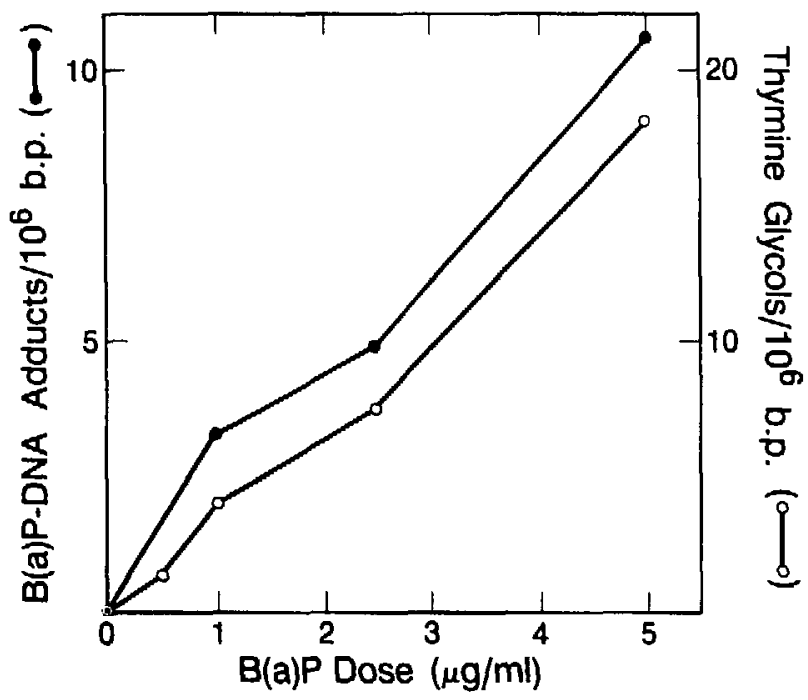

Fis. 1. Dose response for the production of B(a)P-DNA adducts and thymine glycols in DNA from B(a)P treated HMEC.

(XBL 8611-9059)

metabolism of $B(a) P$ represents a large fraction of the total damage produced, not all of this damage appears to play a role in cell killing. Its overall importance, however, may be related to the carcinogenic potential of $B(a) P$.

Repair of DNA damage was also investigated in the HMEC. Thymine glycols produced by $B(a) P$ metabolism were removed from the DNA of HMEC with about the same efficiency as was found for thymine glycols produced by ionizing radiation, even though there was a significantly greater initial frequency of this damage following exposure to the carcinogen (Table 1). By 45 minutes posttreatment,
Table 1. Production and removal of thymine glycols in human mammary epithelial cells.

\begin{tabular}{|c|c|c|c|}
\hline & \multicolumn{2}{|c|}{ Thymine glycols $/ 10^{6}$ bases } & \multirow{2}{*}{$\begin{array}{l}\% \text { remaining } \\
\text { after } 45 \mathrm{~min}\end{array}$} \\
\hline & Initial & After $45 \mathrm{~min}$ & \\
\hline $\begin{array}{l}B(a) P \\
(1 \mu g / m l)\end{array}$ & 4 & 1.8 & 45 \\
\hline $\begin{array}{l}\text { Gamma-rays } \\
\text { (400 rads) }\end{array}$ & 0.11 & 0.07 & 59 \\
\hline
\end{tabular}

approximately half of the initial thymine glycols had been removed. During this same repair time, little or no loss of $B(a) P$ adducts from the DNA was observed.

We have previously shown that both catalase and superoxide dismutase (SOD), which break down hydrogen peroxide and superoxide anions respectively, inhibited the formation of thymine glycols in DNA when cell cultures were exposed to NOH-2-NA. We are currently investigating whether incubation of HMEC with $B(a) P$ in the presence of either catalase, SOD, or both, will modify survival or extent of DNA damage. Our initial results indicate that SOD, but not catalase, protects the cells against the toxic effects of $B(a) P$. This may be due to a decrease in the total amount of damage produced by the carcinogen or specifically due to a decrease in the amount of indirect damage.

\section{REFERENCES}

1. Kaneko, M., and Leadon, S.A. Production of thymine glycols in DNA by $\mathrm{N}$-hydroxy-2naphthylamine as detected by a monoclonal antibody. Cancer Res. 46, 71-75 (1986).

\section{Differentiation}

\section{EXPRESSION OF DIFFERENTIATED FUNCTION IN HUMAN MAMMARY EPITHELIAL CELLS IN CULTURE}

\section{Jack Bartley, Gordon Parry, Gerri Levine, and Martha Stampfer}

Because of the potential relationship between normal cell differentiation and the development of cancer, we are continuing and expanding our studies on regulation of normal differentiated function of human mammary epithelial cells (HMEC) in cul- ture. These investigations into modulation of normal differentiation will provides a basis for studying the role of perturbations of differentiation in the development of breast cancer. Access to normal breast epithelial cells, to normal cells from the 
same specirnens but transformed chemically by carcinogen treatment in vitro, and to cells isolated from breast tumors provides an exceptional opportunity for studies of this type.

We reported in the previous Annual Report that human marnmary epithelial cells in long term culture ( $>6$ passages) in a serum-free medium (MCDB 170) can be induced by use of a medium containing serum and epithelial conditioned medium (MM) to express some characteristics of early prelactation. These characteristics include increased glycogen synthesis and synthesis and secretion of lactoferrin and a milk protein related to casein. Synthesis of $\beta$-casein and $\alpha$-lactalbumin are not detectable under these conditions. Recently, we have demonstrated that medium $M M$ can induce synthesis of these major milk proteins if cells are placed on an extracellular matrix laid down previously by HMEC grown in MCDB 170 . These conditions may approach those of the prelactational staie in vivo in that cells involved in glandular development during pregnancy lay down a matrix that, at the instant of parturition, is supportive of lactation.

We are pursuing these observations and developing other markers suitable for assessing the stage of differentiated function of HMEC in culture to verify this hypothesis. The gene products currently being examined for use as markers include specific cell-surface antigens, the mammary-specific thioesterase responsible for medium-chain triglyceride synthesis, apolipoprotein synthesis, and synthesis and secretion of a human mammary autocrine growth factor.

The expression of surface antigens is being determined by the use of monoclonal antibodies developed in response to the human milk-fat globule membrane, which is derived from the apical plasma membrane of mammary epithelial cells. After screening six antibodies for their ability to bind the cell surface of HMEC (see previous Annual Report), three were selected on the basis of the immunocytochemistry for further study (Table 1). The results indicate that antibody $\mathrm{P} 2 \mathrm{Cl}$ may be useful in distinguishing between normal cells and those of the transformed lines $A 1$ and $B 5$, but the expression is not modulated by the different media (MCDB 170 and MM). Based on the results with expression of milk protein and glycogen synthesis, the surface component reacting with $\mathrm{P} 2 \mathrm{C} 1$ is not modulated by the trarisition from the quiescent to the early prelactational state. In contrast, expression of the surface molecules with affinity for antibodies P2A1 and P3B1 is modulated by the growth
Table 1. Binding of monoclonal antibodies to surface antigens to HMEC in culture.

\begin{tabular}{|c|c|c|c|c|}
\hline \multirow[t]{2}{*}{ Specimen } & \multirow[t]{2}{*}{ Medium } & $P_{2} A_{1}$ & $P_{3} B_{1}$ & $P_{2} C_{1}$ \\
\hline & & \multicolumn{3}{|c|}{ (\% of cells binding antibody) } \\
\hline \multirow[t]{3}{*}{184} & MCDB 170 & ++ & $0 /+$ & $0 /+$ \\
\hline & $M M$ & ++++ & + & $0 /+$ \\
\hline & $N M / 170^{b}$ & ++++ & $+++t$ & $0 /+$ \\
\hline \multirow[t]{3}{*}{$184 \mathrm{~A} 1$} & MCDB170 & $0 /+$ & + & ++ \\
\hline & $M M$ & ++ & + & ++ \\
\hline & NM/170 & ++++ & +++ & + \\
\hline 184B5 & MCDB 170 & ++++ & $+t+t$ & ++ \\
\hline
\end{tabular}

$0 /+=0-10 \%,+=10-25 \%,++=25-50 \%,+++=50-75 \%$, $++++=75-100 \%$

b Cells grown in MCDB 170 but treated with neuraminidase prior to exposure to antibody.

medium. However, one of these, P2A1, reacts with a surface component on $A 1$ cells that responds to the medium, whereas the component reacting with P3B1 on these cells does not. The differential staining and the differences in response to growth medium may help to identify stages of differentiation in normal cells and to distinguish between normal and transformed cells in mixed cultures. In those cultures showing fewer than $10 \%$ positive cells, treatment of the cells with neuraminidase (designated NM in the table) may help to amplify differences between normal and transformed cells.

These promising results at the cellular level led us to attempt identification of the molecules and the epitopes involved in the antibody binding. Analyses of the plasma membranes by polyacrylamide gel electrophoresis followed by immunoblotting has failed to reveal protein components on normal specimen-184 cells and the transformants that react with any of the antibodies on Western blots. From the results with immunocytochemistry, an indetectable reaction between antibodies $\mathrm{P} 3 \mathrm{~B} 1$ and $\mathrm{P} 2 \mathrm{C}$ 1 and the surface proteins from normal cells is not surprising, but the lack of affinity between B5 surface proteins and all three antibodies is inexplicable at this time. In an attempt to uncover the basis for this paradox, we recently adapted a technique for immunoblotting cell surface gangliosides separated on silica gel by thin-layer chromatography. Aithough these sturies have not clarified the lack of correlation between the results with B5 cells at the cellular level (immunocytochemistry) and the immunoblots of separated surface-membrane proteins, they have provided at least a partial explana. tion for the reactivity of the normal cells and $A 1$ 
transformants with antibody P2A1. When glycolipids extracted from these cells are separated on silica gel probed with antibodies, two bands show affinity for antibody $\mathrm{P} 2 \mathrm{~A} 1$ but not for $\mathrm{P} 2 \mathrm{C} 1$. The reactivity of one of these bands is specifically enhanced by treatment of the intact cells or the cell lysates with neuraminidase. Thiese results correspond to those observed under the microscope (Table 1) and indicate that the epitope reacting with antibody P2A1 is the carbohydrate moiety on a glycolipid with migration on TLC corresponding to a complex ganglioside. The possibility that a portion of the binding could also involve a glycoprotein with the same carbohydrate epitope is under investigation. This finding clarifies the specific reactions taking place with normal and $\mathrm{A} 1$ cells, but adds complexity to the overall analyses and the use of cell surface antigens for cell identification. The expression of the carbohydrate linkage solely as part of a gariglioside, soiely as part of a glycoprotein, or in both lipids and proteins on cells could be influenced by the different growth conditions and be different in various transformed cell lines.

The study of the molecules involved in the expression of glycolipids and glycoproteins on the surface of these cells is being actively pursued to provide a rational basis for identification of the stages of differentiated function in HMEC in culture.

Studies have been initiated with the other potential markers of differentiated function in HMEC. The studies with the apolipoproteins are being done in collaboration with Trudy Forte of the Lipoproteins and Structural Biology Group; those on the mammary specific thioesterase with Stuart Smith of Children's Hospital Medical Center in Oakland; and those on the mammary specific growth factor with Henri Rochefort of Unite d'Endocrinologie Cellulaire et Moleculaire, INSERM, Montpellier, France.

\section{SPECIALIZED MEMBRANE BIOGENESIS IN MAMMARY EPITHELIAL CELLS}

\section{Gordon Parry, Betsey L. Cullen, and Lenny Moss}

Epithelial cells in the mammary gland exhibit an array of secretory processes that are responsible for the transport of milk components into the alveolar lumen. One such process, the secretion of fat droplets, is particularly unusual in that fat droplets are synthesized in the epithelial cells, move intracellularly into the apical region of the cell and then bud off from the apical surface encapsulated by apical plasma membrane. This process is of interest in several respects. Firstly, the apical membrane of the epithelial cell is highly differentiated and adapted to participate in the process of fat secretion. As such, it represents a good model to study regulatory aspects of membrane differentiation. Secondly, certain of the apical membrane differentiation antigens are frequently expressed on mammary carcinoma cells, and knowledge of the normal mechanisms by which these antigens are regulated may have implications for a better understanding of tumor antigen expression.' Thirdly, because the apical membrane of the cell is lost during secretion, active membrane biosynthesis must accompany fat secretion, and the cell represents a good model for studying membrane biogenesis in polarized epithelial cells. For our studies we have focused on the milk fat globule membrane of mice.
Experiments have been carried out using primary cultures of cells established from mammary glands of late pregnant mice and also a mouse cell line, COMMA-1-D, that differentiates in an appropriate milieu. When fat globule membranes are purified from mouse milk and the protein composition analyzed by SDS-polyacrylamide gel electrophoresis, four major proteins are identifiable with molecular weights of $55,67,90$, and $150 \mathrm{kDa}$. By analogy with proteins in the bovine membrane, the $67-\mathrm{kDa}$ component was identified as butyrophilin and the $150-\mathrm{kD}$ a one as xanthine oxidase. In addition, a high molecular weight carbohydrate rich glycoprotein, PAS-0, is also present (Fig. 1). A polyclonal antibody was raised in rabbits that quantitatively immunoprecipitates each of these components. The serum also precipitates the soluble components of milk, namely, the caseins, transferrin, and whey proteins. Using this antibody, membrane biosynthesis was probed in cultured cells radiolabeled with ${ }^{35} \mathrm{~S}$ methionine. Synthesis of the proteins in culture was found to be dependent upon the presence of the lactogenic hormones, insulin, hydrocortisone, and prolactin (data not shown). Additionally, synthesis was also dependent upon the substratum used for cell culture. In an 


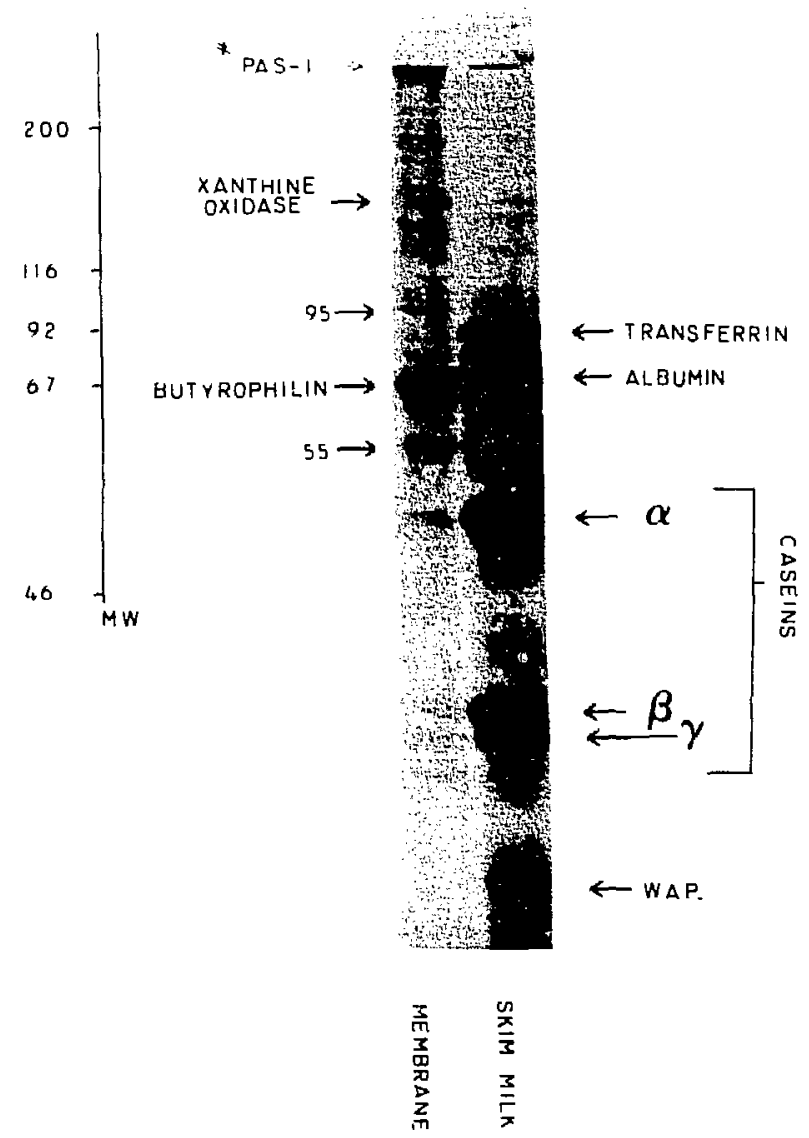

Fig. 1. SDS-polyacrylamide gel analysis of mouse milk fat globule membrane components and skim milk components. The gel was stained with Coomassie blue.

(XBB 8612-10016)

analagous fashion to that described earlier for some skim milk components, ${ }^{2,3}$ the quantities of each of the membrane proteins made were elevated significantly when cultures were established on collagen gels that were floated in the culture medium. Cultures maintained on plastic tissue culture dishes or attached collagen gels did not produce significant quantities of the membrane components (Fig. 2).

This observation was then utilized in experiments designed to examine the process of assembly of the milk fat glubule membrane. Cultures were established on attached collagen gels and then specialized membrane biosynthesis was promoted by releasing the gels from the culture mediun. At various times following release the cells were radiolabeled for 24-hour periods and the levels of each of the membrane proteins determined by immunoprecipitation and SDS-PAGE analysis of the immunoprecipitates. The kinetics of assembly of the apical membrane were thus established (Fig. 3).

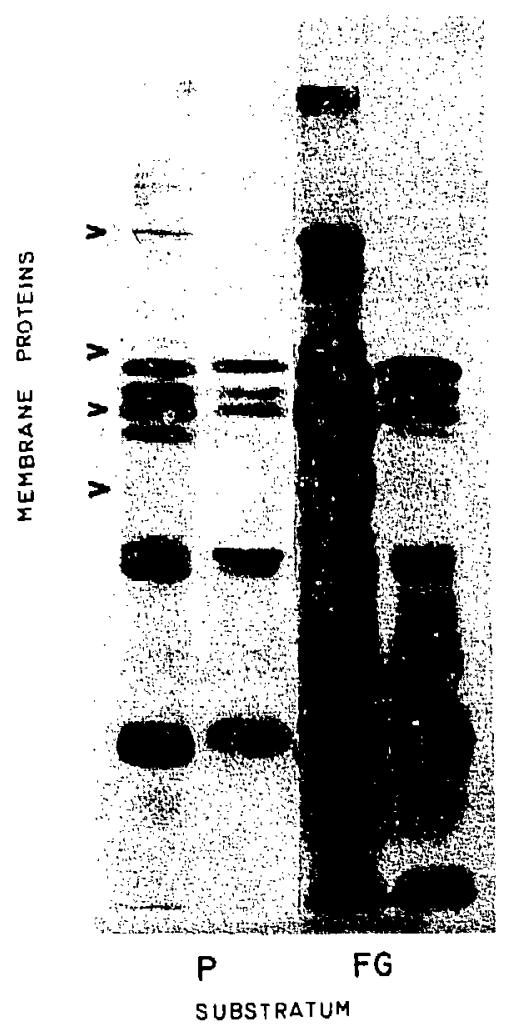

Fig. 2. Autoradiogram of ${ }^{35} \mathrm{~S}$ methionine-labeled cultures of mouse mammary epithelial cells maintained on tissue culture plastic $(P)$ and floating collagen gels (FG). Arrow heads denote the positions of the 55-, 95- and 150-kDa components. The remaining unmarked bands are skim milk proteins. The tracks on the left of each pair of samples are a samples of cellular material; those on the right is secreted material. (XBB860-10017)

It was found that the four components studied did not accumulate in the membrane with identical kinetics. Butyrophilin and the 55-kDa molecule were first inserted, followed by xanthine oxidase and the 95-kDa molecule. This observation is consistent with the view that butyrophilin and the 55$\mathrm{kDa}$ molecules are required for association of xanthine oxidase and the 95-kDa component with the membrane. This possibility is currently being investigated further.

The biosynthesis and secretion of the membrane in cultures maintained on floating collagen gels was then studied in pulse-chase experiments. Significantly, although the membrane proteins were actively made in culture, only a small fraction of the newly synthesised molecules were secreted into the culture medium. Instead the membrane proteins were turned over rapidly $\left(T_{1 / 2}=4\right.$ hours, data not shown). The studies thus reveal an unexpected control of the levels of the membrane components, namely control of the rate of degradation. The sig- 

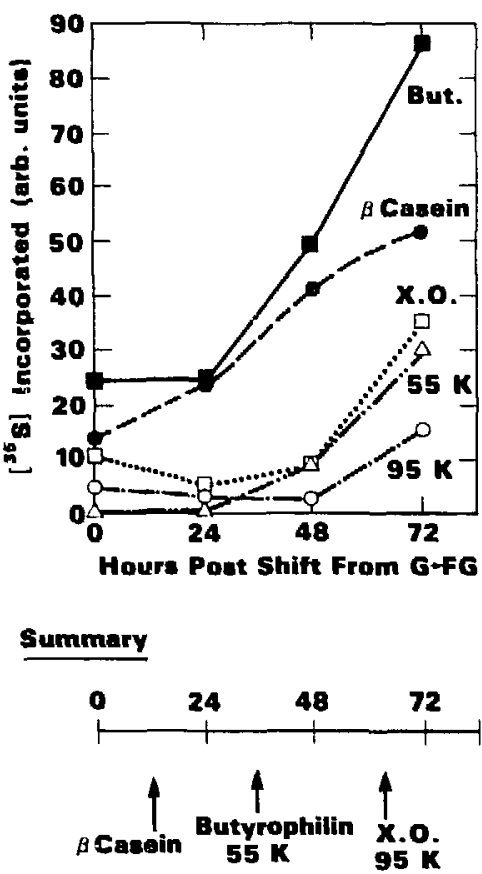

Fig. 3. Kinetics of induction of membrane differentiation in cultured cells maintained on floating gels.

(XBL 8511-8574) nificance of this in determining the composition of the apical membrane is currently being investigated, as is the mechanism by which the membrane is being selectively turned over.

\section{REFERENCES}

1. Parry, G., Moss, L., Cullen, B., Ding, N., Greenwalt, D., and Riviken, R. Expression of multiple epitopes on high molecular weight glycoproteins (PAS-0 like molecules) on human mammary carcinoma cells and milk fat globule membranes. J. Immunol. (submitted) (1986).

2. Lee, E.Y.H, Parry, G., and Bissell, M.J. Modulation of secreted proteins of mouse mammary epithelial cells by collagenous substrata. J. Cell Biol. 98, 146-155 (1984).

3. Lee, E.Y.H., Lee, W.H., Kaetzel, C.S., Parry, G., and Bissell, M.J. Interaction of mouse mammary epithelial cells with collagenous substrata: regulation of casein gene expression and secretion. Proc. Natl. Acad. Sci. USA 82, 1419-1423 (1985).

\title{
INFLUENCE OF EXTRACELLULAR MATRIX AND ITS COMPONENTS ON CASEIN GENE EXPRESSION IN MOUSE MAMMARY EPITHELIAL CELLS
}

\author{
Ming Liang Li, Judith Aggeler, Deborah A. Farson, Carroll Hatier, and \\ Mina J. Bissell
}

Study of the development and regulation of the mammary gland poses a challenging opportunity for understanding tissue-specific gene expression, because the gland changes continuously in its spetial organization and functional differentiation from embryo to aging adult. A primary regulator of these changes is the complex interaction of hormones with the gland during different stages of life.

Most cells in intact higher organisms are in contact with complex extracellular matrices (ECMs) that contain different collagen types - proteoglycans and glycoproteins. The ECM was postulated to play a role in morphogenesis and differentiation decades ago, but our knowledge of its biochemistry and its supramolecular organization has been arrived at much more recently. There has also been rapid recognition that ECMs must play a fundamental role in development and maintenance of the differentiated state in mammary gland.
When primary mouse mammary epithelial cells are cultured on plastic, they rapidly lose their ability to synthesize and secrete most milk proteins even in the presence of lactogenic hormones, whereas cells cultured on released type I collagen gels show greatly enhanced mRNA levels and rates of secretion of $\beta$-casein and of some other milk proteins. ${ }^{1,2}$ We show here that cells cultured on a reconstituted basement membrane from Engelbreth-HolmSwarm (EHS) tumor allows more than $90 \%$ of cells to produce high levels of $\beta$-casein. By comparison $30-40 \%$ of cells on released type I collagen gels and only $2-10 \%$ of cells on plastic express $\beta$-casein after 6 days in culture (Fig. 1). Because only $40 \%$ of cells from late pregnant gland produced $\beta$-casein before culture, the EHS matrix can both induce and maintain an elevated level of casein gene expression. Individual basal larnina components were also evaluated (Fig. 2). Type IV collagen and fibronectin 

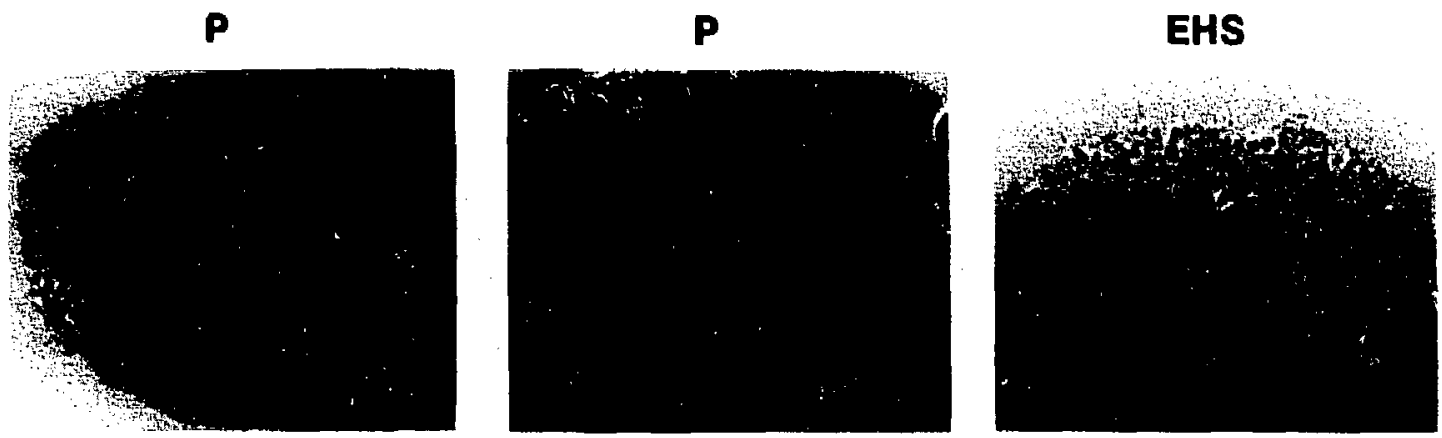

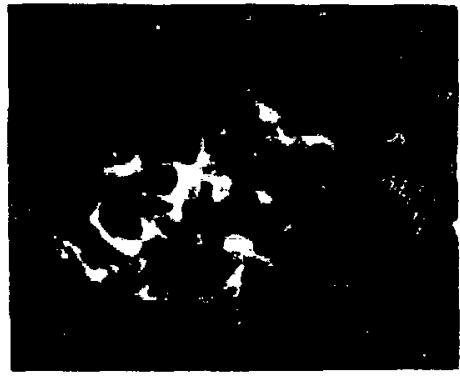

DAY 0

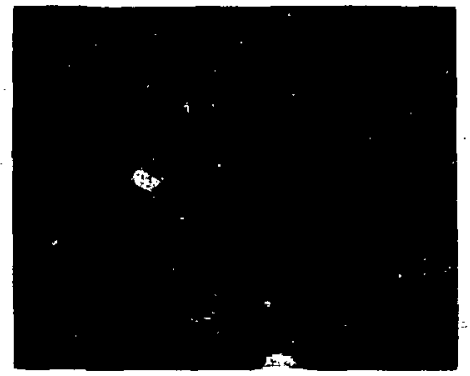

DAY 6

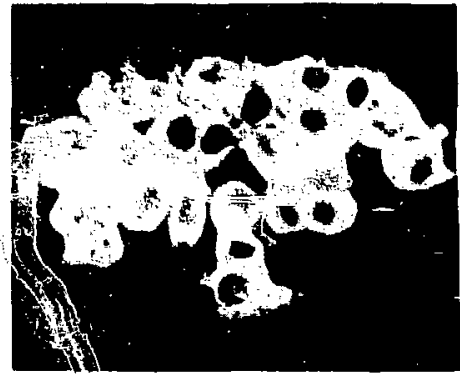

DAY 6

Fig. 1. Immunofluorescent localization of p-casein in PMME cells. Cytocentrifuged preparations of dissociated PMME cells were stained with anti- $\beta$-casein. Day 0; epithelial cells from 16-18 day pregnant mouse gland before plating. Day 6, P; the same cell preparation after plating on tissue culture plastic for 6 days. Day 6, EHS; the same cells after plating on EHS for 6 days.

(XBB 866-4524)

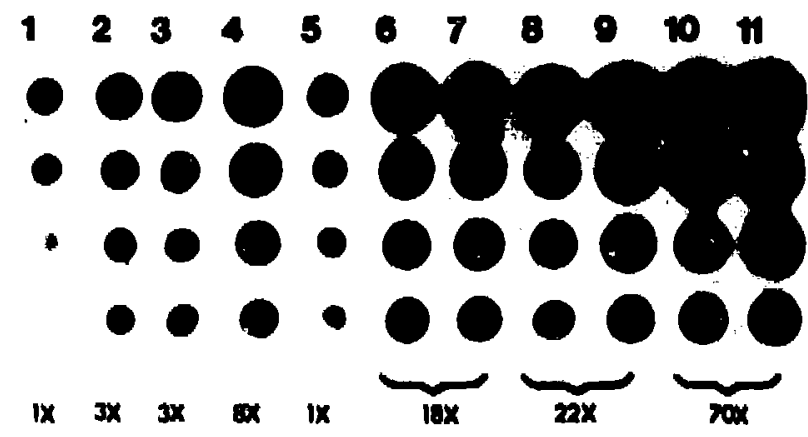

Fig. 2. B-casein mRNA levels on different substrata. Cyloplasmic RNA was extracted (as described in Refs 2, 4) 6 days post-seeding dotted onto nitrocellulose and hybridized with nick-translated $\beta$-casein probe. Top dot: $4 \mu \mathrm{g}$ RNA; other dots are 1/2 dilutions. Lane 1, $P$; lane 2, $R G$; lane 3, laminin; lane 4, HSPG; lane 5, type $\mathrm{N}$ collagen; lanes 6 and 7, EHS on plastic; lanes 8 and 9, EHS on flat gel; lanes 10 ana 11, EHS on released gel.

(XBB-866-4519)

had little effect on morphology and $\beta$-casein mRNA levels. In contrast both laminin and heparan sulfate proteoglycan (HSPG) increased $\beta$-casein mRNA lev- els (1.5- to 4-fold and 2- to 8-fold, respectively). in HSPG-treated cells, the increase in MRNA was not accompanied by increased secretion of $\beta$-casein (not shown). Profound morphological differences were evident between cells on plastic and on EHS, the latter forming ducts, ductules and lumina, and resembling secretory alveoli (Fig. 3).

The additional increase in $\beta$-casein message observed in PMME on floating EHS-collagen gels (Fig. 2, lanes 10 and 11) is intriguing and further emphasizes the correlation between changes in cell shape and epithelial function (for reviews see Ref. 3 ). However, the increase in $\beta$-casein message in cells on HSPG without an obvious morphological change argues against a simple correlation between shape and mRNA levels. Cell-associated HSPG has been shown to span the plasma membrane, connecting the ECM and the cytoskeleton, and laminin also has been reported to have connections to actin via a cell-surface receptor. Perhaps HSPG and laminin in our cultures can increase $\beta$-casein mRNA levels by altering cytoskeletal interactions with polysomes, leading to increased message stability. 


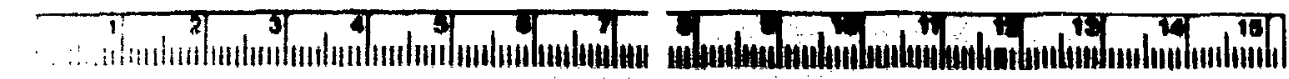
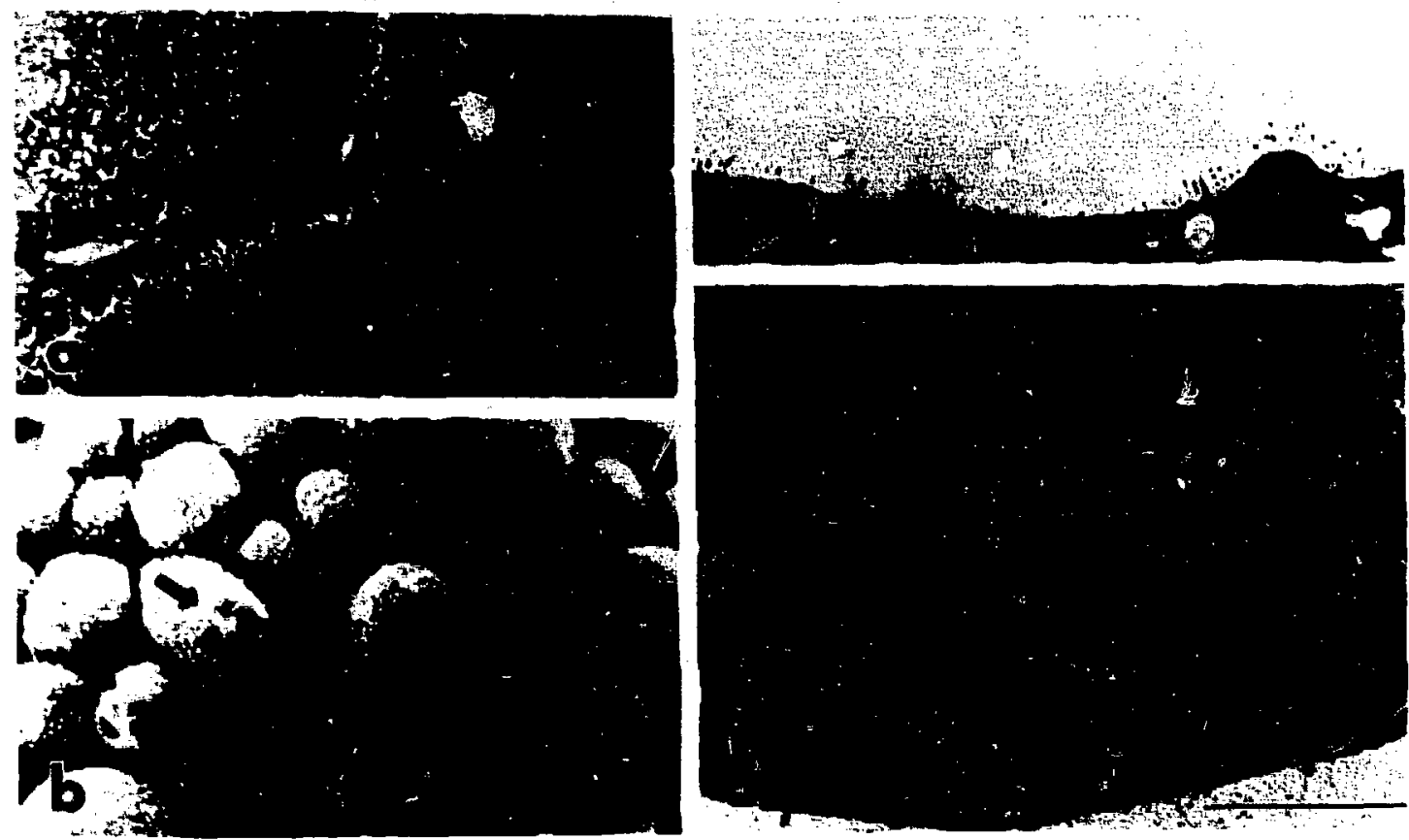

Fig. 3. Electron microscopy of PMME cullured on EHS matrix for 7 days. Cells on coverslips (a,c) spread to form a flat epithelial layer while cells on EHS $(b, d)$ are rounded and covered with apical microvilli. By TEM cells on EHS are seen to form hollow alveolar-like structures with the base of the cells facing out and their apices in. Evidence of secretory activity includes extensive rough endoplasmic reticulum (large arrow), Golgi apparatus (arrowheads), numerous milochondria, and lipid droplets (D). Some of the granular material (G) which fills the lumen may be secreted milk proteins. Bar $=10 \mu \mathrm{m}$.

(XBB 8611-9698)

While these results emphasize the vital role of the extracellular matrix in regulation of gene expression, they also point out the complexity of the factors involved.

Basement membranes in vivo are known to be heterogeneous. ihe complex interactions between, and precise compositional ratios of ECM components are being analyzed both in vitro and in culture. Attachment, spreading, and undoubtedly functional differentiation of cells are influenced by composition, concentration and spatial interactions of ECM molecules. The aim of our studies is not only to establish that the ECM is an "informational" entity, receiving, imparting, and integrating structural and functional signals, but also to understand the mechanism(s) by which the ECM modulates gene expression. While we have come a long way in establishing the former, the latter aim will require careful quantitative analysis of the chemistry and biochemistly of the ECM and a detailed kinetic analysis of cellular functions in response to cellECM inte:'actions.

\section{REFERENCES}

1. Li:e, E.Y-H., Parry, G., and Bissell, M.J. I. Cell Biol. 98, 146-155 (1984).

2. Lee, E.Y-H., Lee, W-H., Kaetzel, C.S., Parry, (J., and Bissell, M.). Proc. Natl. Acad. Sci. J.S.A. 82, 1419-1423 (1985).

3. Bissell, M.J., and Hall, H.G. In The Mammary Gland: Development, Regulation and Function Neville, M., and Daniel, C., Eds. (Plenum Press, New York, in press), (1987).

4. Li, M.L., Aggeler, I., Farson, D.A., Hatier, C., Hassell, I. and Bissell, M.J. Proc. Natl. Acad. Sci. USA (in press), (1986). 


\title{
HIGH SERUM LEVELS INTERFERE WITH THE NORMAL DIFFERENTIATED STATE OF AVIAN TENDON CELLS BY ALTERING TRANSLATIONAL REGULATION
}

\author{
Janice Valmassoi and Richard I. Schwarz
}

Characterization of the control mechanisms that regulate the differentiated state requires an understanding of the normal variations in cellular function that occur during development. For instance, the production of type 1 procollagen by embryonic chick tendon cells varies from $50 \%$ of the total protein synthesis to less than $1 \%$ in the adult, a change that reflects the drop in the overall growth rate of the organism. ${ }^{1}$ We have used primary avian tendon (PAT) cells in culture and their ability to express collagen to examine the regulation of the differentiated state in a structural tissue. One important finding is that the ability of these cells to maintain quantitative in ovo levels of collagen synthesis in culture is directly related to the cell culture conditions. ${ }^{2}$

One element of the cell culture environment found to be detrimental to maintaining the differentiated state is the presence of high serum levels. ${ }^{3}$ Serum levels in excess of $0.5 \%$ have been shown to inhibit the percentage of procollagen synthesized by PAT cells. ${ }^{4}$ High serum rapidly changes the pattern of proteins produced by the cell, and reversibly blocks the induction of procollagen production by ascorbate normally observed in PAT cells. ${ }^{3}$ Ascorbate added to cells grown in low-sodium medium specifically induces procollagen synthesis from $12 \%$ to $50 \%$ of total protein production. 2,5

The focus of this study was to pinpoint the mechanism whereby high serum levels alter the regulation of the collagen pathway. A short discussion of the ascorbate induction process in PAT cells is necessary in order to place the effects of high serum in perspective. Upon ascorbate addition the secretion rate constant increases, resulting in a decrease in the half life of procollagen within the cell from 120 minutes to 20 minutes. ${ }^{6}$ This causes the internal pool of procollagen to drop. Following a 4-hour delay, synthesis of procollagen begins to increase; rising by 6-fold within 48 hours. ${ }^{3}$ This results in the reestablishment of the pool size of procollagen. In the induction process, mRNA levels also increase by 6 -fold but kinetically follow changes in translation and secretion rates. Therefore, transcriptional regulation cannot be the primary control point in the biosynthetic pathway. The theory most consistent with our data is that ascorbate specifically influences the protein syn- thetic machinery of the cell through a posttranslational modification that allows procollagen to be secreted at the faster rate. This effect of increasing the secretion rate causes the cells to respond to a decreasing internal pool of procollagen first by translating procollagen molecules more efficiently and later by increasing mRNA levels. These three steps, secretion, translation, and transcription, are part of a tightly coupled pathway for the control of procollagen production, and serum blocks the linkage between these steps at a specific point.

One method for examining the coupling between secretion and translation rates, and the effects of high serum on that coupling, is to look at pool size. Using a continuous label to saturate the internal procollagen pool we observed a distinct difference in the pool size on SDS PAGE between cells grown in low and high serum medium (Fig. 1). In low serum the size of the internal pool of procollagen is identical for cells grown with or without ascorbate. The corresponding medium from these cells was also applied and run on the gel (with and without ascorbate). The relative amount of procollagen found in the medium of ascorbate-induced cells compared with the level from scorbutic cells indicates an increase in the secretion rate. The pori size does not change in these cells, reflecting the coupling between secretion and translation rates. For cells grown in high serum, the pool size is much smaller in ascorbate-induced cells than uninduced cells (Fig. 1). Most of the labeled procollagen in uninduced cells is found inside the cell while most of the labeled procollagen in ascorbate-induced cells is present in the medium (Fig. 1). These cells are responding to the presence of ascorbate by showing a stimulation of secretion rates but not a parallel increase in translation rates. A pulse-chase experiment showed that cells grown for 48 hours in high serum medium with ascorbate secrete at rates identical to their low serum counterparts (Fig. 2). Therefore, the first step in the path of ascorbate induction of procollagen is not affected by serum concentration.

Ascorbate can increase the secretion rates of PAT cells grown in high serum but the synthetic machinery is not induced to produce the expected levels of procollagen (Table 1). We can also observe the inhibitory effects of serum on transla- 


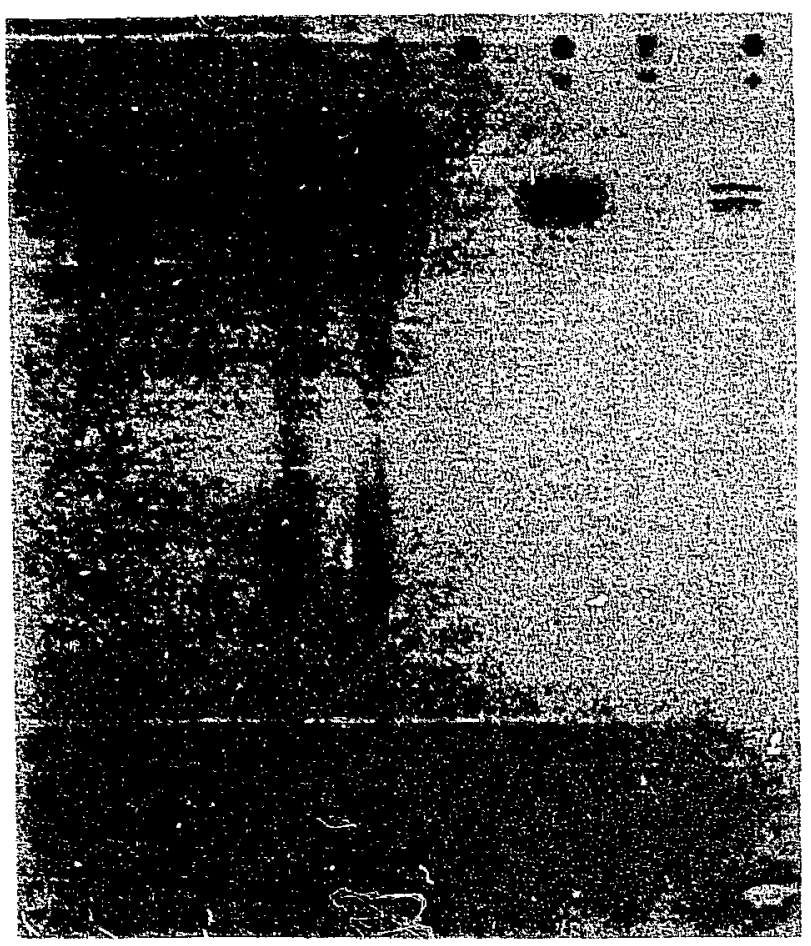

Fig. 1. Autoradinaram of an SDS polyacrylamide gel of cellular proteins (lar 4) and proteins in the medium (lanes 5-8). Cells were gruvn in low serum medium (lanes $1,2 \& 5,6$ ) and high serum medium(lanes 3,4 \& 7,8 ). The odd number lanes were from ceils grown without ascorbate (-) and the even numbered with ascorbate( +$)$. The arrows indicale the mobility of the procollagen $\alpha_{i}$ band (upper) and the procollagen $\alpha_{2}$ band (lower). PAT cells were labeled for $3 \mathrm{hr}$ with ${ }^{3} \mathrm{H}$-prinline $(100 \mu \mathrm{Ci} / 2 \mathrm{ml})$ and the medium was separated from the cell layer. An equal number of cells were applied to each well.

(XBB 863-1642)

tion of procollagen by adding serum to ascorbate induced cultures initially grown in low serum. In this case serum rapidly alters the translational products of the ascorbate-induced cell. The production of noncollagen proteins is stimulated in preference to procollagen. Synthesis of noncollagen proteins increased by 6-fold but procollagen synthesis only rises 2 -fold over a period of 6 hours. The most striking change occurs within the first hour when the ratio of procollagen synthesis to noncollagen synthesis drops dramatically by 5 -fold (Fig. 3). The differential effect of serum on the proteins produced by the cell can be seen as a change in the translational regulation within the cell.

Normally upon ascorbate induction procollagen mRNA levels increase with a 12-hour lag followed by a slow, linear 6-fold increase after 72 hours. Ascorbate addition to cells grown in high serum showed no increase in procollagen mRNA levels

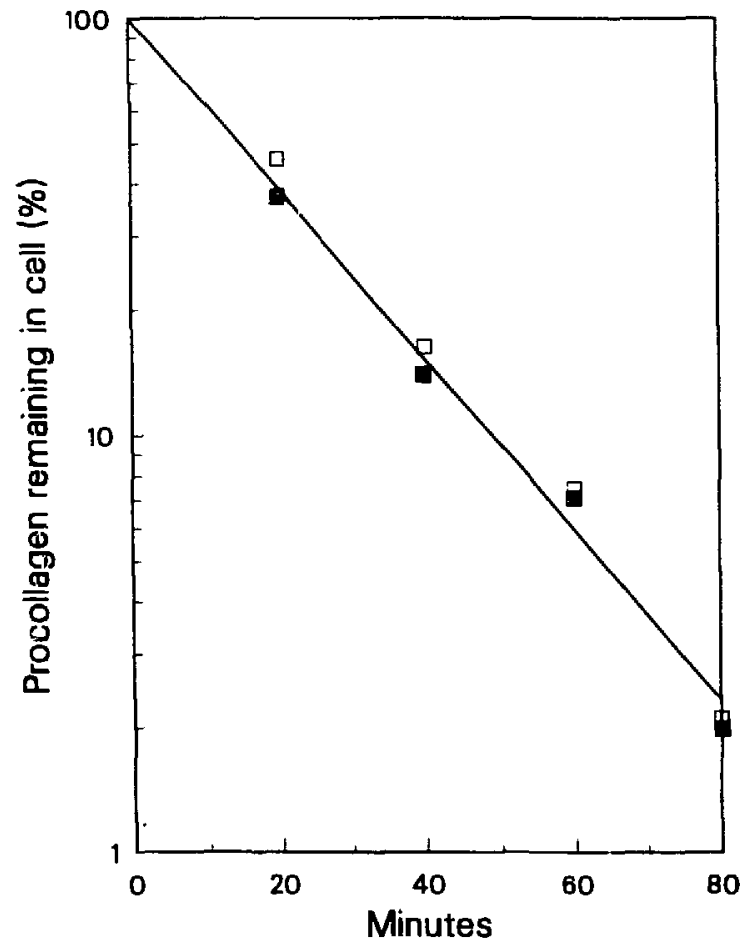

Fig. 2. Rate of procollagen secretion in high (ㅁ) and low (D) serum medium containing ascorbate. Cells grown for $48 \mathrm{hr}$ in the appropriate medium were pulsed $(100 \mu \mathrm{Ci} / 2 \mathrm{ml})$ for $2 \mathrm{hr}$ and then chased with the same medium containing $100 \times$ normal concentration of unlabeled proline. At each point the medium was changed and assayed for secreted procollagen counts.

(XCG 865-7266)

over those of uninduced cells (Table 1). Again one can test if the inhibitory effect of serum on procollagen is due to changes in mRNA levels. High serum addition to ascorbate-induced PAT cultures showed no change in procollagen mRNA levels over a period of 6 hours. Cells grown in high serum for 48 hours will not respond to ascorbate by increasing procollagen mRNA. The level of procollagen mRNA remains at the level of uninduced rells (Table 1 ).

In summary, we see that PAT cells grown under conditions favorable for retaining the differentiated state $(0.2 \%$ serum and ascorbate, producing $50 \%$ of total protein as procollagen) respond to high serum levels by disrupting their normal pattern of protein production. The fact that the ascorbate induction process does not proceed through the linked pathway has allowed us to pinpoint the effects of serum on procollagen production. Even in high serum PAT cells maintain their ability to respond to ascorbate by increasing the secretion rate. The expected next step of increasing the translation rate of pro- 
Table 1. Ascorbate $(+C)$ induction $(48 \mathrm{hr})$ in the presence of low and high serum.

\begin{tabular}{lccc}
\hline & $\begin{array}{c}\text { Fold increase } \\
\text { procollagen } \\
\text { mRINA }\end{array}$ & $\begin{array}{c}\text { \% procollagen } \\
\text { synthesis }\end{array}$ & $\begin{array}{c}\text { Absolute } \\
\text { procollagen } \\
\text { synthesis/ } \\
\text { cell }\end{array}$ \\
\hline Low serum - C & 1 & $1 \%$ & 377 \\
Low serum +C & $4.06 \pm 1.06$ & $52 \%$ & 2,127 \\
High serum -C & $1.34 \pm 0.37$ & $5 \%$ & 380 \\
High serum +C & $1.76 \pm 0.56$ & $8 \%$ & 597 \\
\hline \hline
\end{tabular}

- All values have been normalized to the amount of procollagen mRNA in cells grown in low serum medium without ascorbate.

b Cells were ${ }^{3} \mathrm{H}$-proline labeled ( $50 \mu \mathrm{Ci} /$ flask) for $2 \mathrm{hr}$ and the values shown were corrected for the high amount of proline residues in the procollagen molecule.

c The values shown are the cpm incorporated per $2 \times 10^{5}$ cells.

collagen does not occur. Even though Kao et al. ${ }^{7}$ have shown that tendon cells grown in high serum can respond to ascorbate by increasing the number of membrane-bound polysomes, thereby allowing for a more efficient translation of available mRNAs, the selective production of procollagen does not occur. Therefore, although there is potential for feedback control, in high serum the drop in the internal pool of procollagen is not sufficient to induce synthesis of procollagen or increase mRNA levels due to a specific block at the translational step.

\section{REFERENCES}

1. Schwarz, R.I., Farson, D.A., and Bissell, M.J. In Vitro 15, 941-948 (1979).

2. Schwarz, R.I., Mandell, R.B., and Bissell, M.J. Mol. Cell. Biol. 1, 843-853 (1981)

3. Schwarz, R.I., and Bissell, M.J. Proc. Natl. Acad. Sci. U.S.A. 74, 4453-4457 (1977).

4. Schwarz, R.I., Colarusso, L., and Doty, P. Exp. Cell Res. 102, 63-71 (1976).
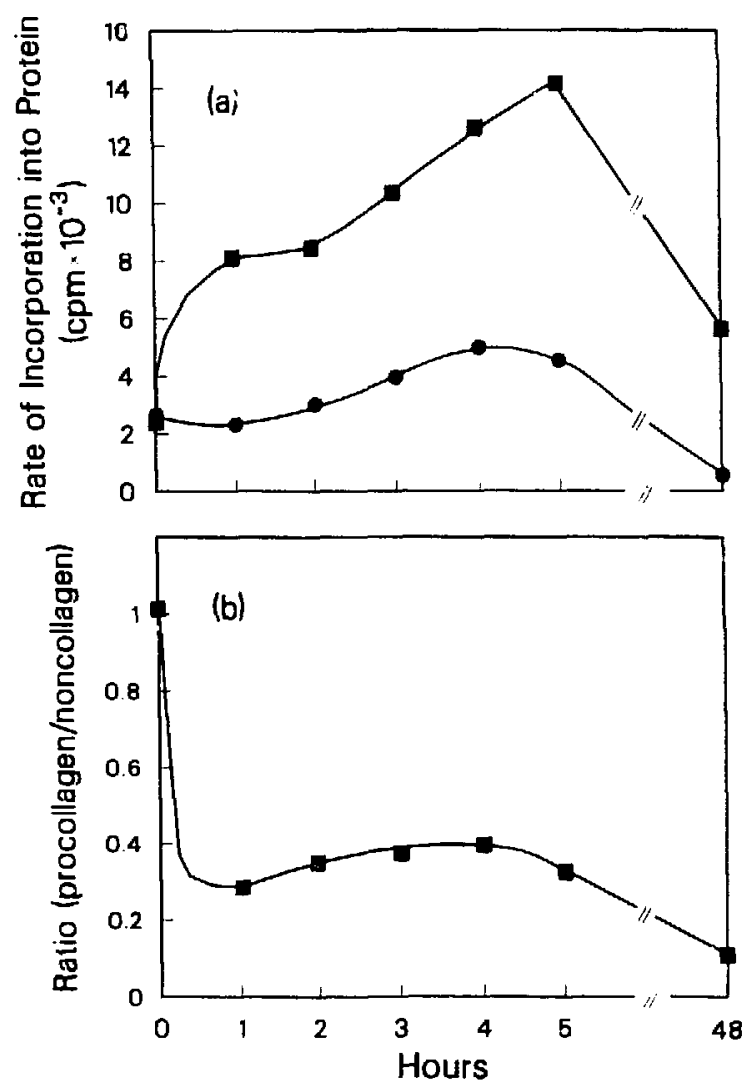

Fig. 3. The effect of high serum on the ratic st procollagen to noncollagen protein production. At zero time, fully induced PAT cells grown in low serum (0.2\%) medium with ascorbate had serum levels in medium increased to 3\%. For each time point, cells were labeled in $2 \mathrm{ml}$ of high serum medium with ${ }^{3} \mathrm{H}$-proline $(50 \mu \mathrm{Ci})$ for $2 \mathrm{hr}$.

(XCG 865-7268)

5. Schwarz, R.I., and Rowe, L.B. Mol. Cell. Biol. 3, 241-249 (1983).

6. Schwarz, R.I. J. Biol. Chem. 260, 3045-3049 (1985).

7. Kao, W.W.-Y., Flaks, J.G., and Prockop, D.J. Arch Biochem. and Biophys. 173, 638-648 (1976). 


\section{APPENDICES}

\section{APPENDIX A. List of Contracts and Grants Supporting Portions of Work Presented in this Annual Report}

\section{INVESTIGATOR}

E.J. Ainsworth

E.I. Ainsworth

E.L. Alpen

E.L. Alpen

J.C. Bartley

M.J. Bissell

G. Brecher

T.F. Budinger

T.F. Budinger

T.F. Budinger

T.F. Budinger

T.F. Budinger

I.R. Castro

A. Chatterjee

\section{CONTRACT OR GRANT}

NASA P.O. T3516-G

Life-Shortening Effects of HzE Particles on

Mice/hieavy lon Cell Transformation

AFFRI P.O. N861014

Murine Bone-Marrow Damage Produced by Charged

Particles

PHS Grant CA 30236

Advanced Design Research Heavy lon Medical Accelerator

PHS Grant RR 05918

Biomedical Research Support Grant

PHS Grant CA 33889

Hurnan Mammary Cells: Modulation of Differentiated

States

Monsanto 1856-29

Mechanisms of Extracellular Matrix Influences on Gene

Expression

PHS Grant AM 27454

Kinetics of Transfused Stem Cells in Normal Mice

PHS Grant HL 25840

Cardiovascular Flow and Metabolism

PHS Grant AG 05890

Cerebral Blood Flow Patterns in Alzheimer's Disease

PHS Grant HL 07367

Quantitative Cardiovascular Research, Training Grant

IBM Instruments

P.O. 4521310

NMR Imaging Project

UCSF Subcontract 8378-01

PET Studies

PHS Grant CA 19138

Treatment of Cancer with Heavy Charged Particles

PHS Grant CA 27024

Bragg Peak Localization by Radioactive Beams 
G.K. Clemons

P.K. Cooper

S.B. Curtis

P.W. Durbin

P.W. Durbin

S.N. Ebbe

M.S. Esposito

T.M. Forte

R.M. Glaeser •

R.M. Glaeser

L.S. Gold

R. Goth-Goldstein

R. Goth-Goldstein

H.G. Hall

1. Hosoda

R.H. Huessman

R.M. Krauss

R.M. Krauss
PHS Grant HL 22469

Radioassay of Erythropoietin

PHS Grant CA 32986

Inducible Responses to Carcinogenic DNA Damage

PHS Grant CA 17411

Response of Rat Tumor Cells to Heavy lons

NRC IAG 60-85-012

Development of Metabolic Models for Alkaline Earth and Actinide Radionuclides

PHS Grant ES 02698

Biological Testing of New Actinide-Chelating Agents

PHS Grant AM 21355

Kinetics of Megasaryocyte and Platelet Turnover

PHS Grant GM 29002

Comparative Analysis of Mitotic and Meiotic

Recombination

PHS Grant HL 07279

Lipoprotein Methodology, Structure and Function

Training Grant

PHS Grant GM 23325

Biological Structure Analysis by Electron Microscopy

PHS Grant GM 36884

Membrane Proteins: High Resolution Electron

Microscopy

PHS IAG 222-Y01-ES-10066

Quantitative Species Extrapolation in Carcinogenesis

PHS Grant ES 01916

Alkylating-Carcinogens Mutagenesis in Mammalian Cells

PHS Grant ES 03603

Inducible Resistance to Alkylating Carcinogens

PHS Grant HD 17892

Extracellular Matrix and Epithelial Lumen

Morphogenesis

PH5 Grant GM 16841

Structure and Function of Helix

Stabilizing Protein

PHS Grant CA 38086

Scatter Compensation in Emission Tomography

PHS Grant HL 33577

Plasma Lipoproteins in Coronary Artery Disease

USC Medical Center 8072.22

Lipid and Protein Analysis 
R.M. Krauss

S.A. Leadon

R.P. Liburdy

R.P. Liburdy

1. Llacer

J.T. Lyman

I.T. Lyman

M.F. Maestre

M.F. Maestre

C.A. Mathis

R.K. Mortimer

A.V. Nichols

1.C. Owicki

G. Parry

W. Schimmerling

W. Schimmerling

W. Schimmerling
Stanford University Contract P.O. \#PR 4699-3

D \& G Analyses

PHS Grant CA 40453

DNA Repair in Specific Sequences of Mammalian Cells

ONR Contract N00014-84-F-0186

Mechanisms of Microwave Interactions with Liposome Membranes

Liposome Technologies Incorporated 1856-31

Electromagnetic Field-Facilitated Drug Delivery in Liposomes

PHS Grant CA-39501

Algorithms and Processing Architectures for Tomography

PHS IAG Y01-CM-20110

Evaluation of Treatment Planning for Particle Beam

Radiotherapy

PHS Grant CA 22286

AAPM B682.19

Charged Particle Beam Dosimetry Task Group: Dosimetry Protocol

PHS Grant Al 08427

Physical Structure of Viruses, Chromosomes and Cell Nuclei

PHS Grant RR 02757

Jasco $500 C$ Circular Dichrograph with FDCD and MCD

PHS Grant NS 22899

${ }^{122}$ I Blood Flow and Blood Volume Agents for PET

Studies

PHS Grant GM 30990

Yeast RAD Genes in Repair, Recombination and Meiosis

PHS Grant HL 18574

Lipoprotein Subclasses: Structure Origin, and Metabolism

PH5 Grant Al 19605

Immunological Recognition of Model Membranes

PHS Grant CA 44398

Proteoglycan Influence on Mammary Cell Differentiation

PHS Grant CA 23247

Physical Characteristics of Heavy Ion Beams

NASA P.O. L22395A

To Measure the Production of Neutrons by High Energy Heavy lons

NASA P.O. L9550C

Iron and Oxygen Fragmentation Experiments 
R.I. Schwarz

B.A. Singer

M.R. Stampier

M.R. Stampfer

T.S. Teniorde

T.S. Tenforde

C.A. Tobias
PHS Grant CA 37958

Manipulation of the Differentiated State by

Oncogenesis

PHS Grant CA 42736

Alkylation of Polynucleotides in vitro and in vivo

PHS Grant CA 24844

Characterization of Human Mammary Cells

PHS Grant CA 30028 (R.Ham, Univ. Colorado)

P.O. 426881

Defined Medium for Human Mammary Epithelial Cells

EPRI Contract RP 799-21

Microprocessor-Controlled Personal Dosimeter for

Recording Low-intensity Power Frequency Magnetic

Fields

EPRI Contract RP 2572.5

Assessment of Biological Effects Associated with

Magnetic Fields from a Superconducting Magnet Energy

Storage System.

PHS Grant CA 15184

Heavy Ion Radiobiology Related to Oncology 


\section{Appendix B: 1986 Publications}

\section{CONTRIBUTIONS TO JOURNALS}

Afzal, S.M.J., Tenforde, T.S., Parr, S.S., Curtis, S.B. PLD repair in rat rhabdomyosarcoma tumor cells irradiated in vivo and in vitro with high-LET and low-LET radiation. Radiat. Res. 107, 354-366 (1986).

Austin, M.A., Krauss, R.M. Genetic control of low density lipoprotein subclasses. Lancet $i, 592-595$ (1986).

Austin-Seymour, M.M., Chen, G.T.Y., Castro, J.R., Saunders, W.M., Pitluck, S., Woodruff, K.H., Kessler, M. Dose volume histogram analysis of liver radiation tolerance. Int. J. Radiat. Oncol. Biol. Phys. 12, 31-35 (1986).

Babiak, J., Nichols, A.V., Gong, E.L., McMahan, C.A., Kuehl, T.J., Mott, G., McGill, H.C., Jr. Effects of dietary polyunsaturated and saturated fats on lipoproteins in the baboon. Atherosclerosis 57,1-17 (1985).

Barnard, J., LaBelle, M., Linn, S. Levels of uracil DNA glycosylase and AP endonuclease in murine B- and $T$ lymphocytes do not changes with age. Exp. Cell Res. $163,500-508$ (1986).

Bassel, I., Mortimer, R.K. Identification of mutations preventing $n$-hexadecane uptake among n-alkane non-utilizing mutants of Yarrowia (Saccharomycopsis) lipolytica. Current Genetics 9, 579-586 (1985).

Blakely, E.A., Chang, P.T., Lommel, Leora Cell-cycledependent recovery from heavy-ion damage in $G_{1}$ phase cells. Radiation Research 104 (Suppl. 8), S145-S157 (1985).

Brecher, G., Mulcahy, K., Tjio, J.H., Raveché, E. Enhanced proliferation of transfused marrow and reversal of normal growth inhibition of female marrow in male hosts 2 months after sublethal irradiation. Ann. N.Y. Acad. Sci. 459, 232-238 (1986).

Carter, V.C., Howlett, A.R., Martin, G.S., Bissell, M.J. The tyrosine phosphorylation substrate P36 is developmentally regulated in embryonic avian limb, and is induced in cell culture. I. Cell Biol. 103, 2017-2024 (1986).

Castro, J.R., Chen, G.T.Y., Blakely, E.A. Current considerations in heavy charged-particle radiotherapy: $A$ clinical research trial of the University of California Lawrence Berkeley Laboratory, Northern California Oncology Group and Radiation Therapy Oncology Group. Radiation Research 104 (Suppl. 8), S263-S271 (1985).
Castro, I.R., Saunders, W.M., Austin-Seymour, M.M., Woodruff, K.H., Gauger, G., Chen, G.T.Y., Collier, J.M., Zink, S.R. Heavy charged particle irradiation of malignant glioma of the brain. Int. J. Rad. Oncol. Biol., Phys. 11, 1795-1800 (1985).

Chatteriee, A. Magee, J.L. Theoretical investigation of the production of strand breaks in DNA by water radicals. Radiat. Prot. Dosimetry 13, 1-4 (1985).

Chen, C.-H., Forte, T.M., Cahoon, B.E., Thrift, R.N., Albers, J.J. Synthesis and secretion of lecithincholesterol acyltransferase by the human hepatoma cell line Hep G2. Biochim. Biophys. Acta 877, 433-439 (1986).

Chen, G.T.Y., Goitein, M., Urie, M., Holley, W. Degradation of Bragg peaks from inhomogeneities. Physics in Medicine and Biology 31, 1-17 (1986).

Clemons, K., Fitzsimmons, S.L., DeManincor, D. Immunoreactive erythropoietin concentrations in fetal and neonatal rats and the effects of hypoxia. Blood 68 , 892-899 (1986).

Cronkite, E.P., Bullis, J., Brecher, G. Marrow transfusions increase pluripotential stem cells in normal hosts. Exp. Hemat. 12, 802-805 (1985).

Curtis, S.B. Lethal and potentially lethal lesions induced by radiation-a unified repair model. Radiation Research 106, 252-270 (1986).

Dickson, M.R., Downing, K.H., Wu, W.H., Glaeser, R.M. Three dimensional structure of the surface layer protein of Aquaspirillum serpens VHA determined by electron crystallography. J. Bacteriol. 167, 1025-1034 (1986).

Durbin, P.W., leung, N., Schmidt, C.T. ${ }^{238} \mathrm{Pu}(\mathrm{IV})$ in monkeys. Overview of metabolism. U.S. Nuclear Regula. tory Commission, NUREG/CR.4355, Vol. 1 (1985).

Ebbe, S., Phalen, E., Threatte, G., Londe, H. Modulation of radiation-induced hemopoietic suppression by acute thrombocytopenia. Ann. N.Y. Acad. Sci. 459, 179-189 (1985).

Erkenbrack, D.E., Rosenberg, L.L. Binding of thyroid hormones to nuclear extracts of thyroid cells. Endocrinoiogy 119, 311-317 (1986). 
Esposito, M.S., Maleas, D.T., Bjornstad, K.A., Holbrook, L. The REC 46 gene of Saccharomyces cerevisiae controls mitotic chromosomal stability, recombination, and sporulation: Cell type and life cycle stage-specific expression of the rec46- 1 mutation. Current Genetics $10,425-433$ (1986).

Fabrikant, I.I., Lyman, J.T., Frankel, K.A. Heavy chargedparticle Bragg peak radiosurgery for intracranial vascular disorders. Radiation Research 104 (Suppl. 8), S244-S258 (1985).

Foster, D.L., Mowbray, S., Koshland, D.E. The fatty acid monolayer technique fur preparing frozen-hydrated specimens. J. Electron Microscopy Technique 2, 59-65 (1985).

Frankel, K., Schimmerling, W., Rasmussen, J.O., Crowe, K.M. Bistirlich, J., Bowman, H., Hashimoto, O., Murphy, D.L., Ridout, J., Sullivan, I.P., Yoo, E., McDonald, W.J., Salomon, M., Xu, I.-S. Measurements of n-p correlations in the reaction of relativistic neon with uranium. Zeitschrift fur Physik A, 391-397 (1986).

Fry, R.J.M., Powers-Risius, P., Alpen, E.L., Ainsworth, E.J. High LET-radiation carcinogenesis. Radiation Research 104 (Suppl. 8), S188-S195 (1985).

Genzel-Boroviczeny, O., Forte, T.M., Austin, M.A. Highdensity lipoprotein subclass distribution and human cord blood lipid levels. Pediatric Research 20 , 487-491 (1986).

Glaeser, R.M., Baldwin, J., Ceska, T.A., Hendersan, R. Electron diffraction analysis of the $M_{412}$ intermediate of bacteriorhodopsin. Biophys. I. 50, 913-920 (1986).

Glaeser, R.M., Jap, B.K. Absorption flattening in the circular dichroism spectra of small membrane fragments. Biochemistry 24, 6398-6401 (1985).

Gold, L.S., de Veciana, M., Backman, G., Magaw, R., Lopidero, P., Smith, M., Blumenthal, M., Levinson, R., Bernstein, L., Ames, B.N. Chronological supplement to the carcinogenic potency database: standardized results of animal bioassays published through December 1982. Environ. Health Perspect. 67, $161-200(1986)$.

Gold, L.S., Ward, J.M., Bernstein, L., Stern, B. Association between carcinogenic potency and tumor pathology in rodent carcinogenesis bioassays. Fundamental and Applied Toxicology 6, 677-690 (1986).

Grant, R.A., Schmid, M.F., Chiu, W., Deatherage, J.F., Hosoda, J. Alignment and merging of electron microscope images of frozen hydrated crystals of the $T 4$ DNA helix destabilizing protein gp32*I. Biophys. I. 49, $241-258$ (1986).
Green, A.R., Searle, S., Gillespie, D.A.F., Bissell, M.J., Wyke, I.A. Expression of integrated Rous sarcoma viruses: DNA rearrangements $5^{\prime}$ to the provirus are comimon in transformed rat cells but not seen in infected but untransformed cells. The EMBO journal 5, 707-711 (1986). (EMBO is European Molecular Biology Organization.)

Gullberg, G.T., Huesman, R.H., Malko, J.A., Pelc, N.J., Budinger, T.F. An attenuated projector-backprojector for iterative SPECT reconstruction. Phys. Med. Biol. 30 (8), $799-816$ (1985).

Hall, H.G. DNA differences found between Africanized and European honeybees. Proc. Nat'l. Acad. Sci. (USA) 83, 4874-4877 (1986).

Hall, H.G., Bissell, M.J. Characterization of the intermediate filament proteins of murine mammary gland epithelial cells: response to collagen substratum. Exp. Cell Research 162, 379-389 (1986).

Hanson, W.R., Crouse, D.A., Fry, R.J.M., Ainsworth, E.J. Relative biological effectiveness measurements using murine lethality and survival of intestinal and hematopoietic stem cells after Fermilab neutrons compared to IANUS reactor neutrons and ${ }^{60}$ Cobalt gamma rays. Radiation Research 100, 290-297 (1984).

Hlatky, L., Alpen, E.L, Yee, M.K. Differences in the $x$-ray sensitivity of cells in different regions of the sandwich, a diffusion limited system for cell growth. Radiation Research 108, 62-73 (1986).

International Experimental Study Group: Chiu, W., Downing, K.H., Dubochet, J., Glaeser, R.M., Heide, H.G., Knapek, E. Kopf, D.A.., Lambik, M.K., Lepault, I., Robertson, J.D., Zeitler, E., Zemlin, F. Cryoprotection in electron microscopy. I. Microscopy 141, 385-391 (1986).

Jagust, W.J., Friedland, R.P. Positron emission tomography in the study of Alzheimer's disease. V.A. Practifioner 2, 55-64 (1985).

lagust, W.J., Friedland, R.P., Budinger, T.F. Positron emission tomography with $\left({ }^{18} \mathrm{~F}\right)$ fluorodeoxyglucose differentiates normal pressure hyrirocephalus from Alzheimer-lype dementia. 1. Neurol. Neurosurg. Psychiat. 48, 1091-1096 (1985).

Jap, B.K., Kong, S.-H. Secondary structure of halorhodopsin. Biochemistry 25, 502-505 (1986).

Jernigan, T.L., Sargent III, T., Pfefferbaum, A., Kusubov, N., Stahl, S. 18-Fluorodeoxyglucose PET in schizophrenia. Psychiat. Res. 16, 317-329 (1986). 
Johnston, J.R., Mortimer, R.K. Electrophoretic caryotyping of laboratory and commercial strains of Saccharomyces and other yeasts. Int. 1. Systematic Bacteriology $36,569-572(1986)$.

Kahlon, T.S., Adamson, G.L., Glines, L.A., Orr, J., Lindgren, F.T. Partial speculk volume and preferential hydration of low density lipoprotein subfractions. Lipids 21, 235-238 (1986).

Kaneko, M., Leadon, S.A. Production of ihymine glycols in DNA by $\mathrm{N}$-hydroxy-2-naphthylamine, as detected by a monoclonal antibody. Cancer Research 46, 71-75 (1986).

Kim, M.H., Ulibarri, L., Keller, D., Maestre, M.F., Bustamante, $C$. The psi-type circular dichroism of large molecular aggregates. J. Chem. Phys. 84, 2981-2989 (1986).

Kokka, N., Clemons, G., Lomax, P. Acute effects of cholinesterase inhibitors on neuroendocrine activity. Proc. West. Pharmacol. Soc. 29, 345-348 (1986).

Leadon, S.A. Differential repair of DNA damage in specific nucleotide sequences in monkey cells. Nucleic Acids Res. 14, 8979-8995 (1986).

Leadon, S.A., Hanawalt, P.C. Cell-cycle-dependent repair of damage in alpha and bulk DNA of monkey cells. Mutation Research 166, 71-77 (1986).

Levantis, P., Gillespie, D.A.F., Hart, K., Bissell, M.J., Wyke, J.A. The control of expression of an integrated Rous sarcoma provirus in rat cell: Studies on the role of $5^{\prime}$ genomic duplications reveal unexpected patterns of gene transcription and its regulation. I. Virol. 57, 907-916 (1986).

Liburdy, R.P., Tenforde, T.S., Magin, R.L. Magnetic fieldinduced drug permeability in liposome vesicles. Radiation Research 108, 102-111 (1986).

Lyman, I.T. Complication probability as assessed from dose-volume histograms. Radiation Res. 104 (Suppl. 8). 513-\$19 (1985).

Lyman, I.T., Kanstein, L.L., Yeater, F., Fabrikant, I.I., Frankel, K.A. A helium-ion beam for stereotactic radiosurgery of central nervous system disorders. Medical Phys. 13, 695-699 (1986).

Madhani, H.D., Leadon, S.A., Smith, C.A., Hanawalt, P.C. Alpha DNA in African Greek monkey cells is organized into extremely long tandem arrays. 1. Biol. Chem. 261, 3214-2318 (1986).
Martis, M.J., Schwarz, R.I. A simple fractionation of egg yolk yields a protein component that stimulates cell proliferation and differentiation in primary avian tendon cells. In Vitro Cellular and Developmental Biol.

Mathis, C.A., Lagunas-Solar, M.C., S.zzent III, T., Yano, Y., Vuletich, A., Harris, L.J. A ${ }^{122} \times \mathrm{ee}^{-122}$ I generator for remote radio-iodinations. Appl. Radiat. Isot. (Int. ). Radiat. Appl. Instrum. Part A) 37, 258-260 (1986).

Mathis, C.A., Shulgin, A.T., Sargent III, Thornton. Synthesis of ${ }^{122} \mid$ - and ${ }^{225}$-labelled meta-dimethoxy-N,Ndimethyliodophenylisopropylamines. I. Labelled Compds. and Radiopharmaceuticals 23, 115-125 (1986).

Mathis, C.A., Shuigin, A.T., Yano, Y., Sargent ill, T. ${ }^{18} \mathrm{~F}$ labelled N,N-Dimethylamphetamine analogues for brain imaging studies. App!. Radiat. Isot. (Int.). Radiat. Appl. Instrum. Part A) 37, 865-872 (1986).

Mays, C.W., Lloyd, R.D., Jones, C.W., Bruenger, F.W., Taylor, G.N., Durbin, P.W., White, D., Raymond, K.N. Decorporation of $\mathrm{Pu}$ and $\mathrm{Am}$ from beagles with delayed daily injections of 3,4,3-LICAM(C) or ZnDTPA. Health Phys. 50, 530-534 (1986).

Mazoyer, B.M., Huesman, R.H., Budinger, T.F., Knittel, B.L. Dynamic PET dala analysis. J. Comp. Assisted Tomography 10, 645-653 (1986).

Mazzeo, R.S., Brooks, G.A., Schoeller, D.A., Budinger, T.F. Disposal of blood $\left(1-{ }^{13} \mathrm{C}\right)$ lactate in humans during rest and exercise. I. Appl. Physiol. 60, 232-241 (1986).

Meecham, W.J., Char, D.H., Chen, G.T.Y., Juster, R., Castro, I.R., Stone, R.D., Saunders, W.M. Correlation of visual field, treatment fields and dose in helium ion irradiation of uveal melanoma. Am l. Ophthalmol. $100,658-665$ (1985).

Mei, M.-T., Craise, L.M., Yang, T.C-H. Induction of proline prototrophs in $\mathrm{CHO}-\mathrm{K} 1$ cells by heavy ions. Interntl. J. Radiat. Biol. 50, 213-224, 1986.

Metivier, H., Masse, R., Durbin, P.W., Raymond, K.N. Promotion by tetrameric catechoylamide ligands and CaNa, - DTPA of the dissociation in vitro of the $\mathrm{Pu}$ transferrin complex formed after intravenous injection of Pu-Tri-N-butylphosphate. Health Phys. 49, 1302-1305 (1985).

Mickols, W., Katz, I., Maestre, M.F., Tinoco, Ir., I., Bustamante, C. Imaging differential polarization microscope with electronic readout. Rev. Scient. Instr. 56, 2228-2236 (1985). 
Miller, M.E., Chandra, M., Garcia, I.F. Clinical applications of measurements of serum immunoreactive levels of erythropoietin. Ann. N.Y. Acad. Sci. 375-381 (1985).

Millward, G.R., Thomas, I.M., Glaeser, R.M. Probing the structure of zeolites by fourier transform electron microscopy: Zeolite-L as a test case. I. Chem. Soc. Chem. Commun., 962-964 (1985).

Moerlein, S.M. Regiospecific incorporation of no-carrieradded radiobromine and radioiodine into aromatic rings via halogenodegermylation. I. Chem. Soc., Perkin Trans. 1, 1687-1692 (1985).

Moerlein, S.M., Coenen, H.H. Regiospecific no-carrieradded radiobromination and radioiodination of aryltrimethyl group IVb organometallics. J. Chem. Soc,, Perkin Trans. 1, 1941-1947 (1985).

Moerlein, S.M., Laufer, P., Stoecklin, G. Design, synthesis, and evaluation of radiobrominated neuroleptics for in vivo mapping of cerebra! dopaminergic receptor areas and non-invasive pharmacokinetic studies. Acta Pharm. Suecica, Suppl. 1, 481-483 (1985).

Moerlein, S.M., Laufer, P., Stoecklin, G. Synthesis of nocarrier-added radiobrominated $\mathrm{N}$-alkylated analogues of spiperone. 1. Lab. Comp. Radiopharm. 22, 1007-1022 (1985).

Moerlein, S.M., Laufer, P., Stoecklin, G. Effect of lipophilicity on the in vivo localization of radiolabelled spiperone analogues. Int. J. Nucl. Med. Biol. 12, 353-356 (1986).

Moerlein, S.M., Stoecklin, G. Synthesis of high specific activity ${ }^{75} \mathrm{BrO}$ and ${ }^{77} \mathrm{Br}$-bromperidol and tissue distribution studies in the rat. J. Med. Chem. 28, 1319-1324 (1985).

Moerlein, S.M., Stoecklin, G., Wienhard, K., Pawllk, G., Heiss, W.D. Receptor-specific positron emission tomography radiopharmaceuticals: ${ }^{75} \mathrm{Br}$-labeled butyrophenone neuroleptics. Trans. Amer. Nucl. Soc., 50, 34-36 (1985).

Mortimer, R.K., Johnston, J.R. Genealogy of principal strains of the yeast genetic stock center. Genetics $118,35-43,1986$.

Mowbray, S., Foster, D.L., Jap, B.K., Koshland, D.E. Proteolytic fragments identified with functional domains of the aspartate receptor. I. Biol. Chem. 260. $11711-11718$ (1985).

Musliner, T.A., Giotas, C., Krauss, R.M. Presence of multiple subpopulations of lipoproteins of inlermediate density in plasma from normal and hyperlipidemic subjects. Arteriosclerosis 6, 79-87 (1986).
Phillips, C.L., Mickols, W.E., Maestre, M.F., Tinoco, I. Ir. Circular differential scattering and circular differential absorption of DNA-protein condensates and of dyes bound to DNA-protein condensates. 1. Biochemistry 25, 7803-7811 (1986).

Preston, B.D., Singer, B., Loeb, L.A. Site-specific mutagenesis by polymerase-catalyzed incorporation of alkyl-deoxyribonucleoside triphosphate (dNTP) adducts in $\times 174$ DNA. Proc. Nat"i. Acad. Sci. (USA) 83, 8501-8505 (1986).

Raveche, E., Santoto, T., Brecher, G., Tijo, I. Role of T cells in sex differences in syngeneic transfers. Exp. Hemat. 13, 975-980 (1985).

Reichl, D., Forte, T.M., Hing, I.-L., Rudra, D.N., Pflug, I. Human interstitial fluid lipoproteins: particle size, cholesterol, and apolipoprotein distributions, and electron microscopic structure. I. Lipid Res. 26, 1399-1411 (1985).

Rosen, M.A., Jones R.M., Yano, Y., Budinger, T.F. "Ccholine: synthesis, purification and brain uptake inhibition by 2 dimethylaminoethanol. J. Nucl. Med. 26 , 1424-1428 (1985).

Saunders, W., Castro, I.R., Chen, G.T.Y., Collier, I.M., Zink, S.R., Pitluck, S., Phillips, T.L., Char, D., Gutin, P.H., Gauger, G.E., Tobias, C.A., and Alpen, E.L. Helium-ion radiation therapy at the Lawrence Berkeley Laboratory: Recent results of a Northern California Oncology Group clinical trial. Radiat. Res. 104 (Suppl. 108), S227-\$234 (1985).

Saunders, W.M., Castro, J.R., Chen, G.T.Y., Gutin, P.H., Collier, J.M., Zink, S.R., Phillips, T.L., Gauger, G.E. Early results of ion beam radiation therapy for sacral chordoma: A Northern California Oncology Group study, J. Neurosurg, 64, 243-247 (1986).

Schaefer, E.I., Gregg, R.E., Chiselli, G., Forte, T.M., Ordovas, I.M., Zech, L.A., Brewer, Ir., H.B. Familial apolipoprotein E deficiency. J. Clin. Invest. 78, 1206-1219 (1986).

Schimmerling, W., Rapkin, M., Wong, M., Howard, I. The propagation of relativistic heavy lons in multielement beam lines. Med. Phys. 13, 217-228 (1986).

Schooley, J.C. Neuraminidase increases DNA synthesis of spleen cells induced by native and asialylated erythropoietin. Exper. Hematol. 13, 994-998 (1985).

Shahrokh, Z. and Nichols, A.V. Factors influencing interaction of human plasma low-density lipoproteins with discoidal complexes of apolipoprotein $A-I$ and phosphatidylcholine. Biochim. Biophys. Acta 878, 152-158 (1986). 
Singer, B. $\mathrm{O}^{4}$-Methyldeoxythymidine replacing deoxythymidine in poly(d(A-T) renders the polymer resistant to the $3^{\prime}-5^{\prime}$ exonuclease activity of the Klenow and $T_{4}$ DNA polymerases. Nucleaic Acids Res. 14. 6735-6743 (1986).

Singer, B. O-alkyl pyrimidines in mutagenesis and carcinogenesis: Occurrence and significance. Cancer Res. $46,4879-4885$ (1986).

Singer, B. In vivo formation and persistence of modified nucleosides resulting from alkylating agents. Environmental Health Perspectives 62, 41-48 (1985).

Singer, B., Chavez, F., Spengler, S.J. $\mathrm{O}^{4}$-methyl-, $\mathrm{O}^{4}-$ ethyl- and $\mathrm{O}^{4}$-isopropylthymidine $5^{\prime}$-triphosphates as analogues of thymidine $5^{\prime}$-triphosphate; Kinetics of incorporation by Escherichia coli DNA polymerase I. Biochemistry 25, 1201-1205 (1986).

Singer, B., Spengler, S.J., Fraenkel-Conrat, H., Kusmierek, J.T. $\mathrm{O}^{4}$-methyl, ethyl or isopropy! substitutents on thymidine in poly(dA-DT) all lead to transitions upon replication. Proc. Nat'l. Acad. Sci. (USA) 83, 28-32 (1986).

Sosinsky, G., Schekman, R., Glaeser, R.M. Morphological observations on the formation and stability of the crystalline arrays in the plasma membrane of Saccharomyces cerevisiae. 1. Ultrastructure and Molec. Structure Research 94, 37-51 (1986).

Stampfer, M.R. Isolation and growth of human mammary epithelial cells. J. Tissue Cult. Methods 9, 107-116 (1985).

Steely, H.T., Gray, D.M., Lang, D., Maestre, M.F. Circular dichroism of double-stranded RNA in the presence of salt and ethanol. Biopolymers 25, 91-117 (1986).

Stoecklin, G., Moerlein, S.M., Kloster, G., Voges, R., Schuer, F.J., Wienhard, K., Pawlik, G., Feinendegen, L.E., Heiss, W.D. Noninvasive measurement of regional cerebral pharmacokinetics of some centrally active agents with PET. Nuklearmedizin, Suppl. 17, 75-82 (1986).

Tenforde, T.S. Thermoregulation in rodents exposed to high-intensity stationary magnetic fields. Bioelectromagnetics $7(3), 341-346$ (1986).

Thrift, R.N., Forte, T.M., Cahoon, B.E., Shore, V.G. Characterization of lipoproteins produced by the human liver cell line. Hep G2, under defined conditions. I. Lipid Research 27, 236-250 (1986).

Tobias, C.A. The repair-mistepair model in radiobiology: Comparison to other models. Radiat. Research 104 (Suppl. 8). 577-595 (1985).
Tobias, C.A. Summary of the Symposium. Radiation Research 104 (Suppl. 8), S321-331 (1985).

Valk, P.E., Hale, J.D., Crooks, L.E., Kaufman, L., Higgins, C.B. Magnetic resonance imaging of aortoiliac atherosclerosis with 3-D vessel reconstruction. I. Comput. Assis' Tomogr. 10, 439-444 (1986).

Valk, P.E., Hale, J.D., Crooks, L.E., Kaufman, L., Roos, M.S. Magnetic resonance imaging of blood flow: correlation of phase shift and signal intensity with image appearances. Amer. J. Roentgenol. 146, 931-939 (1986).

Wardrop, K.J., Kramer, I.W., Abdowitz, J.L., Clemons, G.K.R., Adamson, J.W. Quantitative studies of erythropoiesis in the clinically normal phlebotomized, and feline leukemia virus-infected cat. Am. J. Vet. Res. 57, 2274-2277 (1986).

Widness, I.A., Teramo, K.A., Clemons, G.K., Cavalieri, R.L., Garcia, I.F., Susa, J.B., Schwartz, R. Temporal response of immunoreactive erythropoietin to acute hypoxemia in fetal sheep. Pediatric Res. 20, 15-19 (1986).

Widness, J.A., Teramo, K.A., Clemons, G.K., Coustan, D.R., Cavalieri, R.L., Oh, W., Welch, G.P., Schwartz, R. Correlation of the interpretation of fetal heart rate records with cord plasma erythropoietin levels. Brit. J. Obst. Gyn. 92, 326-332 (1985).

Williams, P.T., Krauss, R.M., Wood, P.D., Lindgren, F.T., Giotas, C., Vranizan, K.M. Lipoprotein subfractions of runners and sedentary men. Metabolism 35, 45-53 (1986).

Williams, R.E., Spengler, S.J. Fibers of RecA protein and complexes of RecA protein and single stranded $\phi \times 174$ DNA as visualized by negative-stain electron microscopy. I. Mol. Biol. 187, 109-118 (1986).

Wright, H.A., Magee, J.L., Hamm, R.N., Chatterjee, A., Turner, I.E., Klots, C.E. Calculations of physical and chemical reactions produced in irradiated water containing DNA. Rad. Prot. Dosimetry 13, 508 (1985).

Wulf, H., Kraft-Weyrather, W., Miltenburger, H.G., Blakely, E.A., Tobias, C.A., Kraft, G. Heavy-ion effects on mammalian cells: Inactivation measurements with different cell lines. Radiat. Res. 104 (Suppl. 8), S122-S134 (1985).

Yang, T.C., Craise, L.M., Mei, M., Tobias, C.A. Neoplastic cell transformation by heavy charged particles. Radiation Res. 104 (Suppl. 8), S177-S187 (1985). 


\section{CONTRIBUTIONS TO BOOKS AND PROCEEDINGS}

Bartsch, H., Singer, B. Meeting Report: International Meeting on the Role of Cyclic Nucleic Adducts in Carcinogenesis and Mutagenesis. Cancer Research 45, 5205-5209 (1985).

Bastacky, J. High-voltage electron microscopy of unsectioned human lung alveolar walls. Pages 224-225 in Proceedings of the 44th Annual Meeting of the Electron Microscopy Society of America, G.W. Bailey, San Francisco Press, Inc., 1986.

Bastacky, J., Hayes, T.L. Scanning electron microscope laboratory safety. Pages 1-9 in Electron Microscopy Safety Handbook, V.C. Barber and D.L. Clayton, Eds., San Francisco Press, Inc. (First Edition) (1985).

Bastacky, J., Hook, G.R., Finch, G.L., Hayes, T.L. Lowtemperature scanning electron microscopy (LTSEM) of frozen-hydrated lung. Pages 204-205 in Proceedings of the 44th Annual Meeting of the Electron Microscopy Society of America; G.W. Bailey, Ed., San Francisco Press, Inc. (1986).

Bernstein, L., Gold, L.S., Ames, B.N., Pike, M.C., Hoel, D.G. Letter to the Editor: Toxicity and Carcinogenic potency. Risk Analysis, 5(4), 163-264 (1985).

Bissell, M.I., Lee, E.Y-H, M., Hall, G. The role of matrix in regulating differentiation of endocrine sensitive cells. In Proceedings. The Second NIADDK Sympoisum on the Study of Benign Prostatic Hyperplasia, U.S. Government Printing Office (1986).

Brady, L.W., Shields, J.A., Augsberger, I.J., Day, J.L., Markoe, A.M., Castro, J.R., Suit, H.D. In Diagnostic Imaging in Ophthalmology. C.F. Gonzalez, M.H. Becker, J.C. Flanagan, Eds., Springer-Verlag, New York, (1986).

Broerse, I.J., Lyman, J.T., Zoetelief, J. Dosimetry of external beams of nuclear particles. Pages 229-290 in The Dosimetry of lonizing Radialion Volume I, K.R. Kase, B.E. Bjarngard, F.H. Altix, Eds., Academic Press, New York (1985).

Budinger, T.F. Health effects of in vivo nuclear magnetic resunance. IEEE Engineering in Med. and Piol. Maga. zine, 31-38. Sept. 1985.

Budinger, T.F. Potential hazards of NMR in vivo imaging and spectroscopy. In New Concepts in Cardiac Imaging. G. Pbhost and L. Sugg, Eds., Year Book Medical Publ. Inc., Chicago, IL (1985).

Cahoon, J.L., Huesman, R.H., Derenzo, S.E., Geyer, A.B., Uber, B.T., Turko, B.T., Budinger, T.F. The electronics for the Donner 600-crystal positron tomograph. IEEE Trans. Nucl. Sci. NN-33(1), 570-574 (1986).
Chatterjee, A. Interaction of ionizing radiation with matter. In A Textbook of Modern Radiation Chemistry, M.A.J. Rodgers, Ed., Verlag Chemie International (1986).

Chung, B.H., Segrest, I.P., Ray, M.J., Brunzell, J.D., Hokanson, J.E., Krauss, R.M., Beaudrie, K., Cone, I.T. Single vertical spin density gradient ultracentrifugation. Pages 181-209 in Methods in Enzymology: Plasma Lipoproteins, J.P. Segrest, J.J. Albers, Eds., Volume 128; Academic Press, New York (1986).

Clemons, G.K. Comparison of radioimmunoassay and bioassay of erythropoietin. In Humoral and Cellular Regulation of Erythropoiesis; E.D. Zanjani, M. Tavassoli, and J.L. Ascensao, Eds., Academic Press, New York (1986).

Curtis, M.P. Cornelius A. Tobias, Symposium Honorary Chairman. Radiation Research 104 (Suppl. 8), S1-52 (1985).

Derenzo, S.E. Mathematical removal of positron range blurring in high resolution tomography. Presented at the 1985 IEEE Nuclear Science Symposium (San Francisco, CA, October 21, 1985). IEEE Trans. Nucl. Sci. NS-33 (1), 565-569 (1986).

Derenzo, S.E., Budinger, T.F., Huesman, R.H. Detectors for high resolution dynamic PET. Pages 21-31 in The Metabolism of the Human Brain Studied with Positron Emission Tomography (presented at VII Nobel Conference, Stockholm, Sweden, 1984) T. Greitz, et al. Eds., Raven Press, New York (1985).

Downing, K.H. Effects of illumination spot size on highresolution image contrast for radiation-sensitive specimens. Pages 14-17 in Proceedings of the 44th Annual Meeting of the Electron Microscopy Society of America, G.W. Bailey Ed., San Francisco Press, Inc. (1986).

Ebbe, S., Phalen, E., Yee, T. Postirradiation thrombocytopoiesis: Suppression, recovery, compensatory states, and macromegakaryocytosis. Pages 71-89 in Megakaryocyle Development and Function, Alan R. Liss, Inc., New York (1986).

Esposito, M.S., Bjornstad, K., Holbrook, L.L., Maleas, D. REC genes governing mitotic recombination, chromosomal stability and sporulation: Cell type and life cycle stage-specific expression of rec mutants. Pages 103-109 in Mecharisms of Yeast Recombination, Banbury Center, Cold Spring Harbor Laboratory Press, Cold Spring Harbor, New York (1986).

Forte, T.M., Nordhausen, R.W. Electron microscopy of negatively stained lipoproteins. Pages $442-457$ in Methods in Enzymology: Plasma Lipoproteins, I.P. Segrest and I.J. Albers, Eds., Vol. 128 Academic Press, New York (1986). 
Friedland, R.P., Budinger, T.F., Jagust, W.J., Koss, E., Derenzo, S.E., Huesman, R.H., Yano, Y. Positron tomography and the differential diagnosis and pathophysiology of Alzheimer's disease. Pages 124-133 in Senile Dementia of the Alzheimer Type, J. Traber and W. Gipsen, Eds., Springer-Verlag Co., Heidelberg (1985).

Goodman, D.S., Dell, R.B., Palmer, R.H., Blum, C.B., Ramakrishnan, R., Nichols, A.V. Lack of relationship in humans of the parameters of body cholesterol metabolism with plasma levels of HDL cholesterol, of $\mathrm{HDL}$ subfractions, or of the major HDL apoproteins. In Atherosclerosis VII, N.H. Fidge, P.J. Nestel, Eds., Elsevier, Amsterdam (1986).

Guzik, T.G., Wefel, J.P., Crawford, H.J., Greiner, D.E., Lindstrom, P.J., Schimmerling, W., Symons, T.J.M. Implication of new measuremerits of ${ }^{16} \mathrm{O}+$ $P^{12,13} C_{,}{ }^{14,15} \mathrm{~N}$ for the abundances of $\mathrm{C}, \mathrm{N}$ isotopes at the cosmic ray source. Pages $80-83$ in Proceedings of the Nineteenth ICRC Conference, Washington, D.C., NASA, Volume 2 (1985).

Hooper, K., Gold, L.S. The exposure-potency index (EPI): Ranking the carcinogenic hazards of volatile industrial chemicals. Pages 1-11, in Cartcer Prevention: Stategies in the Workplace, C. Becker, Ed., Hemisphere Publ., Washington D.C. (1985).

Hooper, K., Gold, L.S. Ranking the carcinogenic hazards of occupational exposures: Exposure-potency index (EPI) values for nine volatile industrial chemicals. Pages 217-228 in Monitoring of occupational Genotoxicants: Proceedings of a Satellite Symposium to the Fourth International Conference on Environmental Mutagens, M. Sorsa and H.N. Norppa, Eds., Alan R. Liss, Inc., New York (1986).

Jap, B.K. 3-D Structure Analysis of PhoE PORIN. Pages 164-165 in Proceedings of the 44th Annual Meeting of the Electron Microscopy Society of Anterica; G.W. Bailey, Ed., San Francisco Press, Inc., 1986.

Kahlon, T.S., Glines, L.A., Lindgren, F.T. Analytic ultracentrifugation of plasmra lipoproteins. Pages 26-45 in Methods in Enzymology: Plasma Lipoproteins, J.P. Segrest and 1.). Albers, Eds., Vol. 129 Academic Press, New York (1986).

Krauss, R.M. Contraceptive steroid effects on serum lipoproteins and lipoprotein subclasses. Pages 321-338 in Contraceptive Steroids: Pharmacology and Safety, R. Blye, A. Gregoire, Eds., Plerium Press, New York (1986).
Krauss, R.M., Nichols, A.V. Metabolic interrelationships of HDL subclasses. Pages 17-27 in Advances in Experimental Medicine and Biology, Volume 201: Lipoprotein Deficiency Syndromes, A. Angel and I. Frohlich, Eds., Plenum Press, New York (1986).

Lawrence, J.H., Tobias, C.A., Linfoot, J.A., Castro, I.R., Saunders, W.M., Chen, G.T., Collier, J.M., Char, D., Gauger, G. Heavy particle irradiation of intracranial lesions. Pages 1113-1132, in Neurosurgery I., R,H. Wilkins, S.S. Rengachary, Eds., MCGraw-Hill, New York, Philadelphia (1985).

Leven, R.M., Koveleski, J., Nachmias, V.T. Megakaryocyte motility. Pages 293-300 in Megakaryocyte Development and Function, Alan R. Liss, Inc., New York (1986).

Liburdy, R.P., Tenforde, T.S. Membrane responses to magnetic and electromagnetic fields. Pages 44-53 in Biophysical Effects of Steady Magnetic Fields, G. Moret, J. Kiepenheurer, N. Buccaro, Eds., SpringerVerlag, Heidleberg, FRG (1986).

Lo, C.C., Fujita, T.Y., Geyer, A.B., Tenforde, T.S. A wide dynamic range portable $60-\mathrm{Hz}$ magnetic dosimeter with data acquisition capabilities. IEEE Trans. Nucl. Sci. 33, 643-646 (1986).

Magee, J.L., Chatterjee, A. The tract reactions of radiation chemistry. In Kinetics of Nonhornogeneous Processes, G.R. Freeman, Ed., John Wiley and Sons, Inc. (1986).

Mathis, C.A., Jones, R.M., Chasko, J.H. Overall radioHPLC design. Pages 125-148 (Chapter 6) in Analytical and Chromatographic Techniques in Radiopharmaceutical Chemistry, D.M. Wieland, M.K. Tobes, T.J Mangner, Eds., Springer-Veriag, New York (1986).

Nachmias, V.T., Leven, R.M. Effects of local anesthetics and $\mathrm{pH}$ change on plateiet. Pages 199-211 in The Cytoskeleton: A Target for Toxic Agents, J. Olmstead et al., Eds., Plenum Press, New York (1986).

Nichols, A.V., Franceschini, G., Sirtori, C.R., Gong, E.L. The Al-Milano HDL Particles. Pages 83-93 in Human Apolipoprotein Mutants (Proceedings of the NATO Advanced Research Workshop, Limone sul Garda Italy, 1985), C.R. Sirtori, Ed., Plenum Publ. Corp., New York (1986).

Nichols, A.V., Krauss, R.M., Musliner, T.A. Nondenaturing polyacrylamide gradient gel electrophoresis. Pages 417-431 in Methods in Enzymology: Plasma Lipoproteins, J.P. Segrest and J.J. Albers, Eds., Vol. 128; Academic Press, New York (1986). 
Singer, B., Holbrook, S.R., Fraenkel-Conrat, H., Kusmierek, I.T. Neutral reactions of haloaldehydes with polynucleotides: Mechanisms, Monomer and Polymer Products. In Role of Cyclic Nucleic Acid Adducts in Carcinogenesis, B. Singer and H. Bartsch, Eds., IARC Scientific Publications, Oxford University Press (1985).

Singer, B. Spengler, S.J. Replication and transcription of polynucleotides containing ethenocytosine, ethenoadenine and their hydrated intermediates. In Role of Cyclic Nucleic Acid Adducts in Carcinogenesis, B. Singer and $H$. Bartsch, Eds., IARC Scientific Publications, Oxford University Press (1985).

Stampfer, MR., Bartley, I.C. Long term growth of normal human mammary epithelial cells and establishment of partially transformed cell lines. Pages 51-56 in Growth and Differentiation of Cells in Defined Environments (Proceedings of International Symposium Sept. 2-6, 1984, Fukuoka, Japan). H. Murakami, I. Yamane, D.W. Barnes, J.P. Mather, I. Hayashi, G.H., Eds., Springer-Verlag, New York (1985).

Tenforde, T.S. Interaction of time-varying ELF magnetic fields with living matter. Pages 197-225 in Handbook of Biological Effects of Electromagnetic Fields, C. Polk and E. Postow, Eds., CRC Press, Boca Raton, FL (1986).

Tenforde, T.S. Magnetic field applications in modern technology and medicine. Pages 21-26 in Proceedings Symposium on Biological Effects of Static and Extremely-Low-Frequency Magnetic Fields (Neuherberg. West Germany-May 13-15, 1985) J. Bernhardt, Ed. (1986).

Tenforde, T.S. Assessment of biological effects associated with magnetic fields from a superconducting magnetic energy storage plant Final Report for Electric Power Research institute Project RP2572-5. Palo Alto, CA (1986).

Tenforde, T.S. Biological effects of extremely-lowfrequency magnetic fields. Pages $21-40$ in IEEE Special Publication Biological Effects of Power Frequency Electric and Magnefic Fields, IEEE Publ. No. 86TH0139-6-PWR, Piscataway, N) (1986).

Tenforde, T.S., Budinger, T.F. Biological effects and physical safety aspects of NMR imaging and in vivo spectroscopy. Pages 493-548 in NMR in Medicine: Instrumentation and Clinical Applications, S.R. Thomas, R.L. Dixon, Eds., Amer. Assoc. of Physicists in Medicine, NY (1986).

Tenforde, T.S., Liburdy, R.P. Membrane responses to static magnetic fields. Pages $1381-1384$ in Proceedings of the Eighth Annual Conference of the IEEE Engineering in Medicine and Biology Society, Volume 3. G.V. K'ndrashe and C.J. Robinson, Eds., IEEE Cata$\log$ No. 86CH2368-9, Piscataway, NI (1986).
White, M.R., Hertz-Picciotto,l. Human health: Analysis of climate related to health. In Characterization of Information Requirements for Studies of $\mathrm{CO}_{2}$ Effects: Water Resources, Agriculture, Fisheries, Forests and Human Health, M.R. White, Ed. U.S. Dept. of Energy, DOE/ER-0236 (1986)

\section{LBL REPORTS ISSUED}

Baker, J.R., Budinger, T.F. Advanced model for medical imaging. LBL-22056, August 1986, 17 p.

Budinger, T.F. Safety of NMR in vivo imaging and spectroscopy. LBL-22052, July 1986, $12 \mathrm{p}$.

Budinger, T.F. Single-photon emission computer tomog. raphy: physics potentials, and limitations. LBL-22095, lune 1986, $59 \mathrm{p}$.

Cahoon, J.L., Huesman, R.H., Derenzo, S.E., Geyer, A.B., Uber, B.T., Turko, B.T., Budinger, T.F. The electronics for the Donner 600-crystal positron tomograph. (Presented at the 1985 Nuclear Science Symposium, San Francisco, CA, October 23-28, 1985). LBL-20681, October 1985, $5 \mathrm{p}$.

Castro, I.R. Heavy ion radiotherapy at Lawrence Berkeley Laboratory. LBL-21906, August 1986, 8 p.

Collier, M., Renner, T., Kessler, M., Pitluck, S., Henderson, $S$. Characterization of the new neon radiotherapy beams at the Lawrence Berkeley Laboratory. LBL. 22118, August 1986, 26 p.

Derenzo, S.E. Potential improvements in instrumentation for PET. LBL-20215, September 1904, $13 \mathrm{p}$.

Derenzo, S.E. Mathematical removal of positron range blurring in high resolution tomography. (Presented at the 1985 IEEE Nuclear Science Symposium, San Francisco, CA, October 10, 1985.) LBL-20678, October 1985, 5 p.

Derenzo, S.E., Budinger, T.F. Recent developments in positron emission tomography (PET) instrumentation. LBL-21556, April 1986, 24 p.

Durbin, P.W. Metabolic models for uranium. LBL-20422, October 1985, $57 \mathrm{p}$.

Ebbe, S., Phalen, E., Yee, T. Postirradiation thrombocytopoiesis: suppression, recovery, compensatory states, and macromegakaryocytosis. LBL-20529. November 1985, $19 \mathrm{p}$.

Masenieisu, A.C. An inverse problem for $"$ " vivo NMR spatial localization. LBL-20682 (Ph.D. thesis), November 1985, $122 \mathrm{p}$. 
Hlatky, L. A two-dimensional diffusion limited system for cell growth. LBL-20560 (Ph.D. thesis), November 1985, $73 \mathrm{p}$.

Klein, 5.B. SEN1 and $x$-ray microanalysis of cellular differentiation in sea urchin embryos: A frozen hydrated study. LBL-21128 (Ph.D. thesis), December 1985, 211 p.

Krauss, R.M. Lipid metabolism. LBL-21398, April 1986, 5 p.

Leven, R.M., Koveleski, J., Nachmias, V.T. Megakaryocyte motility. Presented at the International Conference on Megakaryocytes: Develepment and Function. Woods Hole, Maine, September 18-21, 1985. LBL-20517, November 1985, $8 \mathrm{p}$.
Lyman, J.T. Tolerance doses for treatment planning. LBL-22416, October 1985.

Strickland, R.M., Grosse, D.J., Stubin, A.I., Ostrander, G.K., Sibley, T.H. Potential effects of increased atmospheric $\mathrm{CO}_{2}$ on marine fisheries from the northeast pacific ocean. LBL-20556, December 1985, 26 p.

Tenforde, T.S., Liburdy, R.P. Membrane responses to static magnetic fields. LBL-21722, June 1986, 4 p.

White, M.R., Hertz-Picciotto, I., Johnston, M.E. Carbon dioxide increase and human health: Datà and research requirements for deiermining consequences. 


\section{APPENDIX C: Biology and Medicine Division Staff September 30, 1986}

The accomplishments of the Biology and Medicine Division are due in large measure to the capability and dedication of its staff. Listed below are those who have participated in the Division's programs during fiscal year 1986 as full- or part-tine employees, consultants, and participating guests. The guest staff includes visiting scientists, postdoctoral trainees, resident physicians, graduate and undergraduate students, and summer research participants.

\section{DIVISION HEAD}

Paul H. Silverman (Acting Head; effective 8/19/86)

Edward L. Alpen (Division Head until 8/18/86)

Thomas L. Hayes, Deputy (Until 8/18/86)

\section{DIVISION ADMINISTRATION STAFF}

Igor R. Blake

Mary P. Curtis

Janice C. DeMoor

Diane E. Duhnke

- Michael B. Fizer

Wendell Hom

Allan W. Long

Georgia A. Peterson

Louise Ray

Dolores Ruff

Robert W. Springsteen

- Baird Whaley

Mary L. Worth

\section{DIVISION SCIENTIFIC STAFF}
S. Javed Judith Agg
Lawrence zgerbeck
E. John Airisworth
Julius J. Almasi
- Bruce N. Ames
- Nelly Auersperg Melissa A. Austin
Mary H. Barcellos-tioff
John C. Bartley
John B. Bassel
S. Jacob Bastacky
- Alan J. Beartien
Eugene V. Benton
- Peter A. Berardo
Leslie Bernstein
Anthony M. Berson
Stephen E. Bicknese
Mina !. Bissell
Eleanor A. Blakely
William F. Blakely

- George Brecher Kathleen M. Brennan

- Michael F. Bruno

5t Thomas F. Budinger Vincent P. Carabilio

- Joseph R. Castro Aloke Chatterjee William T. Chu Gisela K. Clemons J. Michael Collier Priscilla K. Cooper Stanley B. Curtis Maya M. Das Stefhen E. Derenzo David S. Dolberg Kenneth $H$. Downing Patricia W. Durbin

- Shirley N. Ebbe Barry L. Engelstad David E. Erkenbrack Michael S. Esposito

- Jacob I. Fabrikant Wendy L. Fitch James Fontanesi Trudy M. Forte David L. Foster Heinz Fraenkel-Conrat Kenneth A. Frankel

- Michae 1 e!:-8

H. Robert Fr.uviand

Comelius T. Gaffey John C, Game

- Grant E. Gauger Peter S. Geissler Orsolya Genzel

- Robert M. Claeser Michael Goitein Lois S. Gold Joseph D. Goldsteir. Regine Goth-Goldstein

- Martin H. Graham Dale E. Greenwalt

- Michael P. Hagen

"Left Biology \& Medicine Division prior 'o September 30, 1986.

'Retired during Fiscal Year 1986.

tFaculty UC Berkeley

'Faculty UC San Francisco

"Faculty UC Davis 
- H. Glenn Hall Sheri D. Henderson Lynn R. Hlatky

- Eugene V. Holahan Libby L. Holbrook William R. Holley

- Gregory R. Hook

- Yoshio Hosobuchi Junko Hosoda Jerry Howard Anthony R. Howlett Mirko 1. Hrovat John P. Hiuberty Ronald H. Huesman

- William J. Jagust Bing K. Jap John R. Johnston Talwinder S. Kahlon Jonathan A. Kans Leon N. Kapp Parris M. Kidd

- Yohsukj Kinouchi

- Patrice A Koehl

- Gerhard Kraft

- Wilma Kraft-Weyrather

- Ronald M. Krauss

- Jaroslaw T. Kusmierek

Michael La Belle

* John H. Lawrence Steven A. Leadon Harold Lecar Roben M. Leven Larissa V. Levin Ming Li Roben P. Liburdy Tz-Hong Lin Frank T. Lindgren David E. Linstadt Francoise Livolant

- Helen F. Londe

- Susan T. Lovett Bemhard A. Ludewigt John T. Lyman

Marcos F. Maestre

- John L. Magee Andrew C. Magyarosy Neela B. Manley Vera Martin

- Hugo A. Massaldi Chester A. Mathis

- Bernard M. Mazoyer

- Elisabeth Mazoyer Mark R. Mc Call Joyce C. McCann

- Howard C. Mel Stephea M. Moerlein

- Robert K. Mortimer

- Richard A. Mushlin Thomas A. Musliner

- Yen H. Nguyen

- Alex V. Nichols

- William G. Owen

- John C. Owicki

- Chung S. Park Charles S. Parkins Gordon Parry Richard Payne Ashol Petrossian Pausla L. Peiti Mark H. Phillips

- Theudore L. Phillips John D. Port

Gian M. Ratto

- Gary V. Richieri Adrian Rodriguez Mark S. Roos Ruth J. Roots

Thomton W. Sargent, III David Schild Walter Schimmerling John C. Schooley Richard I. Schwarz I. Philip Seab

- Zahra Shahrokh Alexander T. Shuigin Bea A. Singer

- Jerome R. Singer Thomas H. Slone Sylvia I. Spengler Martha R. Stampfer Henry H. Stauffer Andrew W. Stoker Thomas Swain

Thomas S. Tenforde

- Comelius A. Tobias Sylvanus A. Tyler

Peter E. Valk Karen M. Vranizan

In Philip R. Weinstein John R. Wetterau

- Margaret R. White Paul T. Williams Kay H. Woodruff Mervyn Wong

Tracy C. Yang

Yukio Yano

Michael I. Yezzi

DIVISION SUPPORT STAFF

Linda D. Abe lames R. Abney Frederick E. Abrams Gerald L. Adamson Mari Aker Charles M. Allison 
Georganne M. Backman

Kari P. Baken

John R. Baker

Ashley I. Barboza

- Violet Barghe-Sharghi

Josephine L. Bärr

George M. Basile

Maren Bell

Sindy E. Berger

Kathleen A. Bjornstad

Patricia !. Blanche

Nicolas R, Bolo

- Yvonne C. Bopp

Mitch C. Brenner

- Robert S. Bridwell

S. Kay Bristol

Gerald L. Brooks

Mary Cabbage

Veronica G. Cabras

John L. Cahoon

Mardel M. Carnahan

Dorothy A. Carpenter

- Albert C. Casselhoff

- Lisa M. Caylor Rosemarie L. Celli Polly Y. Chang

Frank Chavez

Li-How Chen

Marcia Chen

- Melody L-Y Cheng

Amy Choi

- Paul A. Chous

Ling-Fong Chung

Margaret A. Chung

Gary M. Cole

Miguel M. Colina

C. Rebecca Contopoulou

Vincent E. Cook

Douglas Corley

Laurie M. Craise

Freddie L. Crenshaw, Ir.

Christopher Cullander

Betsey L. Cullen

Maria N. DaCosta

- Sahar M. Dajani

Kanu B. Dalal

- Minh N. Dang

Lori L. Debay

Randy ). De Guzman

- Gres T. De Lory

Darlene J. De Manincor

Uyen Nina Dinh

Niedra A. Dodson

- Annette C. Drew

- Bill M. Du Four

- Katherine A. Dukes

- Eva L. Edwards Susan L. Eisenberg

- Julie A. Ellison

- Nina Engineer

Dennis Fantin
Deborah A. Farson

- Sherry L. Fitzsimmons Myrtle L. Foster Marilyn A. Fowler Rochelle I. Frank Roscoe Frazier John Frey

+ Charlie M. Fuller

Lance A. Gee

Sherry L. Gee

- John C. Gilbert

Christine Giotas

Queen E. Gipson

Brian 1. Glassner

Laura A. Glines

Michael A. Glotzer

Tennessee W.-Y Gock

Kaarin Keer Goncz

Elaine L. Gong

Edwin H. Goodwin

Sara P. Goolsby

Kirsten K. Gow

- Roberl W. Gribben

Angela Habrek-Davidson

Helena B. Hansen

William A. Hare

Linda J. Harrison

- Andrew C. Hasenfeld

Carroll Hatie?

Virginia C. Havens

Lilian E. Hawkins

Linda G. Hayashi

- Donna K. Hendrix Mark D. Hertle

- Peter Hertz-Herskovits

- Sharon A. Hibdon

Christene S. Ho

Christine S. Hong

Laura J. Horn

Midori M. Hosobuchi

Mildred K. Hughes

Jacquelyn \}. Iler

- Daniel Young-Bin Im

Gayla P. Ivery

Nylan $M$. leung

- Jay A. Johnson Reese M. Jones Jay 5. loseph James $W$. Judnick

- David M. Kang Norbert V. Kang Peter M. Kang Aaron B. Kantor Kristina S. Kavanau

- Angela H. Kaya

- Leah A. Kenaga Marc L. Kessler Chris B. Kingsley

- Paul M. Kleinman Brian L. Knittel 
Seok-Hwan Kong

Vida W. Kong

- Renee l. Kopa

- Lori E. Kopp

Brian S. Korotzer

Kari L. Koszdin

- Susanne E. Kueh! Birgetta R. Kullgren Natalia Kusubov

- Clifford E. Lai Mark T, Lasartemay Ann Lee

- Charles Y. Lee Krung-Dall Lee Samuel Lee Gerri A. Levine Lynette L. Levy Richard P. Levy Lily J. Li Rebecca $\mathrm{H}$. Lichtenberg

- Vincent Ling

Eng $H$. Lo Leora Lommel Donna Louie

- Janice K. Louie janet S. Lowe

- Karen E. Lowe

Tommy J. McKey

- Velma 8. McNeal

Renae !. Magaw

Eva J. Mah

Lynn I. Mahlmann

Dimitrios T. Maleas

Sam I. Mansour

Marc S. Mendonca

Roger L. Miller

Barbara Modlinski

Herbert W. Moise, III

- David A. Morgenstern Alyssa M. Morimoto Lenny Moss

- Kimberly Mulcahy

Steve 5. Neben

- Frie Nemariame Rocky H. Nevin Robert W. Nordhausen

- Allen J. Norton

Virginia I. Obie Christene Y. Oh Mary Lou Olbrich Lucinda S. Olney Eutimio C. Oregel Joseph R. Orr

Annie C. Pang

- Kwan S. Park

- Shannon S. Parr Timothy E. Peterson Huar N. Phan

- Cynthia L. Phillips Patricia P. Powers-Risius
John C. Prioleau

lose A. Ramirez

Ross A. Ramos

Monica H. Reimers

David Reisman

Bryan T. Rogers

- Anne M. Ross

- David W. Rowen

- Lisa R. Sawrey-Kubicek Janis S. Scherer

- Siniion A. Schiogolev James B. Schmidt Annelie D. Schumarn lanet F. Selmek

- Ajit S. Shah

- Ellen S. Sher

Michael Sieweke Mohindar Singh Karen C. Sitney Karen Smith Margaret M. Snowden Marcia E. Somers

- Elmer M. Soriano Dorothy S. Sprague Karen A. Springsteen

- Lore S. Stein David P. Suchard Shirley C. Sutton

Joseph R. Taylor

- Richard Thrift

- Laura M. Tracewell

- JoAnn Trejo Sally P. Tubach Julia A. Twitchell

Vladimir Vacata Janice Valmassoi Dresden D. Van Dusen

Peter I. Walian Angela $Y$. Wang Karly S. Wang

- Marian M. Wertheimer Monroe Whitney Eric D. Wieder

- Evelyn F. Williams Elizabeth C. Williamson

- Jason Wong Sam T.S. Wong

- Patrick K. Yang Marilyn Yee Tamlyn K. Yee Andy S. Yu 
Paul H. Silverman, Chair john C. Bartley Eleanor A. Blakely Thomas F. Budinger Trudy M. Forte Aloke Chatteriee Robert K. Mortimer Thomas S. Tenforde Cornelius A. Tobias Igor R. Blake

\section{DIVISION STAFF COMMITTEE}

Tony W. Sargent, Chair

E. John Ainsworth Mina I. Bissell Aloke Chatterjee lohn T. Lyman

\section{EQUIPMENT COMMITTEE}

Cornelius T. Gaffey, Chair Paul H. Silverman John C. Bartley Shirley N. Ebbe Adrian Rodriguez Tony W. Sargent Frank T. Upham

- Allan W. Long

\section{SPACE COMMITTEE}

Thomas S. Tenforde, Chair Gisela K. Clemons Michael S. Esposito Tracy C. Yang Yukio Yano

- Allan W. Long

\section{SALARY COMMITTEE}

Paul H. Silverman, Chair John C. Bartley Thomas F. Budinger Trudy Forte Tom S. Teniorde Cornelius A. Tobias

- Igor R. Blake

\section{HUMAN USE COMMITTEE}

Henry $H$. Stauffer, Chair Thomas F. Budinger Shirley N. Ebbe Kathleen E. Handron Walter Schimmerling + Janice C. DeMoor lacob I. Fabrikant, Chair

Edward L. Alpen

Aloke Chatteriee

Henry H. Stauffer

Paola 5. Timiras

Joseph D. Goldstein (Consultant)

* Janice C. DeMoor

ANIMAL WELFARE AND RESEARCH COMMITTEE

John C. Bartley, Chair

Hermann Bonasch

Kathleen Brennan

Gisela K. Clemons

lanice C. DeMoor

Steve F. Sapontzis

Kent A. Zimmerman

- Robert W. Springsteen

\section{DONNER LIBRARY COMMITTEE}

Ronald M. Krauss, Chair

Alan J. Bearden

Edward L. Bennett

Kathleen M. Brennan

Thomas F. Budinger

1. Michael Collier

Stanley B. Curtis

Junko Hosoda

Frank T. Lindgren

- Dorothy F. Denney

- Carol Backhus

\section{DONNER SEMINAR COMMITTEE}

Bing Jap. Chair

Robert K. Mortimer

Ruth J. Roots

\section{SAFETY COMMITTEE}

John T. Lyman, Chair lacob Bastacky

Eleanor A. Blakely

Kathleen M. Brennan

Aloke Chatterjee

Gisela K. Clemons

Michael S. Esposito

Deborah A. Farson

Regine Goth-Goldstein

Herbert W. Moise

Robert W. Nordhausen

Patricia Powers-Risius

Mark S. Roos

Robert W. Springsteen

Yukio Yano

- Allan W. Long

- Ex officio, nonvoting member

** Executive Officer 